\title{
Dielectric Properties of Low-Level Liquid Waste
}

\author{
Final Report \\ November 1997
}

\author{
By: \\ M. A. Ebadian \\ L. E. Lagos
}

Work Performed Under Contract No.: DE-FG21-95EW55094

For

U.S. Department of Energy

Office of Fossil Energy

Federal Energy Technology Center

P.O. Box 880

Morgantown, West Virginia 26507-0880

\author{
By \\ Florida International University \\ Hemispheric Center for Environmental Technology (HCET) \\ Center for Engineering \& Applied Sciences \\ 10555 West Flagler Street \\ EAS-2100 \\ Miami, Florida 33174
}




\section{Disclaimer}

This report was prepared as an account of work sponsored by an agency of the United States Government. Neither the United States Government nor any agency thereof, nor any of their employees, makes any warranty, express or implied, or assumes any legal liability or responsibility for the accuracy, completeness, or usefulness of any information, apparatus, product, or process disclosed, or represents that its use would not infringe privately owed rights. Reference herein to any specific commercial product, process, or service by trade name, trademark, manufacturer, or otherwise does not necessarily constitute or imply its endorsement, recommendation, or favoring by the United States Government or any agency thereof. The views and opinions of authors expressed herein do not necessarily state or reflect those of the United States Government or any agency thereof. 


\section{DISCLAIMER}

Portions of this document may be illegible in electronic image products. Images are produced from the best available original document. 
LIST OF FIGURES .. $\mathrm{v}$

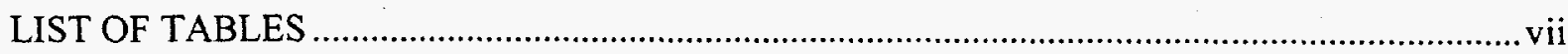

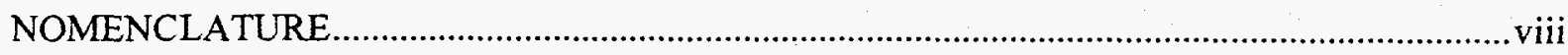

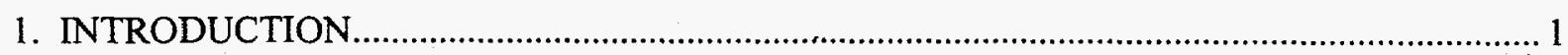

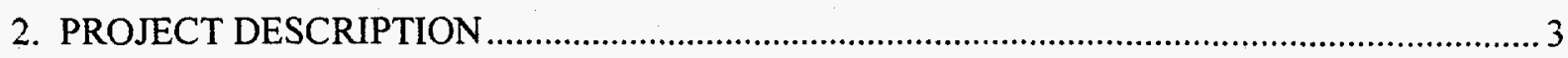

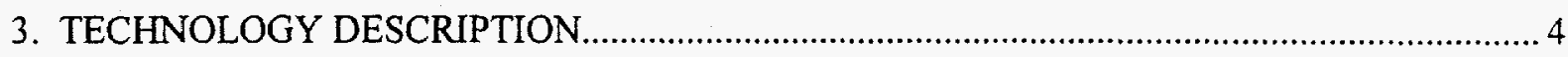

3.1 CHARACTERISTICS OF LOW-LEVEL LIQUID WASTE SIMULANTS

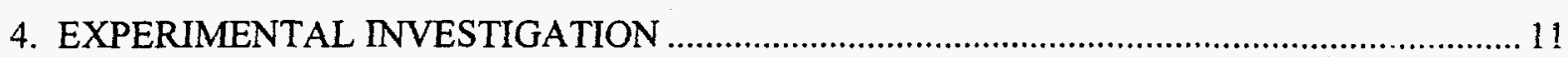

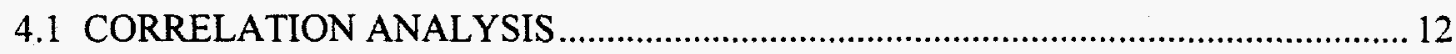

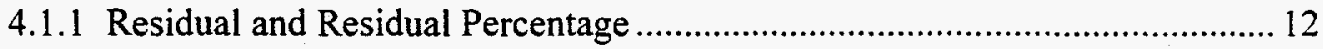

4.1.2 Coefficient of Determination .................................................................. 12

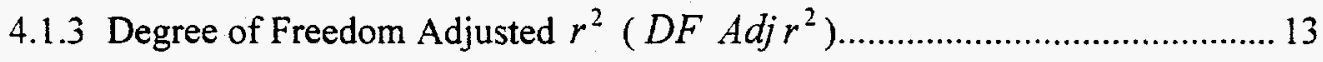

4.1.4 Fit Standard Error ( Root MSE ) ............................................................... 13

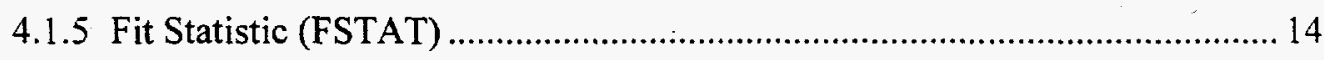

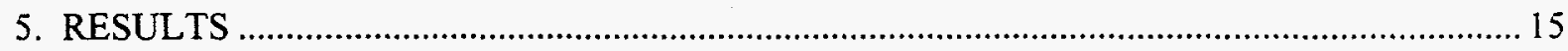

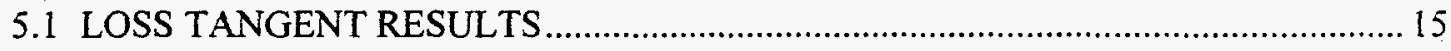

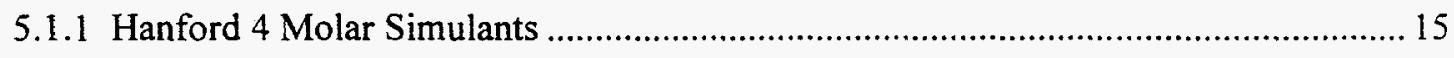

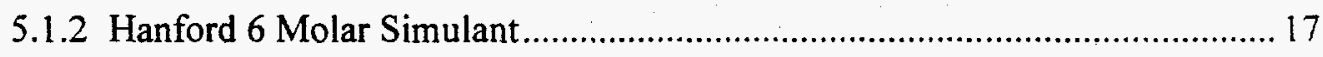

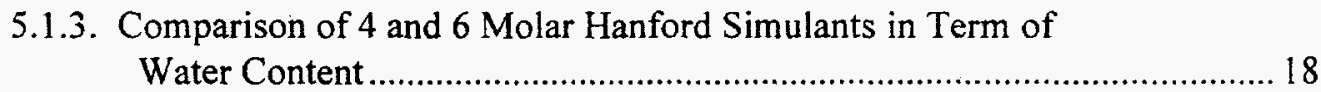

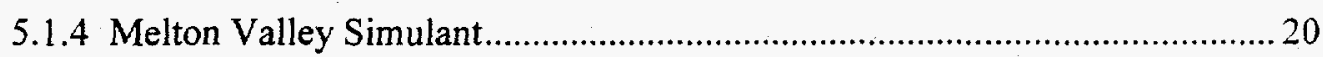

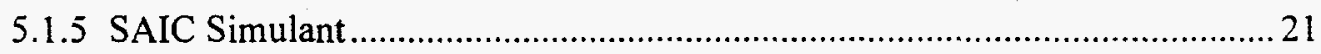

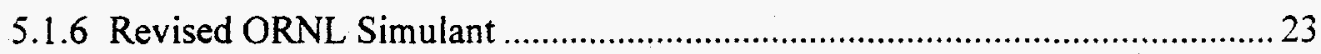

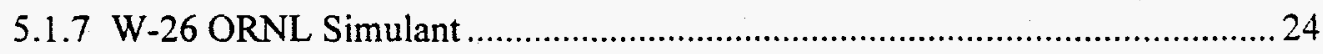

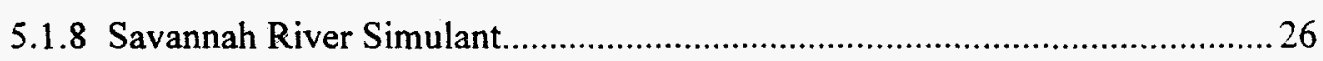

5.2 ANALYSIS OF THE CORRELATED RESULTS FOR THE LOSS

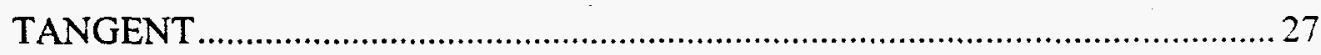

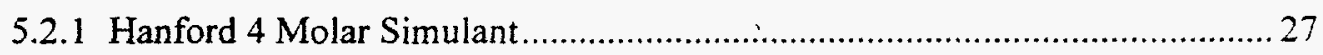

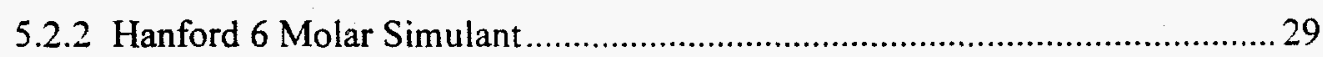

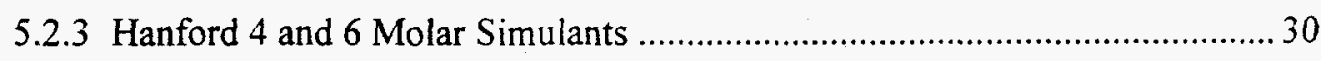

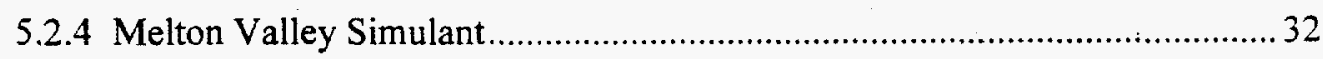

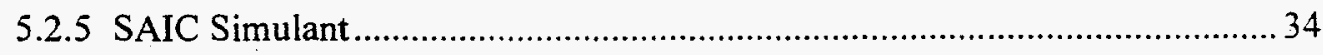

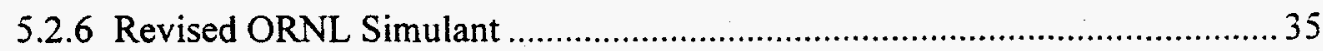

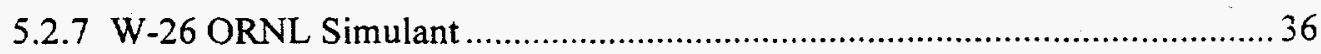

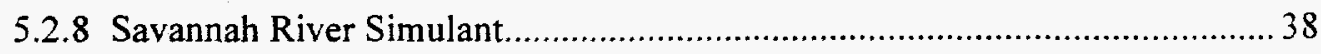




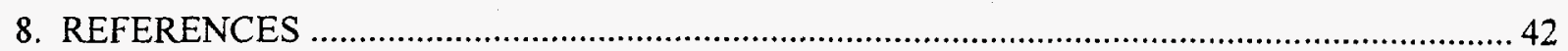
APPENDIX
A. Dielectric Constant and Dielectric Loss versus Frequency Graphs
B. Tabulated Results for Loss Tangent Dielectric Constant and Dielectric Loss
C. Correlation Graphs for the Dielectric Constant and Dielectric Loss 


\section{LIST OF FIGURES}

Figure 1. HCET investigator performing dielectric measurements.....................................

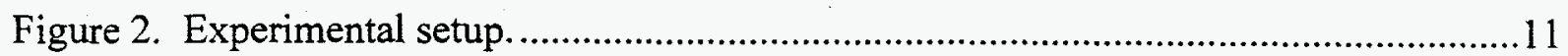

Figure 3. Loss tangent versus frequency for the Hanford 4 Molar simulant. Full scale.

Figure 4. Loss tangent versus frequency for the Hanford 4 Molar simulant. Scaled down.

Figure 5. Loss tangent versus frequency for the Hanford 6 Molar simulant. Full scale.

Figure 6. Loss tangent versus frequency for the Hanford 6 Molar simulant. Scaled down.

Figure 7. Loss tangent versus frequency for the Hanford 4 and 6

Molar simulants at $20^{\circ} \mathrm{C}$.

Figure 8. Loss tangent versus frequency for the Hanford 4 and 6

Molar simulants at $100^{\circ} \mathrm{C}$

Figure 9. Loss tangent versus frequency for the Melton Valley

simulant. Full Scale.

Figure 10. Loss tangent versus frequency for the Melton Valley simulant. Scaled down.

Figure 11. Loss tangent versus frequency for the SAIC simulant. Full scale.

Figure 12. Loss tangent versus frequency for the SAIC simulant. Scaled down.

Figure 13. Loss tangent versus frequency for the Revised ORNL

simulant. Full scale.

Figure 14. Loss tangent versus frequency for the Revised ORNL

simulant. Scaled down.

Figure 15. Loss tangent versus frequency for the W-26 ORNL

simulant. Full scale.

Figure 16. Loss tangent versus frequency for the W-26 ORNL simulant. Scaled down.

Figure 17. Loss tangent versus frequency for the Savannah River simulant. Full scale.

Figure 18. Loss tangent versus frequency for the Savannah River simulant. Scaled down. 
Figure 19. Correlation data for the loss tangent. Simulant: .Hanford 4 Molar. 28

Figure 20. Residual percentage (\%) for loss tangent Hanford 4 Molar simulant. ................ 28

Figure 21. Correlation data for the loss tangent. Simulant: Hanford 6 Molar. .................... 29

Figure 22. Residual percentage (\%) for the loss tangent. Simulant: Hanford 6 Molar........ 30

Figure 23. Correlation data for the Hanford 4 and Hanford 6 Molar simulants.................. 31

Figure 24. Residual percentage (\%) for the Hanford 4 and Hanford 6 simulants................. 31

Figure 25. Correlation data for the Melton Valley simulant............................................... 32

Figure 26. Residual percentage (\%'s) for the Melton Valley simulant. ................................ 33

Figure 27. Correlation data for the SAIC simulant................................................................ 34

Figure 28. Residual percentage (\%) for SAIC simulant. .................................................... 35

Figure 29. Correlation data for the Revised ORNL simulant. ............................................. 35

Figure 30. The residual percentages (\%'s) for the Revised ORNL simulant........................ 36

Figure 31. Correlation data for the W-26 ORNL simulant................................................... 37

Figure 32. Residual percentage (\%s) for the W-26 ORNL simulant.................................. 37

Figure 33. Correlation data for the Savannah River simulant............................................... 38

Figure 34. Residual percentage (\%s) for the Savannah River simulant................................. 39 


\section{LIST OF TABLES}

Table 1. Hanford 4 Molar ${ }^{*}$ Simulant............................................................................6

Table 2. Hanford 6 Molar* Simulant...........................................................................

Table 3. Melton Valley Simulant..............................................................................

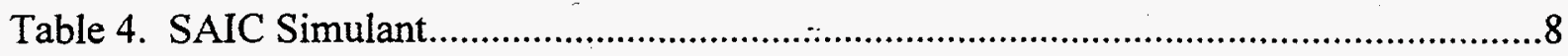

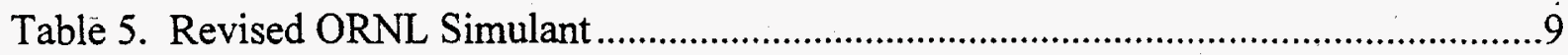

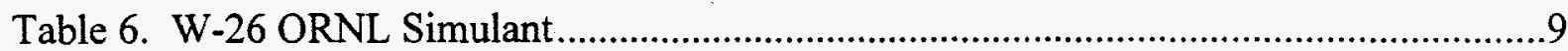

Table 7. Savannah River Simulant (Batch \#95QAB400) .............................................. 10

Table 8. Correlation Equations for the Loss Tangent .....................................................40 
E electric field vector [v], eq. (1)

$\mathrm{H}$ magnetic field vector [A $\left.\mathrm{m}^{-1}\right]$, eq. (2)

$Z \quad$ residual real data value

$\hat{Z} \quad$ estimated value of residual

$\bar{Z} \quad$ mean of the experimental data

$r \quad$ coefficient of determination

W weight

\section{Greek Symbols}

$\beta \quad$ microwave frequency

$\Delta \quad$ dimensionless node interval

$\varepsilon \quad$ complex dielectric permittivity, $\varepsilon^{\prime}-\mathrm{j} \varepsilon^{\prime \prime}\left[\mathrm{Fm}^{-1}\right]$

$\varepsilon^{\prime} \quad$ real part of the complex dielectric permittivity, or dielectric constant $\left[\mathrm{Fm}^{-1}\right]$

$\varepsilon^{\prime \prime} \quad$ imaginary part of the complex dielectric permittivity on dielectric loss $\left[\mathrm{Fm}^{-1}\right]$

$\mu \quad$ magnetic permeability [Henries $\mathrm{m}^{-1}$ ] on viscosity [NS $\mathrm{m}^{-2}$ ]

$\mu^{\prime} \quad$ real part of magnetic permeability [Henries $\mathrm{m}^{-1}$ ]

$\mu^{\prime \prime} \quad$ imaginary part of magnetic permeability [Henries $\mathrm{m}^{-1}$ ]

$\sigma \quad$ electric conductivity $\left[\mathrm{ohm}^{-1}\right]$

$\omega \quad$ microwave single frequency $2 \pi f\left[\mathrm{rad} \mathrm{s}^{-1}\right]$ 


\section{INTRODUCTION}

The purpose of this study was to develop a data collection containing values for the dielectric properties of various low-level liquid waste (LLLW) simulants measured as a function of frequency, temperature, and composition. The investigation was motivated by current interest in the use of microwave processing for the treatment of radioactive waste. A large volume of transuranic liquid and sludge produced by the U.S. Department of Defense (DOD) during the production of nuclear fuel bars is stored at several U.S. Department of Energy (DOE) sites around the United States. Waste storage and disposal space is scarce, expensive, and must be minimized. Thus, several DOE sites are pursuing the use of microwave heating as a means of achieving volume reduction and solidification of low-level liquid wastes.

It is important to know which microwave frequencies should be employed to achieve the most efficient processing at a range of different temperatures. The dielectric properties of the LLLW simulants can be utilized to determine the optimum frequencies for use with a particular LLLW or with other LLLWs of similar composition. Furthermore, nonlinear thermal processes, such as thermal runaway, which occur in the material being treated cannot be modeled without a knowledge of the temperature dependence of the dielectric properties. Often, this data does not exist; however, when it does, only very limited data near room temperature are available. The data collection generated in this study can be used to predict the behavior of a variety of microwave thermal treatment technologies, which have the potential of substantially reducing the volume of the LLLWs that are currently stored at many DOE sites. This information should help the users of the microwave reduction and solidification technology to optimize microwave processes used in the treatment of LLLW.

The microwave reduction and solidification technology has clear advantages over other methods of reducing LLLWs. These include the incineration of combustibles, the evaporation of combustibles, the evaporation of liquids, and the compaction of noncombustibles. The handling of radioactive liquid waste is generally carried out within closed systems consisting of highly corrosion-resistant, welded, leak-tight pipes, tanks, and other apparatus. High power microwave processing is a promising technology for reducing risks to the environment and human health, thereby supporting the DOE's decontamination and decommissioning (D\&D) objectives.

The microwave system is designed to heat the waste in its final disposal container, thus eliminating the need to transport high pressure materials after melting by conventional methods. The microwave energy heats the liquid waste directly because as the microwave field interacts with the material, energy is transferred from the field to the molecular bonds of the material, causing these bonds to vibrate. The energy is then dissipated as heat and transported within the material. Microwave heating requires no local heating elements; the radioactive material becomes its own heating element. Furthermore, moving parts required in other methods are unnecessary.

After microwave processing, the resultant waste form is a brick-like ceramic, a stone-like monolith, or an amorphous glass form. Compared to the original wet sludge slurry, the 
refractory, high-density waste forms render a volume reduction of a factor of 5 to 10 . Moreover, the processed wastes show an enhanced leach resistance for regulated metals. Therefore, compared to the conventional thermal treatment technologies, microwave waste processing offers numerous advantages, including the production of a high-density, leachresistant, robust waste form; volume and toxicity reduction; favorable economics; incontainer treatment; positive public acceptance; isolated equipment; and instantaneous energy control. 


\section{PROJECT DESCRIPTION}

The product of this study is a collection of data for the dielectric properties of several LLLW simulants. More specifically, we have focused on the dielectric permittivity of these simulants and used the measurements to generate values for a very useful quantity, the loss tangent. Correlations of the collected data were also performed in order to develop prediction formulas for the dielectric properties of the tested surrogates.

When Maxwell's equations are introduced to determine electric and magnetic fields, the dielectric properties of materials $(\varepsilon, \mu, \sigma)$ are defined. The term $\varepsilon$ represents complex dielectric permittivity, $\mu$ denotes complex magnetic permeability, and $\sigma$ represents electric conductivity. Permittivity and permeability are usually given in complex forms, such as $\varepsilon=$ $\varepsilon^{\prime}-\mathrm{j} \varepsilon^{\prime \prime}$ and $\mu=\mu^{\prime}-\mathrm{j} \mu^{\prime \prime}$. These describe, respectively, the effect of the presence of the material on the electric and magnetic fields. In the expression for complex permittivity, $\varepsilon^{\prime}$, the dielectric constant, represents the ability of the material to store energy in the form of electric fields, whereas $\varepsilon^{\prime \prime}$, the dielectric loss, is a direct measure of how much energy a material can dissipate in the form of heat. The ratio $\varepsilon^{\prime \prime} / \varepsilon^{\prime}$ is referred to the loss tangent, or $\tan \delta\left(\right.$ since $\varepsilon=\varepsilon^{\prime}-\mathrm{j} \varepsilon^{\prime \prime}=|\varepsilon| \mathrm{e}^{-\mathrm{j} \delta}$, then $\tan \delta=\varepsilon^{\prime \prime} / \varepsilon^{\prime}$.) When $\varepsilon^{\prime \prime} / \varepsilon^{\prime}$ is plotted against the frequencies for the various temperatures, the minima in each curve represent the optimal frequencies for microwave processing at that temperature.

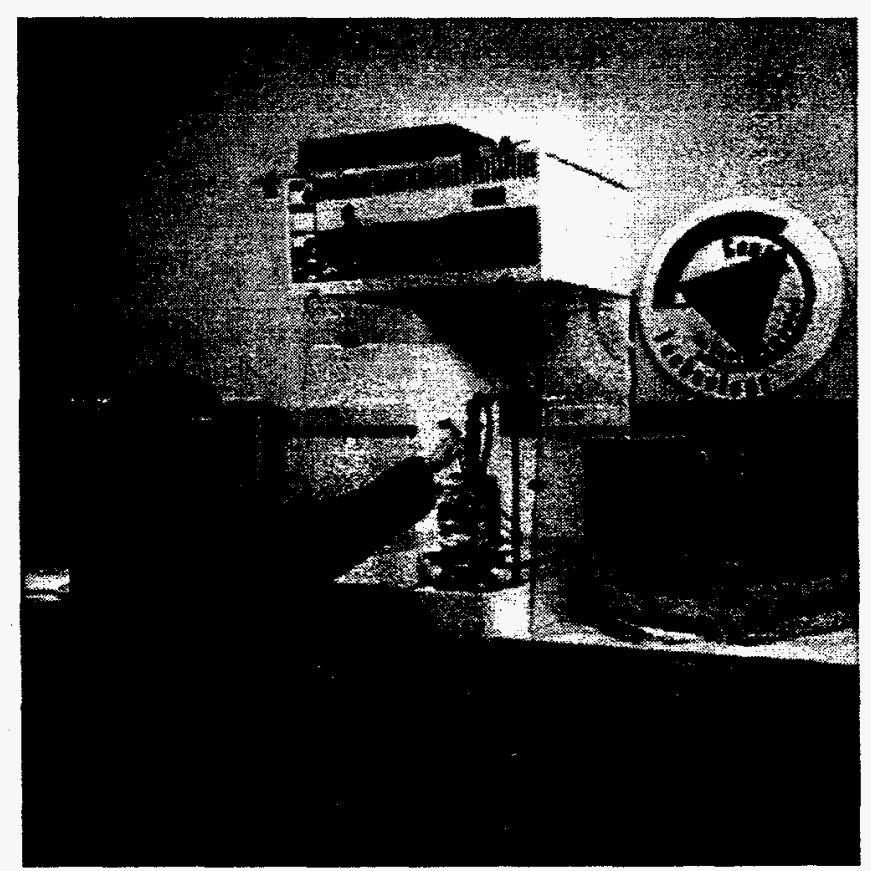

Figure 1. HCET investigator performing dielectric measurements. 


\section{TECHNOLOGY DESCRIPTION}

Maxwell's Equations 1 and 2are used to determine the electric and magnetic fields show the case for a simple propagation of a plane wave within a uniform material. Practically, we may assume that the electric and magnetic fields resulting from the microwaves are perpendicular to each other and the propagation direction. Microwaves are electromagnetic waves having frequencies from $300 \mathrm{MHz}$ to $300 \mathrm{GHz}$. Therefore, microwaves should satisfy Maxwell's Equations, which describe the electromagnetic fields, thus:

$$
\begin{aligned}
& \nabla \times \mathbf{E}=-i \mu \omega \mathbf{H} \\
& \nabla \times \mathbf{H}=(\sigma+\mathrm{I} \varepsilon \omega) \mathbf{E}
\end{aligned}
$$

where $\mathrm{E}$ and $\mathrm{H}$ are electric and magnetic fields vectors respectively; $\mu, \sigma, \varepsilon$ are dielectric properties (permeability, conductivity, and permittivity, respectively) and $\omega$ represents microwave angular frequency.

Permitivitty and permeability are given in the following complex forms:

$$
\varepsilon=\varepsilon^{\prime}-i \varepsilon^{\prime \prime} \text { and } \mu=\mu^{\prime}-\mathrm{i} \mu^{\prime \prime}
$$

where $\varepsilon$ represents a complex number composed of $\varepsilon^{\prime}$ the real part of the complex dielectric permittivity or the dielectric constant, $\varepsilon^{\prime \prime}$ the imaginary part of the complex dielectric permittivity or the dielectric loss. By the same token, the dielectric permeability, $\mu$, is also represented by a complex number composed of real and imaginary parts.

These properties describe, respectively, the effects of the presence of the material on the electric and magnetic fields. The real part of $\varepsilon, \varepsilon^{\prime}$ is always referred to as the dielectric constant, which represents the ability of a material to store electric energy. The imaginary part of $\varepsilon, \varepsilon^{\prime \prime}$, is referred to as loss factor or loss tangent, representing the ability of the substance to dissipate the stored energy as heat. The real part of $\mu, \mu^{\prime}$ describes the ability of a material to store the energy in the magnetic field, while the imaginary part of $\mu, \mu$ ", reports the energy dissipation during the magnetization cycles. Electric conductivity, $\sigma$, has the following relation with the loss factor:

$$
\sigma=\omega \varepsilon^{\prime \prime}=2 \pi \beta \varepsilon^{\prime \prime}
$$

where $\beta$ and $\omega$ are the frequency and angular frequency of the microwave, respectively. In general, the dielectric properties, $\varepsilon, \mu, \sigma$, may be the function of the spatial coordinates; the 
temperature; and the magnitude, direction, and frequency of the electric and magnetic fields. For uniform materials, these properties are usually a function of the temperature.

Measurement of the complex permitivitty is required in order to model the interaction of the materials with microwave energy. White (1990) has suggested that the dielectric constant would vary over a wide range with temperature variation during the solidification process. The dielectric characterization of saline by Stogryn (1971), and HCET's previous measurements with water-based materials simulating biological tissues (Stuchly and Stuchly 1980), guided HCET in predicting the variation in dielectric properties of the liquid radioactive waste with temperature and composition.

The three main principal methods that have been used for measuring the dielectric properties of materials are 1) the reflection method (Atley et al. 1982), 2) the transmission method (Franceschetti 1967), and 3) the perturbation method (Piotrowski 1974). In our study, the open ended coaxial line reflection method (Stuchly and Stuchly 1980; Athey et al. 1982) was used because it was not necessary to fill a special container, but the line was simply immersed in the material. Section 4 of this report explain in detail the measurement of dielectric properties and the steps taken to obtain the data presented in this report.

\subsection{CHARACTERISTICS OF LOW-LEVEL LIQUID WASTE SIMULANTS}

In order to obtain the simulants used for the permittvity measurements in this study, a survey was conducted among Environmental Management (EM) 30 and 50 personnel. This survey allowed the identification of simulants which represent waste material similar to the type of LLLWs targeted for treatment using microwave technology. Tables 1 through 7 show the chemical composition of the seven low-level liquid waste simulants identified and used in this study. 
Table 1.

Hanford 4 Molar* Simulant

\begin{tabular}{|l|l|}
\hline Constituent & Grams per Liter of Simulant \\
\hline $\mathrm{H}_{2} \mathrm{O}$ & 1170.5 \\
\hline $\mathrm{Al}\left(\mathrm{NO}_{3}\right)_{3} \mathrm{x} 9 \mathrm{H}_{2} \mathrm{O}$ & 102.63 \\
\hline $\mathrm{Ca}\left(\mathrm{NO}_{3}\right)_{2} \mathrm{x}_{4} \mathrm{H}_{2} \mathrm{O}$ & 0.161 \\
\hline $\mathrm{Cr}\left(\mathrm{NO}_{3}\right)_{3} \mathrm{x}_{2} \mathrm{O}$ & 2.872 \\
\hline $\mathrm{Fe}\left(\mathrm{NO}_{3}\right)_{3} \times 9 \mathrm{H}_{2} \mathrm{O}$ & 0.166 \\
\hline $\mathrm{KOH}$ & 0.556 \\
\hline $\mathrm{Mg}\left(\mathrm{NO}_{3}\right)_{2} \times 6 \mathrm{H}_{2} \mathrm{O}$ & 0.0005 \\
\hline $\mathrm{Mn}\left(\mathrm{NO}_{3}\right)_{2}$ & 0.306 \\
\hline $\mathrm{Na} \mathrm{MoO}_{4} \times \mathrm{H}_{2} \mathrm{O}$ & 4.138 \\
\hline $\mathrm{Sr}\left(\mathrm{NO}_{3}\right)_{2}$ & 2.558 \\
\hline $\mathrm{CsNO}$ & 3.333 \\
\hline $\mathrm{NaH}_{2} \mathrm{PO}_{4}$ & 25.958 \\
\hline $\mathrm{NaIO}_{3}$ & 3.384 \\
\hline $\mathrm{Na}_{2} \mathrm{CO}_{3}$ & 9.063 \\
\hline $\mathrm{NaCl}$ & 0.92 \\
\hline $\mathrm{NaF}$ & 9.337 \\
\hline $\mathrm{Na}_{2} \mathrm{SO}_{4}$ & 9.231 \\
\hline $\mathrm{NaNO}_{3}$ & 4.36 .05 \\
\hline $\mathrm{NaNO}_{2}$ & 30.677 \\
\hline $\mathrm{NaOH}$ & 150.48 \\
\hline $\mathrm{Na}_{4} \mathrm{EDTA}$ & 7.828 \\
\hline
\end{tabular}

*This simulant is 4 Molar in the Nitrate ion. 
Table 2.

Hanford 6 Molar* Simulant

\begin{tabular}{|c|c|}
\hline Constituent & Grams per Liter of Simulant \\
\hline$\overline{\mathrm{H}_{2} \mathrm{O}}$ & 670.5 \\
\hline $\mathrm{Al}\left(\mathrm{NO}_{3}\right)_{3} \times 9 \mathrm{H}_{2} \mathrm{O}$ & 102.63 \\
\hline $\mathrm{Ca}\left(\mathrm{NO}_{3}\right)_{2} \times 4 \mathrm{H}_{2} \mathrm{O}$ & 0.161 \\
\hline $\mathrm{Cr}\left(\mathrm{NO}_{3}\right)_{3} \times 9 \mathrm{H}_{2} \mathrm{O}$ & 2.872 \\
\hline $\mathrm{Fe}\left(\mathrm{NO}_{3}\right)_{3} \times 9 \mathrm{H}_{2} \mathrm{O}$ & 0.166 \\
\hline $\mathrm{KOH}$ & 0.556 \\
\hline $\mathrm{Mg}\left(\mathrm{NO}_{3}\right)_{2} \times 6 \mathrm{H}_{2} \mathrm{O}$ & 0.0005 \\
\hline $\mathrm{Mn}\left(\mathrm{NO}_{3}\right)_{2}$ & 0.306 \\
\hline $\mathrm{Na}_{2} \mathrm{MoO}_{4} \times \mathrm{H}_{2} \mathrm{O}$ & 4.138 \\
\hline $\mathrm{Sr}\left(\mathrm{NO}_{3}\right)_{2}$ & 2.558 \\
\hline $\mathrm{CsNO}_{3}$ & 3.333 \\
\hline $\mathrm{NaH}_{2} \mathrm{PO}_{4}$ & 25.958 \\
\hline $\mathrm{NaIO}_{3}$ & 3.384 \\
\hline $\mathrm{Na}_{2} \mathrm{CO}_{3}$ & 9.063 \\
\hline $\mathrm{NaCl}$ & 0.92 \\
\hline $\mathrm{NaF}$ & 9.337 \\
\hline $\mathrm{Na}_{2} \mathrm{SO}_{4}$ & 9.231 \\
\hline $\mathrm{NaNO}_{3}$ & 4.36 .05 \\
\hline $\mathrm{NaNO}_{2}$ & 30.677 \\
\hline $\mathrm{NaOH}$ & 150.48 \\
\hline $\mathrm{Na}_{4}$ EDTA & 7.828 \\
\hline
\end{tabular}

*This simulant is 6 Molar in the Nitrate ion. 
Table 3.

Melton Valley Simulant

\begin{tabular}{|l|l|}
\hline Constituent & Percentage by Weight \\
\hline $\mathrm{H}_{2} \mathrm{O}$ & $75.15 \%$ \\
\hline $\mathrm{NaNO}_{3}$ & $23.91 \%$ \\
\hline $\mathrm{KCl}$ & $0.39 \%$ \\
\hline $\mathrm{Na}_{2} \mathrm{CO}_{3}$ & $0.18 \%$ \\
\hline $\mathrm{Na}_{2} \mathrm{HPO}_{4}$ & $0.58 \%$ \\
\hline $\mathrm{Na}_{2} \mathrm{SO}_{4}$ & $0.58 \%$ \\
\hline $\mathrm{NaOH}$ & $0.11 \%$ \\
\hline
\end{tabular}

Table 4.

SAIC Simulant

\begin{tabular}{|l|l|}
\hline Constituents & Percentage by Weight \\
\hline $\mathrm{CaSO}_{4} \times 2 \mathrm{H}_{2} \mathrm{O}$ & $5 \%$ \\
\hline Diatomaceous earth & $9 \%$ \\
\hline $\mathrm{Ca}(\mathrm{OH})_{2}$ & $10 \%$ \\
\hline $\begin{array}{l}\text { Metal oxides [Iron oxide }\left(\mathrm{Fe}_{2} \mathrm{O}_{3}\right)-50 \% \\
\text { Aluminum oxide }\left(\mathrm{Al}_{2} \mathrm{O}_{3}\right)-25 \% ; \text { Magnesium } \\
\text { oxide }(\mathrm{MgO})-25 \%\end{array}$ & $14 \%$ \\
\hline Portland cement & $8 \%$ \\
\hline $\mathrm{NaNO}_{3}$ & $14 \%$ \\
\hline $\mathrm{H}_{2} \mathrm{O}$ & $40 \%$ \\
\hline
\end{tabular}


Table 5.

Revised ORNL Simulant

\begin{tabular}{|l|l|}
\hline Constituent & Percentage by Weight \\
\hline $\mathrm{NaNO}_{3}$ & $41.4 \%$ \\
\hline $\mathrm{H}_{2} \mathrm{O}$ & $38.6 \%$ \\
\hline $\mathrm{Ca}\left(\mathrm{NO}_{3}\right)_{2}$ & $11.4 \%$ \\
\hline Bentonite Clay & $2.61 \%$ \\
\hline $\mathrm{NaCO}_{3}$ & $2.06 \%$ \\
\hline $\mathrm{NaOH}$ & $7.67 \%$ \\
\hline $\mathrm{Sea} \mathrm{Sand}$ & $1.34 \%$ \\
\hline $\mathrm{NaCl}$ & $0.83 \%$ \\
\hline $\mathrm{Al}\left(\mathrm{NO}_{3}\right)_{3}$ & $0.09 \%$ \\
\hline
\end{tabular}

Table 6.

W-26 ORNL Simulant

\begin{tabular}{||l|l|}
\hline Constituents & Percentage by Weight \\
\hline $\mathrm{H}_{2} \mathrm{O}$ & $67.63 \%$ \\
\hline $\mathrm{NaNO}_{3}$ & $29.40 \%$ \\
\hline $\mathrm{KNO}_{3}$ & $1.03 \%$ \\
\hline $\mathrm{Al}(\mathrm{OH})_{3}$ & $0.55 \%$ \\
\hline $\mathrm{NaCl}$ & $0.52 \%$ \\
\hline $\mathrm{SiC}$ (surrogate for $\mathrm{U}_{2} \mathrm{O}_{3}$ and $\left.\mathrm{ThO}\right)$ & $0.22 \%$ \\
\hline $\mathrm{CaHPO}$ & $0.17 \%$ \\
\hline $\mathrm{Ca}(\mathrm{OH})_{2}$ & $0.14 \%$ \\
\hline $\mathrm{Na}(\mathrm{OH})$ & $0.10 \%$ \\
\hline $\mathrm{WyOming}$ Bentonite clay & $0.084 \%$ \\
\hline $\mathrm{CaCO}$ & $0.075 \%$ \\
\hline $\mathrm{Mg}(\mathrm{OH})_{2}$ & $0.030 \%$ \\
\hline $\mathrm{Fe}_{2} \mathrm{O}_{3}$ & $0.030 \%$ \\
\hline $\mathrm{Sr}_{3}\left(\mathrm{NO}_{3}\right)_{2}$ & 0.0029 \\
\hline $\mathrm{CsNO}$ & $0.00017 \%$ \\
\hline
\end{tabular}


Table 7.

Savannah River Simulant

(Batch \#95QAB400)

\begin{tabular}{|l|l|}
\hline Constituent & Percent by Weight (Dry Basis) \\
\hline $\mathrm{Al}$ & 6.76 \\
\hline $\mathrm{Ba}$ & 0.012 \\
\hline $\mathrm{Ca}$ & 2.67 \\
\hline $\mathrm{Cr}$ & .18 \\
\hline $\mathrm{Cs}$ & $<0.01$ \\
\hline $\mathrm{Cu}$ & 0.026 \\
\hline $\mathrm{Fe}$ & 26.8 \\
\hline $\mathrm{K}$ & 0.14 \\
\hline $\mathrm{Mg}$ & 1.31 \\
\hline $\mathrm{Mn}$ & 2.80 \\
\hline $\mathrm{Na}$ & 10.2 \\
\hline $\mathrm{Nd}$ & $<0.01$ \\
\hline $\mathrm{Ni}^{*}$ & 0.24 \\
\hline $\mathrm{Pb}$ & 0.10 \\
\hline $\mathrm{Si}$ & 0.81 \\
\hline $\mathrm{Sr}$ & 0.81 \\
\hline $\mathrm{Ti}$ & 0.044 \\
\hline $\mathrm{Zn}$ & 0.17 \\
\hline $\mathrm{Zr}$ & 0.07 \\
\hline $\mathrm{SO}_{4}$ & 0.33 \\
\hline $\mathrm{C}_{2} \mathrm{O}_{4}$ & 0.194 \\
\hline $\mathrm{CO}$ & 1.94 \\
\hline $\mathrm{NO}_{3}$ & 1.96 \\
\hline $\mathrm{F}$ & 0.007 \\
\hline $\mathrm{NO}_{2}$ & 5.86 \\
\hline $\mathrm{OH}_{3}$ & 2.95 \\
\hline
\end{tabular}

*The sample tested was $76.94 \% \mathrm{H}_{2} \mathrm{O}$ by weight. 


\section{EXPERIMENTAL INVESTIGATION}

The dielectric properties of seven LLLW simulants were measured over the temperature range of $20^{\circ}$ to $100^{\circ} \mathrm{C}$ at increments of twenty degrees using the open-ended coaxial line reflection method. A diagram of the experimental setup is shown in Figure 2. The sample holder consisted of a $150 \mathrm{ml}$ Pyrex beaker immersed in an ethylene glycol bath inside a double jacket vessel that was connected to a.Cole Palmer Polystat chiller charged with ethylene glycol and set to maintain the sample at the target temperature. The double jacket vessel was placed in a portable fume hood to protect the investigators from ethylene glycol fumes while conducting measurements at higher temperatures.

The measurements were performed by immersing the delectric probe just below the surface of the sample. The dielectric probe was connected to a probe cable; both are components of the Hewlett-Packard dielectric probe kit (HP-85070B). The cable was connected to the Hewlett-Packard network analyzer (HP-8720C). The network analyzer, combined with a computer equipped with a HP-IB card, displayed and recorded the readings for the magnitude and phase of the complex reflection coefficient, which can be converted to complex permittivity. The computer displayed plots of the dielectric constant, $\varepsilon^{\prime}$; the dielectric loss, $\varepsilon^{\prime \prime}$; and the loss tangent, $\tan \delta$, verses the frequency. It also generated the same information in tabular form. The measurements were performed in duplicate and then averaged in order to obtain the best values.

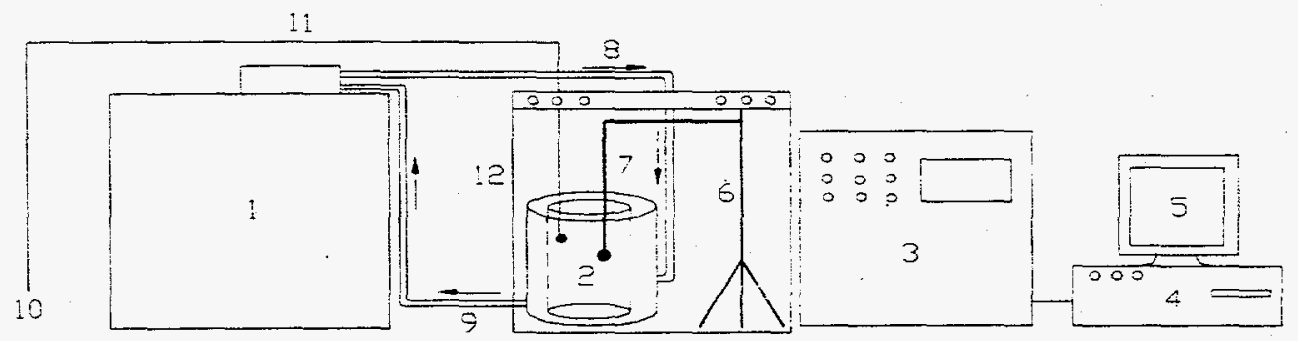

1. Cole Palmer Polystat chiller

2. Double jacket vessel

3. HP 8720C Network Analyzer

4. Gateway $2000486 / 33$ C PC

5. Monitor

6. Stand
7. HP probe

8. Feed line to double-jacket vessel

9. Return line to chiller

10. Temperature controller

11. Thermocouple wire

12. Portable fume hood

Figure 2. Experimental setup. 


\subsection{CORRELATION ANALYSIS}

The correlation presented in this section represent the correlated data obtained from the measurements performed on the seven simulants tested. For each case, the best correlation result is given for each simulant. In addition, data of the Hanford 4 molar and Hanford 6 molar simulants, which have the same constituents but different water content, has been correlated. The best fitted equation and its coefficients are presented in Figures 19 through 34. These equations give predictions for the permittivity of each simulant at various temperatures and frequency ranges.

For each simulate, the result of correlation is included in two figures: 1) the comparison graph of the data by the correlated equation (correlated results shown on the grid line curve) and 2) the experimental data (real data results shown by points). The other graphs presented are the residual graphs.

To perform the correlation, TableCurve $3 D$, a commercially availabie computer software, was used. The most important statistical parameters used in the generation of the correlation are presented and explained below.

\subsubsection{Residual and Residual Percentage}

The residual and residual percentage (\%) represent how accurate the correlated results are to the real experimental data (Jandel Scientific 1993). In this study, the residual percentage (\%) graphs are presented, by the following two equations:

$$
\begin{aligned}
& \text { Residual }=\hat{Z}_{i}-Z_{i} \\
& \text { Residual } \%=\frac{\hat{Z}_{i}-Z_{i}}{Z_{i}} \times 100 \%
\end{aligned}
$$

where $\hat{Z}_{i}$ is the estimated value and $Z_{i}$ is the real data value (Jandel Scientific 1993).

\subsubsection{Coefficient of Determination}

In order to make the results of correlation more agreeable, it is not enough to draw a conclusion only by looking at the residual or residual percentage (\%). Therefore, other statistic standards are usually applied for the determination of the correlation results. Thus, the coefficient of determination $r^{2}$ is defined by the following equations:

$$
r^{2}=1-\frac{S S E}{S S M}
$$

where: 


$$
\begin{gathered}
S S E=\sum_{i=1}^{n} w_{i}\left(\hat{Z}_{i}-Z_{i}\right)^{2} \\
\operatorname{SSM}=\sum_{i=1}^{n} w_{i}\left(\hat{Z}_{i}-\bar{Z}\right)^{2}
\end{gathered}
$$

where $\mathrm{w}_{\mathrm{i}}$ denotes weights and $\bar{Z}$ is the mean of the experimental data.

The $r^{2}$ coefficient of determination is by far the best parameter for fit measurement used and it is the default value used in data-fitting in TableCurve 3D [8]. The closest that $r^{2}$ is to one, the better the correlated results.

\subsubsection{Degree of Freedom Adjusted $r^{2}\left(D F A d j r^{2}\right)$}

The Degree of Freedom Adjusted can be calculated by the following equation as:

$$
\text { DOF adj } r^{2}=1-\frac{\operatorname{SSE}(n-1)}{\operatorname{SSM}(D O F-1)}
$$

where DOF is the Degree of Freedom.

This is a coefficient of determination that has been adjusted for degrees of freedom. The standard $r^{2}$ value increases with increasing terms in an equation even though there has been no real improvement in the standard error of the fit. The DOE Adjusted $r^{2}$ will furnish the same familiar $r^{2}$ sort criteria but will better represent Least Squares sort criteria. As with the unadjusted $r^{2}$, the closer to 1.0 , the better this statistical measure of the fit will be. It is recommended that either this method or the fit standard error be used for approximating functions.

\subsubsection{Fit Standard Error (Root MSE)}

The Fit Standard Error is defined as:

$$
\text { FitStE } r r=\sqrt{M S E}
$$

where MSE denotes the mean square root: 


$$
M S E=\frac{S S E}{D O F}
$$

This sort criterion is the actual Least Squares error of fit. It is known as the Fit Standard Error or Root MSE. The closer this value is to zero, the better the least-square fit will be.

\subsubsection{Fit Statistic (FSTAT)}

$$
\text { Fstat }=\frac{M S R}{M S E}=\frac{\frac{S S M-S S E}{m-1}}{\frac{S S E}{D O F}}
$$

where: MRS is Mean Square Regression

$$
M S R=\frac{S S M-S S E}{m-1}
$$

The Fit statistic is a measure of the extent to which the given equation represents the data. If an additional parameter makes a statistically significant contribution to a model, the Fstatistic increases. Otherwise, a decrease occurs. The higher the F-statistic, the more efficiently a given equation models the data. 


\section{RESULTS}

In this study we measured the dielectric permittivity of the seven LLLW simulants whose components are tabulated in the section entitled "Technology Description." The data were obtained over'a temperature range from $20^{\circ}$ to $100^{\circ} \mathrm{C}$ at increments of twenty degrees over the frequency range from 0.1 to $20.0 \mathrm{GHz}$. Graphs showing $\varepsilon^{\prime}$, $\varepsilon^{\prime \prime}$ and $\varepsilon^{\prime \prime} / \varepsilon^{\prime}$ versus the frequency for the five temperatures were constructed. In addition, a correlation was performed to determine the values for $\varepsilon^{\prime}, \varepsilon^{\prime \prime}$, and $\varepsilon^{\prime \prime} / \varepsilon^{\prime}$ at temperatures between the measured values.

In Section 3.2, the chemical composition of the seven simulants used in this study is presented, while in Section 5.2, graphs of the loss tangent versus the frequency for seven simulants are provided. Two graphs are prosented for each simulant. The first graph shows the full sweep measured; the second graph shows a scaled-down version so that the reader can better appreciate the difference of the loss tangent within the five temperature measured. In Section 5.2, the correlation for the data obtained is presented in the form of graphs, this graphs contain a prediction formula for the dielectric properties of the simulant.

Graphs showing the dielectric constant versus frequency and dielectric loss versus frequency and presented in Appendix A for all the simulants tested. All the tabulated data obtained in this study for the dielectric constant, the dielectric loss, and the loss tangent are presented in Appendix B. Appendix C presents the correlated data for the dielectric constant and the dielectric loss.

\subsection{LOSS TANGENT RESULTS}

For all seven simulants studied, it was found that the values for $\varepsilon^{\prime}, \varepsilon^{\prime \prime}$ and $\varepsilon^{\prime \prime} / \varepsilon^{\prime}$ changed rapidly with increasing frequency in the low frequency range, whereas their rate of change declined considerably in the higher frequency range. For each simulant, a scaled-down graph is presented to show all of the values for the loss tangent versus frequency at the five temperatures. Another graph is scaled down to show more detail in the higher frequency range.

\subsubsection{Hanford 4 Molar Simulants}

Figures 3 and 4 show the loss tangent versus the frequency for the Hanford 4 Molar simulants.

For the most part the loss tangent increases with increasing temperature and decreases with increasing frequency. At $20^{\circ} \mathrm{C}$, optimal processing could be obtained over a wide range of frequencies. For the higher temperatures, a more careful selection of frequency is clearly necessary in order to achieve the best efficiency. 


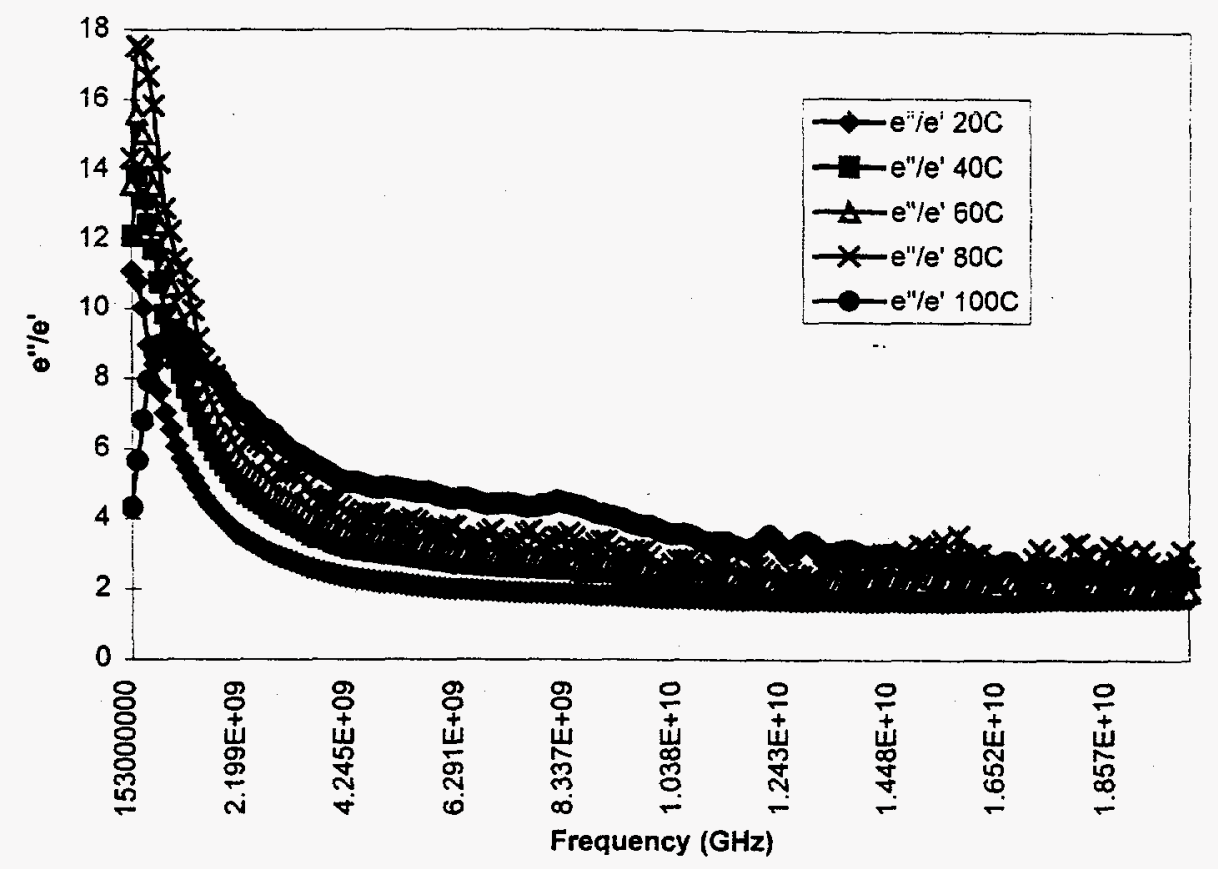

Figure 3. Loss tangent versus frequency for the Hanford 4 molar simulant. Full scale.

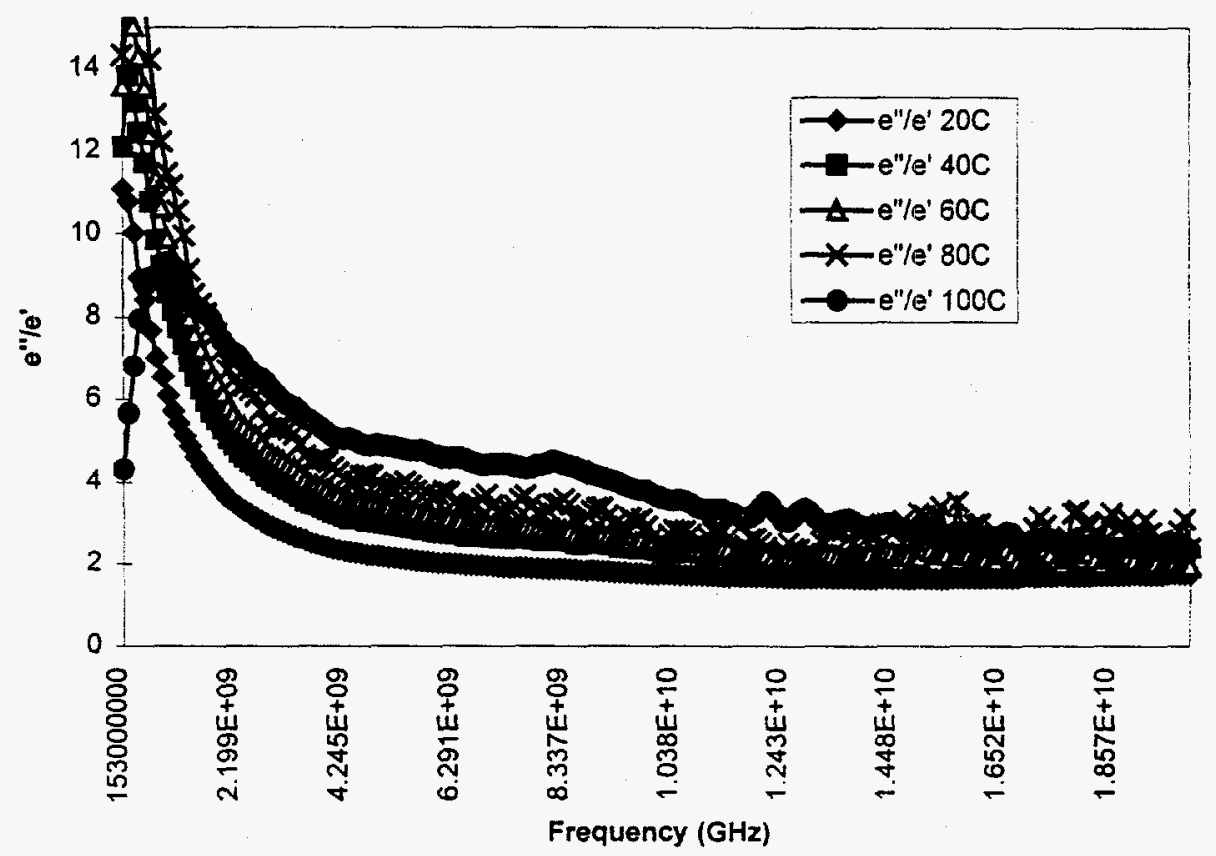

Figure 4. Loss tangent versus frequency for the Hanford 4 molar simulant. Scaled down. 


\subsubsection{Hanford 6 Molar Simulant}

Figures 5 and 6 show the loss tangent versus the frequency for the Hanford 6 Molar simulant. The behavior of this simulant is similar to that of the Hanford 4 Molar simulant.

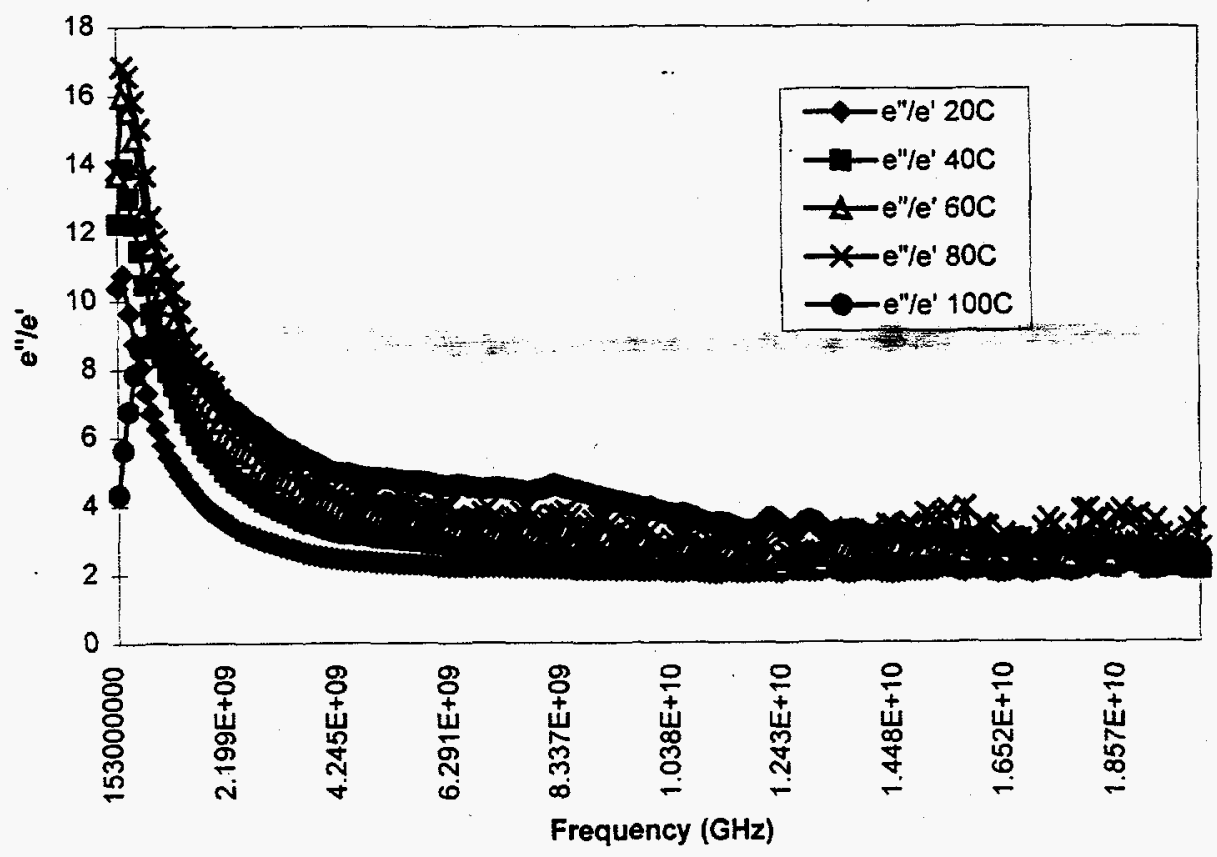

Figure 5. Loss tangent versus frequency for the Hanford 6 Molar simulant. Full scale. 


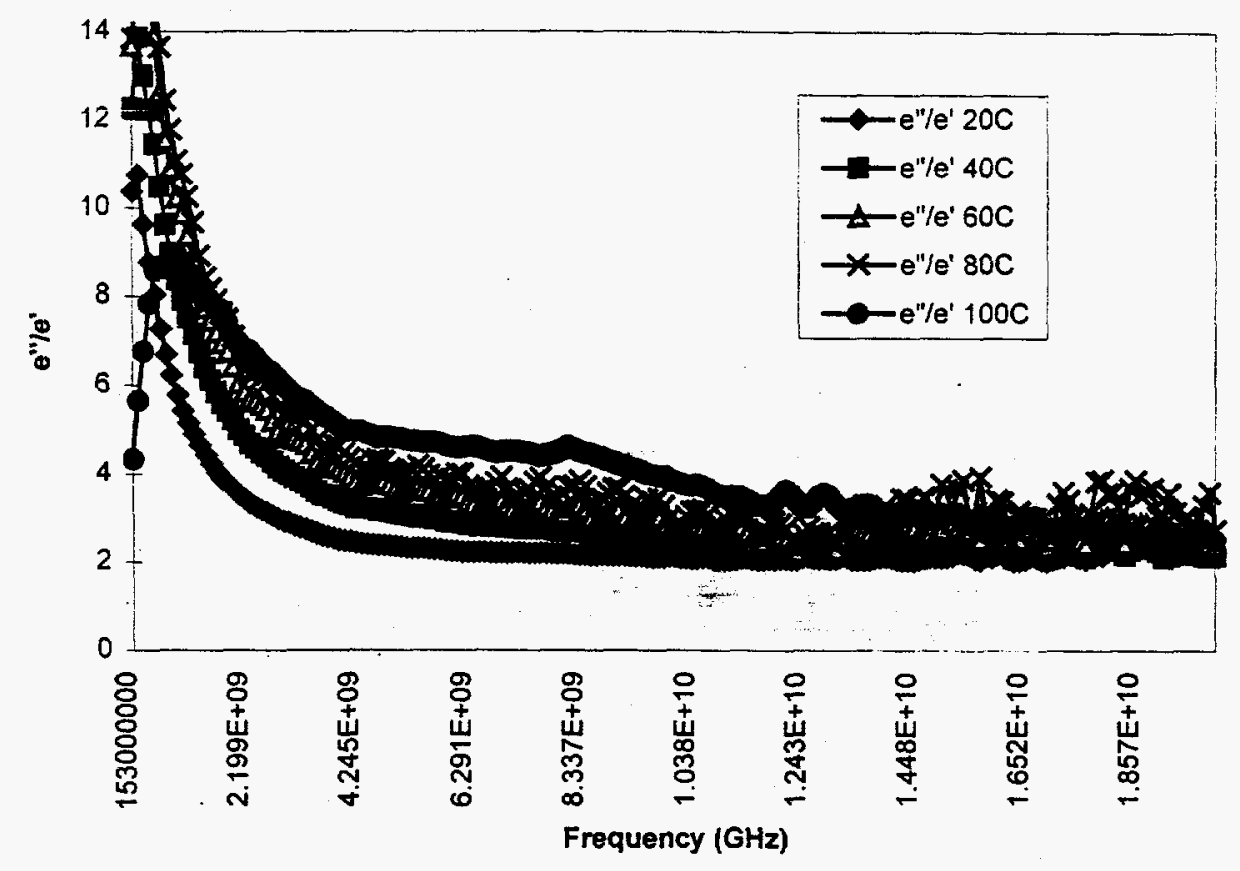

Figure 6. Loss tangent versus frequency for the Hanford 6 molar simulant. Scaled down.

\subsubsection{Comparison of 4 and 6 Molar Hanford Simulants in Term of Water Content}

A comparison of the Hanford simulants was conducted. The Hanford 4 molar simulant had 59.4 percent water, compared to the Hanford 6 molar simulant, which had 45.6 percent water. The distribution of the loss tangent is presented in this section for temperatures of $20^{\circ}$ and $100^{\circ} \mathrm{C}$. These two temperature setting represent both extremes of the temperature range used for this study. 


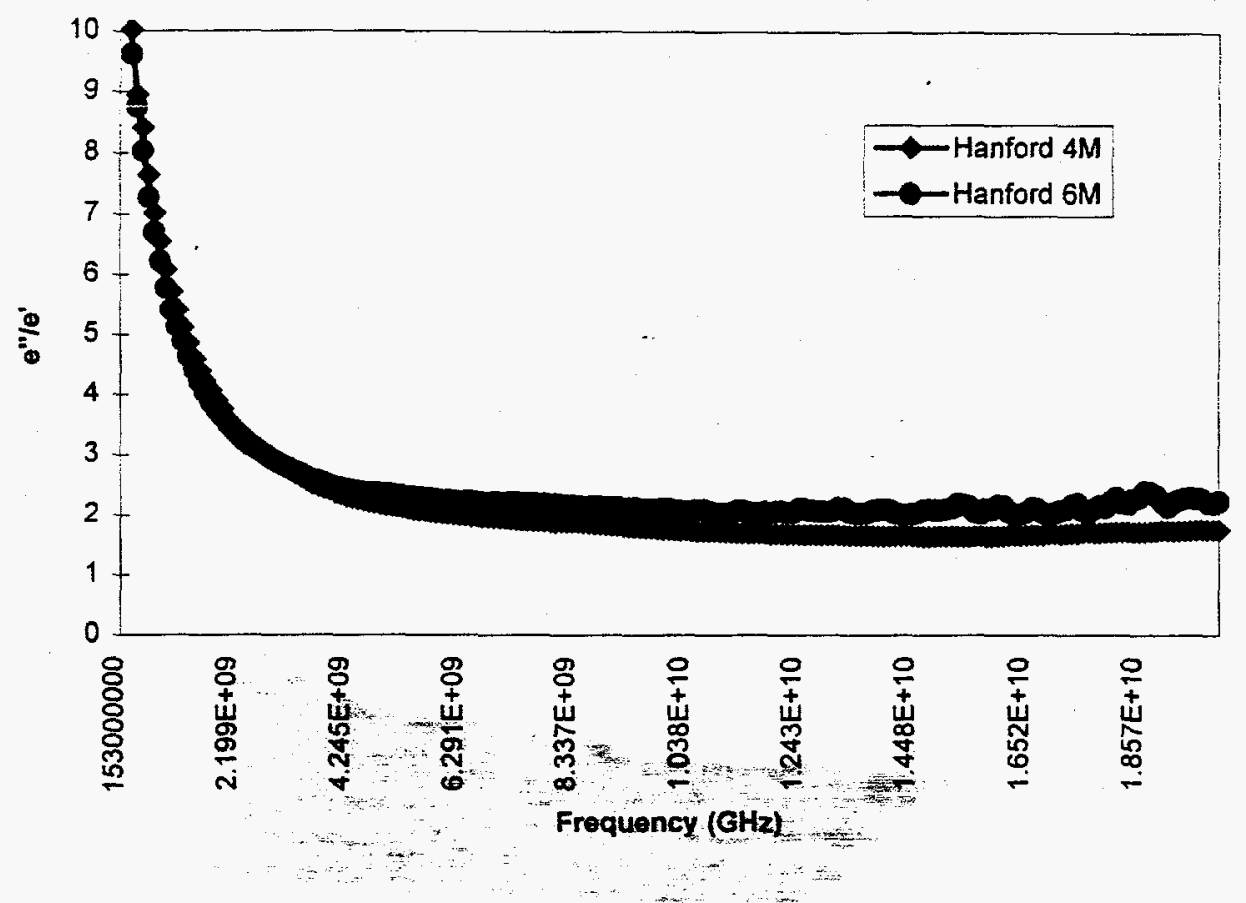

Figure 7. Loss tangent versus frequency for the Hanford 4 and 6 Molar simulants at $20^{\circ} \mathrm{C}$.

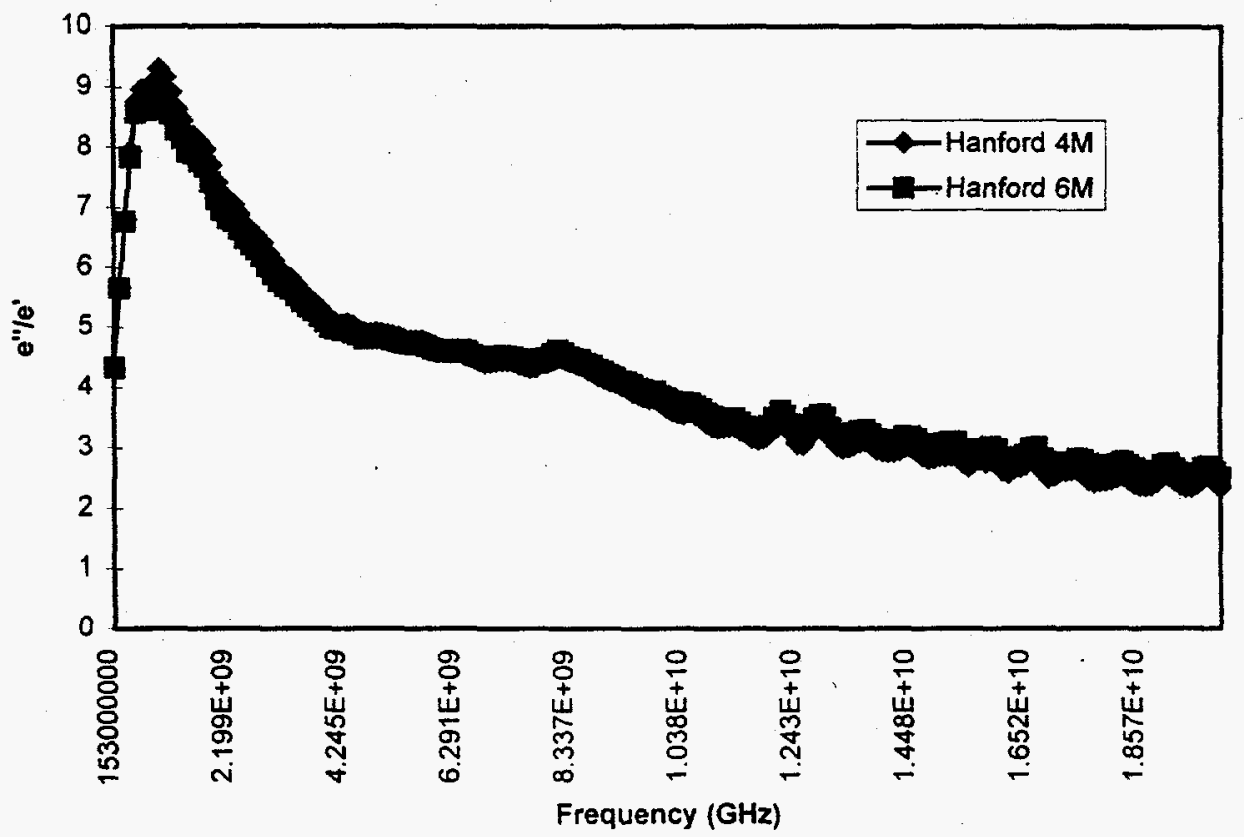

Figure 8. Loss tangent versus frequency for the Hanford 4 and 6 molar simulants at $100^{\circ} \mathrm{C}$. 
At $20^{\circ} \mathrm{C}$, a difference in the loss tangent was observed between the 4 molar and 6 molar simulants (see Figure 7). However, as the temperature increased to $100^{\circ} \mathrm{C}$, the water was driven off, and the value of the loss tangent of both simulants became closer (see Figure 8). Also, at $20^{\circ} \mathrm{C}$ the values of the dielectric constant and dielectric loss were higher for the simulant with the higher water constant (Hanford 4 molar), but as the temperature was increased to $100^{\circ} \mathrm{C}$, the values for the dielectric constant and dielectric loss of both simulants became closer. It can be concluded that moisture constant in the simulants plays an important role in the permittivity measurements.

\subsubsection{Melton Valley Simulant}

Figures 9 and 10 show the loss tangent versus frequency for the Melton Valley simulant. It can be concluded that the loss tangent increases with temperature for this simulant, and the best efficiency is found in the higher frequency range.

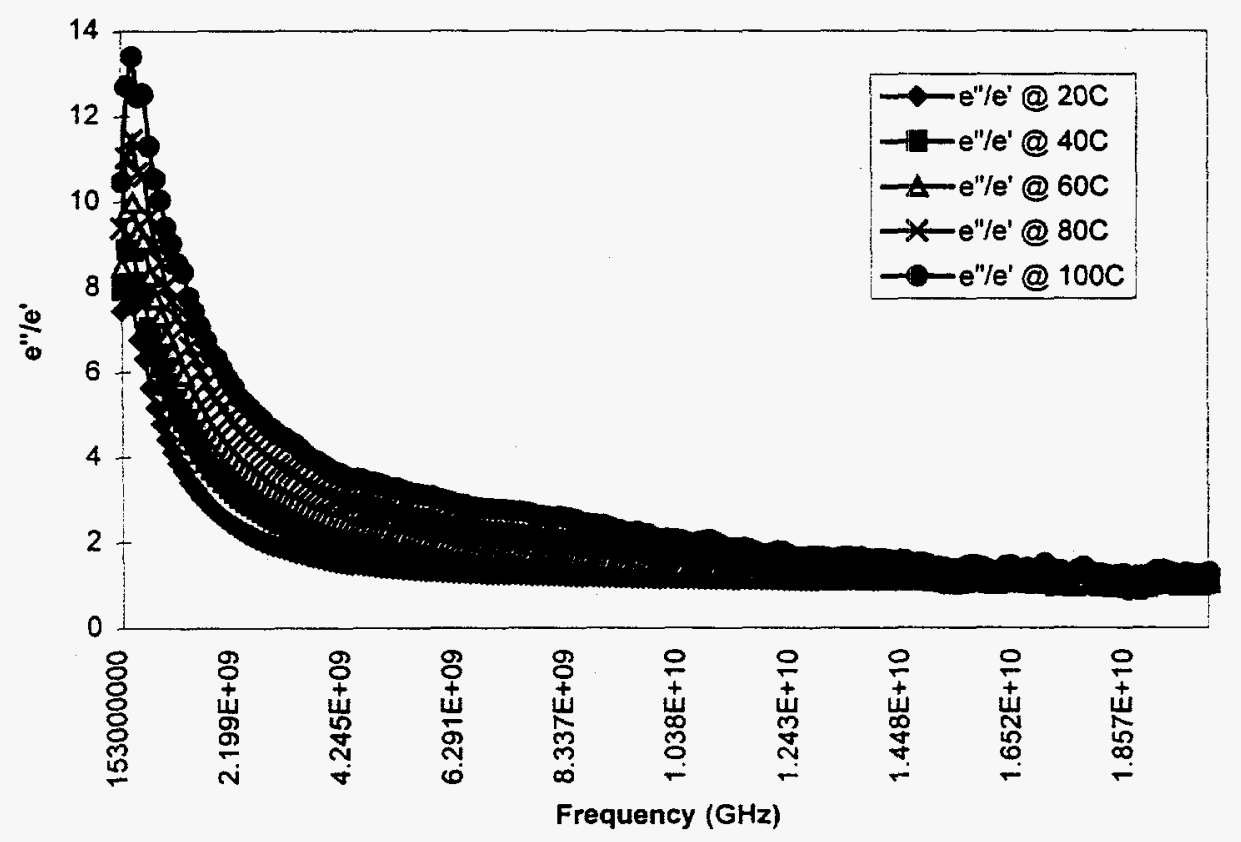

Figure 9. Loss tangent versus frequency for the Melton Valley simulant. Full Scale. 


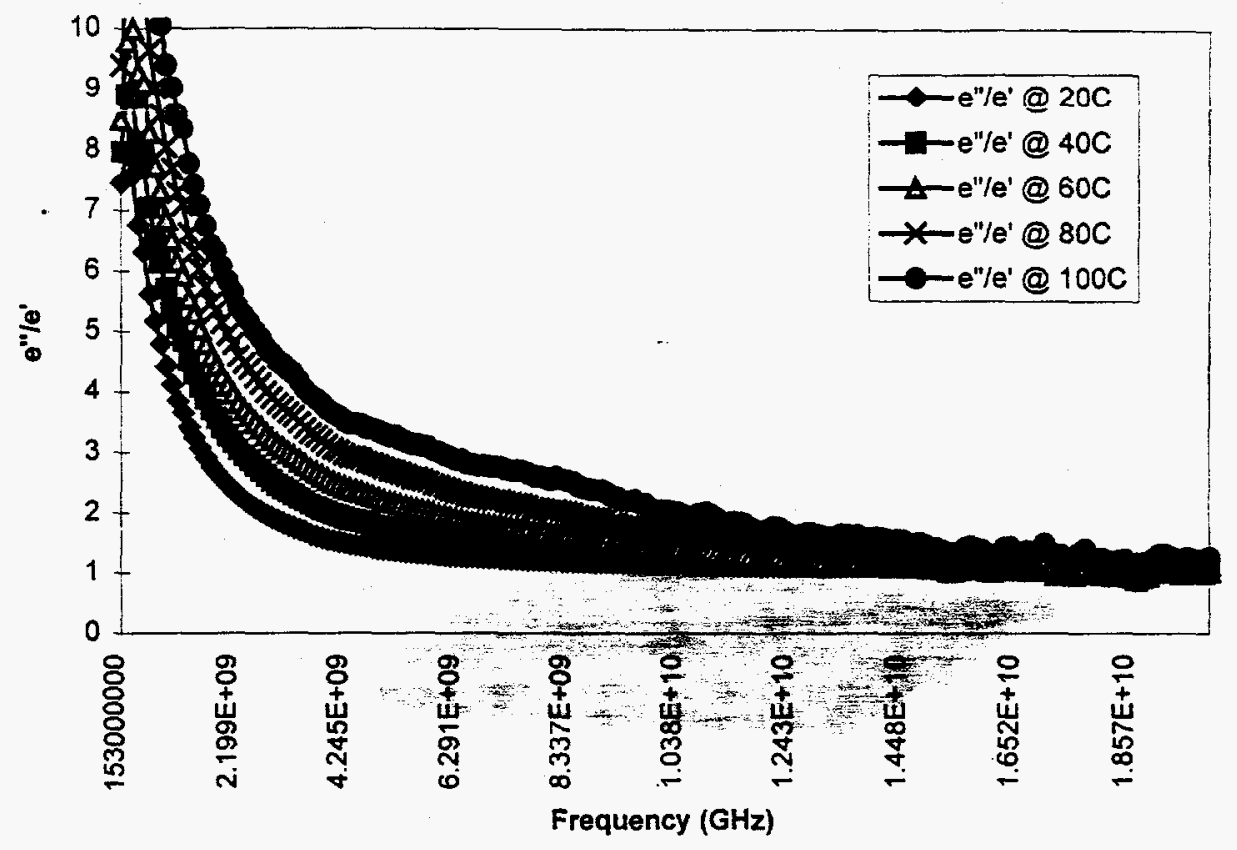

Figure 10. Loss tangent versus frequency for the Melton Valley simulant. Scaled down.

\subsubsection{SAIC Simulant}

Figures 11 and 12 show the loss tangent versus the frequency for the SAIC simulant. This plot shows that good efficiency can be achieved over most of the higher frequency range. 


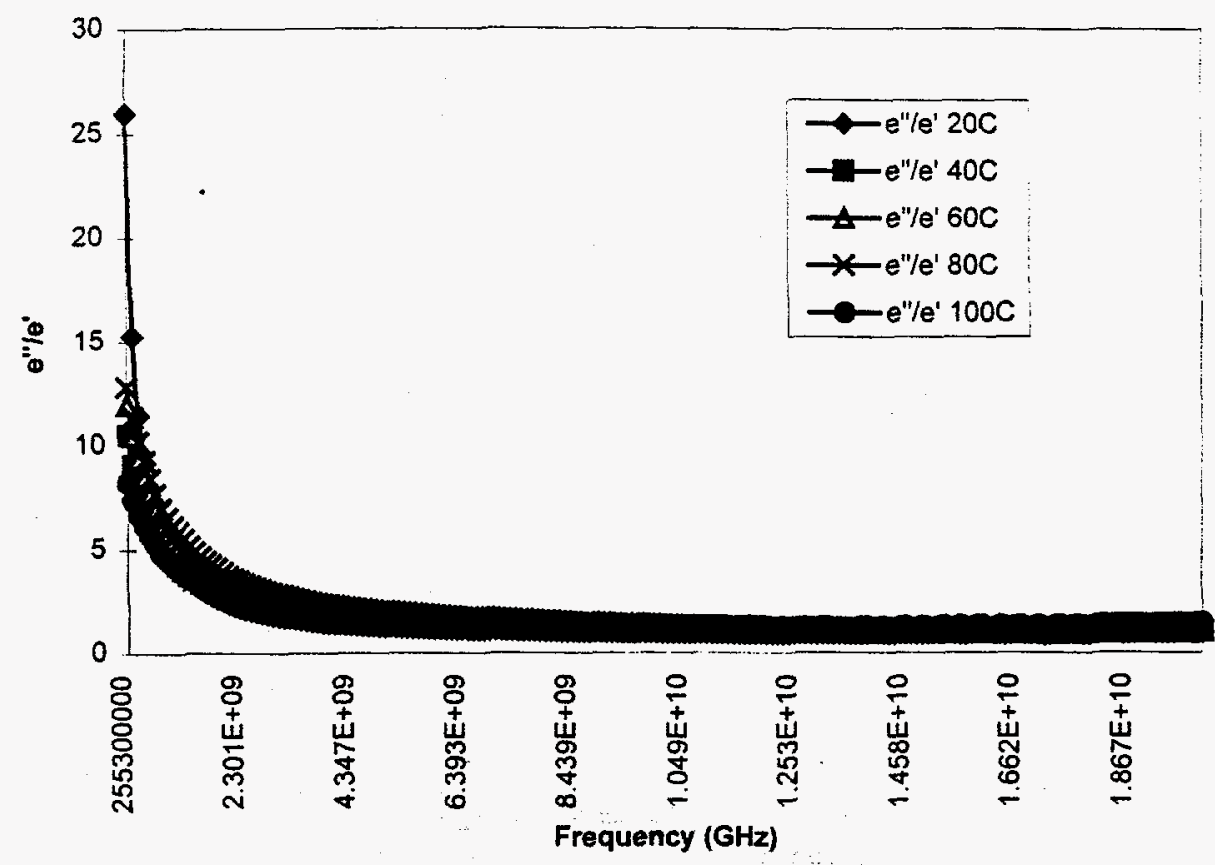

Figure 11. Loss tangent versus frequency for the SAIC simulant-Full scale.

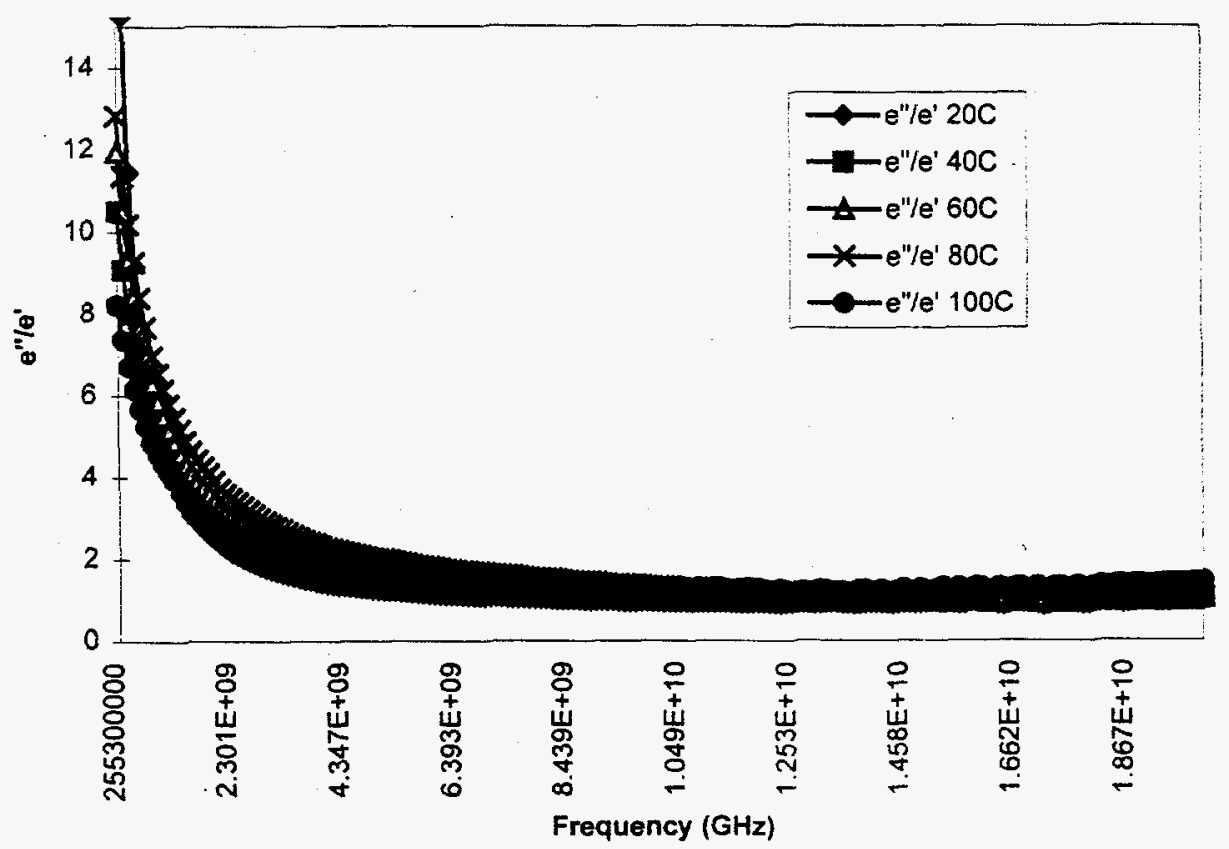

Figure 12. Loss tangent versus frequency for the SAIC simulant. Scaled down. 


\subsubsection{Revised ORNL Simulant}

Figures 13 and 14 show the loss tangent versus frequency for the Revised ORNL simulant. These plots show that the loss tangent increases with increasing temperature over most of the frequency range. Thus, the most efficient processing would be achieved in the higher frequency ranges.

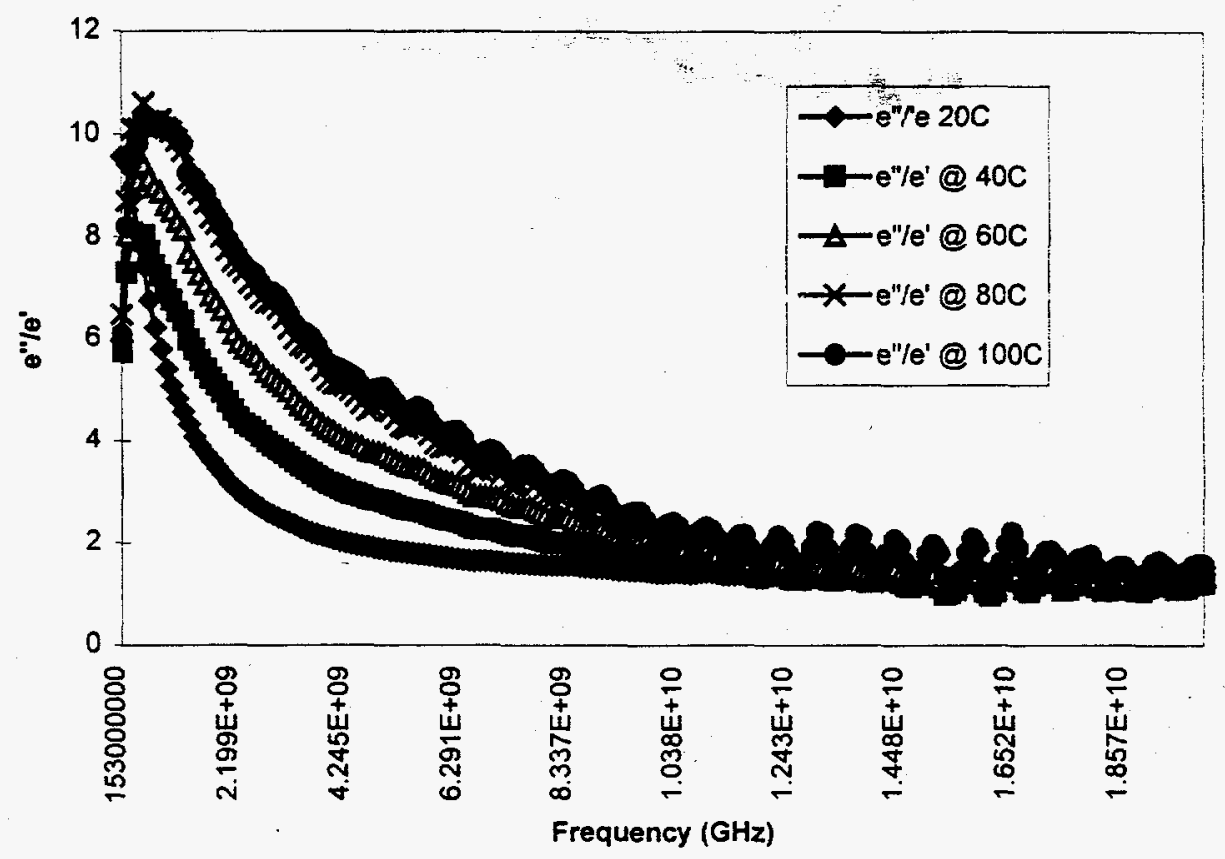

Figure 13. Loss tangent versus frequency for the Revised ORNL simulant. Full scale. 


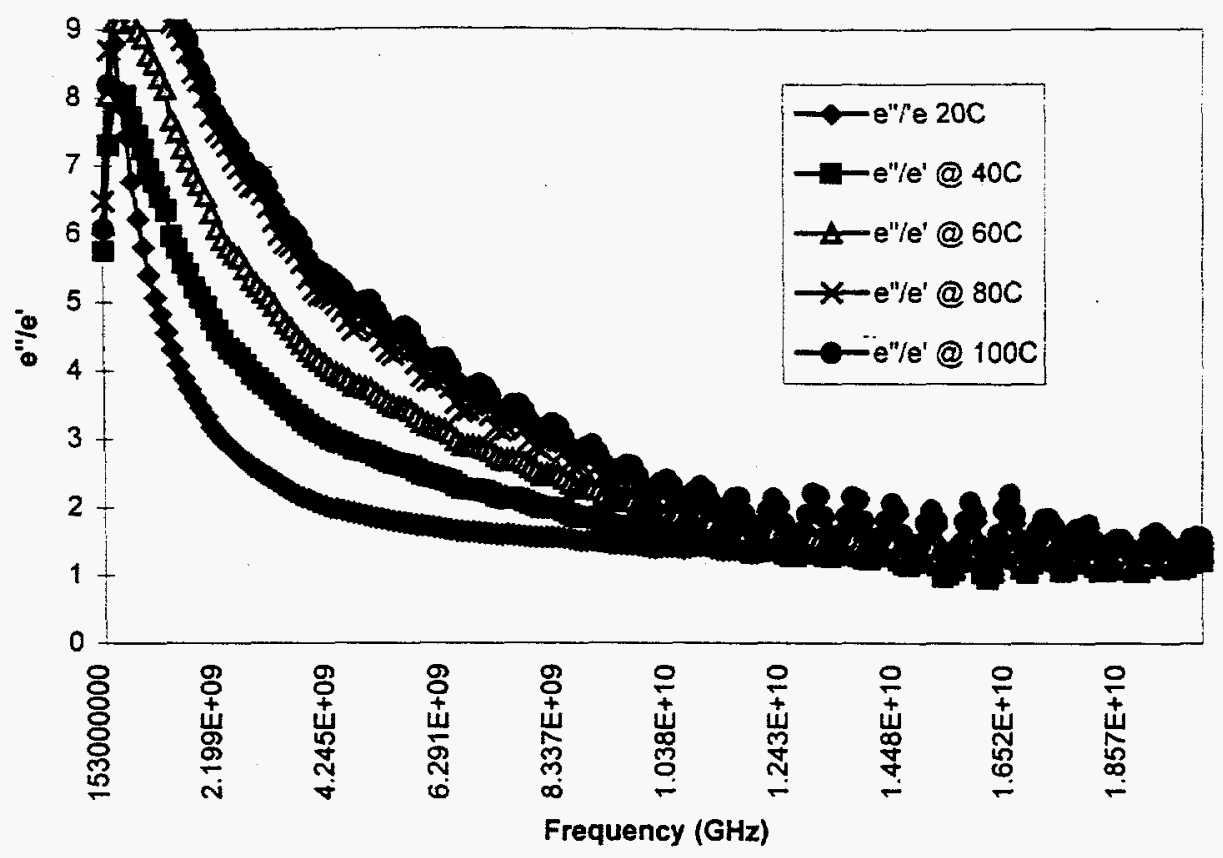

Figure 14. Loss tangent versus frequency for the Revised ORNL simulant. Scaled down.

\subsubsection{W-26 ORNL Simulant}

Figures 15 and 16 show the loss tangent versus frequency for the W-26 ORNL simulant. Again, the values for the loss tangent generally increase with increasing frequency, and the greatest efficiency would be achieved at the higher frequencies. 


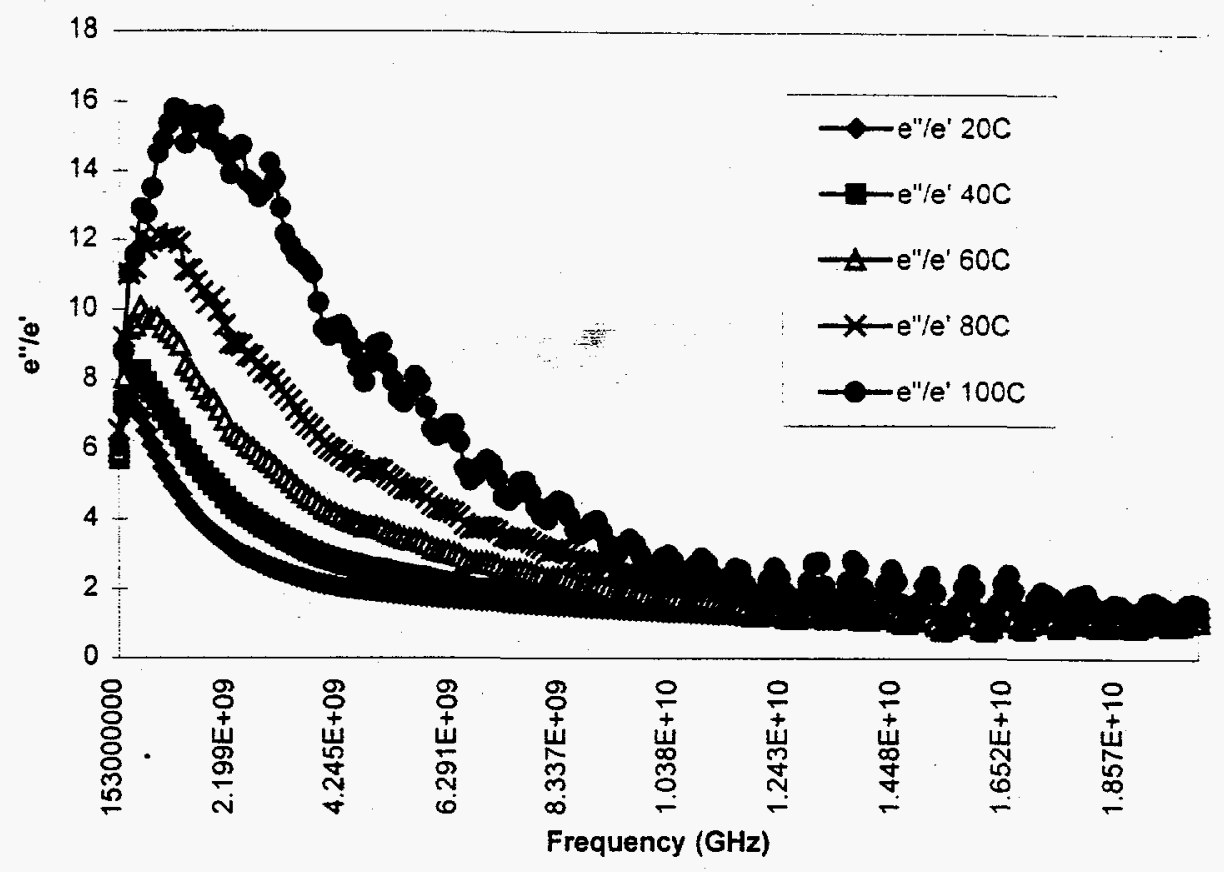

Figure 15. Loss tangent versus frequency for the W-26 ORNL simulant. Full scale.

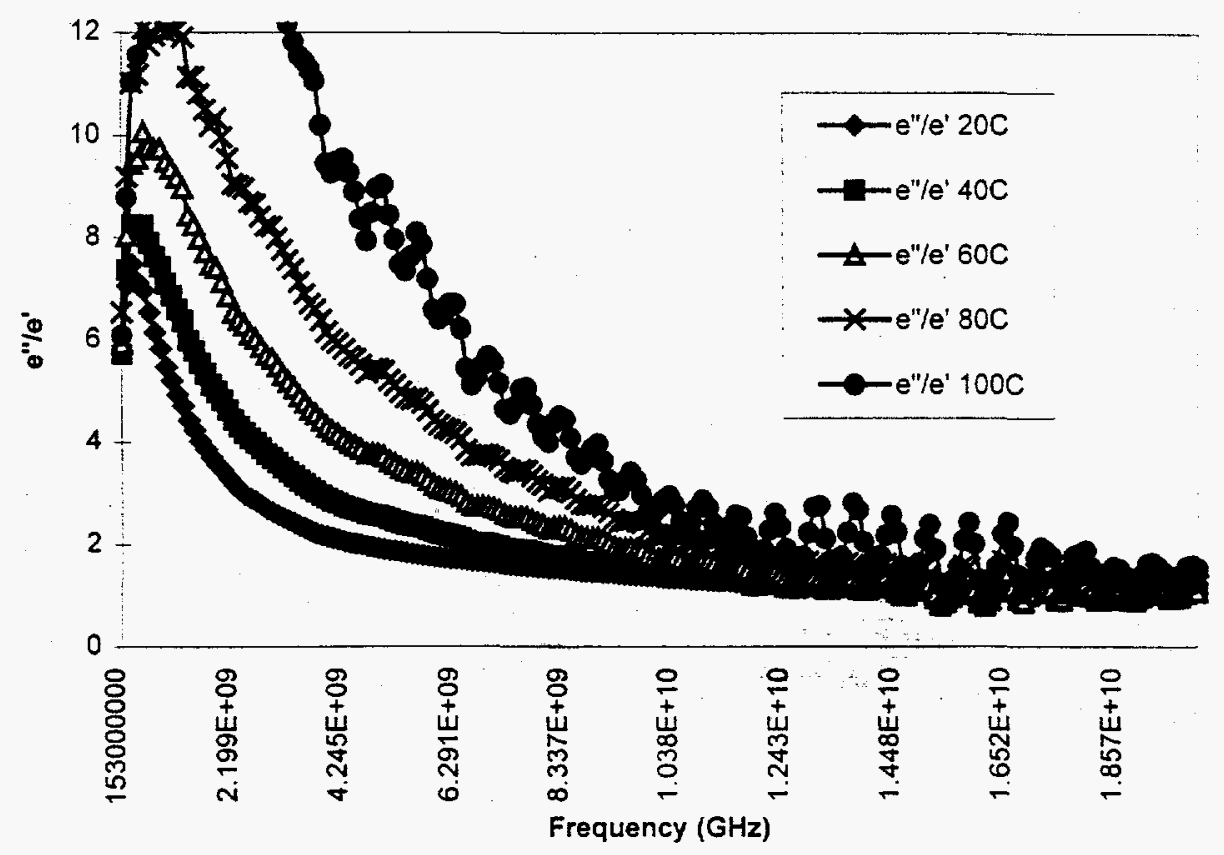

Figure 16. Loss tangent versus frequency for the W-26 ORNL simulant. Scaled down. 


\subsubsection{Savannah River Simulant}

Figures 17 and 18 show the loss tangent versus frequency for the Savannah River simulant. Figure 18 , which is highly scaled down, shows a minimum for the curve representing $20^{\circ} \mathrm{C}$ in the region of $4 \mathrm{GHz}$. All the measurements for this simulant show some rise in the loss tangent at the higher frequencies.

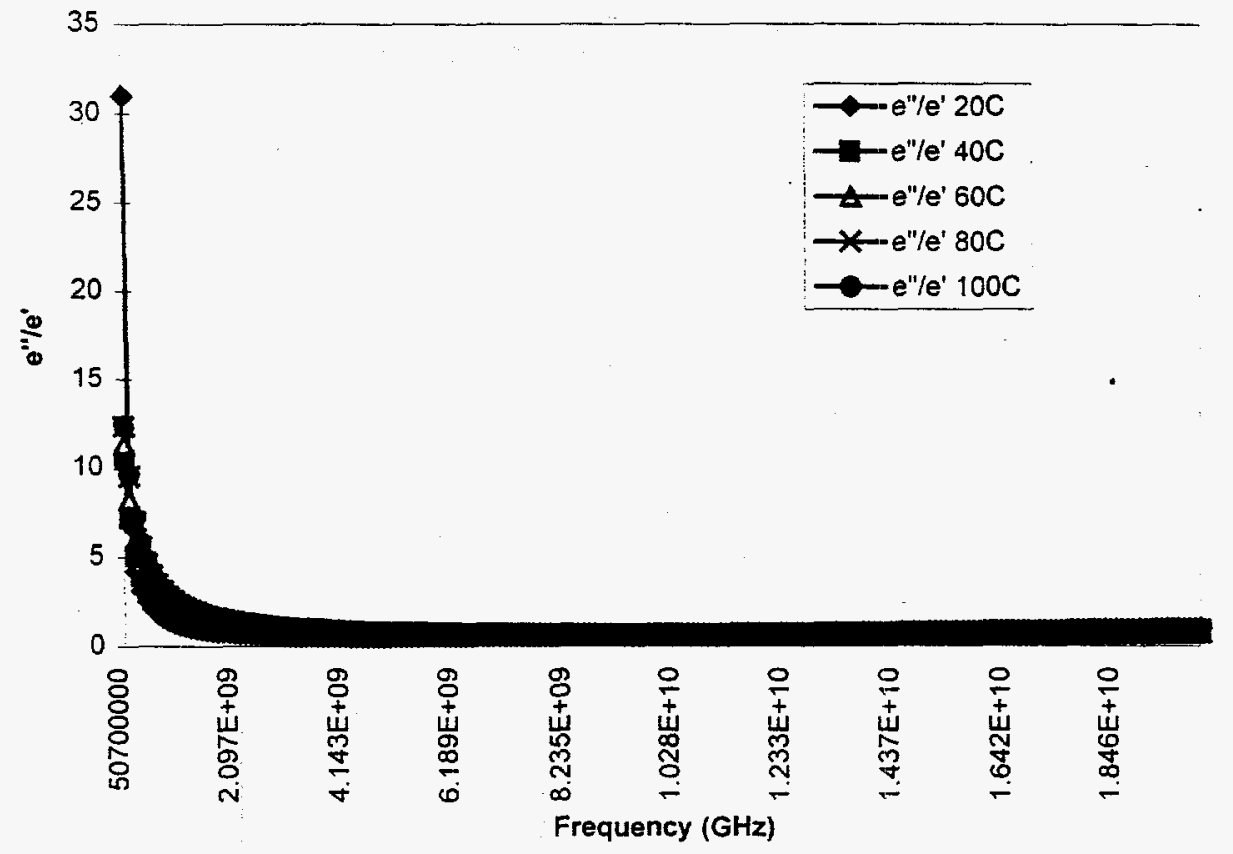

Figure 17. Loss tangent versus frequency for the Savannah River simulant. Full scale. 


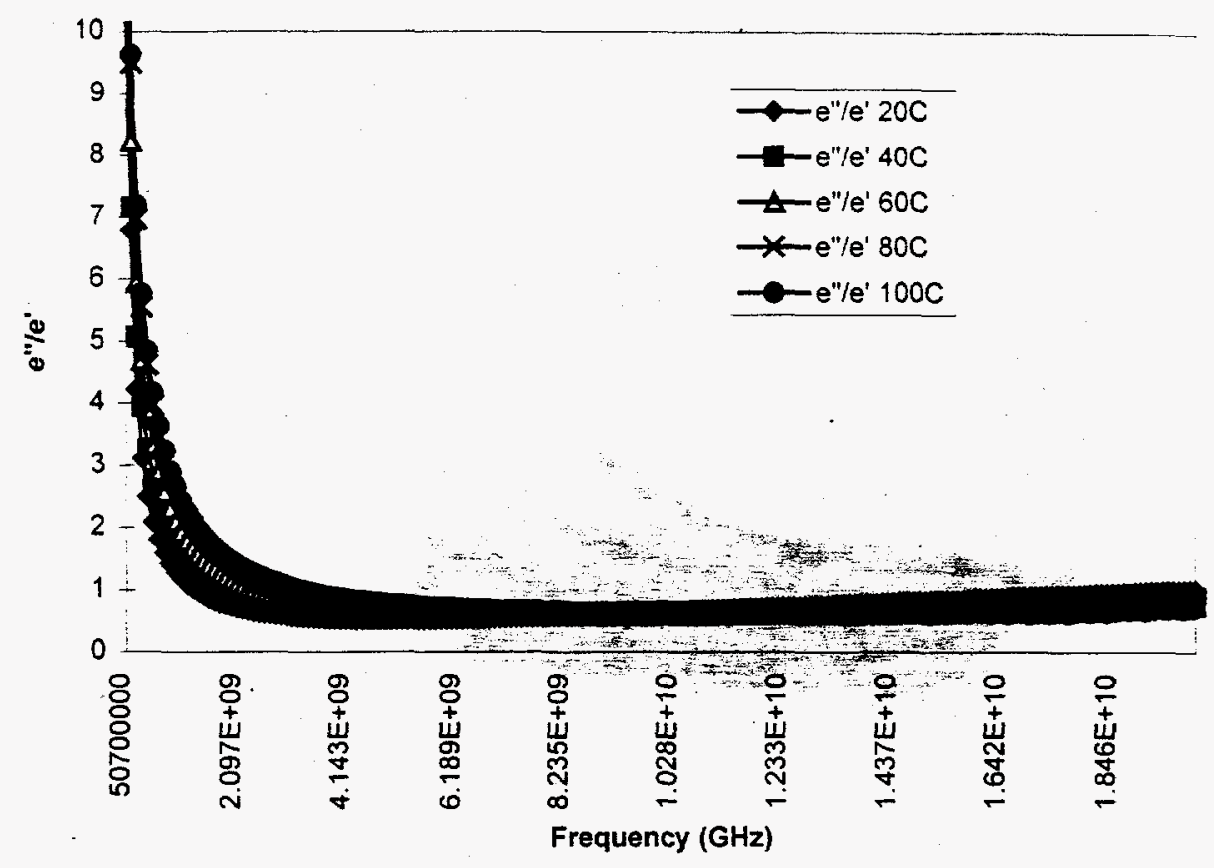

Figure 18. Loss tangent versus frequency for the Savannah River simulant. Scaled down.

\subsection{ANALYSIS OF THE CORRELATED RESULTS FOR THE LOSS TANGENT}

\subsubsection{Hanford 4 Molar Simulant}

Figures 19 and 20 are the correlation results of the loss tangent, $\tan \delta$. Figure 19 presents the correlated results for the temperature and frequency range defined in this study along with a prediction correlation formula and coefficients to predict the loss tangent at other frequency and temperature ranges. Figure 20 shows the loss tangent correlated residual percentage (\%) results, most of which are within $\pm 20 \%$. 
$H:$ daniexpdataiHan $4 m_{\text {Ian. }} x / \mathrm{s}$

Rank 1 Eqn $312 z=a+b, x+c y+d / x^{\wedge} 2+e y^{\wedge} 2+f y / x+g / x^{\wedge} 3+h y^{\wedge} 3+i y^{\wedge} 2 / x+j y / x^{\wedge} 2$

$M=0.95506894$ of $\lambda$ dej $\quad 2=0.95412501$ FitStdE $r=0.4650958$ 1 Fstat $=1: 26.5848$

$a=2.0941009 b=3.0969287 e+c 9 c=0.05425234 ; d=-1.7702512 e+18$ e $=0.0012261603$

$f=2.0583849 e+08 g=1.37351^{-} e+26 n=-6.0485628 \theta-06 i=-1595610.41=.4 .25 C 9056 \theta+15$

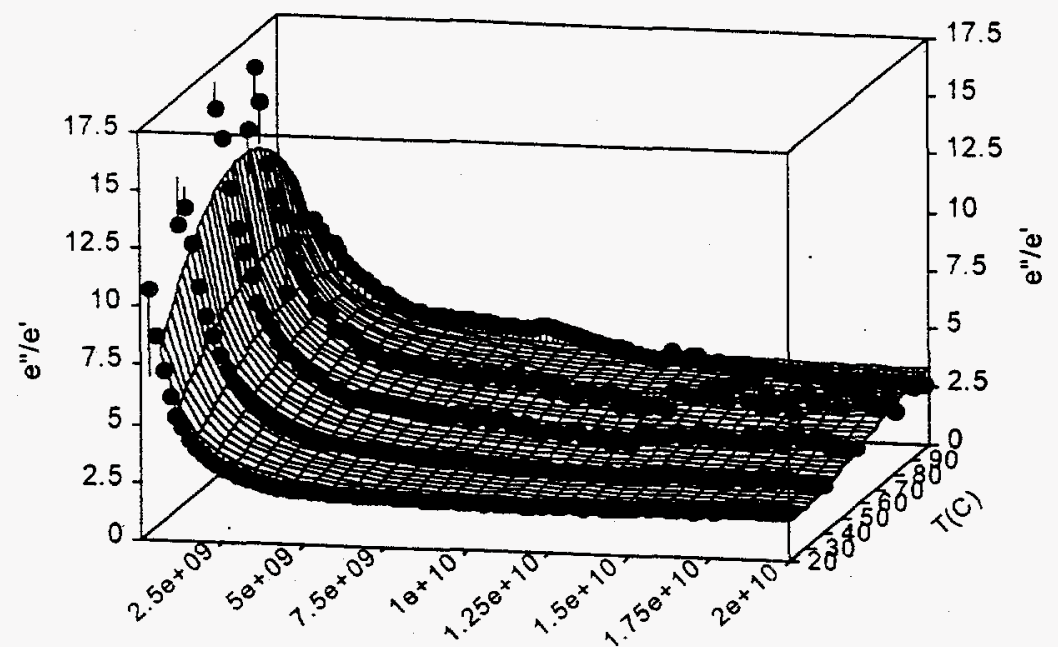

Frequency

$$
\text { F }
$$

Figure 19. Correlation data for the loss tangent. Simulants. Hanford 4 Molar.

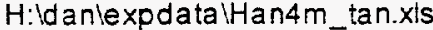

Rank 1 Eqn $312 z=a+b / x+c y+d / x^{\wedge} 2+e y^{\wedge} 2+f y / x+g / x^{\wedge} 3+h y^{\wedge} 3+i y^{\wedge} 2 / x+j y / x^{\wedge} 2$

$r^{\wedge} 2=0.95506894$ of Adj $r^{\wedge} 2=0.95412501$ FitSidE r $=0.46509581$ Fs $t a l=1126.5848$

$a=2.0941009 b=30969287 e+C 9 \quad c=-0054252341 d=-1.7702512 e+18 e=0.0012261603$

$f=2.0583849 e+08 g=1.3735: 7 e+25 . \gamma=-6.0185628 e-06 i=-1595610.4 j=-42509056 e+15$

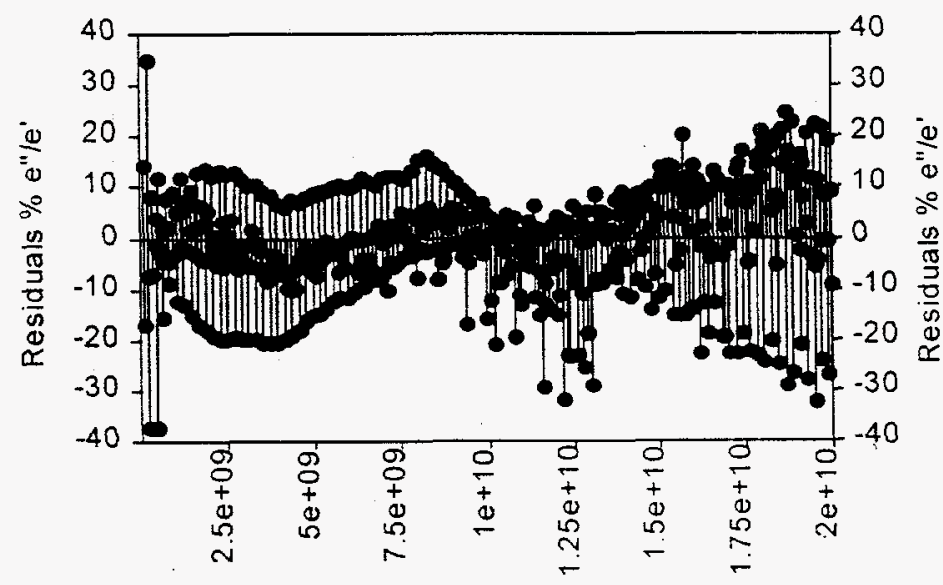

Frequency

Figure 20. Residual percentage (\%) for loss tangent Hanford 4 Molar simulant. 


\subsubsection{Hanford 6 Molar Simulant}

Figures 21 and 22 present the correlation results of the loss tangent, $\tan \delta$. Figure 22 shows the loss tangent correlated residual percentage $(\%)$ results, most of which are within $\pm 20 \%$.

$H:$ IdanlexpdatalHan6m_tan.xls

Rank 1 Eqn $312 z=a+b / x+c y+d / x^{\wedge} 2+e y^{\wedge} 2+f y / x+g / x^{\wedge} 3+h y^{\wedge} 3+i y^{\wedge} 2 / x+j y / x^{\wedge} 2$

$r^{\wedge} 2=0.95506894$ of Adj $r^{\wedge} 2=0.95412501$ FitStdE $r=0.4650958$ i f stat $=1126.5848$

$a=2.0941009 b=3.0969287 \theta+09 \quad c=-0.054252341 d=-1.7702512 \theta+18 \quad \theta=0.0012261603$

$f=2.0583849 \theta+0 B \quad g=1.373517 \theta+26 \quad n=-8.0185628 \theta-06 \quad i=-i 595610.4 j=-4.2509056 \theta+15$

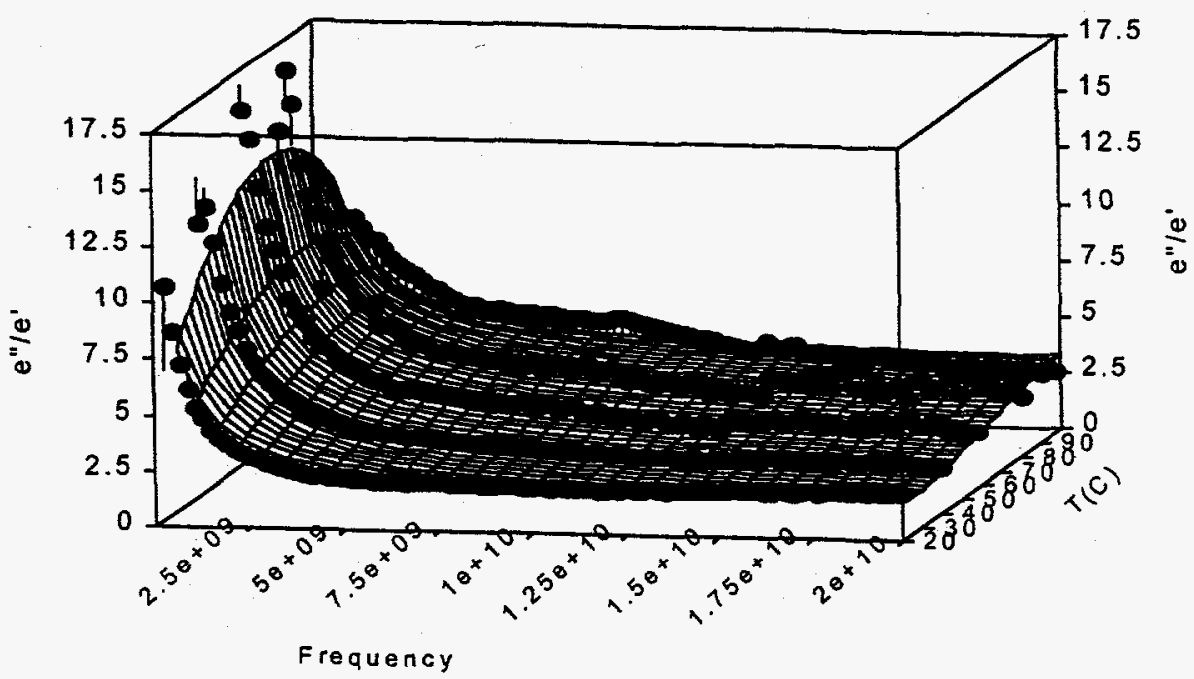

Figure 21. Correlation data for the loss tangent. Simulant: Hanford 6 Molar. 


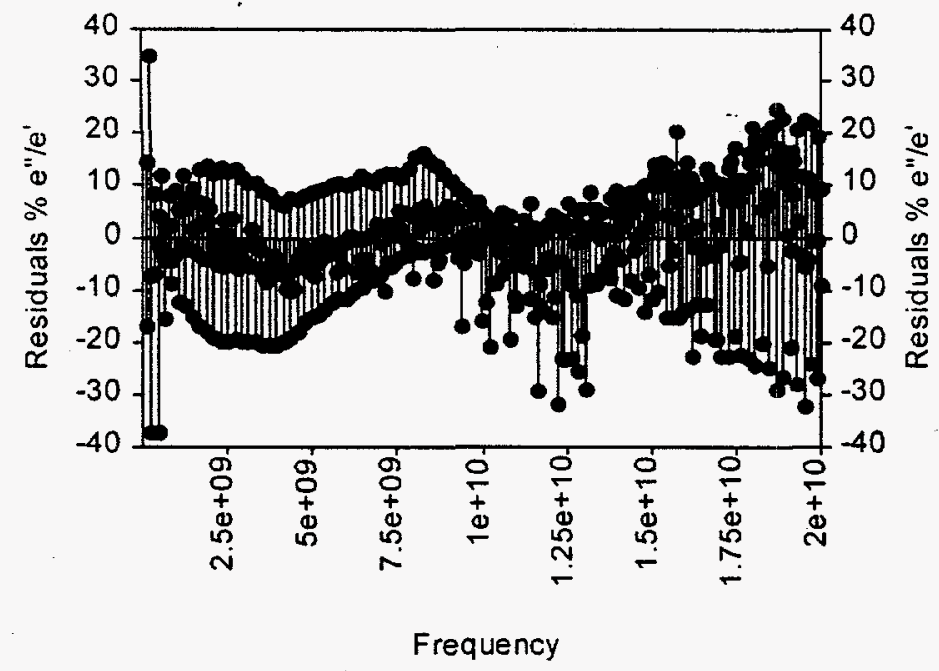

Figure 22. Residual percentage (\%) for the loss tangent. Simulant: Hanford 6 Molar.

\subsubsection{Hanford 4 and 6 Molar Simulants}

Figures 23 and 24 present the correlation results of the loss tangent, tan $\delta$. Figure 23 represents the correlation data for the Hanford 4 and Hanford 6 molar simulants. Here, the loss tangent $\left(\mathrm{e}^{\prime \prime} / \mathrm{e}^{\prime}\right)$ is plotted versus frequency and temperature. Figure 24 shows the loss tangent correlated residual percentage (\%) results, most of which are within $\pm 20 \%$. The Hanford 4 and 6 simulants have the same constituents but different water content. It can be seen that the correlated results are not as satisfactory as the individual results presented earlier. 
Rank I Eqn $312 z=a+b / x+c y+d / x^{\wedge} 2+e y^{\wedge} 2+f y / x+g / x^{\wedge} 3+h y^{\wedge} 3+i y^{\wedge} 2 / x+j y / x^{\wedge} 2$

$\mathrm{r}^{\wedge} 2=0.94798339$ DF Adj $\mathrm{r}^{\wedge}=0.94757922$ FitStdE $\mathrm{tr}=0.51009434$ Fstat $=2608.1466$

$a=1.2137988 b=4.7200435 e+09 c=-0.011662272 d=-1.9093669 e+18 e=0.0005347558$

$f=1.4485955 \mathrm{e}+08 \mathrm{~g}=1.6252442 \mathrm{e}+26 \mathrm{~h}=-2.7124281 \mathrm{e}-06 \mathrm{i}=-1032868.1 \mathrm{j}=-5.0617205 \mathrm{e}+15$

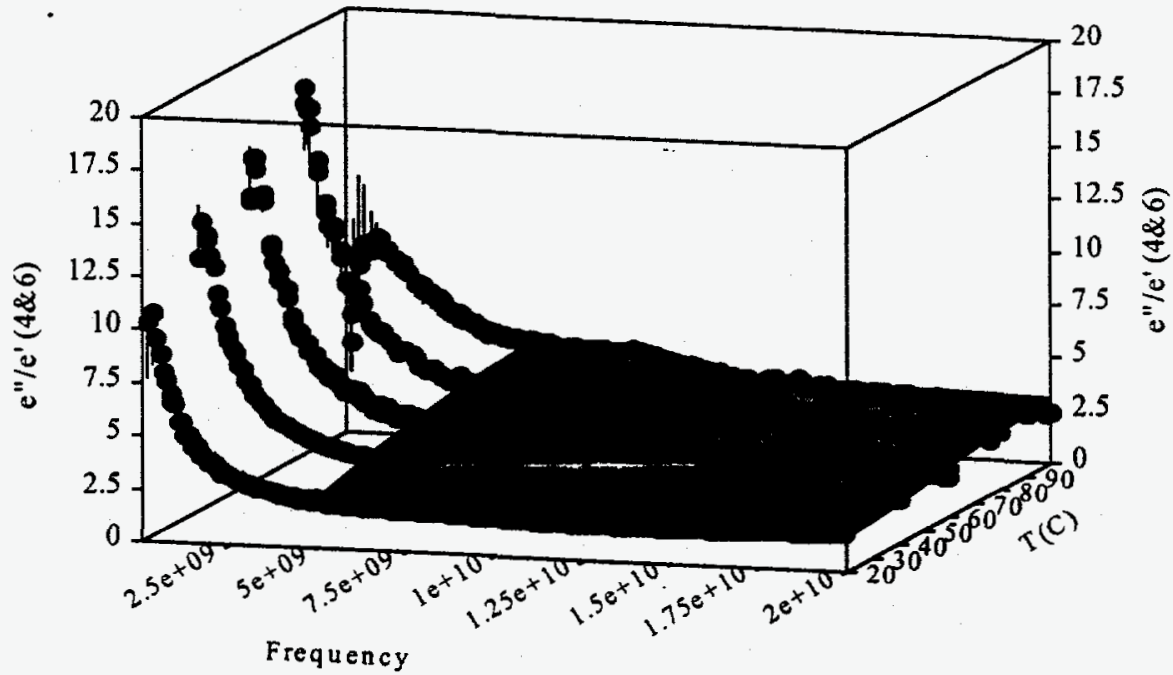

Figure 23. Correlation data for the Hanford 4 and Hanford 6 Molar simulants.

$H:$ ddanlexpdatalHan4m_tan.xls

Rank 1 Egn $312 z=a+b / x+c y+d / x^{\wedge} 2+e y^{\wedge} 2+f y / x+g / x^{\wedge} 3+h y^{\wedge} 3+i y^{\wedge} 2 / x+j y / x^{\wedge} 2$

$r^{\wedge} 2=0.94798339$ DF Adj $r^{\wedge} 2=0.94757922$ FitStdErr $=0.51009434$ Fstat $=2608.1466$

$a=1.2137988 b=4.7200435 e+09 c=-0.011662272 d=-1.9093669 e+18 e=0.0005347558$

$f=1.4485955 e+08 g=1.6252442 e+26 h=-27124281 e-06 i=-1032868.1 j=-5.0617205 e+15$

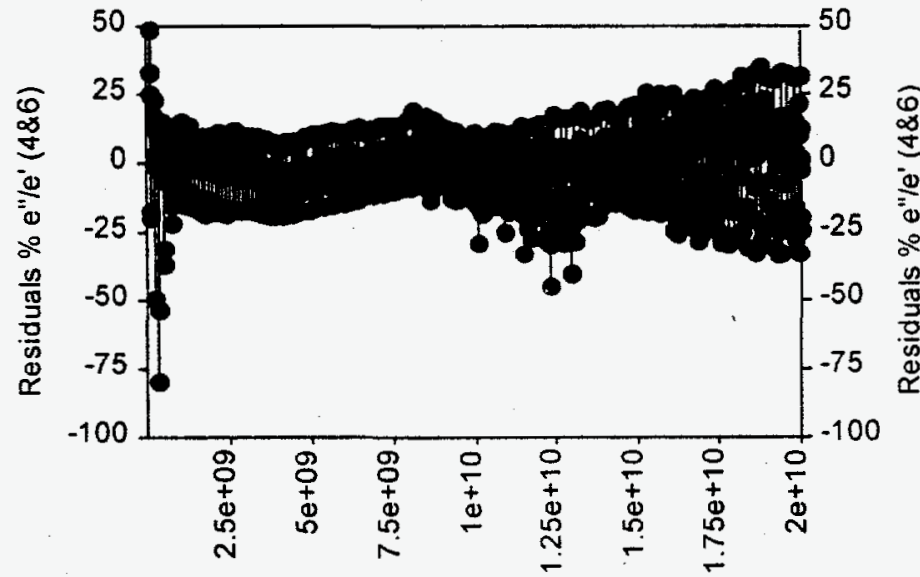

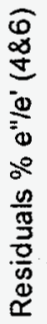

Frequency

Figure 24. Residual percentage (\%) for the Hanford 4 and Hanford 6 simulants. 


\subsubsection{Melton Valley Simulant}

Figures 25 and 26 present the correlation results of the loss tangent, $\tan \delta$. Figure 26 shows the loss tangent correlated residual \% results, most of which are within $\pm 20 \%$. For higher temperatures, the correlated equation yields a better prediction.

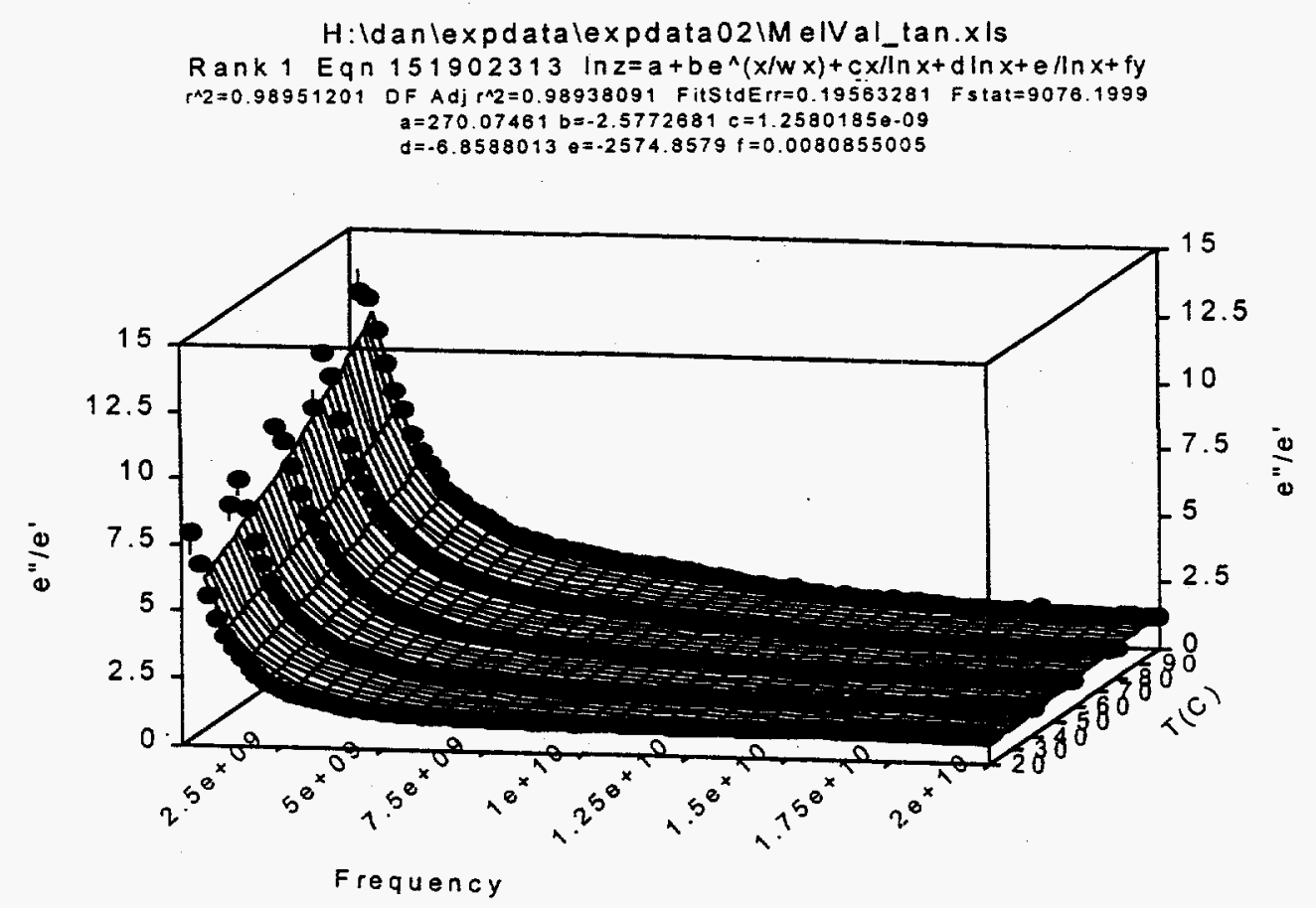

Figure 25. Correlation data for the Melton Valley simulant. 
$H:$ ld anlexpdatalexpdata $02 \mathrm{M}$ elV al ta $n \times x / s$

Rank 1 En 151902313 ln $z=a+b e^{\wedge}(x / w x)+c x / \ln x+d \ln x+e / \ln x+f y$

:2 $2=0.98951201$ DF Adj r"2=0.98938091 Fitstd Err $=0.19563281$ Fstat $=9076.1999$

$a=27007461 b=-2.5772531 c=1.2580185 e-09$

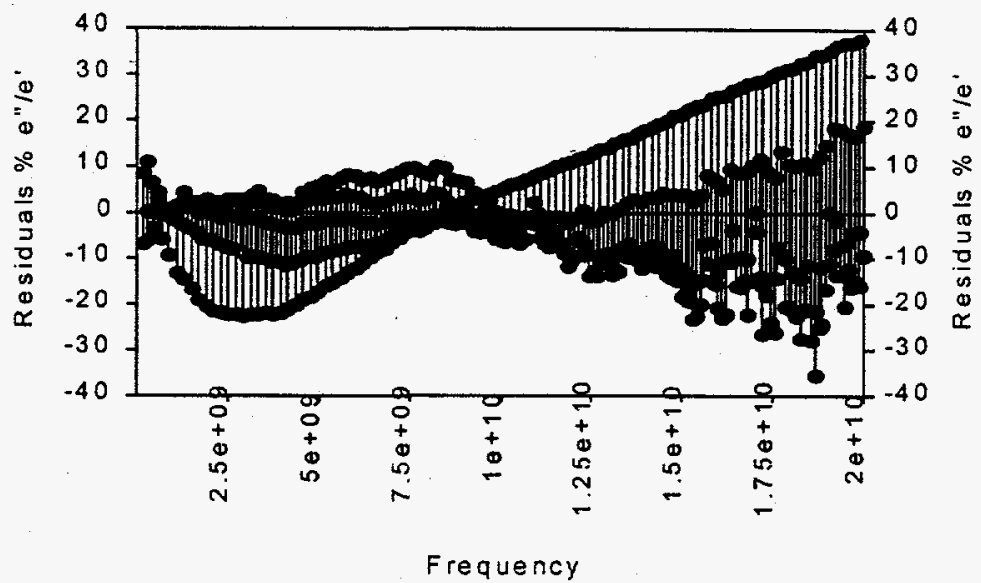

Figure 26. Residual percentage (\%'s) for the Melton Valley simulant. 


\subsubsection{SAIC Simulant}

Figures 27 and 28 present the correlation results of the loss tangent, tand. The residual distributions is quite even, most of them are within $\pm 20 \%$, as shown in Figure 28 .

$\mathrm{H}:$ dan $\backslash$ expdatalSAIC tan.xls

Rank 5 Eqn $312 z=a+b / x+c y+d / x^{\wedge} 2+$ ey $y^{\wedge} 2+f y / x+g / x^{\wedge} 3+h y^{\wedge} 3+i y^{\wedge} 2 / x+j y / x^{\wedge} 2$ $r^{\wedge} 2=0.96740625$ DF Adj $r^{\wedge} 2=0.96671862$ FitStdE $r=0.27706339$ Fstat $=1566.4828$ $a=1.2234916 b=3.2686078 e+09 c=-0.050087265 d=6.826625 e+17 e=0.0010858854$ $f=38555353 \quad g=-5.7553721 e+25 h=-6.3392515 e-06 \quad i=-159682.62 j=-1.6477636 e+16$

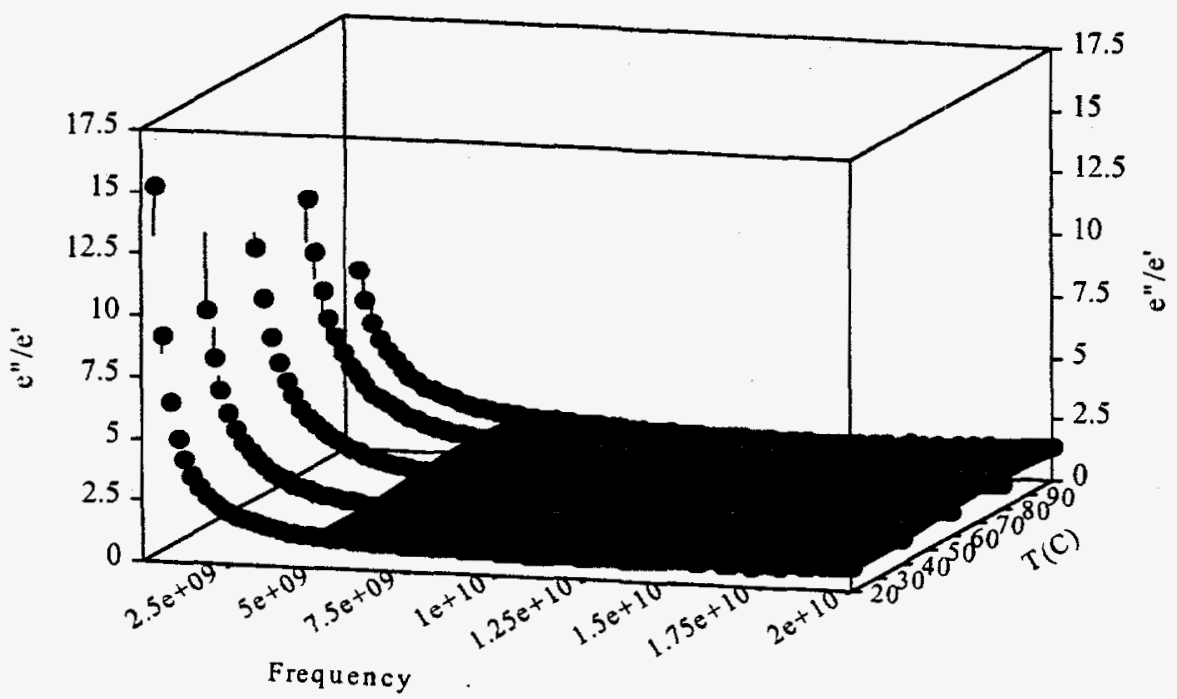

Figure 27. Correlation data for the SAIC simulant. 
HildaniexpdatalSAIC_tan.xis

Rank 5 Eqก $312 z=a+b / x+c y+d / x^{\wedge} 2+e y^{\wedge} 2+f y i x+g / x^{\wedge} 3+h y^{\wedge} 3+i y^{\wedge} 2 / x+j y / x^{\wedge} 2$ $r^{\wedge} 2=0.96740625$ DF Adi ${ }^{\wedge} 2=096671852$ FitSIdE $r=0.27706339$ Fs tat $=1560.4828$ $a=122349150=3.2686078 \mathrm{e}+09 \mathrm{c}=-0050087255 \mathrm{~d}=6.826625 \mathrm{e}+17 \mathrm{e}=0.0010858854$ $f=38555353 g=-5.7553721 e+23 h=-6.3392515 e-06 i=.159682 .62 j=-1.6477636 e+16$

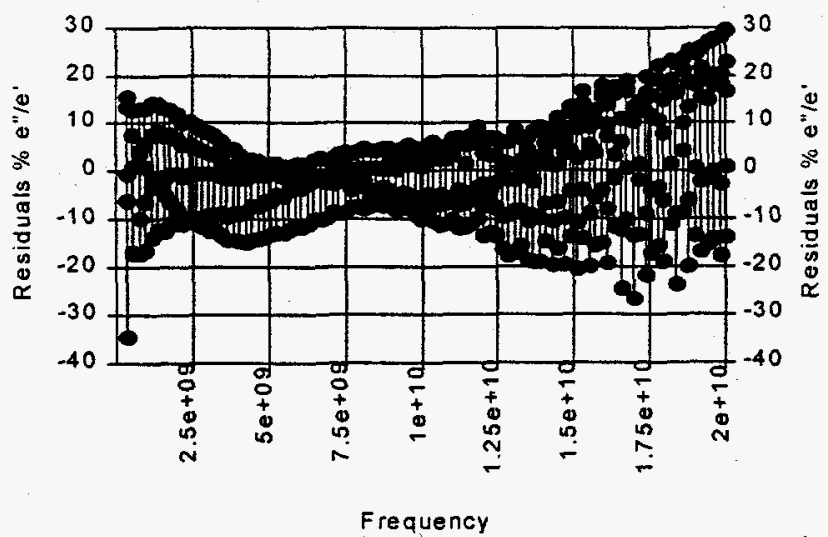

Figure 28. Residual percentage (\%) for SAIC simulant.

\subsubsection{Revised ORNL Simulant}

Figures 29 and 30 present the correlation results of the loss tangent, $\tan \delta$. The loss tangent residual percentages are shown in Figure 30; most of them are within $\pm 20 \%$.

H:Idanlexpdatalexpdata02IRevORNL_tan.xis

Rank 1 Eqn $317 z=a+b \ln x+c / y+d(\ln x)^{\wedge} 2+e / y^{\wedge} 2+f(\ln x) / y+g(\ln x)^{\wedge} 3+h / y^{\wedge} 3+i(\ln x) / y^{\wedge} 2+j(\ln x)^{\wedge} 2 / y$

$r=0.98429638$ OF Adj $m_{2}=0.98398347$ FitSidE $r=0.2748409$ F stat $=3322.017$

$a=-3079.1012 b=424.55782 \quad c=0777.7504 d=-19.255885 \quad \&=28461.427$

$\{=-740.87875 g=0.28803185 n=.3252 .4825 i=-1152.4983 j=19.137838$

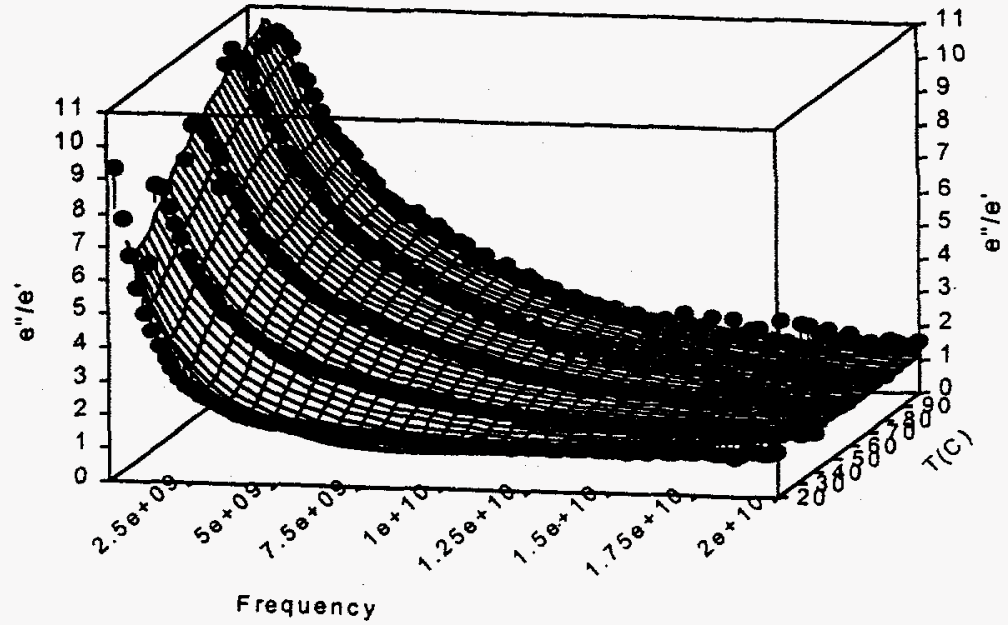

Figure 29. Correlation data for the Revised ORNL simulant. 
H:ldanlexpdatalexpdata02IReVORNL tan. $x$ 's

Rank 1 Ean $317 z=a+b \ln x+c / y+d(\ln x)^{\wedge} 2+e / y^{\wedge} 2+f(\ln x) / y+g(\ln x)^{\wedge} 3+h / y^{\wedge} 3+i(\ln x) / y^{\wedge} 2+j(\ln x)^{\wedge} 2 / y$ $r^{\wedge} 2=0.98429638$ DF Adj $r^{\wedge} 2=0.98396647$ FitStdE $T=0.2748409$ Fstai $=3322.017$ $a=3079.1012 b=424.55782 c=6777.7504 d=19.255865 e=28461.427$ $f=74057875 \mathrm{~g}=0.28803185 \mathrm{c}=-32524625 i-1162.4983 \mathrm{f}=10.137 \varepsilon 36$

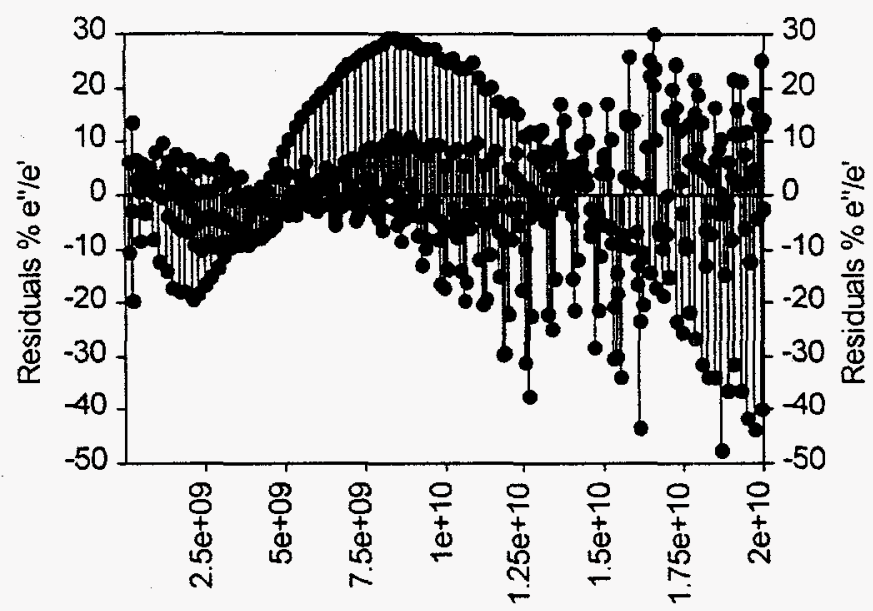

Frequency

Figure 30. The residual percentages (\%'s) for the Revised ORNL simulant.

\subsubsection{W-26 ORNL Simulant}

Figures 31 and 32 are the correlation results of the loss tangent, $\tan \delta$. Figure 32 shows the residual percentages of the loss tangent, most of which are within $\pm 20 \%$. 
H:Idanlexpdatalexpdata02lw26_tan.xis

Rank 1 Eqn $1303 z=\left(a+c \ln x+e y+g(\ln x)^{\wedge} 2+i y^{\wedge} 2+k y \ln x\right) /\left(1+b \ln x+d y+f(\ln x)^{\wedge} 2+h y^{\wedge} 2+j y \ln x\right)$ $r * 2=0.9870617$ OF Adj $r_{2}=0.98678208$ FitStdErr $=0.35594882$ Fstat $=3631.4004$

$a=2.4435891 \quad b=0.098267834 \quad c=-0.2208818 d=0.0009688775 \theta=0.0055026321 \quad f=0.0023322573$

$g=0.0050325871 \mathrm{~h}=1.1617444 \theta-07 \mathrm{i}=1.8833406 \mathrm{e} \cdot 06 \mathrm{i}=-4.776237 \mathrm{e}-0.0 \mathrm{k}=-0.00024725254$

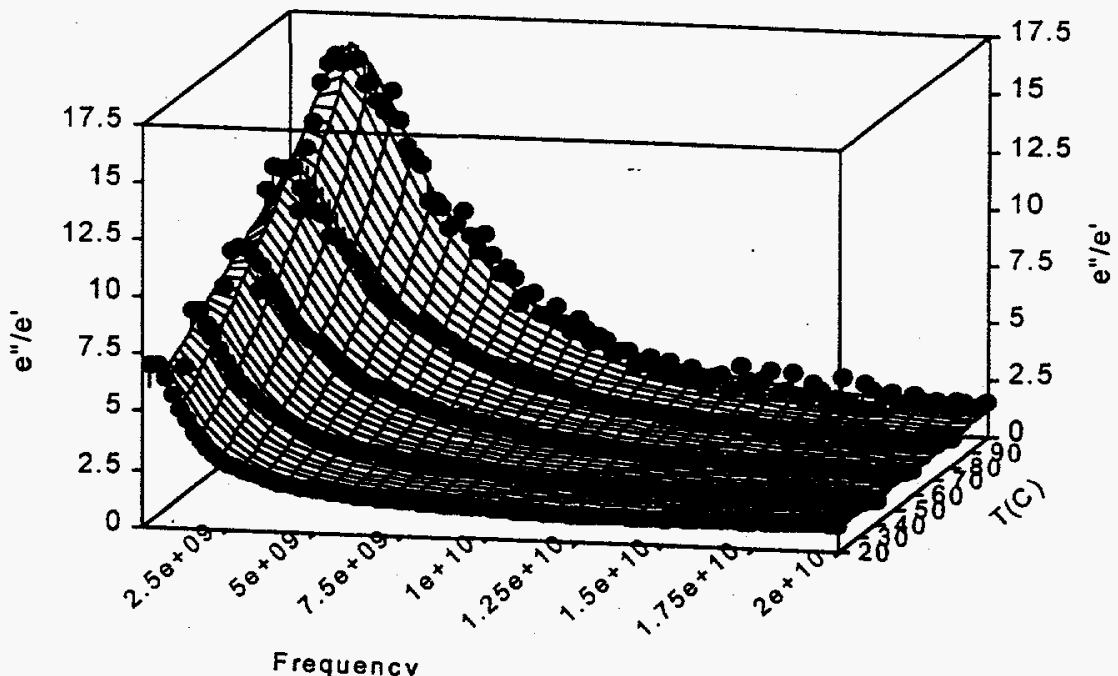

Figure 31. Correlation data for the W-26 ORNL simulant.

H: Idanlexpdatalexpdata02lw26 tan.xls

Rank 1 Eqn $1303 z=\left(a+c \ln x+e y+g(\ln x)^{\wedge} 2+i y^{\wedge} 2+k y \ln x\right) /\left(1+b \ln x+d y+f(\ln x)^{\wedge} 2+h y^{\wedge} 2+j y \ln x\right)$ $r^{\wedge} 2=0.9870617$ DF Adj $r^{\wedge} 2=0.98676208$ FitStdErr $=0.35594882$ Fstat $=3631.4004$ $a=2.4435891 b=-0.096267834 \quad c=-0.2208618 d=0.0009688775 \quad e=0.0055026321 f=0.0023322573$ $\mathrm{g}=0.0050325871 \mathrm{~h}=1.1617444 \mathrm{e}-07 \mathrm{i}=1.8833406 \mathrm{e}-06 \mathrm{j}=-4.776232 \mathrm{e}-05 \mathrm{k}=-0.00024782524$

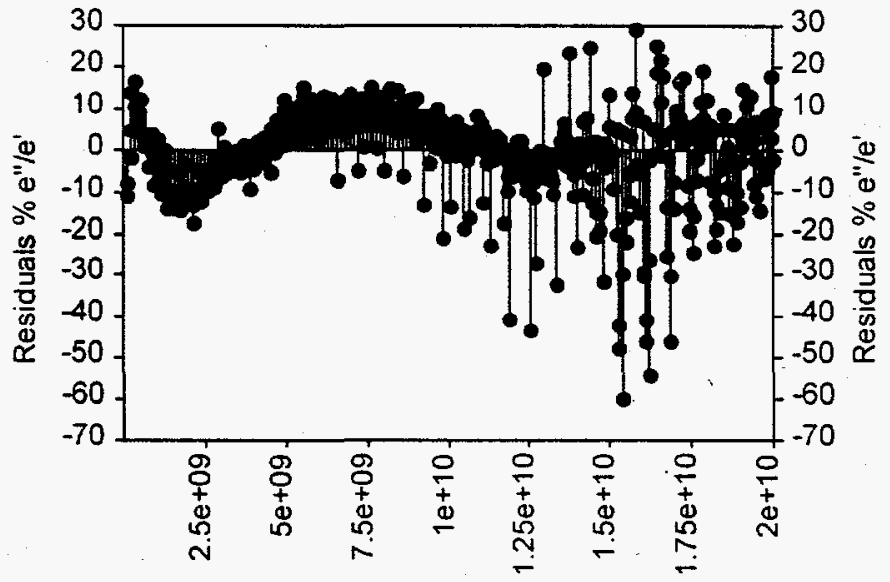

Frequency

Figure 32. Residual percentage (\%s) for the W-26 ORNL simulant. 


\subsubsection{Savannah River Simulant}

Figures 33 and 34 present the correlation results of the loss tangent, $\tan \delta$. Figure 34 shows the loss tangent correlated residual percentage results, most of which are within $\pm 5 \%$.

H:ldanlexpdatalexpdata021SavRiv_tan. $x$ is

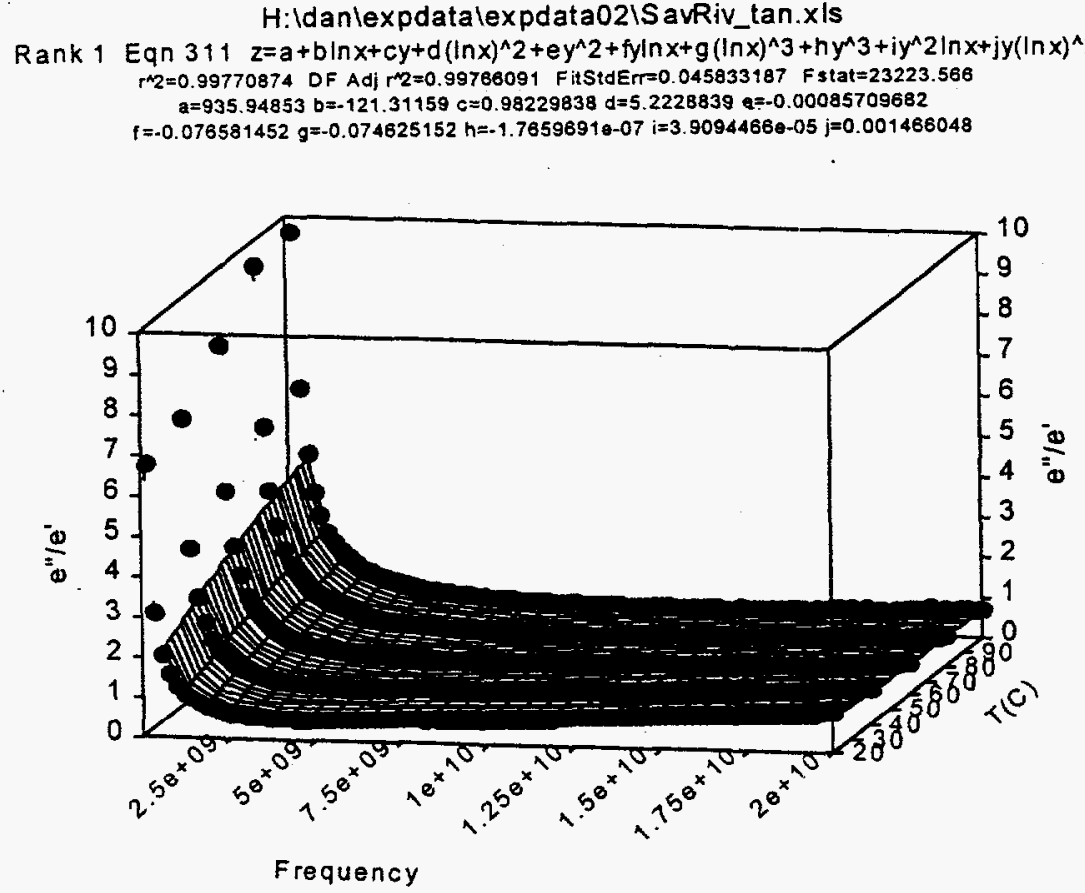

Figure 33. Correlation data for the Savannah River simulant. 
H:Idanlexpdatalexpdata02ISavRiv_tan.xis

Rank 1 Eqn $311 z=a+b \ln x+c y+d(\ln x)^{\wedge} 2+e y^{\wedge} 2+f y \ln x+g(\ln x)^{\wedge} 3+h y^{\wedge} 3+i y^{\wedge} 2 \ln x+j y(\ln x)^{\wedge} 2$ $r^{\prime 2} 2=0.99770874$ DF Adj $r^{\wedge} 2=0997660$ S: FitSidErr=0.04583312i Fstat=23223.566

$a=93594853 b=-12131159 c=0.982298338 d=52223339 \theta=-0.00085709682$

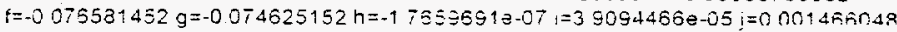

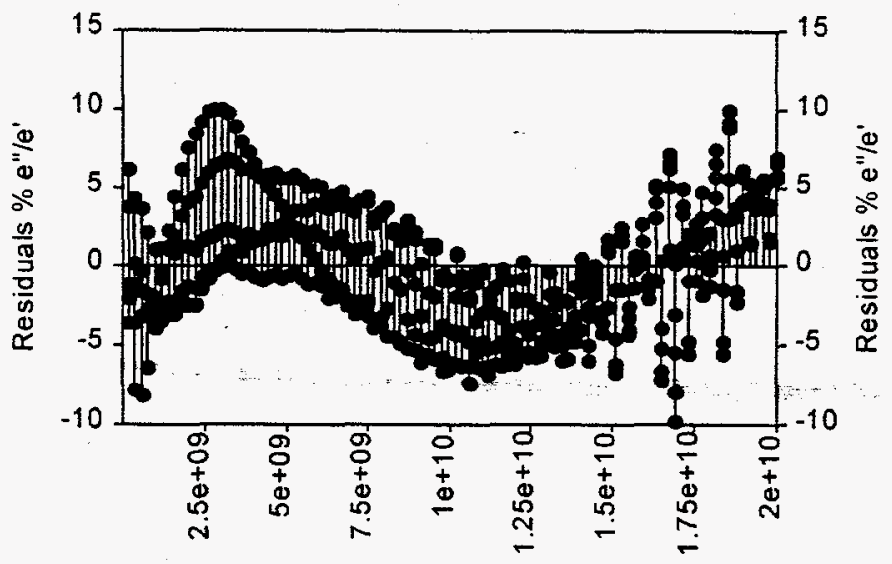

Frequency

Figure 34. Residual percentage (\%s) for the Savannah River simulant. 


\section{CONCLUDING REMARKS}

The correlated equations with its coefficients, and the residuals are included in this analysis. It can be seen that the determination standards $r^{2}$, Degree of Freedom Adjusted $r^{2}$, and the Fit Statistic are quite high, the correlated equations for different simulants can be used to predict the dielectric properties for the measured low-level liquid waste (LLLW) within the frequency range from $0.1 \sim 20 \mathrm{GHz}$ and temperature range from $20 \sim 100^{\circ} \mathrm{C}$. The correlation equation for the loss tangent are provided in Table 8 . Here, the $\mathrm{x}$ axis is represented by the frequency, the $\mathrm{y}$ axis is represented by the temperature and the $\mathrm{z}$ axis gives the desired loss tangent value. The coefficient for these equations can be obtained from the figures presented in section 5.2 (Figures 19 through 34). Similar equations are presented for the dielectric constant and the dielectric loss, these equations and constants are given with each graph in Appendix C.

Table 8.

Correlation Equations for the Loss Tangent

\begin{tabular}{|l|c|c|}
\hline Simulants & $\begin{array}{c}\text { Correlation Equations for the Loss Tangent } \\
\text { Hanford 4 Molar Simulant }\end{array}$ & $\begin{array}{c}z=a+b / x+c y+d / x^{2}+e y^{2}+f y / x \\
+g / x^{3}+h y^{3}+i y^{2} / x+j y / x^{2}\end{array}$ \\
\hline Hanford 6 Molar Simulant & $\begin{array}{c}z=a+b / x+c y+d / x^{2}+e y^{2}+f y / x \\
+g / x^{3}+h y^{3}+i y^{2} / x+j y / x^{2}\end{array}$ & 21 \\
\hline $\begin{array}{l}\text { Hanford 4 and 6 Molar } \\
\text { Simulant }\end{array}$ & $\begin{array}{c}z=a+b / x+c y+d / x^{2}+e y^{2}+f y / x \\
+g / x^{3}+h y^{3}+i y^{2} / x+j y / x^{2}\end{array}$ & 23 \\
\hline Melton Valley Simulant & $\begin{array}{c}\ln z=a+b e^{(x / w x)}+c x / \ln x+d \ln x \\
+e / \ln x+f y\end{array}$ & 25 \\
\hline SAIC Simulant & $\begin{array}{c}z=a+b / x+c y+d / x^{2}+e y^{2}+f y / x \\
+g / x^{3}+h y^{3}+i y^{2} / x+j y / x^{2}\end{array}$ \\
\hline Revised ORNL Simulant & $\begin{array}{c}z=a+b \ln x+c / y+d(\ln x)^{2}+e / y^{2}+f(\ln x) / y \\
+g(\ln x)^{3}+h / y^{3}+i(\ln x) / y^{2}+j(\ln x)^{2} / y\end{array}$ & 29 \\
\hline W-26 ORNL & $\begin{array}{c}z=\left(a+c \ln x+e y+g(\ln x)^{2}+i y^{2}+k y \ln x\right) / \\
\left(1+b \ln x+d y+f(\ln x)^{2}+h y^{2}+j y \ln x\right)\end{array}$ & 31 \\
\hline Savannah River Simulant & $\begin{array}{c}z=a+b \ln x+c y+d(\ln x)^{2}+e y^{2}+f y \ln x \\
+g(\ln x)^{3}+h y^{3}+i y^{2} \ln x+j y(\ln x)^{2}\end{array}$ & 33 \\
\hline
\end{tabular}


The dielectric constants contained in this study were measured over a temperature range from $20^{\circ}$ to $100^{\circ} \mathrm{C}$ at increments of twenty degrees. Testing has shown, as expected, that the water contained in the simulants is driven off before the temperature of $120^{\circ} \mathrm{C}$ is reached and that the remaining material is in the form of either solids or melted compounds mixed with high melting solids. A continuation of this study would involve measurements up to $200^{\circ} \mathrm{C}$, the upper limit of the instrument employed. To carry this out, an oven would have to be incorporated into the setup to replace the ethylene glycol bath to achieve reliable temperature control in the samples.

Attempts were made to obtain measurements over the originally chosen frequency range $(0.1$ $20.0 \mathrm{GHz})$ in two steps, one at a low range $(0.1-4.0 \mathrm{GHz})$ and one at the higher range $(4.0-20.0$ $\mathrm{GHz}$ ), in order to be able to produce a larger number of data points and therefore more detailed information about the behavior of the dielectric properties in each range. This approach led to problems because it proved to be impossible to control the loss of moisture due to the evaporation that occurred between the two steps. The measurements appear to be very sensitive to moisture content. 


\section{REFERENCES}

Athey, T.W., Stuchly, M.A. and Stuchly, S.S., "Measurement of Radio Frequency Permitivity of Biological Tissue with an Open-Ended Coaxial Line: Part I," IEEE Transactions on Microwave Theory and Techniques, Vol. MTT-30, 1982, pp. 82-86.

Franceschetti, G. "A Complete Analysis of the Reflection and Transmission Methods for Measuring the Complex Permeability and Permitivitty of Materials at Microwaves," Alta Frequenza, Vol. 36, 1967, pp. 757-764.

Jandel Scientific, TableCurve 3D, Vol. 1, User's Manual, San Rafael, CA, 1993.

Piotrowski, M. "Resonance Method for the Decontamination of the Complex Dielectric Constant of Biologic Materials in the Microwave Band," in Biologic Effects and Health Hazards of Microwave Radiation, Polish Medical Publisher, Warsaw, 1974, pp. 243-253.

Stogryn, A. "Equations for Calculating the Dielectric Constant of Saline Water," IEEE Transactions on Microwave Theory and Techniques, Vol. MTT-19, 1971, pp. 733-736.

Stuchly, M.A. and Stuchly, S.S., "Coaxial Line Reflection Methods for Measuring Dielectric Properties of Biological Substances at Radio and Microwave Frequencies -- A Review," IEEE Transactions on Instrumentation and Measurement, Vol. IM-29, 1980, pp. 176183.

Von Hippel, A.R., (ed.), Dielectric Materials and Applications, MIT Press, Cambridge, Massachusetts, 1954.

White, T.L., Youngblood, E.L., Berry, J.B., and Mattus, A.J., First Results of In-Can Microwave Processing Experiments for Radioactive Liquid Wastes at the Oak Ridge National Laboratory, Materials Research Society Spring Meeting, San Francisco, California, April 16-21, 1990. 


\section{DIELECTRIC CONSTANT AND \\ DIELECTRIC LOSS VERSUS FREQUENCY GRAPHS}




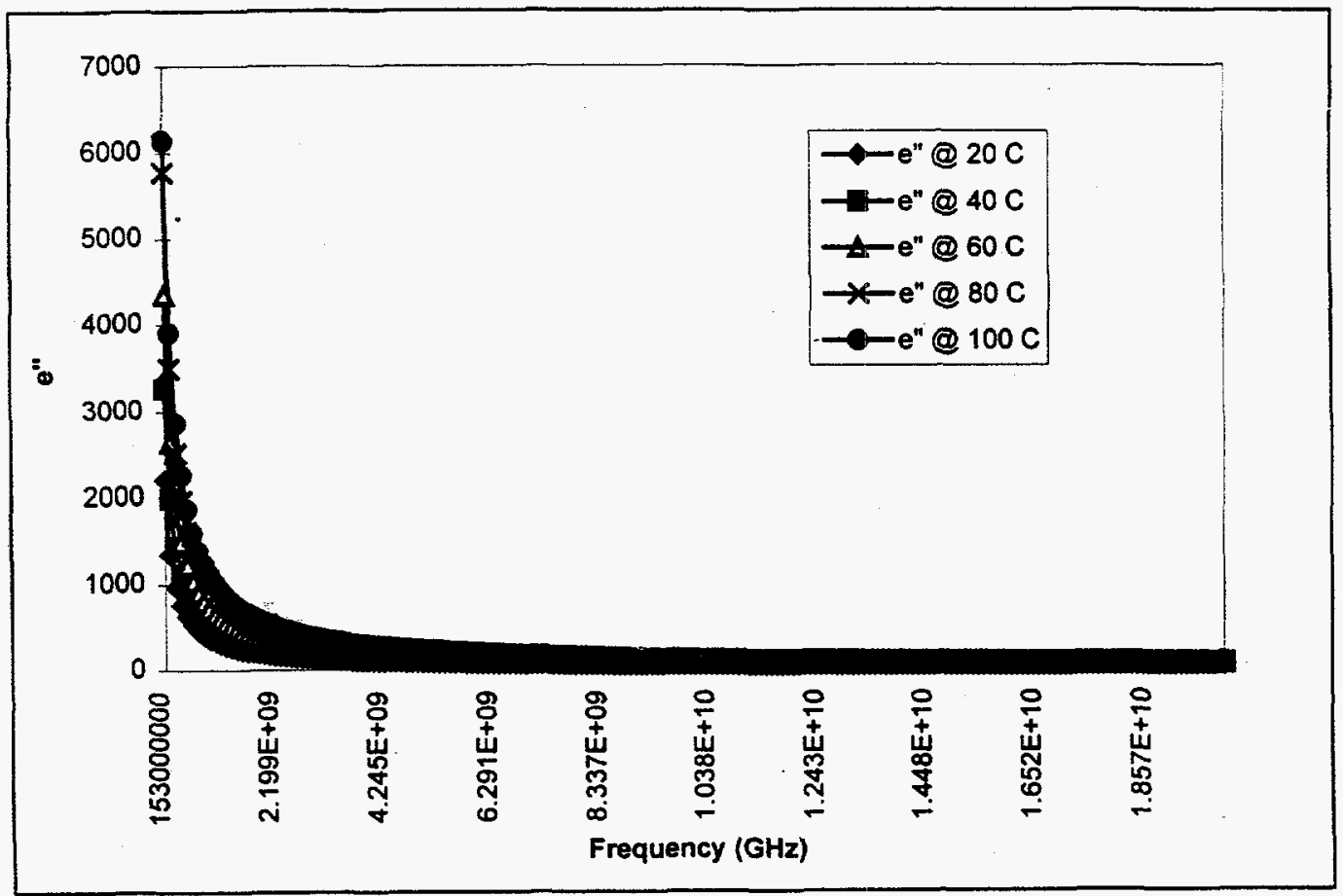

Figure A.1.a. Dielectric loss vs. frequency for the Hanford 4 molar simulant. Full scale.

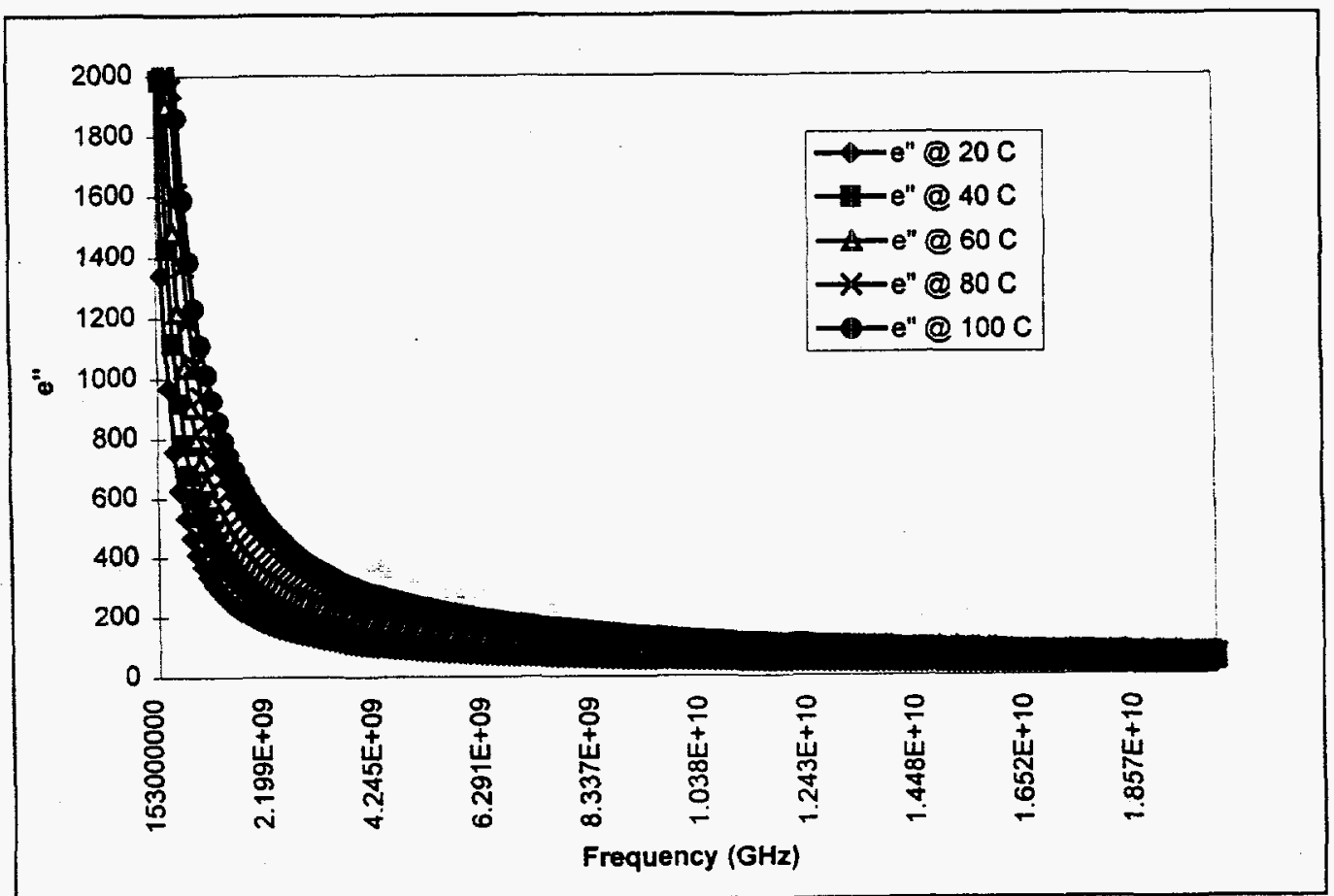

Figure A.1.b. Dielectric loss vs. frequency for the Hanford 4 molar simulant. Scaled down. 


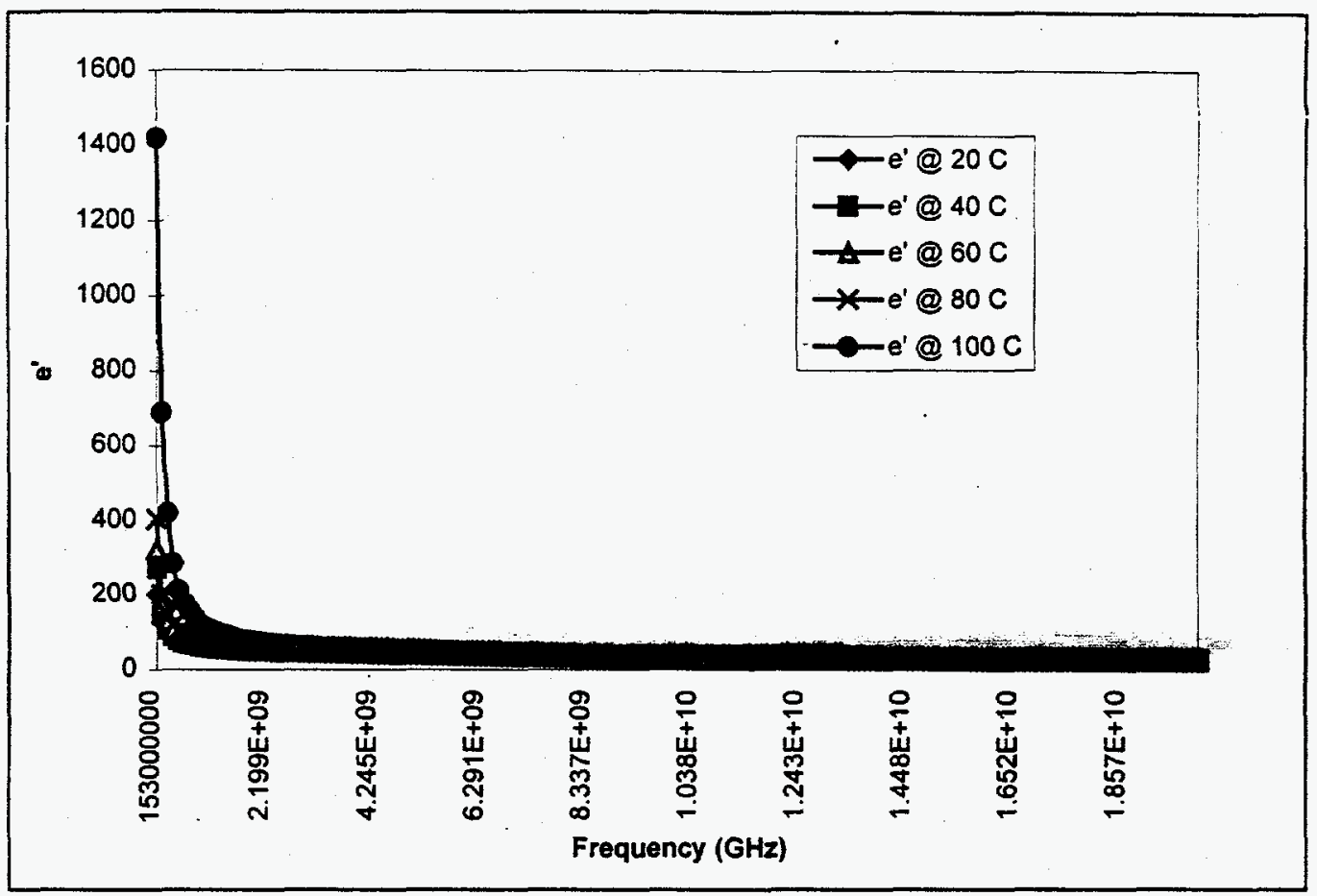

Figure A.1.c. Dielectric constant vs. frequency for the Hanford 4 molar simulant. Full scale.

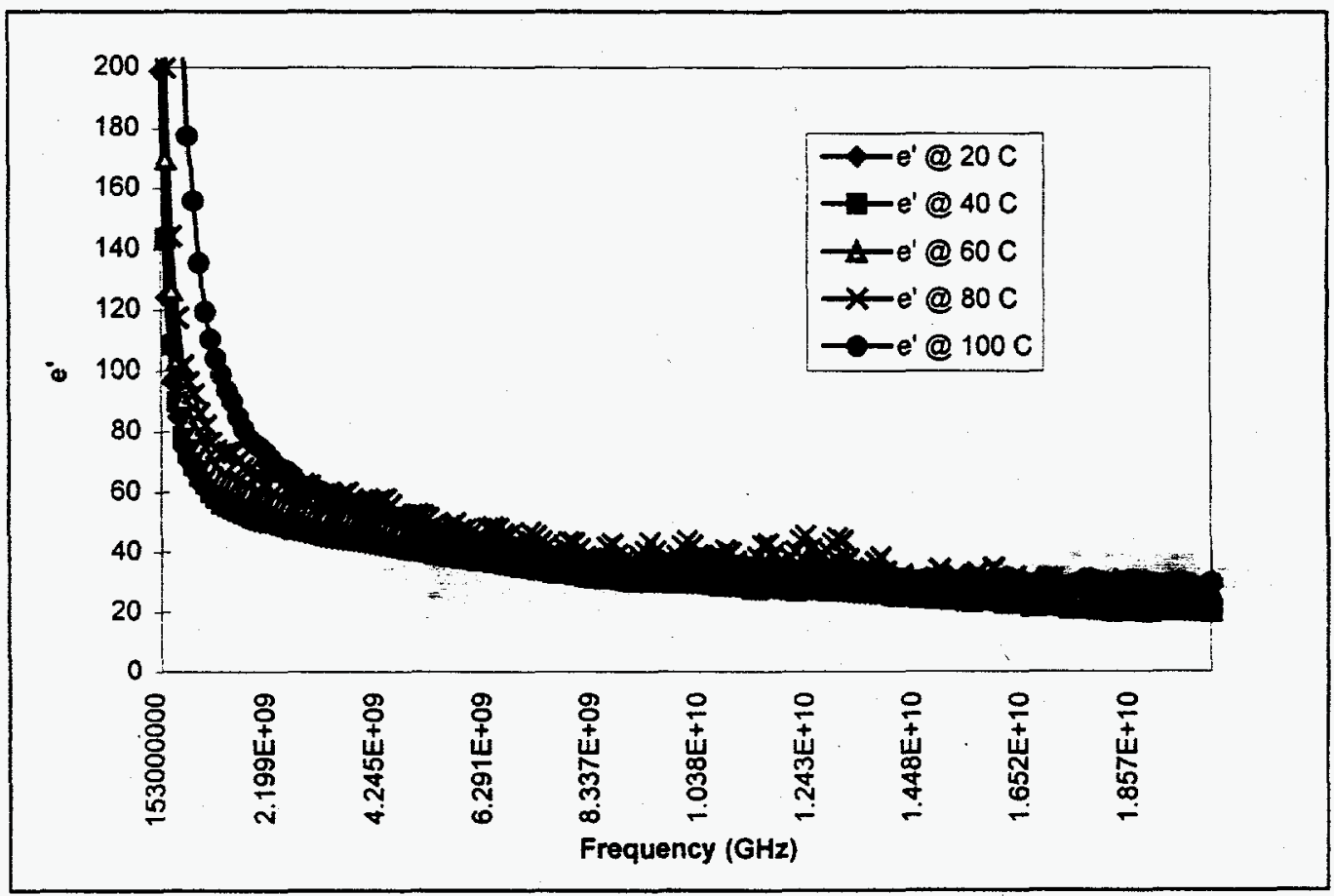

Figure A.1.d. Dielectric constant vs. frequency for the Hanford 4 molar simulant. Scaled down. 


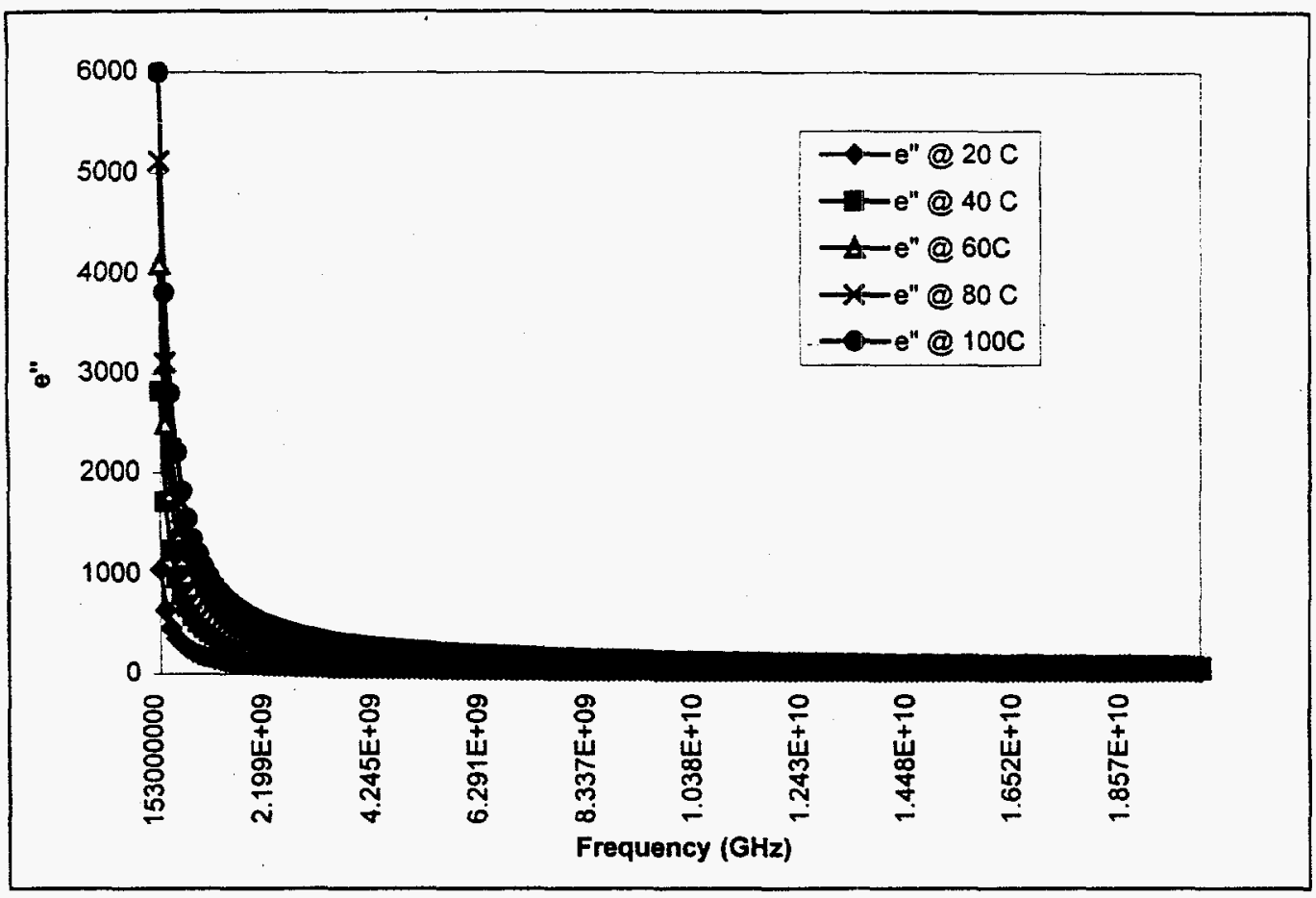

Figure A.2.a. Dielectric loss vs. frequency for the Hanford 6 molar simulant. Full scale.

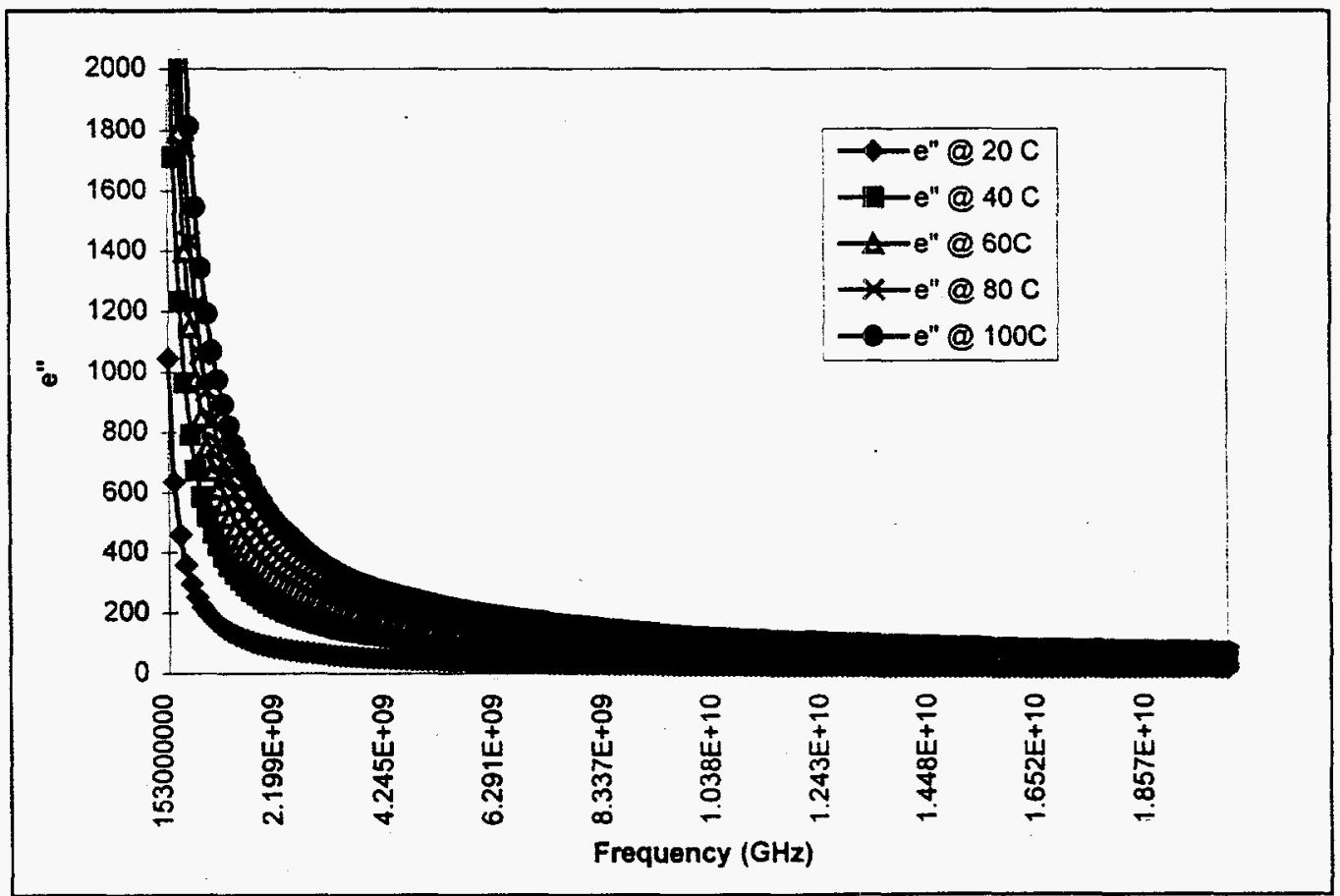

Figure A.2.b. Dielectric loss vs. frequency for the Hanford 6 molar simulant. Scaled down. 


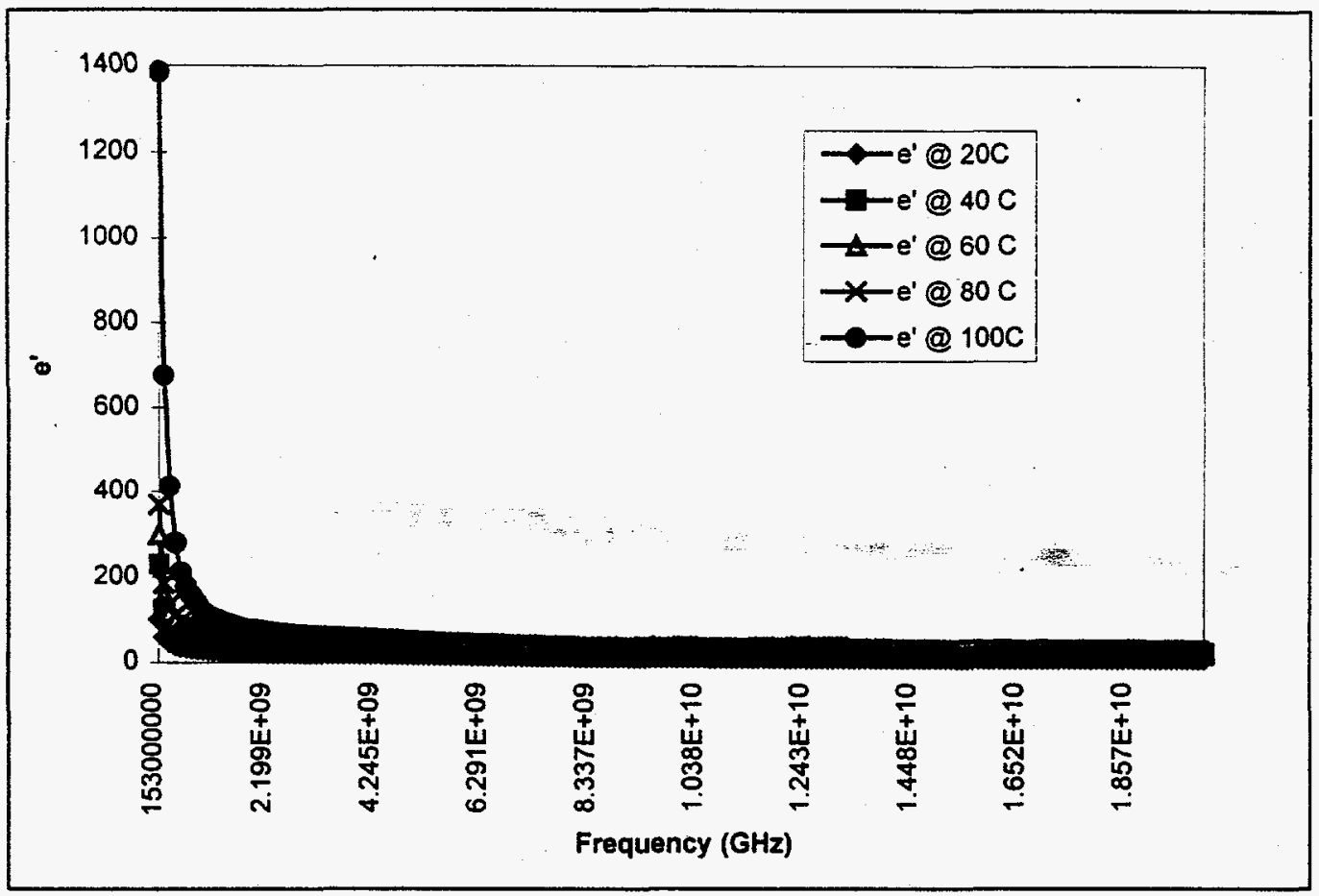

Figure A.2.c. Dielectric constant vs. frequency for the Hanford 6 molar simulant. Full scale.

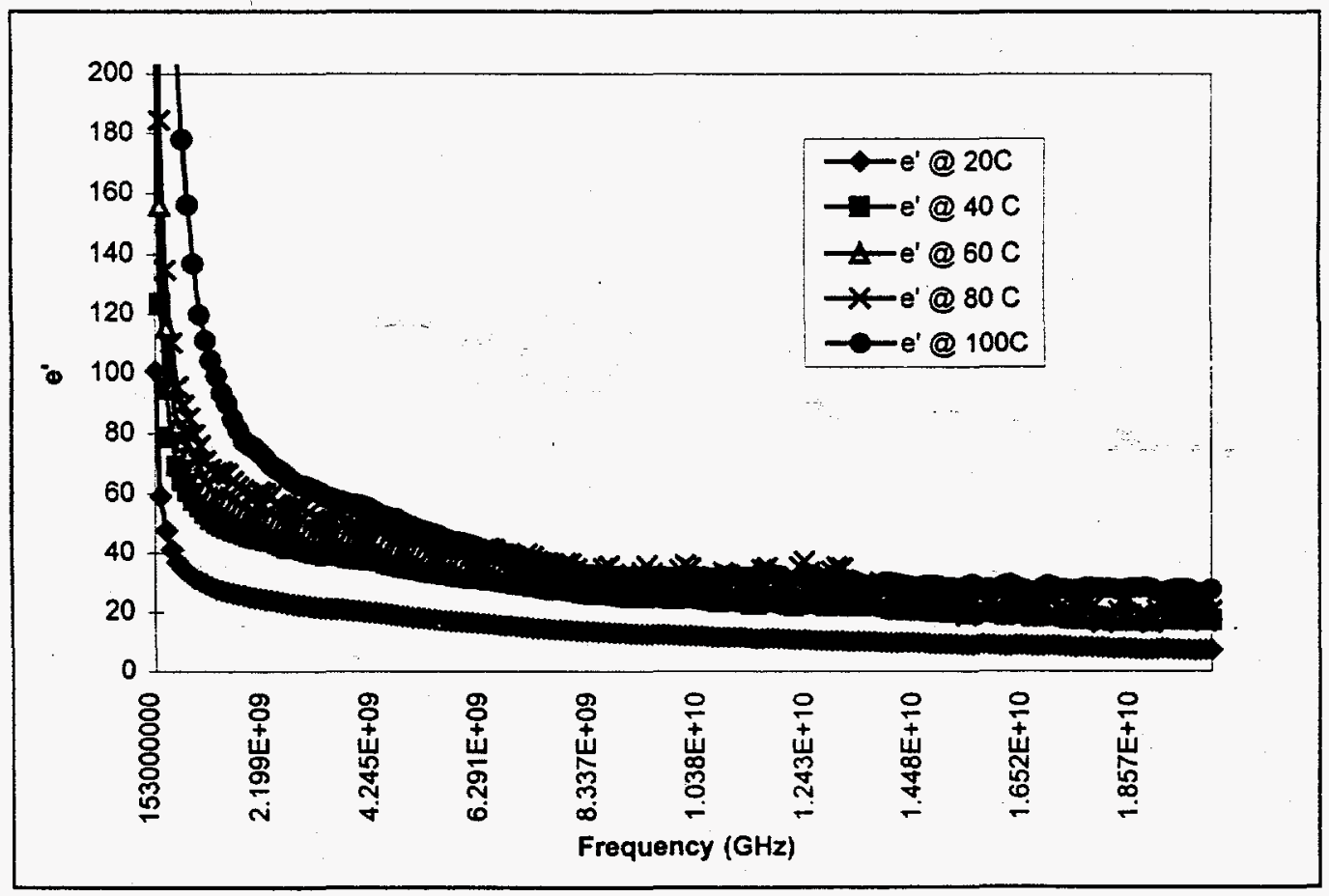

Figure A.2.d. Dielectric constant vs. frequency for the Hanford 6 molar simulant. Scaled down. 


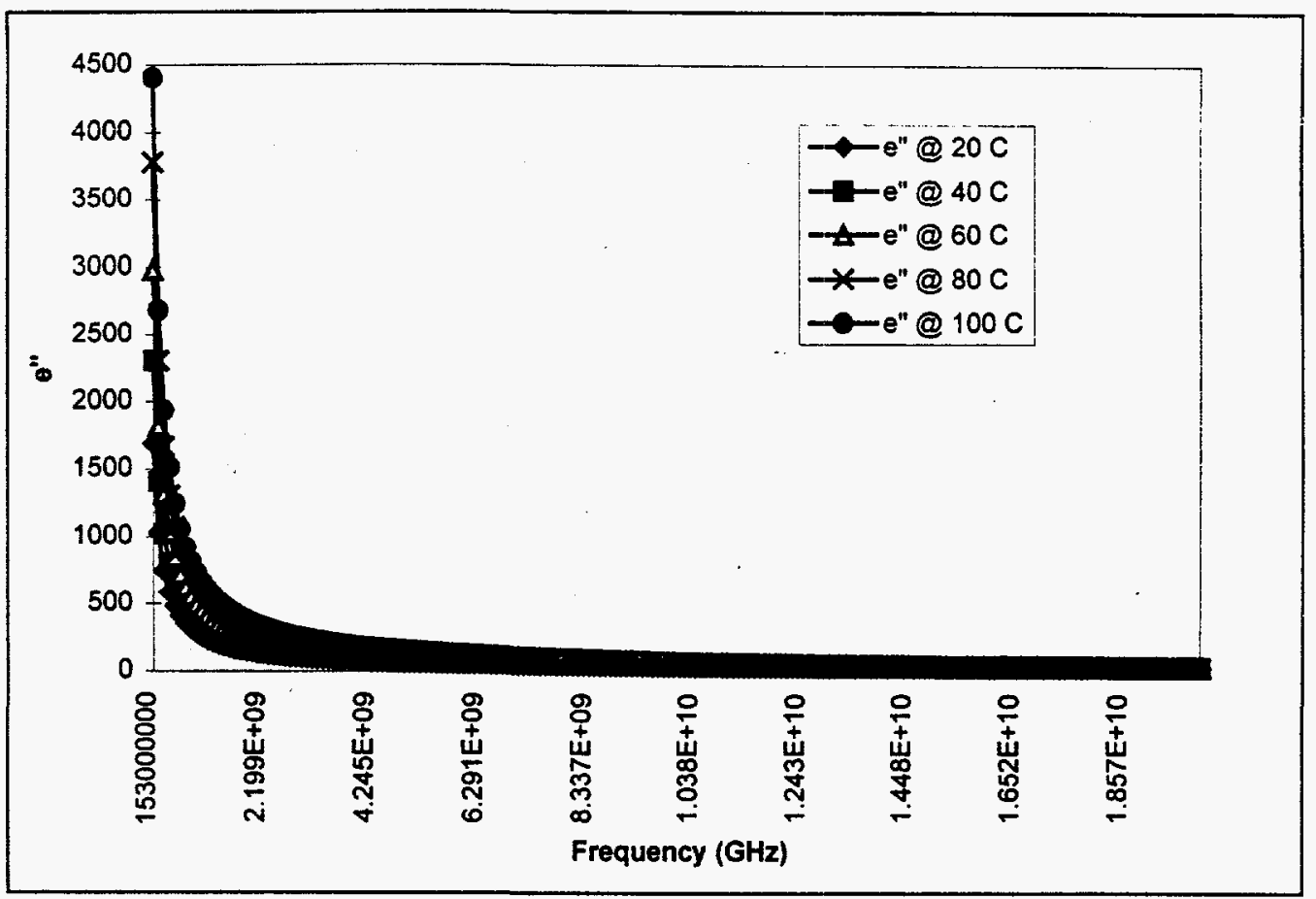

Figure A.3.a. Dielectric loss vs. frequency for the Melton Valley simulant. Full scale.

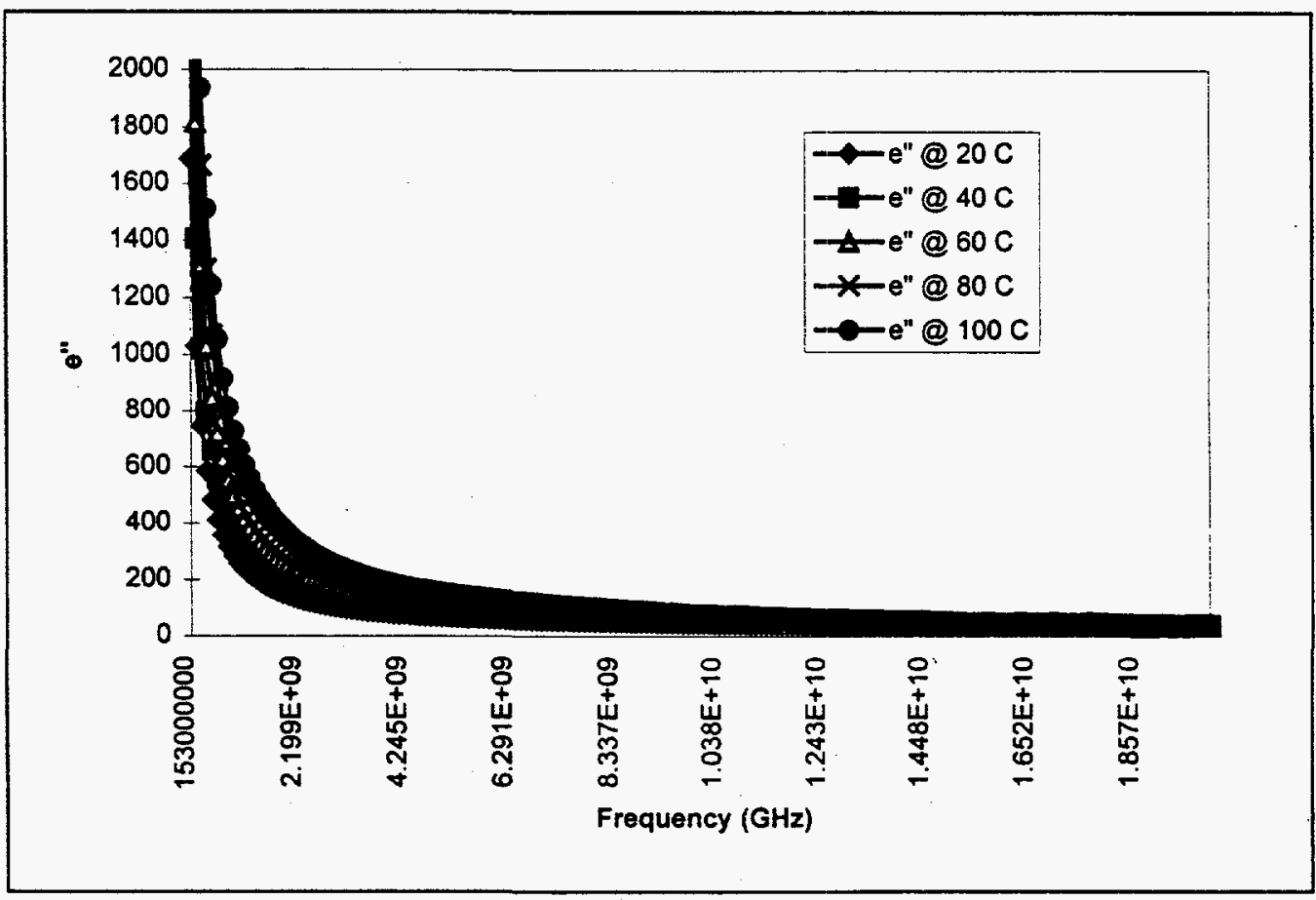

Figure A.3.b. Dielectric loss vs. frequency for the Melton Valley simulant. Scaled down. 


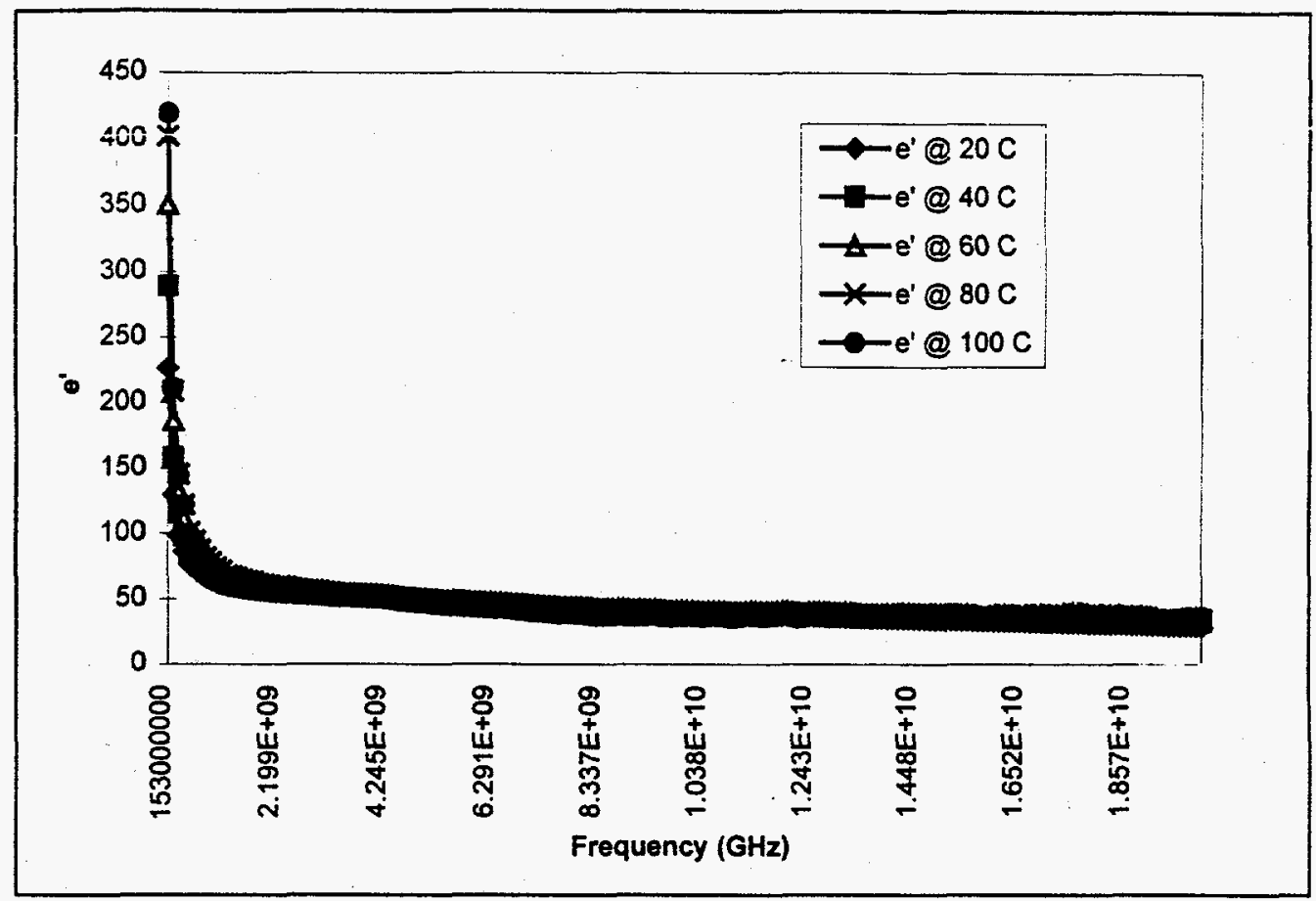

Figure A.3.c. Dielectric constant vs. frequency for the Melton Valley simulant. Full scale.

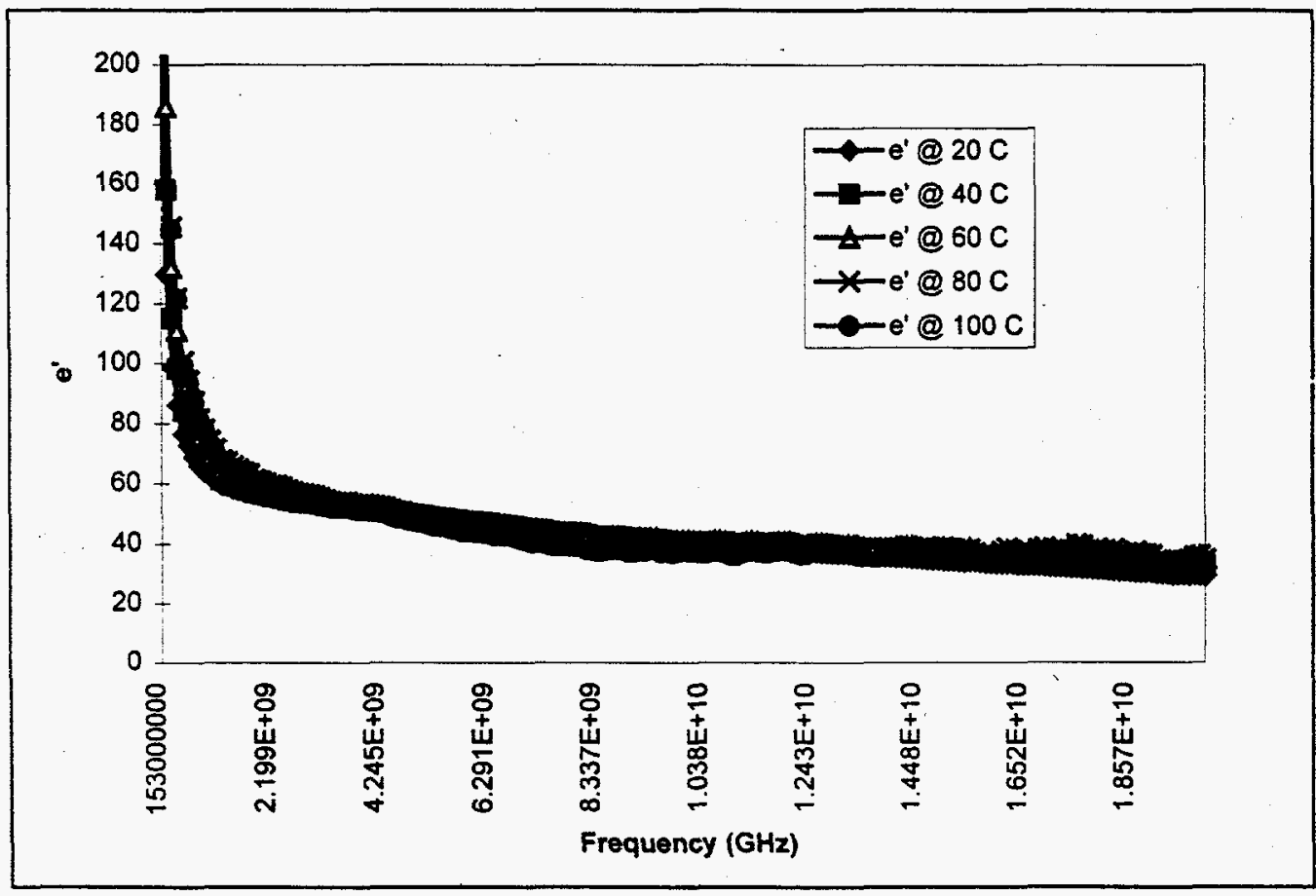

Figure A.3.d. Dielectric constant vs. frequency for the Melton Valley simulant. Scaled down. 


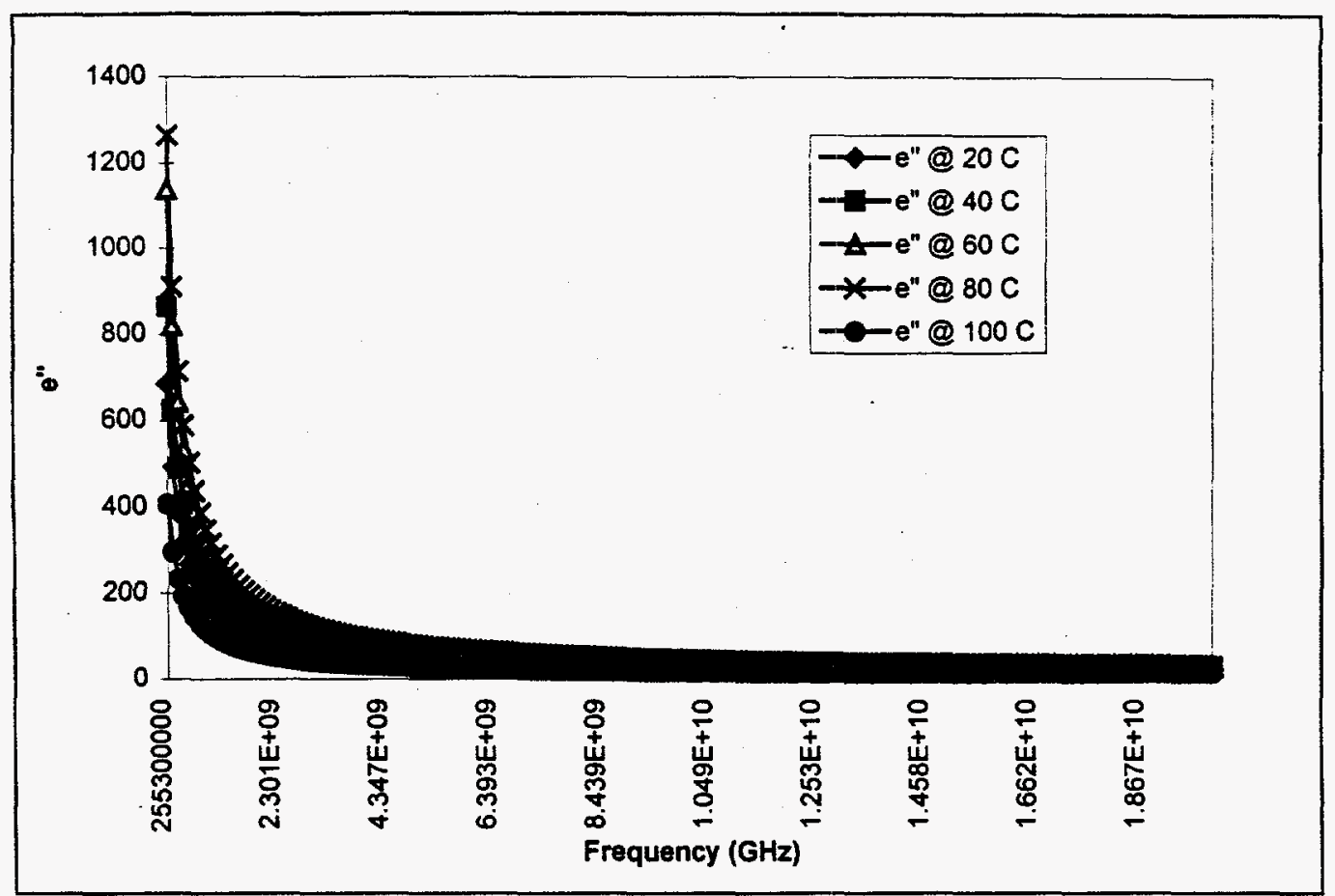

Figure A.4.a. Dielectric loss vs. frequency for the SAIC simulant. Full scale.

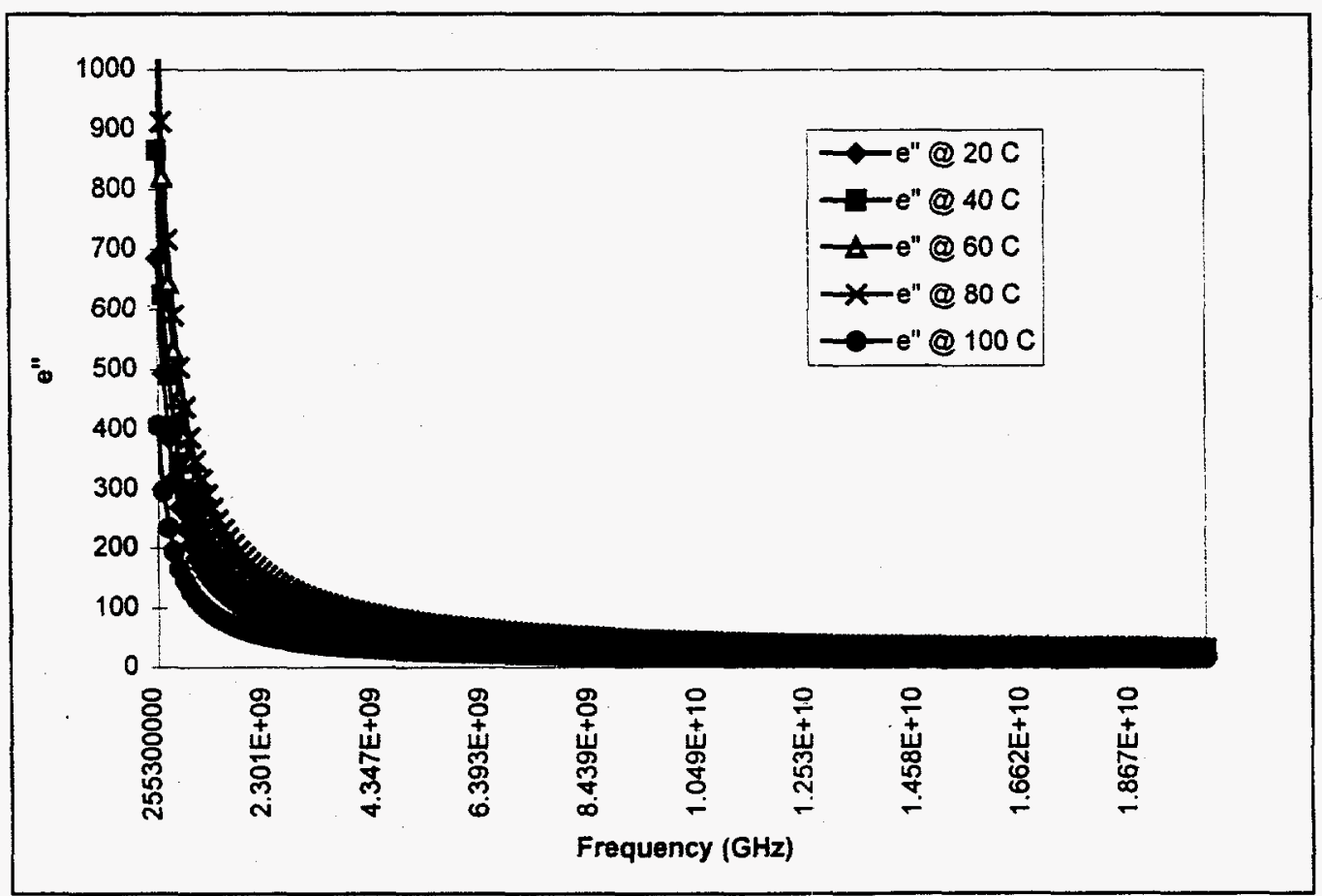

Figure A.4.b. Dielectric loss vs. frequency for the SAIC simulant. Scaled down. 


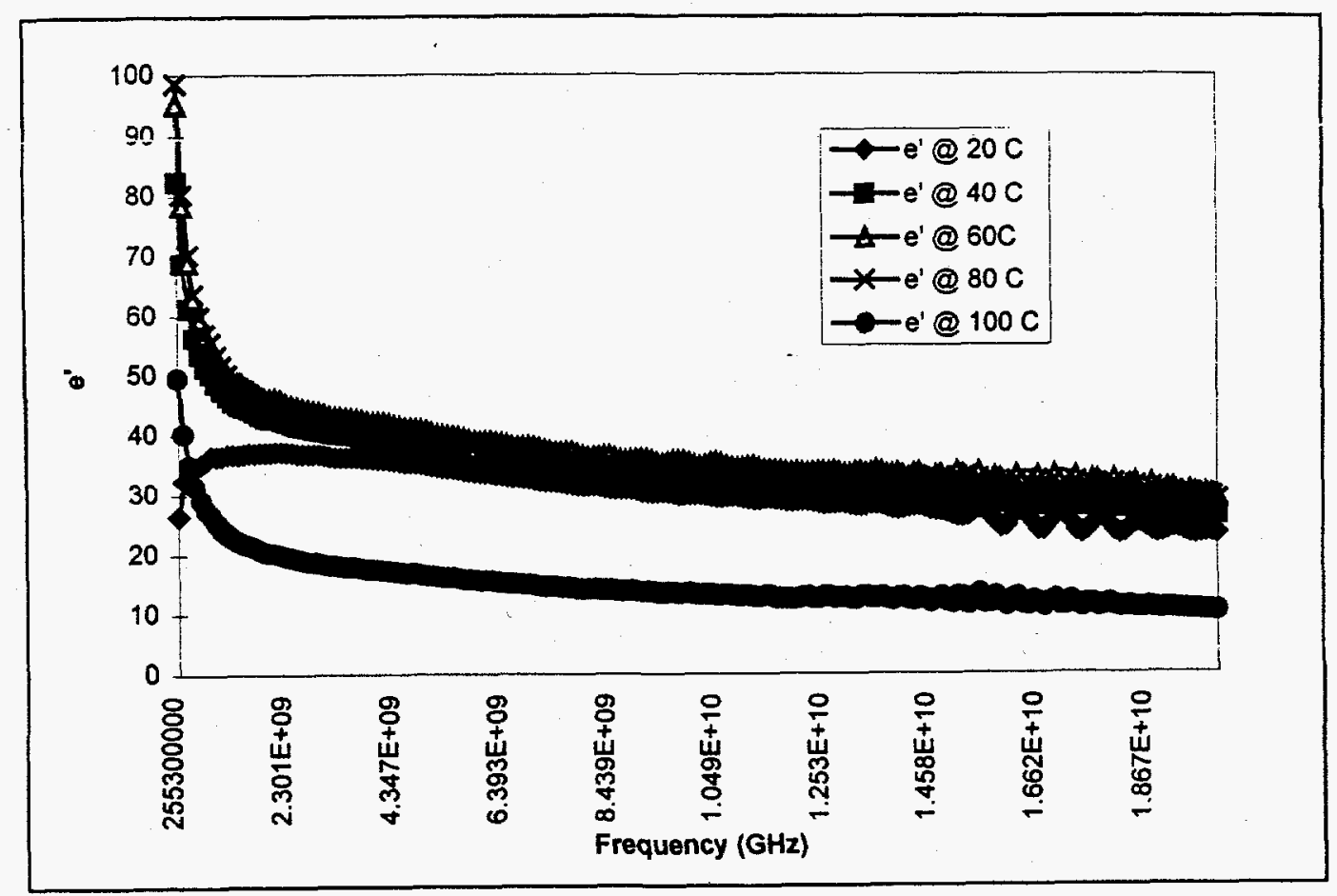

Figure A.4.c. Dielectric constant vs. frequency for the SAIC simulant. Full scale.

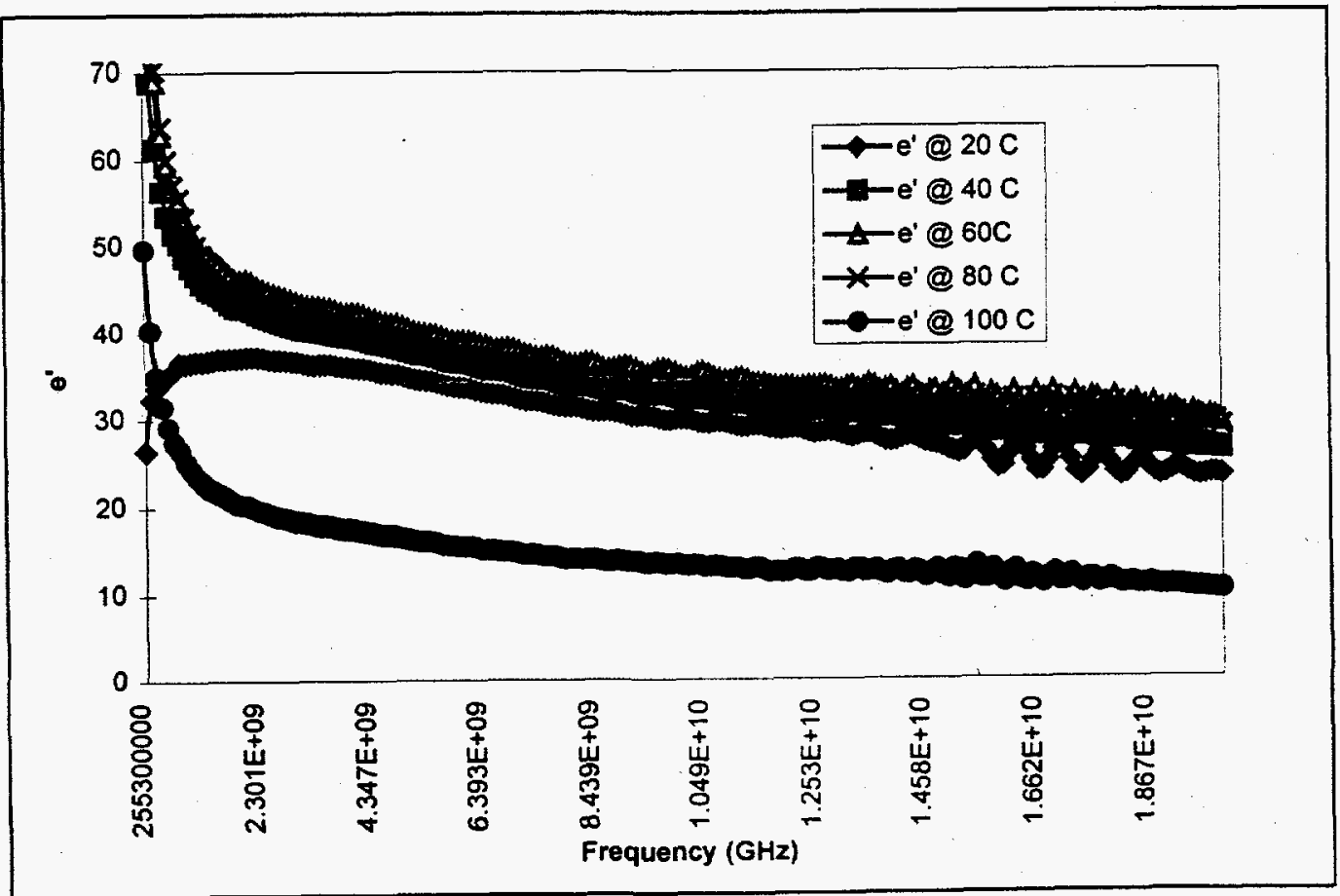

Figure A.4.d. Dielectric constant vs. frequency for the SAIC simulant. Scaled down. 


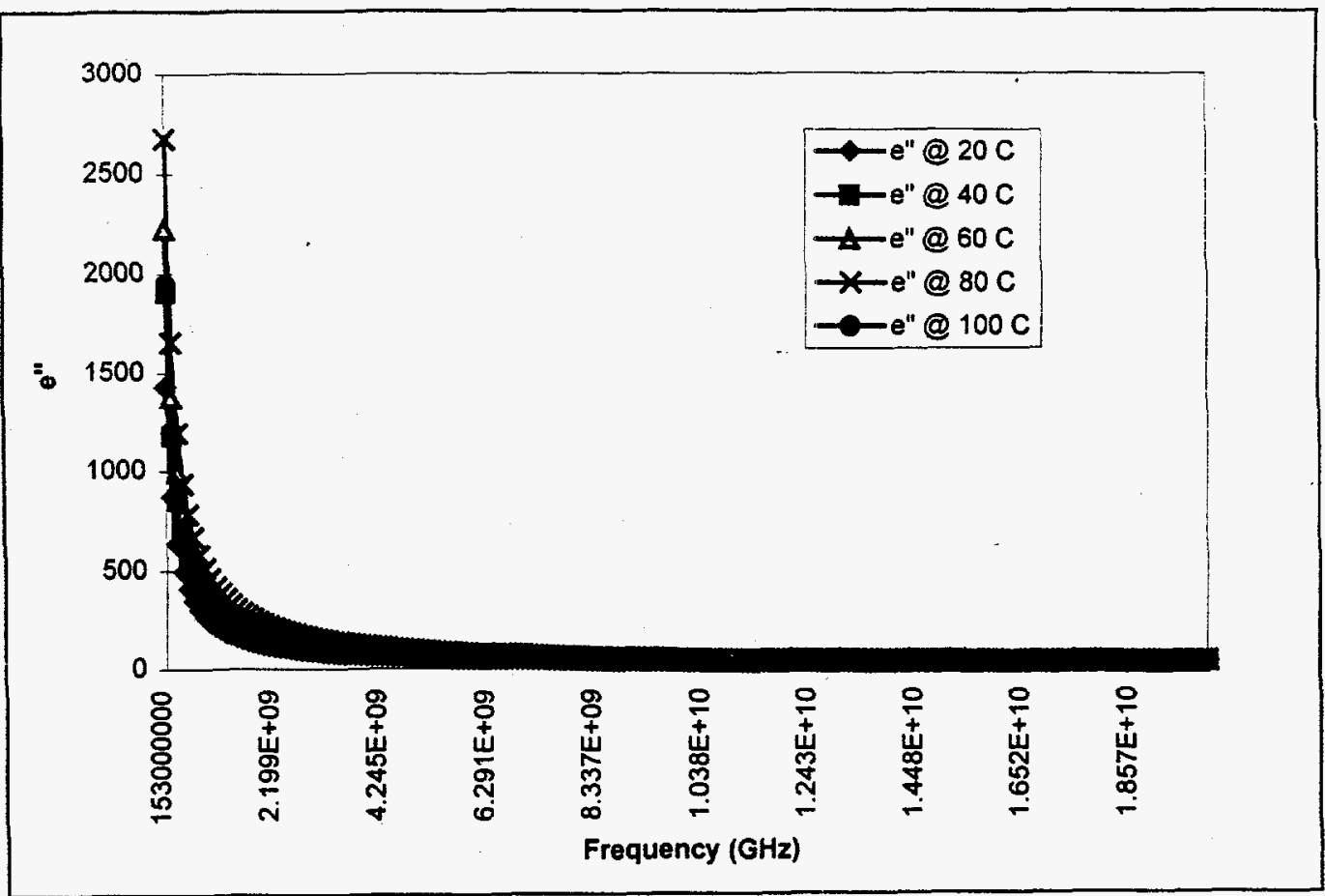

Figure A.5.a. Dielectric loss vs. frequency for the Revised ORNL simulant. Full scale.

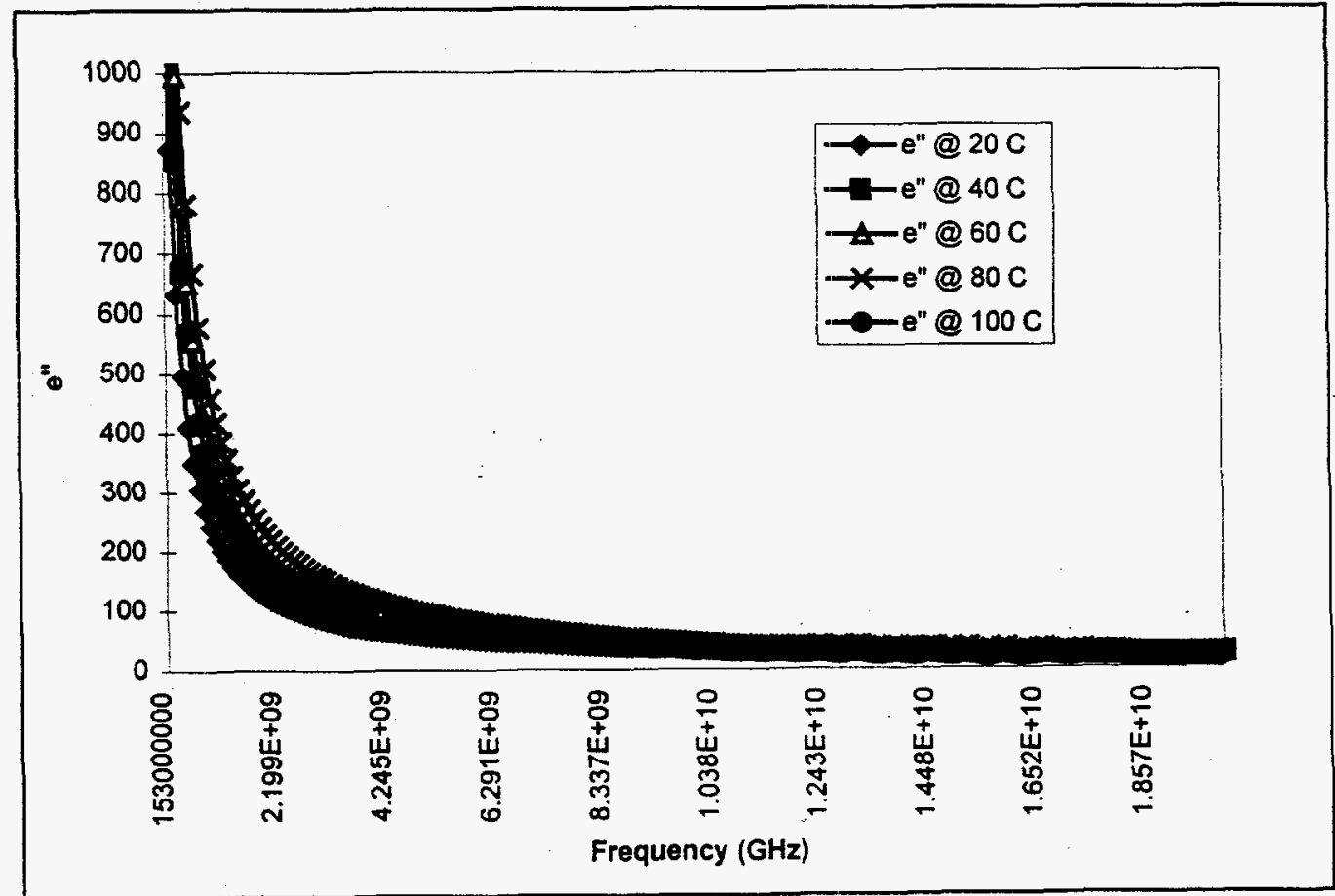

Figure A.5.c. Dielectric loss vs. frequency for the Revised ORNL simulant. Scaled down. 


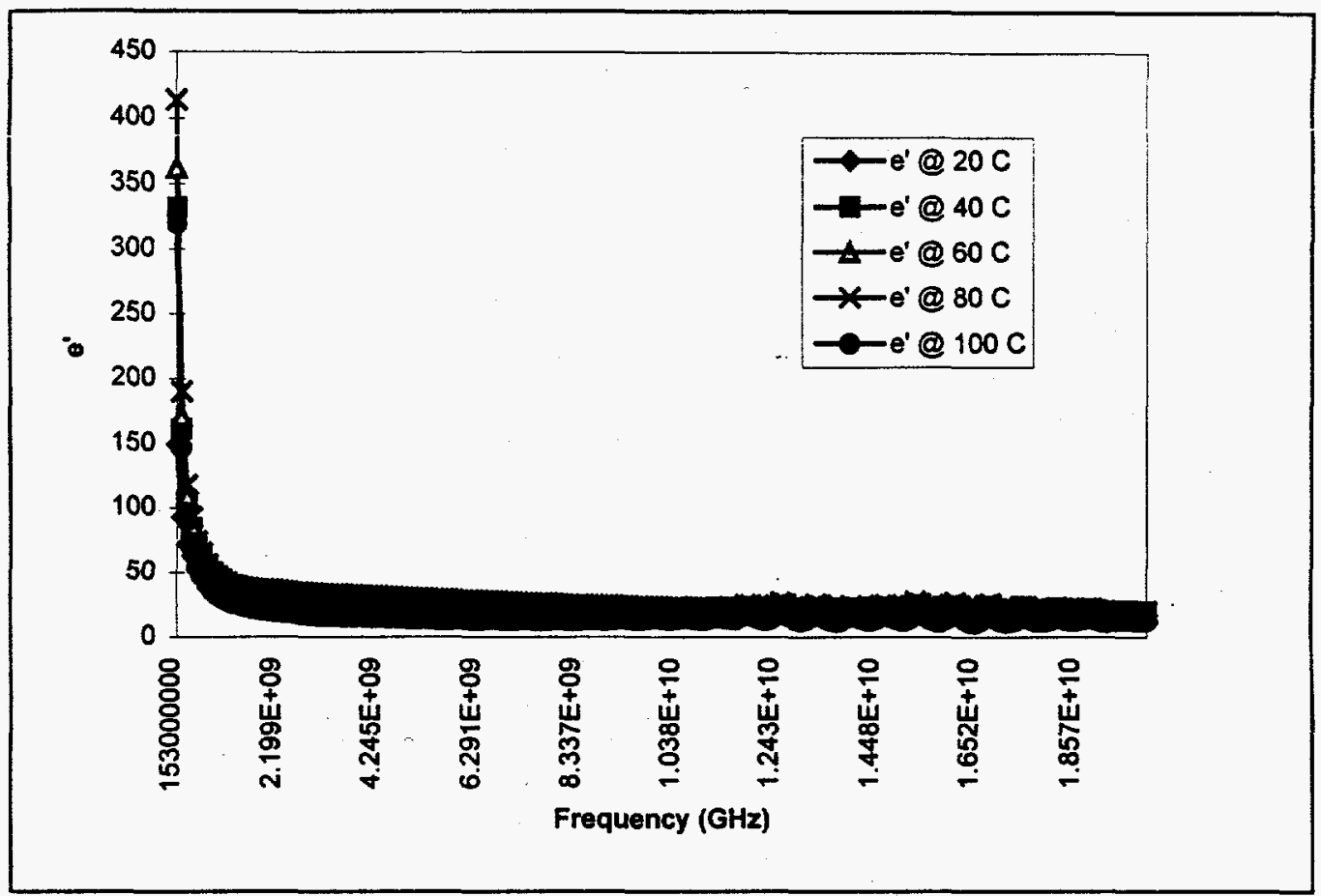

Figure A.5.c. Dielectric constant vs. frequency for the Revised ORNL simulant. Full scale.

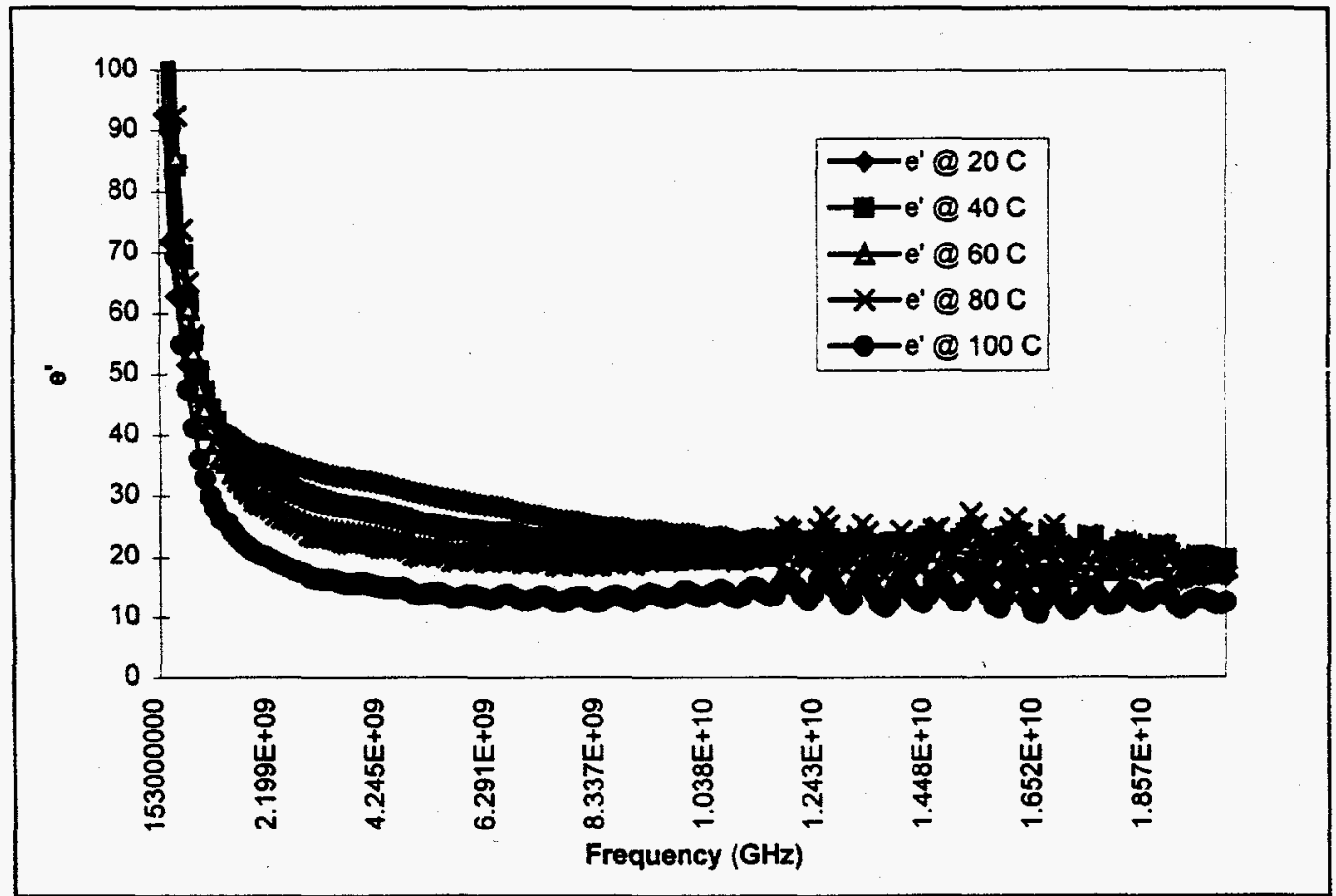

Figure A.5.d. Dielectric constant vs. frequency for the Revised ORNL simulant. Scaled down. 


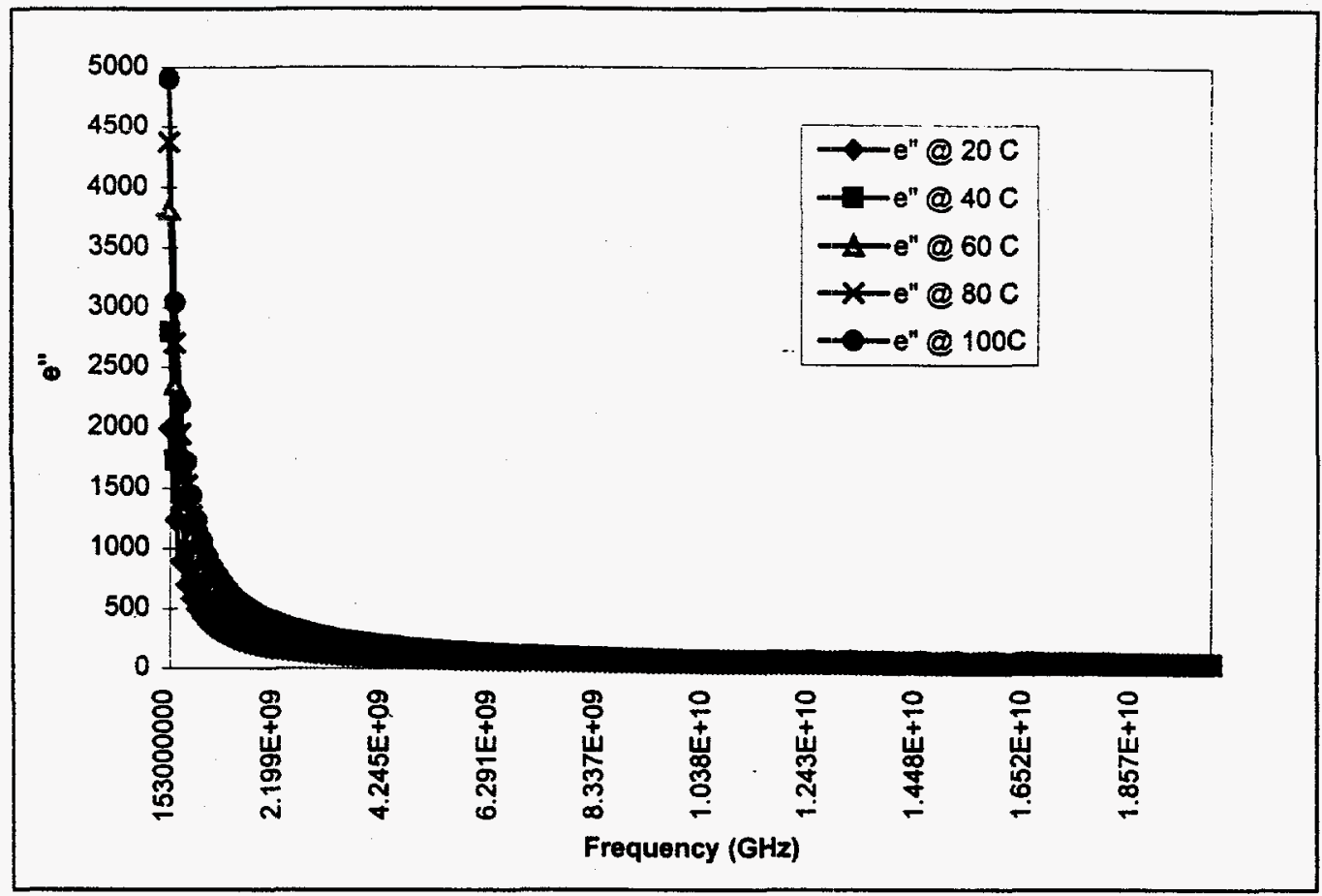

Figure A.6.a. Dielectric loss vs. frequency for the W-26 ORNL simulant. Full scale.

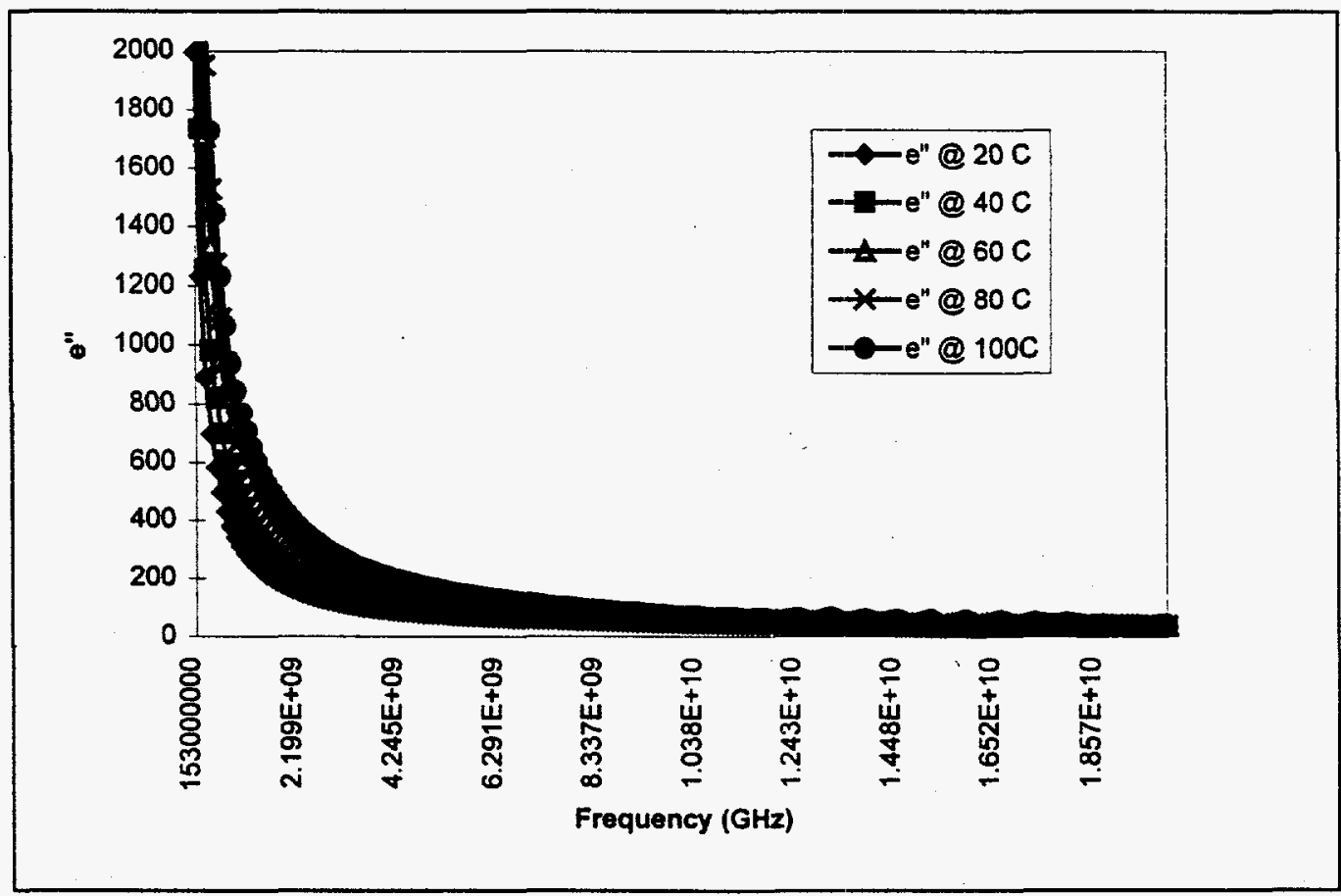

Figure A.6.b. Dielectric loss vs. frequency for the W-26 ORNL simulant. Scaled down. 


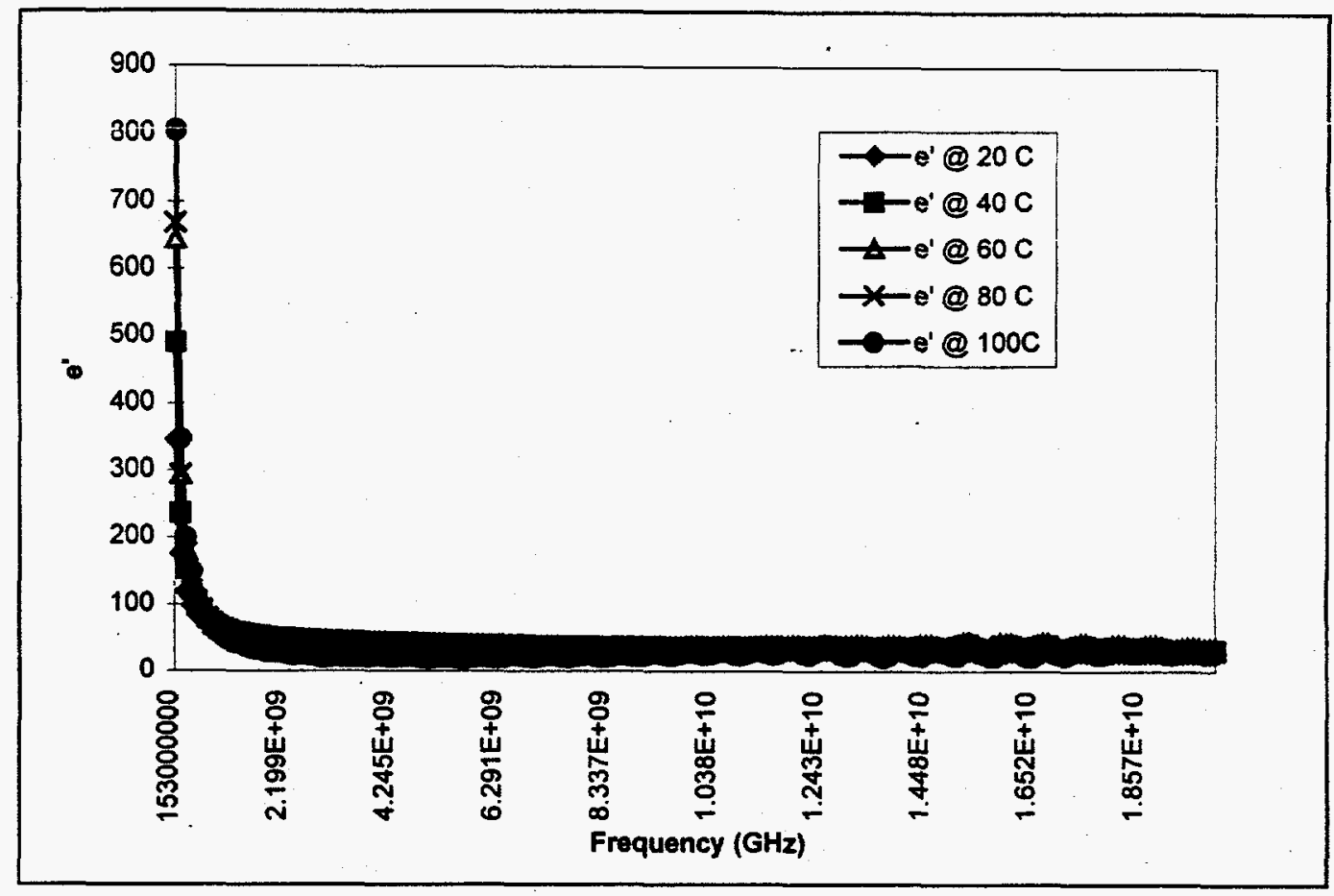

Figure A.6.c. Dielectric constant vs. frequency for the W-26 ORNL simulant. Full scale.

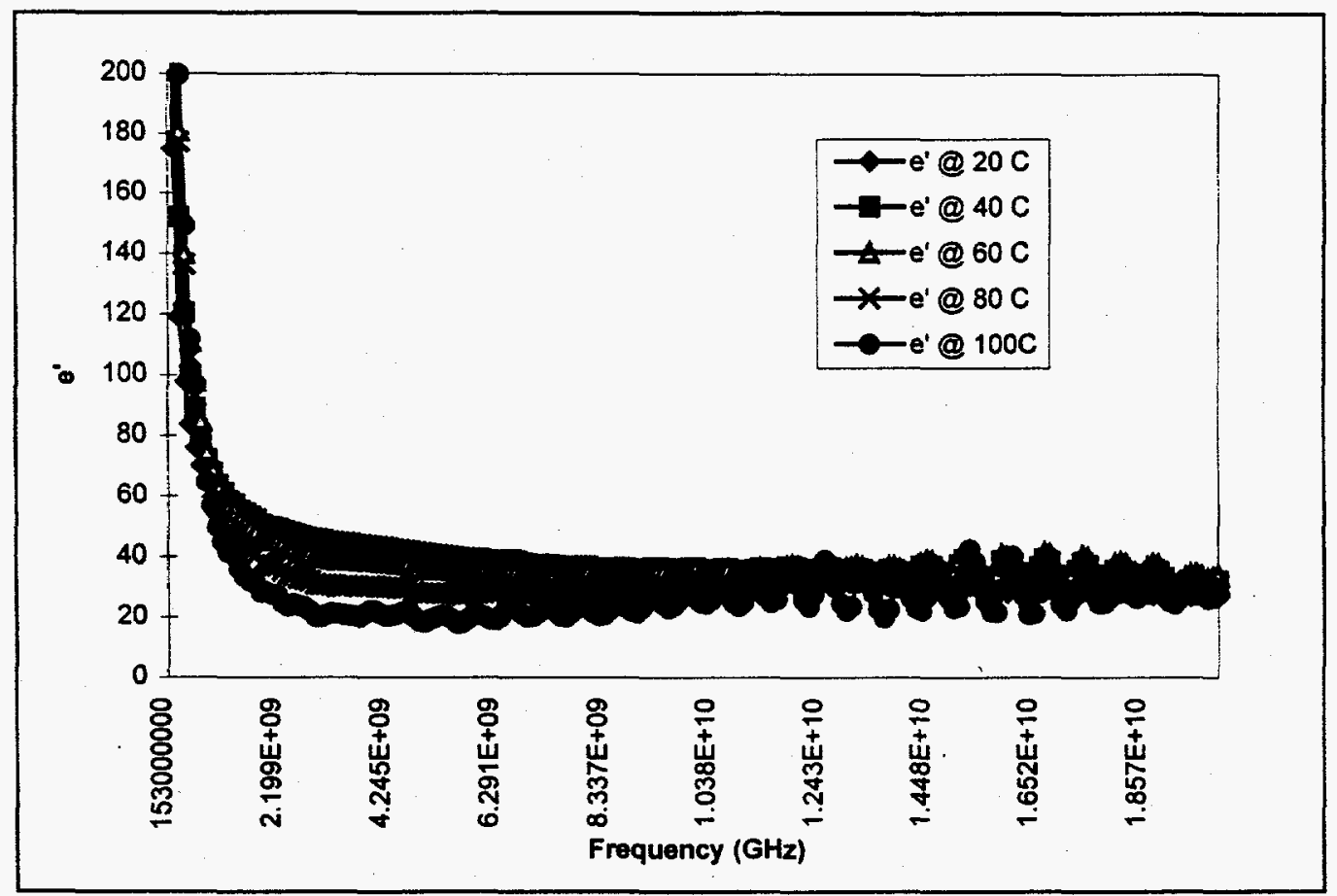

Figure A.6.d. Dielectric constant vs. frequency for the W-26 ORNL simulant. Scaled down. 


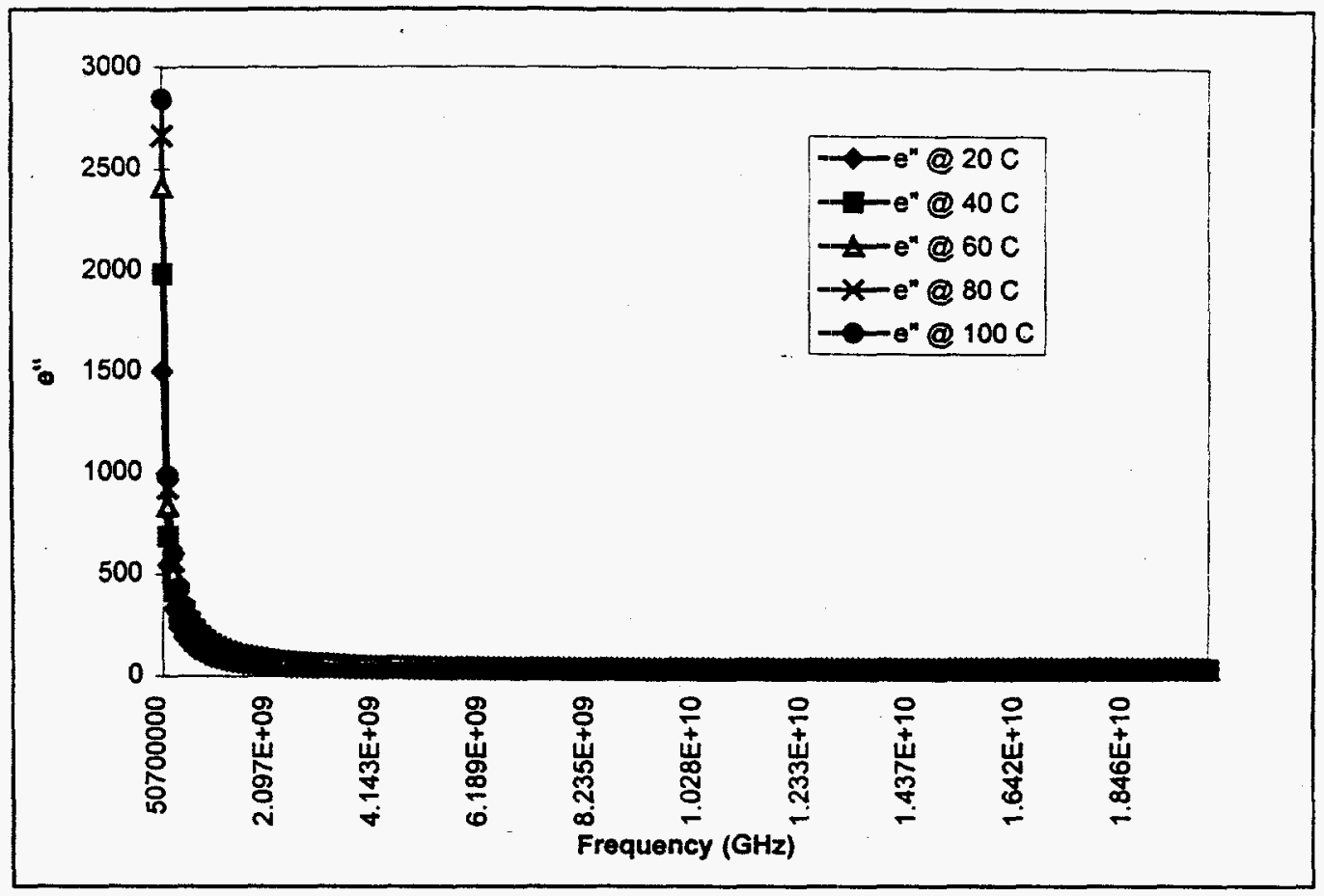

Figure A.7.a. Dielectric loss vs. frequency for the Savannah River simulant. Full scale.

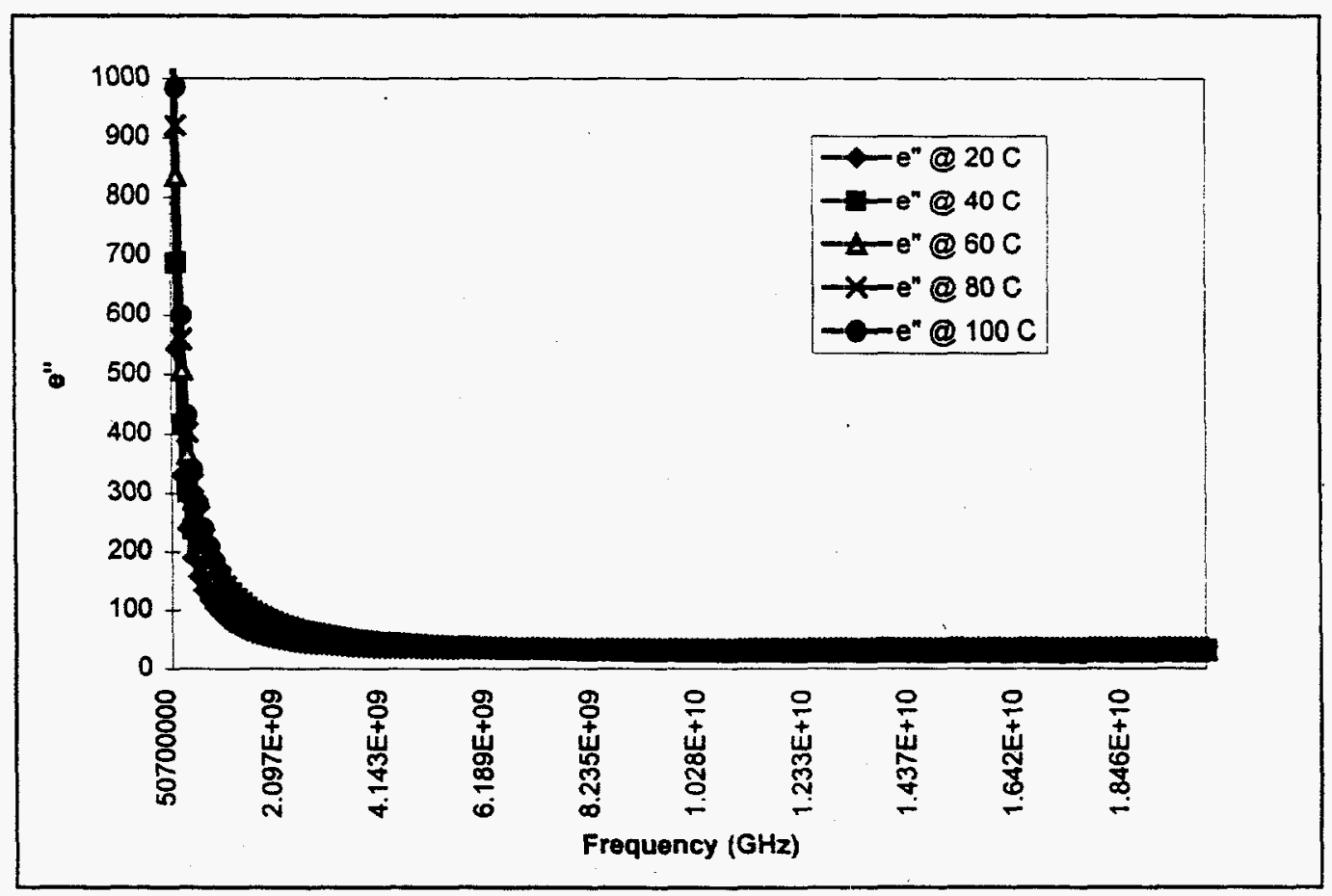

Figure A.7.b. Dielectric loss vs. frequency for the Savannah River simulant. Scaled down. 


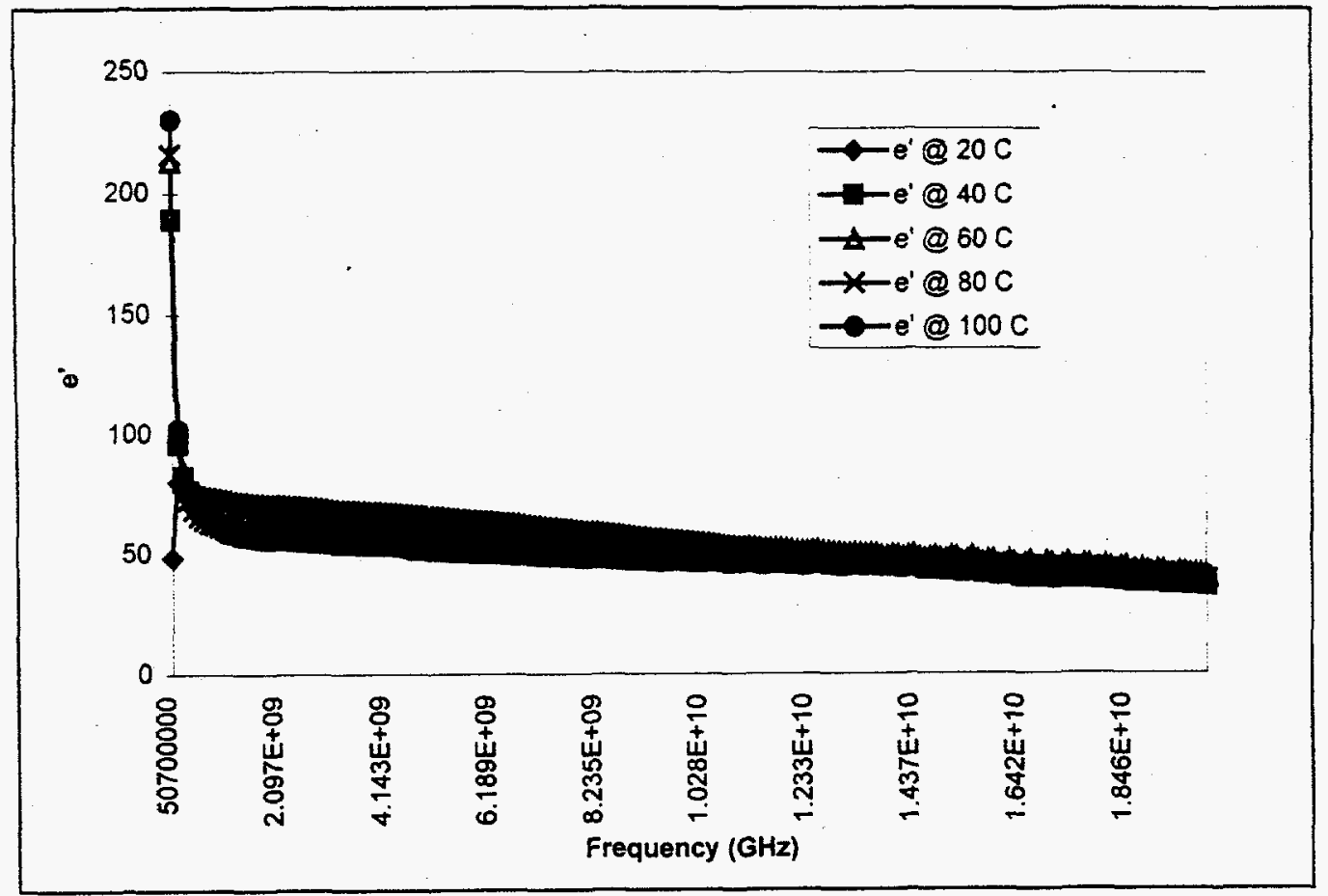

Figure A.7.c. Dielectric constant vs. frequency for the Savannah River simulant. Full scale.

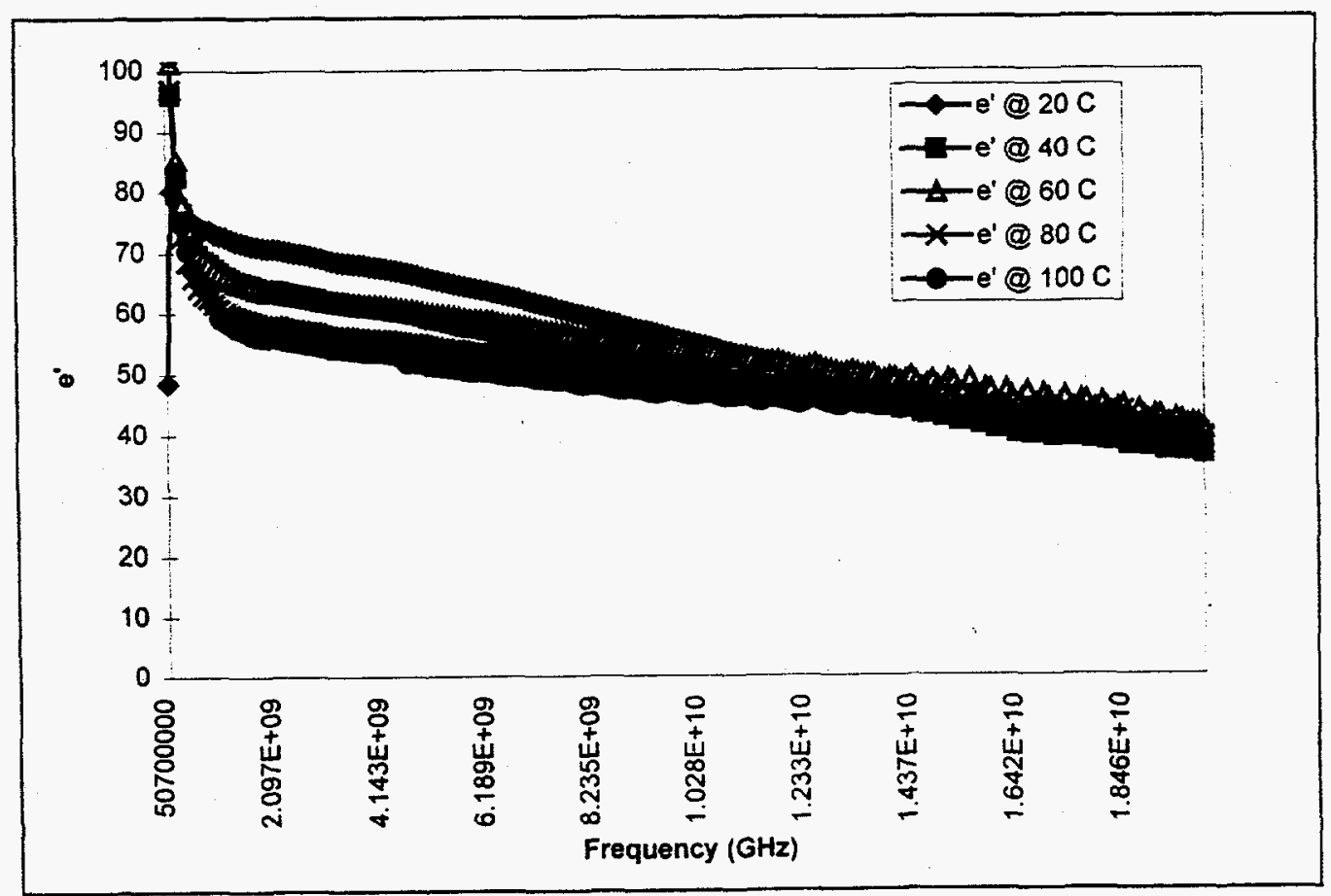

Figure A.7.d. Dielectric loss vs. frequency for the Savannah River simulant. Scaled down. 
TABULATED RESULTS FOR LOSS TANGENT, DIELECTRIC CONSTANT, AND DIELECTRIC LOSS 
Table 1. Dielectric Constant for Hanford 4M Simulant

\begin{tabular}{|c|c|c|c|c|c|}
\hline Frequency & $\mathrm{e}^{\prime} @ 20 \mathrm{C}$ & $e^{t} @ 40 c$ & $\bar{C}$ & $\mathrm{e}^{\prime} @$ & @100C \\
\hline $1.53 \mathrm{E}+08$ & 198.8575 & 270.3677 & 320.7074 & 402.9337 & 1419.842 \\
\hline $2.55 \mathrm{E}+08$ & 124.2155 & 143.5933 & 169.532 & 199.6412 & 689.4328 \\
\hline $3.58 \mathrm{E}+08$ & 96.62798 & 108.4729 & 126.3347 & 144.1173 & 420.9687 \\
\hline $4.6 \mathrm{E}+08$ & 84.84679 & 89.60063 & 103.7154 & 117.4237 & 284.8156 \\
\hline $5.62 \mathrm{E}+08$ & 74.46346 & 78.3095 & 90.02729 & 101.6916 & 212.5836 \\
\hline $6.65 \mathrm{E}+08$ & 69.75643 & 72.44598 & 83.36565 & 96.31848 & 177.311 \\
\hline $7.67 \mathrm{E}+08$ & 66.18846 & 68.84869 & 79.52852 & 92.30133 & 155.6548 \\
\hline $8.69 \mathrm{E}+08$ & 62.83495 & 65.0585 & 74.8873 & 85.71706 & 135.6266 \\
\hline $9.71 \mathrm{E}+08$ & 60.74841 & 62.70668 & 72.36627 & 82.05273 & 119.3228 \\
\hline $1.07 \mathrm{E}+09$ & 58.79125 & 59.95642 & 68.75328 & 76.44173 & 110.3758 \\
\hline $1.18 \mathrm{E}+09$ & 56.90724 & 57.98796 & 66.27603 & 74.00732 & 104.0247 \\
\hline $1.28 \mathrm{E}+09$ & 55.44015 & 56.26929 & 64.37692 & 72.46273 & 98.84793 \\
\hline $1.38 \mathrm{E}+09$ & 54.30958 & 55.43801 & 63.81259 & 73.10627 & 93.52054 \\
\hline $1.48 \mathrm{E}+09$ & 53.64628 & 54.63049 & 62.84035 & 72.74561 & 89.83873 \\
\hline $1.59 \mathrm{E}+09$ & 52.82432 & 53.76626 & 61.58675 & 70.14546 & 84.77519 \\
\hline $1.69 \mathrm{E}+09$ & 51.87058 & 53.0258 & 60.80892 & 67.85505 & 80.92936 \\
\hline $1.79 \mathrm{E}+09$ & 51.06159 & 52.3653 & 60.16007 & 66.92807 & 77.52104 \\
\hline $1.89 E+09$ & 50.46592 & 51.39429 & 58.79424 & 65.19102 & 75.82886 \\
\hline $1.99 \mathrm{E}+09$ & 49.91249 & 50.85893 & 58.22349 & 65.3256 & 74.6111 \\
\hline $2.1 E+09$ & 49.94468 & 50.82319 & 58.56358 & 66.82742 & 72.90152 \\
\hline $2.2 \mathrm{E}+09$ & 49.36027 & 50.16933 & 57.79177 & 66.26324 & 70.72926 \\
\hline $2.3 \mathrm{E}+09$ & 48.8052 & 50.03126 & 57.64889 & 65.47302 & 68.57728 \\
\hline $2.4 \mathrm{E}+09$ & 48.22308 & 49.24603 & 56.41697 & 62.57125 & 67.40107 \\
\hline $2.51 \mathrm{E}+09$ & 47.72968 & 48.59069 & 55.58798 & 61.13365 & 66.41566 \\
\hline $2.61 E+09$ & 47.25594 & 47.69174 & 54.51638 & 60.37112 & 65.0521 \\
\hline $2.71 \mathrm{E}+09$ & 46.78081 & 47.50363 & 54.53228 & 61.47765 & 62.96508 \\
\hline $2.81 E+09$ & 46.42739 & 47.4535 & 54.71778 & 62.64006 & 61.75412 \\
\hline $2.92 \mathrm{E}+09$ & 46.01113 & 47.10456 & 54.28043 & 62.17121 & 61.41821 \\
\hline $3.02 \mathrm{E}+09$ & 45.56462 & 46.34208 & 53.15263 & 60.027 .69 & 60.88928 \\
\hline $3.12 \mathrm{E}+09$ & 45.08565 & 45.96732 & 52.54845 & 57.76786 & 60.36689 \\
\hline $3.22 \mathrm{E}+09$ & 44.74699 & 45.60358 & 52.17025 & 56.84316 & 59.5021 \\
\hline $3.32 \mathrm{E}+09$ & 44.487 & 45.07481 & 51.40706 & 56.43211 & 58.37091 \\
\hline $3.43 \mathrm{E}+09$ & 44.27688 & 44.99108 & 51.63939 & 57.7697 & 57.80877 \\
\hline $3.53 \mathrm{E}+09$ & 44.04945 & 45.14579 & 52.13039 & 59.3356 & 57.47841 \\
\hline $3.63 \mathrm{E}+09$ & 43.95301 & 45.07441 & 52.22615 & 59.85189 & 56.81442 \\
\hline $3.73 \mathrm{E}+09$ & 43.61804 & 44.39832 & 51.12166 & 57.69346 & 56.28413 \\
\hline $3.84 \mathrm{E}+09$ & 43.31669 & 44.08243 & 50.57142 & 55.63667 & 56.01831 \\
\hline $3.94 \mathrm{E}+09$ & 43.05547 & 44.04169 & 50.54599 & 55.13123 & 55.75773 \\
\hline $4.04 \mathrm{E}+09$ & 42.79697 & 43.87368 & 50.5905 & 56.11195 & 55.37571 \\
\hline $4.14 \mathrm{E}+09$ & 42.50431 & 43.56371 & 50.30526 & 56.71352 & 54.80982 \\
\hline $4.25 \mathrm{E}+09$ & 42.28299 & 43.48488 & 50.52445 & 57.42696 & 53.87457 \\
\hline $4.35 E+09$ & 41.97117 & 43.30425 & 50.41846 & 57.71585 & 52.55518 \\
\hline $4.45 \mathrm{E}+09$ & 41.59363 & 42.51819 & 49.20096 & 55.7971 & 52.02567 \\
\hline
\end{tabular}




\begin{tabular}{|c|c|c|c|c|c|}
\hline Frequency & $\mathrm{e}^{\prime} @ 20 \mathrm{C}$ & $e^{\prime} @ 40 \mathrm{C}$ & $e^{\prime} @ 60 \mathrm{C}$ & $\mathrm{e}^{\prime} @ 80 \mathrm{C}$ & $\mathrm{e}^{\prime} @ 100 \mathrm{C}$ \\
\hline $4.55 \mathrm{E}+09$ & 41.29029 & 41.84721 & 48.08885 & 52.89943 & 51.7472 \\
\hline $4.65 \mathrm{E}+09$ & 41.00919 & 41.60053 & 47.83102 & 51.67468 & 51.19196 \\
\hline $4.76 \mathrm{E}+09$ & 40.6596 & 41.03431 & 46.99119 & 51.33894 & 50.23983 \\
\hline $4.86 E+09$ & 40.39502 & 40.74681 & 46.86334 & 52.65176 & 49.04396 \\
\hline $4.96 \mathrm{E}+09$ & 40.07684 & 40.46598 & 46.73998 & 52.79685 & 48.46402 \\
\hline $5.06 \mathrm{E}+09$ & 39.75546 & 40.14061 & 46.3516 & 52.33352 & 47.78743 \\
\hline $5.17 \mathrm{E}+09$ & 39.44144 & 39.79174 & 45.83317 & 51.42322 & 47.25031 \\
\hline $5.27 \mathrm{E}+09$ & 39.18802 & 39.2152 & 44.94134 & 49.20433 & 46.68505 \\
\hline $5.37 \mathrm{E}+09$ & 38.87762 & 39.06737 & 44.70551 & 47.47822 & 46.15098 \\
\hline $5.47 \mathrm{E}+09$ & 38.6443 & 38.89102 & 44.57533 & 48.07843 & 45.5793 \\
\hline $5.57 \mathrm{E}+09$ & 38.38517 & 38.42684 & 44.05299 & 48.9435 & 44.81765 \\
\hline $5.68 \mathrm{E}+09$ & 38.10451 & 38.36361 & 44.2689 & 49.821 & 43.98962 \\
\hline $5.78 \mathrm{E}+09$ & 37.82517 & 37.69357 & 43.33183 & 47.61455 & 43.79572 \\
\hline $5.88 \mathrm{E}+09$ & 37.60801 & 37.71062 & 43.45015 & 47.78145 & 43.7678 \\
\hline $5.98 \mathrm{E}+09$ & 37.36262 & 37.42076 & 42.92975 & 46.61226 & 43.35502 \\
\hline $6.09 \mathrm{E}+09$ & 37.14096 & 37.09663 & 42.49049 & 44.9968 & 43.12332 \\
\hline $6.19 \mathrm{E}+09$ & 36.878 & 36.99787 & 42.39584 & 45.02856 & 42.55522 \\
\hline $6.29 \mathrm{E}+09$ & 36.68849 & 37.10379 & 42.84166 & 48.01135 & 41.84339 \\
\hline $6.39 \mathrm{E}+09$ & 36.36497 & 36.67763 & 42.39812 & 48.40819 & 41.14944 \\
\hline $6.5 E+09$ & 36.16526 & 36.60127 & 42.67216 & 47.9779 & 40.89782 \\
\hline $6.6 \mathrm{E}+09$ & 35.82384 & 36.10319 & 41.9742 & 46.18852 & 40.8233 \\
\hline $6.7 \mathrm{E}+09$ & 35.57949 & 35.88825 & 41.5929 & 45.59117 & 40.51937 \\
\hline $6.8 \mathrm{E}+09$ & 35.28364 & 35.63172 & 41.06809 & 43.68999 & 40.36209 \\
\hline $6.9 \mathrm{E}+09$ & 35.02745 & 35.08358 & 40.25068 & 42.0903 & 39.66757 \\
\hline $7.01 \mathrm{E}+09$ & 34.79476 & 35.01702 & 40.33293 & 44.62388 & 39.06853 \\
\hline $7.11 \mathrm{E}+09$ & 34.57164 & 34.73974 & 40.29649 & 46.57688 & 38.40481 \\
\hline $7.21 \mathrm{E}+09$ & 34.42282 & 34.26612 & 40.00635 & 45.36502 & 37.95522 \\
\hline $7.31 \mathrm{E}+09$ & 34.19808 & 34.05195 & 39.71072 & 43.57117 & 37.61625 \\
\hline $7.42 \mathrm{E}+09$ & 33.92792 & 33.65367 & 38.98053 & 42.26415 & 37.46543 \\
\hline $7.52 \mathrm{E}+09$ & 33.70417 & 33.09713 & 38.04553 & 40.20439 & 37.25101 \\
\hline $7.62 \mathrm{E}+09$ & 33.53444 & 32.94894 & 37.75976 & 39.15504 & 37.1159 \\
\hline $7.72 \mathrm{E}+09$ & 33.27461 & 33.09606 & 38.11884 & 41.29863 & 36.65917 \\
\hline $7.83 \mathrm{E}+09$ & 33.12408 & 32.87328 & 37.99717 & 43.37428 & 35.6737 \\
\hline $7.93 E+09$ & 32.96951 & 32.66676 & 38.02405 & 43.11761 & 35.34007 \\
\hline $8.03 E+09$ & 32.79739 & 32.36681 & 37.82362 & 41.08045 & 34.59292 \\
\hline $8.13 E+09$ & 32.40897 & 31.78922 & 36.67902 & 38.57102 & 33.63317 \\
\hline $8.23 \mathrm{E}+09$ & 32.16588 & 31.50158 & 36.16847 & 38.04096 & 33.40621 \\
\hline $8.34 \mathrm{E}+09$ & 31.85106 & 31.3188 & 35.86105 & 37.03819 & 33.25521 \\
\hline $8.44 E+09$ & 31.65863 & 31.18446 & 35.7715 & 37.86529 & 33.0575 \\
\hline $8.54 \mathrm{E}+09$ & 31.4427 & 31.52872 & 36.56093 & 41.63111 & 32.9776 \\
\hline $8.64 \mathrm{E}+09$ & 31.31633 & 31.66019 & 37.02995 & 42.66135 & 32.97222 \\
\hline $8.75 \mathrm{E}+09$ & 31.18795 & 31.02415 & 36.22608 & 39.28166 & 32.9714 \\
\hline $8.85 \mathrm{E}+09$ & 31.04938 & 30.70922 & 35.66964 & 37.15709 & 33.11715 \\
\hline $8.95 \mathrm{E}+09$ & 30.8116 & 30.34145 & 34.90711 & 36.34598 & 33.05216 \\
\hline $9.05 \mathrm{E}+09$ & 30.78406 & 30.1137 & 34.63596 & 36.45005 & 32.96265 \\
\hline
\end{tabular}




\begin{tabular}{|c|c|c|c|c|c|}
\hline Freq & $\mathrm{e}^{\prime} @ 20 \mathrm{C}$ & $e^{\prime} @ 40 \mathrm{C}$ & $\bar{C}$ & $\mathrm{C}$ & $e^{\prime} @ 100 \mathrm{C}$ \\
\hline $9.16 \mathrm{E}+09$ & 30.6287 & 30.43621 & 35.39031 & 38.45933 & 32.86163 \\
\hline $9.26 \mathrm{E}+09$ & 30.46262 & 30.25596 & 35.33281 & 40.32688 & 32.84051 \\
\hline $9.36 \mathrm{E}+09$ & 30.43751 & 30.70406 & 36.36074 & 43.08851 & 32.84569 \\
\hline $9.46 \mathrm{E}+09$ & 30.26962 & 30.35319 & 35.97816 & 40.12464 & 33.07134 \\
\hline $9.56 \mathrm{E}+09$ & 30.16984 & 29.92448 & 34.98569 & 36.40663 & 33.10147 \\
\hline $9.67 \mathrm{E}+09$ & 30.09878 & 29.90542 & 34.90665 & 36.80038 & 33.06588 \\
\hline $9.77 \mathrm{E}+09$ & 29.86557 & 29.68317 & 34.51162 & 36.94777 & 33.03234 \\
\hline $9.87 \mathrm{E}+09$ & 29.84163 & 29.61417 & 34.80649 & 38.64902 & 32.57637 \\
\hline $9.97 \mathrm{E}+09$ & 29.72081 & 29.88757 & 35.50703 & 41.91084 & 32.91441 \\
\hline $1.01 \mathrm{E}+10$ & 29.57607 & 29.9979 & 35.90607 & 43.81517 & 33.22629 \\
\hline $1.02 \mathrm{E}+10$ & 29.51581 & 30.15968 & 36.19273 & 42.30949 & 33.33926 \\
\hline $1.03 E+10$ & 29.37255 & 30.09904 & 35.99199 & 39.01195 & 33.32415 \\
\hline $1.04 \mathrm{E}+10$ & 29.26744 & 29.67555 & 35.07323 & 36.98296 & 33.20805 \\
\hline $1.05 \mathrm{E}+10$ & 29.24758 & 29.39732 & 34.77737 & 37.71555 & 32.72811 \\
\hline $1.06 \mathrm{E}+10$ & 29.0845 & 29.1314 & 34.60799 & 38.30263 & 32.72856 \\
\hline $1.07 \mathrm{E}+10$ & 28.8995 & 28.40333 & 33.83601 & 38.74431 & 32.93132 \\
\hline $1.08 \mathrm{E}+10$ & 28.72487 & 28.64204 & 34.27473 & 40.62776 & 33.46667 \\
\hline $1.09 \mathrm{E}+10$ & 28.57364 & 28.93438 & 34.5508 & 39.77088 & 33.65496 \\
\hline $1.1 \mathrm{E}+10$ & 28.45737 & 29.03385 & 34.52383 & 37.07762 & 33.52757 \\
\hline $1.11 \mathrm{E}+10$ & 28.37685 & 28.73757 & 33.97605 & 35.08922 & 33.12761 \\
\hline $1.12 \mathrm{E}+10$ & 28.3336 & 28.30993 & 33.60487 & 35.45368 & 32.85969 \\
\hline $1.13 \mathrm{E}+10$ & 28.24137 & 27.51639 & 32.97383 & 36.25889 & 32.83421 \\
\hline $1.14 \mathrm{E}+10$ & 28.1183 & 27.36991 & 33.31461 & 38.43703 & 33.47811 \\
\hline $1.15 \mathrm{E}+10$ & 28.04092 & 28.25791 & 34.76497 & 42.15563 & 33.95346 \\
\hline $1.16 \mathrm{E}+10$ & 27.92038 & 29.27576 & 35.90735 & 42.84805 & 34.22153 \\
\hline $1.17 \mathrm{E}+10$ & 27.79314 & 29.63988 & 35.99712 & 39.76398 & 34.0324 \\
\hline $1.18 \mathrm{E}+10$ & 27.73124 & 28.88766 & 34.66973 & 36.3858 & 33.37971 \\
\hline $1.19 \mathrm{E}+10$ & 27.63107 & 27.93919 & 33.48595 & 36.03058 & 32.50202 \\
\hline $1.2 \mathrm{E}+10$ & 27.50941 & 27.25975 & 33.2967 & 37.99913 & 31.29598 \\
\hline $1.21 \mathrm{E}+10$ & 27.43695 & 26.68176 & 33.36755 & 40.18912 & 30.02388 \\
\hline $1.22 \mathrm{E}+10$ & 27.30299 & 27.46484 & 34.94785 & 43.88034 & 30.85183 \\
\hline $1.23 \mathrm{E}+10$ & 27.1675 & 29.1664 & 37.00526 & 45.53902 & 32.08552 \\
\hline $1.24 E+10$ & 27.0771 & 29.08247 & 36.0588 & 41.01776 & 33.42149 \\
\hline $1.25 \mathrm{E}+10$ & 26.99021 & 27.97669 & 34.05007 & 37.28528 & 33.05217 \\
\hline $1.26 \mathrm{E}+10$ & 26.91808 & 27.61974 & 33.81806 & 38.99109 & 31.41885 \\
\hline $1.27 \mathrm{E}+10$ & 26.85644 & 26.9014 & 33.70015 & 40.27479 & 29.90195 \\
\hline $1.28 \mathrm{E}+10$ & 26.81332 & 26.92404 & 34.78258 & 42.79678 & 29.36127 \\
\hline $1.29 \mathrm{E}+10$ & 26.6867 & 27.34234 & 35.70528 & 44.76396 & 29.92006 \\
\hline $1.3 \mathrm{E}+10$ & 26.57291 & 28.1266 & 36.13083 & 43.79115 & 31.35186 \\
\hline $1.31 E+10$ & 26.35825 & 27.88753 & 34.78317 & 37.53 & 32.43513 \\
\hline $1.32 \mathrm{E}+10$ & 26.227 & 26.83203 & 32.29696 & 32.3258 & 32.29325 \\
\hline $1.33 \mathrm{E}+10$ & 26.19038 & 26.66046 & 31.68296 & 32.50265 & 31.53217 \\
\hline $1.35 \mathrm{E}+10$ & 26.07542 & 27.11966 & 32.66876 & 33.6392 & 30.71475 \\
\hline $1.36 \mathrm{E}+10$ & 25.96972 & 27.37159 & 33.6266 & 34.51131 & 30.25471 \\
\hline $1.37 E+10$ & 25.90757 & 27.06255 & 33.63981 & 36.60953 & 30.24063 \\
\hline
\end{tabular}




\begin{tabular}{|c|c|c|c|c|c|}
\hline Frequency & $e^{\prime} @ 20 C$ & $e^{\prime} @ 40 C$ & $e^{\prime} @ 60 \mathrm{C}$ & $e^{\prime} @ 80 \mathrm{C}$ & $e^{\prime} @ 100 C$ \\
\hline $1.38 \mathrm{E}+10$ & 25.84909 & 27.01093 & 33.74406 & 38.1677 & 31.0015 \\
\hline $1.39 \mathrm{E}+10$ & 25.79222 & 26.28413 & 32.35968 & 33.39861 & 31.69377 \\
\hline $1.4 \mathrm{E}+10$ & 25.7171 & 25.4135 & 30.6901 & 30.1229 & 31.63726 \\
\hline $1.41 E+10$ & 25.57397 & 25.03708 & 29.82939 & 31.02586 & 31.28235 \\
\hline $1.42 \mathrm{E}+10$ & 25.46289 & 25.59722 & 30.56937 & 32.10623 & 30.79626 \\
\hline $1.43 \mathrm{E}+10$ & 25.33585 & 25.79165 & 31.0087 & 29.70589 & 30.42867 \\
\hline $1.44 \mathrm{E}+10$ & 25.21397 & 25.00401 & 29.96355 & 29.08776 & 30.05091 \\
\hline $1.45 \mathrm{E}+10$ & 25.2284 & 24.58234 & 29.46593 & 31.03228 & 30.28772 \\
\hline $1.46 \mathrm{E}+10$ & 25.21272 & 24.89926 & 30.00395 & 29.41471 & 30.69136 \\
\hline $1.47 \mathrm{E}+10$ & 25.13907 & 24.68228 & 29.83961 & 26.39843 & 30.99487 \\
\hline $1.48 \mathrm{E}+10$ & 25.02887 & 24.34063 & 29.534 & 29.99693 & 31.01528 \\
\hline $1.49 \mathrm{E}+10$ & 24.8671 & 24.74812 & 30.51158 & 34.05878 & 30.53112 \\
\hline $1.5 \mathrm{E}+10$ & 24.66083 & 25.20084 & 31.47414 & 29.06885 & 30.03004 \\
\hline $1.51 \mathrm{E}+10$ & 24.65609 & 23.87442 & 29.20796 & 27.28742 & 30.14681 \\
\hline $1.52 \mathrm{E}+10$ & 24.53987 & 23.99361 & 29.49286 & 32.24066 & 30.18461 \\
\hline $1.53 \mathrm{E}+10$ & 24.56818 & 23.93289 & 29.54149 & 27.75925 & 30.10143 \\
\hline $1.54 \mathrm{E}+10$ & 24.55823 & 23.88037 & 29.26193 & 23.54979 & 30.83383 \\
\hline $1.55 \mathrm{E}+10$ & 24.46075 & 23.16313 & 28.55451 & 29.235 & 31.29898 \\
\hline $1.56 \mathrm{E}+10$ & 24.22603 & 24.05307 & 29.99687 & 32.91968 & 30.05651 \\
\hline $1.57 \mathrm{E}+10$ & 24.02671 & 25.00525 & 31.24432 & 25.71477 & 29.35307 \\
\hline $1.58 \mathrm{E}+10$ & 24.02529 & 24.01441 & 29.85323 & 29.37451 & 29.91064 \\
\hline $1.59 \mathrm{E}+10$ & 23.9706 & 23.84344 & 29.76966 & 34.68655 & 29.8928 \\
\hline $1.6 \mathrm{E}+10$ & 23.88519 & 23.53713 & 29.61235 & 27.91455 & 29.76804 \\
\hline $1.61 \mathrm{E}+10$ & 23.82774 & 22.66222 & 28.30711 & 24.57649 & 31.09462 \\
\hline $1.62 \mathrm{E}+10$ & 23.70664 & 22.86397 & 28.32196 & 30.39048 & 31.3334 \\
\hline $1.63 \mathrm{E}+10$ & 23.46761 & 23.68876 & 29.31829 & 31.66887 & 30.22029 \\
\hline $1.64 E+10$ & 23.47171 & 24.00232 & 29.59608 & 28.53707 & 29.5609 \\
\hline $1.65 \mathrm{E}+10$ & 23.41523 & 22.96963 & 28.01641 & 29.394 & 29.2733 \\
\hline $1.66 \mathrm{E}+10$ & 23.20905 & 21.88462 & 26.88989 & 30.6161 & 28.85421 \\
\hline $1.67 \mathrm{E}+10$ & 23.19566 & 21.89851 & 27.19591 & 29.28084 & 28.86557 \\
\hline $1.68 \mathrm{E}+10$ & 23.14145 & 22.70787 & 28.48376 & 29.17102 & 30.48058 \\
\hline $1.69 \mathrm{E}+10$ & 22.91899 & 23.5228 & 29.62761 & 31.23354 & 31.31281 \\
\hline $1.7 \mathrm{E}+10$ & 22.74058 & 23.01668 & 28.60571 & 31.17484 & 30.34324 \\
\hline $1.71 \mathrm{E}+10$ & 22.74065 & 22.17755 & 26.89035 & 27.25094 & 29.04355 \\
\hline $1.72 \mathrm{E}+10$ & 22.61404 & 21.7582 & 25.95822 & 24.44851 & 28.75338 \\
\hline $1.73 \mathrm{E}+10$ & 22.43703 & 21.1112 & 25.20646 & 25.17339 & 28.78668 \\
\hline $1.74 \mathrm{E}+10$ & 22.45845 & 21.04465 & 25.81281 & 28.4693 & 29.0507 \\
\hline $1.75 \mathrm{E}+10$ & 22.21217 & 22.04197 & 27.76968 & 28.92869 & 29.99308 \\
\hline $1.76 \mathrm{E}+10$ & 21.94574 & 22.52964 & 28.60133 & 28.76964 & 30.90991 \\
\hline $1.77 \mathrm{E}+10$ & 21.96113 & 21.50469 & 26.66933 & 27.51845 & 30.9201 \\
\hline $1.79 \mathrm{E}+10$ & 21.88148 & 20.71877 & 24.81679 & 23.00853 & 30.19295 \\
\hline $1.8 \mathrm{E}+10$ & 21.81803 & 20.825 & 24.42291 & 21.25157 & 29.83763 \\
\hline $1.81 \mathrm{E}+10$ & 21.71902 & 20.81983 & 24.82709 & 23.24469 & 29.69542 \\
\hline $1.82 \mathrm{E}+10$ & 21.64358 & 20.17762 & 24.94521 & 24.04841 & 29.68114 \\
\hline $1.83 \mathrm{E}+10$ & 21.55122 & 20.91646 & 26.51788 & 26.78515 & 29.58 \\
\hline
\end{tabular}




\begin{tabular}{|r|r|r|r|r|r|}
\hline Frequency & $e^{\prime} @ 20 \mathrm{C}$ & $\mathbf{e}^{\prime} @ 40 \mathrm{C}$ & $\mathrm{e}^{\prime} @$ @ 60 C & $\mathbf{e}^{\prime} @$ @ 80 C & $\mathrm{e}^{\prime} @ 100 \mathrm{C}$ \\
\hline $1.84 \mathrm{E}+10$ & 21.40615 & 22.07432 & 28.39296 & 30.62028 & 30.12158 \\
\hline $1.85 \mathrm{E}+10$ & 21.27543 & 21.22831 & 27.13465 & 25.49066 & 30.52754 \\
\hline $1.86 \mathrm{E}+10$ & 21.28434 & 20.32472 & 25.45423 & 22.49949 & 30.53778 \\
\hline $1.87 \mathrm{E}+10$ & 21.14532 & 20.50149 & 25.37754 & 25.12964 & 30.14198 \\
\hline $1.88 \mathrm{E}+10$ & 21.07693 & 20.12526 & 25.06871 & 23.34292 & 30.0038 \\
\hline $1.89 \mathrm{E}+10$ & 21.04613 & 19.9173 & 25.19385 & 21.07464 & 29.59412 \\
\hline $1.9 \mathrm{E}+10$ & 20.80926 & 20.44313 & 26.15904 & 26.48664 & 28.97916 \\
\hline $1.91 \mathrm{E}+10$ & 20.77293 & 21.65063 & 27.88701 & 28.73562 & 28.75488 \\
\hline $1.92 \mathrm{E}+10$ & 20.76318 & 21.63373 & 28.35555 & 23.84855 & 29.33268 \\
\hline $1.93 \mathrm{E}+10$ & 20.6053 & 20.78344 & 27.09321 & 25.07382 & 29.81161 \\
\hline $1.94 \mathrm{E}+10$ & 20.49016 & 20.35521 & 26.39776 & 27.31464 & 29.943 \\
\hline $1.95 \mathrm{E}+10$ & 20.38669 & 21.2264 & 27.90021 & 26.87749 & 29.73552 \\
\hline $1.96 \mathrm{E}+10$ & 20.26377 & 20.54382 & 27.05007 & 25.02233 & 29.19837 \\
\hline $1.97 \mathrm{E}+10$ & 20.15467 & 20.42197 & 26.92744 & 27.78322 & 28.21305 \\
\hline $1.98 \mathrm{E}+10$ & 20.16713 & 20.60361 & 27.18537 & 25.42896 & 27.95032 \\
\hline $1.99 \mathrm{E}+10$ & 20.1204 & 20.61412 & 27.1963 & 21.90008 & 28.598 \\
\hline $2 \mathrm{E}+10$ & 20.05618 & 20.06602 & 26.54198 & 25.62997 & 29.70891 \\
\hline
\end{tabular}


Table 2. Dielectric Loss for Hanford 4M Simulant

\begin{tabular}{|c|c|c|c|c|c|}
\hline & & & C & C & \\
\hline 8 & 0.789 & 3268.7825 & 4347.7021 & 9.373 & 72 \\
\hline .08 & 38.945 & 84.7412 & 642.1542 & 3501.3281 & 903.327 \\
\hline $3.58 \mathrm{E}+08$ & 88.1721 & 1428.3204 & 1899.4608 & 512.1689 & 866.040 \\
\hline 108 & 8.7456 & 15.0812 & 1481.9623 & 959.2966 & 259.769 \\
\hline $5.62 E+08$ & 626.0177 & 916.77975 & 1218.2583 & 1609.9412 & 1861.1148 \\
\hline $6.65 E+08$ & 533.0594 & 779.58932 & 1035.6961 & 1368.1258 & 587.828 \\
\hline $7.67 \mathrm{E}+08$ & 464.0386 & 677.86079 & 900.17872 & 1187.9548 & 383.945 \\
\hline $8.69 \mathrm{E}+08$ & 411.2912 & 599.46712 & 795.71548 & 1047.8946 & 230.943 \\
\hline $9.71 E+08$ & 369.6174 & 537.77023 & 713.90774 & 939.43329 & 1109.6742 \\
\hline $1.07 \mathrm{E}+09$ & 335.9699 & 488.31603 & 648.21713 & 852.56419 & 1011.488 \\
\hline $1.18 \mathrm{E}+09$ & 308.3073 & 447.09469 & 593.22265 & 780.25613 & 926.41758 \\
\hline+09 & 284.6326 & 412.93727 & 547.81876 & 720.76873 & 53.4020 \\
\hline $1.38 \mathrm{E}+09$ & 264.5612 & 383.04062 & 507.8212 & 667.28327 & 790.484 \\
\hline $1.48 \mathrm{E}+09$ & 247.0863 & 357.52527 & 473.84568 & 622.36112 & $\overline{39.66621}$ \\
\hline $1.59 E+09$ & 232.299 & 335.4922 & 444.46486 & 583.19031 & 93.75967 \\
\hline $1.69 \mathrm{E}+09$ & 219.0981 & 315.52058 & 417.98543 & 548.23136 & 654.16585 \\
\hline $1.79 \mathrm{E}+09$ & 207.5023 & 298.58209 & 395.45388 & 518.96779 & 616.94658 \\
\hline $1.89 E+09$ & 196.93 & 283.67668 & 375.83509 & 494.39344 & 584.2139 \\
\hline $1.99 E+09$ & 87.4545 & 269.89388 & 357.35771 & 470.77226 & 53.21833 \\
\hline $2.1 \mathrm{E}+09$ & 178.9404 & 257.42057 & 340.51044 & 448.32617 & 526.50103 \\
\hline $2.2 \mathrm{E}+09$ & 171.7897 & 246.43284 & 325.72345 & 28.21083 & 502. \\
\hline $2.3 \mathrm{E}+09$ & 164.9842 & 236.2509 & 312.43475 & 410.70882 & 82.96676 \\
\hline $2.4 \mathrm{E}+09$ & 158.7156 & 227.35163 & 300.65653 & 395.15561 & 463.72484 \\
\hline $2.51 \mathrm{E}+09$ & 153.0134 & 218.74061 & 288.87831 & 379.41472 & 445.17576 \\
\hline $2.61 \mathrm{E}+09$ & 147.3466 & 10.61929 & 278.40168 & 366.06801 & 427.23106 \\
\hline $2.71 \mathrm{E}+09$ & 142.4692 & 203.37873 & 268.59973 & 353.70439 & 410.63868 \\
\hline $2.81 E+09$ & 137.9729 & 196.82528 & 259.77366 & 341.72356 & 395.81582 \\
\hline $2.92 \mathrm{E}+09$ & 33.7611 & 190.76408 & 251.74575 & 330.2709 & 382.40956 \\
\hline$E+09$ & 29.8738 & 84.75561 & 243.54249 & 318.78784 & 371.08276 \\
\hline+09 & 26.1428 & 38 & 236.09582 & 308.89786 & 360.26288 \\
\hline$E+09$ & 2.6086 & 74.14514 & 229.5567 & 300.42455 & 349.72501 \\
\hline $3.32 \mathrm{E}+09$ & 119.2571 & 169.21893 & 222.86713 & 291.44463 & 339.10601 \\
\hline $3.43 \mathrm{E}+09$ & 116.2055 & 164.58341 & 216.55751 & 283.5669 & 329.04068 \\
\hline $3.53 \mathrm{E}+09$ & 113.2752 & 60.30334 & 210.69562 & 275.45299 & 319.8655 \\
\hline $3.63 \mathrm{E}+09$ & 10.6379 & 156.70177 & 205.9111 & 268.32699 & 311.61519 \\
\hline $3.73 \mathrm{E}+09$ & 108.2746 & 153.09411 & 200.93996 & 261.28538 & 304.33132 \\
\hline $3.84 E+09$ & 105.9452 & 149.43858 & 195.99894 & 254.77075 & 297.50575 \\
\hline $3.94 \mathrm{E}+09$ & 103.7527 & 146.17892 & 191.63928 & 249.21985 & 290.308 \\
\hline $4.04 E+09$ & 101.6839 & 143.24013 & 187.81104 & 244.69862 & 283.1123 \\
\hline $4.14 \mathrm{E}+09$ & 99.74407 & 140.43552 & 184.06087 & 240.02645 & 277.22406 \\
\hline $4.25 \mathrm{E}+09$ & 97.8327 & 137.6272 & 180.04367 & 234.56041 & 271.63487 \\
\hline & 6 & 13521454 & 688 & 229.51929 & 265.7861 \\
\hline
\end{tabular}




\begin{tabular}{|c|c|c|c|c|c|}
\hline & & $\overline{0 C}$ & $60 \mathrm{C}$ & (@) $80 \mathrm{C}$ & @100C \\
\hline 09 & 94.46069 & 132.52018 & 173.0145 & 223.78068 & 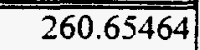 \\
\hline +09 & .86357 & 130.01458 & 169.64829 & 219.48044 & $55.7049^{7}$ \\
\hline $\bar{E}+09$ & 91.31409 & 127.57177 & 166.47942 & 15.48578 & 0.71146 \\
\hline $6 E+09$ & 89.77734 & 125.76915 & 164.15155 & 212.55294 & $\overline{245.4812}$ \\
\hline $86 E+09$ & 88.40364 & 123.60257 & 161.25401 & 209.02557 & 40.7014 \\
\hline $4.96 \mathrm{E}+09$ & 86.95132 & 121.7744 & 158.68029 & 205.50555 & 236.7081 \\
\hline $5.06 \mathrm{E}+09$ & 85.64498 & 119.74174 & 155.73251 & 200.78432 & 32.6598 \\
\hline $5.17 \mathrm{E}+09$ & 4.42311 & 117.55277 & 152.7119 & 196.25702 & 28.9389 \\
\hline $5.27 E+09$ & 83.13161 & 115.65958 & 150.09184 & 192.87226 & 225.1005 \\
\hline $5.37 \mathrm{E}+09$ & 82.02913 & 113.96986 & 147.94198 & 190.57477 & 221.1561 \\
\hline $5.47 \mathrm{E}+09$ & 80.85709 & 112.5106 & 146.14601 & 188.27778 & 17.3003 \\
\hline $5.57 \mathrm{E}+09$ & 79.71158 & 110.9014 & 143.91684 & 185.4173 & 13.694 \\
\hline $5.68 \mathrm{E}+09$ & 78.73079 & 109.31325 & 141.64324 & 182.53196 & 10.2021 \\
\hline $5.78 \mathrm{E}+09$ & 77.60667 & 107.51942 & 138.98406 & 177.99268 & 207.3215 \\
\hline $5.88 \mathrm{E}+09$ & 76.64987 & 105.77174 & 136.52166 & 174.22827 & 04.3492 \\
\hline $5.98 \mathrm{E}+09$ & 75.71374 & 104.39778 & 134.60944 & 172.17476 & 01.0328 \\
\hline $6.09 \mathrm{E}+09$ & 74.82026 & 103.18464 & 133.08383 & 170.82613 & 197.73994 \\
\hline $\mathrm{E}+09$ & 73.9 & 101.9992 & 131.72219 & 169.1916 & 194.92025 \\
\hline $6.29 \mathrm{E}+09$ & 73.09841 & 100.90174 & 130.25008 & 167.46632 & 191.9804 \\
\hline $6.39 \mathrm{E}+09$ & 72.2582 & 99.60389 & 128.30271 & 164.897 & 89.138 \\
\hline $6.5 \mathrm{E}+09$ & 71.44397 & 98.124531 & 126.04394 & 61.34423 & 86.41485 \\
\hline $6.6 \mathrm{E}+09$ & 70.64588 & 96.911776 & 124.42125 & 158.50297 & 183.73056 \\
\hline $6.7 \mathrm{E}+09$ & 69.90596 & 95.652123 & 122.74359 & 156.68842 & 80.74272 \\
\hline $6.8 \mathrm{E}+09$ & 69.18135 & 94.872506 & 121.75996 & 156.70596 & 178.30878 \\
\hline $6.9 \mathrm{E}+09$ & 68.3735 & 94.033143 & 120.70116 & 155.48678 & 175.76911 \\
\hline $7.01 \mathrm{E}+09$ & 67.64443 & 92.908882 & 119.12346 & 153.42256 & 173.55139 \\
\hline $7.11 \mathrm{E}+09$ & 66.91019 & 91.65595 & 117.17295 & 150.83839 & 170.92919 \\
\hline $7.21 \mathrm{E}+09$ & 66.20231 & 90.369184 & 115.30199 & 148.37636 & 169.04443 \\
\hline $7.31 \mathrm{E}+09$ & 65.51994 & 89.251807 & 113.78313 & 145.68211 & 167.08127 \\
\hline $7.42 \mathrm{E}+09$ & 64.8823 & 88.463776 & 112.71268 & 144.44796 & 164.70467 \\
\hline $7.52 \mathrm{E}+09$ & 64.24672 & 87.688744 & 111.79376 & 144.36296 & 162.50837 \\
\hline $7.62 \mathrm{E}+09$ & 63.60482 & 86.95275 & 110.85153 & 143.6758 & 160.81416 \\
\hline $7.72 \mathrm{E}+09$ & 63.055 & 86.114971 & 109.72841 & 141.94765 & 159.47205 \\
\hline $7.83 \mathrm{E}+09$ & 2.53334 & 85.173927 & 108.35319 & 140.15971 & 157.79331 \\
\hline $7.93 \mathrm{E}+09$ & 61.95871 & 83.953278 & 106.57031 & 137.75985 & 156.2645 \\
\hline $8.03 \mathrm{E}+09$ & 61.47818 & 82.934566 & 105.14274 & 135.23916 & 154.63052 \\
\hline $8.13 \mathrm{E}+09$ & 60.93479 & 82.25927 & 104.23268 & 133.59308 & 152.41013 \\
\hline $8.23 E+09$ & 60.43519 & 81.205792 & 102.74749 & 132.56243 & 149.74827 \\
\hline $8.34 \mathrm{E}+09$ & 59.90117 & 80.653289 & 102.07593 & 132.97168 & 147.56177 \\
\hline $8.44 E+09$ & 59.2895 & 79.800147 & 100.91772 & 130.95329 & 145.79759 \\
\hline $8.54 \mathrm{E}+09$ & 58.69689 & 78.804316 & 99.421159 & 128.2828 & 144.26041 \\
\hline $8.64 \mathrm{E}+09$ & 58.06603 & 78.344239 & 98.767567 & 127.74378 & 142.28363 \\
\hline $8.75 \mathrm{E}+09$ & 57.52925 & 77.362239 & 97.394418 & 125.25627 & 140.56141 \\
\hline $8.85 \mathrm{E}+09$ & 57.04209 & 76.542719 & 96.284315 & 122.99583 & 138.8702 \\
\hline $8.95 \mathrm{E}+09$ & 56.48915 & 76.06457 & 95.559829 & 122.67653 & 137.26555 \\
\hline
\end{tabular}




\begin{tabular}{|c|c|c|c|c|c|}
\hline & & & & & \\
\hline 09 & 473 & 75.453686 & 7721 & 122.77177 & 135.59574 \\
\hline $6 \mathrm{E}+09$ & 55.65816 & 75.150899 & 94.25969 & 122.56495 & 34.02367 \\
\hline $26 E+09$ & 55.00332 & 74.222051 & 92.759792 & 4268 & 2.3772 \\
\hline $36 E+09$ & 54.68663 & 73.415176 & 91.63367 & I17.49248 & 131.28013 \\
\hline $9.46 \mathrm{E}+09$ & 54.31722 & 72.913217 & 91.231053 & 117.17358 & 29.689 \\
\hline $9.56 \mathrm{E}+09$ & 53.86524 & 71.795109 & 89.663098 & 113.90159 & 128.23555 \\
\hline $9.67 \mathrm{E}+09$ & 53.4677 & 70.955994 & 88.434363 & 112.35941 & 126.78761 \\
\hline $9.77 \mathrm{E}+09$ & 53.03248 & 71.390162 & 88.949887 & 114.7615 & 125.52715 \\
\hline $9.87 E+09$ & 52.68567 & 70.624223 & 87.662323 & 113.46833 & 396 \\
\hline $9.97 \mathrm{E}+09$ & 2.25604 & 70.478992 & 87.353176 & 112.12635 & 122.8635 \\
\hline $1.01 \mathrm{E}+10$ & 51.8895 & 69.832148 & 86.628175 & 110.56902 & 121.6141 \\
\hline $1.02 \mathrm{E}+10$ & 1.55051 & 68.476161 & 84.871979 & 108.14284 & 120.10138 \\
\hline $1.03 \mathrm{E}+10$ & 51.27473 & 67.791171 & 84.166446 & 106.6837 & 119.02266 \\
\hline $1.04 \mathrm{E}+10$ & 50.93593 & 67.161678 & 83.300371 & 105.04993 & 118.03095 \\
\hline $1.05 \mathrm{E}+10$ & 50.63555 & 66.932615 & 82.836661 & 105.79628 & 117.31016 \\
\hline $1.06 \mathrm{E}+10$ & .32608 & 67.507619 & 83.637712 & 108.44228 & 116.16754 \\
\hline $1.07 \mathrm{E}+10$ & 50.03003 & 66.996525 & 82.79663 & 106.52861 & 417 \\
\hline $1.08 \mathrm{E}+10$ & 49.73397 & 65.991624 & 81.358123 & 103.39405 & 113.94623 \\
\hline $1.09 \mathrm{E}+10$ & 49.35221 & 65.101226 & 80.265842 & 101.30735 & 112.87337 \\
\hline $1.1 \mathrm{E}+10$ & 49.05093 & 64.418894 & 5209 & 99.824991 & 111.60467 \\
\hline $1.11 \mathrm{E}+10$ & 48.75962 & 63.961494 & 78.949629 & 99.124837 & 110.7387 \\
\hline $1.12 \mathrm{E}+10$ & 48.46002 & 63.924693 & 78.897111 & 100.47488 & 10.24597 \\
\hline $1.13 \mathrm{E}+10$ & 48.13601 & 64.108517 & 78.985536 & 102.49362 & 110.09659 \\
\hline $1.14 \mathrm{E}+10$ & 47.85939 & 63.727969 & 78.375649 & 102.0062 & 109.78496 \\
\hline $1.15 \mathrm{E}+10$ & 47.60625 & 63.165572 & 77.436431 & 99.42727 & 109.84463 \\
\hline $1.16 \mathrm{E}+10$ & 47.31791 & 63.015102 & 77.324514 & 98.099504 & 108.28335 \\
\hline$E+10$ & 634 & 98 & 77.480923 & 97.504167 & 106.6797 \\
\hline $\mathrm{E}+10$ & 46.87254 & 62.403995 & 76.706452 & 96.023734 & 105.40622 \\
\hline $1.19 \mathrm{E}+10$ & 46.64065 & 61.349839 & 75.312659 & 95.103033 & 105.31228 \\
\hline $1.2 \mathrm{E}+10$ & 46.40921 & 61.051402 & 74.744167 & 96.574618 & 105.9924 \\
\hline $1.21 \mathrm{E}+10$ & 46.10506 & 60.543216 & 74.095914 & 97.320044 & 106.20147 \\
\hline $1.22 \mathrm{E}+10$ & 45.87448 & 60.204124 & 73.667904 & 96.667586 & 105.45088 \\
\hline $1.23 \mathrm{E}+10$ & 45.57329 & 60.968079 & 74.578132 & 96.812934 & 104.16941 \\
\hline $1.24 \mathrm{E}+10$ & 45.31693 & 61.989996 & 76.164841 & 97.542598 & 102.65504 \\
\hline $1.25 \mathrm{E}+10$ & 45.07617 & 60.899881 & 74.56561 & 94.375121 & 101.04619 \\
\hline $1.26 \mathrm{E}+10$ & 44.95899 & 59.704517 & 73.022616 & 93.908875 & 99.827146 \\
\hline $1.27 \mathrm{E}+10$ & 44.73693 & 58.450459 & 71.590498 & 95.307611 & 98.773142 \\
\hline $1.28 \mathrm{E}+10$ & 44.60396 & 57.980175 & 71.443755 & 96.925243 & 98.848791 \\
\hline $1.29 \mathrm{E}+10$ & 44.42547 & 58.178672 & 71.843791 & 97.429984 & 8.64983 \\
\hline $1.3 \mathrm{E}+10$ & 44.23278 & 58.873583 & 72.590553 & 96.567976 & 98.844613 \\
\hline $1.31 \mathrm{E}+10$ & .94469 & 098 & 74.256461 & 96.404499 & 98.227165 \\
\hline $1.32 \mathrm{E}+10$ & 43.64179 & 59.628652 & 73.153378 & 92.971755 & 96.922518 \\
\hline $1.33 \mathrm{E}+10$ & 43.46359 & 57.699822 & 70.74896 & 91.701848 & 95.156884 \\
\hline $1.35 \mathrm{E}+10$ & 43.3195 & 56.933769 & 70.486169 & 95.185488 & 94.02 \\
\hline $1.36 \mathrm{~F}$ & 43.1497 & 56.894515 & 71.15308 & 97.205152 & 3.80742 \\
\hline
\end{tabular}




\begin{tabular}{|c|c|c|c|c|c|}
\hline & & $\bar{C}$ & $\overline{\mathbf{C}}$ & $\bar{C}$ & \\
\hline & 42.95113 & 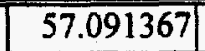 & 091 & 4 & 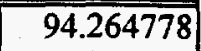 \\
\hline & 3836 & 08 & 3581 & 5.244094 & \\
\hline $1.39 \mathrm{E}+10$ & 42.52115 & 615 & 70.963769 & 1.918895 & $\overline{503}$ \\
\hline & 661 & 728 & 339 & 86.20893 & 3.129511 \\
\hline+10 & 2.17772 & 5.711779 & 6.933101 & 3.871802 & 1.614819 \\
\hline & 03293 & 351 & 6.293143 & 7.021059 & $\overline{0.45}$ \\
\hline+10 & 41.94 & 5.724069 & 68.09407 & 89.422633 & 0.239523 \\
\hline-10 & 41.82898 & 5.050446 & 8.942542 & 87.076936 & 0.71985 \\
\hline+10 & 1.65597 & 55.176196 & 67.387658 & 85.736738 & 90.97371 \\
\hline 10 & .48324 & 679 & 66.147735 & 5.232583 & .2 \\
\hline & .28264 & 024 & 65.621708 & 0.925501 & $\overline{8.88}$ \\
\hline & 10235 & 603 & 64.435514 & 9.444786 & 7.528441 \\
\hline & 581 & & 343 & 8.195958 & 68 \\
\hline+10 & .82747 & .450186 & 67.97508 & 94.590822 & 6.05996 \\
\hline & 37 & 99 & 7557 & 7.408343 & 24 \\
\hline+10 & 40.60871 & 5.748896 & 68.458437 & 87.822782 & 5.744434 \\
\hline+10 & 9654 & 889 & 382 & 88.813051 & 6.22 \\
\hline 10 & 40.25779 & 479 & 935 & 80.21472 & 02 \\
\hline+10 & 40.17263 & 53.536926 & 3.839333 & 76.929543 & 83.866012 \\
\hline & .07892 & 39 & 913 & 8.163996 & 3.07 \\
\hline+10 & .77517 & 3.096037 & 64.77023 & 90.519929 & 82.221841 \\
\hline & 18 & 556 & 6.675339 & 2.85033 & 2.44 \\
\hline+10 & 39.50481 & 54.088098 & 66.019158 & 4.272746 & 3.667682 \\
\hline+10 & 39.42737 & 51 & 63.79366 & 83.035227 & 2.78 \\
\hline+10 & 39.39238 & 903 & 52.173252 & 73.663766 & 1.44 \\
\hline+10 & 39.31226 & 485 & 59.166089 & 70.185073 & 30.98 \\
\hline+10 & .14258 & 01 & 58.679908 & 76.929608 & 0. \\
\hline+10 & 97802 & 331 & 60.718973 & 79.546389 & 79.22371 \\
\hline+10 & .96848 & 507 & 62.767575 & 78.692397 & 9.643524 \\
\hline+10 & 82551 & 562 & 63.475975 & 80.41617 & 0.703277 \\
\hline+10 & 51811 & 781 & 60.38071 & 76.178991 & 0.200688 \\
\hline+10 & 1887 & 455 & 58.393221 & 71.86971 & 78.834719 \\
\hline+10 & 62111 & 274 & 58.273036 & 1.932422 & 8.079365 \\
\hline+10 & 41737 & 49.808795 & 58.975817 & 75.944425 & 77.19531 \\
\hline+10 & 3069 & 35 & 59.03847 & 8.786854 & 5.866126 \\
\hline+10 & 29223 & 49.66 & 59.34156 & 77.138155 & 5.318542 \\
\hline+10 & 16235 & 50.1 & 59.817525 & 74.484851 & 146 \\
\hline$E+10$ & .06297 & 48.845981 & 57.664982 & 72.770921 & 76.582055 \\
\hline $1.75 \mathrm{E}+10$ & 38.06664 & 48.054273 & 56.531162 & 72.67491 & 76.516701 \\
\hline $1.76 \mathrm{E}+10$ & 37.7665 & 48.26165 & 56.854386 & 71.695366 & 76.085471 \\
\hline+10 & 37.44212 & 48.57 & 57.222166 & 73.629597 & 75.086009 \\
\hline+10 & & & 56.390085 & 75.089806 & 73.809666 \\
\hline+10 & 37.35015 & 47.631547 & 55.539636 & 70.45221 & 73.144297 \\
\hline & & & & 69.221213 & 73.550212 \\
\hline-10 & 20911 & 48.72832 & 56.806597 & 71.017581 & $T$ \\
\hline
\end{tabular}




\begin{tabular}{|r|r|r|r|r|r|}
\hline Frequency & $\mathrm{e}^{\prime \prime} @ 20 \mathrm{C}$ & $\mathrm{e}^{\prime \prime} @ 40 \mathrm{C}$ & $\mathrm{e}$ " @ 60 C & $\mathrm{e}^{\prime \prime} @ 80 \mathrm{C}$ & $\mathrm{e}$ " @ 100 C \\
\hline $1.83 \mathrm{E}+10$ & 37.16039 & 47.725856 & 55.787148 & 72.537009 & 74.742207 \\
\hline $1.84 \mathrm{E}+10$ & 36.92305 & 47.821096 & 55.864349 & 73.944486 & 74.286507 \\
\hline $1.85 \mathrm{E}+10$ & 36.68188 & 48.942379 & 57.515972 & 77.386512 & 73.480084 \\
\hline $1.86 \mathrm{E}+10$ & 36.64845 & 47.704049 & 55.179518 & 73.060187 & 72.535838 \\
\hline $1.87 \mathrm{E}+10$ & 36.46677 & 48.614654 & 55.786378 & 73.051393 & 71.526826 \\
\hline $1.88 \mathrm{E}+10$ & 36.41156 & 48.530077 & 55.49623 & 69.2329 & 71.576704 \\
\hline $1.89 \mathrm{E}+10$ & 36.46557 & 47.735264 & 54.892884 & 65.128756 & 72.497731 \\
\hline $1.9 \mathrm{E}+10$ & 36.41548 & 46.774922 & 54.24743 & 68.765839 & 73.16245 \\
\hline $1.91 \mathrm{E}+10$ & 36.25736 & 46.489127 & 54.151187 & 76.198223 & 72.84257 \\
\hline $1.92 \mathrm{E}+10$ & 36.08991 & 47.374606 & 55.175903 & 73.987537 & 72.160568 \\
\hline $1.93 \mathrm{E}+10$ & 35.9057 & 47.061992 & 54.553516 & 70.044789 & 71.24224 \\
\hline $1.94 \mathrm{E}+10$ & 35.75581 & 46.308225 & 53.08213 & 70.627912 & 70.323744 \\
\hline $1.95 \mathrm{E}+10$ & 35.68829 & 46.874994 & 53.498495 & 70.147279 & 69.939638 \\
\hline $1.96 \mathrm{E}+10$ & 35.65604 & 46.455184 & 53.459922 & 62.308423 & 70.235851 \\
\hline $1.97 \mathrm{E}+10$ & 35.58794 & 45.944813 & 53.174287 & 64.485086 & 71.114607 \\
\hline $1.98 \mathrm{E}+10$ & 35.51936 & 45.397879 & 52.727301 & 71.59444 & 70.770369 \\
\hline $1.99 \mathrm{E}+10$ & 35.44807 & 45.229972 & 52.495654 & 68.052792 & 69.867054 \\
\hline $2 \mathrm{E}+10$ & 35.30132 & 44.60667 & 51.436542 & 61.704081 & 69.515849 \\
\hline
\end{tabular}


Table 3. Tangent for Hanford 4M Simulant

\begin{tabular}{|c|c|c|c|c|c|}
\hline & $\mathrm{C}$ & $\overline{\mathrm{DC}}$ & $\overline{D C}$ & $80 \mathrm{C}$ & 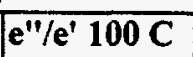 \\
\hline+08 & 1.06716 & 12.09014 & 13.5566 & 14.31842 & 3207 \\
\hline 08 & 77921 & 32197 & $\overline{58499}$ & 7.53811 & .661 \\
\hline $3.58 \mathrm{E}+08$ & .01958 & .16753 & 5.03515 & 17.43142 & $\overline{8082}$ \\
\hline $6 \bar{E}+08$ & $\overline{42538}$ & 2.44502 & .28874 & 6.6857 & $\overline{9341}$ \\
\hline $5.62 E+08$ & 407046 & 11.70713 & 13.5321 & 15.83161 & 7547 \\
\hline $6.65 \mathrm{E}+08$ & 7.641725 & 10.76097 & 12.42354 & 4.20419 & 8.9550 \\
\hline $7.67 \mathrm{E}+08$ & 7.010869 & 9.84566 & 11.31894 & 12.8704 & .8911 \\
\hline $8.69 E+08$ & 6.54558 & 9.214278 & 10.62551 & 12.22504 & .07597 \\
\hline $9.71 \mathrm{E}+08$ & 084396 & 575964 & 9.8652 & 11.44914 & 299 \\
\hline $1.07 \mathrm{E}+09$ & 714624 & 8.144516 & 9.428163 & 1.15312 & $\overline{1640}$ \\
\hline $1.18 E+09$ & 17717 & 0129 & 950787 & 0.54296 & 9957 \\
\hline $1.28 \mathrm{E}+09$ & 134052 & 7.338591 & 8.509553 & 946751 & $6 \overline{63348}$ \\
\hline 09 & 33 & 0935 & 95801 & 127579 & .4525 \\
\hline $1.48 \mathrm{E}+09$ & 4.605842 & 6.544428 & 7.540468 & 8.555308 & .23326 \\
\hline $1.59 E+09$ & 397576 & 39828 & 7.216891 & 4013 & 183 \\
\hline $1.69 E+09$ & 4.223937 & 5.950322 & 6.873752 & 8.079448 & 8.083171 \\
\hline $1.79 E+09$ & 063765 & 5.70 & 6.573362 & 7.754113 & 7.95 \\
\hline $1.89 \mathrm{E}+09$ & 902237 & 5.519615 & 6.39238 & 7.583766 & 7.70437 \\
\hline $1.99 \mathrm{E}+09$ & 3.755663 & 5.306716 & 6.13769 & 7.206551 & 41469 \\
\hline $2.1 \mathrm{E}+09$ & 382772 & 5.065022 & 5.814372 & 6.708716 & 222 \\
\hline $2.2 E+09$ & 3.480324 & 4.912022 & 5.636157 & 6.462268 & .109024 \\
\hline $2.3 E+09$ & 380462 & 22066 & 419614 & 6.272947 & 0 \\
\hline $2.4 \mathrm{E}+09$ & .291279 & 4.616648 & 5.329186 & 6.315291 & .88008 \\
\hline $2.51 \mathrm{E}+09$ & 3.205833 & 4.501698 & 5.196777 & 6.206316 & 6.70287 \\
\hline $2.61 \mathrm{E}+09$ & 3.118054 & 4.416263 & 5.106753 & 6.063628 & 6.567522 \\
\hline $2.71 \mathrm{E}+09$ & 3.045463 & 81331 & 4.925518 & 5.75338 & .5216 \\
\hline $2.81 \mathrm{E}+09$ & 2.9718 & 147751 & 4.747519 & 5.455351 & 6.40954 \\
\hline $2.92 E+09$ & 2.907147 & 4.049801 & .637874 & 5.31228 & 6.22632 \\
\hline $3.02 \mathrm{E}+09$ & 2.850321 & 986779 & 4.581946 & $\overline{5.31068}$ & .09438 \\
\hline $3.12 \mathrm{E}+09$ & 2.79785 & 97973 & 92917 & 5.347227 & 5.96788 \\
\hline $3.22 \mathrm{E}+09$ & 2.740041 & 3.818672 & 4.400145 & 5.28514 & 5.87752 \\
\hline $3.32 \mathrm{E}+09$ & 2.680717 & 179 & 4.335341 & 5.164518 & 5.809503 \\
\hline $3.43 \mathrm{E}+09$ & 2.624519 & 558134 & 4.19365 & 4.908575 & 5.69188 \\
\hline $3.53 \mathrm{E}+09$ & 2.571545 & 3.550793 & 4.041704 & 4.642289 & .56496 \\
\hline $3.63 \mathrm{E}+09$ & 2.517185 & 3.476513 & 3.942682 & 4.483183 & 5.484791 \\
\hline $3.73 E+09$ & 2.482335 & 3.448196 & 3.930623 & 4.528856 & 5.407053 \\
\hline $3.84 \mathrm{E}+09$ & 2.445828 & 3.38998 & 3.875686 & 4.579187 & 5.310867 \\
\hline $3.94 \mathrm{E}+09$ & 2.409744 & 3.319103 & 3.791384 & 4.520484 & 5.206597 \\
\hline $4.04 E+09$ & 2.375959 & 3.264831 & 3.712377 & 4.3609 & 5.112572 \\
\hline $4.14 \mathrm{E}+09$ & 2.346681 & 3.223681 & 3.658879 & 4.232261 & 5.057927 \\
\hline $4.25 \mathrm{E}+09$ & 2.31376 & 3.164944 & 3.563496 & 4.0845 & 5.041987 \\
\hline $4.35 \mathrm{E}+09$ & 2.289886 & 3.122432 & 3.507185 & 3.976712 & 5.0572 \\
\hline
\end{tabular}




\begin{tabular}{|c|c|c|c|c|c|}
\hline & $\overline{0 C}$ & $\overline{\mathrm{OC}}$ & C & $\bar{C}$ & C \\
\hline $4.45 E+09$ & 2.271038 & 3.116788 & 3.516487 & 4.010615 & 5.010116 \\
\hline $.55 \mathrm{E}+09$ & 2.249041 & 3.106888 & 3.527809 & 149014 & 941426 \\
\hline $4.65 E+09$ & 2.226674 & 3.06659 & 3.480575 & 4.170046 & 397477 \\
\hline $4.76 \mathrm{E}+09$ & 2.208023 & 3.064975 & 3.493241 & 4.14019 & .886187 \\
\hline $4.86 \mathrm{E}+09$ & 2.188479 & 3.033429 & 3.440941 & 3.969963 & 907872 \\
\hline $4.96 \mathrm{E}+09$ & 2.169615 & 3.009303 & 3.394958 & 3.892383 & .884205 \\
\hline $5.06 E+09$ & 2.154295 & 2.983057 & 3.359809 & 3.836629 & 8.86864 \\
\hline $5.17 \bar{E}+09$ & 2.140467 & 2.954201 & 3.331908 & 3.816506 & .845237 \\
\hline $5.27 \mathrm{E}+09$ & 2.121353 & 2.949356 & 3.339728 & 3.919823 & .821684 \\
\hline $5.37 \mathrm{E}+09$ & 2.109932 & 2.917265 & 3.309256 & 4.013941 & .792015 \\
\hline $5.47 E+09$ & 2.092342 & 2.892971 & 3.27863 & 3.916055 & .767521 \\
\hline $5.57 \mathrm{E}+09$ & 2.076625 & 2.88604 & 3.266903 & 3.788394 & 1.768098 \\
\hline $5.68 E+09$ & 2.06618 & 2.8494 & 3.199611 & 3.663755 & 1.77844 \\
\hline $5.78 \mathrm{E}+09$ & 2.05172 & 2.852461 & 3.207436 & 3.738199 & 7.733831 \\
\hline $5.88 E+09$ & 2.038126 & 2.804826 & 3.14203 & 3.646358 & .668941 \\
\hline $5.98 \mathrm{E}+09$ & 2.026457 & 2.789836 & 3.135575 & 3.693766 & 4.63689 \\
\hline $6.09 \mathrm{E}+09$ & 2.014494 & 2.78151 & 3.132085 & 3.796406 & 4.585453 \\
\hline $6.19 \mathrm{E}+09$ & 2.004719 & 2.756894 & 3.10696 & 3.757429 & 4.580407 \\
\hline $6.29 E+09$ & 1.992407 & 2.719445 & 3.040267 & 3.488057 & .588072 \\
\hline $6.39 E+09$ & 1.987027 & 2.715658 & 3.026141 & 3.406386 & 4.596379 \\
\hline $6.5 \mathrm{E}+09$ & 1.975486 & 2.680905 & 2.953775 & 3.362887 & 4.558063 \\
\hline $6.6 \mathrm{E}+09$ & 1.972035 & 2.684299 & 2.964232 & 3.431653 & 4.500629 \\
\hline $6.7 \mathrm{E}+09$ & 1.964782 & 2.665277 & 2.951071 & 3.436815 & $4 . \overline{4606}$ \\
\hline $6.8 \mathrm{E}+09$ & 1.96072 & 2.662586 & 2.964831 & 3.58677 & 4.41773 \\
\hline $6.9 \mathrm{E}+09$ & 1.951998 & 2.680261 & 2.998736 & 3.694123 & 4.431053 \\
\hline $7.01 \mathrm{E}+09$ & 1.944098 & 2.65325 & 2.953504 & 3.438127 & 4.44223 \\
\hline $7.11 \mathrm{E}+09$ & 1.935407 & 2.63836 & 2.907771 & 3.238482 & 4.450724 \\
\hline $7.21 \mathrm{E}+09$ & 1.92321 & 2.637275 & 2.882092 & 3.270722 & 4.453786 \\
\hline $7.31 \mathrm{E}+09$ & 1.915895 & 2.621049 & 2.8653 & 3.343544 & $\overline{4.441731}$ \\
\hline $7.42 \mathrm{E}+09$ & 1.912357 & 2.628651 & 2.891512 & 3.417742 & 4.396178 \\
\hline $7.52 E+09$ & 1.906195 & 2.649437 & 2.93842 & 3.590726 & 4.362523 \\
\hline $7.62 \mathrm{E}+09$ & 1.896702 & 2.639015 & 2.935706 & 3.669408 & 4.33275 \\
\hline $7.72 \mathrm{E}+09$ & 1.894989 & 2.60197 & 2.878587 & 3.437103 & 4.35012 \\
\hline $7.83 E+09$ & 1.887851 & .590978 & 2.851612 & 3.231401 & 4.423239 \\
\hline $7.93 \mathrm{E}+09$ & 1.879273 & 2.569991 & 2.802708 & 3.194979 & 4.421737 \\
\hline $8.03 \mathrm{E}+09$ & 1.874484 & 2.562333 & 2.779817 & 3.292056 & 4.47000 \\
\hline $8.13 E+09$ & 1.880183 & 2.587646 & 2.841752 & 3.463561 & 4.531542 \\
\hline $8.23 E+09$ & 1.878861 & 2.577832 & 2.840803 & 3.484729 & 4.482648 \\
\hline $8.34 \mathrm{E}+09$ & 1.880665 & 2.575236 & 2.846429 & 3.590123 & 4.43725 \\
\hline $8.44 \mathrm{E}+09$ & 1.872776 & 2.558972 & 2.821177 & 3.458399 & 4.410425 \\
\hline $8.54 \mathrm{E}+09$ & 1.866789 & 2.499446 & 2.719328 & 3.081417 & 4.374497 \\
\hline $8.64 \mathrm{E}+09$ & 1.854177 & 2.474535 & 2.667235 & 2.994368 & 4.315258 \\
\hline $8.75 \mathrm{E}+09$ & 1.844599 & 2.493613 & 2.688517 & 3.18867 & 4.263131 \\
\hline $5 E+09$ & 1.837141 & 2.492499 & 2.699335 & 3.310158 & 4.193302 \\
\hline $8.95 E+09$ & 1.833373 & 2.506953 & 2.737547 & 3.375244 & 4.1529 \\
\hline
\end{tabular}




\begin{tabular}{|c|c|c|c|c|c|}
\hline & & & & & \\
\hline 9 & 52 & 05627 & 1777 & -1 & 12617 \\
\hline & & 9128 & 63432 & 6872 & \\
\hline \begin{tabular}{|l|}
$E+09$ \\
\end{tabular} & 056 & 53138 & 25316 & $\overline{69}$ & UJUY! \\
\hline & 796685 & 57 & 20127 & $7 \overline{72677}$ & 68 \\
\hline $\bar{E}+09$ & 94447 & 2.40216 & 535734 & 2024 & 14 \\
\hline & 1.7854 & 21 & 62851 & 128594 & 87401 \\
\hline $9.67 E+09$ & 776408 & 2.37268 & 2.533453 & 053213 & 83439 \\
\hline 09 & 75706 & 772 & 7389 & $\overline{106047}$ & 012 \\
\hline $9.87 \mathrm{E}+09$ & 765509 & 2.384812 & 518562 & 935866 & 81072 \\
\hline-09 & & 358138 & 160166 & 675354 & 81 \\
\hline $1.01 \mathrm{E}+10$ & 54442 & 2.327901 & 2.412633 & 523533 & 6601 \\
\hline+10 & 39 & 70454 & 5001 & 555995 & .602 \\
\hline $1.03 \mathrm{E}+10$ & 45669 & 2.25227 & 2.338477 & 734642 & 3.57166 \\
\hline-10 & 61 & 99 & 041 & 840496 & 5 \\
\hline $1.05 \mathrm{E}+10$ & 1.731273 & 2.276827 & 2.381913 & 805111 & 3.58 \\
\hline $5 \mathrm{E}+10$ & 1.73034 & 449 & 6717 & 31197 & .54 \\
\hline $1.07 E+10$ & 731173 & 2.358756 & 2.446997 & 2.749529 & .49 \\
\hline+10 & 91 & 1013 & 2.373706 & 544911 & .40 \\
\hline $1.09 \mathrm{E}+10$ & 27194 & 49961 & 2.323125 & 17275 & \\
\hline $1.1 E+10$ & $\overline{663}$ & 218751 & 2.30117 & 6923 & 74 \\
\hline $1.11 E+10$ & ) & .22571 & 323685 & $82493^{\prime}$ & 91 \\
\hline $1.12 \mathrm{E}+10$ & 10338 & 258031 & 347788 & 83397 & .35505 \\
\hline $1.13 \mathrm{E}+10$ & $\overline{445}$ & 2.32983 & 395401 & 82671 & \\
\hline $1.14 \mathrm{E}+10$ & 02073 & 328396 & 352591 & .65385 & 27 \\
\hline $1.15 \mathrm{E}+10$ & 7742 & 24 & 27427 & $\overline{358}$ & \\
\hline $\mathrm{E}+10$ & 4745 & 52467 & 2.153445 & 289474 & \\
\hline $7 \mathrm{E}+10$ & 3452 & 2.125376 & 2.15242 & 452073 & 65 \\
\hline $8 E+10$ & 0244 & 2.16023 & 212491 & 639045 & 179 \\
\hline $1.19 \mathrm{E}+10$ & 978 & 95835 & 249083 & 539509 & 17 \\
\hline $1.2 \mathrm{E}+10$ & 031 & 617 & 244792 & 1495 & 14 \\
\hline $1.21 \mathrm{E}+10$ & 1.6804 & 269086 & 220598 & 421552 & .53723 \\
\hline $1.22 \mathrm{E}+10$ & 1.6802 & 192043 & 938 & 202982 & 97 \\
\hline $3 E+10$ & 677493 & 090353 & & 5934 & 61 \\
\hline $1.24 \mathrm{E}+10$ & 3626 & 2.131524 & 2.11224 & .378057 & .07152 \\
\hline $1.25 \mathrm{E}+10$ & 0093 & .176808 & 2.189882 & .531163 & 3.05717 \\
\hline $1.26 \mathrm{E}+10$ & 0215 & 2.161661 & .159279 & 2.40847 & 3.17730 \\
\hline $1.27 \mathrm{E}+10$ & 1.665781 & 2.172767 & .124338 & 2.366433 & 3032 \\
\hline $1.28 \mathrm{E}+10$ & 6635 & 153472 & .054009 & 264779 & .36663 \\
\hline $1.29 \mathrm{E}+10$ & 4705 & 2.127787 & 2.012134 & 2.176528 & 3.29711 \\
\hline $1.3 \mathrm{E}+10$ & 64581 & 2.093164 & 2.009103 & 2.205194 & 3.15275 \\
\hline $1.31 \mathrm{E}+10$ & 1.667208 & 2.162188 & 2.134839 & 2.568732 & 3.02841 \\
\hline $1.32 \mathrm{E}+10$ & 1.664002 & 2.222294 & 2.265024 & 2.876085 & 3.00132 \\
\hline $1.33 E+10$ & 59525 & 2.164247 & 2.233029 & 2.821366 & 3.01777 \\
\hline$E+10$ & & & 6.15700 & 2.8296 & 3.06 \\
\hline 1.30 & 1.001 & $2.0^{7}$ & 2.110910 & 316617 & \\
\hline
\end{tabular}




\begin{tabular}{|c|c|c|c|c|c|}
\hline & & & & & \\
\hline 10 & 1.65786 & 508 & 2.129533 & 443 & 1715 \\
\hline+10 & 1.653379 & 2.137761 & 2.13263 & 2.521611 & $\overline{05009}$ \\
\hline $1.39 \mathrm{E}+10$ & 1.648604 & 2.195035 & 2.192969 & 2.752177 & 9701 \\
\hline $1.4 \mathrm{E}+10$ & 1.64601 & 2.245371 & 2.253148 & 2.861907 & .9436 \\
\hline $1.41 \mathrm{E}+10$ & 1.649244 & 2.225171 & 2.243864 & 2.703287 & 9286 \\
\hline+10 & 1.650752 & 2.14929 & 2.168614 & 2.71041 & 2.93717 \\
\hline $1.43 \mathrm{E}+10$ & 1.655362 & 2.160547 & 2.195967 & 3.010266 & 9656 \\
\hline $1.44 \mathrm{E}+10$ & 1.65896 & 2.241658 & 2.30088 & 2.993594 & 3.01887 \\
\hline $1.45 \mathrm{E}+10$ & 1.651153 & 2.244546 & 2.286969 & 2.762824 & 3.0036 \\
\hline $1.46 \mathrm{E}+10$ & 1.645329 & 2.199812 & 2.204634 & 2.897618 & 2.94089 \\
\hline $1.47 \mathrm{E}+10$ & 1.642171 & 2.219205 & 2.199148 & 3.065543 & .86763 \\
\hline $1.48 \mathrm{E}+10$ & 1.642198 & 2.218784 & 2.18174 & 2.64843 & .8221 \\
\hline $1.49 \mathrm{E}+10$ & 1.646626 & 2.17571 & 2.122714 & 2.589522 & .8352 \\
\hline $1.5 \mathrm{E}+10$ & 1.655559 & 2.200331 & 2.159712 & 3.254027 & .86579 \\
\hline $1.51 \mathrm{E}+10$ & 1.646505 & 2.34915 & 2.380774 & 3.203247 & 2.86085 \\
\hline $1.52 \mathrm{E}+10$ & 1.654805 & 2.323489 & 2.321187 & 2.723976 & 2.873 \\
\hline $1.53 \mathrm{E}+10$ & 1.648333 & 2.299997 & 2.247936 & 3.199403 & 2.86432 \\
\hline $1.54 \mathrm{E}+10$ & 1.639279 & 2.294038 & 2.241799 & 3.406175 & 2.75322 \\
\hline $1.55 \mathrm{E}+10$ & 1.64233 & 2.311299 & 2.235701 & 2.631419 & 2.67951 \\
\hline $1.56 \mathrm{E}+10$ & 1.654374 & 2.172747 & 2.086948 & 2.678154 & 2.76407 \\
\hline $1.57 \mathrm{E}+10$ & 1.655456 & 2.123396 & 2.073024 & 3.520153 & 2.80113 \\
\hline $1.58 \mathrm{E}+10$ & 1.649062 & 2.262711 & 2.233438 & 2.820484 & 2.75633 \\
\hline $1.59 \mathrm{E}+10$ & 1.648052 & 2.268468 & 2.217666 & 2.429551 & 2.79892 \\
\hline $1.6 \mathrm{E}+10$ & 1.650703 & 2.253514 & 2.154292 & 2.974622 & 2.78104 \\
\hline $1.61 \mathrm{E}+10$ & 1.653215 & 2.2 & 2.196383 & 2.997327 & 2.61930 \\
\hline $1.62 \mathrm{E}+10$ & 1.65828 & 2.190454 & 2.089053 & 2.309443 & 2.584685 \\
\hline $1.63 \mathrm{E}+10$ & 1.667941 & 2.08815 & 2.001478 & 2.429187 & 2.65945 \\
\hline $1.64 \mathrm{E}+10$ & 1.660639 & 2.104331 & 2.051588 & 2.787476 & 2.68001 \\
\hline $1.65 \mathrm{E}+10$ & 1.664237 & 2.252562 & 2.240386 & 2.677159 & 2.72068 \\
\hline $1.66 \mathrm{E}+10$ & 1.672861 & 2.3907 & 2.360589 & 2.626597 & 2.79693 \\
\hline $1.67 E+10$ & 1.664885 & 2.298183 & 2.220213 & 2.601667 & 2.77842 \\
\hline $1.68 \mathrm{E}+10$ & 1.668818 & 2.169445 & 2.050053 & 2.463736 & 2.58639 \\
\hline $1.69 \mathrm{E}+10$ & 1.685114 & 2.100612 & 1.966849 & 2.303051 & 2.49352 \\
\hline $1.7 \mathrm{E}+10$ & 1.689375 & 2.164031 & 2.06168 & 2.43608 & 2.54406 \\
\hline $1.71 \mathrm{E}+10$ & 1.683359 & 2.235221 & 2.195526 & 2.891161 & 2.6121 \\
\hline $1.72 \mathrm{E}+10$ & 1.693294 & 2.282586 & 2.286042 & 3.155127 & 2.61946 \\
\hline $1.73 E+10$ & 1.700865 & 2.377438 & 2.373103 & 2.958873 & 2.63191 \\
\hline $1.74 \mathrm{E}+10$ & 1.694817 & 2.321064 & 2.233967 & 2.556119 & 2.636152 \\
\hline $1.75 \mathrm{E}+10$ & 1.713774 & 2.180126 & 2.035715 & 2.512209 & 2.55114 \\
\hline $1.76 \mathrm{E}+10$ & 1.720904 & 2.142141 & 1.987823 & 2.492049 & 2.46152 \\
\hline $1.77 \mathrm{E}+10$ & 1.704926 & 2.258866 & 2.145617 & 2.675644 & 2.42838 \\
\hline $1.79 E+10$ & 1.709993 & 2.317129 & 2.272255 & 3.263564 & 2.444599 \\
\hline $1.8 \mathrm{E}+10$ & 1.711894 & 2.287229 & 2.274079 & 3.315153 & 2.45141 \\
\hline $1.81 \mathrm{E}+10$ & 1.713435 & 2.347098 & 2.289082 & 2.977937 & 2.47682 \\
\hline $1.82 \mathrm{E}+10$ & 1.719175 & 2.414968 & 2.277255 & 2.953109 & 2.5095 \\
\hline
\end{tabular}




\begin{tabular}{|r|r|r|r|r|r|}
\hline Frequency & $e^{\prime \prime} / e^{\prime} 20 \mathrm{C}$ & $e^{\prime \prime} / e^{\prime} 40 \mathrm{C}$ & $e^{\prime \prime} / e^{\prime} 60 \mathrm{C}$ & $e^{\prime \prime} / e^{\prime} 80 \mathrm{C}$ & $e^{\prime \prime} / e^{\prime} 100 \mathrm{C}$ \\
\hline \hline $1.83 \mathrm{E}+10$ & 1.724282 & 2.281737 & 2.103756 & 2.708105 & 2.526549 \\
\hline $1.84 \mathrm{E}+10$ & 1.724881 & 2.166368 & 1.967542 & 2.414886 & 2.466223 \\
\hline $1.85 \mathrm{E}+10$ & 1.724143 & 2.305524 & 2.119651 & 3.035877 & 2.407035 \\
\hline $1.86 \mathrm{E}+10$ & 1.72185 & 2.347095 & 2.167794 & 3.247193 & 2.375282 \\
\hline $1.87 \mathrm{E}+10$ & 1.724579 & 2.371274 & 2.198258 & 2.906981 & 2.372997 \\
\hline $1.88 \mathrm{E}+10$ & 1.727555 & 2.411401 & 2.213765 & 2.965906 & 2.385588 \\
\hline $1.89 \mathrm{E}+10$ & 1.732649 & 2.396673 & 2.178821 & 3.090385 & 2.449734 \\
\hline $1.9 \mathrm{E}+10$ & 1.749965 & 2.28805 & 2.073755 & 2.596247 & 2.524657 \\
\hline $1.91 \mathrm{E}+10$ & 1.745414 & 2.147241 & 1.941807 & 2.6517 & 2.533225 \\
\hline $1.92 \mathrm{E}+10$ & 1.738169 & 2.18985 & 1.945859 & 3.102392 & 2.460074 \\
\hline $1.93 \mathrm{E}+10$ & 1.742547 & 2.264398 & 2.01355 & 2.793543 & 2.389748 \\
\hline $1.94 \mathrm{E}+10$ & 1.745024 & 2.275006 & 2.010857 & 2.585716 & 2.348587 \\
\hline $1.95 \mathrm{E}+10$ & 1.750568 & 2.208334 & 1.917495 & 2.609889 & 2.352057 \\
\hline $1.96 \mathrm{E}+10$ & 1.759596 & 2.261273 & 1.976332 & 2.490113 & 2.405472 \\
\hline $1.97 \mathrm{E}+10$ & 1.765741 & 2.249774 & 1.974725 & 2.321008 & 2.520628 \\
\hline $1.98 \mathrm{E}+10$ & 1.76125 & 2.203395 & 1.939547 & 2.815469 & 2.532006 \\
\hline $1.99 \mathrm{E}+10$ & 1.761798 & 2.194126 & 1.93025 & 3.107422 & 2.443075 \\
\hline $2 \mathrm{E}+10$ & 1.760122 & 2.08458 & 1.937932 & 2.407497 & 2.339899 \\
\hline
\end{tabular}


Table 4. Dielectric Constant for Hanford 6M Simulant

\begin{tabular}{|c|c|c|c|c|c|}
\hline Icy & @20C & Q40 C & $50 \mathrm{C} / \mathrm{e}$ & $e^{\prime} @ 80 \mathrm{C}$ & $e^{\prime} @ 100 \mathrm{C}$ \\
\hline$\overline{000}$ & $\overline{58}$ & $721 !$ & 941 & 369.3682 & 10050 \\
\hline 000 & .15286 & 123.48 & 55.8887 & 987 & 576.05 \\
\hline 0000 & .64038 & 4.86777 & 5.3478 & 34.7424 & 13.90 \\
\hline 000 & .04289 & 78.78785 & 94.99173 & 110.1385 & 281.4036 \\
\hline$\overline{000}$ & 36.85796 & 69.20107 & 82.6266 & 95.4198 & 11.808 \\
\hline 000 & 4.63163 & 64.32712 & 77.36074 & 89.41846 & 178.0002 \\
\hline 766800000 & 2.76607 & 60.8824 & 73.54556 & 5.21626 & 156.041 \\
\hline 500 & 1.23049 & 57.78894 & 68.89943 & 79.53973 & 136.888 \\
\hline 000 & 30.28644 & 55.67266 & 65.80535 & 75.92077 & 119.72 \\
\hline F09 & 9.35993 & 53.39365 & 61.91882 & 70.95762 & 111.072 \\
\hline$E+09$ & 28.41292 & 51.50986 & 59.74376 & 68.21636 & 104.4 \\
\hline+09 & 27.57093 & 50.01161 & 58.46357 & 66.80383 & 99.22122 \\
\hline & 27.09747 & 49.25313 & 58.48984 & 67.20819 & $\overline{93.491}$ \\
\hline-09 & 26.5809 & 48.47398 & 57.76436 & 66.4035 & 90.0240 \\
\hline & 6.18267 & 47.65297 & 56.1644 & 64.0704 & 4.856 \\
\hline+09 & 25.79812 & 46.94942 & 54.79177 & 62.2652 & 81.37 \\
\hline & 25.4776 & 46.36611 & 53.73946 & 61.19839 & $\overline{7.75}$ \\
\hline+09 & 25.02602 & 45.4414 & 52.58396 & 59.35915 & 76.40239 \\
\hline & 24.6453 & 215 & 52.59075 & 105 & 5.3 \\
\hline+09 & 24.4164 & 44.7947 & 53.32179 & 60.69218 & 73.621 \\
\hline 409 & 4.06164 & $44.20467 \mid$ & 52.635 & 60.12688 & 1.85601 \\
\hline-09 & 23.86112 & 43.98557 & 52.03935 & 59.22576 & 69.61453 \\
\hline+09 & 3.55311 & 43.22806 & 50.3484 & 56.56512 & 68.36864 \\
\hline+09 & 23.24667 & 42.60765 & 49.21129 & 55.49923 & 7.25164 \\
\hline & .78655 & 1.79014 & 48.39284 & 54.57169 & 65.77072 \\
\hline & 46 & 41 & 5958 & 55.17911 & 64.0471 \\
\hline+09 & 22.33761 & 025 & 49.62991 & 56.18079 & 62.95348 \\
\hline+09 & 22.1301 & 41.03961 & 49.07458 & 55.70104 & 2.58461 \\
\hline+09 & 21.84812 & 0.34815 & 47.7111 & 53.77704 & 61.95007 \\
\hline+09 & 21.62687 & 39.93936 & 46.44453 & 51.67486 & 61.32278 \\
\hline+09 & 21.41157 & 39.60243 & 45.76809 & 50.8386 & 60.42664 \\
\hline+09 & 21.17193 & 39.07742 & 45.26204 & 50.70603 & 59.12399 \\
\hline+09 & 21.00202 & 38.99791 & 45.93545 & 51.51309 & 58.50818 \\
\hline-09 & 20.88525 & 39.06344 & 46.8859 & 52.55135 & 57.8976 \\
\hline+09 & 20.79352 & 38.9834 & 46.95873 & 53.18369 & 57.39799 \\
\hline+09 & 20.53865 & 38.28839 & 45.47135 & 51.42015 & 56.81353 \\
\hline+09 & 20.37642 & 37.97667 & 44.38669 & 49.38569 & 56.50701 \\
\hline+09 & 20.23561 & 37.85622 & 44.08899 & 48.84166 & 56.247 \\
\hline+09 & 20.04506 & 37.69217 & 4.19838 & 49.76533 & 55.87268 \\
\hline & 19.85012 & 37.41084 & 44.49392 & 50.23476 & 55.10097 \\
\hline+09 & 19.70609 & 37.30411 & 45.10233 & 50.58304 & 54.148 \\
\hline & 19.50739 & 37.05697 & 45.10331 & 50.77226 & 53.04879 \\
\hline & & & & & 12 \\
\hline
\end{tabular}


\begin{tabular}{|l|l|l|l|l|l|}
\hline Frequency & $e^{\prime} @ 20 \mathrm{C}$ & $\mathrm{e}^{\prime} @ 40 \mathrm{C}$ & $\mathrm{e}^{\prime} @ 60 \mathrm{C}$ & $\mathrm{e}^{\prime} @ 80 \mathrm{C}$ & $\mathrm{e}^{\prime} @ 100 \mathrm{C}$ \\
\hline
\end{tabular} \begin{tabular}{|l|l|l|l|l|l|}
\hline \hline $4.552 \mathrm{E}+09$ & 19.03644 & 35.74856 & 41.86399 & 46.66549 & 52.00659 \\
\hline
\end{tabular}

\begin{tabular}{|l|l|l|l|l|l|}
\hline $4.654 \mathrm{E}+09$ & 18.83192 & 35.48451 & 41.27679 & 45.41498 & 51.71182 \\
\hline
\end{tabular}

\begin{tabular}{|r|r|r|r|r|r|}
\hline $4.757 \mathrm{E}+09$ & 18.51363 & 34.954 & 40.68624 & 45.18048 & 50.42515 \\
\hline
\end{tabular}

\begin{tabular}{|r|r|r|r|r|r|}
\hline $4.859 \mathrm{E}+09$ & 18.32658 & 34.68196 & 41.07243 & 46.30621 & 49.49638 \\
\hline
\end{tabular}

\begin{tabular}{|r|r|r|r|r|r|}
\hline $4.961 \mathrm{E}+09$ & 18.12514 & 34.38308 & 41.44281 & 46.18408 & 48.84031 \\
\hline
\end{tabular}

\begin{tabular}{|r|r|r|r|r|r|}
\hline $5.063 \mathrm{E}+09$ & 17.93806 & 34.05523 & 41.13531 & 45.6413 & 48.07737 \\
\hline $5.166 \mathrm{E}+09$ & 17.82339 & 33.72747 & 40.1789 & 44.94663 & 47.55975 \\
\hline
\end{tabular}

\begin{tabular}{|r|r|r|r|r|r|}
\hline $5.166 \mathrm{E}+09$ & 17.82339 & 33.72747 & 40.1789 & 44.94663 & 47.55975 \\
\hline $5.268 \mathrm{E}+09$ & 17.59449 & 33.19402 & 38.73995 & 43.17621 & 46.9884 \\
\hline
\end{tabular}

\begin{tabular}{|r|r|r|r|r|r|}
\hline $5.37 \mathrm{E}+09$ & 17.43661 & 33.01147 & 38.11488 & 41.55031 & 46.40074 \\
\hline $5.473 \mathrm{E}+09$ & 1723005 & 32.83988 & 38.11425 & 41.76301 & 45.7671 \\
\hline
\end{tabular}

\begin{tabular}{|r|r|r|r|r|r|}
\hline $5.473 \mathrm{E}+09$ & 17.23005 & 32.83988 & 38.11425 & 41.76301 & 45.7671 \\
\hline $5.575 \mathrm{E}+09$ & 17.04443 & 32.43967 & 38.11497 & 42.65462 & 44.88681 \\
\hline $5.677 \mathrm{E}+09$ & 16.90908 & 32.31553 & 38.6812 & 43.228 & 44.18302 \\
\hline
\end{tabular}

\begin{tabular}{|r|r|r|r|r|r|}
\hline $5.677 \mathrm{E}+09$ & 16.90908 & 32.31553 & 38.6812 & 43.2289 & 44.18302 \\
\hline $5.78 \mathrm{E}+09$ & 16.73385 & 31.76157 & 37.86183 & 41.2155 & 43.78455 \\
\hline $5.882 \mathrm{E}+09$ & 16.63346 & 31.71888 & 37.49323 & 41.32835 & 43.59636 \\
\hline $5.984 \mathrm{E}+09$ & 16.52701 & 31.4543 & 36.44824 & 40.49623 & 43.1016 \\
\hline
\end{tabular}

\begin{tabular}{|r|r|r|r|r|r|}
\hline $5.882 \mathrm{E}+09$ & 16.63346 & 31.71888 & 37.49323 & 41.32835 & 43.59636 \\
\hline $6.984 \mathrm{E}+09$ & 16.52701 & 31.4543 & 36.44824 & 40.49623 & 43.10116 \\
\hline $6.086 \mathrm{E}+09$ & 16.34251 & 31.13468 & 35.76649 & 39.02174 & 42.65789 \\
\hline $6.291 \mathrm{E}+09$ & 16.23213 & 31.01213 & 35.98384 & 38.77122 & 42.02597 \\
\hline $6.393 \mathrm{E}+09$ & 16.12262 & 31.02773 & 37.00137 & 41.2134 & 41.22008 \\
\hline
\end{tabular}

\begin{tabular}{|r|r|r|r|r|r|}
\hline $6.291 \mathrm{E}+09$ & 16.12262 & 31.02773 & 37.00137 & 41.2134 & 41.22008 \\
\hline $6.393 \mathrm{E}+09$ & 15.93663 & 30.63977 & 37.09366 & 41.65563 & 40.62793 \\
\hline $6.496 \mathrm{E}+09$ & 15.80855 & 30.56237 & 37.33709 & 41.24126 & 40.31454 \\
\hline
\end{tabular}

\begin{tabular}{|r|r|r|r|r|r|}
\hline $6.496 \mathrm{E}+09$ & 15.80855 & 30.56237 & 37.33709 & 41.24126 & 40.31454 \\
\hline $6.598 \mathrm{E}+09$ & 15.61655 & 30.14972 & 36.17713 & 39.60381 & 40.21625 \\
\hline $6.7 \mathrm{E}+09$ & 15.48885 & 29.92857 & 35.12479 & 39.14799 & 39.90271 \\
\hline $6.803 \mathrm{E}+09$ & 15.3969 & 29.66636 & 34.30121 & 37.63469 & 39.73843 \\
\hline $6.905 \mathrm{E}+09$ & 15.15944 & 29.15189 & 33.80769 & 36.10021 & 38.99507 \\
\hline $7.007 \mathrm{E}+09$ & 15.068 & 29.11006 & 34.62072 & 38.04904 & 38.39394 \\
\hline $7.109 \mathrm{E}+09$ & 14.90203 & 28.88897 & 35.21065 & 39.77563 & 37.86109 \\
\hline $7.212 \mathrm{E}+09$ & 14.63166 & 28.48523 & 34.94291 & 38.72089 & 37.46549 \\
\hline $7.314 \mathrm{E}+09$ & 14.52575 & 28.3129 & 34.16538 & 37.09467 & 37.13724 \\
\hline $7.416 \mathrm{E}+09$ & 14.43307 & 27.97721 & 32.72927 & 35.95818 & 36.82424 \\
\hline $7.519 \mathrm{E}+09$ & 14.26803 & 27.45593 & 31.37838 & 34.25081 & 36.59268 \\
\hline $7.621 \mathrm{E}+09$ & 14.14851 & 27.28047 & 31.34994 & 33.23349 & 36.50121 \\
\hline $7.723 \mathrm{E}+09$ & 14.07321 & 27.36198 & 32.32215 & 34.77486 & 36.01939 \\
\hline $7.826 \mathrm{E}+09$ & 13.91431 & 27.19968 & 32.82522 & 36.51165 & 35.28595 \\
\hline $7.928 \mathrm{E}+09$ & 13.81159 & 27.05286 & 32.89475 & 36.42 & 34.75131 \\
\hline $8.03 \mathrm{E}+09$ & 13.65995 & 26.83621 & 32.14134 & 34.63975 & 33.93176 \\
\hline $8.132 \mathrm{E}+09$ & 13.58008 & 26.37248 & 30.40514 & 32.50116 & 32.87894 \\
\hline $8.235 \mathrm{E}+09$ & 13.51981 & 26.08231 & 29.55737 & 32.04055 & 32.68568 \\
\hline $8.337 \mathrm{E}+09$ & 13.39539 & 25.87633 & 29.50098 & 31.16809 & 32.64722 \\
\hline $8.439 \mathrm{E}+09$ & 13.26773 & 25.81352 & 30.20935 & 31.82711 & 32.53432 \\
\hline $8.542 \mathrm{E}+09$ & 13.25959 & 26.09365 & 31.60232 & 34.78691 & 32.26762 \\
\hline $8.644 \mathrm{E}+09$ & 13.18286 & 26.16717 & 31.97941 & 35.56267 & 32.27307 \\
\hline $8.746 \mathrm{E}+09$ & 13.01694 & 25.67157 & 30.65529 & 32.90445 & 32.13337 \\
\hline $8.849 \mathrm{E}+09$ & 12.96332 & 25.40364 & 29.36726 & 31.13284 & 32.20147 \\
\hline $8.951 \mathrm{E}+09$ & 12.88908 & 25.05679 & 28.31173 & 30.27867 & 32.12998 \\
\hline $9.053 \mathrm{E}+09$ & 12.77266 & 24.79592 & 28.44492 & 30.28674 & 32.10398 \\
\hline
\end{tabular}




\begin{tabular}{|c|c|c|c|c|c|}
\hline & & & & $\bar{C}$ & \\
\hline+09 & 12.69155 & 25.04339 & 29.95553 & 31.78591 & 32.023 \\
\hline $58 \mathrm{E}+09$ & .63571 & 24.97196 & 0.65866 & 3.38731 & $\overline{32.022}$ \\
\hline $9.36 \mathrm{E} \div 09$ & 12.58674 & 25.30321 & 0065 & .52902 & .888 \\
\hline $.462 \mathrm{E}+09$ & 12.44756 & 25.03513 & 30.39672 & 33.06546 & 1.882 \\
\hline $9.565 E+09$ & 12.47813 & 24.73127 & 28.76131 & .24509 & .03 \\
\hline $367 \mathrm{E}+09$ & 12.42465 & 24.64393 & 28.27349 & 30.43345 & 1.907 \\
\hline $9.769 \mathrm{E}+09$ & 12.33533 & 24.40802 & 28.27876 & 30.34294 & $\overline{1.833}$ \\
\hline $872 E+09$ & 12.24711 & 24.31845 & 29.59382 & 31.68245 & 31.363 \\
\hline $9.974 \mathrm{E}+09$ & 12.11446 & 24.49865 & 31.24679 & 4.37243 & .732 \\
\hline $1.008 \mathrm{E}+10$ & 12.03222 & 382 & 31.76265 & 5.87051 & 31.813 \\
\hline $1.018 \mathrm{E}+10$ & 12.05768 & 24.8154 & 31.20126 & 34.72312 & 31.863 \\
\hline $1.028 \mathrm{E}+10$ & 12.00655 & 24.78378 & 29.81565 & 32.14461 & 31.879 \\
\hline $1.038 \mathrm{E}+10$ & 11.98209 & 24.43736 & 28.35305 & 30.53591 & $\overline{31.826}$ \\
\hline $1.049 \mathrm{E}+10$ & 11.85714 & 24.11543 & 28.24148 & 30.71958 & 31.31 \\
\hline $1.059 \mathrm{E}+10$ & 11.68743 & 23.81485 & 28.90062 & 30.98432 & 31.4593 \\
\hline $1.069 \mathrm{E}+10$ & 11.49674 & 23.30662 & 29.22231 & 31.60108 & 1.562 \\
\hline $1.079 \mathrm{E}+10$ & 11.51937 & 23.58385 & 29.9794 & 33.38476 & 32.036 \\
\hline $1.089 \mathrm{E}+10$ & 416 & 23.93253 & 29.59883 & 32.63824 & 2.19 \\
\hline $1.1 \mathrm{E}+10$ & 11.5887 & 23.97045 & 28.38976 & 30.4592 & 32.090 \\
\hline $1.11 \mathrm{E}+10$ & 11.57087 & 23.71063 & 27.15364 & 28.86529 & 31.8450 \\
\hline $1.12 \mathrm{E}+10$ & 11.4709 & 23.31504 & 26.92942 & 28.83186 & $\overline{31.748}$ \\
\hline $1.13 \mathrm{E}+10$ & 11.17939 & 22.5843 & 27.24992 & 29.00397 & 31.7152 \\
\hline $1.141 \mathrm{E}+10$ & 11.06695 & 22.50114 & 28.70627 & 30.93187 & 32.2971 \\
\hline $1.151 \mathrm{E}+10$ & 11.15474 & 23.2532 & 30.56881 & 34.38543 & 32.8010 \\
\hline $1.161 \mathrm{E}+10$ & 11.25365 & 24.03527 & 31.00741 & 34.98575 & 32.9277 \\
\hline $1.171 \mathrm{E}+10$ & 11.29832 & 4.32736 & 29.65851 & 32.41605 & 32.688 \\
\hline $1.182 \mathrm{E}+10$ & 11.11998 & 23.68841 & 27.59656 & 29.85923 & 32.1586 \\
\hline $1.192 \mathrm{E}+10$ & 11.01514 & 22.97117 & 26.83987 & 29.49661 & 31.4891 \\
\hline $1.202 \mathrm{E}+10$ & 10.82267 & 22.40292 & 27.81291 & 30.52638 & 30.4686 \\
\hline $1.212 \mathrm{E}+10$ & 10.68215 & 22.00452 & 29.23342 & 31.93156 & 29.408 \\
\hline $1.222 \mathrm{E}+10$ & 10.75335 & 22.65025 & 31.16177 & 35.23718 & 30.0030 \\
\hline $1.233 \mathrm{E}+10$ & 10.91771 & 23.92385 & 32.34019 & 37.07374 & 30.8063 \\
\hline $1.243 E+10$ & 10.63136 & 23.68399 & 30.00795 & 33.52008 & 31.7755 \\
\hline $1.253 \mathrm{E}+10$ & 10.52236 & 22.92989 & 27.48772 & 30.90981 & 31.3295 \\
\hline $1.263 \mathrm{E}+10$ & 10.50265 & 22.71659 & 27.7233 & 32.32067 & 29.9574 \\
\hline $1.274 \mathrm{E}+10$ & 10.36896 & 22.15055 & 28.67881 & 32.66286 & 28.6164 \\
\hline $1.284 \mathrm{E}+10$ & 10.31678 & 22.11845 & 30.49517 & 33.82746 & 28.0684 \\
\hline $1.294 \mathrm{E}+10$ & 10.30262 & 22.40853 & 31.3105 & 35.25488 & 28.4301 \\
\hline $1.304 \mathrm{E}+10$ & 10.3345 & 22.9972 & 30.8666 & 34.79389 & 29.8655 \\
\hline $1.315 \mathrm{E}+10$ & 10.07651 & 22.72889 & 28.19884 & 29.52007 & 30.7952 \\
\hline $1.325 \mathrm{E}+10$ & 10.02143 & 22.06456 & 25.15627 & 25.4335 & 30.7207 \\
\hline $1.335 \mathrm{E}+10$ & .1801 & 22.01545 & 24.1991 & 25.84666 & 29.8663 \\
\hline $1.345 \mathrm{E}+10$ & 10.17694 & 22.30924 & 24.94685 & 26.63893 & 29.1550 \\
\hline $1.355 \mathrm{E}+10$ & 10.10534 & 22.39839 & 26.12003 & 26.88514 & 28.82017 \\
\hline $1.366 \mathrm{E}+1$ & 10.028 & 22.19272 & 26.709 & 28.5 & 28.781 \\
\hline
\end{tabular}




\begin{tabular}{|c|c|c|c|c|c|}
\hline & & & & & \\
\hline+10 & 41 & 974 & 27.2861 & .08022 & \\
\hline $6 \mathrm{E}+10$ & & $\overline{494}$ & & & \\
\hline+10 & (1) & 9281 & 709 & 23.894 & 1 \\
\hline $407 E+10$ & 67 & 76925 & 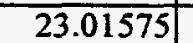 & .7056 & $\overline{72}$ \\
\hline & & & & & \\
\hline+10 & 9.770654 & & J & 0.38272 & \\
\hline & & & & & 425 \\
\hline $448 \mathrm{E}+10$ & 711007 & 48851 & 89971 & 69 & .41 \\
\hline 10 & & & & & 3.6 \\
\hline $468 \mathrm{E}+10$ & 146025 & .49629 & .30548 & .0412 & .0 \\
\hline & & & $\overline{56}$ & 354 & .052 \\
\hline $488 \mathrm{E}+10$ & 30511 & 20.43042 & 23.6068 & .67343 & 3.65 \\
\hline & 21 & $\overline{929}$ & 4.02717 & 2.21559 & 3.0 \\
\hline $1.509 \mathrm{E}+10$ & 49369 & .86439 & 22.01859 & 1.18719 & 3.00 \\
\hline+10 & & 322 & $\overline{924}$ & 5.3852 & 7.919 \\
\hline$E+10$ & 522 & 029 & 343 & Su & 7.86 \\
\hline$E+10$ & 23 & 12340 & 2.14129 & - 500 & 8.5 \\
\hline $\mathrm{E}+10$ & & & 1 & & . \\
\hline+10 & & & 351 & .6557 & .163 \\
\hline $\mathrm{E}+10$ & $12 \mid$ & & 6901 & 68 & .569 \\
\hline $1.58 \mathrm{E}+10$ & 79 & 75 & 3.29736 & 550 & 7.8350 \\
\hline+10 & 44) & & 3.31877 & 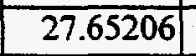 & 7.61 \\
\hline+10 & 28 & $\overline{931}$ & 3.29504 & 1.73359 & 7.639 \\
\hline+10 & 6537 & & 8 & 51 & 9.003 \\
\hline+10 & 29 & 536 & .20764 & 01 & $\overline{9.426}$ \\
\hline$i+10$ & $4 / 6921$ & & ת & 25.008 & 8.4 \\
\hline+10 & 06 & & 104 & 2.23218 & 7.666 \\
\hline $2 \bar{E}+10$ & & & & .36146 & 7.2 \\
\hline $2 E+10$ & 34 & 5 & 58 & 3.93361 & 6.5351 \\
\hline+10 & & & & .6876 & 6.39 \\
\hline$E+10$ & 1 & 8 & 1 & 3201 & 625 \\
\hline $3 \mathrm{E}+10$ & 770691 & 7039 & 23.3 & .073 & $8.9053-$ \\
\hline $3 \mathrm{E}+10$ & 869 & 17 & 22348 & 4.2403 & 254 \\
\hline$E+10$ & 560908 & t & 52 & 20.9122 & 541 \\
\hline $1.724 \mathrm{E}+10$ & 2929 & 8.2508 & 19.42131 & 8.91223 & 7.5395 \\
\hline $1.734 \mathrm{E}+10$ & & $7-7009$ & 18.58482 & 9.43385 & $7312^{\prime}$ \\
\hline $4 \mathrm{E}+10$ & & & 19.3738 & 21.67215 & 27.0852 \\
\hline+10 & 95 & 98 & 21.4635 & 22.16931 & \\
\hline $1.765 \mathrm{E}+10$ & 34039 & 19.02952 & 2.39284 & 22.38823 & 8.0690 \\
\hline $1.775 \mathrm{E}+10$ & & 18.27689 & & 21.1591 & 8.0768 \\
\hline+10 & 37 & 7087 & 8.28744 & 7.27651 & 7.7272 \\
\hline+10 & & & & 6.24266 & 27.657 \\
\hline $1.806 \mathrm{E}+10$ & (1002 & 10.02214 & 17.54297 & 17.6112 & \\
\hline & & & & 35 & 27.4 \\
\hline & & & & & \\
\hline
\end{tabular}




\begin{tabular}{|r|r|r|r|r|r|}
\hline Frequency & $\mathrm{e}^{\prime} @ 20 \mathrm{C}$ & $\mathrm{e}^{\prime} @ 40 \mathrm{C}$ & $\mathrm{e}^{\prime} @ 60 \mathrm{C}$ & $\mathrm{e}^{\prime} @ 80 \mathrm{C}$ & $\mathrm{e}^{\prime} @ 100 \mathrm{C}$ \\
\hline \hline $1.836 \mathrm{E}+10$ & 7.895181 & 18.46715 & 22.33796 & 23.13536 & 27.41834 \\
\hline $1.846 \mathrm{E}+10$ & 7.627209 & 17.84227 & 20.88966 & 18.76536 & 27.73207 \\
\hline $1.857 \mathrm{E}+10$ & 7.475326 & 17.10802 & 18.95777 & 16.96545 & 27.96493 \\
\hline $1.867 \mathrm{E}+10$ & 7.321266 & 17.05727 & 18.19887 & 18.75347 & 27.96872 \\
\hline $1.877 \mathrm{E}+10$ & 7.305457 & 16.8537 & 17.76895 & 17.44293 & 28.0082 \\
\hline $1.887 \mathrm{E}+10$ & 7.316681 & 16.72613 & 18.07128 & 16.07809 & 27.60567 \\
\hline $1.898 \mathrm{E}+10$ & 7.508554 & 17.27493 & 19.50226 & 20.44625 & 26.71912 \\
\hline $1.908 \mathrm{E}+10$ & 7.788457 & 18.36326 & 21.59204 & 21.91739 & 26.33765 \\
\hline $1.918 \mathrm{E}+10$ & 7.638118 & 18.38008 & 21.93838 & 18.51767 & 26.82373 \\
\hline $1.928 \mathrm{E}+10$ & 7.407452 & 17.66489 & 20.80408 & 19.97304 & 27.37739 \\
\hline $1.939 \mathrm{E}+10$ & 7.318977 & 17.30348 & 20.31136 & 21.74305 & 27.6878 \\
\hline $1.949 \mathrm{E}+10$ & 7.307208 & 17.82209 & 21.33614 & 20.62351 & 27.68644 \\
\hline $1.959 \mathrm{E}+10$ & 7.275138 & 17.44639 & 20.57579 & 19.55656 & 27.32581 \\
\hline $1.969 \mathrm{E}+10$ & 7.176878 & 17.26776 & 20.33007 & 21.71618 & 26.2813 \\
\hline $1.979 \mathrm{E}+10$ & 7.245208 & 17.46989 & 20.47321 & 19.30404 & 25.80646 \\
\hline $1.99 \mathrm{E}+10$ & 7.285662 & 17.59562 & 20.67264 & 16.72102 & 26.45416 \\
\hline $2 \mathrm{E}+10$ & 7.192217 & 17.17303 & 20.47478 & 20.14777 & 27.35173 \\
\hline
\end{tabular}


Table 5. Dielectric Loss for Hanford 6M Simulant

\begin{tabular}{|c|c|c|c|c|c|}
\hline & & $\mathrm{C}$ & U. & $80 \mathrm{C}$ & \\
\hline+08 & 1045.97 & 2821.668 & 4097.968 & 5112.725 & 5999.674 \\
\hline+08 & 635.7872 & 712.295 & 2489.424 & 105.414 & $\overline{815.51}$ \\
\hline $3.58 \mathrm{E}+08$ & 458.9237 & 1232.782 & $\overline{789.964}$ & 231.471 & 2799. \\
\hline $4.6 E+08$ & 359.4271 & 963.0468 & 1397.281 & 1742.122 & 2205.99 \\
\hline $5.62 \mathrm{E}+08$ & 296.1813 & 792.157 & 1149.083 & 433.372 & 315.14 \\
\hline $6.65 \mathrm{E}+08$ & 252.0847 & 673.8562 & 977.1453 & 1219.288 & $\overline{546.36}$ \\
\hline $7.67 \mathrm{E}+08$ & 219.5812 & 586.0806 & 848.8815 & 059.917 & 344.66 \\
\hline $8.69 \mathrm{E}+08$ & 194.6399 & 518.5276 & 749.9607 & 935.8942 & 193.59 \\
\hline $9.71 \mathrm{E}+08$ & 174.9413 & 465.3831 & 672.7895 & 40.2594 & $\overline{073.6}$ \\
\hline $1.07 \mathrm{E}+09$ & 159.1463 & 422.7254 & 610.9923 & 763.4188 & 76.031 \\
\hline $1.18 \mathrm{E}+09$ & 146.0708 & 387.2287 & 559.674 & 699.2557 & 392.928 \\
\hline $1.28 \mathrm{E}+09$ & 135.0227 & 357.7369 & 517.0031 & 646.2396 & 321.654 \\
\hline $1.38 \mathrm{E}+09$ & 125.7049 & .332 .0458 & 479.3158 & 599.0984 & 761.043 \\
\hline $1.48 \mathrm{E} \div 09$ & 117.5064 & 310.0232 & 446.9217 & 558.9878 & 712.817 \\
\hline $1.59 \mathrm{E}+09$ & 110.5554 & 290.9961 & 418.8413 & 523.7955 & 669.3798 \\
\hline $1.69 \mathrm{E}+09$ & 104.1564 & $\overline{273.8013}$ & 393.907 & 492.4551 & 631.982 \\
\hline $1.79 \mathrm{E}+09$ & 98.80888 & 259.2456 & 373.0088 & 66.4135 & 597.1817 \\
\hline $1.89 E+09$ & 94.00582 & 246.379 & 354.8408 & 443.9656 & 566.5727 \\
\hline $1.99 \mathrm{E}+09$ & 89.66656 & 234.5487 & 337.7871 & 422.4794 & $\overline{537.297}$ \\
\hline $2.1 \mathrm{E}+09$ & 85.79257 & 223.7948 & 321.8798 & 402.2859 & 511.927 \\
\hline $2.2 \mathrm{E}+09$ & 82.27702 & 214.3019 & 307.547 & 84.4025 & 489.623 \\
\hline $2.3 \mathrm{E}+09$ & 79.0294 & 205.4966 & 294.6532 & 368.4414 & 470.873 \\
\hline $2.4 \mathrm{E}+09$ & 76.18043 & 197.8331 & 283.4545 & 354.2881 & $\$ 52.740$ \\
\hline $2.51 \mathrm{E}+09$ & 73.4981 & 190.4061 & 272.7027 & 340.3949 & 434.955 \\
\hline $2.61 E+09$ & 70.752 & 183.3561 & 263.0517 & 328.5285 & 418.288 \\
\hline $2.71 E+09$ & 68.49968 & 177.121 & 254.0424 & 317.3432 & $\overline{402.540^{\prime}}$ \\
\hline $2.81 \mathrm{E}+09$ & 66.47055 & 171.5056 & 245.6491 & 306.6512 & 388.044 \\
\hline $2.92 \mathrm{E}+09$ & 64.55919 & 166.262 & 237.6714 & 296.7516 & 375.3071 \\
\hline $3.02 \mathrm{E}+09$ & 62.68706 & 161.0653 & 229.6071 & 286.7756 & 364.200 \\
\hline $3.12 \mathrm{E}+09$ & 60.88937 & 156.2364 & 222.6036 & 277.7636 & 353.564 \\
\hline $3.22 \mathrm{E}+09$ & 59.26111 & 151.9133 & 216.7481 & 270.256 & 343.2087 \\
\hline $3.32 \mathrm{E}+09$ & 638 & 147 & 210.7948 & 262.6615 & 332.833 \\
\hline $3.43 \mathrm{E}+09$ & 56.27422 & 143.6419 & 205.0196 & 255.6906 & 322.9442 \\
\hline $3.53 \mathrm{E}+09$ & 54.96393 & 139.9789 & 199.3834 & 248.503 & 314.087 \\
\hline $3.63 \mathrm{E}+09$ & 53.75874 & 136.7986 & 194.4104 & 242.2016 & 306.2373 \\
\hline $3.73 E+09$ & 52.62091 & 133.7192 & 189.5196 & 236.152 & 299.0974 \\
\hline $3.84 \mathrm{E}+09$ & 51.48329 & 130.538 & 184.8813 & 230.3574 & 292.2564 \\
\hline $3.94 \mathrm{E}+09$ & 50.44556 & 127.7065 & 181.1316 & 225.294 & 285.6149 \\
\hline $4.04 \mathrm{E}+09$ & 49.50149 & 125.1762 & 178.0333 & 221.2589 & 278.7586 \\
\hline $4.14 \mathrm{E}+09$ & 48.60846 & 122.7449 & 174.6166 & 217.3425 & 273.0252 \\
\hline $4.25 \mathrm{E}+09$ & 47.75468 & 120.2858 & 170.6878 & 212.431 & 267.598 \\
\hline $4.35 \mathrm{E}+09$ & 46.97029 & 118.2013 & 167.2636 & 207.9376 & 262.2707 \\
\hline $4.45 E+09$ & $46.0904 t$ & 115.8282 & 163.3777 & 202.9316 & 257. \\
\hline
\end{tabular}




\begin{tabular}{|c|c|c|c|c|c|}
\hline & e & & $e^{\prime \prime a}$ & & \\
\hline 9 & 45.26643 & 255 & 195 & $\overline{278}$ & ג \\
\hline $65 \mathrm{E}+09$ & 44.54048 & 5388 & 57.4717 & 95.5488 & 17.9697 \\
\hline $6 \mathrm{E}+09$ & 43.86995 & 9.9368 & 55.6092 & 92.8417 & 11 \\
\hline $4.86 \mathrm{E}+09$ & 43.1772 & 8.0734 & 152.9983 & 189.9011 & $\overline{38.551}$ \\
\hline $96 \mathrm{E}+09$ & 42.58207 & 106.4669 & 150.3195 & 186.9188 & $\overline{34.441}$ \\
\hline $5.06 \mathrm{E}+09$ & 41.97051 & 104.6648 & 147.1933 & 182.5482 & 30.595 \\
\hline $5.17 \mathrm{E}+09$ & 41.2879 & 102.7234 & 144.0925 & 178.3947 & 26.700 \\
\hline $5.27 \mathrm{E}+09$ & 40.70731 & 101.0855 & 141.6156 & 175.5816 & 22.750 \\
\hline $5.37 \mathrm{E}+09$ & 40.107 & 99.5598 & 139.8494 & 173.4838 & 18.690 \\
\hline $5.47 E+09$ & 39.60008 & 98.28915 & 138.5014 & 171.321 & 214.9482 \\
\hline $5.57 \mathrm{E}+09$ & 39.06219 & 96.87843 & 136.5138 & 168.8281 & 211.286 \\
\hline $5.68 \mathrm{E}+09$ & 38.57243 & 95.47069 & 134.1901 & 166.4254 & 207.8741 \\
\hline $5.78 E+09$ & 38.05568 & 93.86395 & 131.2672 & 162.4239 & 204.82 \\
\hline $5.88 \mathrm{E}+09$ & 37.51943 & 92.31063 & 128.7772 & 158.8232 & 201.86 \\
\hline $5.98 \mathrm{E}+09$ & 37.06745 & $91: 08789$ & 127.0932 & 156.9889 & $\overline{98.604}$ \\
\hline $6.09 E+09$ & 36.63727 & 90.01413 & 125.9903 & 156.0058 & 95.6305 \\
\hline $6.19 \mathrm{E}+09$ & 36.20412 & 89.02749 & 125.0675 & 154.4451 & $\overline{92.913}$ \\
\hline $6.29 E+09$ & 35.87747 & 88.10136 & 123.8728 & 152.7475 & 90.237 \\
\hline $6.39 E+09$ & 35.5185 & 86.96471 & 121.7941 & 150.6361 & 187.626 \\
\hline $6.5 \mathrm{E}+09$ & 35.10305 & 85.57712 & 119.2504 & 147.4873 & 185.0047 \\
\hline $6.6 \mathrm{E}+09$ & 34.64759 & 84.44589 & 117.3139 & 144.5245 & 82.3974 \\
\hline $6.7 \mathrm{E}+09$ & 34.22091 & 83.36899 & 115.8323 & 142.6896 & 179.6865 \\
\hline $6.8 \mathrm{E}+09$ & 33.8979 & 82.69018 & 115.2233 & 142.5741 & 177.5602 \\
\hline $6.9 \mathrm{E}+09$ & 33.54384 & 81.91004 & 114.474 & 141.455 & $\overline{75.1448}$ \\
\hline $7.01 E+09$ & 33.1603 & 80.89191 & 113.0653 & 139.2871 & 173.0504 \\
\hline $7.11 \mathrm{E}+09$ & 2.84449 & 79.82055 & 111.0106 & 136.8763 & 40 \\
\hline $7.21 \mathrm{E}+09$ & 32.40879 & 78.61516 & 108.8126 & 134.6478 & 168.5864 \\
\hline $7.31 \mathrm{E}+09$ & 32.05032 & 49 & 93 & & 166.5167 \\
\hline $7.42 \mathrm{E}+09$ & .72643 & 76.83858 & 106.1528 & 130.5781 & 164.1405 \\
\hline $7.52 \mathrm{E}+09$ & 31.42557 & 76.18267 & 105.5759 & 130.3585 & 162.0615 \\
\hline $7.62 \mathrm{E}+09$ & .11757 & 75.51293 & 105.0436 & 129.8588 & 160.1743 \\
\hline $7.72 \mathrm{E}+09$ & 30.79533 & 74.77376 & 104.105 & 128.2343 & 158.8459 \\
\hline $7.83 \mathrm{E}+09$ & 30.44791 & 73.87722 & 102.5484 & 126.2555 & 157.230 \\
\hline $7.93 E+09$ & 30.13596 & $72.79121 \mid$ & 100.3898 & 124.0878 & 155.5671 \\
\hline $8.03 \mathrm{E}+09$ & 29.84011 & 71.87033 & 98.79214 & 121.8349 & 154.0012 \\
\hline $8.13 E+09$ & 29.52723 & 71.19134 & 97.94457 & 120.1651 & 151.689 \\
\hline $8.23 \mathrm{E}+09$ & 29.24219 & 70.30561 & 96.96698 & 119.1758 & 149.0547 \\
\hline $8.34 \mathrm{E}+09$ & 28.94083 & 69.77452 & 96.78532 & 119.4259 & 146.7521 \\
\hline $8.44 \mathrm{E}+09$ & 28.59708 & 69.03978 & 95.83756 & 117.8223 & $145.07 \cdot 17$ \\
\hline $8.54 \mathrm{E}+09$ & 28.33332 & 68.20663 & 94.24233 & 115.5489 & 143.4824 \\
\hline $8.64 \mathrm{E}+09$ & 28.21015 & 67.75833 & 93.16972 & 114.9154 & 141.4625 \\
\hline $8.75 \mathrm{E}+09$ & 27.93619 & 66.87776 & 91.49907 & 112.6704 & 139.8192 \\
\hline $8.85 E+09$ & 27.68382 & 66.1194 & 90.52713 & 110.7936 & 138.0541 \\
\hline $8.95 E+09$ & 27.45034 & 65.66898 & 90.21859 & 110.3717 & 136.5228 \\
\hline $9.05 \mathrm{E}+09$ & 27.22877 & 65.1820 & 89.8794 & 110.4435 & 4.849 \\
\hline
\end{tabular}




\begin{tabular}{|c|c|c|c|c|c|}
\hline cy $1 \mathrm{e}$ & @20 C & e"@40C & $50 \mathrm{C}$ & (@) 80C & E \\
\hline 09 & 24 & 2 & 89.74457 & & 133.362 \\
\hline & .77473 & 2673 & 08705 & 74 & .62 \\
\hline & 5.65606 & 84 & 61652 & 1648 & 0.58 \\
\hline+09 & 6.46247 & .87402 & 5.72884 & 15.5713 & $\overline{29.02}$ \\
\hline-09 & .14756 & 476 & 003 & 2.8978 & 27.680 \\
\hline-09 & 25.92825 & 1.21164 & 83.60076 & 01.7373 & 26.27 \\
\hline $9.77 \mathrm{E}+09$ & 25.85033 & 1.48405 & 84.62576 & 03.7034 & 25.09 \\
\hline-09 & 25.64256 & 60.8847 & 3.72776 & 102.7173 & 23.622 \\
\hline+09 & 25.57771 & 60.74587 & 83.23059 & 101.846 & $\overline{122.4}$ \\
\hline & 25.3236 & 0.12478 & .90574 & 100.527 & 21.28 \\
\hline+10 & 25.03651 & 58.96012 & 79.7802 & 8.13408 & 19.956 \\
\hline-10 & 24.85901 & 8.34683 & .01536 & 6.75673 & 119. \\
\hline+10 & 24.61798 & 57.76377 & 78.55241 & 95.54537 & 18.1078 \\
\hline & .47132 & 14 & 99 & .26151 & 17.3 \\
\hline+10 & 24.32698 & 57.89618 & 79.87724 & 98.21304 & 6.1 \\
\hline & 24.0 & & 86 & 6.52737 & 13.1 \\
\hline $1.08 \mathrm{E}+10$ & 23.74005 & 56.49288 & 76.78421 & 94.06089 & 14.002 \\
\hline 10 & .60942 & & 075 & 668 & \\
\hline $1.1 \mathrm{E}+10$ & 23.53714 & 55.20308 & 74.25564 & 90.46897 & 111.79 \\
\hline+10 & 3.4237 & 93 & 202 & 89.8371 & 10.9 \\
\hline+10 & 23.32538 & 54.7241 & 75.06087 & 91.17961 & 10.438 \\
\hline+10 & 23.2018 & 78382 & .85594 & 92.7302 & 109.99 \\
\hline+10 & 22.94795 & 54.41364 & 75.3025 & 92.20032 & $\overline{09.733}$ \\
\hline+10 & 22.83257 & 53.99778 & 3.91428 & 90.43856 & 09.584 \\
\hline+10 & 22.8663 & 53.88588 & 72.98538 & 89.41018 & 08.04 \\
\hline+10 & 22.97539 & 3.89273 & 72.60008 & 88.20555 & 106.762 \\
\hline+10 & 22.88285 & .34328 & 1.95581 & 86.35515 & 05. \\
\hline+10 & .52108 & 37561 & 331 & 85.67712 & 05.411 \\
\hline+10 & 2.3151 & 772 & 1.95735 & 87.1774 & 06.177 \\
\hline+10 & .05714 & 134 & 1.96705 & 87.6525 & 106.329 \\
\hline$E+10$ & 1.95056 & .39977 & 1.44784 & 87.49575 & 05.527 \\
\hline+10 & 2.28286 & 52.07908 & 71.64442 & 88.25409 & 04.1 \\
\hline$E+10$ & 22.44636 & .78602 & 1.96126 & 88.67633 & 02.581 \\
\hline$E+10$ & 2.13595 & 1.79903 & 0.10947 & 85.55562 & 101.26 \\
\hline $\mathrm{E}+10$ & 81552 & 73595 & 69.5441 & 85.36243 & 00.251 \\
\hline$E+10$ & .42446 & 49.68806 & 9.81677 & 86.31912 & 9.5049 \\
\hline $8 \mathrm{E}+10$ & 1.25564 & 49.35353 & 70.74707 & 87.18267 & 9.4012 \\
\hline $1.29 \mathrm{E}+10$ & 21.20684 & 9.50283 & 70.91701 & 87.18268 & 98.9572 \\
\hline $1.3 \mathrm{E}+10$ & 21.37229 & 50.02691 & 70.10805 & 86.88456 & 98.917 \\
\hline $1.31 \mathrm{E}+10$ & 21.44485 & 0.92784 & 69.98234 & 86.83222 & 98.3805 \\
\hline+10 & 21.20062 & 50.31605 & 68.41086 & 83.53633 & 97.1180 \\
\hline $1.33 \mathrm{E}+10$ & 20.83682 & 48.80366 & 67.24161 & 82.18806 & 95.5478 \\
\hline $5 \mathrm{E}+10$ & 61768 & 3.16865 & 68.66845 & 85.05368 & 94.4101 \\
\hline+10 & & & 70.47979 & 86.62895 & $94.1748^{\prime}$ \\
\hline+10 & 0.39153 & 8.15662 & 70.50379 & 85.62204 & 7.78 \\
\hline
\end{tabular}




\begin{tabular}{|c|c|c|c|c|c|}
\hline & & & $\bar{C}$ & $\mathrm{e}^{\prime \prime} @ 80 \mathrm{C}$ & $100 \mathrm{C}$ \\
\hline $1.38 \mathrm{E}+10$ & 20.46045 & 48.61203 & 69.39289 & 85.59594 & 60 \\
\hline+10 & 20.38419 & 48.53807 & 66.80938 & 82.29956 & .129 \\
\hline $1.4 \mathrm{E}+10$ & 20.28472 & 48.04882 & 64.33688 & 77.52692 & .250 \\
\hline $1.41 E+10$ & 20.06204 & 46.94283 & 62.42394 & 75.29513 & 91.6774 \\
\hline $1.42 \mathrm{E}+10$ & 19.83225 & 46.27952 & 62.50753 & 77.6631 & 90.4233 \\
\hline $1.43 \mathrm{E}+10$ & 19.70002 & 46.68794 & 64.56084 & 79.54532 & 90.19 \\
\hline $1.44 \mathrm{E}+10$ & 19.63807 & 46.88954 & 65.23022 & $77.3195 \mathrm{I}$ & 0.468 \\
\hline $1.45 E+10$ & 19.61537 & 46.30927 & 63.37933 & 76.29872 & 90.5941 \\
\hline $1.46 \mathrm{E}+10$ & 19.70678 & 46.10502 & 61.89591 & 76.3538 & 39.969 \\
\hline $1.47 \mathrm{E}+10$ & 19.72743 & 46.12317 & 61.38651 & 72.98185 & 88.6218 \\
\hline $1.48 \mathrm{E}+10$ & 19.52333 & 45.40302 & 60.59354 & 71.45733 & 87.369 \\
\hline 1.49 & 19.46612 & 45.20615 & 61.53443 & 78.3903 & $\overline{6.431}$ \\
\hline $1.5 \mathrm{E}+10$ & 19.49312 & 46.33997 & 65.22947 & 83.25403 & 85.993 \\
\hline $1.51 \mathrm{E}+10$ & 19.45537 & 46.84185 & 66.27212 & 77.22728 & 36.0219 \\
\hline $1.52 \mathrm{E}+10$ & 19.57801 & 46.65189 & 64.74571 & 78.08405 & $\overline{6.442}$ \\
\hline $1.53 \mathrm{E}+10$ & 19.68549 & 46.3326 & 62.47927 & 79.27476 & 86.1394 \\
\hline $1.54 \mathrm{E}+10$ & 19.47528 & 46.00495 & 61.37373 & 71.94165 & 4.63 \\
\hline $1.55 \mathrm{E}+10$ & 19.06816 & 44.74489 & 59.92058 & 69.1257 & 83.9158 \\
\hline+10 & 18.73625 & 43.6694 & 59.7551 & 7.90604 & 83.182 \\
\hline $1.57 \mathrm{E}+10$ & 18.8442 & 44.32904 & 62.30903 & 78.88847 & 81.96245 \\
\hline 1.58 & 19.02602 & 45.37742 & 64.04101 & 73.18847 & 82.07 \\
\hline $1.59 \mathrm{E}+10$ & 18.93202 & 45.25073 & 62.75831 & 75.03955 & 83.2014 \\
\hline $1.6 \mathrm{E}+10$ & 18.8 & 44.50492 & 60.18821 & 73.76655 & 82.2028 \\
\hline $1.61 \mathrm{E}+10$ & 18.47385 & 43.57064 & 58.20237 & 65.71071 & 81.3277 \\
\hline $1.62 \mathrm{E}+10$ & 18.02788 & 41.76896 & 55.56254 & 63.27775 & 81.02076 \\
\hline $1.63 \mathrm{E}+10$ & 18.00778 & 41.27256 & 55.60813 & 68.59423 & 80.16577 \\
\hline $1.64 \mathrm{E}+10$ & 18.14789 & 42.13617 & 57.86299 & 69.93176 & 78.76292 \\
\hline $1.65 \mathrm{E}+10$ & 18.21832 & 43.07889 & 59.57353 & 69.3742 & 78.77396 \\
\hline $1.66 \mathrm{E}+10$ & 18.38299 & 43.59349 & 59.77461 & 71.26615 & 79.41482 \\
\hline+10 & 17.92837 & 42.01338 & 56.55272 & 7.69261 & 79.40613 \\
\hline$E+10$ & 7.58087 & 41.002 & 54.58506 & 64.00762 & 78.66158 \\
\hline $1.69 \mathrm{E}+10$ & 17.54016 & 1.04879 & 54.65128 & 64.33831 & 78.43832 \\
\hline $1.7 \mathrm{E}+10$ & 7.75143 & 1.42186 & 55.54347 & 67.52591 & 77.56803 \\
\hline $1.71 \mathrm{E}+10$ & 17.82523 & 41.24009 & 55.89361 & 68.94391 & 76.00156 \\
\hline $1.72 \mathrm{E}+10$ & 17.87007 & 41.31735 & 56.15689 & 67.47222 & 75.25375 \\
\hline $1.73 \mathrm{E}+10$ & 18.04135 & 41.75659 & 56.24132 & 65.96281 & 75.5467 \\
\hline $1.74 \mathrm{E}+10$ & 17.72693 & 40.68661 & 54.05348 & 64.84142 & 76.34657 \\
\hline $1.75 \mathrm{E}+10$ & 17.29069 & 39.90032 & 52.95151 & 64.39619 & 76.62203 \\
\hline $1.76 \mathrm{E}+10$ & 17.15021 & 39.92281 & 53.51399 & 63.64995 & 76.65389 \\
\hline $1.77 \mathrm{E}+10$ & 17.32598 & 40.2367 & 54.11705 & 65.34911 & 76.13616 \\
\hline $1.79 \mathrm{E}+10$ & 17.36437 & 39.80032 & 53.13903 & 66.0235 & 75.20979 \\
\hline $1.8 \mathrm{E}+10$ & 17.36442 & 39.54342 & 51.93303 & 62.48685 & 74.36045 \\
\hline $1.81 E+10$ & 17.6692 & 40.59099 & 52.8184 & 62.35459 & 74.45922 \\
\hline $1.82 \mathrm{E}+10$ & 17.52099 & 40.4686 & 52.72288 & 63.8872 & 74.91024 \\
\hline $1.83 E+10$ & 17.22337 & 39.63177 & 52.51969 & 64.88958 & 74.95125 \\
\hline
\end{tabular}




\begin{tabular}{|r|r|r|r|r|r|}
\hline Frequency & $e^{\prime \prime} @ 20 \mathrm{C}$ & $e^{\prime \prime} @ 40 \mathrm{C}$ & $\mathrm{e}^{\prime \prime} @ 60 \mathrm{C}$ & $\mathrm{e}^{\prime \prime} @ 80 \mathrm{C}$ & $\mathrm{e}$ " @ 100 C \\
\hline $1.84 \mathrm{E}+10$ & 17.30825 & 39.74024 & 53.73318 & 66.42031 & 74.475 \\
\hline $1.85 \mathrm{E}+10$ & 17.30502 & 40.44912 & 55.47213 & 68.7881 & 73.94499 \\
\hline $1.86 \mathrm{E}+10$ & 17.18091 & 39.53911 & 52.81862 & 65.18014 & 73.00928 \\
\hline $1.87 \mathrm{E}+10$ & 17.52381 & 40.16603 & 52.13605 & 65.84652 & 72.06381 \\
\hline $1.88 \mathrm{E}+10$ & 17.37637 & 40.0456 & 50.67453 & 62.68423 & 71.74851 \\
\hline $1.89 \mathrm{E}+10$ & 17.03091 & 39.42153 & 49.98888 & 58.9414 & 72.36829 \\
\hline $1.9 \mathrm{E}+10$ & 16.75504 & 38.65453 & 50.423 & 61.78645 & 72.69055 \\
\hline $1.91 \mathrm{E}+10$ & 16.72028 & 38.44234 & 51.83288 & 67.36811 & 72.16935 \\
\hline $1.92 \mathrm{E}+10$ & 16.93449 & 39.11959 & 53.80056 & 65.33814 & 71.30819 \\
\hline $1.93 \mathrm{E}+10$ & 16.72906 & 38.78644 & 52.61443 & 62.21999 & 70.66942 \\
\hline $1.94 \mathrm{E}+10$ & 16.7285 & 38.24312 & 49.92742 & 63.121 & 69.72313 \\
\hline $1.95 \mathrm{E}+10$ & 16.81702 & 38.60724 & 49.44262 & 62.56609 & 69.20248 \\
\hline $1.96 \mathrm{E}+10$ & 16.54775 & 38.27541 & 48.8786 & 56.26829 & 69.53162 \\
\hline $1.97 \mathrm{E}+10$ & 16.35008 & 37.84966 & 49.33386 & 58.05477 & 69.90976 \\
\hline $1.98 \mathrm{E}+10$ & 16.06616 & 37.28497 & 49.8886 & 63.16077 & 69.42284 \\
\hline $1.99 \mathrm{E}+10$ & 16.02876 & 37.09062 & 50.02592 & 59.7085 & 68.67617 \\
\hline $2 \mathrm{E}+10$ & 16.07662 & 36.69628 & 48.6184 & 54.99943 & 68.5069 \\
\hline
\end{tabular}


Table 6. LossTangent for Hanford 6M Simulant

\begin{tabular}{|c|c|c|c|c|c|}
\hline$y$ & $\overline{\mathrm{DC}}$ & $\overline{0 C}$ & 60C & $80 \mathrm{C}$ & C \\
\hline+08 & 10.37609 & 12.26961 & 3.66928 & .84181 & 288 \\
\hline 08 & 74821 & .86699 & $\overline{6923}$ & 84076 & 6438 \\
\hline $8 E+08$ & 533083 & .99474 & .51797 & .561 & $\overline{764}$ \\
\hline $6 \mathrm{E}+08$ & 757354 & .22329 & 4.7095 & .81756 & 83925 \\
\hline $5.62 E+08$ & 8.03575 & 11.44718 & 3.90694 & 5.02175 & $\overline{5697}$ \\
\hline $6.65 E+08$ & 7.279032 & 0.47546 & 2.63102 & 3.63575 & 68741 \\
\hline $7.67 \mathrm{E}+08$ & 6.701482 & 9.626437 & 11.54225 & 12.43797 & 617382 \\
\hline $8.69 E+08$ & 232368 & 8.972783 & 0.88486 & 1.76637 & .71949 \\
\hline $9.71 \mathrm{E}+08$ & 776225 & 8.359276 & 10.22393 & 11.06758 & .9678 \\
\hline $1.07 \mathrm{E}+09$ & 5.420527 & 7.917148 & 9.867634 & 10.7588 & .78736 \\
\hline $1.18 \mathrm{E}+09$ & 5.141 & 7.517564 & 9.367907 & 10.25056 & .5473 \\
\hline $1.28 \mathrm{E}+09$ & 897285 & 7.153077 & 8.843168 & 9.673692 & 8.2810 \\
\hline $1.38 \mathrm{E}+09$ & 638989 & 6.741619 & 8.194855 & 8.914068 & 14026 \\
\hline $1.48 \mathrm{E}+09$ & 4.420709 & 6.395661 & 7.73698 & .418047 & .918072 \\
\hline $1.59 \mathrm{E}+09$ & 222466 & 567 & .457417 & 312 & 888 \\
\hline $1.69 \mathrm{E}+09$ & 4.037365 & 5.831835 & 7.189164 & 7.908994 & 7.766669 \\
\hline$E+09$ & 66 & & .94106 & & 680 \\
\hline $1.89 \mathrm{E}+09$ & 3.756323 & 5.421907 & 6.74808 & 7.479312 & 7.415641 \\
\hline $1.99 \mathrm{E}+09$ & 82 & 5.220065 & 422937 & 99 &. \\
\hline $2.1 \mathrm{E}+09$ & 3.513727 & 4.99601 & 6.036554 & 6.628299 & 6.95350 \\
\hline $2.2 \mathrm{E}+09$ & 9427 & 4.847946 & 5.843013 & .39318 & 81395 \\
\hline $2.3 \mathrm{E}+09$ & 3.312058 & 4.671909 & 5.662124 & 6.220965 & 6.764013 \\
\hline $2.4 \mathrm{E}+09$ & 3.23441 & 4.576497 & 5.629862 & 6.263367 & .6220 \\
\hline $2.51 \mathrm{E}+09$ & 161661 & 4.468824 & 5.541466 & 6.133327 & 46757 \\
\hline $2.61 \mathrm{E}+09$ & 3.104989 & 4.387545 & 5.435756 & .02012 & 6.35 \\
\hline $2.71 E+09$ & 3.039774 & 4.26249 & 5.188818 & 5.751148 & .28507 \\
\hline $2.81 E+09$ & 2.975724 & 4.136626 & 4.949619 & 5.458293 & 6.1639 \\
\hline $2.92 \mathrm{E}+09$ & 2.917258 & 4.051258 & 4.843065 & 5.327577 & 5.99679 \\
\hline $3.02 E+09$ & 2.869219 & 3.991888 & 4.812446 & 5.332676 & .87893 \\
\hline $3.12 \mathrm{E}+09$ & 2.81545 & 3.91184 & 4.79289 & 5.375217 & 5.76563 \\
\hline $3.22 \mathrm{E}+09$ & 767714 & 835959 & 735791 & 5.315962 & 5.67975 \\
\hline $3.32 \mathrm{E}+09$ & 2.725136 & 3.778101 & 4.657209 & 5.180084 & 5.62941 \\
\hline $3.43 \mathrm{E}+09$ & 2.679466 & 683322 & .463211 & 4.963604 & 5.519642 \\
\hline $3.53 E+09$ & 2.63171 & .583372 & 4.252524 & 4.72877 & 5.42487 \\
\hline $3.63 \mathrm{E}+09$ & 2.58536 & 3.50915 & .140028 & 4.554058 & 53253 \\
\hline $3.73 E+09$ & 2.562044 & .492421 & 167891 & 4.592597 & 5.26454 \\
\hline $3.84 \mathrm{E}+09$ & 2.526611 & 3.43732 & 165241 & 4.664456 & 5.17203 \\
\hline $3.94 \mathrm{E}+09$ & 2.492911 & 373461 & 4.108319 & 4.612743 & 5.07783 \\
\hline $4.04 \mathrm{E}+09$ & 2.46951 & 3.321015 & 4.02805 & 4.446045 & 4.98917 \\
\hline $4.14 \mathrm{E}+09$ & 2.448774 & 3.280999 & 3.924505 & 4.326535 & 4.95499 \\
\hline $4.25 \mathrm{E}+09$ & 2.423347 & 3.224466 & 3.784457 & 4.199649 & 4.94190 \\
\hline $4.35 \mathrm{E}+09$ & 2.40782 & 3.189719 & 3.708455 & 4.095497 & 4.94395 \\
\hline $4.45 E+09$ & 2.39163 & 3.188328 & 3.758039 & 4.117415 & 4.90141 \\
\hline
\end{tabular}




\begin{tabular}{|c|c|c|c|c|c|}
\hline & & & & & \\
\hline 9 & 883 & 78464 & 6558 & 4.269275 & 558 \\
\hline & & 309 & & $\overline{5821}$ & \\
\hline & 503 & 45185 & 24610 & $\overline{68254}$ & \\
\hline & & 5126 & 25086 & $\overline{0984}$ & 95 \\
\hline-09 & 37 & 3.096491 & .627156 & $\overline{047257}$ & 8001 \\
\hline & & & 7827 & 999626 & $\overline{796}$ \\
\hline+09 & 316501 & 3.045689 & .586272 & 969035 & 7666 \\
\hline & 4 & 291 & 4 & 66628 & $\overline{740}$ \\
\hline+09 & 2.30016 & .015915 & 3.669155 & 175271 & 7130 \\
\hline & 15 & 982 & 49 & 02219 & 6965 \\
\hline $5.57 \mathrm{E}+09$ & 291787 & 2.986419 & 3.581631 & 958027 & 7070 \\
\hline & 81167 & 54329 & 3.46913 & 849863 & 704 \\
\hline $5.78 E+09$ & 274174 & 2.955268 & 3.467005 & 940845 & 67808 \\
\hline & 01 & 74 & 43468 & 842959 & 03 \\
\hline+09 & 242841 & 881 & 486951 & .87663 & 00 \\
\hline+09 & 2.24184 & 391121 & 522579 & 3.99792 & $\overline{586}$ \\
\hline+09 & 30399 & 70731 & 3.475658 & 3.9835 & \\
\hline $9 \mathrm{E}+09$ & 225288 & 2.83944 & 3.347791 & 706259 & 61517 \\
\hline+09 & 28 & 95 & 422 & 16225 & 01 \\
\hline $6.5 \mathrm{E}+09$ & 2.22051 & .800081 & 3.193887 & .576208 & .58903 \\
\hline$E+09$ & 45 & 8008 & .24 & & \\
\hline$E+09$ & 209391 & 785599 & 3.297736 & 3.644876 & 1.50311 \\
\hline 09 & 06 & & 3.35916 & & 468 \\
\hline $8+09$ & 212736 & 309768 & 386033 & 18398 & .4914 \\
\hline+09 & 2.20071 & 2.77 .883 & 265828 & 660725 & .507 \\
\hline+09 & 204027 & 763011 & 3.152756 & 3.441209 & .5096 \\
\hline+09 & 976 & 59858 & 3.114009 & .477395 & .49977 \\
\hline+09 & 206449 & 740853 & 3.135316 & .557438 & 4.48 \\
\hline$E+09$ & 98176 & 2.74647 & 3.24336 & 631388 & 45740 \\
\hline $2 \mathrm{E}+09$ & 02517 & .774725 & 364607 & 80599 & 79 \\
\hline+09 & & & & 07468 & $\overline{38819}$ \\
\hline 09 & 24 & & 56 & 7559 & 001 \\
\hline+09 & & & 575 & 7951 & 45589 \\
\hline+09 & 933 & .690703 & 3.051848 & .07133 & .47658 \\
\hline+09 & .184497 & .678111 & 3.073678 & 7198 & .53855 \\
\hline $8+09$ & 74305 & 2.699456 & .221317 & .697255 & 1.61356 \\
\hline+09 & 914 & 2605528 & .280637 & .719529 & 4.56 \\
\hline+09 & 60507 & 2.6964 & 280749 & .831671 & .49508 \\
\hline+09 & 155386 & 2.674559 & .172447 & 3.701947 & 4.45903 \\
\hline+09 & 816 & 2.613917 & .982133 & .321621 & 4.44663 \\
\hline+09 & & & 2.913429 & 3.231348 & 4.38329 \\
\hline $5 E+09$ & 2.146142 & 2.605129 & 2.984773 & 3.424169 & 4.35121 \\
\hline$\div+09$ & & 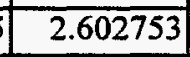 & $\overline{586}$ & 3.558736 & 4.28719 \\
\hline & & & & & 4.24907 \\
\hline 9.0J2 & 2.131802 &. .62874 & 772 & 594 & \\
\hline
\end{tabular}




\begin{tabular}{|c|c|c|c|c|c|}
\hline & & & & & \\
\hline & 130176 & 678 & $\overline{5927}$ & 5 & 46145 \\
\hline & 974 & 69 & 73154 & 27197 & 1102 \\
\hline+09 & 11779 & 6909 & $7 \overline{740973}$ & 3811 & $\overline{99496}$ \\
\hline & 125917 & 32 & 820332 & $\overline{92795}$ & $\overline{04702}$ \\
\hline $.56 \mathrm{E}+09$ & 095472 & 503097 & 2.930326 & 0213 & 9859 \\
\hline 09 & 08684 & 42 & 2.95686 & $\overline{34294}$ & 9576 \\
\hline $9.77 E+09$ & 095633 & 2.51901 & 2.992555 & 3.41771 & $\overline{92949}$ \\
\hline-09 & 3764 & 503643 & 29231 & 242087 & 94163 \\
\hline $97 \mathrm{E}+09$ & 111336 & 2.47956 & 2.663653 & 2.963013 & 85911 \\
\hline+10 & 48 & 42 & & 802496 & 81229 \\
\hline $1.02 E+10$ & 076396 & 2.375949 & 2.556954 & 826188 & $\overline{76476}$ \\
\hline+10 & 53 & 234 & $\longdiv { 1 3 }$ & $\overline{10045}$ & .733 \\
\hline $1.04 \mathrm{E}+10$ & 2.054565 & .363748 & 2.77051 & 3.128951 & .71097 \\
\hline-10 & & & & & 7 \\
\hline $1.06 \mathrm{E}+10$ & 2.081465 & .431095 & 763859 & .169765 & 69215 \\
\hline & 24 & & & & \\
\hline $1.08 \mathrm{E}+10$ & 2.060881 & 2.395406 & 2.561232 & 2.81748 & .55855 \\
\hline & 2.034565 & 37 & 639 & कבר1 & 0884 \\
\hline $1.1 \mathrm{E}+10$ & 2.031042 & 2.302964 & 2.615578 & 2.970169 & 48376 \\
\hline$E+10$ & 2.024369 & 64 & 2.73341 & 3.11228 & .484 \\
\hline$E+10$ & 03344 & .347159 & 2.787319 & 3.16246 & .47853 \\
\hline+10 & $7 \overline{5408}$ & 48 & 13 & 97156 & 46826 \\
\hline $8+10$ & 73557 & 2.418261 & 623207 & 2.980755 & 397 \\
\hline$E+10$ & 46894 & $\overline{322}$ & 417964 & .630142 & .34087 \\
\hline+10 & 01 & $\overline{24}$ & 05 & 617 & 2 \\
\hline $\mathrm{E}+10$ & 33523 & 215314 & 2.447866 & 2.721046 & $\overline{3.266}$ \\
\hline $2+10$ & 2.057814 & .251873 & 2.60742 & .892076 & .28126 \\
\hline $8+10$ & .044556 & .280059 & 2.657364 & 2.904643 & .34755 \\
\hline$E+10$ & 61886 & 23256 & 2.587194 & .855806 & .48481 \\
\hline$\overline{E+10}$ & 064859 & $5 \overline{5882}$ & 2.461807 & 2.745011 & .61556 \\
\hline $2 E+10$ & 41275 & 269281 & 292805 & 483052 & .51722 \\
\hline$E+10$ & 340984 & 176869 & 337 & $\overline{80501}$ & .37969 \\
\hline $\mathrm{E}+10$ & 11335 & 228764 & 2.398073 & 2.645469 & 3.2283 \\
\hline $\mathrm{E}+10$ & 03706 & 259018 & .550574 & 2.767912 & 2322 \\
\hline $\mathrm{E}+10$ & 77144 & 233432 & .508507 & 2.64111 & .34645 \\
\hline $1.27 \mathrm{E}+10$ & 66212 & 2.243198 & 2.434437 & 2.64273 & .47719 \\
\hline $1.28 \mathrm{E}+10$ & 60297 & .231329 & 2.319943 & .577275 & .54138 \\
\hline$E+10$ & 58392 & 2.209107 & 2.264959 & 2.472925 & 3.48071 \\
\hline $1.3 \mathrm{E}+10$ & 2.068052 & 2.175348 & 2.271324 & 2.497121 & 31209 \\
\hline$\overline{E+10}$ & 28203 & $\overline{10665}$ & 2.481745 & 2.941464 & .19467 \\
\hline $1.32 \mathrm{E}+10$ & & & 2.719436 & 3.2845 & .16132 \\
\hline$E+10$ & 46819 & $10 / 9$ & 2.778682 & 179832 & .19917 \\
\hline & & & & 3.192834 & 3.23820 \\
\hline$E+10$ & 24426 & 2.140201 & 2.698304 & 3.222187 & 3.26767 \\
\hline $1.07 \mathrm{E}$ & $\overline{03}$ & 2.16 & 01 & 3.004049 & \\
\hline
\end{tabular}




\begin{tabular}{|c|c|c|c|c|c|}
\hline & & & & & \\
\hline 0 & 46 & 9 & 2.543159 & 0 & 255 \\
\hline & 96 & 4 & 9 & 3.097125 & $\overline{579}$ \\
\hline $\mathrm{E}+10$ & 506 & 77972 & 485 & 3.24451 & 1114 \\
\hline 10 & 38 & 260209 & 712227 & 3.047685 & $\overline{084678}$ \\
\hline $1.42 \mathrm{E}+10$ & 33847 & 2.187687 & 2.68683 & 3.03417 & 09285 \\
\hline $1.43 \mathrm{E}+10$ & & 2.192372 & .757851 & .411924 & 12082 \\
\hline $1.44 \mathrm{E}+10$ & 2.013799 & 2.260331 & 2.872516 & 3.38995 & 18260 \\
\hline+10 & & 260255 & 767691 & 89071 & $\overline{878}$ \\
\hline $1.46 \mathrm{E}+10$ & 2.046132 & 2.222569 & 2.61201 & .228403 & 13564 \\
\hline-10 & & 0318 & 95 & 68521 & $\overline{5344}$ \\
\hline $8 E+10$ & 00349 & 2.250955 & 649412 & 2.990655 & 0728 \\
\hline-10 & & 689 & 39 & 93889 & $\overline{670}$ \\
\hline $1.5 \mathrm{E}+10$ & 91442 & .234404 & 714821 & 747549 & 06475 \\
\hline+10 & & & & 99 & 4 \\
\hline $1.52 \mathrm{E}+10$ & 27053 & 2.340522 & 877684 & 3.075965 & 09613 \\
\hline+10 & & 92 & 02 & 36 & 169 \\
\hline $1.54 \mathrm{E}+10$ & 74158 & 2.332499 & 2.698785 & 3.850231 & .96097 \\
\hline$E+10$ & & 12 & & 14 & 86385 \\
\hline $1.56 \mathrm{E}+10$ & 65552 & 2.186709 & 2.511932 & 3.036595 & .95353 \\
\hline $1.57 \mathrm{E}+10$ & $49 !$ & 127874 & .495454 & 911448 & 97291 \\
\hline $1.58 \mathrm{E}+10$ & 09 & 2.265388 & 2.748853 & 3.107764 & 94854 \\
\hline $1.59 \mathrm{E}+10$ & 36 & 269747 & 691322 & 100 & .01320 \\
\hline $1.6 \mathrm{E}+10$ & 757 & 2.27422 & 2.583735 & 394126 & .97405 \\
\hline $1.61 \mathrm{E}+10$ & 56807 & 308202 & .641243 & 339891 & 2.8040 \\
\hline $1.62 \mathrm{E}+10$ & 9] & 2.179599 & .501 & 512 & 5330 \\
\hline $1.63 \mathrm{E}+10$ & 2.012561 & 2.076257 & 2.408118 & 2.742804 & 8.81530 \\
\hline $1.64 \mathrm{E}+10$ & 19] & 89. & 3983 & 3.14552 & .84689 \\
\hline $1.65 \mathrm{E}+10$ & 75 & 994 & 332 & 601 & $\overline{8883}$ \\
\hline $1.66 \mathrm{E}+10$ & & 362029 & 901739 & 2.97766 & .99281 \\
\hline $1.67 \mathrm{E}+10$ & & 278885 & 2.665529 & 983682 & .008279 \\
\hline $1.68 \mathrm{E}+10$ & 48265 & 45087 & .437177 & 758753 & .81310 \\
\hline $1.69 \mathrm{E}+10$ & 86 & 065827 & 344534 & 5986 & .71362 \\
\hline $1.7 \mathrm{E}+10$ & 2484 & 130845 & 2.4853 & 785683 & .73845 \\
\hline $1.71 \mathrm{E}+10$ & & 209606 & .712818 & 3.296828 & 2.75952 \\
\hline $1.72 \mathrm{E}+10$ & & 263865 & 2.8915 & 3.567651 & .73253 \\
\hline $1.73 \mathrm{E}+10$ & & 350886 & .026197 & 394222 & 2.76598 \\
\hline $1.74 \mathrm{E}+10$ & & 306365 & 2.79003 & 991923 & 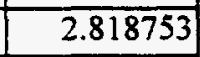 \\
\hline $1.75 \mathrm{E}+10$ & 8023 & 148646 & 2.46705 & .904745 & .794742 \\
\hline $1.76 \mathrm{E}+10$ & 578 & & 2.389781 & 2.843009 & .73 \\
\hline $1.77 \mathrm{E}+10$ & 107 & 2.201506 & 2.644062 & 3.088464 & 2.71170 \\
\hline $1.79 \mathrm{E}+10$ & $104 / 03$ & 2.278096 & 2.905766 & 3.821577 & 2.71248 \\
\hline $3 E+10$ & 41032 & 2.254075 & .956137 & 3.847082 & 2.688652 \\
\hline $\mathrm{E}+10$ & & 2.343223 & 801 & 3.540622 & 2.681064 \\
\hline 20 & & & & & 2.728518 \\
\hline 710 & 2.20916 & 2.261435 & 2.620714 & 3.251852 & 2.70 \\
\hline
\end{tabular}




\begin{tabular}{|r|r|r|r|r|r|}
\hline Frequency & $\mathrm{e}^{\prime \prime} / \mathrm{e}^{\prime} 20 \mathrm{C}$ & $\mathrm{e}^{\prime \prime} / \mathrm{e}^{\prime} 40 \mathrm{C}$ & $\mathrm{e}^{\prime \prime} / \mathrm{e}^{\prime} 60 \mathrm{C}$ & $\mathrm{e}^{\prime \prime} / \mathrm{e}^{\prime} 80 \mathrm{C}$ & $\mathrm{e}^{\prime \prime} / \mathrm{e}^{\prime} 100 \mathrm{C}$ \\
\hline \hline $1.84 \mathrm{E}+10$ & 2.192255 & 2.151942 & 2.405465 & 2.870944 & 2.716247 \\
\hline $1.85 \mathrm{E}+10$ & 2.268854 & 2.267039 & 2.655483 & 3.665697 & 2.666407 \\
\hline $1.86 \mathrm{E}+10$ & 2.298349 & 2.311145 & 2.78612 & 3.841934 & 2.0610145 \\
\hline $1.87 \mathrm{E}+10$ & 2.39355 & 2.354776 & 2.864796 & 3.511165 & 2.576586 \\
\hline $1.88 \mathrm{E}+10$ & 2.378547 & 2.376072 & 2.851858 & 3.593676 & 2.561697 \\
\hline $1.89 \mathrm{E}+10$ & 2.327683 & 2.356884 & 2.766206 & 3.665946 & 2.621501 \\
\hline $1.9 \mathrm{E}+10$ & 2.23146 & 2.237608 & 2.585495 & 3.021897 & 2.720544 \\
\hline $1.91 \mathrm{E}+10$ & 2.146803 & 2.093438 & 2.400555 & 3.073729 & 2.740159 \\
\hline $1.92 \mathrm{E}+10$ & 2.217102 & 2.128368 & 2.452349 & 3.528421 & 2.658399 \\
\hline $1.93 \mathrm{E}+10$ & 2.258409 & 2.19568 & 2.529044 & 3.115198 & 2.581306 \\
\hline $1.94 \mathrm{E}+10$ & 2.285633 & 2.21014 & 2.458104 & 2.903043 & 2.518189 \\
\hline $1.95 \mathrm{E}+10$ & 2.301429 & 2.166258 & 2.317318 & 3.033727 & 2.499508 \\
\hline $1.96 \mathrm{E}+10$ & 2.274561 & 2.193888 & 2.37554 & 2.877208 & 2.544541 \\
\hline $1.97 \mathrm{E}+10$ & 2.27816 & 2.191926 & 2.426645 & 2.673342 & 2.660058 \\
\hline $1.98 \mathrm{E}+10$ & 2.217488 & 2.134241 & 2.436775 & 3.271894 & 2.690134 \\
\hline $1.99 \mathrm{E}+10$ & 2.200042 & 2.107946 & 2.41991 & 3.570864 & 2.596044 \\
\hline $2 \mathrm{E}+10$ & 2.235281 & 2.136855 & 2.374551 & 2.729802 & 2.504664 \\
\hline
\end{tabular}


Table 7. Dielectric Constant for the Melton Valley Simulant

\begin{tabular}{|c|c|c|c|c|c|}
\hline Frequency & $e^{\prime} @ 20 \mathrm{C}$ & $e^{\prime} @ 40 C$ & $e^{\prime} @ 60 \mathrm{C}$ & $\mathrm{e}^{\prime} @ 80 \mathrm{C}$ & $e^{\prime} @ 100 \mathrm{C}$ \\
\hline $1.53 \mathrm{E}+08$ & 226.284627 & 289.1865115 & 350.794242 & 402.1176815 & 420.320315 \\
\hline $2.55 \mathrm{E}+08$ & 129.678184 & 157.961016 & 185.854052 & 208.29391 & 211.0737625 \\
\hline $3.58 \mathrm{E}+08$ & 98.542862 & 115.1013925 & 131.923054 & 145.325015 & 144.580243 \\
\hline $4.6 \mathrm{E}+08$ & 86.4504105 & 98.224947 & 111.003729 & 121.7433075 & 121.5397275 \\
\hline $5.62 \mathrm{E}+08$ & 76.4828185 & 84.2510495 & 93.1290735 & 100.6355505 & 99.2436225 \\
\hline $6.65 E+08$ & 72.7809015 & 79.232699 & 86.9261535 & 94.175106 & 93.492661 \\
\hline $7.67 \mathrm{E}+08$ & 69.0109085 & 74.3441315 & 80.986949 & 87.561319 & 87.115834 \\
\hline $8.69 \mathrm{E}+08$ & 65.96654 & 70.235168 & 75.8468285 & 81.5865755 & 80.9788685 \\
\hline $9.71 \mathrm{E}+08$ & 64.0979035 & 67.5832205 & 72.610267 & 78.0140155 & 77.600886 \\
\hline $1.07 \mathrm{E}+09$ & 62.5732295 & 65.234074 & 69.4615625 & 74.1735625 & 73.413048 \\
\hline $1.18 \mathrm{E}+09$ & 61.3400225 & 63.493734 & 67.326427 & 71.5004985 & 70.687202 \\
\hline $1.28 \mathrm{E}+09$ & 59.74618 & 61.282307 & 64.356811 & 67.925794 & 66.834043 \\
\hline $1.38 \mathrm{E}+09$ & 59.346614 & 60.7353635 & 63.754012 & 67.2862595 & 66.4242385 \\
\hline $1.48 \mathrm{E}+09$ & 58.3203945 & 59.5330875 & 62.311613 & 65.8323695 & 65.01978 \\
\hline $1.59 \mathrm{E}+09$ & 57.88326 & 58.9747355 & 61.6320355 & 64.770446 & 63.979277 \\
\hline $1.69 E+09$ & 57.382015 & 58.2747105 & 60.7630585 & 63.8222275 & 62.993135 \\
\hline $1.79 \mathrm{E}+09$ & 57.163073 & 57.8729625 & 60.1998315 & 63.3197385 & 62.6364705 \\
\hline $1.89 \mathrm{E}+09$ & 56.664371 & 57.1026475 & 59.0026295 & 61.5346525 & 60.503361 \\
\hline $1.99 \mathrm{E}+09$ & 56.3599675 & 56.6935525 & 58.5076295 & 60.8922105 & 59.8940135 \\
\hline $2.1 \mathrm{E}+09$ & 56.001856 & 56.2595185 & 57.853048 & 60.190184 & 59.1534935 \\
\hline $2.2 \mathrm{E}+09$ & 55.49141 & 55.6971425 & 57.2537135 & 59.4309025 & 58.371357 \\
\hline $2.3 E+09$ & 55.253236 & 55.519064 & 57.2381395 & 59.451753 & 58.5222045 \\
\hline $2.4 \mathrm{E}+09$ & 55.069104 & 55.1956545 & 56.7569555 & 58.7299815 & 57.594322 \\
\hline $2.51 \mathrm{E}+09$ & 54.6978975 & 54.626067 & 55.969489 & 57.7683285 & 56.48895 \\
\hline $2.61 \mathrm{E}+09$ & 54.372726 & 54.230067 & 55.5042285 & 57.2417915 & 55.8636215 \\
\hline $2.71 E+09$ & 54.0035525 & 53.8552385 & 55.005364 & 56.6306225 & 55.191308 \\
\hline $2.81 E+09$ & 53.7783905 & 53.7217195 & 54.8885295 & 56.526649 & 55.0896255 \\
\hline $2.92 \mathrm{E}+09$ & 53.5467495 & 53.541547 & 54.58997 & 56.0990755 & 54.5114545 \\
\hline $3.02 \mathrm{E}+09$ & 53.324824 & 53.495742 & 54.3139345 & 55.7825365 & 54.197182 \\
\hline $3.12 \mathrm{E}+09$ & 53.0103985 & 53.0789535 & 53.8280205 & 55.1223435 & 53.385103 \\
\hline $3.22 \mathrm{E}+09$ & 52.7809315 & 52.780253 & 53.4014475 & 54.6297155 & 52.8402905 \\
\hline $3.32 \mathrm{E}+09$ & 52.5833745 & 52.4467775 & 52.907003 & 53.8946715 & 51.9749795 \\
\hline $3.43 \mathrm{E}+09$ & 52.3684385 & 52.27907 & 52.7551175 & 53.8017875 & 51.979221 \\
\hline $3.53 E+09$ & 52.1566145 & 52.1164125 & 52.6509605 & 53.803949 & 52.0999985 \\
\hline $3.63 \mathrm{E}+09$ & 52.0639975 & 52.099671 & 52.6129005 & 53.755536 & 52.0734495 \\
\hline $3.73 \mathrm{E}+09$ & 51.926698 & 51.8600575 & 52.3072045 & 53.254202 & 51.504781 \\
\hline $3.84 \mathrm{E}+09$ & 51.789805 & 51.708975 & 52.165887 & 53.0592065 & 51.262713 \\
\hline $3.94 \mathrm{E}+09$ & 51.647537 & 51.578441 & 52.032031 & 53.031858 & 51.297843 \\
\hline $4.04 \mathrm{E}+09$ & 51.4905395 & 51.480963 & 51.92854 & 52.828363 & 51.124935 \\
\hline $4.14 \mathrm{E}+09$ & 51.3011725 & 51.2809755 & 51.635845 & 52.3912565 & 50.6292875 \\
\hline $4.25 \mathrm{E}+09$ & 51.1096085 & 51.088657 & 51.474704 & 52.314319 & 50.591758 \\
\hline $4.35 \mathrm{E}+09$ & 50.890476 & 50.850233 & 51.2312035 & 52.01775 & 50.336315 \\
\hline $4.45 \mathrm{E}+09$ & 50.6054635 & 50.446558 & 50.6659465 & 51.17102 & 49.3277225 \\
\hline
\end{tabular}




\begin{tabular}{|c|c|c|c|c|c|}
\hline Frequency & $e^{\prime} @ 20 c$ & $e^{\prime} @ 40 \mathrm{C}$ & $e^{\prime} @ 60 \mathrm{C}$ & $\mathrm{e}^{\prime} @ 80 \mathrm{C}$ & $e^{\prime} @ 100 \mathrm{C}$ \\
\hline $4.55 E+09$ & 50.3547045 & 50.122145 & 50.1993265 & 50.5789215 & 48.5887645 \\
\hline $4.65 \mathrm{E}+09$ & 50.154047 & 49.928896 & 50.0201435 & 50.4542745 & 48.5487795 \\
\hline $4.76 \mathrm{E}+09$ & 49.8923785 & 49.65746005 & 49.6534195 & 49.921958 & 47.857613 \\
\hline $4.86 \mathrm{E}+09$ & 49.737746 & 49.5192255 & 49.4474805 & 49.646999 & 47.5308415 \\
\hline $4.96 \mathrm{E}+09$ & 49.451292 & 49.2273735 & 49.1807125 & 49.340815 & 47.219416 \\
\hline $5.06 \mathrm{E}+09$ & 49.1738285 & 48.9976805 & 49.0028395 & 49.127171 & 46.9781125 \\
\hline $5.17 E+09$ & 48.981889 & 48.782711 & 48.646413 & 48.6466655 & 46.2899115 \\
\hline $5.27 \mathrm{E}+09$ & 48.7200335 & 48.5153365 & 48.3057135 & 48.285619 & 45.9401895 \\
\hline $5.37 \mathrm{E}+09$ & 48.536652 & 48.3643495 & 48.1447745 & 48.093928 & 45.7618355 \\
\hline $5.47 \mathrm{E}+09$ & 48.3177035 & 48.2273385 & 48.0898335 & 48.072086 & 45.6747125 \\
\hline $5.57 \mathrm{E}+09$ & 48.106314 & 48.001133 & 47.7752615 & 47.530954 & 44.9753685 \\
\hline $5.68 \mathrm{E}+09$ & 47.8589965 & 47.7593905 & 47.5211905 & 47.254898 & 44.6460255 \\
\hline $5.78 \mathrm{E}+09$ & 47.5845295 & 47.4378675 & 47.184561 & 46.7745585 & 43.960672 \\
\hline $5.88 \mathrm{E}+09$ & 47.383617 & 47.3330825 & 47.17352 & 46.922175 & 44.184066 \\
\hline $5.98 \mathrm{E}+09$ & 47.2220265 & 47.1976145 & 47.007544 & 46.633304 & 43.8377245 \\
\hline $6.09 \mathrm{E}+09$ & 47.0162135 & 47.022627 & 46.818217 & 46.5077875 & 43.7164165 \\
\hline $6.19 \mathrm{E}+09$ & 46.819989 & 46.8171145 & 46.6423185 & 46.358019 & 43.54929 \\
\hline $6.29 \mathrm{E}+09$ & 46.5850475 & 46.652395 & 46.6096575 & 46.3015125 & 43.494516 \\
\hline $6.39 \mathrm{E}+09$ & 46.2718555 & 46.3203075 & 46.223353 & 45.750064 & 42.758854 \\
\hline $6.5 \mathrm{E}+09$ & 46.055378 & 46.160682 & 46.165176 & 45.8285225 & 42.986341 \\
\hline $6.6 \mathrm{E}+09$ & 45.796351 & 45.9761 & 46.0188445 & 45.6269305 & 42.7106925 \\
\hline $6.7 \mathrm{E}+09$ & 45.600254 & 45.7803355 & 45.7538285 & 45.4065515 & 42.464706 \\
\hline $6.8 \mathrm{E}+09$ & 45.433247 & 45.5456235 & 45.3566855 & 44.9266585 & 41.9251225 \\
\hline $6.9 \mathrm{E}+09$ & 45.207451 & 45.235087 & 45.0033735 & 44.5116645 & 41.4241745 \\
\hline $7.01 \mathrm{E}+09$ & 44.977528 & 44.996676 & 44.7532515 & 44.239718 & 41.089033 \\
\hline $7.11 \mathrm{E}+09$ & 44.6658725 & 44.6096875 & 44.2650815 & 43.6153395 & 40.365899 \\
\hline $7.21 \mathrm{E}+09$ & 44.4987955 & 44.5104635 & 44.139644 & 43.5487445 & 40.387024 \\
\hline $7.31 \mathrm{E}+09$ & 44.291216 & 44.3635565 & 43.9901635 & 43.4005405 & 40.1998335 \\
\hline $7.42 \mathrm{E}+09$ & 44.0315125 & 44.0396875 & 43.637056 & 42.9933185 & 39.687693 \\
\hline $7.52 \mathrm{E}+09$ & 43.8036845 & 43.8078575 & 43.362273 & 42.6157165 & 39.300449 \\
\hline $7.62 \mathrm{E}+09$ & 43.6033505 & 43.654117 & 43.21369 & 42.4550765 & 39.2174255 \\
\hline $7.72 \mathrm{E}+09$ & 43.430468 & 43.620049 & 43.3164925 & 42.693318 & 39.479333 \\
\hline $7.83 \mathrm{E}+09$ & 43.2416765 & 43.51742 & 43.2692935 & 42.5487925 & 39.296217 \\
\hline $7.93 \mathrm{E}+09$ & 43.0760985 & 43.397999 & 43.1625765 & 42.4272025 & 39.147978 \\
\hline $8.03 \mathrm{E}+09$ & 42.8973985 & 43.308398 & 43.0932205 & 42.4439955 & 39.1877075 \\
\hline $8.13 E+09$ & 42.618038 & 42.901268 & 42.555945 & 41.591616 & 38.0410955 \\
\hline $8.23 \mathrm{E}+09$ & 42.408141 & 42.5944395 & 42.2171635 & 41.2883195 & 37.832554 \\
\hline $8.34 \mathrm{E}+09$ & 42.1805525 & 42.3822785 & 41.9747165 & 41.0023985 & 37.5508455 \\
\hline $8.44 \mathrm{E}+09$ & 42.012802 & 42.2958425 & 41.8494065 & 40.8758665 & 37.4369465 \\
\hline $8.54 \mathrm{E}+09$ & 41.8930675 & 42.2709595 & 41.989339 & 41.170384 & 37.9159065 \\
\hline $8.64 \mathrm{E}+09$ & 41.748793 & 42.293584 & 42.1454725 & 41.3464305 & 38.1122505 \\
\hline $8.75 \mathrm{E}+09$ & 41.6272645 & 42.215182 & 42.0696985 & 41.2128215 & 37.990492 \\
\hline $8.85 E+09$ & 41.457685 & 42.018922 & 41.856229 & 40.980883 & 37.746251 \\
\hline $8.95 E+09$ & 41.1858165 & 41.6970415 & 41.4968475 & 40.4967795 & 37.153109 \\
\hline $9.05 \mathrm{E}+09$ & 41.0160385 & 41.5227045 & 41.311369 & 40.4071545 & 37.195388 \\
\hline
\end{tabular}




\begin{tabular}{|c|c|c|c|c|c|}
\hline Frequency & $e^{\prime} @ 20 \mathrm{C}$ & $\mathrm{e}^{\prime} @ 40 \mathrm{C}$ & $e^{\prime} @ 60 \mathrm{C}$ & $e^{\prime} @ 80 \mathrm{C}$ & $e^{\prime} @ 100 \mathrm{C}$ \\
\hline $9.16 \mathrm{E}+09$ & 40.920965 & 41.7296015 & 41.782039 & 41.0776775 & 37.9849245 \\
\hline $9.26 \mathrm{E}+09$ & 40.683689 & 41.4878815 & 41.559404 & 40.7274485 & 37.515829 \\
\hline $9.36 \mathrm{E}+09$ & 40.606118 & 41.490194 & 41.6964765 & 41.0286845 & 38.086384 \\
\hline $9.46 \mathrm{E}+09$ & 40.476574 & 41.497618 & 41.7301005 & 40.9804925 & 37.97751 \\
\hline $9.56 \mathrm{E}+09$ & 40.316617 & 40.9962115 & 40.8547245 & 39.9689655 & 36.898724 \\
\hline $9.67 \mathrm{E}+09$ & 40.1763505 & 40.8006255 & 40.701529 & 39.9479755 & 37.104283 \\
\hline $9.77 \mathrm{E}+09$ & 39.9215215 & 40.54475 & 40.464003 & 39.619922 & 36.6533845 \\
\hline $9.87 E+09$ & 39.868749 & 40.5915665 & 40.6346815 & 40.010053 & 37.2883515 \\
\hline $9.97 \mathrm{E}+09$ & 39.702447 & 40.5999365 & 40.822299 & 40.2033955 & 37.4795745 \\
\hline $1.01 \mathrm{E}+10$ & 39.570322 & 40.635378 & 40.903147 & 40.2172765 & 37.413521 \\
\hline $1.02 \mathrm{E}+10$ & 39.537791 & 40.5019235 & 40.620078 & 40.055489 & 37.322079 \\
\hline $1.03 \mathrm{E}+10$ & 39.4018745 & 40.299701 & 40.248361 & 39.7073305 & 37.0323375 \\
\hline $1.04 \mathrm{E}+10$ & 39.2531785 & 40.0667515 & 39.938067 & 39.3355045 & 36.6852625 \\
\hline $1.05 \mathrm{E}+10$ & 39.1578665 & 40.0755005 & 40.175439 & 39.619577 & 37.1154535 \\
\hline $1.06 \mathrm{E}+10$ & 39.028608 & 40.35936 & 40.722305 & 40.2005735 & 37.6491665 \\
\hline $1.07 \mathrm{E}+10$ & 38.9263375 & 40.434921 & 40.6994135 & 40.029024 & 37.273943 \\
\hline $1.08 \mathrm{E}+10$ & 38.8541905 & 40.308754 & 40.3249705 & 39.600972 & 36.7341995 \\
\hline $1.09 \mathrm{E}+10$ & 38.737612 & 39.930404 & 39.702699 & 38.895595 & 35.988027 \\
\hline $1.1 \mathrm{E}+10$ & 38.617183 & 39.633653 & 39.353129 & 38.670649 & 35.93143 \\
\hline $1.11 \mathrm{E}+10$ & 38.4936295 & 39.707393 & 39.70269 & 39.1419045 & 36.6558475 \\
\hline $1.12 \mathrm{E}+10$ & 38.350204 & 39.9149355 & 40.2892525 & 39.843881 & 37.4284785 \\
\hline $1.13 \mathrm{E}+10$ & 38.212317 & 39.964626 & 40.5887165 & 40.076535 & 37.5634515 \\
\hline $1.14 \mathrm{E}+10$ & 38.0972955 & 39.871551 & 40.3769825 & 39.763291 & 37.1939055 \\
\hline $1.15 \mathrm{E}+10$ & 38.0191255 & 39.6513365 & 39.960809 & 39.435189 & 36.916504 \\
\hline $1.16 \mathrm{E}+10$ & 37.9357295 & 39.506334 & 39.705351 & 39.3168675 & 36.918079 \\
\hline $1.17 \mathrm{E}+10$ & 37.8474035 & 39.5923005 & 40.0577435 & 39.780226 & 37.479965 \\
\hline $1.18 \mathrm{E}+10$ & 37.692489 & 39.716502 & 40.537161 & 40.3586875 & 38.172911 \\
\hline $1.19 \mathrm{E}+10$ & 37.606451 & 39.689502 & 40.501842 & 40.285644 & 38.1157105 \\
\hline $1.2 \mathrm{E}+10$ & 37.4716115 & 39.635034 & 40.4367455 & 40.1124035 & 37.768129 \\
\hline $1.21 \mathrm{E}+10$ & 37.3570095 & 39.214394 & 39.667088 & 39.138317 & 36.6484705 \\
\hline $1.22 \mathrm{E}+10$ & 37.289768 & 38.8168765 & 38.868354 & 38.4695225 & 36.0795425 \\
\hline $1.23 \mathrm{E}+10$ & 37.194426 & 38.59643 .45 & 38.571213 & 38.468578 & 36.292764 \\
\hline $1.24 \mathrm{E}+10$ & 37.0867825 & 38.9759505 & 39.625499 & 39.5757265 & 37.4278765 \\
\hline $1.25 \mathrm{E}+10$ & 36.9586775 & 39.086205 & 39.997531 & 39.8311645 & 37.553565 \\
\hline $1.26 \mathrm{E}+10$ & 36.8680005 & 39.170959 & 39.992323 & 39.8752545 & 37.570538 \\
\hline $1.27 \mathrm{E}+10$ & 36.715733 & 38.998555 & 39.780042 & 39.5602005 & 37.082786 \\
\hline $1.28 \mathrm{E}+10$ & 36.643625 & 38.7491685 & 39.352364 & 39.175045 & 36.6593395 \\
\hline $1.29 \mathrm{E}+10$ & 36.5627805 & 38.6809795 & 39.194422 & 39.0094335 & 36.644029 \\
\hline $1.3 \mathrm{E}+10$ & 36.4272915 & 38.4535415 & 38.996957 & 38.971767 & 36.629486 \\
\hline $1.31 \mathrm{E}+10$ & 36.2924915 & 38.571246 & 39.4131965 & 39.4061455 & 37.043734 \\
\hline $1.32 \mathrm{E}+10$ & 36.078347 & 38.2749005 & 39.110876 & 38.8594645 & 36.3682135 \\
\hline $1.33 \mathrm{E}+10$ & 35.9869845 & 38.07792 & 38.6284785 & 38.2249415 & 35.680271 \\
\hline $1.35 \mathrm{E}+10$ & 35.9388095 & 37.974456 & 38.2521435 & 38.017992 & 35.513357 \\
\hline $1.36 \mathrm{E}+10$ & 35.891338 & 37.9230415 & 38.2522085 & 38.1211655 & 35.662049 \\
\hline $1.37 \mathrm{E}+10$ & 35.728053 & 37.6167535 & 38.019358 & 37.74063 & 35.1898265 \\
\hline
\end{tabular}




\begin{tabular}{|c|c|c|c|c|c|}
\hline Frequency & $e^{\prime} @ 20 c$ & $e^{\prime} @ 40 \mathrm{C}$ & $e^{\prime} @ 60 \mathrm{C}$ & $e^{\prime} @ 80 \mathrm{C}$ & $e^{\prime} @ 100 \mathrm{C}$ \\
\hline $1.38 \mathrm{E}+10$ & 35.640818 & 37.671178 & 38.187611 & 37.9867085 & 35.599704 \\
\hline $1.39 \mathrm{E}+10$ & 35.4766775 & 37.5584835 & 38.184864 & 38.0456785 & 35.5667565 \\
\hline $1.4 \mathrm{E}+10$ & 35.3684655 & 37.6329105 & 38.3388915 & 38.0514495 & 35.6127945 \\
\hline $1.41 E+10$ & 35.2211225 & 37.681198 & 38.582789 & 38.072689 & 35.5459305 \\
\hline $1.42 \mathrm{E}+10$ & 35.15219 & 37.7462285 & 38.6584335 & 38.2377835 & 35.7661845 \\
\hline $1.43 \mathrm{E}+10$ & 35.068548 & 37.8954935 & 38.932999 & 38.812246 & 36.438164 \\
\hline $1.44 \mathrm{E}+10$ & 34.979645 & 37.7856905 & 38.91477 & 38.774877 & 36.270243 \\
\hline $1.45 \mathrm{E}+10$ & 34.8163095 & 37.3284065 & 38.3294505 & 38.049836 & 35.631641 \\
\hline $1.46 \mathrm{E}+10$ & 34.6768815 & 37.0724765 & 38.018896 & 37.9406865 & 35.7138425 \\
\hline $1.47 \mathrm{E}+10$ & 34.525713 & 37.076221 & 38.2003025 & 38.1989855 & 35.890162 \\
\hline $1.48 \mathrm{E}+10$ & 34.442929 & 37.050213 & 38.311557 & 37.9219225 & 35.497289 \\
\hline $1.49 \mathrm{E}+10$ & 34.384114 & 37.057637 & 38.333176 & 38.0204405 & 35.6740635 \\
\hline $1.5 \mathrm{E}+10$ & 34.2994445 & 37.2194245 & 38.538278 & 38.742949 & 36.7418255 \\
\hline $1.51 \mathrm{E}+10$ & 34.07215 & 36.758114 & 38.0447285 & 38.3187765 & 36.27118 \\
\hline $1.52 \mathrm{E}+10$ & 34.0068575 & 36.554422 & 37.6901445 & 37.9701035 & 36.0410165 \\
\hline $1.53 \mathrm{E}+10$ & 33.8004345 & 36.1891815 & 37.527666 & 37.447627 & 35.855807 \\
\hline $1.54 \mathrm{E}+10$ & 33.7582145 & 36.3116405 & 37.472181 & 37.4722595 & 35.650866 \\
\hline $1.55 \mathrm{E}+10$ & 33.8065225 & 36.829638 & 38.28679 & 38.1564925 & 36.2752315 \\
\hline $1.56 \mathrm{E}+10$ & 33.783376 & 36.4061385 & 37.1794255 & 36.915044 & 34.9758275 \\
\hline $1.57 \mathrm{E}+10$ & 33.5801305 & 35.6989275 & 35.800995 & 35.9994495 & 33.958368 \\
\hline $1.58 \mathrm{E}+10$ & 33.318424 & 35.167092 & 35.4672945 & 35.8007 & 33.969133 \\
\hline $1.59 \mathrm{E}+10$ & 33.309402 & 35.3864325 & 36.0047735 & 36.2026435 & 34.359161 \\
\hline $1.6 \mathrm{E}+10$ & 33.178107 & 35.2059205 & 35.924308 & 35.907975 & 34.1816965 \\
\hline $1.61 \mathrm{E}+10$ & 33.120509 & 36.0293275 & 37.4204185 & 37.4891445 & 35.8618265 \\
\hline $1.62 \mathrm{E}+10$ & 33.136745 & 36.301926 & 37.7792645 & 38.390407 & 36.794499 \\
\hline $1.63 \mathrm{E}+10$ & 32.986218 & 35.8305635 & 36.8466335 & 37.0346005 & 35.0544685 \\
\hline $1.64 \mathrm{E}+10$ & 32.8033015 & 35.247271 & 35.8113695 & 35.594551 & 33.418066 \\
\hline $1.65 \mathrm{E}+10$ & 32.745125 & 35.4748865 & 36.4731785 & 36.856266 & 34.788243 \\
\hline $1.66 \mathrm{E}+10$ & 32.6692655 & 35.8434155 & 37.2380875 & 37.608074 & 35.4696545 \\
\hline $1.67 \mathrm{E}+10$ & 32.5140645 & 35.566296 & 36.6943125 & 36.5508615 & 34.2414025 \\
\hline $1.68 \mathrm{E}+10$ & 32.411453 & 35.7546225 & 37.4739985 & 37.998034 & 36.1437305 \\
\hline $1.69 \mathrm{E}+10$ & 32.398328 & 35.866807 & 37.9701985 & 38.5797265 & 36.7041015 \\
\hline $1.7 \mathrm{E}+10$ & 32.2038175 & 34.608534 & 35.379005 & 35.1033365 & 32.6261985 \\
\hline $1.71 \mathrm{E}+10$ & 32.1005525 & 34.265526 & 35.0285055 & 35.1023585 & 33.0760815 \\
\hline $1.72 \mathrm{E}+10$ & 32.074206 & 35.3069765 & 37.4215415 & 38.053713 & 36.552113 \\
\hline $1.73 \mathrm{E}+10$ & 31.8920115 & 35.2741135 & 37.2710445 & 37.4149355 & 35.4056555 \\
\hline $1.74 \mathrm{E}+10$ & 31.784891 & 35.266147 & 37.3880995 & 37.4736905 & 35.7061875 \\
\hline $1.75 \mathrm{E}+10$ & 31.727939 & 35.930925 & 38.947529 & 39.729374 & 38.195188 \\
\hline $1.76 \mathrm{E}+10$ & 31.5627845 & 35.784214 & 38.6704415 & 39.2020885 & 37.031418 \\
\hline $1.77 \mathrm{E}+10$ & 31.456903 & 34.6041755 & 36.264616 & 36.064735 & 33.78321 \\
\hline $1.79 E+10$ & 31.345821 & 34.808771 & 36.752692 & 37.2368935 & 35.454631 \\
\hline $1.8 \mathrm{E}+10$ & 31.194439 & 34.990013 & 37.441309 & 38.171528 & 36.348347 \\
\hline $1.81 \mathrm{E}+10$ & 31.2185755 & 34.9455045 & 37.3339635 & 37.541423 & 35.7458045 \\
\hline $1.82 \mathrm{E}+10$ & 31.0250125 & 34.548917 & 36.600683 & 36.8805885 & 35.1520575 \\
\hline $1.83 \mathrm{E}+10$ & 30.87671 & 34.800919 & 37.4642225 & 38.171779 & 36.6982745 \\
\hline
\end{tabular}




\begin{tabular}{|r|r|r|r|r|r|}
\hline Frequency & $\mathbf{e}^{\prime} @ 20 \mathrm{C}$ & $\mathbf{e}^{\prime} @ 40 \mathrm{C}$ & $\mathbf{e}^{\prime} @ 60 \mathrm{C}$ & $\mathrm{e}^{\prime} @ 80 \mathrm{C}$ & $\mathrm{e}^{\prime} @ 100 \mathrm{C}$ \\
\hline \hline $1.84 \mathrm{E}+10$ & 30.7235805 & 34.005236 & 36.211172 & 36.6850275 & 35.126089 \\
\hline $1.85 \mathrm{E}+10$ & 30.6954105 & 33.9459365 & 35.9226975 & 36.0695605 & 34.325044 \\
\hline $1.86 \mathrm{E}+10$ & 30.514308 & 33.7390445 & 35.546447 & 36.0526285 & 34.7771035 \\
\hline $1.87 \mathrm{E}+10$ & 30.303802 & 34.077603 & 36.409933 & 37.3229015 & 36.0704265 \\
\hline $1.88 \mathrm{E}+10$ & 30.175467 & 33.553671 & 35.9921505 & 36.422894 & 34.939754 \\
\hline $1.89 \mathrm{E}+10$ & 30.2568135 & 34.0761785 & 36.348832 & 36.472267 & 34.9983855 \\
\hline $1.9 \mathrm{E}+10$ & 30.0982295 & 33.7848545 & 35.654122 & 36.0215905 & 34.3375155 \\
\hline $1.91 \mathrm{E}+10$ & 29.948813 & 32.826258 & 34.12345 & 34.391367 & 32.643946 \\
\hline $1.92 \mathrm{E}+10$ & 29.872373 & 32.174747 & 32.9472165 & 33.042409 & 31.395252 \\
\hline $1.93 \mathrm{E}+10$ & 29.697583 & 32.3036215 & 33.115594 & 33.252554 & 31.6305785 \\
\hline $1.94 \mathrm{E}+10$ & 29.4085835 & 31.8210315 & 32.7852135 & 33.0538325 & 31.5875635 \\
\hline $1.95 \mathrm{E}+10$ & 29.4971795 & 32.4551395 & 33.785778 & 34.329275 & 32.880644 \\
\hline $1.96 \mathrm{E}+10$ & 29.424031 & 32.633071 & 34.1543085 & 34.2625395 & 32.613666 \\
\hline $1.97 \mathrm{E}+10$ & 29.3427325 & 32.9344965 & 34.5617965 & 34.664196 & 33.059955 \\
\hline $1.98 \mathrm{E}+10$ & 29.312059 & 33.27515 & 35.3702585 & 35.694499 & 33.99301 \\
\hline $1.99 \mathrm{E}+10$ & 29.1769495 & 33.2125745 & 35.624734 & 35.8523855 & 34.172358 \\
\hline $2 \mathrm{E}+10$ & 29.015792 & 32.612379 & 34.5908585 & 34.5528005 & 32.7210825 \\
\hline
\end{tabular}


Table 8. Dielectric Loss for Melton Valley Simulant

\begin{tabular}{|c|c|c|c|c|c|}
\hline Frequency & $\mathrm{e}^{\prime \prime} @ 20 \mathrm{C}$ & $\mathrm{e}^{\prime \prime} @ 40 \mathrm{C}$ & $\mathrm{e}^{\prime \prime} @ 60 \mathrm{C}$ & $e^{\prime \prime} @ 80 \mathrm{C}$ & e"@100 C \\
\hline 0000 & 1686.8404 & 35863 & 2978.855237 & 3775.16588 & 4405.00723 \\
\hline 255300000 & 1030.10501 & 1405.74631 & 1817.063458 & 2299.939514 & 2679.994357 \\
\hline 357600000 & 746.562488 & 1019.1009 & 1316.346262 & 1663.768024 & 1936.204733 \\
\hline 459900000 & 584.622715 & 797.772653 & 1029.301126 & 1300.42541 & 1511.995314 \\
\hline 562200000 & 482.318702 & 656.529291 & 846.7118855 & 1068.896242 & 1243.030696 \\
\hline 664500000 & 409.450438 & 558.142783 & 719.328945 & 907.910364 & 1055.781923 \\
\hline 766800000 & 356.338609 & 485.472918 & 625.627072 & 789.357545 & 918.159178 \\
\hline 869100000 & 316.055217 & 430.522257 & 554.580009 & 699.3867065 & 813.6271215 \\
\hline 971400000 & 283.386352 & 385.882473 & 497.058261 & 627.084436 & 729.360017 \\
\hline 1073700000 & 257.750372 & 350.70919 & 451.7419365 & 569.83331 & 662.3650935 \\
\hline 1176000000 & 236.077293 & 320.778571 & 413.2610585 & 520.870636 & 605.078796 \\
\hline 1278300000 & 218.222669 & 296.28909 & 381.6807465 & 481.0013505 & 558.5140445 \\
\hline 1380600000 & 202.585582 & 274.58263 & 353.5718155 & 445.1596695 & 516.677829 \\
\hline 1482900000 & 189.368912 & 256.437301 & 330.270403 & 415.9341705 & 482.693317 \\
\hline 1585200000 & 177.987218 & 240.823834 & 310.2250075 & 390.3865585 & 453.2194785 \\
\hline 1687500000 & 167.228539 & 226.07232 & 291.1883745 & 366.2361715 & 425.3120015 \\
\hline 1789800000 & 158.580295 & 214.11832 & 275.8134015 & 346.635619 & 402.573711 \\
\hline 1892100000 & 150.755495 & 203.263107 & 261.9308295 & 328.878005 & 382.2378935 \\
\hline 1994400000 & 143.921408 & 193.709976 & 249.5822065 & 313.112565 & 363.5191725 \\
\hline 20967 & 137.151784 & 184.226577 & 237.296185 & 297.4595155 & 455 \\
\hline 2199000000 & 131.569379 & 176.431907 & 227.1805465 & 284.630047 & 329.6523205 \\
\hline 2301300000 & 126.392573 & 169.260804 & 218.0608775 & 273.3387005 & 316.3542815 \\
\hline 2403600000 & 121.720588 & 162.829895 & 209.8401105 & 262.9914705 & 303.833414 \\
\hline 2505900000 & 117.400078 & 156.673329 & 201.6713395 & 252.540526 & 291.205069 \\
\hline 2608200000 & 113.155535 & 150.655296 & 194.0287145 & 243.045358 & 279.8746085 \\
\hline 2710500000 & 109.466964 & 145.387635 & 187.1172695 & 234.2682475 & 269.793242 \\
\hline 2812800000 & 106.027852 & 140.53523 & 180.7277665 & 226.227977 & 260.6682885 \\
\hline 2915100000 & 102.867091 & 136.066732 & 175.0459085 & 219.051629 & 252.6681485 \\
\hline 3017400000 & 99.989674 & 131.718523 & 169.7069155 & 212.2676885 & 245.0173555 \\
\hline 3119700000 & 97.284159 & 127.933556 & 164.7064995 & 205.917241 & 237.7843205 \\
\hline 3222000000 & 94.6948985 & 124.223588 & 159.864766 & 199.6985065 & 230.7426515 \\
\hline 3324300000 & 92.2600955 & 120.677021 & 155.139304 & 193.5431885 & 223.4899345 \\
\hline 3426600000 & 89.9637965 & 117.308697 & 150.5990505 & 187.833362 & 216.8143515 \\
\hline 3528900000 & 87.8377995 & 114.21225 & 146.4641055 & 182.531341 & 210.5092695 \\
\hline 3631200000 & 85.8389965 & 111.453333 & 142.9203025 & 178.1387525 & 205.3923235 \\
\hline 3733500000 & 84.0681125 & 108.881135 & 139.4375455 & 173.656058 & 200.062063 \\
\hline 3835800000 & 82.398221 & 106.455639 & 136.2044775 & 169.4276325 & 195.1039595 \\
\hline 3938100000 & 80.765299 & 104.101506 & 133.0038435 & 165.3607825 & 190.3232865 \\
\hline 4040400000 & 79.259279 & 101.851416 & 130.06456 & 161.693469 & 186.094849 \\
\hline 4142700000 & 77.944971 & 99.91847 & 127.3536165 & 158.264846 & 181.9274865 \\
\hline 4245000000 & 76.6730665 & 97.999394 & 124.668971 & 154.9782795 & 177.9806295 \\
\hline 4347300000 & 75.409305 & 96.2222465 & 122.226436 & 152.155503 & 174.6150465 \\
\hline 4449600000 & 74.189159 & 94.446415 & 119.797908 & 149.100075 & 171.0278825 \\
\hline
\end{tabular}




\begin{tabular}{|c|c|c|c|c|c|}
\hline Frequency & $\mathrm{e}^{\prime \prime} @ 20 \mathrm{C}$ & $e^{\prime \prime} @ 40 \mathrm{C}$ & $e^{\prime \prime 60 C}$ & $e^{\prime \prime @ 80 C ~}$ & $\mathrm{e}^{\prime \prime} @ 100 \mathrm{C}$ \\
\hline 0000 & 4435 & 92.779998 & 117.3979935 & 174095 & 167.777293 \\
\hline 4654200000 & 71.9888275 & 91.10084 & 115.0610585 & 143.1627675 & 164.905399 \\
\hline 4756500000 & 70.8243555 & 89.410038 & 112.843687 & 140.4289485 & 162.1326285 \\
\hline 4858800000 & 69.86246 & 87.963301 & 110.835143 & 137.895725 & 159.408705 \\
\hline 4961100000 & 68.921881 & 86.539119 & 108.749491 & 135.273793 & 156.4310775 \\
\hline 5063400000 & 68.02404 & 85.2122665 & 106.903546 & 132.8919245 & 153.6181415 \\
\hline 5165700000 & 67.2235045 & 84.050941 & 105.3321785 & 130.874958 & 151.3135775 \\
\hline 5268000000 & 66.25665 & 82.5891235 & 103.279399 & 128.01114 & 147.799933 \\
\hline 5370300000 & 65.477248 & 81.472803 & 101.7183825 & 125.91997 & 145.382287 \\
\hline 5472600000 & 64.700513 & 80.33644 & 100.2072285 & 123.9978395 & 143.24047 \\
\hline 5574900000 & 63.8947175 & 79.213252 & 98.702266 & 122.0021635 & 140.8459515 \\
\hline 5677200000 & 63.200133 & 78.133749 & 97.14855 & 120.048954 & 138.5093985 \\
\hline 5779500000 & 62.47283 & 77.044293 & 95.5855795 & 117.8252285 & 135.846556 \\
\hline 5881800000 & 61.8066935 & 76.0153755 & 94.195693 & 116.044467 & 133.899374 \\
\hline 5984100000 & 61.177259 & 75.0291705 & 92.8243445 & 114.295311 & 131.849351 \\
\hline 6086400000 & 60.537315 & 73.988614 & 91.3372025 & 112.299594 & 129.4707725 \\
\hline 6188700000 & 59.9668285 & 73.050426 & 89.843621 & 110.441225 & 127.3616455 \\
\hline 6291000000 & 59.405691 & 72.0625765 & 88.4209645 & 108.666996 & 125.403321 \\
\hline 6393300000 & 58.8861125 & 71.1852505 & 87.13079 & 106.8176485 & 123.200691 \\
\hline 6495600000 & 58.312611 & 70.2491175 & 85.7437705 & 105.0326795 & 121.234189 \\
\hline 6597900000 & 57.740205 & 69.492633 & 84.793496 & 103.8009995 & 119.824295 \\
\hline 6700200000 & 57.2368845 & 68.721078 & 83.7488495 & 102.3712725 & 118.292597 \\
\hline 6802500000 & 56.751368 & 68.002192 & 82.66722 & 101.004803 & 116.7118055 \\
\hline 6904800000 & 56.2525955 & 67.200376 & 81.4119625 & 99.31981 & 114.8478465 \\
\hline 7007100000 & 55.8164835 & 66.450653 & 80.298848 & 97.9485675 & 113.2746125 \\
\hline 7109400000 & 55.381916 & 65.77773335 & 79.339037 & 96.6255545 & 111.6913175 \\
\hline 7211700000 & 54.891119 & 65.0496115 & 78.3841965 & 95.3043705 & 110.1745705 \\
\hline 7314000000 & 54.472292 & 64.4588475 & 77.5383815 & 94.206479 & 108.979958 \\
\hline 7416300000 & 53.997141 & 63.775787 & 76.579526 & 92.934423 & 107.4708775 \\
\hline 7518600000 & 53.5179985 & 63.0289255 & 75.552665 & 91.605004 & 105.8887055 \\
\hline 7620900000 & 53.117347 & 62.3494685 & 74.5175635 & 90.2657865 & 104.3390565 \\
\hline 7723200000 & 52.755114 & 61.7580725 & 73.6930725 & 89.2536405 & 103.1389925 \\
\hline 7825500000 & 52.3605885 & 61.3051215 & 73.1112695 & 88.4586625 & 102.197704 \\
\hline 7927800000 & 51.9963145 & 60.8167235 & 72.5005285 & 87.5729 & 101.0712835 \\
\hline 8030100000 & 51.594834 & 60.240029 & 71.656564 & 86.5181285 & 99.8782425 \\
\hline 8132400000 & 51.2272375 & 59.8778895 & 71.0953815 & 85.742544 & 98.768181 \\
\hline 8234700000 & 50.778203 & 59.153946 & 70.021773 & 84.2501065 & 96.955623 \\
\hline 8337000000 & 50.418113 & 58.545501 & 69.1515825 & 83.2394315 & 95.7568485 \\
\hline 8439300000 & 50.030117 & 57.956646 & 68.2616635 & 82.1125715 & 94.2819425 \\
\hline 8541600000 & 49.6942885 & 57.4090175 & 67.4332355 & 80.9354725 & 92.8567625 \\
\hline 8643900000 & 49.352759 & 56.9552455 & 66.8571445 & 80.326297 & 92.0550455 \\
\hline 8746200000 & 49.0163345 & 56.5049775 & 66.2764185 & 79.4352235 & 90.9292005 \\
\hline 8848500000 & 48.7293365 & 56.0720955 & 65.609439 & 78.600002 & 89.8461535 \\
\hline 8950800000 & 48.4126095 & 55.653982 & 64.968794 & 77.756876 & 88.607179 \\
\hline 9053100000 & 48.0528635 & 54.942758 & 63.9350725 & 76.340285 & 86.919176 \\
\hline
\end{tabular}




\begin{tabular}{|c|c|c|c|c|c|}
\hline ncy & "a $20 \mathrm{C}$ & "10 $40 \mathrm{C}$ & e"@60C & @ 80C & $\mathrm{e}^{\prime \prime @ 1}$ \\
\hline 5400000 & 17555 & 38356 & $\overline{63 .}$ & 75.59373 & 233947 \\
\hline 9257700000 & 47.4216395 & 54.029709 & 62.5705645 & 74.588209 & 84.792367 \\
\hline 9360000000 & 47.1978145 & 53.6413345 & 62.0543644 & 73.857678 & $\overline{8} \overline{3} . \overline{94674}$ \\
\hline 9462300000 & 46.9256015 & 53.380457 & 61.8352475 & 73.7063215 & 83.7612845 \\
\hline 9564600000 & 46.6772535 & 52.903013 & 60.9927705 & 72.560945 & 82.195014 \\
\hline 9666900000 & 46.3430485 & 52.214028 & 59.9293415 & 71.231194 & 80.5081635 \\
\hline 9769200000 & 46.1181995 & 51.791312 & 59.080294 & 70.37654 & 79.438207 \\
\hline 9871500000 & 45.8291525 & 51.111382 & 58.147176 & 69.118954 & 77.974585 \\
\hline 9973800000 & 45.5892525 & 50.735981 & 57.5023255 & 68.3719375 & 77.137473 \\
\hline $1.0076 \mathrm{E}+10$ & 45.3178745 & 50.6015285 & 57.481966 & 68.229676 & 76.9596095 \\
\hline $1.0178 \mathrm{E}+10$ & 45.1238705 & 50.323136 & 57.2266625 & 67.757069 & 76.3518 \\
\hline $1.0281 \mathrm{E}+10$ & 44.8857615 & 49.821605 & 56.5300355 & 66.9200035 & 75.4464245 \\
\hline $1.0383 \mathrm{E}+10$ & 44.6498985 & 49.3729075 & 55.7579925 & 65.911532 & 74.1829755 \\
\hline $1.0485 \mathrm{E}+10$ & 44.429739 & 48.950563 & 55.1002565 & 65.0515075 & 73.1357985 \\
\hline $1.0588 \mathrm{E}+10$ & 44.249264 & 48.8383205 & 55.172668 & 65.1640925 & 73.3693245 \\
\hline $1.069 \mathrm{E}+10$ & 44.0485925 & 49.035077 & 55.8015405 & 65.779968 & 73.9258445 \\
\hline $1.0792 \mathrm{E}+10$ & 43.866948 & 49.0302145 & 55.955011 & 65.7920725 & 73.850115 \\
\hline $1.0895 \mathrm{E}+10$ & 43.699209 & 48.826847 & 55.58115 & 65.1822195 & 73.008824 \\
\hline $1.0997 \mathrm{E}+10$ & 43.4631815 & 48.116916 & 54.4659425 & 63.779891 & 71.347075 \\
\hline $1.1099 \mathrm{E}+10$ & 43.2899645 & 47.5059105 & 53.477937 & 62.679588 & 70.142272 \\
\hline $1.1201 \mathrm{E}+10$ & 43.0670195 & 47.128478 & 52.9210285 & 61.9610555 & 69.3729725 \\
\hline $1.1304 \mathrm{E}+10$ & 42.8852745 & 47.0677725 & 53.039871 & 61.974201 & 69.229228 \\
\hline $1.1406 \mathrm{E}+10$ & 42.725993 & 47.090748 & 53.2467515 & 62.032774 & 69.184637 \\
\hline $1.1508 \mathrm{E}+10$ & 42.547515 & 46.7593515 & 52.6940195 & 61.238299 & 68.2852455 \\
\hline $1.1611 \mathrm{E}+10$ & 42.4302845 & 46.3144135 & 51.7736825 & 60.1754905 & 67.189252 \\
\hline $1.1713 \mathrm{E}+10$ & 42.307771 & 45.8555015 & 50.8258605 & 59.185938 & 66.2033585 \\
\hline $1.1815 \mathrm{E}+10$ & 42.199834 & 45.437789 & 50.1775125 & 58.3954545 & 65.434482 \\
\hline $1.1918 \mathrm{E}+10$ & 41.914635 & 45.323617 & 50.2663865 & 58.3250515 & 65.1764965 \\
\hline $1.202 \mathrm{E}+10$ & 41.7572905 & 45.3789 & 50.54529 & 58.537571 & 65.376399 \\
\hline $1.2122 \mathrm{E}+10$ & 41.579003 & 45.3637135 & 50.722762 & 58.458194 & 65.063183 \\
\hline $1.2224 \mathrm{E}+10$ & 41.4060295 & 44.851593 & 49.8314265 & 57.330056 & 63.6790175 \\
\hline $1.2327 \mathrm{E}+10$ & 41.2229505 & 43.9242335 & 47.887402 & 55.22695 & 61.6340585 \\
\hline $1.2429 \mathrm{E}+10$ & 41.176539 & 43.606845 & 47.133411 & 54.675051 & 61.290897 \\
\hline $1.2531 \mathrm{E}+10$ & 41.0073905 & 43.516818 & 47.239397 & 54.684889 & 61.229449 \\
\hline $1.2634 \mathrm{E}+10$ & 40.877285 & 43.567395 & 47.5333235 & 54.7155185 & 61.4611975 \\
\hline $1.2736 \mathrm{E}+10$ & 40.712007 & 43.703643 & 48.07816 & 55.137146 & 61.7172275 \\
\hline $1.2838 \mathrm{E}+10$ & 40.5510085 & 43.4284595 & 47.7177465 & 54.576931 & 61.077768 \\
\hline $1.2941 \mathrm{E}+10$ & 40.4596745 & 43.1291755 & 47.116913 & 53.837304 & 60.358374 \\
\hline $1.3043 \mathrm{E}+10$ & 40.3404805 & 42.672423 & 46.1970435 & 52.766949 & 59.30986 \\
\hline $1.3145 \mathrm{E}+10$ & 40.274495 & 42.538128 & 45.7802775 & 52.51485 & 59.1714805 \\
\hline $1.3247 \mathrm{E}+10$ & 40.13522 & 42.5144105 & 45.826104 & 52.4081585 & 58.9184535 \\
\hline $1.335 \mathrm{E}+10$ & 40.0135065 & 42.640444 & 46.306938 & 52.570462 & 58.8790095 \\
\hline $1.3452 \mathrm{E}+10$ & 39.880635 & 42.521591 & 46.146235 & 52.299595 & 58.4815285 \\
\hline $1.3554 \mathrm{E}+10$ & 39.7544475 & 42.302852 & 45.7800285 & 51.9364985 & 58.146453 \\
\hline $1.3657 \mathrm{E}+10$ & 39.696255 & 42.093755 & 45.4749465 & 51.5984485 & 57.7091125 \\
\hline
\end{tabular}




\begin{tabular}{|c|c|c|c|c|c|}
\hline Frequency & $\mathrm{e}^{\prime \prime} @ 20 \mathrm{C}$ & $\mathrm{e}^{\prime \prime} @ 40 \mathrm{C}$ & $e^{\prime \prime @ 60 ~ C ~}$ & $e^{\prime \prime @ 80 ~ C ~}$ & e"@100 C \\
\hline $1.3759 \mathrm{E}+10$ & 39.5473175 & 41.548833 & 44.545119 & 50.5476215 & 56.629771 \\
\hline $1.3861 \mathrm{E}+10$ & 39.437743 & 41.496254 & 44.44537 & 50.390723 & 56.4558895 \\
\hline $1.3964 \mathrm{E}+10$ & 39.3494945 & 41.5695015 & 44.6218205 & 50.701304 & 56.735248 \\
\hline $1.4066 \mathrm{E}+10$ & 39.20688 & 41.600588 & 44.917681 & 50.872926 & 56.7680765 \\
\hline $1.4168 \mathrm{E}+10$ & 39.071776 & 41.5473785 & 45.0347655 & 50.72254 & 56.4907235 \\
\hline $1.427 \mathrm{E}+10$ & 39.014464 & 41.523699 & 45.037794 & 50.903111 & 56.6710535 \\
\hline $1.4373 \mathrm{E}+10$ & 38.9547985 & 41.432133 & 44.888724 & 50.9186355 & 56.7580105 \\
\hline $1.4475 \mathrm{E}+10$ & 38.84014 & 40.960103 & 44.105589 & 49.810568 & 55.4614495 \\
\hline $1.4577 \mathrm{E}+10$ & 38.6862425 & 40.2557855 & 42.7528145 & 48.135537 & 53.54083 \\
\hline $1.468 \mathrm{E}+10$ & 38.6849285 & 40.3412195 & 42.6376625 & 48.159772 & 53.6491735 \\
\hline $1.4782 \mathrm{E}+10$ & 38.568468 & 40.32952 & 42.80468 & 48.302962 & 53.663413 \\
\hline $1.4884 \mathrm{E}+10$ & 38.479311 & 39.9653095 & 42.1986815 & 47.2797365 & 52.4133985 \\
\hline $1.4987 \mathrm{E}+10$ & 38.38024 & 39.6243335 & 41.688659 & 46.580745 & 51.939993 \\
\hline $1.5089 \mathrm{E}+10$ & 38.340339 & 39.3853165 & 41.185956 & 46.3246805 & 51.587228 \\
\hline $1.5191 \mathrm{E}+10$ & 38.293062 & 38.819218 & 39.93705 & 44.9388865 & 50.1843265 \\
\hline $1.5293 \mathrm{E}+10$ & 38.0766655 & 37.9483045 & 38.4018165 & 42.863282 & 47.7783655 \\
\hline $1.5396 \mathrm{E}+10$ & 38.0206595 & 38.220589 & 38.8499715 & 43.3046105 & 48.043749 \\
\hline $1.5498 \mathrm{E}+10$ & 38.022621 & 38.870092 & 40.1101025 & 44.5020455 & 49.259868 \\
\hline $1.56 \mathrm{E}+10$ & 38.033785 & 39.480637 & 41.257279 & 45.6202985 & 50.128123 \\
\hline $1.5703 \mathrm{E}+10$ & 37.957574 & 39.192448 & 40.664144 & 45.0208965 & 49.563779 \\
\hline $1.5805 \mathrm{E}+10$ & 37.8638945 & 38.437049 & 39.191203 & 43.639771 & 48.175839 \\
\hline $1.5907 \mathrm{E}+10$ & 37.8127505 & 38.069257 & 38.5903685 & \begin{tabular}{|c|}
43.278885 \\
\end{tabular} & 47.8797715 \\
\hline $\mathrm{E}+10$ & 37.6513 & 37.226087 & 37.30 & 41.3203475 & 3885 \\
\hline $1.6112 \mathrm{E}+10$ & 37.6074705 & 37.7463765 & 38.2050915 & 41.7900245 & 46.0050305 \\
\hline $1.6214 \mathrm{E}+10$ & 37.5590035 & 38.5140055 & 39.7959635 & 43.8387865 & 48.3213115 \\
\hline $1.6316 \mathrm{E}+10$ & 37.520686 & 39.071884 & 40.786457 & 45.504856 & 50.2230885 \\
\hline $1.6419 \mathrm{E}+10$ & 37.419724 & 38.6273495 & 39.869435 & 44.137497 & 48.416701 \\
\hline $1.6521 \mathrm{E}+10$ & 37.3263825 & 38.0565275 & 38.955575 & 43.0192135 & 47.4503975 \\
\hline $1.6623 \mathrm{E}+10$ & 37.327448 & 38.0841585 & 38.983224 & 43.5365255 & 48.2696015 \\
\hline $1.6726 \mathrm{E}+10$ & 37.2349285 & 37.9740965 & 38.9907425 & 42.9073295 & 47.3120365 \\
\hline $1.6828 \mathrm{E}+10$ & 37.156741 & 37.834282 & 38.775445 & 42.049043 & 46.5778055 \\
\hline $1.693 \mathrm{E}+10$ & 37.028155 & 38.571141 & 40.47077 & 44.728922 & 49.775089 \\
\hline $1.7033 \mathrm{E}+10$ & 36.997882 & 38.4061085 & 40.0925455 & 44.342272 & 48.9526865 \\
\hline $1.7135 \mathrm{E}+10$ & 36.922015 & 37.1777465 & 37.5360315 & 40.920894 & 45.2219065 \\
\hline $1.7237 \mathrm{E}+10$ & 36.738983 & 36.5159325 & 36.2790125 & 40.2683745 & 44.8358365 \\
\hline $1.7339 \mathrm{E}+10$ & 36.7925475 & 37.021576 & 37.390164 & 41.727385 & 46.4858755 \\
\hline $1.7442 \mathrm{E}+10$ & 36.6571035 & 36.425108 & 36.590812 & 39.946544 & 44.3819635 \\
\hline $1.7544 \mathrm{E}+10$ & 36.533629 & 36.949919 & 37.3856165 & 41.2538855 & 46.0574 \\
\hline $1.7646 \mathrm{E}+10$ & 36.4858415 & 37.866231 & 39.842977 & 44.5012215 & 49.67098 \\
\hline $1.7749 \mathrm{E}+10$ & 36.4276095 & 37.582617 & 39.194319 & 43.0702845 & 7.5526855 \\
\hline $1.7851 \mathrm{E}+10$ & 36.3535025 & 36.5170265 & 36.7862135 & 40.3530735 & 44.77441 \\
\hline $1.7953 \mathrm{E}+10$ & 36.355063 & 36.5663385 & 37.0050255 & 40.957997 & 45.6223915 \\
\hline $1.8056 \mathrm{E}+10$ & 36.3051595 & 36.183216 & 36.225447 & 40.1128845 & 44.634502 \\
\hline $1.8158 \mathrm{E}+10$ & 36.1935755 & 35.7498415 & 35.5278105 & 38.639998 & 42.8260125 \\
\hline $1.826 \mathrm{E}+10$ & 36.124892 & 35.812143 & 35.673659 & 38.8834635 & 43.2868265 \\
\hline
\end{tabular}




\begin{tabular}{|r|r|r|r|r|r|}
\hline Frequency & $e^{\prime \prime} @ 20 \mathrm{C}$ & $\mathrm{e}$ " @ 40 C & \multicolumn{1}{|c|}{$\mathrm{e}^{\prime \prime} @ 60 \mathrm{C}$} & $\mathrm{e}$ " @ 80 C & $\mathrm{e}^{\prime \prime} @ 100 \mathrm{C}$ \\
\hline \hline $1.8362 \mathrm{E}+10$ & 35.873687 & 35.30067 & 35.0484655 & 38.639238 & 42.8147335 \\
\hline $1.8465 \mathrm{E}+10$ & 35.938487 & 35.3899325 & 34.8475955 & 38.238361 & 42.3747495 \\
\hline $1.8567 \mathrm{E}+10$ & 35.8841105 & 34.377546 & 32.962928 & 35.723453 & 39.638594 \\
\hline $1.8669 \mathrm{E}+10$ & 35.981172 & 34.438728 & 32.18468 & 35.3022855 & 39.5014785 \\
\hline $1.8772 \mathrm{E}+10$ & 35.8998765 & 34.956308 & 33.610333 & 36.7067755 & 40.9213715 \\
\hline $1.8874 \mathrm{E}+10$ & 35.8252265 & 35.2602195 & 34.7061955 & 37.499876 & 41.593233 \\
\hline $1.8976 \mathrm{E}+10$ & 35.771363 & 35.9259045 & 35.961681 & 38.6958595 & 42.8102455 \\
\hline $1.9079 \mathrm{E}+10$ & 35.68458 & 36.098316 & 36.2590595 & 39.216022 & 43.097517 \\
\hline $1.9181 \mathrm{E}+10$ & 35.7609275 & 35.632506 & 35.191888 & 38.1513825 & 41.71753 \\
\hline $1.9283 \mathrm{E}+10$ & 35.7781495 & 35.2756745 & 34.380361 & 37.0945725 & 40.682947 \\
\hline $1.9385 \mathrm{E}+10$ & 35.677617 & 34.9658825 & 33.4441665 & 36.0481675 & 39.4398415 \\
\hline $1.9488 \mathrm{E}+10$ & 35.6956815 & 34.7843505 & 33.1480705 & 36.0464065 & 39.5988565 \\
\hline $1.959 \mathrm{E}+10$ & 35.553893 & 35.1509765 & 34.6668295 & 37.5424865 & 41.2146265 \\
\hline $1.9692 \mathrm{E}+10$ & 35.4270055 & 34.9861805 & 34.568241 & 37.344606 & 40.9323715 \\
\hline $1.9795 \mathrm{E}+10$ & 35.387861 & 35.4308015 & 35.1714915 & 37.9962145 & 41.754264 \\
\hline $1.9897 \mathrm{E}+10$ & 35.3616915 & 35.576539 & 35.5061 & 38.538672 & 42.223024 \\
\hline $1.9999 \mathrm{E}+10$ & 35.2573945 & 35.449758 & 35.6541845 & 38.4532615 & 41.792081 \\
\hline
\end{tabular}


Table 9. Loss Tangent for Melton Valley Simulant

\begin{tabular}{|c|c|c|c|c|c|}
\hline ncy & $e^{\prime \prime / / e^{\prime} @ 20 C}$ & $e^{\prime \prime / /} e^{\prime} @ 40 C$ & $e^{\prime \prime / / e^{\prime} @ 60 C}$ & $e^{\prime \prime} / e^{\prime} @ 80 C$ & $e^{\prime \prime} / e^{\prime} @ 100 \mathrm{C}$ \\
\hline $1.53 \mathrm{E}+08$ & 7.454507306 & 7.958042794 & 8.491744972 & 9.388211594 & 10.48011974 \\
\hline $2.55 \mathrm{E}+08$ & 7.943549001 & 8.899324324 & 9.776829953 & 11.04179913 & 12.69695639 \\
\hline $3.58 \mathrm{E}+08$ & 7.576017916 & 8.853940633 & 9.978136698 & 11.44860039 & 13.39190399 \\
\hline $4.6 \mathrm{E}+08$ & 6.762520983 & 8.121894461 & 9.272671601 & 10.68169936 & 12.44033819 \\
\hline $5.62 \mathrm{E}+08$ & 6.306235982 & 7.792535463 & 9.09181047 & 10.62145769 & 12.52504357 \\
\hline $6.65 \mathrm{E}+08$ & 5.625795086 & 7.044348988 & 8.275172845 & 9.640661981 & 11.29267165 \\
\hline $7.67 \mathrm{E}+08$ & 5.163511345 & 6.530077199 & 7.725035697 & 9.014911539 & 10.53952119 \\
\hline $8.69 E+08$ & 4.791144367 & 6.129724877 & 7.311841773 & 8.572325805 & 10.04740047 \\
\hline $9.71 \mathrm{E}+08$ & 4.421148525 & 5.709737863 & 6.84556443 & 8.038099718 & 9.398861979 \\
\hline $1.07 \mathrm{E}+09$ & 4.119179625 & 5.376165683 & 6.503480778 & 7.682431459 & 9.022443715 \\
\hline $1.18 \mathrm{E}+09$ & 3.848666554 & 5.052129561 & 6.138170061 & 7.28485321 & 8.55994832 \\
\hline $1.28 \mathrm{E}+09$ & 3.652495758 & 4.834822708 & 5.930697009 & 7.08127682 & 8.356729885 \\
\hline $1.38 \mathrm{E}+09$ & 3.413599662 & 4.520967912 & 5.545875536 & 6.615907509 & 7.778453177 \\
\hline $1.48 \mathrm{E}+09$ & 3.247044428 & 4.307475251 & 5.300302578 & 6.3180799 & 7.423791914 \\
\hline $1.59 \mathrm{E}+09$ & 3.074934238 & 4.083508497 & 5.033502544 & 6.027232829 & 7.083848079 \\
\hline $1.69 \mathrm{E}+09$ & 2.914302312 & 3.879424154 & 4.792194167 & 5.73837965 & 6.751719874 \\
\hline $1.79 \mathrm{E}+09$ & 2.774173714 & 3.69979884 & 4.581630789 & 5.474369086 & 6.427145524 \\
\hline $1.89 \mathrm{E}+09$ & 2.660498861 & 3.559609158 & 4.439307735 & 5.344598395 & 6.317630743 \\
\hline $1.99 \mathrm{E}+09$ & 2.553610557 & 3.416790225 & 4.265806163 & 5.142079133 & 6.06937407 \\
\hline $2.1 \mathrm{E}+09$ & 2.449057831 & 3.274585029 & 4.101705843 & 4.941993789 & 5.832163497 \\
\hline $2.2 \mathrm{E}+09$ & 2.370986401 & 3.167701225 & 3.967961772 & 4.789260049 & 5.647501402 \\
\hline $2.3 \mathrm{E}+09$ & 2.2875144 & 3.048697003 & 3.809712884 & 4.597655859 & 5.405713681 \\
\hline $2.4 \mathrm{E}+09$ & 2.210324468 & 2.950049166 & 3.697169953 & 4.477976389 & 5.27540569 \\
\hline $2.51 \mathrm{E}+09$ & 2.14633621 & 2.868105606 & 3.603237105 & 4.371608675 & 5.155080224 \\
\hline $2.61 \mathrm{E}+09$ & 2.081108367 & 2.778076892 & 3.495746536 & 4.245942547 & 5.009961778 \\
\hline $2.71 E+09$ & 2.027032638 & 2.699600606 & 3.401800404 & 4.136776838 & 4.888328467 \\
\hline $2.81 \mathrm{E}+09$ & 1.971569826 & 2.615985319 & 3.292632689 & 4.002147323 & 4.731712843 \\
\hline $2.92 \mathrm{E}+09$ & 1.92107069 & 2.541329857 & 3.206558064 & 3.904727967 & 4.635138629 \\
\hline $3.02 \mathrm{E}+09$ & 1.875105561 & 2.462224433 & 3.124555734 & 3.805271359 & 4.520850466 \\
\hline $3.12 \mathrm{E}+09$ & 1.835190109 & 2.410250147 & 3.059865438 & 3.735640176 & 4.454132467 \\
\hline $3.22 \mathrm{E}+09$ & 1.794111923 & 2.353599707 & 2.993641062 & 3.655492339 & 4.366793773 \\
\hline $3.32 \mathrm{E}+09$ & 1.754548779 & 2.300942522 & 2.932301873 & 3.591137734 & 4.299952336 \\
\hline $3.43 \mathrm{E}+09$ & 1.717901069 & 2.243894096 & 2.854681359 & 3.491210436 & 4.171173545 \\
\hline $3.53 \mathrm{E}+09$ & 1.684116202 & 2.191483336 & 2.78179361 & 3.392526839 & 4.040485136 \\
\hline $3.63 E+09$ & 1.648720817 & 2.139232943 & 2.71644979 & 3.313868036 & 3.944281116 \\
\hline $3.73 E+09$ & 1.618976668 & 2.099518199 & 2.665742642 & 3.26088931 & 3.88433965 \\
\hline $3.84 \mathrm{E}+09$ & 1.591012382 & 2.058745875 & 2.610987473 & 3.193180669 & 3.805962425 \\
\hline $3.94 \mathrm{E}+09$ & 1.563778327 & 2.018314319 & 2.556191656 & 3.118140468 & 3.710161585 \\
\hline $4.04 \mathrm{E}+09$ & 1.539297894 & 1.978428715 & 2.504683552 & 3.060732149 & 3.640001674 \\
\hline $4.14 \mathrm{E}+09$ & 1.51936042 & 1.948451039 & 2.466380021 & 3.020825546 & 3.593325039 \\
\hline $4.25 \mathrm{E}+09$ & 1.500169317 & 1.91822216 & 2.421946341 & 2.962444747 & 3.517976772 \\
\hline $4.35 E+09$ & 1.481796024 & 1.892267563 & 2.38578108 & 2.925068904 & 3.468967613 \\
\hline $4.45 \mathrm{E}+09$ & 1.466030619 & 1.872207317 & 2.364466003 & 2.913760074 & 3.467175735 \\
\hline
\end{tabular}




\begin{tabular}{|c|c|c|c|c|c|}
\hline requency & $0 \mathrm{C}$ & $40 \mathrm{C}$ & $\vec{C}$ & $80 \mathrm{C}$ & C \\
\hline $4.55 E+09$ & 1.451193969 & 1.851077962 & 2.338636824 & 2.888899272 & 3.453005952 \\
\hline $4.65 E+09$ & 1.435354309 & 1.824611544 & 2.30029445 & 2.837475495 & 3.39669505 \\
\hline $4.76 \mathrm{E}+09$ & 1.419542576 & 1.800535853 & 2.272626702 & 2.812969565 & 3.387812687 \\
\hline $4.86 \mathrm{E}+09$ & 1.404616526 & 1.776346462 & 2.241472 & 2.777523874 & 3.353795135 \\
\hline $4.96 \mathrm{E}+09$ & 1.393732665 & 1.75794711 & 2.211222357 & 2.741620563 & 3.312854981 \\
\hline $5.06 \mathrm{E}+09$ & 1.383338293 & 1.739108171 & 2.1815786 & 2.705059579 & 3.269993904 \\
\hline $5.17 \mathrm{E}+09$ & 1.372415517 & 1.722965765 & 2.165260952 & 2.69031714 & 3.268824083 \\
\hline $5.27 \mathrm{E}+09$ & 1.359946725 & 1.702330221 & 2.138036922 & 2.651123516 & 3.217225149 \\
\hline $5.37 E+09$ & 1.349026876 & 1.684563193 & 2.112760597 & 2.618209309 & 3.176933036 \\
\hline $5.47 \mathrm{E}+09$ & 1.339064325 & 1.665786305 & 2.083750789 & 2.57941458 & 3.13610009 \\
\hline $5.57 \mathrm{E}+09$ & 1.328198155 & 1.650237131 & 2.065970188 & 2.566793915 & 3.13162418 \\
\hline $5.68 \mathrm{E}+09$ & 1.320548645 & 1.635987147 & 2.044320628 & 2.540455256 & 3.102390346 \\
\hline $5.78 \mathrm{E}+09$ & 1.312881112 & 1.624109537 & 2.025780838 & 2.519002472 & 3.09018379 \\
\hline $5.88 \mathrm{E}+09$ & 1.304389521 & 1.605967148 & 1.996791696 & 2.473126342 & 3.030490087 \\
\hline $5.98 \mathrm{E}+09$ & 1.295523795 & 1.589681413 & 1.974669098 & 2.450937446 & 3.00766868 \\
\hline $6.09 E+09$ & 1.287583803 & 1.573468322 & 1.950890238 & 2.414640645 & 2.961605339 \\
\hline $6.19 \mathrm{E}+09$ & 1.280795442 & 1.560335932 & 1.926225451 & 2.38235428 & 2.924540113 \\
\hline $6.29 \mathrm{E}+09$ & 1.275209411 & 1.544670461 & 1.89705244 & 2.346942684 & 2.883198447 \\
\hline $6.39 \mathrm{E}+09$ & 1.272611869 & 1.53680436 & 1.884995015 & 2.334808723 & 2.881290761 \\
\hline $6.5 \mathrm{E}+09$ & 1.266141188 & 1.521838813 & 1.857325758 & 2.29186266 & 2.820295614 \\
\hline $6.6 \mathrm{E}+09$ & 1.260803617 & 1.511494733 & 1.842582032 & 2.27499414 & 2.805487057 \\
\hline $6.7 \mathrm{E}+09$ & 1.255187844 & 1.501104727 & 1.83042277 & 2.254548498 & 2.785668574 \\
\hline $6.8 \mathrm{E}+09$ & 1.249115389 & 1.493056561 & 1.822602756 & 2.248215344 & 2.783815492 \\
\hline $6.9 \mathrm{E}+09$ & 1.244321329 & 1.485580784 & 1.809019106 & 2.231320961 & 2.77248365 \\
\hline $7.01 \mathrm{E}+09$ & 1.240986021 & 1.476790263 & 1.794257295 & 2.214041407 & 2.756808915 \\
\hline $7.11 \mathrm{E}+09$ & 1.239915687 & 1.474516796 & 1.792361706 & 2.215403012 & 2.766972129 \\
\hline $7.21 \mathrm{E}+09$ & 1.233541681 & 1.461445386 & 1.775823033 & 2.188452769 & 2.727969521 \\
\hline $7.31 \mathrm{E}+09$ & 1.229866708 & 1.45296844 & 1.762629991 & 2.170629165 & 2.710955457 \\
\hline $7.42 \mathrm{E}+09$ & 1.226329461 & 1.448143496 & 1.754919626 & 2.161601529 & 2.707914453 \\
\hline $7.52 \mathrm{E}+09$ & 1.221769335 & 1.438758458 & 1.742359424 & 2.149559166 & 2.694338314 \\
\hline $7.62 \mathrm{E}+09$ & 1.218194161 & 1.428260901 & 1.724397141 & 2.126148248 & 2.660527946 \\
\hline $7.72 \mathrm{E}+09$ & 1.214702867 & 1.415818504 & 1.701270538 & 2.09057634 & 2.612480624 \\
\hline $7.83 \mathrm{E}+09$ & 1.21088248 & 1.408748991 & 1.689680223 & 2.078993487 & 2.600700826 \\
\hline $7.93 \mathrm{E}+09$ & 1.207080407 & 1.401371605 & 1.67970808 & 2.06407434 & 2.581775322 \\
\hline $8.03 \mathrm{E}+09$ & 1.202749719 & 1.390954914 & 1.662826848 & 2.038406787 & 2.548713586 \\
\hline $8.13 E+09$ & 1.202008349 & 1.395713747 & 1.670633363 & 2.061534325 & 2.596354803 \\
\hline $8.23 \mathrm{E}+09$ & 1.197369227 & 1.388771555 & 1.658609134 & 2.040531257 & 2.562756482 \\
\hline $8.34 \mathrm{E}+09$ & 1.195292854 & 1.381367474 & 1.647458 & 2.030111275 & 2.550058387 \\
\hline $8.44 \mathrm{E}+09$ & 1.190830285 & 1.370268153 & 1.631126202 & 2.008827666 & 2.518419671 \\
\hline $8.54 \mathrm{E}+09$ & 1.186217469 & 1.358119574 & 1.605960873 & 1.965866349 & 2.449018659 \\
\hline $8.64 E+09$ & 1.182136188 & 1.346663964 & 1.58634227 & 1.942762556 & 2.415366301 \\
\hline $8.75 \mathrm{E}+09$ & 1.177505538 & 1.338498967 & 1.575395614 & 1.927439583 & 2.393472569 \\
\hline $8.85 E+09$ & 1.175399362 & 1.334448692 & 1.567495223 & 1.917967507 & 2.380266944 \\
\hline $8.95 \mathrm{E}+09$ & 1.175468004 & 1.334722561 & 1.56563204 & 1.920075546 & 2.384919631 \\
\hline $9.05 \mathrm{E}+0 \mathrm{~s}$ & 1.171562766 & 1.323197963 & 1.547638678 & 1.88927644 & 2.336826706 \\
\hline
\end{tabular}




\begin{tabular}{|c|c|c|c|c|c|}
\hline Frequency & $e^{\prime \prime} / e^{\prime} @ 20 C$ & $e^{\prime \prime} / e^{\prime} @ 40 \mathrm{C}$ & $e^{\prime \prime / e} @ 60 C$ & $e^{\prime \prime} / e^{\prime} @ 80 C$ & $e^{\prime \prime} / e^{\prime} @ 100 \mathrm{C}$ \\
\hline $9.16 \mathrm{E}+09$ & 1.167659548 & 1.304550105 & 1.512575212 & 1.840262999 & 2.270215043 \\
\hline $9.26 \mathrm{E}+09$ & 1.165617983 & 1.302300986 & 1.505569341 & 1.831399013 & 2.260175751 \\
\hline $9.36 \mathrm{E}+09$ & 1.162332595 & 1.292867768 & 1.488239996 & 1.800147358 & 2.204114389 \\
\hline $9.46 \mathrm{E}+09$ & 1.159327405 & 1.286349906 & 1.481790045 & 1.798570905 & 2.205549666 \\
\hline $9.56 \mathrm{E}+09$ & 1.157767119 & 1.29043663 & 1.492918414 & 1.815432151 & 2.227584184 \\
\hline $9.67 \mathrm{E}+09$ & 1.153490746 & 1.279735969 & 1.472410078 & 1.783098971 & 2.169780871 \\
\hline $9.77 \mathrm{E}+09$ & 1.155221489 & 1.277386394 & 1.460070424 & 1.776291735 & 2.167281633 \\
\hline $9.87 \mathrm{E}+09$ & 1.14950064 & 1.25916259 & 1.430974081 & 1.727539676 & 2.09112449 \\
\hline $9.97 \mathrm{E}+09$ & 1.148273115 & 1.249656659 & 1.408600861 & 1.700650819 & 2.058120297 \\
\hline $1.01 \mathrm{E}+10$ & 1.145249071 & 1.245257974 & 1.405318911 & 1.696526516 & 2.056999915 \\
\hline $1.02 \mathrm{E}+10$ & 1.141284562 & 1.242487557 & 1.408826997 & 1.691580123 & 2.045754204 \\
\hline $1.03 \mathrm{E}+10$ & 1.139178328 & 1.236277287 & 1.404530125 & 1.685331214 & 2.037311971 \\
\hline $1.04 \mathrm{E}+10$ & 1.137484917 & 1.232266297 & 1.396111447 & 1.675624422 & 2.022146509 \\
\hline $1.05 \mathrm{E}+10$ & 1.13463125 & 1.221458557 & 1.371491087 & 1.64190313 & 1.970494541 \\
\hline $1.06 \mathrm{E}+10$ & 1.133764853 & 1.210086595 & 1.354851303 & 1.620974201 & 1.94876358 \\
\hline $1.07 \mathrm{E}+10$ & 1.131588414 & 1.212691302 & 1.371064979 & 1.643306817 & 1.983311626 \\
\hline $1.08 \mathrm{E}+10$ & 1.129014591 & 1.216366413 & 1.387602032 & 1.661375193 & 2.010391298 \\
\hline $1.09 \mathrm{E}+10$ & 1.12808216 & 1.222798722 & 1.399933793 & 1.675825232 & 2.0286976 \\
\hline $1.1 E+10$ & 1.125488141 & 1.214041915 & 1.384030797 & 1.649310075 & 1.985645297 \\
\hline $1.11 \mathrm{E}+10$ & 1.124600747 & 1.196399635 & 1.346960042 & 1.601342316 & 1.913535678 \\
\hline $1.12 \mathrm{E}+10$ & 1.122993231 & 1.18072289 & 1.313527187 & 1.555095888 & 1.853480966 \\
\hline $1.13 \mathrm{E}+10$ & 1.1222893 & 1.177735843 & 1.306763938 & 1.546396189 & 1.842994327 \\
\hline $1.14 \mathrm{E}+10$ & 1.121496748 & 1.181061354 & 1.318740238 & 1.560051305 & 1.860106812 \\
\hline $1.15 \mathrm{E}+10$ & 1.119108197 & 1.179262936 & 1.31864246 & 1.552884633 & 1.849721347 \\
\hline $1.16 \mathrm{E}+10$ & 1.118478149 & 1.172328809 & 1.303947231 & 1.530526065 & 1.819955258 \\
\hline $1.17 \mathrm{E}+10$ & 1.117851347 & 1.158192399 & 1.268814867 & 1.487823071 & 1.766366604 \\
\hline $1.18 \mathrm{E}+10$ & 1.119582047 & 1.144053144 & 1.237815162 & 1.446911635 & 1.714160128 \\
\hline $1.19 \mathrm{E}+10$ & 1.114559707 & 1.141954792 & 1.241088899 & 1.447787492 & 1.709964097 \\
\hline $1.2 \mathrm{E}+10$ & 1.114371355 & 1.144918912 & 1.249984126 & 1.459338406 & 1.730993849 \\
\hline $1.21 \mathrm{E}+10$ & 1.113017438 & 1.156812815 & 1.27871151 & 1.493630756 & 1.77533147 \\
\hline $1.22 \mathrm{E}+10$ & 1.110385817 & 1.155466309 & 1.282056516 & 1.490272098 & 1.764961889 \\
\hline $1.23 \mathrm{E}+10$ & 1.108309898 & 1.138038632 & 1.241532176 & 1.435637938 & 1.698246474 \\
\hline $1.24 \mathrm{E}+10$ & 1.110275312 & 1.118814152 & 1.189471734 & 1.381529939 & 1.637573454 \\
\hline $1.25 \mathrm{E}+10$ & 1.10954702 & 1.113354904 & 1.181057826 & 1.372917154 & 1.630456363 \\
\hline $1.26 \mathrm{E}+10$ & 1.108747001 & 1.11223713 & 1.188561202 & 1.372167255 & 1.635888139 \\
\hline $1.27 \mathrm{E}+10$ & 1.108843639 & 1.120647752 & 1.208600031 & 1.393752946 & 1.664309351 \\
\hline $1.28 \mathrm{E}+10$ & 1.106632013 & 1.120758488 & 1.212576365 & 1.393155541 & 1.666090247 \\
\hline $1.29 \mathrm{E}+10$ & 1.106580899 & 1.114996984 & 1.202133125 & 1.380109865 & 1.647154411 \\
\hline $1.3 \mathrm{E}+10$ & 1.107424649 & 1.109713731 & 1.184632009 & 1.353978869 & 1.619183518 \\
\hline $1.31 \mathrm{E}+10$ & 1.109719761 & 1.102845576 & 1.161546933 & 1.332656349 & 1.597341145 \\
\hline $1.32 \mathrm{E}+10$ & 1.112446199 & 1.110764756 & 1.171697203 & 1.348658793 & 1.620053553 \\
\hline $1.33 \mathrm{E}+10$ & 1.111888286 & 1.119820726 & 1.198777166 & 1.375292151 & 1.650183921 \\
\hline $1.35 \mathrm{E}+10$ & 1.109681583 & 1.119741939 & 1.206369912 & 1.375653796 & 1.646747405 \\
\hline $1.36 \mathrm{E}+10$ & 1.107633477 & 1.115492068 & 1.196794389 & 1.36240584 & 1.630485478 \\
\hline $1.37 \mathrm{E}+10$ & 1.111066842 & 1.119016159 & 1.1960998 & 1.36718567 & 1.639937398 \\
\hline
\end{tabular}




\begin{tabular}{|c|c|c|c|c|c|}
\hline Frequency & $e^{\prime \prime} / e^{\prime} @ 20 C$ & $e^{\prime \prime} / e^{\prime} @ 40 C$ & $e^{\prime \prime} / e^{\prime} @ 60 C$ & $e^{\prime \prime / e} @ 80 \mathrm{C}$ & $e^{\prime \prime} / e^{\prime} @$ \\
\hline $1.38 \mathrm{E}+10$ & 1.109607459 & 1.102934264 & 1.166480904 & 6001 & 37131 \\
\hline $1.39 \mathrm{E}+10$ & 1.111652663 & 1.104843703 & 1.163952555 & 1.324479546 & 1.587321844 \\
\hline $1.4 E+10$ & 1.112558714 & i.104605011 & 1.163878734 & 1.332440805 & 1.593114183 \\
\hline $1.41 \mathrm{E}+10$ & 1.113163841 & 1.104014474 & 1.164189582 & 1.336205226 & 1.597034476 \\
\hline $1.42 \mathrm{E}+10$ & 1.111503323 & 1.100702776 & 1.164940258 & 1.326503143 & 1.579445062 \\
\hline $1.43 \mathrm{E}+10$ & 1.112520085 & 1.0957424 & 1.156802588 & 1.31152191 & 1.555266437 \\
\hline $1.44 \mathrm{E}+10$ & 1.113641905 & 1.096503265 & 1.153513794 & 1.313186255 & 1.564864357 \\
\hline $1.45 \mathrm{E}+10$ & 1.115573148 & 1.097290424 & 1.150697138 & 1.309087587 & 1.55652246 \\
\hline $1.46 \mathrm{E}+10$ & 1.115620576 & 1.08586718 & 1.124514886 & 1.268704956 & 1.499161845 \\
\hline $1.47 \mathrm{E}+10$ & 1.120467186 & 1.088061793 & 1.116160337 & 1.260760498 & 1.494815585 \\
\hline $1.48 \mathrm{E}+10$ & 1.119778983 & 1.088509802 & 1.117278528 & 1.273747711 & 1.511760884 \\
\hline $1.49 \mathrm{E}+10$ & 1.119101426 & 1.078463516 & 1.100839688 & 1.243534685 & 1.469229837 \\
\hline $1.5 \mathrm{E}+10$ & 1.118975557 & 1.064614352 & 1.081746802 & 1.202302514 & 1.413647588 \\
\hline $1.51 \mathrm{E}+10$ & 1.125269142 & 1.071472723 & 1.082566695 & 1.208929009 & 1.42226495 \\
\hline $1.52 \mathrm{E}+10$ & 1.126039417 & 1.061956827 & 1.0596152 & 1.183533421 & 1.392422617 \\
\hline $1.53 E+10$ & 1.126514084 & 1.048609085 & 1.023293495 & 1.144619444 & 1.332514019 \\
\hline $1.54 \mathrm{E}+10$ & 1.126263935 & 1.052571255 & 1.036768356 & 1.155644497 & 1.347618007 \\
\hline $1.55 \mathrm{E}+10$ & 1.124712576 & 1.055402499 & 1.047622496 & 1.166303362 & 1.357947723 \\
\hline $1.56 \mathrm{E}+10$ & 1.125813625 & 1.084450003 & 1.10968038 & 1.235818614 & 1.43322193 \\
\hline $1.57 \mathrm{E}+10$ & 1.130358144 & 1.097860657 & 1.135838375 & 1.250599582 & 1.459545376 \\
\hline $1.58 \mathrm{E}+10$ & 1.136425135 & 1.092983435 & 1.104995561 & 1.218964182 & 1.41822398 \\
\hline $1.59 \mathrm{E}+10$ & 1.135197519 & 1.07581506 & 1.071812561 & $1.1954620 \mathrm{l} 1$ & 1.393508168 \\
\hline $1.6 \mathrm{E}+10$ & 1.134823635 & 1.057381442 & 1.038435577 & 1.150728982 & 1.336560007 \\
\hline $1.61 \mathrm{E}+10$ & 1.135473809 & 1.047656982 & 1.020969113 & 1.114723343 & 1.282841255 \\
\hline $1.62 \mathrm{E}+10$ & 1.133454825 & 1.060935596 & 1.053381108 & 1.141920337 & 1.31327543 \\
\hline $1.63 \mathrm{E}+10$ & 1.137465532 & 1.090462448 & 1.106924924 & 1.228711945 & 1.432715732 \\
\hline $1.64 \mathrm{E}+10$ & 1.140730423 & 1.095896176 & 1.113317797 & 1.240007129 & 1.448818163 \\
\hline $1.65 \mathrm{E}+10$ & 1.139906551 & 1.072773763 & 1.068060877 & 1.167215732 & 1.363977982 \\
\hline $1.66 \mathrm{E}+10$ & 1.14258608 & 1.062514773 & 1.046864289 & 1.157637732 & 1.36087036 \\
\hline $1.67 \mathrm{E}+10$ & 1.14519452 & 1.067698939 & 1.062582723 & 1.173907474 & 1.38172017 \\
\hline $1.68 \mathrm{E}+10$ & 1.146407753 & 1.058164773 & 1.03472932 & 1.106611016 & 1.288682846 \\
\hline $1.69 \mathrm{E}+10$ & 1.14290327 & 1.075399352 & 1.065856161 & 1.159389297 & 1.356117899 \\
\hline $1.7 \mathrm{E}+10$ & 1.148866342 & 1.109729424 & 1.133229877 & 1.263192517 & 1.500410368 \\
\hline $1.71 \mathrm{E}+10$ & 1.150198739 & 1.084989809 & 1.071585298 & 1.165759104 & 1.367208703 \\
\hline $1.72 \mathrm{E}+10$ & 1.145437022 & 1.034241278 & 0.969468682 & 1.058198302 & 1.22662776 \\
\hline $1.73 \mathrm{E}+10$ & 1.153660298 & 1.049539516 & 1.003196033 & 1.115260108 & 1.312950568 \\
\hline $1.74 \mathrm{E}+10$ & 1.15328706 & 1.032863273 & 0.978675367 & 1.065989057 & 1.242976823 \\
\hline $1.75 \mathrm{E}+10$ & 1.151465558 & 1.028359804 & 0.959897007 & 1.038372402 & 1.205842998 \\
\hline $1.76 \mathrm{E}+10$ & 1.155976638 & 1.058182555 & 1.030321234 & 1.135174762 & 1.341319957 \\
\hline $1.77 \mathrm{E}+10$ & 1.158016398 & 1.086071737 & 1.08078682 & 1.194249299 & 1.407583397 \\
\hline $1.79 \mathrm{E}+10$ & 1.159755953 & 1.049075433 & 1.000912083 & 1.083685284 & 1.262864927 \\
\hline $1.8 \mathrm{E}+10$ & 1.165434102 & 1.045050726 & 0.988347536 & 1.072998623 & 1.255143501 \\
\hline $1.81 \mathrm{E}+10$ & 1.162934532 & 1.035418333 & 0.970308095 & 1.068496644 & 1.248664077 \\
\hline $1.82 \mathrm{E}+10$ & 1.166593422 & 1.03476012 & 0.970687091 & 1.04770557 & 1.218307421 \\
\hline $1.83 \mathrm{E}+10$ & 1.169972189 & 1.029057394 & 0.952206041 & 1.018644258 & 1.179533019 \\
\hline
\end{tabular}




\begin{tabular}{|r|r|r|r|r|r|}
\hline Frequency & $\mathrm{e}^{\prime \prime} / \mathrm{e}^{\prime} @ 20 \mathrm{C}$ & $\mathrm{e} " / \mathrm{e}^{\prime} @ 40 \mathrm{C}$ & $\mathrm{e}^{\prime \prime} / \mathrm{e}^{\prime} @ 60 \mathrm{C}$ & $\mathrm{e}^{\prime \prime} / \mathrm{e}^{\prime} @ 80 \mathrm{C}$ & $\mathrm{e}^{\prime \prime} / \mathrm{e}^{\prime} @ 100 \mathrm{C}$ \\
\hline \hline $1.84 \mathrm{E}+10$ & 1.167627159 & 1.038095133 & 0.967890945 & 1.053269975 & 1.218887007 \\
\hline $1.85 \mathrm{E}+10$ & 1.170809786 & 1.042538111 & 0.970071791 & 1.060128276 & 1.234514062 \\
\hline $1.86 \mathrm{E}+10$ & 1.175976545 & 1.018924706 & 0.927319909 & 0.990869584 & 1.139789977 \\
\hline $1.87 \mathrm{E}+10$ & 1.187348439 & 1.010597136 & 0.883953288 & 0.945861229 & 1.095120916 \\
\hline $1.88 \mathrm{E}+10$ & 1.18970409 & 1.041802788 & 0.933823974 & 1.00779404 & 1.171198043 \\
\hline $1.89 \mathrm{E}+10$ & 1.184038316 & 1.034746883 & 0.954809098 & 1.028175079 & 1.188432906 \\
\hline $1.9 \mathrm{E}+10$ & 1.188487283 & 1.063373072 & 1.008626184 & 1.074240725 & 1.246748487 \\
\hline $1.91 \mathrm{E}+10$ & 1.191519009 & 1.099678069 & 1.062584806 & 1.140286805 & 1.320230005 \\
\hline $1.92 \mathrm{E}+10$ & 1.19712376 & 1.107468102 & 1.068129321 & 1.154618675 & 1.328784684 \\
\hline $1.93 \mathrm{E}+10$ & 1.204749541 & 1.092003709 & 1.03819249 & 1.115540554 & 1.286190419 \\
\hline $1.94 \mathrm{E}+10$ & 1.213170196 & 1.098829323 & 1.020099091 & 1.090589646 & 1.248587644 \\
\hline $1.95 \mathrm{E}+10$ & 1.210138803 & 1.071767093 & 0.981124972 & 1.050019451 & 1.204321196 \\
\hline $1.96 \mathrm{E}+10$ & 1.208328424 & 1.077158092 & 1.015006042 & 1.095729828 & 1.263722591 \\
\hline $1.97 \mathrm{E}+10$ & 1.207351957 & 1.062295897 & 1.000186463 & 1.07732503 & 1.238125445 \\
\hline $1.98 \mathrm{E}+10$ & 1.207279946 & 1.064782623 & 0.994380392 & 1.06448376 & 1.228319116 \\
\hline $1.99 \mathrm{E}+10$ & 1.211973565 & 1.071176792 & 0.996669898 & 1.074926297 & 1.235590005 \\
\hline $2 \mathrm{E}+10$ & 1.215110534 & 1.087003128 & 1.030740087 & 1.112884077 & 1.277221834 \\
\hline
\end{tabular}


Table 10. Dielectric Constant for the SAIC Simulant

\begin{tabular}{|c|c|c|c|c|c|}
\hline cy & $\mathrm{e}^{\prime} @ 20 \mathrm{C}$ & @40 C & @60 C & $\bar{C}$ & $\bar{C}$ \\
\hline $2.55 \mathrm{E}+08$ & 26.411437 & 82.392067 & 95.28453 & 3647 & $\overline{6226}$ \\
\hline-08 & 32.309732 & 68.815553 & 78.35279 & 80.30609 & $\overline{3036}$ \\
\hline $4.6 \mathrm{E}+08$ & 33.694147 & 61.23547 & 8.9541695 & .2945 & .9299 \\
\hline $5.62 E+08$ & 34.565999 & 56.337764 & 62.8504025 & 63.6731 & .49303 \\
\hline $6.65 \mathrm{E}+08$ & 35.108116 & 53.485277 & 59.3657145 & 59.91158 & 9.1297785 \\
\hline $7.67 \mathrm{E}+08$ & 35.707112 & 51.411241 & 56.6837815 & 6.98502 & 27.45763 \\
\hline $8.69 E+08$ & 36.549738 & 50.321383 & 55.3301535 & 55.51803 & 26.5233605 \\
\hline $9.71 \mathrm{E}+08$ & 36.454396 & 48.741676 & 53.3364965 & 53.26234 & 25.2709805 \\
\hline $1.07 \mathrm{E}+09$ & 36.55376 & 47.561098 & 51.763577 & 1.47541 & 24.263129 \\
\hline $1.18 \mathrm{E}+09$ & 36.728138 & 46.552607 & 50.466934 & 50.02516 & 23.458043 \\
\hline $1.28 \mathrm{E}+09$ & 36.717766 & 45.659033 & 49.4336745 & 48.8911 & $\overline{095}$ \\
\hline $1.38 \mathrm{E}+09$ & 36.686155 & 45.01329 & 48.749735 & 48.12641 & 555055 \\
\hline $1.48 E+09$ & 36.826323 & 44.718467 & 48.348493 & 47.65941 & 21.86618 \\
\hline $1.59 \mathrm{E}+09$ & 36.973973 & 44.326681 & 47.782099 & 46.96585 & 21.576513 \\
\hline $1.69 \mathrm{E}+09$ & 36.953393 & 43.875196 & 47.2332315 & 6.34155 & 21.227757 \\
\hline $1.79 \mathrm{E}+09$ & 37.046045 & 43.339523 & 46.594705 & 45.62872 & 20.7735875 \\
\hline $1.89 \mathrm{E}+09$ & 37.043679 & 42.924675 & 46.072184 & 5.05699 & 20.3997 \\
\hline $1.99 \mathrm{E}+09$ & 37.100688 & 42.769259 & 45.972378 & 44.94083 & 20.2616785 \\
\hline $2.1 \mathrm{E}+09$ & 37.17753 & 42.81772 & 46.1181345 & .09108 & 20.2057 \\
\hline $2.2 \mathrm{E}+09$ & 37.188205 & 42.57573 & 45.7959865 & 44.70312 & 9.9355425 \\
\hline $2.3 \mathrm{E}+09$ & 37.161605 & 42.190164 & 45.252644 & 44.06544 & 19.6277755 \\
\hline $2.4 \mathrm{E}+09$ & 37.103969 & 41.915144 & 44.8978115 & 43.64361 & 1437 \\
\hline $2.51 \mathrm{E}+09$ & 36.906577 & 41.649797 & 44.6212745 & 43.32637 & 19.2672415 \\
\hline $2.61 \mathrm{E}+09$ & 36.867042 & 41.429541 & 44.379474 & 3.07136 & 19.03816 \\
\hline $2.71 E+09$ & 36.853737 & 41.200648 & 44.135089 & 42.80862 & 18.870614 \\
\hline $2.81 E+09$ & 36.80358 & 40.994869 & 43.9522325 & 42.60848 & 8.7774405 \\
\hline $2.92 E+09$ & 36.717312 & 40.7928 & 43.7350965 & 42.34361 & 18.5791545 \\
\hline $3.02 \mathrm{E}+09$ & 36.623819 & 40.581271 & 43.4150175 & 41.96966 & 18.3890235 \\
\hline $3.12 \mathrm{E}+09$ & 36.429884 & 40.376462 & 43.10842 & 41.60573 & 18.264701 \\
\hline $3.22 E+09$ & 36.291186 & 40.2104 & 42.948463 & 41.44296 & 8.1670935 \\
\hline $3.32 \mathrm{E}+09$ & 36.257558 & 0.131229 & 42.931511 & 41.43138 & 18.0195465 \\
\hline $3.43 \mathrm{E}+09$ & 36.282407 & 40.035769 & 42.8406195 & 41.3342 & 17.918625 \\
\hline $3.53 \mathrm{E}+09$ & 36.297633 & 39.928727 & 42.73857 & 41.22564 & 17.9048375 \\
\hline $3.63 E+09$ & 36.277002 & 39.811994 & 42.6329445 & 41.12266 & 17.830046 \\
\hline $3.73 \mathrm{E}+09$ & 36.155875 & 39.643539 & 42.430481 & 40.883 & 17.6176765 \\
\hline $3.84 \mathrm{E}+09$ & 36.069717 & 39.502238 & 42.1879515 & 40.62022 & 17.5244195 \\
\hline $3.94 E+09$ & 35.986244 & 39.432194 & 42.096661 & 40.50683 & 17.5237025 \\
\hline $4.04 \mathrm{E}+09$ & 35.916822 & 39.382166 & 42.12402 & 40.55856 & 17.440415 \\
\hline $4.14 \mathrm{E}+09$ & 35.871906 & 39.276184 & 42.075153 & 40.52992 & 17.2946395 \\
\hline $4.25 \mathrm{E}+09$ & 35.816955 & 39.11034 & 41.875327 & 40.30038 & 17.2135685 \\
\hline $4.35 \mathrm{E}+09$ & 35.701888 & 38.904745 & 41.624608 & 40.01678 & 17.129266 \\
\hline $4.45 \mathrm{E}+09$ & 35.504259 & 38.7221 & 41.407476 & 39.7851 & 16.9528965 \\
\hline $4.55 \mathrm{E}+09$ & 35.363069 & 8.574321 & 1.2152175 & 39.57156 & 16.824096 \\
\hline
\end{tabular}




\begin{tabular}{|c|c|c|c|c|c|}
\hline equency & $\mathrm{e}^{\prime} @ 20 \mathrm{C}$ & $e^{\prime} @$ & @ & & \\
\hline 09 & 35.303706 & 38.465964 & 41.08663 & 991 & $\overline{834}$ \\
\hline $4.76 E+09$ & 35.194675 & 38.3462 & 40.977468 & 0705 & .768402 \\
\hline $4.86 E+09$ & 35.109969 & 8.209357 & 40.9085705 & 39.23931 & .5885 \\
\hline $4.96 E+09$ & 34.918218 & 37.982289 & 40.6762795 & 38.97009 & 16.4416755 \\
\hline $5.06 \mathrm{E}+09$ & 34.72563 & 37.736472 & 40.3436965 & 38.60988 & 6.36855 \\
\hline 5.17 & 34.573095 & 37.554179 & 40.0877295 & 38.35079 & 16.2217365 \\
\hline $5.27 \mathrm{E}+09$ & 34.49807 & 37.414564 & 39.9255345 & 38.18347 & 16.0966325 \\
\hline $5.37 \mathrm{E}+09$ & 34.422956 & 37.343301 & 39.8420755 & 38.08507 & $16.11027 t$ \\
\hline $5.47 \mathrm{E}+09$ & 34.350106 & 37.258017 & 39.762712 & 38.00949 & 16.1044415 \\
\hline $5.57 \mathrm{E}+09$ & 34.183924 & 37.118832 & 39.680766 & 37.93949 & 15.92759 \\
\hline $5.68 \mathrm{E}+09$ & 34.009017 & 36.881629 & 39.470539 & 37.71927 & 15.7205845 \\
\hline $5.78 \mathrm{E}+09$ & 33.846101 & 36.656238 & 39.174993 & 553 & 15.670263 \\
\hline $5.88 \mathrm{E}+09$ & 33.712108 & 36.536612 & 38.9710305 & 37.17697 & 15.6124955 \\
\hline $5.98 \mathrm{E}+09$ & 33.652969 & 36.485363 & 38.907209 & 37.11404 & 15.527182 \\
\hline $6.09 \mathrm{E}+09$ & 33.599106 & 36.447074 & 8.9371795 & 351 & 15.5355 \\
\hline $6.19 \mathrm{E}+09$ & 33.519319 & 36.375673 & 38.9266875 & 37.14122 & 15.539217 \\
\hline $6.29 \mathrm{E}+09$ & 33.310522 & 36.214828 & 38.769311 & 952 & 15.3758575 \\
\hline $6.39 \mathrm{E}+09$ & 33.130761 & 35.987077 & 38.5525375 & 36.78523 & 15.161902 \\
\hline$E+09$ & 32.978974 & 35.777301 & 38.326797 & 36.55979 & 15.109938 \\
\hline $6.6 \mathrm{E}+09$ & 32.894084 & 35.597023 & 38.042526 & 36.24285 & 15.085044 \\
\hline $6.7 \mathrm{E}+09$ & 32.848045 & 35.545928 & 37.9277055 & 36.15342 & 5.0056735 \\
\hline $6.8 \mathrm{E}+09$ & 32.76088 & 35.536128 & 38.0164415 & 36.24621 & 14.969846 \\
\hline $6.9 \mathrm{E}+09$ & 32.584542 & 35.440615 & 38.043 & 36.30108 & 14.980028 \\
\hline $7.01 \mathrm{E}+09$ & 32.404628 & 35.29196 & 37.927042 & 36.17566 & 14.8566975 \\
\hline $7.11 \mathrm{E}+09$ & 32.211702 & 35.093288 & 37.6754315 & 35.93386 & 14.64241 \\
\hline $7.21 \mathrm{E}+09$ & 32.099856 & 34.858635 & 37.384085 & 35.64048 & 14.5691305 \\
\hline $7.31 \mathrm{E}+09$ & 32.091804 & 34.710315 & 37.179052 & 35.41013 & 14.622524 \\
\hline $7.42 \mathrm{E}+09$ & 32.036103 & 34.614481 & 37.0068475 & 35.22709 & 14.546759 \\
\hline $7.52 \mathrm{E}+09$ & 31.90436 & 34.569367 & 36.998348 & 35.24521 & 14.4178785 \\
\hline $7.62 \mathrm{E}+09$ & 31.741112 & 34.52553 & 7.1203165 & 35.38717 & 14.419014 \\
\hline $7.72 \mathrm{E}+09$ & 31.54513 & 34.406474 & 7.052424 & 35.34307 & 14.37517 \\
\hline$E+09$ & 31.407739 & 34.188195 & 36.7131105 & 34.99083 & 14.1759455 \\
\hline $7.93 \mathrm{E}+09$ & 31.35669 & 33.960205 & 36.3782505 & 34.64815 & 14.071235 \\
\hline $8.03 \mathrm{E}+09$ & 31.372581 & 33.828439 & 36.232511 & 34.51586 & 14.144525 \\
\hline $8.13 E+09$ & 31.363292 & 33.750354 & 36.1767695 & 34.4347 & 14.142507 \\
\hline $8.23 E+09$ & 31.172496 & 33.777512 & 36.221263 & 34.50774 & 14.0241 \\
\hline $8.34 \mathrm{E}+09$ & 30.975149 & 33.780396 & 36.360768 & 34.68664 & 14.015868 \\
\hline $8.44 E+09$ & 30.805672 & 33.66427 & 36.352714 & 34.68153 & 14.040757 \\
\hline $8.54 \mathrm{E}+09$ & 30.779184 & 33.466397 & 36.0379655 & 34.35424 & 13.90523 \\
\hline $8.64 E+09$ & 30.800066 & 33.293339 & 35.679458 & 33.98861 & 13.759946 \\
\hline $8.75 \mathrm{E}+09$ & 30.798723 & 33.165446 & 35.537112 & 33.83496 & 13.8174975 \\
\hline $8.85 E+09$ & 30.704396 & 33.174384 & 35.6910915 & 34.00639 & 13.900831 \\
\hline $8.95 \mathrm{E}+09$ & 30.540956 & 33.220154 & 35.807549 & 34.15067 & 13.771888 \\
\hline $9.05 \mathrm{E}+09$ & 30.338415 & 33.285029 & 35.91679 & 34.30542 & 13.7107375 \\
\hline $9.16 \mathrm{E}+09$ & 30.230495 & 33.160403 & 35.8480165 & 34.24495 & 13.754859 \\
\hline
\end{tabular}




\begin{tabular}{|c|c|c|c|c|c|}
\hline acy & $e^{\prime} @ 20 \mathrm{C}$ & @ $40 \mathrm{C}$ & (a)60C & @ $80 \mathrm{C}$ & (a) $100 \mathrm{C}$ \\
\hline 109 & 939 & 894 & 2005 & 33.94268 & 13.69788 \\
\hline $\mathrm{E}+09$ & .282791 & 32.754246 & 35.2056735 & 33.5445 & .517677 \\
\hline $6 \mathrm{E}+09$ & .262692 & 32.632618 & 35.0165685 & 33.39085 & 13.509799 \\
\hline $9.56 \mathrm{E}+09$ & 0.168814 & 32.647456 & 35.2722235 & 33.67058 & 13.6192 \\
\hline $67 \mathrm{E}+09$ & 29.966751 & 32.749457 & 35.571621 & 34.00874 & 13.548043 \\
\hline $77 \mathrm{E}+09$ & 29.783593 & 32.86195 & 35.617394 & 34.08087 & 13.41611 \\
\hline $87 \mathrm{E}+09$ & 29.766193 & 32.775099 & 35.4774985 & 33.91441 & 13.456203 \\
\hline $97 \mathrm{E}+09$ & 29.824362 & 32.50444 & 35.1646535 & 33.56398 & 13.469643 \\
\hline $1.01 E+10$ & 29.873437 & 32.241283 & 34.794682 & 3.17387 & 13.2893655 \\
\hline $1.02 \mathrm{E}+10$ & 29.842664 & 32.142773 & 34.6175155 & 33.02604 & 13.211182 \\
\hline $1.03 E+10$ & 29.718695 & 32.134431 & 34.7900645 & 33.26253 & 13.3209255 \\
\hline $1.04 \mathrm{E}+10$ & 29.522209 & 32.228998 & 35.170686 & 33.67214 & 3.3012965 \\
\hline $1.05 \mathrm{E}+10$ & 29.403263 & 32.338821 & 35.244883 & 33.74024 & 3.1031325 \\
\hline $1.06 \mathrm{E}+10$ & 29.397624 & 32.309324 & 34.977859 & 33.46482 & 13.1270705 \\
\hline $1.07 \mathrm{E}+10$ & 29.535886 & 32.033158 & 34.5831975 & 33.02732 & 13.2257555 \\
\hline $1.08 \mathrm{E}+10$ & 29.563171 & 31.797413 & 34.415071 & 32.83715 & 13.1246225 \\
\hline $1.09 \mathrm{E}+10$ & 29.47 & 31.666849 & 34.3160345 & 2.80157 & 12.98126 \\
\hline $1.1 \mathrm{E}+10$ & 29.288133 & 31.635554 & 34.3439425 & 32.88357 & 13.022027 \\
\hline $1.11 \mathrm{E}+10$ & 29.091538 & 3127 & 34558 & & 13.0 \\
\hline $1.12 \mathrm{E}+10$ & 29.008071 & 31.654688 & 34.6247715 & 33.21077 & 12.8122075 \\
\hline $1.13 \mathrm{E}+10$ & 29.060674 & 31.653579 & 34.2991755 & 09 & 12.801 \\
\hline $1.14 \mathrm{E}+10$ & 29.197406 & 31.530726 & 33.9593875 & 32.40207 & 13.002537 \\
\hline $1.15 \mathrm{E}+10$ & 29.229855 & 31.328082 & 33.9438395 & 32.40033 & 69907 \\
\hline $1.16 \mathrm{E}+10$ & 29.072627 & 31.072598 & 33.9617995 & 32.44438 & 12.6372145 \\
\hline $1.17 \mathrm{E}+10$ & 28.836185 & 30.831127 & 33.719407 & 32.26757 & 12.4850705 \\
\hline $1.18 \mathrm{E}+10$ & 28.688255 & 30.740173 . & 33.5512395 & 32.1282 & 12.518234 \\
\hline $1.19 \mathrm{E}+10$ & 28.659726 & 30.695904 & 33.554077 & 32.15 & 12.4266045 \\
\hline $1.2 \mathrm{E}+10$ & 28.73 & 30.976831 & 33.6866045 & 2.19575 & 12.5075795 \\
\hline $1.21 \mathrm{E}+10$ & 28.83137 & 31.14435 & 33.5653355 & 31.98876 & 12.7920755 \\
\hline $1.22 \mathrm{E}+10$ & 28.823348 & 30.916988 & 33.435863 & 31.81196 & 12.7976545 \\
\hline $1.23 \mathrm{E}+10$ & 28.604279 & 30.657626 & 33.5541095 & 31.95491 & 12.4969675 \\
\hline $4 E+10$ & 28.369157 & 30.726107 & 33.649683 & 32.09696 & 12.5548055 \\
\hline $1.25 \mathrm{E}+10$ & 28.215724 & 30.984685 & 33.6379775 & 32.17276 & 12.888437 \\
\hline $1.26 \mathrm{E}+10$ & 28.331343 & 30.958166 & 33.620902 & 32.28149 & 12.8571375 \\
\hline $7 \mathrm{E}+10$ & 28.417838 & 30.897589 & 33.71807 & 32.42687 & 12.587823 \\
\hline $1.28 \mathrm{E}+10$ & 28.43815 & 30.805375 & 3.546865 & 32.29513 & 12.6304535 \\
\hline $1.29 \mathrm{E}+10$ & 28.338475 & 30.623988 & 33.2651755 & 32.02665 & 2.7081105 \\
\hline $1.3 \mathrm{E}+10$ & 28.103784 & 30.408127 & 33.258064 & 32.01611 & 12.41807 \\
\hline $1.31 \mathrm{E}+10$ & 27.897725 & 30.577211 & 33.570796 & 32.24815 & 12.3848655 \\
\hline $1.32 \mathrm{E}+10$ & 27.844615 & 30.92236 & 33.6450085 & 32.23247 & 12.7961785 \\
\hline $1.33 \mathrm{E}+10$ & 28.014392 & 30.789996 & 33.314773 & 31.9886 & 12.7951195 \\
\hline $1.35 \mathrm{E}+10$ & 28.15575 & 30.453321 & 3.4309755 & 32.31222 & 12.4613205 \\
\hline $1.36 \mathrm{E}+10$ & 28.148807 & 30.436995 & 33.84086 & 32.94808 & 12.530302 \\
\hline $1.37 \mathrm{E}+10$ & 27.976582 & 30.365052 & 33.555325 & 32.72075 & 12.7305145 \\
\hline 10 & 27.641772 & 30.003427 & 33.038669 & 32.09938 & 12.36614 \\
\hline
\end{tabular}




\begin{tabular}{|c|c|c|c|c|c|}
\hline & DC & $40 \mathrm{C}$ & & & \\
\hline$E+10$ & 27.372646 & 14016 & 33.229909 & 8 & \\
\hline+10 & 229 & 8163 & .419041 & 2.16394 & .5446 \\
\hline $1.41 \mathrm{E}+10$ & .46523 & 0.483527 & .9246375 & 906 & .69863 \\
\hline $2 \mathrm{E}+10$ & 7.718107 & 29.915022 & 2.5956305 & .44508 & .19208 \\
\hline $1.43 \mathrm{E}+10$ & 27.880289 & 29.828559 & 3.2517195 & 2588 & .160372 \\
\hline $1.44 \mathrm{E}+10$ & .845847 & 30.074948 & 33.4851485 & 2.61401 & 2.632755 \\
\hline $1.45 \mathrm{E}+10$ & 27.556121 & 29.838595 & 32.6893695 & 4661 & 2.381818 \\
\hline $6 \mathrm{E}+10$ & .28193 & 29.550027 & 32.331588 & 1.30741 & 1.80960 \\
\hline $1.47 \mathrm{E}+10$ & 27.102127 & 30.077433 & 32.996792 & 31.81966 & 2.26050 \\
\hline$E+10$ & 91 & 111 & 33.059297 & 1.81874 & 12.76774 \\
\hline $1.49 \mathrm{E}+10$ & 26.799071 & 29.381413 & 32.1609405 & .07521 & 1.967870 \\
\hline $1.5 \mathrm{E}+10$ & 373 & 3.946991 & 32.4590855 &, 56544 & 11.70575 \\
\hline $1.51 \mathrm{E}+10$ & 26.217941 & 29.883684 & 3.647011 & .12114 & .8185 \\
\hline $1.52 \mathrm{E}+10$ & 5579 & 0 & 32.984252 & 7148 & .56319 \\
\hline $1.53 \mathrm{E}+10$ & 26.253869 & 28.828695 & 31.5596945 & 0.57296 & 11.40058 \\
\hline+10 & 1 & & 32.015117 & 1.56387 & 12.466573 \\
\hline $1.55 \mathrm{E}+10$ & 27.418919 & 30.420786 & 3.4987795 & 2.46209 & 13.21865 \\
\hline $1.56 \mathrm{E}+10$ & 314 & 46 & 10415 & & 11.6487 \\
\hline $1.57 \mathrm{E}+10$ & 26.200236 & 28.340648 & 31.4846575 & 30.67668 & 1.672440 \\
\hline $1.58 \mathrm{E}+10$ & 2 & 586 & 2.726 & 338 & 2.74336 \\
\hline $1.59 \mathrm{E}+10$ & 24.289089 & 8.947535 & 32.31761 & 31.4742 & 1.865626 \\
\hline $1.6 \mathrm{E}+10$ & .55041 & 3.235346 & .737312 & 94 & \\
\hline $1.61 \mathrm{E}+10$ & 8.844009 & 29.048402 & 31.301923 & 30.3141 & 2.210923 \\
\hline $2 \mathrm{E}+10$ & 26.833601 & 29.68 & .97618 & 2.05691 & 2.621585 \\
\hline$E+10$ & 26.890325 & 28.275049 & 1.783513 & 30.81505 & 11.483863 \\
\hline $1.64 \mathrm{E}+10$ & 26.229938 & 27.599305 & .255132 & 9.27765 & 1.28167 \\
\hline $\mathrm{E}+10$ & 134 & 5822 & 958215 & 31.11463 & 12.01592 \\
\hline$E+10$ & 23.879824 & 29.110704 & 2.870173 & 17 & 1.749783 \\
\hline $\begin{array}{c}E+10 \\
\end{array}$ & 3.92148 & 27.870632 & 30.477821 & 29.51543 & 1.118 \\
\hline $8 \mathrm{E}+10$ & .013495 & 40235 & 30.46949 & 29.57771 & 1.794583 \\
\hline $1.69 \mathrm{E}+10$ & 5.292892 & 18 & 189 & 32.1952 & 2.226636 \\
\hline $1.7 \mathrm{E}+10$ & 6.900552 & 397 & 348735 & 1.01445 & 11.26830 \\
\hline $1.71 \mathrm{E}+10$ & 26.531654 & 27.447981 & .0450745 & 9.15114 & 1.333243 \\
\hline $1.72 \mathrm{E}+10$ & 25.149715 & 28.965647 & 31.715191 & 1.01662 & 2.004757 \\
\hline $1.73 \mathrm{E}+10$ & 745067 & 28.823538 & 32.707751 & 1.93678 & 11.50393 \\
\hline $1.74 \mathrm{E}+10$ & 3.300101 & 27.736256 & 0.7099855 & 9.80433 & 1.01484 \\
\hline $1.75 \mathrm{E}+10$ & 883278 & .406256 & 0.353733 & 29.61451 & 11.52965 \\
\hline $1.76 \mathrm{E}+10$ & 24.930725 & 28.789992 & 32.2824565 & 31.60614 & 11.57033 \\
\hline $1.77 \mathrm{E}+10$ & 25.791991 & 27.473477 & 32.0588895 & 1.23132 & 1.0615445 \\
\hline $1.79 \mathrm{E}+10$ & 791479 & 2047 & 30.2358905 & 29.45017 & 1.2680055 \\
\hline $1.8 \mathrm{E}+10$ & & 28.348304 & 30.8121245 & 30.14328 & 11.5635745 \\
\hline $1.81 \mathrm{E}+10$ & 23.701293 & 28.355783 & 32.3011185 & 31.61412 & 11.24500 \\
\hline $1.82 \mathrm{E}+10$ & & & 30.9825255 & 30.23132 & 10.937133 \\
\hline $1.83 \mathrm{E}+10$ & 3.561986 & 27.454788 & 29.539598 & 9.03 & 11.0 \\
\hline $1.04 \mathrm{LT} 10$ & 4. & 27.8 & 1.4 & $30.934 / 4$ & \\
\hline
\end{tabular}




\begin{tabular}{|r|r|r|r|r|r|}
\hline Frequency & $e^{\prime} @ 20 \mathrm{C}$ & $\mathrm{e}^{\prime} @ 40 \mathrm{C}$ & $\mathrm{e}^{\prime} @ 60 \mathrm{C}$ & $\mathrm{e}^{\prime} @ 80 \mathrm{C}$ & $\mathrm{e}^{\prime} @ 100 \mathrm{C}$ \\
\hline \hline $1.85 \mathrm{E}+10$ & 25.036848 & 27.089178 & 31.7847205 & 31.21125 & 10.903837 \\
\hline $1.86 \mathrm{E}+10$ & 24.980494 & 26.664568 & 29.439398 & 28.83609 & 10.9076845 \\
\hline $1.87 \mathrm{E}+10$ & 24.309281 & 27.425854 & 29.739132 & 29.17057 & 10.9673075 \\
\hline $1.88 \mathrm{E}+10$ & 23.620218 & 27.700009 & 31.4360915 & 30.83472 & 10.8117125 \\
\hline $1.89 \mathrm{E}+10$ & 23.298636 & 26.954318 & 30.2962795 & 29.74675 & 10.6917655 \\
\hline $1.9 \mathrm{E}+10$ & 23.482231 & 26.800524 & 29.306294 & 28.91755 & 10.706116 \\
\hline $1.91 \mathrm{E}+10$ & 23.912373 & 27.155951 & 30.8045935 & 30.39757 & 10.7413165 \\
\hline $1.92 \mathrm{E}+10$ & 24.216843 & 26.700667 & 30.7666605 & 30.25564 & 10.696182 \\
\hline $1.93 \mathrm{E}+10$ & 24.082279 & 26.362506 & 29.3171015 & 28.75799 & 10.654245 \\
\hline $1.94 \mathrm{E}+10$ & 23.671214 & 26.831282 & 29.561787 & 29.00495 & 10.555826 \\
\hline $1.95 \mathrm{E}+10$ & 23.270748 & 27.15681 & 30.422705 & 29.92073 & 10.4744595 \\
\hline $1.96 \mathrm{E}+10$ & 23.186256 & 26.753084 & 30.007988 & 29.59796 & 10.465181 \\
\hline $1.97 \mathrm{E}+10$ & 23.326714 & 26.322373 & 29.6317345 & 29.33212 & 10.4176045 \\
\hline $1.98 \mathrm{E}+10$ & 23.492265 & 26.397176 & 29.984881 & 29.60868 & 10.3461985 \\
\hline $1.99 \mathrm{E}+10$ & 23.473464 & 26.380058 & 29.785393 & 29.29729 & 10.284441 \\
\hline $2 \mathrm{E}+10$ & 23.26571 & 26.235878 & 29.32183 & 28.77651 & 10.276752 \\
\hline
\end{tabular}


Table 11. Dielectric Loss for the SAIC Simulant

\begin{tabular}{|c|c|c|c|c|c|}
\hline Frequency & $\mathrm{e}^{\prime \prime} @ 20 \mathrm{C}$ & $\mathrm{e}^{\prime \prime} @ 40 \mathrm{C}$ & $\mathrm{e}^{\prime \prime} @ 60 \mathrm{C}$ & $\mathrm{e}^{\prime \prime} @ 80 \mathrm{C}$ & $\mathrm{e}^{\prime \prime} @ 100 \mathrm{C}$ \\
\hline $2.55 \mathrm{E}+08$ & 685.35707 & 865.11749 & 1137.60801 & 1264.1283 & 407.667019 \\
\hline $3.58 \mathrm{E}+08$ & 493.26111 & 624.6182 & 821.012341 & 912.20095 & 296.386373 \\
\hline $4.6 \mathrm{E}+08$ & 385.65627 & 490.31578 & 644.163912 & 715.77256 & 234.250915 \\
\hline $5.62 \mathrm{E}+08$ & 317.29811 & 404.86647 & 531.38118 & 590.06946 & 193.840439 \\
\hline $6.65 \mathrm{E}+08$ & 268.95222 & 344.81244 & 452.83433 & 503.40765 & 165.731503 \\
\hline $7.67 \mathrm{E}+08$ & 232.91806 & 299.87858 & 393.537612 & 437.55464 & 144.679988 \\
\hline $8.69 \mathrm{E}+08$ & 205.45007 & 265.31569 & 347.937684 & 386.84993 & 128.375105 \\
\hline $9.71 \mathrm{E}+08$ & 184.2227 & 238.56901 & 312.756829 & 347.81465 & 115.813495 \\
\hline $1.07 \mathrm{E}+09$ & 167.10457 & 216.86231 & 284.234607 & 316.10056 & 105.543388 \\
\hline $1.18 \mathrm{E}+09$ & 152.87605 & 198.79756 & 260.405412 & 289.63466 & 96.8898875 \\
\hline $1.28 \mathrm{E}+09$ & 141.17916 & 183.74931 & 240.539253 & 267.54097 & 89.7392215 \\
\hline $1.38 \mathrm{E}+09$ & 131.03556 & 170.59611 & 223.239829 & 248.30958 & 83.480718 \\
\hline $1.48 \mathrm{E}+09$ & 122.15014 & 159.37331 & 208.430146 & 231.85895 & 78.102279 \\
\hline $1.59 \mathrm{E}+09$ & 114.40655 & 149.4698 & 195.318328 & 217.23292 & 73.340289 \\
\hline $1.69 \mathrm{E}+09$ & 107.6809 & 140.93554 & 184.023645 & 204.68086 & 69.2912125 \\
\hline $1.79 \mathrm{E}+09$ & 101.91815 & 133.35971 & 174.099193 & 193.63044 & 65.706129 \\
\hline $1.89 \mathrm{E}+09$ & 96.871378 & 126.72602 & 165.387077 & 183.93851 & 62.475319 \\
\hline $1.99 \mathrm{E}+09$ & 92.275774 & 120.53396 & 157.196825 & 174.79073 & 59.455268 \\
\hline $2.1 \mathrm{E}+09$ & 88.079098 & 115.1234 & 150.012056 & 166.79953 & 56.9390725 \\
\hline $2.2 \mathrm{E}+09$ & 84.299556 & 110.32067 & 143.666227 & 159.76161 & 54.6380545 \\
\hline $2.3 E+09$ & 80.850721 & 105.89241 & 137.803513 & 153.21788 & 52.4276555 \\
\hline $2.4 \mathrm{E}+09$ & 77.851461 & 101.86938 & 132.397149 & 147.16831 & 50.49163 \\
\hline $2.51 \mathrm{E}+09$ & 75.144713 & 98.177343 & 127.500037 & 141.67785 & 48.7735755 \\
\hline $2.61 E+09$ & 72.457572 & 94.653557 & 122.903478 & 136.55404 & 47.0253515 \\
\hline $2.71 \mathrm{E}+09$ & 70.076416 & 91.504287 & 118.702088 & 131.86233 & 45.4595845 \\
\hline $2.81 E+09$ & 67.901321 & 88.522358 & 114.69269 & 127.38931 & 44.0669725 \\
\hline $2.92 E+09$ & 65.816724 & 85.745045 & 110.999637 & 123.26556 & 42.7747215 \\
\hline $3.02 \mathrm{E}+09$ & 63.871907 & 83.120497 & 107.533154 & 119.38421 & 41.4477475 \\
\hline $3.12 \mathrm{E}+09$ & 62.101 & 80.775694 & 104.406858 & 115.84812 & 40.272173 \\
\hline $3.22 \mathrm{E}+09$ & 60.467063 & 78.593928 & 101.433559 & 112.51916 & 39.2534345 \\
\hline $3.32 \mathrm{E}+09$ & 58.862568 & 76.504468 & 98.681273 & 109.42447 & 38.2681725 \\
\hline $3.43 \mathrm{E}+09$ & 57.370671 & 74.481336 & 96.0123135 & 106.45565 & 37.2414205 \\
\hline $3.53 \mathrm{E}+09$ & 56.015126 & 72.570843 & 93.4528635 & 103.58462 & 36.323432 \\
\hline $3.63 E+09$ & 54.730087 & 70.799557 & 91.049794 & 100.91092 & 35.540114 \\
\hline $3.73 E+09$ & 53.598831 & 69.274449 & 89.0087535 & 98.623533 & 34.757358 \\
\hline $3.84 \mathrm{E}+09$ & 52.500364 & 67.81991 & 87.078801 & 96.42351 & 33.970172 \\
\hline $3.94 \mathrm{E}+09$ & 51.46709 & 66.468968 & 85.2456895 & 94.330108 & 33.358225 \\
\hline $4.04 \mathrm{E}+09$ & 50.49638 & 65.12092 & 83.42994 & 92.287656 & 32.7771225 \\
\hline $4.14 \mathrm{E}+09$ & 49.541774 & 63.821001 & 81.7206795 & 90.384324 & 32.1053935 \\
\hline $4.25 \mathrm{E}+09$ & 48.677008 & 62.596698 & 80.1111425 & 88.545178 & 31.4573985 \\
\hline $4.35 \mathrm{E}+09$ & 47.908847 & 61.461744 & 78.509277 & 86.743474 & 30.9323975 \\
\hline $4.45 \mathrm{E}+09$ & 47.121377 & 60.449143 & 77.075377 & 85.091013 & 30.4290405 \\
\hline $4.55 \mathrm{E}+09$ & 46.371254 & 59.496693 & 75.775656 & 83.5914 & 29.8674655 \\
\hline
\end{tabular}




\begin{tabular}{|c|c|c|c|c|c|}
\hline ncy & $\mathrm{e}^{\prime \prime} @ 20 \mathrm{C}$ & $e^{\prime \prime @ 40 C ~}$ & $e^{\prime \prime} @ 60 C$ & $\mathrm{e}^{\prime \prime} @ 80 \mathrm{C}$ & $\mathrm{e}^{\prime \prime}$ \\
\hline $4.65 E+09$ & 45.694755 & 58.550417 & 74.508223 & 82.126626 & 21855 \\
\hline $76 \mathrm{E}+09$ & 45.00312 & 57.576702 & 73.1943135 & 80.633594 & 29.0409565 \\
\hline $4.86 \mathrm{E}+09$ & 44.376092 & 56.60106 & 71.9165215 & 79.192001 & 28.5646335 \\
\hline $4.96 \mathrm{E}+09$ & 43.711831 & 55.6542 & 70.6861125 & 77.835761 & 28.0173385 \\
\hline $5.06 \mathrm{E}+09$ & 43.129806 & 54.827642 & 69.504628 & 76.464585 & 27.6135625 \\
\hline $5.17 \mathrm{E}+09$ & 42.57336 & 54.084113 & 68.370414 & 75.147282 & 27.262007 \\
\hline $5.27 \mathrm{E}+09$ & 41.996721 & 53.340315 & 67.344678 & 73.963555 & 26.835795 \\
\hline $5.37 \mathrm{E}+09$ & 41.401867 & 52.612786 & 66.407197 & 72.90127 & 26.4681205 \\
\hline $5.47 \mathrm{E}+09$ & 40.894269 & 51.843866 & 65.419323 & 71.786073 & 26.2007925 \\
\hline $5.57 \mathrm{E}+09$ & 40.401415 & 51.059532 & 64.3554115 & 70.593829 & 25.845259 \\
\hline $5.68 \mathrm{E}+09$ & 39.892372 & 50.402493 & 63.453263 & 69.570877 & 25.4061265 \\
\hline $5.78 \mathrm{E}+09$ & 39.460639 & 49.770749 & 62.5873775 & 68.57146 & 25.0480305 \\
\hline $5.88 \mathrm{E}+09$ & 39.011395 & 49.21104 & 61.7690465 & 67.607143 & 24.825302 \\
\hline $5.98 \mathrm{E}+09$ & 38.576067 & 48.680116 & 60.99115 & 66.702282 & 24.554002 \\
\hline $6.09 E+09$ & 38.131537 & 48.119656 & 60.2909895 & 65.917486 & 24.2544995 \\
\hline $6.19 \mathrm{E}+09$ & 37.741467 & 47.51609 & 59.570784 & 65.113606 & 24.041993 \\
\hline $6.29 \mathrm{E}+09$ & 37.410048 & 46.933327 & 58.7924705 & 64.235721 & 23.813137 \\
\hline $6.39 \mathrm{E}+09$ & 37.078957 & 46.390428 & 57.948729 & 63.277127 & 23.43679 \\
\hline $6.5 \mathrm{E}+09$ & 36.721419 & 45.946087 & 57.272375 & 62.487848 & 23.108855 \\
\hline $6.6 \mathrm{E}+09$ & 36.348142 & 45.523244 & 56.701287 & 61.771262 & 22.9458905 \\
\hline $6.7 \mathrm{E}+09$ & 36.002284 & 45.07036 & 56.0639715 & 61.0357 & 22.7787795 \\
\hline $6.8 \mathrm{E}+09$ & 35.637219 & 44.611144 & 55.474683 & 60.383086 & 22.528579 \\
\hline $6.9 \mathrm{E}+09$ & 35.270912 & 44.073245 & 54.826151 & 59.6713 & 22.281624 \\
\hline $7.01 \mathrm{E}+09$ & 35.022152 & 43.545684 & 54.1324455 & 58.8664 & 22.0776195 \\
\hline $7.11 E+09$ & 34.732066 & 43.090418 & 53.3718415 & 58.000593 & 21.7663055 \\
\hline $7.21 \mathrm{E}+09$ & 34.434456 & 42.71092 & 52.733616 & 57.240327 & 21.4278895 \\
\hline $7.31 \mathrm{E}+09$ & 34.111133 & 42.392528 & 52.3590935 & 56.749472 & 21.319935 \\
\hline $7.42 \mathrm{E}+09$ & 33.831278 & 42.076428 & 52.0078685 & 56.348574 & 21.2846895 \\
\hline $7.52 \mathrm{E}+09$ & 33.506204 & 41.663061 & 51.43737 & 55.713824 & 21.0519395 \\
\hline $7.62 \mathrm{E}+09$ & 33.244085 & 41.198141 & 50.8026515 & 55.048472 & 20.7755625 \\
\hline $7.72 \mathrm{E}+09$ & 32.994037 & 40.753795 & 50.255515 & 54.411518 & 20.610887 \\
\hline $7.83 \mathrm{E}+09$ & 32.770832 & 40.349982 & 49.6697365 & 53.720828 & 20.3930085 \\
\hline $7.93 E+09$ & 32.495474 & 40.008046 & 49.0669065 & 53.008956 & 20.0802545 \\
\hline $8.03 E+09$ & 32.199478 & 39.763477 & 48.696722 & 52.584083 & 19.9413705 \\
\hline $8.13 E+09$ & 31.92592 & 39.455858 & 48.4696025 & 52.308931 & 19.935871 \\
\hline $8.23 \mathrm{E}+09$ & 31.634266 & 39.119973 & 48.073943 & 51.835473 & 19.7936055 \\
\hline $8.34 E+09$ & 31.419744 & 38.722929 & 47.445907 & 51.167963 & 19.4978215 \\
\hline $8.44 \mathrm{E}+09$ & 31.205191 & 38.355535 & 46.9321855 & 50.566455 & 19.3318015 \\
\hline $8.54 \mathrm{E}+09$ & 31.016691 & 37.935314 & 46.3903305 & 49.928873 & 19.166764 \\
\hline $8.64 E+09$ & 30.811643 & 37.677415 & 46.010229 & 49.46229 & 18.9422895 \\
\hline $8.75 \mathrm{E}+09$ & 30.550232 & 37.471428 & 45.68502 & 49.069638 & 18.7838305 \\
\hline $8.85 \mathrm{E}+09$ & 30.286264 & 37.27861 & 45.501256 & 48.86851 & 18.85845 \\
\hline $8.95 \mathrm{E}+09$ & 30.060642 & 37.052202 & 45.2745595 & 48.611676 & 18.827812 \\
\hline $9.05 \mathrm{E}+09$ & 29.913214 & 36.693508 & 44.701733 & 47.977461 & 18.5326725 \\
\hline $9.16 \mathrm{E}+09$ & 29.742407 & 36.408137 & 44.15346 & 47.361318 & 18.3251595 \\
\hline
\end{tabular}




\begin{tabular}{|c|c|c|c|c|c|}
\hline & $\mathrm{e}^{\prime \prime} @ 20 \mathrm{C}$ & $e^{\prime \prime @ 40 C}$ & @60 C & $e^{\prime \prime @ 80 C}$ & $e^{\prime \prime} @ 100 \mathrm{C}$ \\
\hline $9.26 \mathrm{E}+09$ & 29.616231 & 35.950225 & 43.6219615 & 46.750354 & 18.19340 \\
\hline $6 \mathrm{E}+09$ & 29.440022 & 35.762964 & 43.4520855 & 46.511371 & 8.073171 \\
\hline $9.46 \mathrm{E}+09$ & 29.186926 & 35.630462 & 43.2362115 & 46.265888 & 7.897058 \\
\hline $9.56 \mathrm{E}+09$ & 28.958303 & 35.537758 & 43.048995 & 46.06416 & 17.952088 \\
\hline $9.67 \mathrm{E}+09$ & 28.790797 & 35.397771 & 42.9147445 & 45.902732 & 18.068236 \\
\hline $9.77 \mathrm{E}+09$ & 28.699949 & 35.179984 & 42.6122715 & 45.545444 & 7.8495355 \\
\hline $9.87 E+09$ & 28.602922 & 34.867052 & 41.9860645 & 44.828444 & 17.5387405 \\
\hline $9.97 \mathrm{E}+09$ & 28.47496 & 34.412456 & 41.3990285 & 44.181404 & 17.390853 \\
\hline $1.01 \mathrm{E}+10$ & 28.314002 & 34.139228 & 41.275878 & 44.009094 & 17.3167155 \\
\hline $1.02 \mathrm{E}+10$ & 28.071278 & 34.047241 & 41.182798 & 43.87272 & 17.148501 \\
\hline $1.03 E+10$ & 27.864448 & 34.062169 & 41.0127095 & 43.710575 & 17.17716 \\
\hline $1.04 \mathrm{E}+10$ & 27.732956 & 34.010816 & 40.856865 & 43.545526 & 17.38213 \\
\hline $1.05 E+10$ & 27.66253 & 33.828057 & 40.643025 & 43.268449 & 17.2280095 \\
\hline $1.06 \mathrm{E}+10$ & 27.579952 & 33.426401 & 40.1171925 & 42.653936 & 16.797041 \\
\hline $1.07 \mathrm{E}+10$ & 27.500315 & 33.015656 & 39.5151955 & 41.983084 & 16.624525 \\
\hline $1.08 \mathrm{E}+10$ & 27.287632 & 32.718777 & 39.297998 & 41.715684 & 16.6495925 \\
\hline $1.09 \mathrm{E}+10$ & 27.064677 & 32.649039 & 39.3617825 & 41.792328 & 16.55516 \\
\hline $1.1 \mathrm{E}+10$ & 26.907568 & 32.826626 & 39.3992045 & 41.822225 & 16.5899605 \\
\hline $1.11 \mathrm{E}+10$ & 26.866964 & 32.894161 & 39.2466685 & 41.634846 & 16.803599 \\
\hline $1.12 \mathrm{E}+10$ & 26.913983 & 32.627854 & 38.956927 & 41.263495 & 16.671679 \\
\hline $1.13 \mathrm{E}+10$ & 26.889722 & 32.163068 & 38.536795 & 40.740885 & 16.2019 \\
\hline $1.14 \mathrm{E}+10$ & 26.747703 & 31.803995 & 38.0031825 & 40.152668 & 15.9935545 \\
\hline $1.15 \mathrm{E}+10$ & 26.536845 & 31.621745 & 37.7329955 & 39.88192 & 16.144421 \\
\hline $1.16 \mathrm{E}+10$ & 26.322304 & 31.650363 & 37.967431 & 40.148088 & 6.1522075 \\
\hline $1.17 \mathrm{E}+10$ & 26.202053 & 31.809691 & 38.1936365 & 40.393413 & 15.9805055 \\
\hline $1.18 \mathrm{E}+10$ & 26.246206 & 31.77088 & 37.856686 & 9.982751 & 5.920674 \\
\hline $1.19 \mathrm{E}+10$ & 26.303745 & 31.331913 & 37.185032 & 39.172203 & 15.786037 \\
\hline $1.2 \mathrm{E}+10$ & 26.273863 & 30.852295 & 36.785202 & 38.603531 & 15.4489475 \\
\hline $1.21 \mathrm{E}+10$ & 26.118461 & 30.711158 & 36.627879 & 38.304412 & 15.354582 \\
\hline $1.22 \mathrm{E}+10$ & 25.909407 & 30.752119 & 36.453507 & 38.122082 & 15.6517935 \\
\hline $1.23 \mathrm{E}+10$ & 25.732898 & 30.579895 & 36.319036 & 38.063192 & 15.6614085 \\
\hline $1.24 \mathrm{E}+10$ & 25.723849 & 30.309766 & 36.2551455 & 38.096372 & 15.2555735 \\
\hline $1.25 \mathrm{E}+10$ & 25.805305 & 30.30528 & 36.0880135 & 37.91864 & 15.1867245 \\
\hline $1.26 \mathrm{E}+10$ & 25.914726 & 30.067763 & 35.435636 & 37.157201 & 15.2622345 \\
\hline $1.27 \mathrm{E}+10$ & 25.844597 & 29.857289 & 35.2129465 & 36.746444 & 15.095356 \\
\hline $1.28 \mathrm{E}+10$ & 25.591004 & 29.787558 & 35.2582425 & 36.574956 & 14.857959 \\
\hline $1.29 \mathrm{E}+10$ & 25.268396 & 30.026056 & 35.2699915 & 36.440973 & 15.1992205 \\
\hline $1.3 \mathrm{E}+10$ & 25.213658 & 30.013111 & 35.014871 & 36.212366 & 15.4738135 \\
\hline $1.31 \mathrm{E}+10$ & 25.343908 & 29.83096 & 35.050196 & 36.40558 & 15.185926 \\
\hline $1.32 \mathrm{E}+10$ & 25.503019 & 29.793455 & 35.237566 & 36.774548 & 15.014243 \\
\hline $1.33 E+10$ & 25.566497 & 29.655825 & 34.9086175 & 36.490631 & 15.172809 \\
\hline $1.35 \mathrm{E}+10$ & 25.406238 & 29.159165 & 34.2207005 & 35.741207 & 14.8902835 \\
\hline $1.36 \mathrm{E}+10$ & 25.026926 & 28.905452 & 34.16168 & 35.627029 & 14.476375 \\
\hline $1.37 \mathrm{E}+10$ & 24.654523 & 29.31252 & 34.5745235 & 36.000357 & 14.878404 \\
\hline $1.38 \mathrm{E}$ & 24.641307 & 29.528274 & 34.2883675 & 35.63997 & 15.3782625 \\
\hline
\end{tabular}




\begin{tabular}{|c|c|c|c|c|c|}
\hline ency & "@20C & $e^{\prime \prime} @ 40 \mathrm{C}$ & $\mathrm{e}^{\prime \prime} @ 60 \mathrm{C}$ & $\mathrm{e}^{\prime \prime} @ 80 \mathrm{C}$ & $\mathrm{e}^{\prime \prime} @ 100 \mathrm{C}$ \\
\hline $1.39 \mathrm{E}+10$ & 24.893875 & 29.207206 & 33.8593295 & 35.177547 & $\overline{15.050277}$ \\
\hline $1.4 \mathrm{E}+10$ & 25.204089 & 29.021195 & 34.105885 & 35.475097 & 14.7654865 \\
\hline $1.41 \mathrm{E}+10$ & 25.376282 & 29.11096 & 34.370839 & 35.788003 & 15.120637 \\
\hline $1.42 \mathrm{E}+10$ & 25.261462 & 28.848944 & 33.8276595 & 35.282404 & 15.098827 \\
\hline $1.43 \mathrm{E}+10$ & 24.974203 & 28.348661 & 33.387859 & 34.902606 & 14.4873645 \\
\hline $1.44 \mathrm{E}+10$ & 24.753344 & 28.640259 & 33.8556555 & 35.31732 & 14.6382245 \\
\hline $1.45 \mathrm{E}+10$ & 24.706388 & 29.148348 & 33.9057885 & 35.117172 & 15.3134135 \\
\hline $1.46 \mathrm{E}+10$ & 24.775525 & 28.843177 & 33.1934775 & 34.227146 & 15.028755 \\
\hline $1.47 \mathrm{E}+10$ & 24.716132 & 28.231811 & 33.007314 & 34.073856 & 14.398031 \\
\hline $1.48 \mathrm{E}+10$ & 24.635007 & 28.481822 & 33.766315 & 34.869987 & 14.812527 \\
\hline $1.49 \mathrm{E}+10$ & 24.494773 & 28.685131 & 33.759319 & 34.895768 & 15.236225 \\
\hline $1.5 \mathrm{E}+10$ & 24.556948 & 27.882616 & 32.692952 & 34.011177 & 14.3341485 \\
\hline $1.51 \mathrm{E}+10$ & 25.013952 & 28.164724 & 33.126159 & 34.495787 & 14.4529075 \\
\hline $1.52 \mathrm{E}+10$ & 25.583745 & 29.262939 & 33.9706845 & 35.189311 & 15.6071295 \\
\hline $1.53 \mathrm{E}+10$ & 25.885544 & 28.63767 & 32.9152375 & 33.988045 & 14.9773625 \\
\hline $1.54 \mathrm{E}+10$ & 25.714297 & 27.406158 & 31.807754 & 32.8022 & 13.8812385 \\
\hline $1.55 \mathrm{E}+10$ & 25.05461 & 28.342149 & 33.2682185 & 34.226995 & 14.930197 \\
\hline $1.56 \mathrm{E}+10$ & 24.02931 & 28.648091 & 33.773097 & 34.888735 & 15.1907275 \\
\hline $1.57 \mathrm{E}+10$ & 23.470946 & 27.393189 & 32.0880515 & 33.341969 & 14.072959 \\
\hline $1.58 \mathrm{E}+10$ & 23.923755 & 27.874945 & 32.156656 & 33.319883 & 14.7960765 \\
\hline $1.59 \mathrm{E}+10$ & 24.648087 & 28.917443 & 33.3462355 & 34.341972 & 15.6233795 \\
\hline $1.6 \mathrm{E}+10$ & 25.295823 & 28.094079 & 32.6064925 & 33.498927 & 14.6303805 \\
\hline $1.61 \mathrm{E}+10$ & 25.716347 & 26.850351 & 30.974317 & 31.750757 & 14.1399285 \\
\hline $1.62 \mathrm{E}+10$ & 25.187369 & 27.464103 & 31.781949 & 32.637675 & 14.9276275 \\
\hline $1.63 \mathrm{E}+10$ & 23.655921 & 28.073342 & 33.363569 & 34.376437 & 14.92346 \\
\hline $1.64 \mathrm{E}+10$ & 22.510849 & 26.955571 & 31.5822495 & 32.490926 & 14.101705 \\
\hline $1.65 \mathrm{E}+10$ & 22.599646 & 26.888432 & 30.2070015 & 31.028581 & 14.370233 \\
\hline $1.66 \mathrm{E}+10$ & 23.354484 & 28.224084 & 32.080189 & 32.969311 & 15.1429215 \\
\hline $1.67 \mathrm{E}+10$ & 24.50597 & 27.605808 & 32.4483875 & 33.20218 & 14.5782665 \\
\hline $1.68 \mathrm{E}+10$ & 25.592203 & 26.112293 & 30.1125085 & 30.745765 & 14.032453 \\
\hline $1.69 \mathrm{E}+10$ & 25.560759 & 27.04799 & 30.5873525 & 31.354766 & 14.867427 \\
\hline $1.7 \mathrm{E}+10$ & 24.03171 & 27.769739 & 32.875183 & 33.767915 & 14.8961375 \\
\hline $1.71 \mathrm{E}+10$ & 22.628774 & 26.302265 & 30.980747 & 31.69091 & 13.9318515 \\
\hline $1.72 \mathrm{E}+10$ & 22.337377 & 26.577674 & 29.5289645 & 30.185102 & 14.311062 \\
\hline $1.73 \mathrm{E}+10$ & 22.681978 & 28.092196 & 31.459759 & 32.175996 & 15.087185 \\
\hline $1.74 \mathrm{E}+10$ & 23.606798 & 27.395777 & 32.406115 & 32.970682 & 14.6354625 \\
\hline $1.75 \mathrm{E}+10$ & 24.695682 & 26.107674 & 30.4043745 & 30.902483 & 14.350418 \\
\hline $1.76 \mathrm{E}+10$ & 24.929547 & 26.932275 & 30.2753525 & 30.956053 & 14.869327 \\
\hline $1.77 \mathrm{E}+10$ & 23.978981 & 27.509488 & 32.2600205 & 32.98493 & 14.827998 \\
\hline $1.79 \mathrm{E}+10$ & 22.861029 & 26.811055 & 31.79356 & 32.400484 & 14.5035125 \\
\hline $1.8 \mathrm{E}+10$ & 22.272668 & 26.913924 & 30.0770715 & 30.773203 & 14.7265675 \\
\hline $1.81 \mathrm{E}+10$ & 22.36744 & 27.657438 & 30.8122595 & 31.53222 & 15.004037 \\
\hline $1.82 \mathrm{E}+10$ & 23.061457 & 27.267084 & 32.501902 & 33.056959 & 14.7779375 \\
\hline $1.83 \mathrm{E}+10$ & 23.869412 & 26.370051 & 30.949899 & 31.395933 & 14.6154985 \\
\hline $1.84 \mathrm{E}+10$ & 24.172317 & 26.738239 & 29.771 & 068 & 14.7 \\
\hline
\end{tabular}




\begin{tabular}{|r|r|r|r|r|r|}
\hline Frequency & e" @ 20 C & e" @ 40 C & e" @ 60 C & e" @ 80 C & e" @ 100 C \\
\hline \hline $1.85 \mathrm{E}+10$ & 23.589673 & 27.329644 & 31.6625905 & 32.182817 & 14.844439 \\
\hline $1.86 \mathrm{E}+10$ & 22.81171 & 26.912427 & 31.61948 & 32.061017 & 14.759092 \\
\hline $1.87 \mathrm{E}+10$ & 22.320029 & 26.722385 & 29.887402 & 30.464092 & 14.8175705 \\
\hline $1.88 \mathrm{E}+10$ & 22.309703 & 27.093748 & 30.3664055 & 30.987106 & 14.8670445 \\
\hline $1.89 \mathrm{E}+10$ & 22.81679 & 26.800999 & 31.5774325 & 32.04398 & 14.720112 \\
\hline $1.9 \mathrm{E}+10$ & 23.411189 & 26.075542 & 30.126293 & 30.576378 & 14.537221 \\
\hline $1.91 \mathrm{E}+10$ & 23.631723 & 26.333745 & 29.4882405 & 29.948235 & 14.5839815 \\
\hline $1.92 \mathrm{E}+10$ & 23.317876 & 26.935937 & 30.6924825 & 31.046792 & 14.735682 \\
\hline $1.93 \mathrm{E}+10$ & 22.842097 & 26.700238 & 30.387727 & 30.742161 & 14.773946 \\
\hline $1.94 \mathrm{E}+10$ & 22.591153 & 26.380121 & 29.5448165 & 30.018742 & 14.7451895 \\
\hline $1.95 \mathrm{E}+10$ & 22.631934 & 26.45758 & 30.016391 & 30.571059 & 14.7370465 \\
\hline $1.96 \mathrm{E}+10$ & 22.929888 & 26.255989 & 30.3751035 & 30.922433 & 14.690952 \\
\hline $1.97 \mathrm{E}+10$ & 23.257257 & 25.900598 & 29.810116 & 30.28403 & 14.620394 \\
\hline $1.98 \mathrm{E}+10$ & 23.229836 & 26.00587 & 29.529999 & 29.912025 & 14.607857 \\
\hline $1.99 \mathrm{E}+10$ & 22.883953 & 26.517957 & 29.83353 & 30.111817 & 14.688799 \\
\hline $2 \mathrm{E}+10$ & 22.563887 & 26.580824 & 29.799062 & 30.003524 & 14.767238 \\
\hline
\end{tabular}


Table 12. Loss Tangent for SAIC Simulant

\begin{tabular}{|c|c|c|c|c|c|}
\hline icy & $\bar{C}$ & $\overline{\mathrm{C}}$ & & & \\
\hline $5 E+08$ & 25.9492533 & 10.5000095 & 11.9390631 & 537 & 34 \\
\hline $3.58 \mathrm{E}+08$ & 5.2666422 & 9.07670096 & 10.478406 & 11.35905 & 7.353837253 \\
\hline $4.6 \mathrm{E}+08$ & 11.4457942 & 8.00705516 & 9.34191386 & 10.18248 & 6.706306245 \\
\hline $5.62 E+08$ & 9.17948641 & 7.18641346 & 8.45469812 & 9.267171 & 6.155026456 \\
\hline $6.65 E+08$ & 7.66068495 & 6.44686653 & 7.62787636 & 8.40251 & 5.689418579 \\
\hline $7.67 \mathrm{E}+08$ & 6.52301607 & 5.83293793 & 6.9426845 & 7.678415 & 5.269208676 \\
\hline $8.69 E+08$ & 5.62110912 & 5.27242451 & 6.2883918 & 6.968006 & 4.840076901 \\
\hline $9.71 \mathrm{E}+08$ & 5.05351135 & 4.894559 & 5.86384275 & 6.530218 & 4.582865097 \\
\hline $1.07 \mathrm{E}+09$ & 4.57147432 & 4.55965734 & 5.49101556 & 6.140807 & 4.349949568 \\
\hline $1.18 \mathrm{E}+09$ & 4.16236855 & 4.27038512 & 5.15992139 & 5.78978 & 4.13034819 \\
\hline $1.28 \mathrm{E}+09$ & 3.84498224 & 4.02438014 & 4.8658987 & 5.472181 & 3.935766 \\
\hline $1.38 \mathrm{E}+09$ & 3.57179878 & 3.78990547 & 4.57930344 & 528 & 3.749329563 \\
\hline $1.48 \mathrm{E}+09$ & 3.31692478 & 3.56392616 & 4.31099571 & 4.864915 & 3.571829814 \\
\hline $1.59 \mathrm{E}+09$ & 3.0942455 & 3.37200522 & 4.08768831 & 4.625338 & 3.399079777 \\
\hline $1.69 \mathrm{E}+09$ & 2.9139651 & 3.21219165 & 3.89606299 & 4.41679 & 3.264179577 \\
\hline $1.79 \mathrm{E}+09$ & 2.75112092 & 3.0770922 & 3.73645875 & 4.243609 & 3.162964943 \\
\hline $1.89 \mathrm{E}+09$ & 2.61505828 & 2.95228854 & 3.58973816 & 4.082352 & 3.062548364 \\
\hline $1.99 \mathrm{E}+09$ & 2.48717149 & 2.81823816 & 3.41937554 & 3.889352 & 2.934370319 \\
\hline $2.1 \mathrm{E}+09$ & 2.36914873 & 2.68868599 & 3.25277805 & 3.699169 & 2.817963822 \\
\hline $2.2 \mathrm{E}+09$ & .2 .26683584 & 2.59116335 & 3.13709208 & 3.573836 & 2.740735774 \\
\hline $2.3 \mathrm{E}+09$ & 2.17565205 & 2.50988384 & 3.04520356 & 3.477053 & 2.671095127 \\
\hline $2.4 E+09$ & 2.09819766 & 2.43037176 & 2.94885528 & 3.372047 & 2.590451833 \\
\hline $2.51 \mathrm{E}+09$ & 2.03607919 & 2.35721062 & 2.85738223 & 3.270014 & 2.531424932 \\
\hline $2.61 E+09$ & 1.96537527 & 2.28468758 & 2.76937662 & 3.170414 & 2.470056806 \\
\hline $2.71 \mathrm{E}+09$ & 1.90147382 & 2.22094294 & 2.68951735 & 3.080275 & 2.409014593 \\
\hline $2.81 E+09$ & 1.84496513 & 2.15935215 & 2.60948497 & 2.989764 & 2.346804001 \\
\hline $2.92 \mathrm{E}+09$ & 1.79252567 & 2.1019652 & 2.53799912 & 2.911078 & 2.302296453 \\
\hline $3.02 \mathrm{E}+09$ & 1.74399911 & 2.04824774 & 2.47686537 & 2.844536 & 2.253939558 \\
\hline $3.12 \mathrm{E}+09$ & 1.70467192 & 2.00056395 & 2.42195974 & 2.784427 & 2.204918274 \\
\hline $3.22 \mathrm{E}+09$ & 1.66616387 & 1.95456719 & 2.36175061 & 2.715037 & 2.160688747 \\
\hline $3.32 \mathrm{E}+09$ & 1.62345649 & 1.90635749 & 2.29857442 & 2.641101 & 2.123703418 \\
\hline $3.43 \mathrm{E}+09$ & 1.58122561 & 1.86036982 & 2.24115138 & 2.575486 & 2.078363742 \\
\hline $3.53 \mathrm{E}+09$ & 1.54321707 & 1.81750958 & 2.18661653 & 2.512626 & 2.028693754 \\
\hline $3.63 E+09$ & 1.50867172 & 1.77834743 & 2.1356675 & 2.4539 & 1.993271021 \\
\hline $3.73 E+09$ & 1.48243767 & 1.74743352 & 2.097755 & 2.412336 & 1.972868443 \\
\hline $3.84 \mathrm{E}+09$ & 1.45552471 & 1.71686249 & 2.06406801 & 2.373781 & 1.938447776 \\
\hline $3.94 E+09$ & 1.43018788 & 1.6856523 & 2.02499884 & 2.328745 & 1.903605987 \\
\hline $4.04 \mathrm{E}+09$ & 1.40592561 & 1.6535637 & 1.98057878 & 2.275418 & 1.87937744 \\
\hline $4.14 \mathrm{E}+09$ & 1.38107447 & 1.62492876 & 1.94225508 & 2.230064 & 1.856378301 \\
\hline $4.25 E+09$ & 1.35904933 & 1.60051533 & 1.91308697 & 2.19713 & 1.827476883 \\
\hline $4.35 \mathrm{E}+09$ & 1.34191354 & 1.5798007 & 1.88612652 & 2.167677 & 1.80582154 \\
\hline $4.45 \mathrm{E}+09$ & 1.32720351 & 1.56110188 & 1.86138795 & 2.138766 & 1.794916904 \\
\hline $4.55 E+09$ & 1.31129042 & 1.54239121 & 1.83853588 & 2.112411 & 1.775279017 \\
\hline
\end{tabular}




\begin{tabular}{|c|c|c|c|c|c|}
\hline requency & $1 / e^{\prime} 20 \mathrm{C}$ & $e^{11 / e^{9} 40 \mathrm{C}}$ & $e^{\prime \prime} / e^{\prime} 60 c$ & $/ e^{\prime} 80 \mathrm{C}$ & $e^{\prime \prime} / e^{\prime} 100 \mathrm{C}$ \\
\hline+09 & 33311 & 21357 & 14206 & 8338 & 200 \\
\hline $4.76 \mathrm{E}+09$ & 1.27869118 & 1.50149696 & 1.78620879 & 2.051377 & 1.731885752 \\
\hline $4.86 \mathrm{E}+09$ & 1.26391714 & i. 48134028 & 1.75798178 & 2.01818 & 1.721947538 \\
\hline $4.96 \mathrm{E}+09$ & 1.25183453 & 1.46526716 & 1.73777232 & 1.99732 & 1.704044001 \\
\hline $5.06 \mathrm{E}+09$ & 1.2420165 & 1.45290853 & 1.72281258 & 1.980441 & 1.686987942 \\
\hline $5.17 \mathrm{E}+09$ & 1.23140146 & 1.44016229 & 1.70551974 & 1.959472 & 1.680584998 \\
\hline $5.27 \mathrm{E}+09$ & 1.21736435 & 1.42565645 & 1.68675708 & 1.937057 & 1.667168273 \\
\hline $5.37 \mathrm{E}+09$ & 1.20274004 & 1.40889491 & 1.66676048 & 1.914169 & 1.642934019 \\
\hline $5.47 \mathrm{E}+09$ & 1.19051362 & 1.39148217 & 1.64524299 & 1.888635 & 1.626929596 \\
\hline $5.57 \mathrm{E}+09$ & 1.18188346 & 1.37556947 & 1.62182886 & 1.860695 & 1.622671683 \\
\hline $5.68 \mathrm{E}+09$ & 1.17299398 & 1.3666016 & 1.60761075 & 1.844439 & 1.616105718 \\
\hline $5.78 E+09$ & 1.16588434 & 1.35777024 & 1.59763596 & 1.834171 & 1.59844353 \\
\hline $5.88 E+09$ & 1.1571924 & 1.34689666 & 1.58499905 & 1.818522 & 1.590091859 \\
\hline 5.98 & 1.14629017 & 1.33423686 & 1.56760538 & 1.797225 & 1.581355073 \\
\hline $6.09 E+09$ & 1.13489739 & 1.32026116 & 1.54841697 & 1.774193 & 1.561230697 \\
\hline $6.19 \mathrm{E}+09$ & 1.12596163 & 1.30626008 & 1.53033273 & 1.753136 & 1.547181753 \\
\hline $6.29 \mathrm{E}+09$ & 1.12307 & 1.29596992 & 1.51646932 & 1.735185 & 1.548735542 \\
\hline $6.39 E+09$ & 1.1191701 & 1.28908575 & 1.50311063 & 1.720178 & 1.545768466 \\
\hline $6.5 \mathrm{E}+09$ & 1.11347971 & 1.28422452 & 31676 & 1.709196 & 1.529381193 \\
\hline $6.6 \mathrm{E}+09$ & 1.10500546 & 1.27884975 & 1.49047114 & 1.704371 & 1.521101993 \\
\hline $6.7 \mathrm{E}+09$ & 1.09602518 & 1.26794721 & 17989 & 1.688241 & 1.518011138 \\
\hline $6.8 \mathrm{E}+09$ & 1.08779798 & 1.25537436 & 1.45922871 & 1.665914 & 1.504930578 \\
\hline $6.9 \mathrm{E}+09$ & 1.0824431 & 1.24358013 & 12923 & 1.643789 & 1.487422053 \\
\hline $7.01 E+09$ & 1.08077624 & 1.2338698 & 1.42727834 & 1.627238 & 1.486038166 \\
\hline $7.11 E+09$ & 1.07824374 & 88206 & 66219 & 614093 & 4793 \\
\hline $7.21 \mathrm{E}+09$ & 1.07272931 & 1.22526083 & 1.41058999 & 1.606048 & 1.470773393 \\
\hline $7.31 \mathrm{E}+09$ & 1.06292351 & 1.22132364 & 1.40829555 & 1.602634 & 1.458020175 \\
\hline $7.42 \mathrm{E}+09$ & 1.056036 & 1.21557299 & 1.40535798 & 1.599581 & 1.463191182 \\
\hline $7.52 \mathrm{E}+09$ & 1.05020768 & 1.20520172 & 1.39026126 & 1.580749 & 1.460127404 \\
\hline $7.62 \mathrm{E}+09$ & 1.04735099 & 1.19326598 & 1.36859424 & 1.555605 & 1.44084488 \\
\hline $7.72 \mathrm{E}+09$ & 1.04593126 & 1.18448043 & 1.35633542 & 1.539525 & 1.433783879 \\
\hline $7.83 \mathrm{E}+09$ & 1.04339993 & 1.18023142 & 1.35291551 & 1.535283 & 1.438564257 \\
\hline $7.93 \mathrm{E}+09$ & 1.0363171 & 1.17808612 & 1.34879786 & 1.529922 & 1.427042793 \\
\hline $8.03 \mathrm{E}+09$ & 1.02635732 & 1.17544522 & 1.34400627 & 1.523476 & 1.409829634 \\
\hline $8.13 E+09$ & 1.01793906 & 1.16905021 & 1.33979908 & 1.519076 & 1.40964194 \\
\hline $8.23 E+09$ & 1.01481338 & 1.15816619 & 1.32722989 & 1.502141 & 1.411399341 \\
\hline $8.34 \mathrm{E}+09$ & 1.0143533 & 1.14631367 & 1.30486537 & 1.475149 & 1.391124795 \\
\hline $8.44 \mathrm{E}+09$ & 1.01296902 & 1.13935443 & 1.29102288 & 1.458023 & 1.376834703 \\
\hline $8.54 \mathrm{E}+09$ & 1.00771648 & 1.13353445 & 1.28726275 & 1.453354 & 1.378385255 \\
\hline $8.64 \mathrm{E}+09$ & 1.00037586 & 1.13168028 & 1.28954394 & 1.455261 & 1.376625279 \\
\hline $8.75 \mathrm{E}+09$ & 0.99193178 & 1.12983338 & 1.28555804 & 1.450264 & 1.359423477 \\
\hline $8.85 E+09$ & 0.986382 & 1.12371672 & 1.27486311 & 1.437039 & 1.356641916 \\
\hline $8.95 \mathrm{E}+09$ & 0.98427312 & 1.11535311 & 1.26438588 & 1.423447 & 1.367119163 \\
\hline $9.05 \mathrm{E}+09$ & 0.98598473 & 1.10240275 & 1.24459154 & 1.398539 & 1.351690418 \\
\hline $9.16 \mathrm{E}+09$ & 0.98385445 & 1.09794011 & 1.23168488 & 1.383016 & 1.332268025 \\
\hline
\end{tabular}




\begin{tabular}{|c|c|c|c|c|c|}
\hline ency & $/ e^{\prime} 20 \mathrm{C}$ & $" / e^{t} 40 \mathrm{C}$ & $e^{\prime \prime} / e^{\prime} 60 \mathrm{C}$ & $e^{\prime \prime} / e^{\prime} 80 \mathrm{C}$ & $e^{e / / e}$ \\
\hline 09 & 3 & 6869 & 2807 & 1332 & 60 \\
\hline $9.36 \mathrm{E}+09$ & 0.97217004 & 1.09185733 & 1.23423531 & 1.386557 & 1.337002677 \\
\hline $9.46 \mathrm{E}+09$ & 0.96445242 & 1.09186649 & 1.23473582 & 1.385586 & 1.324746381 \\
\hline $9.56 \mathrm{E}+09$ & 0.95987542 & 1.08853071 & 1.22047863 & 1.368083 & 1.31814072 \\
\hline $9.67 \mathrm{E}+09$ & 0.96075804 & 1.08086589 & 1.20643207 & 1.349734 & 1.333641717 \\
\hline $9.77 \mathrm{E}+09$ & 0.96361608 & 1.07053853 & 1.19638937 & 1.336393 & 1.330454768 \\
\hline $9.87 \mathrm{E}+09$ & 0.96091971 & 1.06382751 & 1.18345617 & 1.321811 & 1.303394416 \\
\hline $9.97 \mathrm{E}+09$ & 0.95475504 & 1.05870017 & 1.17729095 & 1.316334 & 1.29111462 \\
\hline $1.01 \mathrm{E}+10$ & 0.94779861 & 1.05886694 & 1.18626973 & 1.326619 & 1.303050586 \\
\hline $1.02 \mathrm{E}+10$ & 0.94064248 & 1.05925027 & 1.18965204 & 1.328428 & 1.298029276 \\
\hline $1.03 E+10$ & 0.93760673 & 1.05998978 & 1.1788627 & 1.314109 & 1.28948698 \\
\hline $1.04 \mathrm{E}+10$ & 0.939393 & 1.05528616 & 1.16167382 & 1.293221 & 1.306799679 \\
\hline $1.05 \mathrm{E}+10$ & 0.94079796 & 1.04605103 & 1.15316101 & 1.282399 & 1.314800831 \\
\hline $1.06 \mathrm{E}+10$ & 0.93816942 & 1.03457445 & 1.14693105 & 1.27459 & 1.279572697 \\
\hline $1.07 \mathrm{E}+10$ & 0.93108143 & 1.0306713 & 1.14261255 & 1.271162 & 1.256981123 \\
\hline $1.08 \mathrm{E}+10$ & 0.9230279 & 1.02897605 & 1.14188339 & 1.270381 & 1.268576868 \\
\hline $1.09 \mathrm{E}+10$ & 0.91827378 & 1.03101636 & 1.14703762 & 1.274095 & 1.275311472 \\
\hline $1.1 \mathrm{E}+10$ & 0.91871913 & 1.03764979 & 1.14719516 & 1.271827 & 1.273992175 \\
\hline $1.11 \mathrm{E}+10$ & 0.92353194 & 1.03986436 & 1.13564769 & 1.255212 & 1.291478338 \\
\hline $1.12 \mathrm{E}+10$ & 0.92781016 & 1.03074317 & 1.12511723 & 1.242473 & 1.301233921 \\
\hline $1.13 \mathrm{E}+10$ & 0.9252959 & 1.01609578 & 1.12354873 & 1.242551 & 1.265641534 \\
\hline $1.14 \mathrm{E}+10$ & 0.91609862 & 1.00866675 & 1.11907738 & 1.239201 & 1.230033377 \\
\hline $1.15 \mathrm{E}+10$ & 0.90786781 & 1.00937378 & 1.11163015 & 1.230911 & 1.244759966 \\
\hline $1.16 \mathrm{E}+10$ & 0.90539821 & 1.01859404 & 1.11794521 & 1.237443 & 1.278146185 \\
\hline $1.17 E+10$ & 0.90865186 & 1.03173948 & 1.13269004 & 1.251827 & 1.279969184 \\
\hline $1.18 \mathrm{E}+10$ & 0.91487633 & 1.03352966 & 1.12832451 & 1.244475 & 1.271798722 \\
\hline $1.19 \mathrm{E}+10$ & 0.91779473 & 1.02071969 & 1.10821204 & 1.21842 & 1.270341951 \\
\hline $1.2 \mathrm{E}+10$ & 0.91435979 & 0.99597969 & 1.09198308 & 1.199026 & 1.235166844 \\
\hline $1.21 \mathrm{E}+10$ & 0.90590428 & 0.98609083 & 1.09124126 & 1.197433 & 1.200319839 \\
\hline $1.22 \mathrm{E}+10$ & 0.89890345 & 0.99466735 & 1.09025172 & 1.198357 & 1.223020476 \\
\hline $1.23 \mathrm{E}+10$ & 0.89961708 & 0.99746453 & 1.08240202 & 1.191153 & 1.25321671 \\
\hline $1.24 E+10$ & 0.90675408 & 0.98644991 & 1.07742902 & 1.186915 & 1.215118267 \\
\hline $1.25 \mathrm{E}+10$ & 0.91457177 & 0.97807288 & 1.07283541 & 1.178595 & 1.178321662 \\
\hline $1.26 \mathrm{E}+10$ & 0.91470166 & 0.97123849 & 1.05397635 & 1.151037 & 1.187063178 \\
\hline $1.27 \mathrm{E}+10$ & 0.90944978 & 0.96633069 & 1.04433458 & 1.13321 & 1.199203071 \\
\hline $1.28 \mathrm{E}+10$ & 0.89988287 & 0.96695974 & 1.05101453 & 1.132522 & 1.176359899 \\
\hline $1.29 \mathrm{E}+10$ & 0.89166393 & 0.98047504 & 1.06026771 & 1.137833 & 1.196025208 \\
\hline $1.3 \mathrm{E}+10$ & 0.89716239 & 0.98700951 & 1.05282349 & 1.131067 & 1.246072337 \\
\hline $1.31 \mathrm{E}+10$ & 0.90845786 & 0.97559454 & 1.04406805 & 1.12892 & 1.226168019 \\
\hline $1.32 \mathrm{E}+10$ & 0.91590489 & 0.96349228 & 1.04733414 & 1.140916 & 1.17333804 \\
\hline $1.33 \mathrm{E}+10$ & 0.91262009 & 0.9631643 & 1.04784197 & 1.140739 & 1.185827846 \\
\hline $1.35 \mathrm{E}+10$ & 0.90234634 & 0.95750362 & 1.02362255 & 1.10612 & 1.194920193 \\
\hline $1.36 \mathrm{E}+10$ & 0.88909366 & 0.94968153 & 1.00948026 & 1.081308 & 1.155309345 \\
\hline $1.37 \mathrm{E}+10$ & 0.88125571 & 0.96533737 & 1.03037367 & 1.10023 & 1.168719772 \\
\hline $1.38 \mathrm{E}+10$ & 0.89145178 & 0.98416336 & 1.03782533 & 1.110301 & 1.24357 \\
\hline
\end{tabular}




\begin{tabular}{|c|c|c|c|c|c|}
\hline & & & & & \\
\hline$E+10$ & 90944352 & 0.96988744 & 1.01894139 & 5185 & 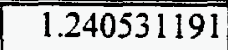 \\
\hline+10 & 9235 & 95032551 & 1.02055247 & 102946 & 1.17703230 \\
\hline $1.41 E+10$ & 92394209 & 0.9549735 & 1.0439246 & 1.13185 & 1.1907296 \\
\hline $1.42 \mathrm{E}+10$ & 0.91137037 & 0.96436314 & 1.03779737 & 1.122032 & 1.238412267 \\
\hline $1.43 \mathrm{E}+10$ & 0.89576559 & 0.95038652 & 1.00409421 & .079711 & 1.191358612 \\
\hline $1.44 \mathrm{E}+10$ & 0.88894205 & 0.95229621 & 01106482 & 082888 & 158751509 \\
\hline $1.45 \mathrm{E}+10$ & 0.89658437 & 0.97686731 & 1.03721145 & .106171 & 1.23676611 \\
\hline $1.46 \mathrm{E}+10$ & 3081295 & 0.97607956 & 02665782 & 1.09326 & 1.272587764 \\
\hline $1.47 \mathrm{E}+10$ & 0.9119628 & 0.93863765 & 1.00031888 & 070843 & 1.174342124 \\
\hline $1.48 \mathrm{E}+10$ & 39 & 41 & 2138636 & 95895 & 160152528 \\
\hline $1.49 \mathrm{E}+10$ & 0.91401577 & 0.97630196 & 04969937 & .122946 & 1.273094073 \\
\hline $1.5 \mathrm{E}+10$ & 22 & 302 & 496 & .077481 & .224538885 \\
\hline $1.51 \mathrm{E}+10$ & 0.95407769 & 0.94247831 & 0.98452011 & 1.054042 & 1.127503055 \\
\hline $1.52 \mathrm{E}+10$ & 8260778 & 98373802 & 1.02990617 & 1.097215 & 1.242289988 \\
\hline $1.53 \mathrm{E}+10$ & 0.98597062 & 0.99337381 & 1.04295171 & 1.111703 & 1.313736428 \\
\hline $1.54 \mathrm{E}+10$ & 0.95249198 & 0.92009191 & 0.97524389 & 1.039233 & 1.11347665 \\
\hline $1.55 \mathrm{E}+10$ & 0.91377089 & 0.93167052 & 0.99311733 & 1.054368 & 1.129479114 \\
\hline $1.56 \mathrm{E}+10$ & 0.88938766 & 0.99860378 & 1.06112471 & 1.126607 & 1.304062789 \\
\hline $1.57 \mathrm{E}+10$ & 9582955 & 0.9665689 & 1.0191647 & 1.086883 & 1.205656949 \\
\hline $1.58 \mathrm{E}+10$ & 0.95221097 & 0.9511406 & 0.98257793 & 1.045383 & 1.16108117 \\
\hline $1.59 \mathrm{E}+10$ & 1478022 & 99896045 & 1.03182864 & 1.091115 & 1.316692338 \\
\hline $1.6 \mathrm{E}+10$ & 1.03036255 & 0.9949968 & 1.06081143 & 1.125034 & 1.302524562 \\
\hline $1.61 \mathrm{E}+10$ & 9506029 & 92433143 & 0.98953398 & 1.047392 & 157973719 \\
\hline $1.62 \mathrm{E}+10$ & 0.93865037 & 0.92516606 & 0.96378498 & 1.018117 & 1.182706206 \\
\hline-10 & 65 & 624 & 1.04971305 & 1.115573 & 1.29951562 \\
\hline $1.64 \mathrm{E}+10$ & 35821207 & 0.9766757 & 1.04386419 & 1.109752 & 1.249965874 \\
\hline $1.65 \mathrm{E}+10$ & 0351117 & 2892271 & 0.94520301 & 0.997234 & 1.195931917 \\
\hline $1.66 \mathrm{E}+10$ & 7800067 & 6954318 & 0.97596652 & 1.028518 & 1.288783023 \\
\hline $1.67 \mathrm{E}+10$ & 1.02443369 & 9049812 & 1.06465574 & 1.124909 & 1.311168813 \\
\hline $1.68 \mathrm{E}+10$ & 1.02313583 & 91492915 & 0.98828368 & 1.039491 & 1.189737052 \\
\hline $1.69 \mathrm{E}+10$ & 7215472 & 0.91600173 & 0.92741813 & 0.973896 & 1.21598667 \\
\hline $1.7 \mathrm{E}+10$ & .89335377 & 99585236 & 1.0294446 & 1.08878 & 1.321950269 \\
\hline $1.71 \mathrm{E}+10$ & 0.8528972 & 95825864 & 1.03114229 & 1.087124 & 1.229290759 \\
\hline $1.72 \mathrm{E}+10$ & 0.88817615 & 0.91755843 & 0.9310669 & 0.973191 & 1.192115876 \\
\hline $1.73 \mathrm{E}+10$ & 0.95522906 & 0.97462692 & 0.96184415 & 1.00749 & 1.31147992 \\
\hline $1.74 \mathrm{E}+10$ & 1.01316288 & 0.98772439 & 1.05523055 & 1.106238 & 1.328703566 \\
\hline $1.75 \mathrm{E}+10$ & 1.0340156 & 0.91908184 & 1.00166836 & 1.043491 & 1.244653286 \\
\hline $1.76 \mathrm{E}+10$ & 99995275 & 0.93547351 & 0.93782679 & 0.979432 & 1.285124798 \\
\hline $1.77 \mathrm{E}+10$ & 0.92970647 & 1.00131074 & 1.0062738 & 1.056149 & 1.340499783 \\
\hline $1.79 \mathrm{E}+10$ & 0.88637914 & 0.98057965 & 1.05151724 & 1.10018 & 1.287141056 \\
\hline $1.8 \mathrm{E}+10$ & 0.8991127 & 0.94940156 & 0.97614403 & 1.020898 & 1.273530732 \\
\hline $1.81 \mathrm{E}+10$ & 0.94372235 & 0.97537204 & 0.95390689 & 0.997409 & 1.334284244 \\
\hline $1.82 \mathrm{E}+10$ & 0.99115269 & 0.99494634 & 1.04903979 & 1.09325 & 1.351171052 \\
\hline $1.83 E+10$ & 1.0130 & 0.96049008 & 1.04774273 & 1.081175 & 1.322060084 \\
\hline 1.075 .10 & 0.59052047 & 0.958697 & 0.94764115 & 0.978902 & 1.332416663 \\
\hline
\end{tabular}




\begin{tabular}{|c|c|c|c|c|c|}
\hline$\overline{\text { acy }}$ & $e^{\prime \prime} / e^{\prime} 20 \mathrm{C}$ & $e^{\prime \prime} / e^{t} 40 C$ & $e^{\prime \prime} / e^{\prime} 60 \mathrm{C}$ & $e^{\prime \prime} / e^{\prime} 80 \mathrm{C}$ & $/ e^{\prime} 100 \mathrm{C}$ \\
\hline $1.85 \mathrm{E}+10$ & 0.9421982 & 1.00887685 & 0.99615759 & 1.031129 & 1.36139590 \\
\hline $1.86 \mathrm{E}+10$ & 0.9131809 & 1.00929546 & 1.07405321 & 1.111836 & 1.353091208 \\
\hline $1.87 E+10$ & $0.9181690 \mathrm{i}$ & 0.974350015 & 1.00498509 & 1.044343 & 1.351067297 \\
\hline $1.88 \mathrm{E}+10$ & 0.94451724 & 0.97811331 & 0.96597268 & 1.004942 & 1.375086925 \\
\hline $1.89 \mathrm{E}+10$ & 0.97931868 & 0.99431191 & 1.04228747 & 1.077226 & 1.376770936 \\
\hline $1.9 \mathrm{E}+10$ & 0.99697463 & 0.97294898 & 1.0279803 & 1.057364 & 1.357842657 \\
\hline $1.91 E+10$ & 0.98826342 & 0.96972279 & 0.95726764 & 0.985218 & 1.357746185 \\
\hline $1.92 \mathrm{E}+10$ & 0.96287844 & 1.00881141 & 0.99758901 & 1.026149 & 1.377658121 \\
\hline $1.93 \mathrm{E}+10$ & 0.94850232 & 1.01281105 & 1.0365188 & 1.068996 & 1.386672261 \\
\hline $1.94 \mathrm{E}+10$ & 0.95437239 & 0.98318526 & 0.99942593 & 1.034952 & 1.3968769 \\
\hline $1.95 \mathrm{E}+10$ & 0.97254865 & 0.97425215 & 0.98664438 & 1.021735 & 1.406950545 \\
\hline $1.96 \mathrm{E}+10$ & 0.9889431 & 0.98141915 & 1.01223393 & 1.044749 & 1.403793398 \\
\hline $1.97 \mathrm{E}+10$ & 0.99702243 & 0.98397656 & 1.00601995 & 1.032453 & 1.40343147 \\
\hline $1.98 \mathrm{E}+10$ & 0.98882915 & 0.98517622 & 0.98482962 & 1.010245 & 1.411905735 \\
\hline $1.99 \mathrm{E}+10$ & 0.97488605 & 1.0052274 & 1.00161613 & 1.027802 & 1.428254487 \\
\hline $2 \mathrm{E}+10$ & 0.96983445 & 1.01314789 & 1.01627566 & 1.042639 & 1.436955762 \\
\hline
\end{tabular}


Table 12. Dielectric Constant for Revised ORNL Simulant

\begin{tabular}{|c|c|c|c|c|c|}
\hline Frequency & $e^{\prime} @ 20 \mathrm{C}$ & $e^{\prime} @ 40 \mathrm{C}$ & $\mathrm{e}^{\prime} @ 60 \mathrm{C}$ & $e^{\prime} @ 80 \mathrm{C}$ & $e^{\prime} @ 100 \mathrm{C}$ \\
\hline $1.53 \mathrm{E}+08$ & 149.2546 & 331.820586 & 361.9038345 & 413.62872 & 319.8025505 \\
\hline $2.55 \mathrm{E}+08$ & 92.67089 & 161.706909 & 171.179664 & 190.02621 & 146.736212 \\
\hline $3.58 \mathrm{E}+08$ & 71.82076 & 105.777244 & 109.001693 & 118.19282 & 90.3036955 \\
\hline $4.6 \mathrm{E}+08$ & 62.71913 & 84.3213445 & 85.5290475 & 92.431052 & 69.273634 \\
\hline $5.62 \mathrm{E}+08$ & 55.1151 & 69.6533045 & 69.151175 & 73.655754 & 54.838466 \\
\hline $6.65 E+08$ & 51.52201 & 61.949908 & 60.73341 & 65.035228 & 47.546334 \\
\hline $7.67 \mathrm{E}+08$ & 48.81211 & 55.6133065 & 53.583508 & 56.361606 & 41.308024 \\
\hline $8.69 E+08$ & 46.38217 & 50.424727 & 47.780255 & 49.439908 & 36.129562 \\
\hline $9.71 \mathrm{E}+08$ & 44.84514 & 47.2470825 & 44.2360805 & 45.171511 & 32.895665 \\
\hline $1.07 \mathrm{E}+09$ & 43.37706 & 44.296099 & 40.9386465 & 41.523294 & 29.9938445 \\
\hline $1.18 \mathrm{E}+09$ & 41.91212 & 42.2178145 & 38.567422 & 38.90574 & 28.009263 \\
\hline $1.28 \mathrm{E}+09$ & 40.83412 & 40.0718245 & 36.2509325 & 36.560287 & 26.2069295 \\
\hline $1.38 \mathrm{E}+09$ & 40.07635 & 39.3472415 & 35.5205205 & 35.825047 & 25.669413 \\
\hline $1.48 \mathrm{E}+09$ & 39.64219 & 37.829124 & 33.855085 & 33.614626 & 24.1423735 \\
\hline $1.59 \mathrm{E}+09$ & 39.08533 & 36.989339 & 32.879158 & 32.31099 & 23.15205 \\
\hline $1.69 \mathrm{E}+09$ & 38.40299 & 36.021852 & 31.8895145 & 31.21266 & 22.201082 \\
\hline $1.79 \mathrm{E}+09$ & 37.80552 & 35.220085 & 31.038601 & 30.453199 & 21.5685895 \\
\hline $1.89 \mathrm{E}+09$ & 37.29735 & 34.2510635 & 29.9244185 & 29.215052 & 20.851459 \\
\hline $1.99 \mathrm{E}+09$ & 36.90479 & 33.551443 & 29.2255065 & 28.665031 & 20.304853 \\
\hline $2.1 \mathrm{E}+09$ & 36.89949 & 33.31349 & 28.9917475 & 28.299257 & 20.0467305 \\
\hline $2.2 \mathrm{E}+09$ & 36.5758 & 32.90656 & 28.637071 & 27.748455 & 19.524731 \\
\hline $2.3 \mathrm{E}+09$ & 36.26129 & 32.4776075 & 28.1090865 & 26.836217 & 19.0061065 \\
\hline $2.4 \mathrm{E}+09$ & 35.85531 & 32.0199145 & 27.5422315 & 26.226628 & 18.648158 \\
\hline $2.51 E+09$ & 35.46265 & 31.447682 & 27.01232 & 26.027104 & 18.284863 \\
\hline $2.61 E+09$ & 35.14452 & 30.841021 & 26.344903 & 25.5074 & 17.8056995 \\
\hline $2.71 E+09$ & 34.83513 & 30.466429 & 26.005588 & 25.091393 & 17.542597 \\
\hline $2.81 \mathrm{E}+09$ & 34.66349 & 30.183925 & 25.7252435 & 24.481204 & 17.2203605 \\
\hline $2.92 \mathrm{E}+09$ & 34.4116 & 29.7663645 & 25.257701 & 23.607054 & 16.671143 \\
\hline $3.02 \mathrm{E}+09$ & 34.11834 & 29.442912 & 24.944796 & 23.328204 & 16.3495985 \\
\hline $3.12 \mathrm{E}+09$ & 33.81091 & 29.17444 & 24.690014 & 23.354191 & 16.238596 \\
\hline $3.22 \mathrm{E}+09$ & 33.5561 & 28.920278 & 24.4214695 & 23.329728 & 16.2197565 \\
\hline $3.32 \mathrm{E}+09$ & 33.35465 & 28.6523915 & 24.2360985 & 23.169879 & 16.150454 \\
\hline $3.43 \mathrm{E}+09$ & 33.2293 & 28.4688495 & 24.125071 & 22.924531 & 15.9652205 \\
\hline $3.53 \mathrm{E}+09$ & 33.11519 & 28.229617 & 23.9361185 & 22.517627 & 15.636966 \\
\hline $3.63 \mathrm{E}+09$ & 33.06231 & 28.190962 & 23.924223 & 22.376515 & 15.51558 \\
\hline $3.73 \mathrm{E}+09$ & 32.83416 & 28.048199 & 23.770843 & 22.32064 & 15.507213 \\
\hline $3.84 \mathrm{E}+09$ & 32.64379 & 27.9236045 & 23.696582 & 22.599629 & 15.649972 \\
\hline $3.94 \mathrm{E}+09$ & 32.47571 & 27.7438825 & 23.5769095 & 22.762116 & 15.6348435 \\
\hline $4.04 E+09$ & 32.33948 & 27.513737 & 23.3960215 & 22.495053 & 15.3911415 \\
\hline $4.14 \mathrm{E}+09$ & 32.15998 & 27.302741 & 23.2041695 & 21.963976 & 15.1042325 \\
\hline $4.25 \mathrm{E}+09$ & 32.01048 & 27.1732635 & 23.083685 & 21.633417 & 14.9331965 \\
\hline $4.35 \mathrm{E}+09$ & 31.78804 & 27.008834 & 22.952226 & 21.568752 & 14.849206 \\
\hline $4.45 \mathrm{E}+09$ & 31.53576 & 26.706189 & 22.6658115 & 21.602139 & 14.760847 \\
\hline
\end{tabular}




\begin{tabular}{|c|c|c|c|c|c|}
\hline ncy & @ $20 \mathrm{C}$ & $\mathrm{e}^{\prime} @ 40 \mathrm{C}$ & $\mathrm{e}^{\prime} @ 60 \mathrm{C}$ & $\mathrm{e}^{\prime} @ 80 \mathrm{C}$ & $e^{\prime} @ 100 \mathrm{C}$ \\
\hline $4.55 \mathrm{E}+09$ & 31.3019 & 26.488249 & 22.486764 & 21.749451 & 14.8006 \\
\hline $4.65 \mathrm{E}+09$ & 31.06306 & 26.3337085 & 22.400212 & 21.730687 & 14.842017 \\
\hline $4.76 \mathrm{E}+09$ & 30.82195 & 25.970365 & 22.0125855 & 20.957563 & 14.3898405 \\
\hline $4.86 \mathrm{E}+09$ & 30.63233 & 25.642016 & 21.69869 & 20.245574 & 13.911858 \\
\hline $4.96 \mathrm{E}+09$ & 30.42123 & 25.411526 & 21.456377 & 19.923397 & 13.6553755 \\
\hline $5.06 \mathrm{E}+09$ & 30.27553 & 25.422147 & 21.481597 & 20.222898 & 13.837903 \\
\hline $5.17 \mathrm{E}+09$ & 30.05583 & 25.2965805 & 21.4010345 & 20.478971 & 13.977429 \\
\hline $5.27 \mathrm{E}+09$ & 29.89649 & 25.13916 & 21.297429 & 20.594742 & 14.0369495 \\
\hline $5.37 \mathrm{E}+09$ & 29.65856 & 24.976217 & 21.2069635 & 20.396347 & 13.900904 \\
\hline $5.47 \mathrm{E}+09$ & 29.49491 & 24.698856 & 20.952417 & 19.782631 & 13.485446 \\
\hline $5.57 \mathrm{E}+09$ & 29.35175 & 24.462821 & 20.7026455 & 19.212969 & 13.118875 \\
\hline $5.68 \mathrm{E}+09$ & 29.1507 & 24.332114 & 20.582615 & 19.208764 & 13.079969 \\
\hline $5.78 \mathrm{E}+09$ & 29.03547 & 24.407696 & 20.697987 & 19.718831 & 13.448086 \\
\hline $5.88 \mathrm{E}+09$ & 28.90537 & 24.4405495 & 20.8154255 & 20.200858 & 13.683602 \\
\hline $5.98 \mathrm{E}+09$ & 28.75456 & 24.301813 & 20.742457 & 20.100521 & 3.6176435 \\
\hline $6.09 E+09$ & 28.58079 & 24.078188 & 20.5547405 & 19.6281 & 13.3594865 \\
\hline $6.19 E+09$ & 28.41856 & 23.9002135 & 20.3912665 & 19.154438 & 13.066554 \\
\hline $6.29 \mathrm{E}+09$ & 28.35752 & 23.779834 & 20.2800765 & 19.032624 & 12.902755 \\
\hline $6.39 \mathrm{E}+09$ & 28.21679 & 23.769865 & 20.3188705 & 19.454834 & 13.1270565 \\
\hline $6.5 \mathrm{E}+09$ & 28.09266 & 23.9431495 & 20.5787095 & 20.310158 & 13.6462695 \\
\hline $6.6 \mathrm{E}+09$ & 27.90603 & 23.993759 & 20.694812 & 20.617247 & 13.8571175 \\
\hline $6.7 \mathrm{E}+09$ & 27.67177 & 23.6694905 & 20.415019 & 20.035067 & 13.4891425 \\
\hline $6.8 \mathrm{E}+09$ & 27.38397 & 23.350845 & 20.113249 & 19.311386 & 13.048017 \\
\hline $6.9 \mathrm{E}+09$ & 27.1719 & 23.107275 & 9.868043 & 3.829497 & 12.759396 \\
\hline $7.01 \mathrm{E}+09$ & 27.00028 & 22.9780385 & 19.775014 & 18.940292 & 12.7648465 \\
\hline $7.11 \mathrm{E}+09$ & 26.8321 & 22.9223425 & 19.752755 & 19.372289 & 12.9993715 \\
\hline $7.21 E+09$ & 26.70275 & 23.023561 & 19.9389455 & 20.00863 & 13.426249 \\
\hline $7.31 \mathrm{E}+09$ & 26.55481 & 22.933007 & 19.9172 & 19.914075 & 13.41059 \\
\hline $7.42 \mathrm{E}+09$ & 26.33646 & 22.762383 & 19.756512 & 19.332971 & 13.0290695 \\
\hline $7.52 \mathrm{E}+09$ & 26.17292 & 22.427828 & 19.41459 & 18.524108 & 12.54083 \\
\hline $7.62 \mathrm{E}+09$ & 26.06053 & 22.2957795 & 19.293294 & 18.385128 & 12.463268 \\
\hline $7.72 \mathrm{E}+09$ & 25.95047 & 22.40005 & 19.4208615 & 18.939226 & 12.7488055 \\
\hline $7.83 E+09$ & 25.90442 & 22.551554 & 19.6154705 & 19.641711 & 13.1438385 \\
\hline $7.93 E+09$ & 25.81431 & 22.5364765 & 19.650341 & 19.826789 & 13.3464535 \\
\hline $8.03 E+09$ & 25.64858 & 22.642225 & 19.8405535 & 19.799011 & 13.3701705 \\
\hline $8.13 \mathrm{E}+09$ & 25.34164 & 22.2581545 & 19.4434095 & 18.813561 & 12.767317 \\
\hline $8.23 E+09$ & 25.1114 & 22.0237295 & 19.225581 & 18.364005 & 12.4557245 \\
\hline $8.34 \mathrm{E}+09$ & 24.86677 & 21.884843 & 19.0890335 & 18.476305 & 12.5505195 \\
\hline $8.44 \mathrm{E}+09$ & 24.78345 & 22.0350125 & 19.277812 & 19.252003 & 13.0193425 \\
\hline $8.54 \mathrm{E}+09$ & 24.7498 & 22.276021 & 19.5816425 & 19.963629 & 13.4514525 \\
\hline $8.64 \mathrm{E}+09$ & 24.74088 & 22.3722205 & 19.7490095 & 20.01287 & 13.5259495 \\
\hline $8.75 \mathrm{E}+09$ & 24.72919 & 22.3043985 & 19.6885645 & 19.43835 & 13.2731375 \\
\hline $8.85 E+09$ & 24.59611 & 22.145014 & 19.535944 & 18.873107 & 12.882451 \\
\hline $8.95 E+09$ & 24.41297 & 21.9234635 & 19.299996 & 18.684704 & 12.69049 \\
\hline $9.05 E+09$ & 24.36792 & 21.952903 & 19.3643435 & 19.38282 & 13.1063815 \\
\hline
\end{tabular}




\begin{tabular}{|c|c|c|c|c|c|}
\hline quency & @ 20 C & $e^{\prime} @ 40 \mathrm{C}$ & $\mathrm{e}^{\prime} @ 60 \mathrm{C}$ & $\mathrm{e}^{\prime} @ 80 \mathrm{C}$ & @ 100 C \\
\hline+09 & 24.3861 & 22.1620315 & 19.6362295 & 20.319328 & 13.68905 \\
\hline $6 \mathrm{E}+09$ & 4.44767 & 22.505842 & 20.0409395 & 5637 & +208 \\
\hline $6 \mathrm{E}+09$ & 24.397 & 22.4541005 & 20.0388205 & 20.357124 & 3.77053 \\
\hline $46 \mathrm{E}+09$ & 24.26623 & 22.3872745 & 19.9720995 & 19.684834 & .437792 \\
\hline $56 \mathrm{E}+09$ & 24.00092 & 22.0661975 & 19.667179 & 9.167677 & 3.125492 \\
\hline $9.67 \mathrm{E}+09$ & 23.82454 & 21.9206925 & 19.5440445 & 19.567014 & 13.19230 \\
\hline$E+09$ & .67525 & 21.8472255 & 19.4890645 & 20.291538 & 3.647696 \\
\hline \begin{tabular}{l|l}
$7 E+09$ \\
\end{tabular} & 3.80106 & 22.303249 & 20.0348185 & 21.374887 & 4.391604 \\
\hline $\mathrm{E}+09$ & .84623 & 22.4368195 & 20.2209345 & 21.233258 & 14.3437 \\
\hline $\mathrm{E}+10$ & .77233 & 22.4487135 & 20.258366 & 20.511774 & 3.878391 \\
\hline$E+10$ & .52854 & 22.1739045 & 20.0026075 & 19.711576 & 35530 \\
\hline $3 E+10$ & .20556 & 21.8688085 & 19.694517 & 19.554455 & 13.282532 \\
\hline $1.04 \mathrm{E}+10$ & 23.11623 & 21.8343705 & 19.6464935 & 20.305025 & 13.673857 \\
\hline+10 & 3.2054 & 22.0346985 & 9.8803105 & 21.377336 & 14.237395 \\
\hline $1.06 \mathrm{E}+10$ & 23.33161 & 22.342845 & 20.2078985 & 21.729394 & 14.61278 \\
\hline$E+10$ & 3.22375 & 22.3810245 & 20.245517 & 20.94648 & 14.17034 \\
\hline $.08 \mathrm{E}+10$ & 22.90792 & 22.0590385 & 19.932213 & 19.68096 & 13.36364 \\
\hline 10 & 22.59477 & 21.76462 & 19.6525475 & 19.16879 & 1458 \\
\hline $1.1 \mathrm{E}+10$ & 22.37691 & 21.671686 & 19.5990815 & 19.90002 & 13.522015 \\
\hline $1.11 \mathrm{E}+10$ & 22.60968 & 22.019629 & 19.9662715 & 21.490426 & 4. \\
\hline $1.12 \mathrm{E}+10$ & 2.90521 & 22.4293755 & 20.371959 & 22.381553 & 15.0585 \\
\hline $1.13 \mathrm{E}+10$ & 23.03609 & 22.530807 & 20.4618695 & 21.846481 & 14.779079 \\
\hline $1.14 \mathrm{E}+10$ & 22.8769 & 22.430566 & 20.394611 & 20.544832 & 14.04662 \\
\hline $1.15 \mathrm{E}+10$ & 22.62793 & 22.2362515 & 20.2550465 & 19.67498 & 3.393234 \\
\hline $1.16 \mathrm{E}+10$ & 22.3792 & 22.0218435 & 20.070305 & 20.139911 & 13.561619 \\
\hline $1.17 \mathrm{E}+10$ & 2.44216 & 22.565813 & 20.665173 & 22.644898 & 15.22388 \\
\hline $1.18 \mathrm{E}+10$ & 22.65964 & 23.135879 & 21.2625485 & 24.755621 & 16.57340 \\
\hline $1.19 \mathrm{E}+10$ & 22.68912 & 22.945599 & 21.0537725 & 24.287909 & 16.2208 \\
\hline $1.2 \mathrm{E}+10$ & 22.51412 & 22.581282 & 20.686879 & 22.150151 & 15.024268 \\
\hline $1.21 \mathrm{E}+10$ & 22.16959 & 22.1213735 & 20.22705 & 19.786544 & 13.555823 \\
\hline $1.22 \mathrm{E}+10$ & 21.71928 & 21.8384005 & 19.99548 & 18.857298 & 12.8251 \\
\hline$E+10$ & 21.55762 & 22.3114555 & 20.5844665 & 21.007389 & 14.09597 \\
\hline $4 E+10$ & 21.8508 & 22.7427715 & 21.016423 & 24.476143 & 16.211883 \\
\hline $1.25 \mathrm{E}+10$ & 22.06119 & 23.0727445 & 21.3182155 & 26.551472 & 17.615646 \\
\hline $1.26 \mathrm{E}+10$ & 22.03775 & 22.8528065 & 21.093072 & 25.174992 & 16.91408 \\
\hline $1.27 \mathrm{E}+10$ & 21.97637 & 22.216641 & 20.398484 & 21.784176 & 14.868582 \\
\hline $1.28 \mathrm{E}+10$ & 21.57527 & 21.8590825 & 20.084853 & 18.923942 & 13.03621 \\
\hline $1.29 \mathrm{E}+10$ & 21.38675 & 21.9952705 & 20.296286 & 17.943864 & 12.128872 \\
\hline $1.3 \mathrm{E}+10$ & 21.34439 & 22.183402 & 20.4915185 & 19.332326 & 12.97669 \\
\hline $1.31 \mathrm{E}+10$ & 21.44796 & 22.5804265 & 20.8771235 & 23.34634 & 15.397658 \\
\hline $1.32 \mathrm{E}+10$ & 21.45982 & 22.328882 & 20.631955 & 25.289221 & 16.75811 \\
\hline $1.33 \mathrm{E}+10$ & 21.34791 & 21.917404 & 20.186022 & 23.929775 & 16.11911 \\
\hline $1.35 \mathrm{E}+10$ & 20.99852 & .2227105 & 19.4949255 & 20.611565 & 14.12395 \\
\hline $1.36 \mathrm{E}+10$ & 20.7193 & 1.1109945 & 19.382006 & 17.820923 & 12.378890 \\
\hline $1.37 \mathrm{E}+10$ & 20.63006 & 21.6802195 & 19.9411825 & 16.871249 & 11.66473 \\
\hline
\end{tabular}




\begin{tabular}{|c|c|c|c|c|c|}
\hline Frequency & $\mathrm{e}^{\prime} @ 20 \mathrm{C}$ & $e^{\prime} @ 40 C$ & $\mathrm{e}^{\prime} @ 60 \mathrm{C}$ & $e^{\prime} @ 80 \mathrm{C}$ & $e^{\prime} @ 100 \mathrm{C}$ \\
\hline $1.38 \mathrm{E}+10$ & 20.77342 & 22.226146 & 20.5504775 & 18.620328 & 12.608348 \\
\hline $1.39 \mathrm{E}+10$ & 20.99595 & 22.3531755 & 20.6774535 & 22.125924 & 14.8419645 \\
\hline $1.4 \mathrm{E}+10$ & 21.06808 & 22.1262935 & 20.445794 & 24.160585 & 16.2506795 \\
\hline $1.41 E+10$ & 21.11836 & 21.3846765 & 19.6448175 & 22.615534 & 15.3868525 \\
\hline $1.42 \mathrm{E}+10$ & 21.06076 & 21.1038235 & 19.328534 & 20.002389 & 13.920032 \\
\hline $1.43 E+10$ & 20.83386 & $21: 5732135$ & 19.7965805 & 18.161001 & 12.724468 \\
\hline $1.44 \mathrm{E}+10$ & 20.58202 & 22.6517655 & 20.866176 & 18.181226 & 12.392135 \\
\hline $1.45 \mathrm{E}+10$ & 20.62499 & 23.2328345 & 21.561896 & 20.6588 & 13.7119885 \\
\hline $1.46 \mathrm{E}+10$ & 20.89495 & 23.1643665 & 21.6735835 & 24.339165 & 16.0595515 \\
\hline $1.47 \mathrm{E}+10$ & 21.05656 & 22.1942475 & 20.755092 & 24.636587 & 16.6062495 \\
\hline $1.48 \mathrm{E}+10$ & 20.93398 & 21.284524 & 19.732205 & 22.333008 & 15.401644 \\
\hline $1.49 \mathrm{E}+10$ & 20.83583 & 21.0583025 & 19.4954395 & 19.882294 & 14.056433 \\
\hline $1.5 \mathrm{E}+10$ & 20.42494 & 21.2962805 & 19.6586465 & 18.012775 & 12.685696 \\
\hline $1.51 E+10$ & 20.20974 & 22.8250415 & 21.113637 & 18.697317 & 12.710595 \\
\hline $1.52 \mathrm{E}+10$ & 20.11836 & 23.7112295 & 22.1729805 & 22.278082 & 14.3029235 \\
\hline $1.53 \mathrm{E}+10$ & 20.79742 & 24.6528135 & 23.3840965 & 27.137957 & 17.612211 \\
\hline $1.54 \mathrm{E}+10$ & 20.83999 & 22.839723 & 21.551858 & 25.399163 & 17.114241 \\
\hline $1.55 \mathrm{E}+10$ & 20.76416 & 21.771821 & 20.391025 & 22.766846 & 15.797339 \\
\hline $1.56 E+10$ & 19.73822 & 19.979158 & 18.614011 & 19.051676 & 13.47051 \\
\hline $1.57 \mathrm{E}+10$ & 19.01246 & 19.8118215 & 18.3733885 & 17.09394 & 12.0141825 \\
\hline $1.58 \mathrm{E}+10$ & 19.01016 & 20.819359 & 19.1828585 & 17.14325 & 11.551469 \\
\hline $1.59 \mathrm{E}+10$ & 19.3444 & 23.062657 & 21.397761 & 21.169419 & 13.438777 \\
\hline $1.6 \mathrm{E}+10$ & 19.72142 & 23.297349 & 21.9142545 & 24.720426 & 15.910023 \\
\hline $1.61 \mathrm{E}+10$ & 20.14114 & 23.9332725 & 22.731935 & 26.294954 & 17.6171415 \\
\hline $1.62 \mathrm{E}+10$ & 19.40359 & 22.236994 & 21.103595 & 23.682127 & 16.031881 \\
\hline $1.63 \mathrm{E}+10$ & 19.03859 & 19.9455625 & 18.5549335 & 19.456999 & 13.0299495 \\
\hline $1.64 \mathrm{E}+10$ & 19.20984 & 18.4697455 & 16.8559955 & 16.14011 & 11.018618 \\
\hline $1.65 E+10$ & 19.24235 & 19.064191 & 17.3673895 & 16.365678 & 10.6713025 \\
\hline $1.66 \mathrm{E}+10$ & 19.29602 & 21.625558 & 19.932495 & 20.279477 & 12.621732 \\
\hline $1.67 \mathrm{E}+10$ & 19.53376 & 23.491795 & 21.828402 & 24.224409 & 15.0526685 \\
\hline $1.68 \mathrm{E}+10$ & 19.60616 & 23.5201575 & 22.195141 & 25.255405 & 16.4303325 \\
\hline $1.69 \mathrm{E}+10$ & 18.84463 & 20.865835 & 19.5620575 & 21.659798 & 14.177192 \\
\hline $1.7 \mathrm{E}+10$ & 18.30983 & 18.783427 & 17.2410675 & 17.817351 & 12.0825745 \\
\hline $1.71 \mathrm{E}+10$ & 18.39168 & 18.2373595 & 16.72617 & 16.71079 & 11.229141 \\
\hline $1.72 \mathrm{E}+10$ & 18.85266 & 19.5694345 & 18.136174 & 18.434659 & 11.972336 \\
\hline $1.73 E+10$ & 18.85503 & 21.1781875 & 19.526048 & 20.534522 & 12.9230795 \\
\hline $1.74 \mathrm{E}+10$ & 19.04166 & 23.031548 & 21.397791 & 23.387199 & 14.917233 \\
\hline $1.75 \mathrm{E}+10$ & 19.00319 & 22.3633435 & 21.0074955 & 23.185993 & 14.948487 \\
\hline $1.76 E+10$ & 18.73237 & 20.705952 & 19.3682565 & 20.737583 & 13.367164 \\
\hline $1.77 \mathrm{E}+10$ & 18.18278 & 19.3023455 & 17.739009 & 18.213404 & 12.024617 \\
\hline $1.79 \mathrm{E}+10$ & 18.28855 & 19.2740455 & 17.677693 & 18.170306 & 12.1135185 \\
\hline $1.8 \mathrm{E}+10$ & 18.54781 & 19.5846995 & 18.0861455 & 18.971945 & 12.269023 \\
\hline $1.81 \mathrm{E}+10$ & 18.5022 & 21.310964 & 19.810108 & 21.321546 & 13.586511 \\
\hline $1.82 \mathrm{E}+10$ & 18.22116 & 22.148688 & 20.5530775 & 22.39976 & 14.5045915 \\
\hline $1.83 \mathrm{E}+10$ & 18.39414 & 21.607874 & 20.27742 & 21.879366 & 14.3005495 \\
\hline
\end{tabular}




\begin{tabular}{|r|r|r|r|r|r|}
\hline Frequency & $\mathrm{e}^{\prime} @ 20 \mathrm{C}$ & $\mathrm{e}^{\prime} @ 40 \mathrm{C}$ & $\mathrm{e}^{\prime} @ 60 \mathrm{C}$ & $\mathrm{e}^{\prime} @ 80 \mathrm{C}$ & $\mathrm{e}^{\prime} @ 100 \mathrm{C}$ \\
\hline \hline $1.84 \mathrm{E}+10$ & 18.03585 & 19.787797 & 18.6036665 & 19.53709 & 12.8780415 \\
\hline $1.85 \mathrm{E}+10$ & 17.79301 & 19.5871635 & 18.13211 & 18.904823 & 12.488563 \\
\hline $1.86 \mathrm{E}+10$ & 17.69814 & 19.697323 & 18.259591 & 19.390097 & 12.72819 \\
\hline $1.87 \mathrm{E}+10$ & 18.36827 & 20.8023675 & 19.5866085 & 21.144096 & 13.706737 \\
\hline $1.88 \mathrm{E}+10$ & 18.25322 & 21.419377 & 20.093142 & 21.855564 & 14.178368 \\
\hline $1.89 \mathrm{E}+10$ & 17.65697 & 21.5836905 & 20.1025385 & 21.740645 & 14.132365 \\
\hline $1.9 \mathrm{E}+10$ & 17.50202 & 20.432709 & 19.1272865 & 20.265123 & 13.2779575 \\
\hline $1.91 \mathrm{E}+10$ & 17.11725 & 18.75477 & 17.512957 & 18.225815 & 11.9574255 \\
\hline $1.92 \mathrm{E}+10$ & 16.14397 & 17.9736435 & 16.630127 & 17.548792 & 11.409773 \\
\hline $1.93 \mathrm{E}+10$ & 16.38152 & 18.6424645 & 17.2839555 & 18.56133 & 12.037423 \\
\hline $1.94 \mathrm{E}+10$ & 16.97027 & 19.318656 & 18.0941375 & 19.539486 & 12.771081 \\
\hline $1.95 \mathrm{E}+10$ & 16.86361 & 19.812196 & 18.6149265 & 20.153285 & 13.1050445 \\
\hline $1.96 \mathrm{E}+10$ & 16.67934 & 19.9895 & 18.678974 & 19.964848 & 13.0029395 \\
\hline $1.97 \mathrm{E}+10$ & 17.05582 & 19.7449965 & 18.4704575 & 19.442945 & 12.7076875 \\
\hline $1.98 \mathrm{E}+10$ & 17.21729 & 19.114438 & 17.7573795 & 18.616375 & 12.137334 \\
\hline $1.99 \mathrm{E}+10$ & 17.03254 & 19.149624 & 17.6864655 & 18.827571 & 12.1055425 \\
\hline $2 \mathrm{E}+10$ & 16.79821 & 19.508136 & 18.0525825 & 19.488389 & 12.455157 \\
\hline
\end{tabular}


Table 13. Dielectric Loss for the Revised ORNL Simulant

\begin{tabular}{|c|c|c|c|c|c|}
\hline ncy & $\mathbf{C}$ & e" @ 40 C & $\mathrm{e}^{\prime \prime} @ 60 \mathrm{C}$ & $e^{\prime \prime @ 80 C}$ & e"@100 C \\
\hline $1.53 \mathrm{E}+08$ & 1428.896 & 1905.133699 & 2223.72142 & 2676.21191 & 2819 \\
\hline $2.55 \mathrm{E}+08$ & 871.8114 & 1180.124271 & 1375.31055 & 1652.2646 & 1202.02291 \\
\hline $3.58 \mathrm{E}+08$ & 631.6136 & 854.334889 & 994.766021 & 1194.32359 & 869.361363 \\
\hline $4.6 E+08$ & 495.5831 & 669.1153575 & 778.779865 & 935.11119 & 680.656218 \\
\hline $5.62 \mathrm{E}+08$ & 409.707 & 559.538676 & 651.255822 & 780.965989 & 568.97301 \\
\hline $6.65 \mathrm{E}+08$ & 348.3537 & 477.7689155 & 555.803024 & 667.10015 & 485.423194 \\
\hline $7.67 \mathrm{E}+08$ & 303.4035 & 413.899089 & 481.32105 & 577.169703 & 420.067462 \\
\hline $8.69 \mathrm{E}+08$ & 269.0006 & 365.0583195 & 424.223398 & 508.54136 & 370.03706 \\
\hline $9.71 \mathrm{E}+08$ & 241.8903 & 328.3287855 & 381.463196 & 457.526777 & 332.781543 \\
\hline $1.07 \mathrm{E}+09$ & 219.9603 & 299.4953305 & 347.858256 & 417.455362 & 303.559196 \\
\hline $1.18 \mathrm{E}+09$ & 201.8917 & 275.8192925 & 320.150825 & 384.26404 & 279.404763 \\
\hline $1.28 \mathrm{E}+09$ & 186.3019 & 254.152691 & 294.885715 & 353.699616 & 257.168039 \\
\hline $1.38 \mathrm{E}+09$ & 173.1138 & 234.9703875 & 272.528434 & 326.670663 & 237.493173 \\
\hline $1.48 \mathrm{E}+09$ & 161.6355 & 218.850513 & 253.714751 & 304.029178 & 220.940051 \\
\hline $1.59 \mathrm{E}+09$ & 152.0275 & 206.038162 & 238.651234 & 285.904125 & 207.877846 \\
\hline $1.69 \mathrm{E}+09$ & 143.4499 & 194.7007 & 225.370199 & 270.174985 & 196.468714 \\
\hline $1.79 \mathrm{E}+09$ & 135.8702 & 183.7785785 & 212.642884 & 255.01632 & 185.405707 \\
\hline $1.89 \mathrm{E}+09$ & 128.918 & 173.8794385 & 201.02371 & 241.070859 & 175.160811 \\
\hline $1.99 \mathrm{E}+09$ & 122.6491 & 165.803854 & 191.57154 & 229.423986 & 166.758135 \\
\hline $2.1 \mathrm{E}+09$ & 117.0233 & 158.3754475 & 182.854883 & 218.717736 & 159.064047 \\
\hline $2.2 \mathrm{E}+09$ & 112.3123 & 151.1065045 & 174.282541 & 208.319019 & 151.587797 \\
\hline $2.3 \mathrm{E}+09$ & 107.881 & 144.325983 & 166.406799 & 199.159651 & 144.762994 \\
\hline $2.4 \mathrm{E}+09$ & 103.8252 & 138.6815225 & 159.751412 & 191.323713 & 139.055882 \\
\hline $2.51 \mathrm{E}+09$ & 100.0897 & 133.9914055 & 154.120524 & 184.424107 & 134.1889 \\
\hline $2.61 E+09$ & 96.30372 & 129.344476 & 148.732197 & 177.81632 & 129.332127 \\
\hline $2.71 \mathrm{E}+09$ & 93.08152 & 124.4666245 & 142.985535 & 170.639163 & 124.116199 \\
\hline $2.81 E+09$ & 90.15948 & 119.7773975 & 137.502808 & 163.965132 & 119.287 \\
\hline $2.92 \mathrm{E}+09$ & 87.45045 & 115.6750985 & 132.642077 & 158.221405 & 115.140012 \\
\hline $3.02 E+09$ & 84.93633 & 112.2777085 & 128.608195 & $153.51226 \mathrm{i}$ & 111.737386 \\
\hline $3.12 \mathrm{E}+09$ & 82.5269 & 109.2124465 & 124.975746 & 149.195376 & 108.582893 \\
\hline $3.22 \mathrm{E}+09$ & 80.19298 & 105.883117 & 121.028811 & 144.3171 & 105.015749 \\
\hline $3.32 \mathrm{E}+09$ & 77.94846 & 102.3190995 & 116.817528 & 138.940205 & 101.185263 \\
\hline $3.43 \mathrm{E}+09$ & 75.88156 & 99.279875 & 113.266133 & 134.479679 & 97.9406995 \\
\hline $3.53 \mathrm{E}+09$ & 73.96535 & 96.770172 & 110.326867 & 130.993357 & 95.3967745 \\
\hline $3.63 \mathrm{E}+09$ & 72.22574 & 94.426469 & 107.577802 & 127.976141 & 93.1638225 \\
\hline $3.73 \mathrm{E}+09$ & 70.69393 & 91.9184665 & 104.585705 & 124.456882 & 90.622283 \\
\hline $3.84 \mathrm{E}+09$ & 69.17686 & 89.593227 & 101.84489 & 121.105606 & 88.162009 \\
\hline $3.94 \mathrm{E}+09$ & 67.72376 & 87.6078965 & 99.497506 & 118.040359 & 85.9782485 \\
\hline $4.04 \mathrm{E}+09$ & 66.37318 & 85.7043605 & 97.2251965 & 115.070203 & 83.8418355 \\
\hline $4.14 \mathrm{E}+09$ & 65.1099 & 83.6817925 & 94.8320745 & 112.114642 & 81.6894 \\
\hline $4.25 \mathrm{E}+09$ & 63.86653 & 81.7512145 & 92.547922 & 109.545199 & 79.793003 \\
\hline $4.35 \mathrm{E}+09$ & 62.71675 & 80.145051 & 90.643782 & 107.511501 & 78.2984635 \\
\hline $4.45 \mathrm{E}+09$ & 61.62254 & 78.649657 & 88.8408315 & 105.36213 & 76.7487975 \\
\hline
\end{tabular}




\begin{tabular}{|c|c|c|c|c|c|}
\hline Frequency & $e^{\prime \prime} @ 20 \mathrm{C}$ & $e^{\prime \prime @ 40 C ~}$ & $e^{\prime \prime @ 60 C ~}$ & $\mathrm{e}^{\prime \prime} @ 80 \mathrm{C}$ & $\mathrm{e}^{\prime \prime} @ 100 \mathrm{C}$ \\
\hline $4.55 \mathrm{E}+09$ & 60.57096 & 77.0465155 & 86.9104645 & 102.791881 & 74.901736 \\
\hline $4.65 \mathrm{E}+09$ & 59.56926 & 75.248588 & 84.7707035 & 99.867188 & 72.822563 \\
\hline $4.76 \mathrm{E}+09$ & 58.57321 & 73.7173545 & 82.9844875 & 97.601005 & 71.1569545 \\
\hline $4.86 \mathrm{E}+09$ & 57.66819 & 72.4155205 & 81.4104075 & 95.7918955 & 69.7981885 \\
\hline $4.96 \mathrm{E}+09$ & 56.70708 & 71.2006855 & 79.981329 & 94.3071345 & 68.6854795 \\
\hline $5.06 \mathrm{E}+09$ & 55.84022 & 69.9634645 & 78.52124 & 92.68369 & 67.5425695 \\
\hline $5.17 \mathrm{E}+09$ & 55.05229 & 68.7173445 & 77.0254725 & 90.798269 & 66.158909 \\
\hline $5.27 \mathrm{E}+09$ & 54.27555 & 67.281399 & 75.313414 & 88.385721 & 64.417981 \\
\hline $5.37 \mathrm{E}+09$ & 53.5707 & 66.1053005 & 73.930167 & 86.465728 & 63.012924 \\
\hline $5.47 \mathrm{E}+09$ & 52.83808 & 65.0518755 & 72.665478 & 84.9505765 & 61.9278945 \\
\hline $5.57 \mathrm{E}+09$ & 52.15313 & 64.0840475 & 71.4926865 & 83.728506 & 61.0261915 \\
\hline $5.68 \mathrm{E}+09$ & 51.50519 & 63.075243 & 70.2865765 & 82.5479445 & 60.1108955 \\
\hline $5.78 \mathrm{E}+09$ & 50.80048 & 62.0540975 & 69.071273 & 81.1047075 & 59.1056205 \\
\hline $5.88 E+09$ & 50.17785 & 61.071505 & 67.9404315 & 79.5706445 & 58.0009 \\
\hline $5.98 \mathrm{E}+09$ & 49.54163 & 59.9784045 & 66.6495745 & 77.6741845 & 56.662068 \\
\hline $6.09 \mathrm{E}+09$ & 48.91662 & 58.916545 & 65.4074835 & 76.0518975 & 55.4561025 \\
\hline $6.19 \mathrm{E}+09$ & 48.23264 & 57.98451 & 64.335207 & 74.9833695 & 54.6642685 \\
\hline $6.29 \mathrm{E}+09$ & 47.60268 & 57.2016955 & 63.425357 & 74.215812 & 54.0705425 \\
\hline $6.39 \mathrm{E}+09$ & 47.01773 & 56.393655 & 62.4575205 & 73.168605 & 53.299063 \\
\hline $6.5 \mathrm{E}+09$ & 46.48058 & 55.4460645 & 61.3805205 & 71.710926 & 52.3113915 \\
\hline $6.6 \mathrm{E}+09$ & 46.0262 & 54.5992655 & 60.4009575 & 70.217164 & 51.2600275 \\
\hline $6.7 \mathrm{E}+09$ & 45.55818 & 53.697375 & 59.335047 & 68.6664155 & 50.103237 \\
\hline $6.8 \mathrm{E}+09$ & 45.0335 & 52.884176 & 58.3545285 & 67.524141 & 49.2387995 \\
\hline $6.9 \mathrm{E}+09$ & 44.41452 & 52.1434925 & 57.4828735 & 66.769644 & 48.6822135 \\
\hline $7.01 \mathrm{E}+09$ & 43.88577 & 51.4585095 & 56.6917435 & 66.1037755 & 48.192154 \\
\hline $7.11 \mathrm{E}+09$ & 43.45508 & 50.729223 & 55.809883 & 64.992218 & 47.3899615 \\
\hline $7.21 \mathrm{E}+09$ & 43.01952 & 49.902282 & 54.864336 & 63.5133065 & 46.3451015 \\
\hline $7.31 \mathrm{E}+09$ & 42.58483 & 49.140361 & 53.9941255 & 62.1467 & 45.3735715 \\
\hline $7.42 E+09$ & 42.16313 & 48.4613265 & 53.198469 & 61.1157555 & 44.639979 \\
\hline $7.52 \mathrm{E}+09$ & 41.74515 & 47.8238065 & 52.435932 & 60.415346 & 44.05839 \\
\hline $7.62 \mathrm{E}+09$ & 41.31207 & 47.317196 & 51.8354585 & 60.0524555 & 43.7793605 \\
\hline $7.72 \mathrm{E}+09$ & 40.94829 & 46.834629 & 51.289524 & 59.617104 & 43.477256 \\
\hline $7.83 \mathrm{E}+09$ & 40.66338 & 46.3153975 & 50.6897275 & 58.661286 & 42.8000595 \\
\hline $7.93 \mathrm{E}+09$ & 40.38507 & 45.6068985 & 49.852504 & 57.2258885 & 41.761622 \\
\hline $8.03 E+09$ & 40.06825 & 44.9722905 & 49.1478305 & 56.1117685 & 41.0274715 \\
\hline $8.13 \mathrm{E}+09$ & 39.68518 & 44.4430795 & 48.510033 & 55.5152275 & 40.5114525 \\
\hline $8.23 \mathrm{E}+09$ & 39.34377 & 43.947692 & 47.8855845 & 55.1146775 & 40.20576 \\
\hline $8.34 E+09$ & 38.93362 & 43.51565 & 47.391602 & 54.8773435 & 39.9885765 \\
\hline $8.44 \mathrm{E}+09$ & 38.49404 & 42.974034 & 46.792331 & 54.1810505 & 39.5173105 \\
\hline $8.54 \mathrm{E}+09$ & 38.10514 & 42.3857335 & 46.143488 & 52.9817315 & 38.7255005 \\
\hline $8.64 \mathrm{E}+09$ & 37.7094 & 41.7966765 & 45.503945 & 51.784633 & 37.8486505 \\
\hline $8.75 \mathrm{E}+09$ & 37.41829 & 41.3009015 & 44.9006265 & 51.0093245 & 37.275888 \\
\hline $8.85 E+09$ & 37.11015 & 40.964091 & 44.4914275 & 50.8637885 & 37.1841415 \\
\hline $8.95 \mathrm{E}+09$ & 36.74976 & 40.649266 & 44.106802 & 50.867068 & 37.1051025 \\
\hline $9.05 E+09$ & 36.36708 & 40.1329735 & 43.533475 & 50.322775 & 36.7012515 \\
\hline
\end{tabular}




\begin{tabular}{|c|c|c|c|c|c|}
\hline & $e^{\prime \prime @ 20 C ~}$ & (a) $40 \mathrm{C}$ & $\bar{C}$ & $\bar{C}$ & $C$ \\
\hline & 57 & $\overline{3}$ & 42.908126 & & \\
\hline & & 07 & x & & \\
\hline & & 11 & & & 4. \\
\hline$E+09$ & .42997 & 3.4324685 & 192765 & 3885 & .4 \\
\hline & 5 & 11235 & 915 & 25 & 1.35 \\
\hline $57 E+09$ & 1.72049 & 7.691252 & 0.726047 & 5.9965035 & 4.3 \\
\hline$E+09$ & 34.15271 & 36.8777435 & .869155 & .8492095 & \\
\hline+09 & 3.87712 & 6.392761 & 39.3499245 & 4.5777365 & 2.7643 \\
\hline $9.97 \mathrm{E}+09$ & 3.57572 & 35.719064 & 38.6250565 & $\overline{3.2353375}$ & 1.8 \\
\hline & & 2 & 5 & & \\
\hline-10 & 3.40836 & .5889545 & 38.3760805 & 3.407847 & 1. \\
\hline & & 41 & 323 & & 53 \\
\hline+10 & .73726 & .7909305 & 7.495775 & 3.2106605 & 1.0 \\
\hline & 02 & 025 & 05 & & $.0 y$ \\
\hline+10 & 91 & 33.902672 & 36 & 0.714695 & 29.9 \\
\hline & 03 & 86 & 245 & .423863 & $\overline{9.7}$ \\
\hline+10 & 64 & .0887855 & 1742 & $\overline{.760699}$ & 9. \\
\hline $9 \mathrm{E}+10$ & 1 & .89 & & 41.340061 & 0. \\
\hline $1.1 \mathrm{E}+10$ & 58 & 3.391169 & 799294 & 41.44219 & $\overline{0.34}$ \\
\hline 10 & & -1 & & & \\
\hline $1.12 \mathrm{E}+10$ & 31.35115 & 32.497761 & 34.916218 & 9.2550345 & 8.90 \\
\hline $3 \mathrm{E}+10$ & 05 & .6756745 & 85 & 8275 & $8.39->$ \\
\hline $1.14 \mathrm{E}+10$ & 88 & 32.522579 & 34.851552 & 139 & 8.2 \\
\hline $1.15 \mathrm{E}+10$ & & .419895 & 5295 & .136167 & 8.7 \\
\hline$E+10$ & 46 & .0845605 & 4.327127 & 0.0307985 & 9. \\
\hline $7 E+10$ & 86 & 37 & 295 & 45 & 9.0 \\
\hline $8 \mathrm{E}+10$ & 79 & 2 & 35 & 25 & 140 \\
\hline $1.19 \mathrm{E}+10$ & & 722145 & 65 & 917 & 33 \\
\hline $1.2 \mathrm{E}+10$ & 99 & 9819155 & 3.1186545 & 35.4193125 & 6.2 \\
\hline $1.21 \mathrm{E}+10$ & 96 & .053431 & 33.090367 & 36.021101 & 6.5 \\
\hline $1.22 \mathrm{E}+10$ & .88555 & .691468 & .682685 & 37.515895 & 27.40 \\
\hline $1.23 \mathrm{E}+10$ & 77 & 3208645 & 3315655 & 9.2051705 & - \\
\hline $1.24 \mathrm{E}+10$ & 31 & .697446 & 31.76175 & 39.2045035 & 8.519163 \\
\hline $1.25 \mathrm{E}+10$ & 12 & 29.526027 & 1.5553785 & 36.865575 & 27. \\
\hline $1.26 \mathrm{E}+10$ & & .3247275 & 56 & 3.896103 & 5.28582 \\
\hline $1.27 \mathrm{E}+10$ & 29.2 & 29.5353615 & 1.444092 & 32.7684715 & 4.276848 \\
\hline $1.28 \mathrm{E}+10$ & & & 1.468953 & 33.713026 & $\overline{563}$ \\
\hline $1.29 \mathrm{E}+10$ & 28.78491 & 9196215 & .8242275 & 36.93177 & 26.865334 \\
\hline $1.3 E+10$ & & 3085385 & & .4019285 & 28. \\
\hline $1.31 \mathrm{E}+10$ & 3.14492 & 0801765 & .0308325 & .0146595 & 88.824908 \\
\hline $1.32 \mathrm{E}+10$ & & & 50.20001 & 36.4293065 & 26.805771 \\
\hline $1.33 \mathrm{E}+10$ & 28.22095 & 28.3654585 & 30.0753675 & 32.735532 & 24.36767 \\
\hline $1.35 \mathrm{E}+10$ & & 4325 & 29.969333 & 0.8148945 & 22.8317 \\
\hline $\mathrm{E}+10$ & & & & & \\
\hline$\overline{E+}$ & 27.9 & 28.7 & .4779515 & 98 & 0.1074 \\
\hline
\end{tabular}




\begin{tabular}{|c|c|c|c|c|c|}
\hline & $\begin{array}{lll}\mathrm{C} & \mathrm{E}\end{array}$ & $\mathrm{C}$ & (a) $60 \mathrm{C}$ & (a) $80 \mathrm{C}$ & $(\omega)$ \\
\hline+10 & 27.47913 & 38 & 30.109986 & 37.064659 & 26.71880 \\
\hline-10 & 53057 & 882 & 45 & 7775 & \\
\hline & & & & 05 & 5.1 \\
\hline+10 & 27.6164 & 27.276721 & 4857 & .6098995 & .9366 \\
\hline & 27.73502 & 175 & 545 & 45 & 1.8775 \\
\hline+10 & 27.85747 & 28.598442 & 30.162307 & 31.366591 & .08270 \\
\hline & 27.78759 & 9.2871435 & .9872275 & 739 & 53. \\
\hline+10 & 27.4319 & 8.3368645 & 0.0121435 & 36.645851 & 6.6582 \\
\hline+10 & 27.0122 & 6.8894085 & 28.505556 & 34.6203485 & $\overline{8.82960}$ \\
\hline-10 & .92229 & 248216 & 5.7722785 & 0.1347385 & 3.01515 \\
\hline & 8.84077 & 25.386005 & 79425 & 7.8972485 & $21.2 .2+3$ \\
\hline+10 & .51842 & 251 & 7.252947 & 27.6245205 & .. \\
\hline 10 & 84] & 1 & 25 & 90 & 22.5030 \\
\hline+10 & 171 & 786 & .835587 & 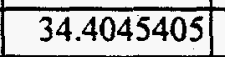 & O \\
\hline+10 & 25.61987 & 26.91 & 3.634424 & 5.71583 & 5.80 \\
\hline & 51 & & & 31.26534 & + \\
\hline+10 & .61316 & 3.161498 & 4.626818 & 7.1071995 & 0.98959 \\
\hline & & & 54 & 5.179719 & 374 \\
\hline+10 & 26.23387 & 4.8355365 & 5.8449595 & 25.921189 & 9.63512 \\
\hline & 58825 & 55 & .0288695 & 9.213051 & .0 \\
\hline+10 & 5.0936 & 8.087166 & 29.431644 & 33.001105 & 4.061 \\
\hline-10 & 11 & 0189 & 514 & 0.401203 & .0 \\
\hline+10 & .89169 & 08 & 251 & 0.250003 & 2.65 \\
\hline+10 & 25.787 & 8 & 346 & 5.1122295 & 0.562879 \\
\hline & 4 & 1 & 35 & 0828 & \\
\hline+10 & .73731 & .7581435 & 5.9379335 & 27.797848 & 21.05547 \\
\hline+10 & 45015 & .9838185 & 7.9788105 & 29.5226045 & 21.75324 \\
\hline+10 & .49795 & .196631 & 29.26275 & 32.394492 & 3.57582 \\
\hline+10 & 1497 & 55 & 45 & 3.356066 & 4.064542 \\
\hline+10 & 1309 & 446755 & 1405 & 1.267198 & 23.1159 \\
\hline+10 & 19 & 35 & .656268 & 7.6932345 & 21.4179 \\
\hline+10 & & & 69 & 27.3800995 & 760 \\
\hline+10 & 65 & 254 & 95 & 26.747371 & 0.070417 \\
\hline+10 & & 0905083 & 25.972338 & 7.8612895 & 0.594693 \\
\hline+10 & .56767 & 9874845 & 7.0522395 & 0.1694875 & 2.066010 \\
\hline$E+10$ & 54086 & 824226 & 27.1719965 & .9415435 & 22.19623 \\
\hline+10 & 43 & - & 25.731526 & 28.5903865 & 21.06757 \\
\hline+10 & 5.356 & .3350335 & .4018765 & 27.590573 & 20.77005 \\
\hline $8+10$ & 25.50703 & 3862535 & 5.5730005 & 27.6909905 & 20.87241 \\
\hline$E+10$ & 25.06896 & 24.3280895 & & 27.3964765 & 20.3028 \\
\hline $1.79 \mathrm{E}+10$ & 24.34449 & 24.818562 & 5.5985125 & 28.2781265 & 20.7258 \\
\hline & & & & 29.9222015 & 21.56271 \\
\hline+10 & 24.05711 & 24.172611 & 3975675 & 28.536264 & 20.7742 \\
\hline & & & & 5.7312125 & 19.7755 \\
\hline & & $20 x$ & & $=0707$ & \\
\hline
\end{tabular}




\begin{tabular}{|r|r|r|r|r|r|}
\hline Frequency & e" @ 20 C & \multicolumn{1}{|c|}{ e" @ 40 C } & e" @ 60 C & e" @ 80 C & e" @ 100 C \\
\hline $1.84 \mathrm{E}+10$ & 23.65681 & 22.193383 & 23.168587 & 25.265084 & 19.0084505 \\
\hline $1.85 \mathrm{E}+10$ & 23.37783 & 22.4362745 & 23.4147515 & 25.7381055 & 19.2166685 \\
\hline $1.86 \mathrm{E}+10$ & 22.61402 & 22.94558 & 23.706185 & 26.428264 & 19.5539575 \\
\hline $1.87 \mathrm{E}+10$ & 22.23525 & 22.60553 & 23.516721 & 26.4791425 & 19.5183475 \\
\hline $1.88 \mathrm{E}+10$ & 23.12055 & 22.586403 & 23.68709 & 26.0315465 & 19.265405 \\
\hline $1.89 \mathrm{E}+10$ & 23.48182 & 22.6812355 & 23.5890645 & 25.43068 & 18.9456655 \\
\hline $1.9 \mathrm{E}+10$ & 23.68889 & 22.9196185 & 23.59539 & 25.605412 & 19.0073915 \\
\hline $1.91 \mathrm{E}+10$ & 23.95918 & 22.7470635 & 23.4821305 & 25.6137895 & 19.0051895 \\
\hline $1.92 \mathrm{E}+10$ & 23.24911 & 22.374854 & 23.0619065 & 25.312973 & 18.704161 \\
\hline $1.93 \mathrm{E}+10$ & 22.40579 & 22.524077 & 23.083795 & 25.6084435 & 18.775199 \\
\hline $1.94 \mathrm{E}+10$ & 22.31985 & 22.375626 & 23.0786085 & 25.3599575 & 18.652855 \\
\hline $1.95 \mathrm{E}+10$ & 22.53326 & 21.692746 & 22.5265285 & 24.322604 & 18.0358405 \\
\hline $1.96 \mathrm{E}+10$ & 22.91337 & 21.934715 & 22.6633855 & 24.3661035 & 18.156972 \\
\hline $1.97 \mathrm{E}+10$ & 22.80849 & 22.1579255 & 22.848516 & 24.7389525 & 18.435618 \\
\hline $1.98 \mathrm{E}+10$ & 23.44783 & 22.738995 & 23.4316315 & 25.637945 & 18.837272 \\
\hline $1.99 \mathrm{E}+10$ & 23.69557 & 23.1669415 & 23.837607 & 26.2139485 & 19.1915655 \\
\hline $2 \mathrm{E}+10$ & 23.65878 & 23.6916555 & 24.276313 & 26.5104525 & 19.4834245 \\
\hline
\end{tabular}


Table 14. Loss Tangent for the Revised ORNL Simulant

\begin{tabular}{|c|c|c|c|c|c|}
\hline & e $20 \mathrm{C}$ & $\overline{0 C}$ & $\overline{60 \mathrm{C}}$ & le'@80C & $\overline{00 \mathrm{C}}$ \\
\hline $1.53 E+08$ & 9.573549 & 5.74145723 & 6.144509131 & 6.47008248 & 6 \\
\hline $2.55 E+08$ & 9.407608 & 7.29792115 & 8.034310381 & 8.694930047 & 3.191726477 \\
\hline $3.58 \mathrm{E}+08$ & 8.794304 & 8.07673617 & 9.126152022 & 10.10487406 & 35112 \\
\hline $4.6 \mathrm{E}+08$ & 7.901625 & 7.93530228 & 9.105442978 & 10.11685115 & 9.825617319 \\
\hline $5.62 \mathrm{E}+08$ & 7.43366 & 8.03319641 & 9.417856197 & 10.60291893 & 10.37543628 \\
\hline $6.65 \mathrm{E}+08$ & 6.76126 & 7.71218119 & 9.151520119 & 10.25752005 & 10.2094768 \\
\hline $7.67 \mathrm{E}+08$ & 6.215742 & 7.44244705 & 8.982634172 & 10.24047652 & 10.16914927 \\
\hline $8.69 \mathrm{E}+08$ & 5.799655 & 7.23966873 & 8.878634019 & 10.28604989 & 10.24194701 \\
\hline $9.71 \mathrm{E}+08$ & 5.393903 & 6.94918645 & 8.623349802 & 10.12865779 & 10.11627347 \\
\hline $1.07 \mathrm{E}+09$ & 5.070889 & 6.76121232 & 8.49706294 & 10.0535224 & 10.12071645 \\
\hline $1.18 \mathrm{E}+09$ & 4.817024 & 6.53324422 & 8.301068827 & 9.876795685 & 9.975441428 \\
\hline $1.28 \mathrm{E}+09$ & 4.562407 & 6.34242873 & 8.134569076 & 9.67442121 & 9.812978625 \\
\hline $1.38 \mathrm{E}+09$ & 4.319599 & 5.97171183 & 7.672422297 & 9.11849933 & 9.251990803 \\
\hline $1.48 \mathrm{E}+09$ & 4.07736 & 5.78523872 & 7.494140127 & 9.044550489 & 9.151546367 \\
\hline $1.59 \mathrm{E}+09$ & 3.889631 & 5.57020394 & 7.25843507 & 8.848510213 & 8.978809479 \\
\hline $1.69 \mathrm{E}+09$ & 3.735385 & 5.4050719 & 7.067219509 & 8.655942316 & 8.849510758 \\
\hline $1.79 \mathrm{E}+09$ & 3.593923 & 5.21800497 & 6.850917137 & 8.374040442 & 8.596097881 \\
\hline $1.89 E+09$ & 3.456492 & 5.07661429 & 6.717714815 & 8.251597807 & 8.400410278 \\
\hline $1.99 \mathrm{E}+09$ & 3.323392 & 4.94178012 & 6.554943351 & 8.003 & 086 \\
\hline $2.1 \mathrm{E}+09$ & 3.171407 & 4.75409354 & 6.307135591 & 7.728744804 & 7.934662812 \\
\hline $2.2 \mathrm{E}+09$ & 3.070673 & 4.59198727 & 6.085906638 & 7.507409638 & 7.763886581 \\
\hline $2.3 \mathrm{E}+09$ & 2.975101 & 4.4438613 & 5.920035822 & 7.421301378 & 7.616656968 \\
\hline $2.4 \mathrm{E}+09$ & 2.895673 & 4.33110221 & 5.800234887 & 7.295017587 & 7.456815923 \\
\hline $2.51 \mathrm{E}+09$ & 2.822399 & 4.26077208 & 5.705564128 & 7.085848277 & 7.338797097 \\
\hline $2.61 E+09$ & 2.74022 & 4.19391031 & 5.645577704 & 6.971166015 & 7.263524076 \\
\hline $2.71 E+09$ & 2.672059 & 4.08536965 & 5.49826195 & 6.800705047 & 7.075132547 \\
\hline $2.81 E+09$ & 2.600993 & 3.96825123 & 5.345053702 & 6.697592651 & 6.927090754 \\
\hline $2.92 \mathrm{E}+09$ & 2.541307 & 3.88610099 & 5.251549874 & 6.702293518 & 6.90654576 \\
\hline $3.02 E+09$ & 2.489462 & 3.81340366 & 5.155712418 & 6.580543633 & 6.834258713 \\
\hline $3.12 \mathrm{E}+09$ & 2.440836 & 3.74342906 & 5.061793242 & 6.388377109 & 6.68671682 \\
\hline $3.22 \mathrm{E}+09$ & 2.389818 & 3.66120675 & 4.955836544 & 6.185974393 & 6.474557648 \\
\hline $3.32 \mathrm{E}+09$ & 2.336959 & 3.57104919 & 4.819980741 & 5.996587423 & 6.265165208 \\
\hline $3.43 E+09$ & 2.283574 & 3.48731602 & 4.694955426 & 5.866191047 & 6.134628676 \\
\hline $3.53 E+09$ & 2.233578 & 3.42796617 & 4.60922129 & 5.81736965 & 6.100721489 \\
\hline $3.63 \mathrm{E}+09$ & 2.184534 & 3.34952986 & 4.496605888 & 5.71921695 & 6.004533669 \\
\hline $3.73 \mathrm{E}+09$ & 2.15306 & 3.27716109 & 4.399747413 & 5.5758654 & 5.843879426 \\
\hline $3.84 \mathrm{E}+09$ & 2.119143 & 3.20851225 & 4.297872579 & 5.358743101 & 5.633365287 \\
\hline $3.94 \mathrm{E}+09$ & 2.085367 & 3.15773744 & 4.220125034 & 5.185825386 & 5.499143532 \\
\hline $4.04 \mathrm{E}+09$ & 2.052389 & 3.11496619 & 4.155629473 & 5.115356032 & 5.44740853 \\
\hline $4.14 \mathrm{E}+09$ & 2.024563 & 3.06495939 & 4.086854929 & 5.10447842 & 5.408378082 \\
\hline $4.25 \mathrm{E}+09$ & 1.995176 & 3.00851661 & 4.009235181 & 5.063702997 & 5.343330412 \\
\hline $4.35 \mathrm{E}+09$ & 1.972967 & 2.96736434 & 3.949237081 & 4.984595354 & 5.272905737 \\
\hline $4.45 \mathrm{E}+09$ & 1.954052 & 2.94499739 & 3.919596327 & 4.877393481 & 5.19948465 \\
\hline
\end{tabular}




\begin{tabular}{|c|c|c|c|c|c|}
\hline ncy & e $20 \mathrm{C}$ & $10 \mathrm{C}$ & le'@60C & $e^{\prime \prime} / e^{\prime} @ 80 \mathrm{C}$ & $\overline{00 \mathrm{C}}$ \\
\hline $4.55 \mathrm{E}+09$ & 1.935057 & 2.9087055 & 3.864960939 & 4.72618279 & 5.060707215 \\
\hline $4.65 E+09$ & .917688 & 2.85750061 & 3.78437059 & 4.595675705 & 4.906513919 \\
\hline $4.76 \mathrm{E}+09$ & 1.900373 & 2.83851823 & 3.769865539 & 4.657078083 & 4.944993935 \\
\hline $4.86 \mathrm{E}+09$ & 1.882592 & 2.82409622 & 3.751858177 & 4.731498129 & 5.017172293 \\
\hline $4.96 \mathrm{E}+09$ & 1.864063 & 2.80190515 & 3.727625079 & 4.733486808 & 5.029922429 \\
\hline $5.06 \mathrm{E}+09$ & 1.844401 & 2.7520675 & 3.655279447 & 4.583106239 & 4.880983015 \\
\hline $5.17 \mathrm{E}+09$ & 1.831668 & 2.71646773 & 3.599147158 & 4.433732106 & 4.733267398 \\
\hline $5.27 \mathrm{E}+09$ & 1.815449 & 2.67635828 & 3.536267875 & 4.291664397 & 4.589172384 \\
\hline $5.37 \mathrm{E}+09$ & 1.806248 & 2.64672991 & 3.486126951 & 4.239275304 & 4.533009076 \\
\hline $5.47 \mathrm{E}+09$ & 1.79143 & 2.63380116 & 3.46811912 & 4.294200124 & 4.592202179 \\
\hline $5.57 \mathrm{E}+09$ & 1.776832 & 2.61965075 & 3.453311631 & 4.357916165 & 4.651785424 \\
\hline $5.68 \mathrm{E}+09$ & 1.76686 & 2.59226317 & 3.414851636 & 4.297410632 & 4.595645104 \\
\hline $5.78 \mathrm{E}+09$ & 1.749601 & 2.54239882 & 3.337100994 & 4.113058708 & 4.395095369 \\
\hline $5.88 \mathrm{E}+09$ & 1.735936 & 2.4987779 & 3.263946322 & 3.938973508 & 4.2387158 \\
\hline $5.98 \mathrm{E}+09$ & 1.722914 & 2.46806296 & 3.213195741 & 3.864287125 & 4.160930487 \\
\hline $6.09 E+09$ & 1.711521 & 2.4468845 & 3.182111859 & 3.874643878 & 4.151065425 \\
\hline $6.19 E+09$ & 1.697223 & 2.42610845 & 3.155037329 & 3.914673532 & 4.183526008 \\
\hline $6.29 \mathrm{E}+09$ & $1: 678661$ & 2.40547077 & 3.127471289 & 3.899399996 & 4.190619949 \\
\hline $6.39 \mathrm{E}+09$ & 1.666303 & 2.37248529 & 3.073867738 & 3.760947279 & 4.060244808 \\
\hline $6.5 \mathrm{E}+09$ & 1.654545 & 2.31573814 & 2.982719616 & 3.530791231 & 3.833384025 \\
\hline $6.6 \mathrm{E}+09$ & 1.649328 & 2.27556114 & 2.918652148 & 3.405748789 & 3.699184011 \\
\hline $6.7 \mathrm{E}+09$ & 1.646377 & 2.26863248 & 2.906440939 & 3.427311584 & 3.71433818 \\
\hline $6.8 \mathrm{E}+09$ & 1.64452 & 2.26476498 & 2.90129797 & 3.496597344 & 3.773661507 \\
\hline $6.9 \mathrm{E}+09$ & 1.634575 & 2.25658337 & 2.893232791 & 3.546013152 & 3.815401097 \\
\hline $7.01 E+09$ & 1.625382 & 2.23946485 & 2.866837085 & 3.490113946 & 3.775380613 \\
\hline $7.11 \mathrm{E}+09$ & 1.619518 & 2.2130907 & 2.825422732 & 3.354906485 & 3.645557903 \\
\hline $7.21 E+09$ & 1.611052 & 2.16744412 & 2.751616729 & 3.174295696 & 3.451827945 \\
\hline $7.31 E+09$ & 1.603658 & 2.14277879 & 2.710929523 & 3.12074249 & 3.383413519 \\
\hline $7.42 \mathrm{E}+09$ & 1.600941 & 2.12900936 & 2.692705524 & 3.161219095 & 3.426183198 \\
\hline $7.52 \mathrm{E}+09$ & 1.594975 & 2.13234231 & 2.700851885 & 3.261444364 & 3.513195698 \\
\hline $7.62 \mathrm{E}+09$ & 1.585235 & 2.12224901 & 2.686708579 & 3.266360479 & 3.512671035 \\
\hline $7.72 E+09$ & 1.57794 & 2.09082698 & 2.640949991 & 3.147811003 & 3.410300361 \\
\hline $7.83 E+09$ & 1.569747 & 2.05375636 & 2.584170872 & 2.986567005 & 3.256283125 \\
\hline $7.93 E+09$ & 1.564445 & 2.0236925 & 2.536979078 & 2.886291295 & 3.129042633 \\
\hline $8.03 E+09$ & 1.562202 & 1.98621339 & 2.477140091 & 2.834069334 & 3.068582521 \\
\hline $8.13 E+09$ & 1.566007 & 1.99670999 & 2.494934492 & 2.950809205 & 3.173059187 \\
\hline $8.23 E+09$ & 1.566769 & 1.99547002 & 2.490722361 & 3.001234072 & 3.22789413 \\
\hline $8.34 E+09$ & 1.565689 & 1.98839215 & 2.482661157 & 2.970147169 & 3.186208866 \\
\hline $8.44 E+09$ & 1.553215 & 1.95026139 & 2.427263582 & 2.814307265 & 3.035276973 \\
\hline $8.54 \mathrm{E}+09$ & 1.539614 & 1.90275155 & 2.356466675 & 2.653912915 & 2.878908467 \\
\hline $8.64 E+09$ & 1.524174 & 1.86823997 & 2.304112771 & 2.587566551 & 2.798225034 \\
\hline $8.75 \mathrm{E}+09$ & 1.513122 & 1.85169313 & 2.280543434 & 2.624159175 & 2.808370515 \\
\hline $8.85 E+09$ & 1.508781 & 1.84981102 & 2.277413751 & 2.695040612 & 2.886418237 \\
\hline $8.95 \mathrm{E}+09$ & 1.505338 & 1.85414435 & 2.285327002 & 2.722390965 & 2.923851049 \\
\hline $9.05 E+09$ & 1.492416 & 1.8281397 & 2.24812553 & 2.596256633 & 2.800258141 \\
\hline
\end{tabular}




\begin{tabular}{|c|c|c|c|c|c|}
\hline & $0 \mathrm{C}$ & $40 \mathrm{C}$ & $60 \mathrm{C}$ & $\overline{\mathbf{C}}$ & \\
\hline 09 & 1.479477 & 1.78443168 & 2.185150973 & 2.427673726 & $\overline{8762}$ \\
\hline & & & 6382 & 651 & $\overline{51354356}$ \\
\hline+09 & 0875 & 72348342 & 092079347 & 321275515 & 344 \\
\hline $\mathrm{E}+09$ & 460053 & 71047 & 083870877 & 395620329 & 566173462 \\
\hline $\mathrm{E}+09$ & 57741 & 5877 & $\longdiv { 8 6 6 4 3 5 1 4 }$ & 2.451837211 & 6206566 \\
\hline $9.67 \mathrm{E}+09$ & 457342 & 1.7194371 & 2.083808548 & .401823022 & .607244058 \\
\hline $8+09$ & 42549 & 1.68798292 & 2.04571928 & 2.259523625 & .455303721 \\
\hline $9.87 \mathrm{E}+09$ & 1.423345 & 1.63172464 & 1.964076914 & 2.085519166 & .27662819 \\
\hline+09 & 09 & 428 & 1902 & 2.03620841 & .218945122 \\
\hline $1.01 \mathrm{E}+10$ & 108678 & 02885 & 3557 & 2.103709289 & .282513215 \\
\hline+10 & & 268 & & 9996 & 368088927 \\
\hline $1.03 \mathrm{E}+10$ & 25559 & 1.61256801 & .92971592 & 2.23187016 & .406960909 \\
\hline 10 & & 04 & 22506 & 87724 & 542936 \\
\hline $1.05 \mathrm{E}+10$ & 401485 & 1.56574425 & 1.870473326 & 1.975023945 & 2.17792984 \\
\hline 10 & 84 & 4 & 09 & 1.873715162 & 692562 \\
\hline $1.07 \mathrm{E}+10$ & 1.403748 & 1.52060894 & 1.807986652 & 1.929864254 & .098265237 \\
\hline+10 & 073 & 322 & 1.8353076 & 071072754 & 236229619 \\
\hline $1.09 \mathrm{E}+10$ & 1.429747 & 1.55746526 & 1.848840767 & 2.156633887 & 2.309784417 \\
\hline+10 & 85 & 1.5 & 1.82658 & 2.08252007 & 2292 \\
\hline $1.11 \mathrm{E}+10$ & 1481 & 1.5014674 & 1.777211284 & 1.903414244 & 2.086247829 \\
\hline $1.12 \mathrm{E}+10$ & 734 & 1.44889281 & 19 & 1.753901282 & .919314803 \\
\hline $1.13 \mathrm{E}+10$ & 6269 & 1.4502665 & 14272418 & 1.764395299 & 1.921345203 \\
\hline $1.14 \mathrm{E}+10$ & .371203 & 1.44992235 & 1.708860836 & 1.866802269 & 2.012434798 \\
\hline $1.15 \mathrm{E}+10$ & 1.377572 & 33] & 79 & 891 & 699701 \\
\hline+10 & 192 & 694254 & 1.710344063 & 1.987635372 & .154209016 \\
\hline+10 & 67 & 1.38969498 & 1.626147988 & 1.75197942 & 797 \\
\hline+10 & 24239 & 1655 & 53420725 & 1.53348378 & 1.693685945 \\
\hline $1.19 \mathrm{E}+10$ & 8931 & 1068 & 76304033 & 49934343 & .664479424 \\
\hline $1.2 \mathrm{E}+10$ & 1.346932 & 37201756 & 1.600949786 & 055126 & .745012112 \\
\hline $1.21 \mathrm{E}+10$ & 66826 & 10377 & 3594627 & 820484765 & .958290878 \\
\hline $1.22 \mathrm{E}+10$ & 75992 & 3992 & 1.634503648 & 989462912 & .136612788 \\
\hline $1.23 E+10$ & 53939 & 98191 & 70677846 & 1.866256225 & 2.0266874 \\
\hline $1.24 \mathrm{E}+10$ & 5252 & 79714 & 1282391 & 1.601743555 & .759151767 \\
\hline $1.25 \mathrm{E}+10$ & 01023 & 59 & 14 & 88456919 & 1.54912231 \\
\hline$E+10$ & 07 & 027 & 708 & 419614 & .494957042 \\
\hline$E+10$ & 17 & & & 1.504232809 & 532761462 \\
\hline $1.28 \mathrm{E}+10$ & 2498 & 1.35459061 & 1.566800265 & .781501233 & .904353203 \\
\hline $1.29 \mathrm{E}+10$ & 922 & 1.36027522 & 1.567982807 & 3183789 & 214990264 \\
\hline$E+10$ & 84 & 1 & 15854 & 2.038136978 & .175039561 \\
\hline $1.31 \mathrm{E}+10$ & 242 & 1.28784886 & 1.486355747 & 1.713958606 & 31939 \\
\hline $1.32 \mathrm{E}+10$ & 1.304758 & 1.275298 & 467641336 & 1.440507262 & .599569325 \\
\hline+10 & 53 & 1.294 & 1.489910568 & 1.367983305 & 1.511725615 \\
\hline+10 & 43923 & 1.33467554 & .537288922 & 1.495029344 & 1.616524152 \\
\hline+10 & 15 & & 793451 & 1.776371011 & .881364004 \\
\hline 1. & $\longrightarrow$ & 1.32 & 380 & 82 & 2.1.58 \\
\hline
\end{tabular}




\begin{tabular}{|c|c|c|c|c|c|}
\hline ncy & e 20 C & $\overline{0 C}$ & $\overline{\mathrm{OC}}$ & /e'@80 & $e^{\prime \prime} / e^{\prime} @ 100 \mathrm{C}$ \\
\hline $1.38 E+10$ & 1.322802 & 1.27418573 & 1.465172087 & 1.990548126 & 2.119135869 \\
\hline $1.39 \mathrm{E}+10$ & 311232 & 1.24165276 & 1.425763791 & 1.663843658 & 1.804030794 \\
\hline $1.4 \mathrm{E}+10$ & 1.308393 & 1.23723926 & 1.420150594 & 1.396797325 & 1.550173025 \\
\hline $1.41 \mathrm{E}+10$ & 1.307696 & 1.27552647 & 1.467300829 & 1.353490017 & 1.490664449 \\
\hline $1.42 \mathrm{E}+10$ & 1.316905 & 1.31255919 & 1.509641368 & 1.476308917 & 1.571662407 \\
\hline $1.43 \mathrm{E}+10$ & 1.337125 & 1.3256459 & 1.523611969 & 1.727139985 & 1.814040556 \\
\hline $1.44 \mathrm{E}+10$ & 1.35009 & 1.29292984 & 1.485045822 & 1.926203381 & 2.060654641 \\
\hline $1.45 \mathrm{E}+10$ & 1.330032 & 1.21969037 & 1.391906514 & 1.773861593 & 1.944155292 \\
\hline $1.46 \mathrm{E}+10$ & 1.292762 & 1.16080915 & 1.315221177 & 1.422413185 & 1.608364188 \\
\hline $1.47 \mathrm{E}+10$ & 1.278571 & 1.1376018 & 1.289913747 & 1.223170203 & 1.38593332 \\
\hline $1.48 \mathrm{E}+10$ & 1.282162 & 1.1926978 & 1.354533997 & 1.249148754 & 1.379203382 \\
\hline $1.49 \mathrm{E}+10$ & 1.272731 & 1.23529667 & 1.397913958 & 1.389403114 & 1.476475646 \\
\hline $1.5 \mathrm{E}+10$ & 1.293508 & 1.29934366 & 1.477760053 & 1.701597672 & 1.778799523 \\
\hline $1.51 \mathrm{E}+10$ & 1.290057 & 1.2351691 & 1.413095574 & 1.840079056 & 1.962706388 \\
\hline $1.52 \mathrm{E}+10$ & 1.273457 & 1.13524248 & 1.291410688 & 1.603182482 & 1.804447391 \\
\hline $1.53 \mathrm{E}+10$ & 1.218665 & 0.9776457 & 1.102773225 & 1.152088957 & 1.341567734 \\
\hline $1.54 \mathrm{E}+10$ & 1.229039 & 1.01408839 & 1.142677258 & 1.067247769 & 1.226440133 \\
\hline $1.55 \mathrm{E}+10$ & 1.263976 & 1.07816218 & 1.211364019 & 1.105981874 & 1.236592726 \\
\hline $1.56 \mathrm{E}+10$ & 1.32909 & 1.24307223 & 1.388468047 & 1.360572634 & 1.457637981 \\
\hline $1.57 \mathrm{E}+10$ & 1.345867 & 1.35762658 & 1.525514442 & 1.708971592 & 1.805856329 \\
\hline $1.58 \mathrm{E}+10$ & 1.32001 & 1.34908889 & 1.534267899 & 1.925020343 & 2.082986372 \\
\hline $1.59 \mathrm{E}+10$ & 1.285494 & 1.20457146 & 1.369139229 & 1.675117481 & 1.905802105 \\
\hline $1.6 \mathrm{E}+10$ & 1.262165 & 1.03470176 & 1.163318195 & 1.22368456 & 1.423985119 \\
\hline $1.61 \mathrm{E}+10$ & 1.280315 & 0.95179036 & 1.060461681 & 0.993050986 & 1.167208625 \\
\hline $1.62 \mathrm{E}+10$ & 1.348635 & 1.05071265 & 1.165409187 & 1.067928929 & 1.244465949 \\
\hline $1.63 \mathrm{E}+10$ & 1.35185 & 1.29142226 & 1.451793589 & 1.428681165 & 1.61592944 \\
\hline $1.64 \mathrm{E}+10$ & 1.32485 & 1.46097403 & 1.659872922 & 1.829145242 & 1.974225897 \\
\hline $1.65 \mathrm{E}+10$ & 1.325096 & 1.47903633 & 1.684925072 & 1.979416495 & 2.209273048 \\
\hline $1.66 \mathrm{E}+10$ & 1.311927 & 1.25455794 & 1.429952171 & 1.644818889 & 1.906595901 \\
\hline $1.67 \mathrm{E}+10$ & 1.300984 & 1.10015754 & 1.244898298 & 1.290731124 & 1.53566924 \\
\hline $1.68 \mathrm{E}+10$ & 1.31934 & 1.03823257 & 1.155940753 & 1.09652704 & 1.303563394 \\
\hline $1.69 \mathrm{E}+10$ & 1.381474 & 1.17761621 & 1.320692826 & 1.264097668 & 1.483203938 \\
\hline $1.7 \mathrm{E}+10$ & 1.376674 & 1.28912866 & 1.46632739 & 1.501198004 & 1.661104386 \\
\hline $1.71 \mathrm{E}+10$ & 1.339268 & 1.37610757 & 1.552796486 & 1.667263507 & 1.834039977 \\
\hline $1.72 \mathrm{E}+10$ & 1.303141 & 1.32796298 & 1.491617775 & 1.636563361 & 1.843083129 \\
\hline $1.73 E+10$ & 1.301555 & 1.21937848 & 1.391576857 & 1.506806161 & 1.717565152 \\
\hline $1.74 \mathrm{E}+10$ & 1.302745 & 1.06385873 & 1.202531888 & 1.222480148 & 1.412298078 \\
\hline $1.75 \mathrm{E}+10$ & 1.334302 & 1.08816615 & 1.209181575 & 1.189967279 & 1.389442256 \\
\hline $1.76 \mathrm{E}+10$ & 1.361655 & 1.17774124 & 1.320356352 & 1.335304658 & 1.561469321 \\
\hline $1.77 \mathrm{E}+10$ & 1.37872 & 1.2603696 & 1.426860993 & 1.504193134 & 1.688443216 \\
\hline $1.79 \mathrm{E}+10$ & 1.331133 & 1.2876675 & 1.44806862 & 1.556282349 & 1.710968865 \\
\hline $1.8 \mathrm{E}+10$ & 1.309663 & 1.30238621 & 1.462996718 & 1.577181585 & 1.757492793 \\
\hline $1.81 \mathrm{E}+10$ & 1.30023 & 1.1342805 & 1.282050936 & 1.338376901 & 1.529037661 \\
\hline $1.82 \mathrm{E}+10$ & 1.317814 & 1.05865639 & 1.191075716 & 1.193370514 & 1.363400065 \\
\hline $1.83 \mathrm{E}+10$ & 1.294835 & 1.0647482 & 1.178140094 & 1.182497816 & 1.360054066 \\
\hline
\end{tabular}




\begin{tabular}{|r|r|r|r|r|r|}
\hline Frequency & $\mathrm{e}^{\prime \prime} / \mathrm{e}^{20 \mathrm{C}}$ & $\mathrm{e}^{\prime \prime} / \mathrm{e}^{\prime} @ \mathbf{4 0} \mathrm{C}$ & $\mathrm{e}^{\prime \prime} / \mathrm{e}^{\prime} @ \mathbf{6 0 \mathrm { C }}$ & $\mathrm{e}^{\prime \prime} / \mathrm{e}^{\prime} @ 80 \mathrm{C}$ & $\mathrm{e}^{\prime \prime} / \mathrm{e}^{\prime} @ 100 \mathrm{C}$ \\
\hline $1.84 \mathrm{E}+10$ & 1.311655 & 1.12156917 & 1.245377464 & 1.293185628 & 1.476035816 \\
\hline $1.85 \mathrm{E}+10$ & 1.313877 & 1.14545807 & 1.291341796 & 1.361457136 & 1.538741367 \\
\hline $1.86 \mathrm{E}+10$ & 1.277762 & 1.16490855 & 1.298286747 & 1.362977435 & 1.536271654 \\
\hline $1.87 \mathrm{E}+10$ & 1.210525 & 1.08668064 & 1.200653038 & 1.252318496 & 1.423996645 \\
\hline $1.88 \mathrm{E}+10$ & 1.266656 & 1.05448459 & 1.178864411 & 1.191071825 & 1.358788614 \\
\hline $1.89 \mathrm{E}+10$ & 1.329889 & 1.05085066 & 1.173437101 & 1.169729812 & 1.34058705 \\
\hline $1.9 \mathrm{E}+10$ & 1.353495 & 1.12171218 & 1.233598399 & 1.263521174 & 1.431499649 \\
\hline $1.91 \mathrm{E}+10$ & 1.39971 & 1.21286817 & 1.340843268 & 1.405357703 & 1.589404801 \\
\hline $1.92 \mathrm{E}+10$ & 1.440111 & 1.24487025 & 1.386754683 & 1.44243397 & 1.639310528 \\
\hline $1.93 \mathrm{E}+10$ & 1.367748 & 1.20821348 & 1.335562048 & 1.379666409 & 1.559735751 \\
\hline $1.94 \mathrm{E}+10$ & 1.315233 & 1.15823927 & 1.275474363 & 1.297882529 & 1.460554122 \\
\hline $1.95 \mathrm{E}+10$ & 1.336206 & 1.09491881 & 1.210132551 & 1.206880397 & 1.376251756 \\
\hline $1.96 \mathrm{E}+10$ & 1.373758 & 1.09731184 & 1.213309976 & 1.220450238 & 1.396374412 \\
\hline $1.97 \mathrm{E}+10$ & 1.337285 & 1.12220458 & 1.237030323 & 1.272387138 & 1.450745307 \\
\hline $1.98 \mathrm{E}+10$ & 1.361877 & 1.18962404 & 1.31954332 & 1.37717171 & 1.55201068 \\
\hline $1.99 \mathrm{E}+10$ & 1.391194 & 1.20978571 & 1.347788059 & 1.392317071 & 1.58535361 \\
\hline $2 \mathrm{E}+10$ & 1.408411 & 1.21444999 & 1.344755688 & 1.360320403 & 1.564285741 \\
\hline
\end{tabular}


Table 15. Dielectric Constant for the Savannah River Simulant

\begin{tabular}{|c|c|c|c|c|c|}
\hline Frequency & $\mathrm{e}^{\prime} @ 20 \mathrm{C}$ & $e^{\prime} @ 40 \mathrm{C}$ & $e^{\prime} @ 60 C$ & $e^{\prime} @ 80 \mathrm{C}$ & $e^{\prime} @ 100 \mathrm{C}$ \\
\hline 50700000 & 48.438693 & 189.45027 & 213.225603 & 215.832103 & $230: 268814$ \\
\hline $1.53 \mathrm{E}+08$ & 80.214862 & 96.087427 & 101.311857 & 96.875616 & 102.361506 \\
\hline $2.55 \mathrm{E}+08$ & 78.533409 & 82.510151 & 85.3576925 & 80.3431575 & 83.3033385 \\
\hline $3.58 \mathrm{E}+08$ & 77.195498 & 76.531423 & 78.119291 & 72.7247915 & 75.035755 \\
\hline $4.6 \mathrm{E}+08$ & 75.760414 & 73.095908 & 74.0403445 & 68.5541775 & 70.315908 \\
\hline $5.62 E+08$ & 75.015618 & 70.947254 & 71.5013265 & 65.898082 & 67.4427715 \\
\hline $6.65 E+08$ & 74.361339 & 69.656268 & 70.012364 & 64.24369 & 65.699619 \\
\hline $7.67 E+08$ & 73.908196 & 68.645296 & 68.8377505 & 63.0370105 & 64.4469455 \\
\hline $8.69 E+08$ & 73.74882 & 68.101188 & 68.219034 & 62.4930415 & 63.779929 \\
\hline $9.71 E+08$ & 73.181166 & 67.326879 & 67.340909 & 61.552802 & 61.537978 \\
\hline $1.07 \mathrm{E}+09$ & 72.841913 & 66.756009 & 66.652378 & 60.790537 & 59.150523 \\
\hline $1.18 \mathrm{E}+09$ & 72.599122 & 66.189493 & 66.045016 & 60.140568 & 58.6345305 \\
\hline $1.28 E+09$ & 72.271254 & 65.744761 & 65.539944 & 59.6531295 & 58.073313 \\
\hline $1.38 \mathrm{E}+09$ & 71.918899 & $65: 322742$ & 65.1289165 & 59.308413 & 57.637475 \\
\hline $1.48 \mathrm{E}+09$ & 71.700526 & 65.123277 & 64.924383 & 59.057711 & 57.38134 \\
\hline $1.59 \mathrm{E}+09$ & 71.461449 & 64.872969 & 64.6480865 & 58.7717915 & 57.034014 \\
\hline $1.69 \mathrm{E}+09$ & 71.167429 & 64.607713 & 64.3744745 & 58.4784055 & 56.4975925 \\
\hline $1.79 \mathrm{E}+09$ & 71.031145 & 64.244147 & 64.015215 & 58.1749445 & 56.2248735 \\
\hline $1.89 E+09$ & 70.858429 & 63.978723 & 63.755268 & 57.897014 & 56.1101865 \\
\hline 1.99 & 70.724407 & 63.809075 & 63.607708 & 57.808155 & 56.013008 \\
\hline $2.1 \mathrm{E}+09$ & 70.648059 & 63.826285 & 63.6639015 & 57.891274 & 56.0928785 \\
\hline $2.2 \mathrm{E}+09$ & 70.494168 & 63.699712 & 63.5406905 & 57.7785225 & 55.974213 \\
\hline $2.3 \mathrm{E}+09$ & 70.312668 & 63.446735 & 63.2831085 & 57.4463695 & 55.690966 \\
\hline $2.4 \mathrm{E}+09$ & 70.179633 & 63.276125 & 63.104346 & 57.289887 & 55.5136655 \\
\hline $2.51 \mathrm{E}+09$ & 69.977041 & 63.087671 & 62.9541225 & 57.180722 & 55.3459705 \\
\hline $2.61 \mathrm{E}+09$ & 69.804004 & 62.929775 & 62.8246515 & 57.011729 & 55.2092455 \\
\hline $2.71 \mathrm{E}+09$ & 69.676013 & 62.745789 & 62.647116 & 56.855831 & 55.0319215 \\
\hline $2.81 \mathrm{E}+09$ & 69.517103 & 62.55408 & 62.491795 & 56.7807555 & 54.891004 \\
\hline $2.92 \mathrm{E}+09$ & 69.308912 & 62.360516 & 62.3385295 & 56.657329 & 54.727604 \\
\hline $3.02 \mathrm{E}+09$ & 69.10105 & 62.155401 & 62.1443375 & 56.4428945 & 54.5187325 \\
\hline $3.12 \mathrm{E}+09$ & 68.874892 & 61.991314 & 61.9859515 & 56.2325885 & 54.335497 \\
\hline $3.22 \mathrm{E}+09$ & 68.678699 & 61.859923 & 61.873144 & 56.1424205 & 54.217435 \\
\hline $3.32 \mathrm{E}+09$ & 68.534774 & 61.746441 & 61.8003875 & 56.1021035 & 54.1684915 \\
\hline $3.43 \mathrm{E}+09$ & 68.39229 & 61.607487 & 61.7043025 & 56.038945 & 54.083063 \\
\hline $3.53 \mathrm{E}+09$ & 68.288043 & 61.463116 & 61.595198 & 55.925671 & 53.9952495 \\
\hline $3.63 \mathrm{E}+09$ & 68.182658 & 61.32238 & 61.4864745 & 55.8434315 & 53.9145 \\
\hline $3.73 E+09$ & 68.074043 & 61.203 & 61.3991855 & 55.7449655 & 53.8165615 \\
\hline $3.84 E+09$ & 67.941464 & 61.086987 & 61.315802 & 55.687423 & 53.648853 \\
\hline $3.94 \mathrm{E}+09$ & 67.804696 & 61.030233 & 61.2753845 & 55.644886 & 53.611617 \\
\hline $4.04 \mathrm{E}+09$ & 67.653759 & 60.94567 & 61.2355465 & 55.6222955 & 53.6070135 \\
\hline $4.14 \mathrm{E}+09$ & 67.503566 & 60.816206 & 61.1558855 & 55.571066 & 53.567436 \\
\hline $4.25 \mathrm{E}+09$ & 67.38276 & 60.657894 & 61.035663 & 55.477261 & 53.4493975 \\
\hline
\end{tabular}




\begin{tabular}{|c|c|c|c|c|c|}
\hline Frequency & $\mathrm{e}^{\prime} @ 20 \mathrm{C}$ & $e^{\prime} @ 40 \mathrm{C}$ & @60 C & $\mathrm{e}^{\prime} @ 80 \mathrm{C}$ & $e^{\prime} @ 100 \mathrm{C}$ \\
\hline $4.35 \mathrm{E}+09$ & 6863 & 60.474754 & 60.868755 & 55.3515165 & 53.2866665 \\
\hline $4.45 \mathrm{E}+09$ & 67.041114 & 60.313877 & 60.752245 & 55.240373 & 53.1451015 \\
\hline $4.55 E+09$ & 66.844661 & 60.186551 & 60.660764 & 55.132147 & 52.6942145 \\
\hline $4.65 \mathrm{E}+09$ & 66.692203 & 60.06526 & 60.554985 & 55.04871 & 51.793188 \\
\hline $4.76 \mathrm{E}+09$ & 66.475931 & 59.900276 & 60.4495145 & 55.0066775 & 51.8562985 \\
\hline $4.86 \mathrm{E}+09$ & 66.291575 & 59.735761 & 60.3317625 & 54.932679 & 51.7794395 \\
\hline $4.96 \mathrm{E}+09$ & 66.0523 & 59.465279 & 60.121314 & 54.6680615 & 51.614874 \\
\hline $5.06 \mathrm{E}+09$ & 65.821814 & 59.212668 & 59.898766 & 54.471114 & 51.391706 \\
\hline $5.17 \mathrm{E}+09$ & 65.599893 & 59.014847 & 59.7245815 & 54.3268665 & 51.25907 \\
\hline $5.27 \mathrm{E}+09$ & 65.462904 & 58.857328 & 59.608389 & 54.246635 & 51.155148 \\
\hline $5.37 \mathrm{E}+09$ & 65.214627 & 58.713666 & 59.540784 & 54.1208005 & 51.0054095 \\
\hline $5.47 \mathrm{E}+09$ & 65.015935 & 58.551609 & 59.4224175 & 53.9771365 & 50.8806795 \\
\hline $5.57 \mathrm{E}+09$ & 64.789471 & 58.368192 & 59.2821205 & 53.9360765 & 50.789257 \\
\hline $5.68 \mathrm{E}+09$ & 64.599162 & 58.097796 & 59.074564 & 53.795455 & 50.6245715 \\
\hline $5.78 \mathrm{E}+09$ & 64.378752 & 57.841624 & 58.8783805 & 53.5848515 & 50.4327485 \\
\hline $5.88 \mathrm{E}+09$ & 64.183775 & 57.684683 & 58.755392 & 53.4087355 & 50.3213425 \\
\hline $5.98 \mathrm{E}+09$ & 63.976724 & 57.581292 & 58.693457 & 53.4044595 & 50.288137 \\
\hline $6.09 \mathrm{E}+09$ & 63.805067 & 57.459651 & 58.6644905 & 53.450942 & 50.292538 \\
\hline $6.19 \mathrm{E}+09$ & 63.63002 & 57.304668 & 58.5951865 & 53.3446205 & 50.239508 \\
\hline $6.29 \mathrm{E}+09$ & 63.394999 & 57.123041 & 58.4475205 & 53.2295635 & 50.123466 \\
\hline $6.39 \mathrm{E}+09$ & 63.188827 & 56.819483 & 58.219287 & 53.025768 & 49.909116 \\
\hline $6.5 \mathrm{E}+09$ & 62.945122 & 56.565985 & 58.0238595 & 52.8761825 & 49.751465 \\
\hline $6.6 \mathrm{E}+09$ & 62.715205 & 56.327852 & 57.8529615 & 52.701722 & 49.599945 \\
\hline $6.7 \mathrm{E}+09$ & 62.546205 & 56.205236 & 57.7691895 & 52.631174 & 49.5414165 \\
\hline $6.8 \mathrm{E}+09$ & 62.299645 & 56.108275 & 57.7589685 & 52.685206 & 49.570719 \\
\hline $6.9 E+09$ & 62.04805 & 55.925107 & 57.657634 & 52.612024 & 49.5129885 \\
\hline $7.01 \mathrm{E}+09$ & 61.8116 & 55.679526 & 57.468411 & 52.445555 & 49.3567785 \\
\hline $7.11 \mathrm{E}+09$ & 61.568761 & 55.383954 & 57.2105875 & 52.1803365 & 49.1316965 \\
\hline $7.21 \mathrm{E}+09$ & 61.322266 & 55.072847 & 56.944743 & 51.9335 & 49.024712 \\
\hline $7.31 \mathrm{E}+09$ & 61.106248 & 54.871982 & 56.833991 & 51.867474 & 48.9824345 \\
\hline $7.42 \mathrm{E}+09$ & 60.89754 & 54.692409 & 56.7659915 & 51.8141435 & 48.926031 \\
\hline $7.52 \mathrm{E}+09$ & 60.645391 & 54.58018 & 56.672298 & 51.7651615 & 48.84317 \\
\hline $7.62 \mathrm{E}+09$ & 60.419229 & 54.440532 & 56.613132 & 51.7218815 & 48.8333155 \\
\hline $7.72 \mathrm{E}+09$ & 60.203043 & 54.24681 & 56.513545 & 51.604492 & 48.7468195 \\
\hline $7.83 \mathrm{E}+09$ & 59.922804 & 53.917398 & 56.2211815 & 51.362555 & 48.4631905 \\
\hline $7.93 \mathrm{E}+09$ & 59.687198 & 53.591342 & 55.91268 & 51.0672745 & 48.168035 \\
\hline $8.03 \mathrm{E}+09$ & 59.503285 & 53.383143 & 55.8130785 & 50.9914005 & 48.069171 \\
\hline $8.13 E+09$ & 59.293295 & 53.245818 & 55.786219 & 50.983183 & 48.1075915 \\
\hline $8.23 \mathrm{E}+09$ & 59.023783 & 53.220371 & 55.7930435 & 50.999631 & 48.089656 \\
\hline $8.34 \mathrm{E}+09$ & 58.795103 & 53.13967 & 55.7638645 & 51.0043325 & 48.0502765 \\
\hline $8.44 \mathrm{E}+09$ & 58.536043 & 52.906772 & 55.6234815 & 50.922537 & 47.9377475 \\
\hline $8.54 \mathrm{E}+09$ & 58.335074 & 52.544659 & 55.303268 & 50.6161715 & 47.6376865 \\
\hline $8.64 \mathrm{E}+09$ & 58.14962 & 52.275066 & 55.0673975 & 50.3928285 & 47.400419 \\
\hline $8.75 \mathrm{E}+09$ & 57.921938 & 52.049161 & 54.906244 & 50.2576205 & 47.2659955 \\
\hline 7 & 57.705 & 52.027274 & 54.9836345 & 50.4151875 & 47.3887715 \\
\hline
\end{tabular}




\begin{tabular}{|c|c|c|c|c|c|}
\hline ncy & $\mathrm{e}^{\prime} @ \mathbf{2 0 C}$ & $e^{\prime} @ 40 C$ & $\mathrm{e}^{\prime} @ 60 \mathrm{C}$ & $\mathrm{e}^{\prime} @ 80 \mathrm{C}$ & $\mathrm{e}^{\prime} @ 100 \mathrm{C}$ \\
\hline $8.95 \mathrm{E}+09$ & 57.449362 & 52.029437 & 55.045632 & 50.490465 & $\overline{47.44566}$ \\
\hline $9.05 \mathrm{E}+09$ & 57.219013 & 52.003721 & 55.022529 & 50.412252 & 47.3822895 \\
\hline $9.16 \mathrm{E}+09$ & 57.009593 & 51.767677 & 54.8506265 & 50.308163 & 47.2525615 \\
\hline $9.26 \mathrm{E}+09$ & 56.784235 & 51.35346 & 54.491495 & 50.048738 & 46.9911145 \\
\hline $9.36 \mathrm{E}+09$ & 56.587604 & 51.055146 & 54.2208855 & 49.818269 & 46.739066 \\
\hline $9.46 E+09$ & 56.381218 & 50.879864 & 54.1003095 & 49.6798585 & 46.6323585 \\
\hline $9.56 \mathrm{E}+09$ & 56.1473 & 50.911879 & 54.1945 & 49.8167945 & 46.778855 \\
\hline $9.67 \mathrm{E}+09$ & 55.928861 & 50.99571 & 54.390906 & 50.0403965 & 46.988636 \\
\hline $9.77 \mathrm{E}+09$ & 55.644419 & 51.019326 & 54.4402835 & 50.047166 & 47.1609215 \\
\hline $9.87 \mathrm{E}+09$ & 55.460643 & 50.801053 & 54.1518215 & 49.770437 & 46.9166565 \\
\hline $9.97 \mathrm{E}+09$ & 55.289971 & 50.310229 & 53.72477 & 49.4035805 & 46.612186 \\
\hline $1.01 E+10$ & 55.064434 & 49.91256 & 53.4141085 & 49.2335675 & 46.446285 \\
\hline $1.02 \mathrm{E}+10$ & 54.906387 & 49.783347 & 53.3112095 & 49.1228085 & 46.3891455 \\
\hline $1.03 E+10$ & 54.691408 & 49.816994 & 53.398526 & 49.244872 & 46.499764 \\
\hline $1.04 \mathrm{E}+10$ & 54.46465 & 49.909686 & 53.5724535 & 49.4277215 & 46.6886255 \\
\hline $1.05 E+10$ & 54.265691 & 49.917383 & 53.593572 & 49.436103 & 46.700584 \\
\hline $1.06 \mathrm{E}+10$ & 54.035724 & 49.720769 & 53.3667575 & 49.1852945 & 46.500886 \\
\hline $1.07 \mathrm{E}+10$ & 53.83282 & 49.228195 & 52.8340355 & 48.774519 & 46.1100655 \\
\hline $1.08 \mathrm{E}+10$ & 53.652392 & 48.819807 & 52.5373675 & 48.646824 & 46.026386 \\
\hline $1.09 \mathrm{E}+10$ & 53.452886 & 48.675611 & 52.4913565 & 48.6790145 & 46.088949 \\
\hline $1.1 \mathrm{E}+10$ & 53.229186 & 48.748961 & 52.590894 & 48.7025675 & 46.132718 \\
\hline $1.11 \mathrm{E}+10$ & 53.010964 & 48.823556 & 52.6993545 & 48.792041 & 46.239676 \\
\hline $1.12 \mathrm{E}+10$ & 52.773706 & 48.743511 & 52.7055875 & 48.802096 & 46.2398085 \\
\hline $1.13 \mathrm{E}+10$ & 52.559268 & 48.474854 & 52.3837015 & 48.437412 & 45.92285 \\
\hline $1.14 \mathrm{E}+10$ & 52.399428 & 48.11 .2183 & 51.914916 & 47.980926 & 45.4511925 \\
\hline $1.15 E+10$ & 52.241864 & 47.791902 & 51.673696 & 47.9182705 & 45.435028 \\
\hline $1.16 \mathrm{E}+10$ & 52.042233 & 47.678205 & 51.785374 & 48.164418 & 45.8892565 \\
\hline $1.17 \mathrm{E}+10$ & 51.823132 & 47.725315 & 51.961416 & 48.2250405 & 45.9976135 \\
\hline $1.18 \mathrm{E}+10$ & 51.61331 & 47.76642 & 51.9917855 & 48.0692465 & 45.850981 \\
\hline $1.19 \mathrm{E}+10$ & 51.31758 & 47.4297 & 51.642224 & 47.827966 & 45.5753105 \\
\hline $1.2 \mathrm{E}+10$ & 51.168815 & 47.237781 & 51.3715925 & 47.628275 & 45.458446 \\
\hline $1.21 \mathrm{E}+10$ & 50.990087 & 47.117674 & 51.0477215 & 47.243975 & 45.1583795 \\
\hline $1.22 \mathrm{E}+10$ & 50.842188 & 46.831928 & 50.768954 & 46.952138 & 44.973432 \\
\hline $1.23 \mathrm{E}+10$ & 50.619251 & 46.643028 & 50.854167 & 47.2662295 & 45.307786 \\
\hline $1.24 \mathrm{E}+10$ & 50.470523 & 46.825865 & 51.194407 & 47.718371 & 45.71887 \\
\hline $1.25 \mathrm{E}+10$ & 50.209489 & 47.094701 & 51.3612625 & 47.817557 & 45.774042 \\
\hline $1.26 \mathrm{E}+10$ & 49.965656 & 46.756197 & 50.896863 & 47.4579255 & 45.474227 \\
\hline $1.27 \mathrm{E}+10$ & 49.683337 & 46.361289 & 50.584382 & 47.162982 & 45.200899 \\
\hline $1.28 \mathrm{E}+10$ & 49.462362 & 46.16822 & 50.332424 & 46.828651 & 44.930424 \\
\hline $1.29 \mathrm{E}+10$ & 49.314255 & 46.034719 & 50.0696615 & 46.423407 & 44.6461015 \\
\hline $1.3 \mathrm{E}+10$ & 49.195577 & 45.663546 & 49.802998 & 46.3815255 & 44.658734 \\
\hline $1.31 \mathrm{E}+10$ & 49.084691 & 45.731338 & 50.0220445 & 46.83757 & 45.1298175 \\
\hline $1.32 \mathrm{E}+10$ & 48.815148 & 46.117641 & 50.373615 & 47.157042 & 45.345013 \\
\hline $1.33 \mathrm{E}+10$ & 48.586956 & 45.856555 & 49.99055 & 46.762643 & 44.934685 \\
\hline $1.35 \mathrm{E}+10$ & 48.264103 & 45.300006 & 49.5483765 & 46.60246 & 44.8307885 \\
\hline
\end{tabular}




\begin{tabular}{|c|c|c|c|c|c|}
\hline \begin{tabular}{l|l} 
lcy & $e$ \\
\end{tabular} & $\mathrm{e}^{\prime} @ 20 \mathrm{C}$ & @ & $\mathrm{e}^{\prime} @ 60 \mathrm{C}$ & $\mathrm{OC}$ & $\overline{\mathrm{JOC}}$ \\
\hline $.36 \mathrm{E}+10$ & 48.022527 & 45.314824 & 49.7747285 & 47.0846595 & 45.28621 \\
\hline $1.37 \mathrm{E}+10$ & 47.89434 & 45.359759 & 49.7216305 & 46.948044 & 45.2408155 \\
\hline $1.38 \mathrm{E}+10$ & 47.81411 & 44.690023 & 48.9231765 & 46.1092315 & 44.552333 \\
\hline $1.39 \mathrm{E}+10$ & 47.653638 & 44.501064 & 48.7895715 & 46.0279235 & 44.51751 \\
\hline $1.4 \mathrm{E}+10$ & 47.435979 & 44.916905 & 49.2574235 & 46.32652 & 44.8220545 \\
\hline $1.41 E+10$ & 47.189584 & 44.877347 & 49.0580285 & 45.9445085 & 44.4571055 \\
\hline $1.42 \mathrm{E}+10$ & 46.867799 & 44.130502 & 48.3560335 & 45.519337 & 43.9905765 \\
\hline $1.43 \mathrm{E}+10$ & 46.675316 & 44.13627 & 48.724456 & 46.3089455 & 44.667427 \\
\hline $1.44 \mathrm{E}+10$ & 46.610113 & 44.581209 & 49.246837 & 46.741737 & 45.137958 \\
\hline $1.45 \mathrm{E}+10$ & 46.495754 & 44.05379 & 48.329167 & 45.6114735 & 44.0635365 \\
\hline $1.46 \mathrm{E}+10$ & 46.308497 & 43.298312 & 47.417045 & 44.6942175 & 43.312754 \\
\hline $1.47 \mathrm{E}+10$ & 46.02482 & 43.819365 & 48.105054 & 45.3104435 & 43.9742115 \\
\hline $1.48 \mathrm{E}+10$ & 45.734858 & 44.396227 & 48.7243025 & 45.724203 & 44.379315 \\
\hline $1.49 \mathrm{E}+10$ & 45.338569 & 43.371482 & 47.6233755 & 44.927704 & 43.5961605 \\
\hline $1.5 \mathrm{E}+10$ & 45.06382 & 42.815304 & 47.3025775 & 45.1164835 & 43.6778235 \\
\hline $1.51 \mathrm{E}+10$ & 45.070144 & 44.049257 & 48.8062115 & 46.497618 & 44.8445075 \\
\hline $1.52 \mathrm{E}+10$ & 45.029429 & 43.838203 & 48.396377 & 45.91864 & 44.3344245 \\
\hline $1.53 \mathrm{E}+10$ & 44.892184 & 42.127036 & 46.237806 & 43.8229905 & 42.350496 \\
\hline $1.54 \mathrm{E}+10$ & 44.851365 & 42.963985 & 47.1100525 & 44.5169615 & 43.0071035 \\
\hline $1.55 \mathrm{E}+10$ & 44.672436 & 44.278034 & 48.8526195 & 46.1255225 & 44.5823185 \\
\hline $1.56 \mathrm{E}+10$ & 44.170903 & 42.269536 & 46.8608205 & 44.8147185 & 43.4880465 \\
\hline $1.57 \mathrm{E}+10$ & 43.800993 & 41.55433 & 45.8208255 & 43.800871 & 42.446591 \\
\hline $1.58 \mathrm{E}+10$ & 43.588141 & 42.906723 & 47.31552 & 44.93739 & 43.4514475 \\
\hline $1.59 \mathrm{E}+10$ & 43.406987 & 42.396895 & 47.053274 & 44.873829 & 43.481777 \\
\hline $1.6 \mathrm{E}+10$ & 43.388721 & 40.845111 & 44.8885715 & 42.6709195 & 41.4447875 \\
\hline $1.61 \mathrm{E}+10$ & 43.448713 & 41.509614 & 45.183597 & 42.558815 & 41.3714035 \\
\hline $1.62 \mathrm{E}+10$ & 43.234132 & 42.707172 & 47.1306005 & 44.9738825 & 43.646159 \\
\hline $1.63 \mathrm{E}+10$ & 42.872923 & 41.186402 & 45.918277 & 44.4227605 & 43.2089135 \\
\hline $1.64 \mathrm{E}+10$ & 42.511712 & 40.073872 & 43.866843 & 41.7595575 & 40.7020715 \\
\hline $1.65 \mathrm{E}+10$ & 42.215737 & 41.664694 & 45.5548315 & 43.025559 & 41.84021 \\
\hline $1.66 \mathrm{E}+10$ & 42.022972 & 41.919131 & 46.7715495 & 44.798832 & 43.541015 \\
\hline $1.67 \mathrm{E}+10$ & 42.02504 & 39.876302 & 44.0671605 & 42.117466 & 41.07609 \\
\hline $1.68 \mathrm{E}+10$ & 42.046755 & 40.324323 & 43.663752 & 41.067839 & 40.162403 \\
\hline $1.69 \mathrm{E}+10$ & 42.006943 & 42.097762 & 46.391593 & 44.1649915 & 43.1406815 \\
\hline $1.7 \mathrm{E}+10$ & 41.693473 & 40.470815 & 45.5020075 & 44.1357205 & 43.083132 \\
\hline $1.71 \mathrm{E}+10$ & 41.320771 & 39.396747 & 43.1512565 & 41.1301845 & 40.2031305 \\
\hline $1.72 \mathrm{E}+10$ & 41.073741 & 41.013303 & 44.7329085 & 42.3409135 & 41.2864955 \\
\hline $1.73 \mathrm{E}+10$ & 40.850999 & 41.016391 & 45.90633 & 44.097002 & 42.9014885 \\
\hline $1.74 \mathrm{E}+10$ & 40.617915 & 39.432758 & 43.949016 & 42.2202105 & 41.1881925 \\
\hline $1.75 \mathrm{E}+10$ & 40.628771 & 39.998084 & 43.481943 & 41.0259875 & 40.2539365 \\
\hline $1.76 \mathrm{E}+10$ & 40.594205 & 41.048399 & 45.3720465 & 43.204172 & 42.3508435 \\
\hline $1.77 \mathrm{E}+10$ & 40.337805 & 39.799867 & 45.108436 & 44.0081755 & 42.932668 \\
\hline $1.79 \mathrm{E}+10$ & 40.092224 & 39.09807 & 43.1623295 & 41.658232 & 40.7434385 \\
\hline $1.8 \mathrm{E}+10$ & 39.843581 & 39.911045 & 43.515293 & 41.427649 & 40.5254995 \\
\hline $1.81 \mathrm{E}+1$ & 39.60506 & 40.069538 & 44.9451915 & 43.382973 & 42.322344 \\
\hline
\end{tabular}




\begin{tabular}{|r|r|r|r|r|r|}
\hline Frequency & $\mathrm{e}^{\prime} @ 20 \mathrm{C}$ & $\mathrm{e}^{\prime} @$ @ 40 C & $\mathrm{e}^{\prime} @ 60 \mathrm{C}$ & \multicolumn{1}{|c|}{$\mathrm{e}^{\prime} @ 80 \mathrm{C}$} & $\mathrm{e}^{\prime} @ 100 \mathrm{C}$ \\
\hline \hline $1.82 \mathrm{E}+10$ & 39.383192 & 38.78122 & 43.7898985 & 42.718639 & 41.7569425 \\
\hline $1.83 \mathrm{E}+10$ & 39.313868 & 38.69399 & 42.2627805 & 40.2723065 & 39.7901625 \\
\hline $1.84 \mathrm{E}+10$ & 39.311864 & 39.787636 & 44.0287395 & 41.9730465 & 41.571959 \\
\hline $1.85 \mathrm{E}+10$ & 39.125537 & 39.070154 & 44.392587 & 43.423982 & 42.477316 \\
\hline $1.86 \mathrm{E}+10$ & 38.883491 & 37.91646 & 41.8097385 & 40.309152 & 39.506044 \\
\hline $1.87 \mathrm{E}+10$ & 38.666255 & 38.486802 & 41.91283 & 39.833382 & 39.0425395 \\
\hline $1.88 \mathrm{E}+10$ & 38.491612 & 38.932189 & 43.627016 & 42.179833 & 41.0437625 \\
\hline $1.89 \mathrm{E}+10$ & 38.235886 & 37.929792 & 42.4865065 & 41.49414 & 40.330817 \\
\hline $1.9 \mathrm{E}+10$ & 38.141114 & 37.755305 & 41.381435 & 39.6666125 & 38.7596675 \\
\hline $1.91 \mathrm{E}+10$ & 38.060399 & 38.610188 & 42.8746635 & 41.1783885 & 40.0898175 \\
\hline $1.92 \mathrm{E}+10$ & 37.872257 & 37.982001 & 42.7009145 & 41.5733125 & 40.432495 \\
\hline $1.93 \mathrm{E}+10$ & 37.664135 & 37.071444 & 40.934692 & 39.4476225 & 38.579223 \\
\hline $1.94 \mathrm{E}+10$ & 37.471777 & 37.378908 & 41.1517445 & 39.3816615 & 38.5738195 \\
\hline $1.95 \mathrm{E}+10$ & 37.240587 & 37.855897 & 42.201992 & 40.8336895 & 39.8378535 \\
\hline $1.96 \mathrm{E}+10$ & 37.151362 & 37.363385 & 41.7794945 & 40.6421805 & 39.60632 \\
\hline $1.97 \mathrm{E}+10$ & 37.0018 & 36.962676 & 41.178836 & 39.9450195 & 39.0092485 \\
\hline $1.98 \mathrm{E}+10$ & 36.84553 & 37.168844 & 41.4335905 & 40.093052 & 39.0669875 \\
\hline $1.99 \mathrm{E}+10$ & 36.620802 & 36.988418 & 41.181224 & 39.778089 & 38.6694795 \\
\hline $2 \mathrm{E}+10$ & 36.439557 & 36.495695 & 40.5067175 & 39.054423 & 37.981711 \\
\hline
\end{tabular}


Table 16. Dielectric Loss for the Savannah River Simulant

\begin{tabular}{|c|c|c|c|c|c|}
\hline \begin{tabular}{|l|l} 
Frequency & $e$ \\
\end{tabular} & e"@20C & @ 40C & $\bar{C}$ & $\mathbf{e}^{\prime \prime} @$ & $\bar{C}$ \\
\hline 50700000 & 1499.63557 & 1982.61234 & 2412.24084 & 2662.977 & 2846.54148 \\
\hline $1.53 E+08$ & 544.19627 & 687.613584 & 834.75092 & 919.94685 & 985.30268 \\
\hline $2.55 E+08$ & 331.363095 & 418.163164 & 507.4387025 & 559.14928 & 598.83242 \\
\hline $3.58 \mathrm{E}+08$ & 240.059268 & 302.214497 & 366.47876 & 402.98486 & 432.533801 \\
\hline $4.6 \mathrm{E}+08$ & 189.376294 & 238.073336 & 288.448858 & 316.64331 & 340.186791 \\
\hline $5.62 \mathrm{E}+08$ & 156.5673 & 196.480659 & 237.728791 & 261.40283 & 280.833563 \\
\hline $6.65 \mathrm{E}+08$ & 133.980702 & 167.819792 & 202.6942125 & 222.49928 & 239.442421 \\
\hline $7.67 \mathrm{E}+08$ & 117.076676 & 146.418302 & 176.5728415 & 193.55129 & 208.720829 \\
\hline $8.69 E+08$ & 104.191384 & 129.918268 & 156.397205 & 171.42889 & 184.790006 \\
\hline $9.71 E+08$ & 94.278233 & 117.320144 & 140.9541555 & 154.3582 & 163.531387 \\
\hline $1.07 \mathrm{E}+09$ & 86.286682 & 107.109622 & 128.4287475 & 140.38696 & 143.211353 \\
\hline $1.18 \mathrm{E}+09$ & 79.709039 & 98.6280175 & 118.0153305 & 128.79945 & 132.175831 \\
\hline $1.28 \mathrm{E}+09$ & 74.329083 & 91.625669 & 109.3808665 & 119.26057 & 122.542062 \\
\hline $1.38 \mathrm{E}+09$ & 69.6480595 & 85.459841 & 101.778184 & 110.93376 & 113.959636 \\
\hline $1.48 \mathrm{E}+09$ & 65.6293705 & 80.1997815 & 95.2833425 & 103.64819 & 106.630285 \\
\hline $1.59 E+09$ & 62.070132 & 75.623552 & 89.619103 & 97.321944 & 100.231041 \\
\hline $1.69 E+09$ & 58.9896225 & 71.7380595 & 84.7626065 & 91.886599 & 94.5780355 \\
\hline $1.79 \mathrm{E}+09$ & 56.353901 & 68.334693 & 80.5238395 & 87.232601 & 89.5098425 \\
\hline $1.89 \mathrm{E}+09$ & 54.1383655 & 65.344555 & 76.784209 & 83.0217 & 85.2491735 \\
\hline $1.99 \mathrm{E}+09$ & 52.0750905 & 62.4661955 & 73.194891 & 79.068039 & 81.209042 \\
\hline $2.1 E+09$ & 50.229494 & 59.947324 & 70.0508265 & 75.587166 & 77.678065 \\
\hline $2.2 \mathrm{E}+09$ & 48.6153185 & 57.84772 & 67.399038 & 72.62577 & 74.667448 \\
\hline $2.3 \mathrm{E}+09$ & 47.1493795 & 55.9500945 & 64.971217 & 69.797318 & 71.8619985 \\
\hline $2.4 \mathrm{E}+09$ & 45.904063 & 54.224472 & 62.762964 & 67.294738 & 69.306268 \\
\hline $2.51 E+09$ & 44.8438365 & 52.6762695 & 60.755397 & 65.065434 & 66.963529 \\
\hline $2.61 \mathrm{E}+09$ & 43.7534305 & 51.1921865 & 58.865554 & 62.869972 & 64.814713 \\
\hline $2.71 \mathrm{E}+09$ & 42.7991015 & 49.854533 & 57.1193555 & 60.910071 & 62.8101295 \\
\hline $2.81 E+09$ & 41.9656535 & 48.5804425 & 55.473115 & 59.100359 & 60.888298 \\
\hline $2.92 E+09$ & 41.1798475 & 47.4199535 & 53.9571655 & 57.401675 & 59.130131 \\
\hline $3.02 \mathrm{E}+09$ & 40.4475955 & 46.3541195 & 52.570903 & 55.81519 & 57.519883 \\
\hline $3.12 \mathrm{E}+09$ & 39.8013245 & 45.432827 & 51.341937 & 54.337572 & 56.0416475 \\
\hline $3.22 \mathrm{E}+09$ & 39.210732 & 44.563344 & 50.1784245 & 52.981927 & 54.621679 \\
\hline $3.32 \mathrm{E}+09$ & 38.600951 & 43.720023 & 49.0562955 & 51.703196 & 53.2840235 \\
\hline $3.43 \mathrm{E}+09$ & 38.0377465 & 42.895677 & 47.9658225 & 50.496217 & 52.017499 \\
\hline $3.53 \mathrm{E}+09$ & 37.564852 & 42.0895375 & 46.9218515 & 49.286778 & 50.799271 \\
\hline $3.63 \mathrm{E}+09$ & 37.0942285 & 41.406935 & 45.996921 & 48.199883 & 49.6829725 \\
\hline $3.73 \mathrm{E}+09$ & 36.766814 & 40.8503995 & 45.238544 & 47.295887 & 48.726798 \\
\hline $3.84 \mathrm{E}+09$ & 36.4584275 & 40.367906 & 44.545086 & 46.487584 & 47.7891805 \\
\hline $3.94 \mathrm{E}+09$ & 36.1615495 & 39.9274545 & 43.8751895 & 45.683313 & 46.955453 \\
\hline $4.04 \mathrm{E}+09$ & 35.91663 & 39.444335 & 43.176013 & 44.855073 & 46.0983945 \\
\hline $4.14 \mathrm{E}+09$ & 35.6819385 & 38.964044 & 42.5207005 & 44.083758 & 45.313568 \\
\hline $4.25 \mathrm{E}+09$ & 35.4691225 & 38.5437035 & 41.920678 & 43.399864 & 44.5907585 \\
\hline
\end{tabular}




\begin{tabular}{|c|c|c|c|c|c|}
\hline & (a) $20 \mathrm{C}$ & $\bar{C}$ & $\bar{C}$ & "@80C & $\overline{\mathbf{C}}$ \\
\hline+09 & 35.292084 & 38.188586 & .3961285 & 42.771825 & 3856 \\
\hline & 653 & 265 & 6628 & 071 & 3381 \\
\hline 09 & $\overline{0646495}$ & 7.7160465 & 0.5789215 & .684228 & 2.3782 \\
\hline+09 & .949921 & 37.480955 & 89467 & 3300 & 1.04579 \\
\hline $76 \mathrm{E}+09$ & 4.844562 & 37.18573 & 9.7541685 & .684699 & 0.6384 \\
\hline $86 \mathrm{E}+09$ & .7485945 & 36.869979 & .2805935 & 0.12383 & 0.086755 \\
\hline $4.96 \mathrm{E}+09$ & 1.6669965 & 36.5485955 & 8.8457155 & 9.579291 & 9.59124 \\
\hline $5.06 \mathrm{E}+09$ & 4.585542 & 36.35686 & 8.5173305 & 39.16395 & 9.159392 \\
\hline $7 \mathrm{E}+09$ & 13475 & 5.2209785 & 362 & 8.74798 & 8.7129 \\
\hline $5.27 \mathrm{E}+09$ & 1.436405 & .113604 & 37.9730485 & 3.393959 & 8.3232 \\
\hline $37 \mathrm{E}+09$ & 3524855 & 95 & 68908 & 41 & $\overline{7.8346}$ \\
\hline $1 E+09$ & 299214 & 36995 & 7.33214 & 1.50510 & 7.393745 \\
\hline $7 \mathrm{E}+09$ & .260841 & 474 & 36.923096 & 7678 & 2. \\
\hline $8 \mathrm{E}+09$ & .2055275 & 5.24357 & 36.641465 & .789444 & 6.545773 \\
\hline $5.78 \mathrm{E}+09$ & re & $\overline{4845}$ & 36.42978 & 1477 & 25305 \\
\hline $5.88 \mathrm{E}+09$ & 4.186212 & .0781475 & 5.2539745 & 5.183598 & 5.944748 \\
\hline & 3 & & & & 11 \\
\hline $6.09 \mathrm{E}+09$ & 1.1443235 & 4.9306405 & 35.8924425 & 35.723107 & 5.389647 \\
\hline $6.19 E+09$ & 1814 & 933 & .61399 & 5.3859 & .086808 \\
\hline $6.29 \mathrm{E}+09$ & 4.173213 & .5240465 & 35.3262065 & 5.063899 & 34.75437 \\
\hline $6.39 \mathrm{E}+09$ & 1963535 & 05 & 051315 & .148005 & 4.4 \\
\hline $6.5 \mathrm{E}+09$ & 2334915 & .3804655 & 9868075 & 4.542427 & 4.16457 \\
\hline $6.6 \mathrm{E}+09$ & .238336 & .4 & 963769 & 45603 & 34.03299 \\
\hline $6.7 \mathrm{E}+09$ & 2688005 & 34.388478 & 65644 & 4.26995 & 3.808127 \\
\hline $6.8 E+09$ & 34.29039 & 34.30644 & 72091 & .04862 & 3.581827 \\
\hline $6.9 \mathrm{E}+09$ & 4.320916 & 0 & 30427 & 3936 & 3.300123 \\
\hline $7.01 E+09$ & 4.382994 & .8556255 & 3755 & 32 & 2.930977 \\
\hline $11 E+09$ & 4031105 & 33.7093 & 4295 & 644 & 2.686934 \\
\hline $21 E+09$ & 1.447479 & 3.747748 & 45 & 2.926 & 2.617193 \\
\hline $7.31 \mathrm{E}+09$ & .4240415 & 33.851349 & 15937 & 2.939228 & 32.59568 \\
\hline $7.42 \mathrm{E}+09$ & 4.471195 & .9252145 & 68471 & 2.979961 & 2.595870 \\
\hline $7.52 \mathrm{E}+09$ & .5086875 & 3.8176395 & 3.8090095 & 2.78031 & 2.36328 \\
\hline $7.62 \mathrm{E}+09$ & 4.540916 & 5 & 0917 & .398129 & 1.99112 \\
\hline $2 \mathrm{E}+09$ & 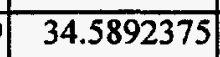 & & .19905 & 2.0770 & 1.673330 \\
\hline $7.83 E+09$ & .5962375 & 3.2271955 & 33.095874 & 62238 & 14510 \\
\hline$\overline{3 \mathrm{E}+09}$ & .632618 & 2785455 & 33.086141 & 31.831914 & 31.25505 \\
\hline $3 E+09$ & .642364 & .4315225 & 3.1887875 & 1.82598 & 11.196527 \\
\hline $3 E+09$ & .6297455 & 19907 & 189485 & 1.846115 & 1.193625 \\
\hline $8.23 \mathrm{E}+09$ & 34.681729 & 33.28817 & 33.0824125 & 31.689743 & 31.00944 \\
\hline $8.34 \mathrm{E}+09$ & 34.731363 & 33.026759 & 32.724915 & 31.29112 & 30.56729 \\
\hline $4 \mathrm{E}+09$ & 9878 & 8494275 & 32.500901 & 30.999679 & 30.24897 \\
\hline $8.54 \mathrm{E}+09$ & 34.763789 & 32.7212345 & 32.4103155 & 30.860053 & 0.044882 \\
\hline $4 E+09$ & 7681795 & 32.8501955 & 32.561732 & 30.944815 & 30.048678 \\
\hline & 34.8187775 & & 8790 & 0.99683 & $\bar{n}$ \\
\hline $8.85 E+U 9$ & $\overline{4.8}$ & 3.0347805 & 32. & 30.9 & 0.01 \\
\hline
\end{tabular}




\begin{tabular}{|c|c|c|c|c|c|}
\hline Frequency & $e^{\prime \prime} @ 20 \mathrm{C}$ & $e^{\prime \prime} @ 40 C$ & $e^{\prime \prime @ 60 ~ C ~}$ & $\mathrm{e}^{\prime \prime} @ 80 \mathrm{C}$ & $e^{\prime \prime} @ 100 \mathrm{C}$ \\
\hline $8.95 \mathrm{E}+09$ & 34.8755025 & 32.889857 & 32.5653175 & 30.855414 & 29.891605 \\
\hline $9.05 E+09$ & 34.938954 & 32.5581345 & 32.1857025 & 30.42157 & 29.467844 \\
\hline $9.16 \mathrm{E}+09$ & 34.950811 & 32.4428985 & 31.982255 & 30.147183 & 29.1269395 \\
\hline $9.26 E+09$ & 35.0032395 & 32.3232895 & 31.9184045 & 30.079444 & 29.009974 \\
\hline $9.36 \mathrm{E}+09$ & 35.0549025 & 32.5133515 & 32.188558 & 30.29917 & 29.14226 \\
\hline $9.46 \mathrm{E}+09$ & 35.0726695 & 32.7102595 & 32.380884 & 30.392526 & 29.1990325 \\
\hline $9.56 \mathrm{E}+09$ & 35.0811465 & 32.715439 & 32.2969345 & 30.244586 & 29.0720115 \\
\hline $9.67 \mathrm{E}+09$ & 35.159371 & 32.5432475 & 32.105277 & 30.08804 & 28.935166 \\
\hline $9.77 \mathrm{E}+09$ & 35.183858 & 32.274454 & 31.8879325 & 29.885561 & 28.8065745 \\
\hline $9.87 E+09$ & 35.207662 & 32.1446575 & 31.6716455 & 29.598642 & 28.5023615 \\
\hline $9.97 \mathrm{E}+09$ & 35.248291 & 32.073047 & 31.6194325 & 29.449799 & 28.339508 \\
\hline $1.01 \mathrm{E}+10$ & 35.3171185 & 32.260798 & 31.934069 & 29.758744 & 28.571003 \\
\hline $1.02 \mathrm{E}+10$ & 35.3150805 & 32.435958 & 32.15021 & 29.939838 & 28.704585 \\
\hline $1.03 \mathrm{E}+10$ & 35.329111 & 32.5046045 & 32.105733 & 29.812785 & 28.580907 \\
\hline $1.04 \mathrm{E}+10$ & 35.365623 & 32.328098 & 31.847004 & 29.532717 & 28.3560735 \\
\hline $1.05 \mathrm{E}+10$ & 35.387418 & 32.0967185 & 31.644327 & 29.369914 & 28.1997205 \\
\hline $1.06 \mathrm{E}+10$ & 35.422995 & 31.9221345 & 31.5240315 & 29.241678 & 28.0250575 \\
\hline $1.07 \mathrm{E}+10$ & 35.4815995 & 31.9550855 & 31.558389 & 29.203731 & 27.918633 \\
\hline $1.08 \mathrm{E}+10$ & 35.528162 & 32.0802115 & 31.7396345 & 29.347191 & 28.013129 \\
\hline $1.09 E+10$ & 35.546886 & 32.190722 & 31.986857 & 29.60579 & 28.247171 \\
\hline $1.1 \mathrm{E}+10$ & 35.537417 & 32.3638375 & 32.088007 & 29.614557 & 28.2820635 \\
\hline $1.11 \mathrm{E}+10$ & 35.5932165 & 32.327379 & 31.88696 & 29.324086 & 28.010074 \\
\hline $1.12 \mathrm{E}+10$ & 35.6481075 & 32.082447 & 31.6563335 & 29.082843 & 27.807034 \\
\hline $1.13 \mathrm{E}+10$ & 35.7160205 & 31.9141825 & 31.7121925 & 29.189154 & 27.8223695 \\
\hline $1.14 \mathrm{E}+10$ & 35.743866 & 31.965487 & 31.8252795 & 29.254528 & 27.6916865 \\
\hline $1.15 \mathrm{E}+10$ & 35.7939195 & 32.0526605 & 31.8506375 & 29.237722 & 27.832513 \\
\hline $1.16 \mathrm{E}+10$ & 35.8015975 & 32.1460085 & 31.9841475 & 29.389402 & 28.312168 \\
\hline $1.17 \mathrm{E}+10$ & 35.8104865 & 32.276763 & 32.137073 & 29.50998 & 28.4630975 \\
\hline $1.18 \mathrm{E}+10$ & 35.832549 & 32.28168 & 32.0265935 & 29.182825 & 28.1283465 \\
\hline $1.19 \mathrm{E}+10$ & 35.8969285 & 32.032361 & 31.6479665 & 28.722968 & 27.645088 \\
\hline $1.2 \mathrm{E}+10$ & 35.96005 & 31.728033 & 31.5590555 & 28.745216 & 27.6442635 \\
\hline $1.21 \mathrm{E}+10$ & 36.0164595 & 31.8139595 & 31.8099265 & 29.09096 & 27.939102 \\
\hline $1.22 \mathrm{E}+10$ & 36.1131645 & 32.0937845 & 32.0439365 & 29.191279 & 28.0297635 \\
\hline $1.23 \mathrm{E}+10$ & 36.0977855 & 32.0629665 & 31.964949 & 29.05502 & 27.862779 \\
\hline $1.24 \mathrm{E}+10$ & 36.09763 & 31.873457 & 31.9704975 & 29.111143 & 27.8864145 \\
\hline $1.25 \mathrm{E}+10$ & 36.0823725 & 31.9972845 & 32.1164365 & 29.175244 & 27.8701145 \\
\hline $1.26 \mathrm{E}+10$ & 36.095907 & 31.847328 & 31.8088 & 28.655384 & 27.388727 \\
\hline $1.27 \mathrm{E}+10$ & 36.212298 & 31.633534 & 31.554266 & 28.423028 & 27.19452 \\
\hline $1.28 \mathrm{E}+10$ & 36.3024165 & 31.612282 & 31.631049 & 28.569507 & 27.3535915 \\
\hline $1.29 \mathrm{E}+10$ & 36.301784 & 32.0298915 & 32.033142 & 28.739821 & 27.616651 \\
\hline $1.3 \mathrm{E}+10$ & 36.3705265 & 32.19415 & 32.074991 & 28.615711 & 27.5093 \\
\hline $1.31 \mathrm{E}+10$ & 36.3632915 & 32.010202 & 32.0683965 & 28.796986 & 27.644989 \\
\hline $1.32 \mathrm{E}+10$ & 36.2932795 & 32.0449415 & 32.3118255 & 29.395809 & 28.026255 \\
\hline $1.33 \mathrm{E}+10$ & 36.294574 & 32.2299265 & 32.41242 & 29.32514 & 27.8964555 \\
\hline $1.35 \mathrm{E}+10$ & 36.351047 & 31.768035 & 31.7026805 & 28.426914 & 27.081191 \\
\hline
\end{tabular}




\begin{tabular}{|c|c|c|c|c|c|}
\hline & $0 \mathrm{C}$ & @ $40 \mathrm{C}$ & $\mathrm{C}$ & $30 \mathrm{C}$ & $\mathbf{C}$ \\
\hline $6 \bar{E}+10$ & 36.4512325 & 31.3929965 & 31.3296285 & 28.059712 & .82202 \\
\hline 101 & 353 & 964778 & 2.037071 & .741812 & 2408 \\
\hline+10 & 489 & .211002 & 32.2962665 & $\overline{8} . \overline{8} 31483$ & 7.65860 \\
\hline $1.39 \mathrm{E}+10$ & 4441 & 31.838582 & 1.853095 & 28.55652 & 7.364319 \\
\hline+10 & .478092 & 762595 & 2.064891 & 29.06776 & 7.788406 \\
\hline 1.41 & .4870725 & 2.337772 & 2.8 & 29.83 & 8.387984 \\
\hline $\bar{E}+10$ & 5.5500865 & .2682615 & 2.612704 & 29.421248 & 27.91280 \\
\hline $1.43 \mathrm{E}+10$ & 36.6640735 & .523279 & 31.715431 & 28.607155 & 27.098 \\
\hline+10 & .736706 & 7467125 & .0630295 & 28.99001 & 27.51844 \\
\hline $5 E+10$ & 5.765864 & .429968 & .755426 & 9.405064 & 8.021468 \\
\hline+10 & .65 & 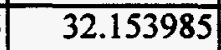 & 8808 & 3.856937 & 7.47725 \\
\hline $1.47 \mathrm{E}+10$ & .4893035 & 31.561148 & 1.7920655 & 8.621324 & 27.20137 \\
\hline+10 & & 95 & 85 & 9.619653 & 8.030 \\
\hline $1.49 \mathrm{E}+10$ & 6.515087 & 2.831029 & 33.35438 & 29.99122 & 28.26240 \\
\hline $5 \mathrm{E}+10$ & 2875 & 31.650818 & 31.961169 & 8.735814 & 27.05 \\
\hline $1.51 \mathrm{E}+10$ & 36.947625 & 31.499476 & 31.858812 & 8.878821 & 27.21356 \\
\hline $1.52 \mathrm{E}+10$ & .1407325 & .8223325 & 33.31513 & .040485 & 28.314 \\
\hline $1.53 \mathrm{E}+10$ & 7.006317 & 32.474184 & 2.883889 & .588205 & 27. \\
\hline $1.54 \mathrm{E}+10$ & .71 & 31.028967 & .3044115 & $3.18300 \mathrm{C}$ & .588301 \\
\hline $5 \mathrm{E}+10$ & 6.676338 & 32.588438 & 3.099396 & .479023 & 27.77443 \\
\hline $1.56 \mathrm{E}+10$ & .5671555 & 3.6382615 & 4.5154855 & 30.786439 & 8.937636 \\
\hline $1.57 \mathrm{E}+10$ & , & .737818 & 38 & 9.321 & 41 \\
\hline $8 E+10$ & 7.051934 & 1.7773035 & $32: 052739$ & 28.833262 & 27.140379 \\
\hline $1.59 \mathrm{E}+10$ & 1825 & .231157 & 6944895 & .202435 & 8.396845 \\
\hline $1.6 \mathrm{E}+10$ & 0376135 & 32.569642 & 3.354694 & 30.26568 & 28.469818 \\
\hline$\overline{E+10}$ & 9616755 & 1.167675 & 70225 & 8.403912 & 6.701764 \\
\hline $2 \mathrm{E}+10$ & .8436605 & .1527995 & .3119055 & 8.781236 & 26.92974 \\
\hline $1.63 E+10$ & 6.640545 & .3657075 & .385345 & 1.271039 & 9.259904 \\
\hline $1.64 \mathrm{E}+10$ & .5681835 & .747217 & 2.639713 & 29.732663 & 27.90685 \\
\hline $\mathrm{E}+10$ & .740828 & .0224435 & 1.8881605 & 7.327213 & 5.676735 \\
\hline $1.66 \mathrm{E}+10$ & 960416 & 32.744349 & $2.90508 \mathrm{i}$ & 29.176493 & 7.424726 \\
\hline $1.67 \mathrm{E}+10$ & 0299325 & 36925 & 3.761377 & 0.832125 & 28.9014 \\
\hline $1.68 \mathrm{E}+10$ & 9706785 & .876216 & 1.3567725 & 8.316543 & 6.470533 \\
\hline $1.69 \mathrm{E}+10$ & 8731155 & 1.904529 & 31.593536 & 7.872192 & 26.00890 \\
\hline $7 \mathrm{E}+10$ & 7251 & 208642 & 34.196114 & 0.95563 & 28.900984 \\
\hline $71 E+10$ & .6076955 & 1.263501 & 2.3606685 & 9.568983 & 27.599071 \\
\hline $2 \mathrm{E}+10$ & 6823115 & 30.9660715 & .765222 & 7.131558 & 25.3723195 \\
\hline $\mathrm{E}+10$ & 89 & 32.956851 & 32.882533 & 28.867985 & 27.05273 \\
\hline $1.74 \mathrm{E}+10$ & 9943485 & 32.813809 & 34.047856 & 30.92913 & 28.8490935 \\
\hline $1.75 \mathrm{E}+10$ & 36.9386965 & 31.319147 & 31.986651 & 28.989469 & 26.96174 \\
\hline $1.76 \mathrm{E}+10$ & 36.888561 & 32.12575 & 31.7899475 & 28.002451 & 26.0801725 \\
\hline $8+10$ & 864 & 5051 & 33.894006 & 30.440939 & 28.239035 \\
\hline $1.79 E+10$ & 36.6604375 & 32.194456 & 33.6326395 & 30.941339 & 28.728601 \\
\hline$E+10$ & 624376 & .763571 & .0164775 & 28.762587 & 26.872584 \\
\hline 815 & 36.78 & 2.8733755 & 32.806 & 28.732532 & 7.00271 \\
\hline
\end{tabular}




\begin{tabular}{|c|c|c|c|c|c|}
\hline & $\bar{C}$ & $\bar{C}$ & $\bar{C}$ & $\mathrm{JC}$ & $\bar{C}$ \\
\hline & 582 & 33. & 34.723233 & 574 & 39. \\
\hline & & & & & \\
\hline 10 & 741 & 65 & 91125 & 64 & 137 \\
\hline & 973 & & 1865 & | & 0.2 \\
\hline+10 & 36.581971 & 0295 & 894656 & 315 & $\overline{462}$ \\
\hline-10 & 36.6079775 & 005 & 34523 & 29.139569 & 27.62738 \\
\hline+10 & 36.642723 & 32.5646545 & 8885 & 158 & 27.438147 \\
\hline-10 & 884 & 345 & 8665 & 30.979025 & 29. \\
\hline+10 & 945 & 9685 & 8915 & 70 & 28.02844 \\
\hline+10 & 36.6983775 & 32.039232 & 9025 & 28.165068 & 26.755621 \\
\hline $1.92 \mathrm{E}+10$ & 36.62 & $\overline{32.72}$ & 58585 & 29.6856 & 8.058058 \\
\hline $1.93 \mathrm{E}+10$ & 6.5634135 & $\overline{32.26}$ & 33.0420025 & 30.008302 & 28.3256 \\
\hline $1.94 \mathrm{E}+10$ & 6.5906855 & 31.8210855 & 81475 & 29.010005 & 7.4316865 \\
\hline $1.95 \mathrm{E}+10$ & 36.584928 & 32.2119055 & 32.6196215 & 29.284159 & 69916 \\
\hline $1.96 \mathrm{E}+10$ & 36.598291 & 2.351828 & 33.2056975 & 30.00591 & 28.3771665 \\
\hline$E+10$ & तो & 15 & 857 & 849 & $6 \overline{6786}$ \\
\hline $8 E+10$ & 36.525668 & 31.941569 & 32.3486005 & 28.905897 & 27.409148 \\
\hline $9 \mathrm{E}+10$ & & & & 9.328156 & 7145 \\
\hline $2 \mathrm{E}+10$ & 36.4157625 & 32.3722965 & 32.8239325 & 29.457248 & 27.805740 \\
\hline
\end{tabular}


Table 17. Loss Tangent for the Savannah River Simulant

\begin{tabular}{|c|c|c|c|c|c|}
\hline requency & $e^{\prime} 20 \mathrm{C}$ & $e^{\prime \prime} / e^{\prime} 40 C$ & $e^{\prime \prime} / e^{\prime} 60 \mathrm{C}$ & $e^{\prime \prime} / e^{\prime} 80 \mathrm{C}$ & $e^{\prime \prime} / e^{\prime} 100 \mathrm{C}$ \\
\hline 000 & 564 & 0805 & 092 & 12.3381876 & 12.361819 \\
\hline $1.53 \mathrm{E}+08$ & 6.784232447 & 7.15612446 & 8.2394198 & 9.49616521 & 62571501 \\
\hline $2.55 \mathrm{E}+08$ & 4.219390183 & 5.06802086 & 5.9448503 & 6.95951342 & 188576482 \\
\hline $3.58 \mathrm{E}+08$ & 3.109757373 & 3.94889426 & 4.6912709 & 5.54123085 & 5.764369273 \\
\hline $4.6 \mathrm{E}+08$ & 2.499673448 & 3.2569995 & 3.8958335 & 4.61887692 & 83797764 \\
\hline $5.62 \mathrm{E}+08$ & 2.08712937 & 2.76939063 & 3.3248165 & 3.96677453 & 4.164027608 \\
\hline $6.65 \mathrm{E}+08$ & 1.801752156 & 2.40925614 & 2.8951202 & 3.46336399 & 3.644502429 \\
\hline $7.67 E+08$ & 1.584082447 & 2.13296921 & 2.5650583 & 3.07043891 & 3.238645787 \\
\hline $8.69 \mathrm{E}+08$ & 1.412787133 & 1.90772395 & 2.2925743 & 2.74316765 & 2.897306541 \\
\hline $9.71 \mathrm{E}+08$ & 1.288285481 & 1.7425454 & 2.093143 & 2.50773633 & 2.657405911 \\
\hline $1.07 E+09$ & 1.184574636 & 1.6044941 & 1.9268442 & 2.30935547 & 2.421134172 \\
\hline $1.18 \mathrm{E}+09$ & 097933925 & 1.49008572 & 1.7868923 & 2.1416401 & 2.254231932 \\
\hline $1.28 \mathrm{E}+09$ & 1.028473693 & 1.39365735 & 1.6689191 & 1.99923401 & 2.110126936 \\
\hline $1.38 \mathrm{E}+09$ & 0.968424996 & 1.30827089 & 1.5627188 & 1.87045564 & 1.977179535 \\
\hline $1.48 \mathrm{E}+09$ & 0.915326213 & 1.23150716 & 1.4676049 & 1.75503229 & 1.858274571 \\
\hline $1.59 \mathrm{E}+09$ & 0.868582058 & 1.16571746 & 1.3862607 & 1.65592951 & 1.757390616 \\
\hline $1.69 \mathrm{E}+09$ & 0.828885114 & 1.1103637 & 1.3167114 & 1.57129112 & 1.674018862 \\
\hline $1.79 \mathrm{E}+09$ & 0.793368895 & 1.06367189 & 1.2578859 & 1.49948748 & 1.591997223 \\
\hline $1.89 \mathrm{E}+09$ & 0.76403565 & 1.02134823 & 1.2043587 & 1.43395478 & 1.519317237 \\
\hline $1.99 \mathrm{E}+09$ & 0.736310034 & 0.97895473 & 1.1507236 & 1.36776617 & 1.449824691 \\
\hline $2.1 \mathrm{E}+09$ & 710981944 & 0.93922628 & 1.1003226 & 1.3056746 & 1.384811532 \\
\hline $2.2 \mathrm{E}+09$ & 0.689636035 & 0.90813158 & 1.0607225 & 1.25696827 & 1.333961551 \\
\hline $2.3 \mathrm{E}+09$ & 0.670567351 & 0.88184356 & 1.0266755 & 1.21499964 & 1.290370839 \\
\hline $2.4 \mathrm{E}+09$ & 0.654093802 & 0.85694995 & 0.9945902 & 1.17463554 & 1.248454185 \\
\hline $2.51 \mathrm{E}+09$ & 0.640836421 & 0.83496932 & 0.9650742 & 1.13789108 & 1.209907937 \\
\hline $2.61 E+09$ & 0.626804027 & 0.81348116 & 0.9369818 & 1.10275504 & 1.173982952 \\
\hline $2.71 \mathrm{E}+09$ & 0.614258767 & 0.79454787 & 0.9117635 & 1.07130737 & 1.141339931 \\
\hline $2.81 \mathrm{E}+09$ & 0.603673801 & 0.77661509 & 0.8876864 & 1.04085192 & 1.109258231 \\
\hline $2.92 \mathrm{E}+09$ & 0.594149386 & 0.76041632 & 0.8655508 & 1.01313767 & 1.080444359 \\
\hline $3.02 \mathrm{E}+09$ & 0.58533981 & 0.74577782 & 0.8459484 & 0.98887895 & 1.055048061 \\
\hline $3.12 \mathrm{E}+09$ & 0.577878576 & 0.73289021 & 0.8282834 & 0.96630039 & 1.031400293 \\
\hline $3.22 \mathrm{E}+09$ & 0.570930039 & 0.7203912 & 0.8109888 & 0.94370578 & 1.007455978 \\
\hline $3.32 \mathrm{E}+09$ & 0.563231613 & 0.70805738 & 0.7937862 & 0.92159103 & 0.98367191 \\
\hline $3.43 \mathrm{E}+09$ & 0.556170096 & 0.69627377 & 0.7773497 & 0.9010915 & 0.961807563 \\
\hline $3.53 E+09$ & 0.550094136 & 0.68479343 & 0.7617778 & 0.88129078 & 0.940810006 \\
\hline $3.63 \mathrm{E}+09$ & 0.544041988 & 0.67523366 & 0.7480819 & 0.86312537 & 0.92151411 \\
\hline $3.73 E+09$ & 0.540100347 & 0.66745747 & 0.7367939 & 0.84843333 & 0.905423844 \\
\hline $3.84 E+09$ & 0.536615281 & 0.6608266 & 0.7264862 & 0.83479502 & 0.890777301 \\
\hline $3.94 E+09$ & 0.533319248 & 0.65422419 & 0.7160329 & 0.82097954 & 0.875844745 \\
\hline $4.04 \mathrm{E}+09$ & 0.530888904 & 0.64720488 & 0.7050809 & 0.80642254 & 0.859932152 \\
\hline $4.14 E+09$ & 0.52859339 & 0.64068522 & 0.6952839 & 0.79328616 & 0.845916314 \\
\hline $4.25 \mathrm{E}+09$ & 0.526382754 & 0.63542766 & 0.6868227 & 0.78230005 & 0.8342612 \\
\hline
\end{tabular}




\begin{tabular}{|c|c|c|c|c|c|}
\hline ncy & $20 \mathrm{C}$ & $e^{\prime} 40 \mathrm{C}$ & $60 \mathrm{C}$ & $e^{\prime \prime} / e^{\prime} 80 \mathrm{C}$ & $e^{\prime \prime} / e^{\prime} 100 \mathrm{C}$ \\
\hline 09 & 6195 & 7981 & 0.6800883 & 0.77273086 & 0.823576326 \\
\hline $4.45 E+09$ & 524628115 & 0.62888292 & 0.6741583 & 0.76376584 & 813779262 \\
\hline $4.55 \mathrm{E}+09$ & 524569187 & 0.6266524 & 0.6689484 & 0.75607844 & 0.804229694 \\
\hline $4.65 \mathrm{E}+09$ & 0.524048081 & 0.62400388 & 0.6636855 & 0.7481194 & 0.79249416 \\
\hline $4.76 \mathrm{E}+09$ & 524168097 & 0.62079397 & 0.6576425 & 0.73963199 & 0.78367381 \\
\hline $4.86 \mathrm{E}+09$ & 0.524178144 & 0.61721787 & 0.6510765 & 0.73041835 & 0.774182878 \\
\hline $4.96 \mathrm{E}+09$ & 0.524841625 & 0.61462077 & 0.6461222 & 0.72399294 & 0.767051112 \\
\hline $5.06 \mathrm{E}+09$ & 0.525441949 & 0.61400476 & 0.6430405 & 0.71898575 & 0.76197884 \\
\hline $5.17 \mathrm{E}+09$ & 0.526120904 & 0.61376044 & 0.6398264 & 0.71323789 & .755241677 \\
\hline $5.27 \mathrm{E}+09$ & 0.526044567 & 0.61361372 & 0.637042 & 0.70776665 & 0.749157846 \\
\hline $5.37 \mathrm{E}+09$ & 0.526760442 & 0.61213022 & 0.632996 & 0.70214669 & 0.741777497 \\
\hline $5.47 \mathrm{E}+09$ & 0.527550888 & 0.60986709 & 0.6282501 & 0.69594614 & 0.734930152 \\
\hline $5.57 \mathrm{E}+09$ & 0.528802608 & 0.60693116 & 0.622837 & 0.68854986 & 0.726967378 \\
\hline $5.68 \mathrm{E}+09$ & 0.529504199 & 0.60662491 & 0.6202579 & 0.68387643 & 0.721897933 \\
\hline $5.78 \mathrm{E}+09$ & 0.531223046 & 0.60744292 & 0.6187293 & 0.68119022 & 0.718851472 \\
\hline $5.88 \mathrm{E}+09$ & 0.532630123 & 0.6081016 & 0.6170323 & 0.67748464 & 0.714304244 \\
\hline $5.98 \mathrm{E}+09$ & 0.534042256 & 0.60846629 & 0.6149908 & 0.67329165 & .709103173 \\
\hline $6.09 E+09$ & 0.535134988 & 0.60791599 & 0.6118257 & 0.66833447 & 0.703675911 \\
\hline $6.19 \mathrm{E}+09$ & 0.536410554 & 0.60586572 & 0.6077972 & 0.66334674 & 0.698390766 \\
\hline $6.29 E+09$ & 0.539052189 & 0.60438041 & 0.604409 & 0.65872978 & 0.693375255 \\
\hline $6.39 \mathrm{E}+09$ & 0.541177221 & 0.60538278 & 0.6029811 & 0.65530421 & 0.689464466 \\
\hline $6.5 \mathrm{E}+09$ & 0.543862501 & 0.60779399 & 29728 & 0.65327005 & .686704924 \\
\hline $6.6 \mathrm{E}+09$ & 0.545933574 & 0.611312 & 0.6043557 & 0.65379378 & 0.686149894 \\
\hline 09 & 54789576 & 62 & 35335 & 0.65113405 & 82421495 \\
\hline $6.8 \mathrm{E}+09$ & 0.550410685 & 0.61143292 & 0.6002893 & 0.64626539 & 0.677452903 \\
\hline $6.9 \mathrm{E}+09$ & 0.553134482 & 0.60952085 & 0.5966327 & 0.64160116 & 0.672553294 \\
\hline $7.01 \mathrm{E}+09$ & 55625472 & 0.60804443 & 0.5931846 & 0.63706318 & 0.667202733 \\
\hline $7.11 \mathrm{E}+09$ & 0.558775423 & 0.60864741 & 0.5924479 & 0.63446215 & 0.665292201 \\
\hline $7.21 \mathrm{E}+09$ & 0.561745044 & 0.6127838 & 0.5942145 & 0.63401393 & 0.665321471 \\
\hline $7.31 \mathrm{E}+09$ & 0.563347332 & 0.616915 & 0.5967544 & 0.6350652 & 0.665456593 \\
\hline $7.42 \mathrm{E}+09$ & 0.56605234 & 0.6202911 & 0.5983947 & 0.63650499 & 0.666227565 \\
\hline $7.52 \mathrm{E}+09$ & 0.569024075 & 0.6195956 & 0.5965703 & 0.63325048 & 0.662595917 \\
\hline $7.62 \mathrm{E}+09$ & 0.571687472 & 0.61628821 & 0.5915751 & 0.62639114 & 0.655108683 \\
\hline $7.72 \mathrm{E}+09$ & 0.574543014 & 0.61437102 & 0.587453 & 0.62159491 & 0.649751734 \\
\hline $7.83 \mathrm{E}+09$ & 0.577346773 & 0.61626111 & 0.5886727 & 0.62228675 & 0.648967395 \\
\hline $7.93 \mathrm{E}+09$ & 0.580235279 & 0.6209687 & 0.5917466 & 0.62333293 & 0.648875359 \\
\hline $8.03 \mathrm{E}+09$ & 82192462 & 0.62625617 & 0.5946418 & 0.62414406 & 0.648992418 \\
\hline$E+09$ & 584041514 & 0.62791556 & 0.5954687 & 0.62463959 & 0.648413785 \\
\hline $8.23 \mathrm{E}+09$ & 0.587589057 & 0.62547813 & 0.5929487 & 0.621372 & 0.644825719 \\
\hline $8.34 \mathrm{E}+09$ & 0.59071864 & 0.62150855 & 0.586848 & 0.61349934 & 0.636152281 \\
\hline $8.44 \mathrm{E}+09$ & 0.593990919 & 0.62089269 & 0.5843018 & 0.60876147 & 0.631005389 \\
\hline $8.54 \mathrm{E}+09$ & 0.595932886 & 0.62273188 & 0.586047 & 0.60968762 & 0.630695668 \\
\hline $8.64 \mathrm{E}+09$ & 0.597908972 & 0.62841041 & 0.5913069 & 0.6140718 & 0.633932761 \\
\hline $8.75 \mathrm{E}+09$ & 0.601132818 & 0.63425489 & 0.5955656 & 0.61675894 & 0.636047061 \\
\hline $8.85 \mathrm{E}+09$ & 0.604005901 & 0.63495121 & 0.5949028 & 0.61445332 & 0.63344101 \\
\hline
\end{tabular}




\begin{tabular}{|c|c|c|c|c|c|}
\hline Frequency & $1 / e^{\prime} 20 \mathrm{C}$ & $e^{\prime \prime} / e^{\prime} 40 \mathrm{C}$ & $e^{\prime \prime} / e^{\prime} 60 \mathrm{C}$ & $e^{\prime \prime} / e^{\prime} 80 \mathrm{C}$ & $100 \mathrm{C}$ \\
\hline $8.95 \mathrm{E}+09$ & 0.607065097 & 0.6321394 & 0.5916058 & 0.61111368 & $630017 t$ \\
\hline$E+09$ & 610617908 & 0.62607318 & 0.584955 & 0.60345587 & 0.621916845 \\
\hline$E+09$ & 0.61306895 & 0.62670185 & 0.5830791 & 0.59925032 & 616409747 \\
\hline $9.26 \mathrm{E}+09$ & 0.616425307 & 0.62942769 & 0.5857502 & 0.60100305 & 0.617350201 \\
\hline $9.36 \mathrm{E}+09$ & 0.619480245 & 0.6368281 & 0.5936561 & 0.60819395 & .623509678 \\
\hline $9.46 \mathrm{E}+09$ & 0.622062998 & 0.64289205 & 0.5985342 & 0.61176756 & 0.626153886 \\
\hline $9.56 \mathrm{E}+09$ & 0.624805588 & 0.64258951 & 0.5959449 & 0.60711626 & 0.621477621 \\
\hline $9.67 \mathrm{E}+09$ & 0.628644508 & 0.63815657 & 0.5902692 & 0.60127501 & 0.61579072 \\
\hline $9.77 \mathrm{E}+09$ & 0.632298057 & 0.63259272 & 0.5857415 & 0.59714791 & 0.610814496 \\
\hline $9.87 \mathrm{E}+09$ & 0.634822463 & 0.63275574 & 0.5848676 & 0.59470328 & 0.607510501 \\
\hline $9.97 \mathrm{E}+09$ & 0.637516907 & 0.63750549 & 0.5885448 & 0.59610657 & 0.607984959 \\
\hline $1.01 \mathrm{E}+10$ & 0.641378041 & 0.64634629 & 0.5978583 & 0.60444013 & 0.615140759 \\
\hline $1.02 \mathrm{E}+10$ & 0.643187112 & 0.65154234 & 0.6030666 & 0.60948953 & 0.61877805 \\
\hline $1.03 \mathrm{E}+10$ & 0.645971868 & 0.65248025 & 0.6012476 & 0.60539877 & 0.614646281 \\
\hline $1.04 \mathrm{E}+10$ & 0.649331692 & 0.64773195 & 0.594466 & 0.59749299 & 0.607344363 \\
\hline $1.05 \mathrm{E}+10$ & 0.652114022 & 0.64299683 & 0.59045 & 0.59409848 & 0.603840853 \\
\hline $1.06 \mathrm{E}+10$ & 0.655547708 & 0.64202818 & .5907054 & 0.59452074 & 0.602677925 \\
\hline $1.07 \mathrm{E}+10$ & 0.659107204 & 0.64912162 & 0.5973117 & 0.59874975 & 0.60547806 \\
\hline $1.08 \mathrm{E}+10$ & 0.662191582 & 1468 & 0.6041345 & 0.60327043 & 0.608631949 \\
\hline $1.09 \mathrm{E}+10$ & 0.66501341 & 0.66133165 & 0.6093738 & 0.60818384 & 0.612883817 \\
\hline $1.1 \mathrm{E}+10$ & 0.667630298 & 0.66388774 & 0.6101438 & 0.60806973 & 0.613058686 \\
\hline $1.11 \mathrm{E}+10$ & 0.671431225 & 0.66212669 & 0.6050731 & 0.60100142 & 0.60575844 \\
\hline $1.12 \mathrm{E}+10$ & 0.67548994 & 8909 & 0.6006258 & 0.5959343 & 0.601365683 \\
\hline $1.13 \mathrm{E}+10$ & 679538012 & 6573 & 0.6053828 & 0.60261588 & 0.605850236 \\
\hline & 0.6 & 9486 & 6130277 & 60971162 & 2045 \\
\hline $1.15 \mathrm{E}+10$ & 0.685157785 & 0.67067138 & 0.6163801 & 0.61015811 & 0.612578317 \\
\hline $1.16 \mathrm{E}+10$ & 0.687933545 & 0.67422858 & 0.617629 & 0.61018907 & 0.616967242 \\
\hline $1.17 \mathrm{E}+10$ & 0.691013552 & 0.67630278 & 0.6184795 & 0.61192235 & 0.618795092 \\
\hline $1.18 \mathrm{E}+10$ & 0.694250165 & 0.67582373 & 0.6159933 & 0.6070997 & 0.613473166 \\
\hline $1.19 \mathrm{E}+10$ & 0.699505488 & 0.67536503 & 0.6128312 & 0.60054754 & 0.606580355 \\
\hline $1.2 \mathrm{E}+10$ & 0.70277278 & 0.67166646 & 0.6143289 & 0.60353258 & 0.608121613 \\
\hline $1.21 \mathrm{E}+10$ & 0.706342389 & 0.67520225 & 0.623141 & 0.61576021 & 0.618691421 \\
\hline $1.22 \mathrm{E}+10$ & 0.710299181 & 0.68529711 & 0.6311719 & 0.62172416 & 0.623251601 \\
\hline $1.23 \mathrm{E}+10$ & 0.713123659 & 0.68741177 & 0.6285611 & 0.61470991 & 0.614966686 \\
\hline $1.24 \mathrm{E}+10$ & 0.715222039 & 0.68068059 & 0.624492 & 0.61006153 & 0.60995415 \\
\hline 1.25 & 0.718636521 & 2431 & 0.6253047 & 0.61013664 & 0.608862868 \\
\hline $1.26 \mathrm{E}+10$ & 0.722414359 & 0.68113599 & 0.6249658 & 0.60380607 & 0.602291206 \\
\hline $1.27 \mathrm{E}+10$ & 0.728862033 & 0.68232646 & 0.6237946 & 0.60265544 & 0.601636706 \\
\hline $1.28 \mathrm{E}+10$ & 0.73394022 & 0.68471953 & 0.6284428 & 0.61008605 & 0.608798873 \\
\hline $1.29 \mathrm{E}+10$ & 0.736131652 & 0.69577684 & 0.6397715 & 0.61908038 & 0.618568029 \\
\hline $1.3 \mathrm{E}+10$ & 0.739304813 & 0.70502957 & 0.6440374 & 0.61696356 & 0.615989249 \\
\hline $1.31 \mathrm{E}+10$ & 0.740827553 & 0.69996207 & 0.6410853 & 0.61482663 & 0.612565938 \\
\hline $1.32 \mathrm{E}+10$ & 0.743483959 & 0.69485215 & 0.6414435 & 0.62335989 & 0.618066975 \\
\hline $1.33 \mathrm{E}+10$ & 0.747002434 & 0.7028423 & 0.6483709 & 0.62710612 & 0.620822322 \\
\hline $1.35 \mathrm{E}+10$ & 0.753169439 & 70128104 & 0.6398329 & 0.60998741 & 0.60407572 \\
\hline
\end{tabular}




\begin{tabular}{|c|c|c|c|c|c|}
\hline & $1 / e^{\prime} 20 \mathrm{C}$ & $\overline{\mathrm{C}}$ & $\bar{C}$ & & \\
\hline $5 E+10$ & 59044448 & 69277543 & 294284 & 0.5959417 & 780 \\
\hline$\overline{7 \mathrm{E}+10}$ & 762080726 & 70469462 & 6443286 & 0.61220467 & 608390591 \\
\hline $1.38 \mathrm{E}+10$ & 765035455 & 0.72212095 & 0.6601425 & 0.62528655 & 620811653 \\
\hline $1.39 \mathrm{E}+10$ & .766666356 & 0.71545665 & 0.6528669 & 0.6204173 & .614686659 \\
\hline $1.4 \mathrm{E}+10$ & 0.768996301 & 0.70714122 & 0.6509657 & 0.62745398 & $\overline{.6199717}$ \\
\hline $1.41 \mathrm{E}+10$ & 0.773201826 & 0.7205812 & 0.6692276 & 0.64939297 & 0.638547746 \\
\hline $1.42 E+10$ & 0.779854989 & 0.73120087 & 0.6744288 & 0.64634613 & 0.63451785 \\
\hline $1.43 E+10$ & 0.785513137 & 0.71422617 & 0.650914 & 0.61774577 & 0.60668368 \\
\hline $1.44 \mathrm{E}+10$ & 0.788170284 & 0.71210973 & 0.6510678 & 0.62021679 & 0.609651936 \\
\hline $1.45 \mathrm{E}+10$ & 0.790735945 & 0.73614479 & 0.6777569 & 0.64468568 & 0.635933262 \\
\hline $1.46 \mathrm{E}+10$ & 0.79163842 & 0.74261522 & 0.6811434 & 0.64565257 & 0.634391743 \\
\hline $1.47 \mathrm{E}+10$ & 0.79281796 & 0.72025571 & 0.6608883 & 0.63167168 & 0.618575549 \\
\hline $1.48 \mathrm{E}+10$ & 0.797643471 & 0.72541299 & 0.6714823 & 0.64778937 & 0.631618131 \\
\hline $1.49 E+10$ & 0.805386853 & 0.75697274 & 0.7003783 & 0.66754401 & 0.648277364 \\
\hline $1.5 \mathrm{E}+10$ & 0.815494281 & 0.73924077 & 0.675675 & 0.63692494 & 418765 \\
\hline $1.51 \mathrm{E}+10$ & 0.819780505 & 0.71509665 & 0.6527614 & 0.62108173 & 0.6068427 \\
\hline $1.52 \mathrm{E}+10$ & 0.824810204 & 0.74871529 & 0.6883807 & 0.65434179 & $\overline{651213}$ \\
\hline $1.53 \mathrm{E}+10$ & 0.824337649 & 0.77086325 & 0.7111905 & 0.6751754 & 0.658599134 \\
\hline $1.54 \mathrm{E}+10$ & 818558198 & 0.72220878 & 0.6644954 & 0.63308466 & 30463 \\
\hline $1.55 \mathrm{E}+10$ & 0.821006 & 0.7359956 & 0.6775357 & 0.63910436 & 0.622992207 \\
\hline $1.56 \mathrm{E}+10$ & 0.8278562 & 0.7958039 & 0.7365532 & 0.68697159 & 415875 \\
\hline $1.57 \mathrm{E}+10$ & 0.835762306 & 0.76376682 & 0.7074957 & 0.66943846 & 0.648912854 \\
\hline $1.58 \mathrm{E}+10$ & 218 & 362 & 0.6774255 & 0.64163188 & 13933 \\
\hline $1.59 \mathrm{E}+10$ & 0.857377706 & 0.78381111 & 0.7160923 & 0.67305232 & 0.653074632 \\
\hline $1.6 \mathrm{E}+10$ & 0.853623086 & 0.7973939 & 0.7430554 & 0.70928117 & 0.686933634 \\
\hline $1.61 E+10$ & 0.850696671 & 0.75085438 & 0.697975 & 0.66740373 & 0.64541597 \\
\hline $1.62 \mathrm{E}+10$ & 0.852189213 & 0.75286651 & 0.6855823 & 0.63995443 & 0.617001487 \\
\hline $1.63 \mathrm{E}+10$ & 0.854631372 & 0.81011466 & 0.7488379 & 0.70394181 & 0.677172882 \\
\hline $1.64 \mathrm{E}+10$ & 0.860190799 & 0.79221736 & 0.7440634 & 0.7119966 & 0.68563729 \\
\hline $1.65 \mathrm{E}+10$ & 0.870311193 & 0.7445739 & 0.6780436 & 0.63513905 & 0.613685627 \\
\hline $1.66 \mathrm{E}+10$ & 0.879528867 & 0.78113139 & 0.7035277 & 0.65127798 & 0.629859605 \\
\hline $1.67 \mathrm{E}+10$ & 0.881139732 & 0.81812231 & 0.7661346 & 0.7320508 & 607622 \\
\hline $1.68 \mathrm{E}+10$ & 0.879275438 & 0.76569709 & 0.718142 & 0.68950651 & 087393 \\
\hline $1.69 \mathrm{E}+10$ & 0.877786215 & 0.75786758 & 0.6810186 & 0.63109242 & 885724 \\
\hline $1.7 \mathrm{E}+10$ & 0.88160684 & 2055778 & 0.7515298 & 0.70137366 & 819011 \\
\hline $1.71 \mathrm{E}+10$ & 0.885939324 & 0.79355539 & 0.7499357 & 0.71891198 & 0.686490596 \\
\hline $1.72 \mathrm{E}+10$ & 0.893084258 & 0.75502506 & 0.6877537 & 0.64078819 & 1542823 \\
\hline $1.73 E+10$ & 0.903145331 & 0.80350441 & 0.7162963 & 0.65464733 & 0.630577981 \\
\hline $1.74 \mathrm{E}+10$ & 0.91078897 & 0.83214594 & 0.7747126 & 0.73256692 & 0.70042145 \\
\hline $1.75 \mathrm{E}+10$ & 0.909175839 & 0.78301619 & 0.7356307 & 0.70661234 & 0.669791587 \\
\hline $1.76 \mathrm{E}+10$ & 0.908714951 & 0.78263101 & 0.7006505 & 0.64814229 & 0.615812351 \\
\hline $1.77 \mathrm{E}+10$ & 0.911424517 & 0.83342115 & 0.7513895 & 0.69171099 & 0.657751692 \\
\hline $1.79 \mathrm{E}+10$ & 0.914402702 & 0.82342828 & 0.7792128 & 0.74274248 & 0.705109879 \\
\hline $1.8 \mathrm{E}+10$ & 0.919203924 & 0.79585918 & 0.7357523 & 0.6942848 & 0.663103091 \\
\hline $1.81 \mathrm{E}+10$ & 0.928769669 & 0.82040815 & 0.7299247 & 0.66229973 & 0.638024999 \\
\hline
\end{tabular}




\begin{tabular}{|r|r|r|r|r|r|}
\hline Frequency & \multicolumn{1}{|c|}{$\mathrm{e}^{\prime \prime} / \mathrm{e}^{\prime} \mathbf{2 0} \mathrm{C}$} & $\mathrm{e}^{\prime \prime} / \mathrm{e}^{\prime} \mathbf{4 0} \mathrm{C}$ & $\mathrm{e}^{\prime \prime} / \mathrm{e}^{\prime} 60 \mathrm{C}$ & $\mathrm{e}^{\prime \prime} / \mathrm{e}^{\prime} 80 \mathrm{C}$ & $\mathrm{e}^{\prime \prime} / \mathrm{e}^{\prime} 100 \mathrm{C}$ \\
\hline \hline $1.82 \mathrm{E}+10$ & 0.935261481 & 0.85746948 & 0.7929508 & 0.73566817 & 0.705031145 \\
\hline $1.83 \mathrm{E}+10$ & 0.936543053 & 0.8304056 & 0.7871172 & 0.75176514 & 0.7166783 \\
\hline $1.84 \mathrm{E}+10$ & 0.936072161 & 0.80971801 & 0.7249609 & 0.66426353 & 0.640026069 \\
\hline $1.85 \mathrm{E}+10$ & 0.93918132 & 0.84620442 & 0.7597549 & 0.69207514 & 0.672984105 \\
\hline $1.86 \mathrm{E}+10$ & 0.940809841 & 0.85456369 & 0.8106881 & 0.7749931 & 0.746078828 \\
\hline $1.87 \mathrm{E}+10$ & 0.946768131 & 0.82706536 & 0.7690849 & 0.73153639 & 0.707622592 \\
\hline $1.88 \mathrm{E}+10$ & 0.951966456 & 0.83644552 & 0.7481577 & 0.68462001 & 0.668509557 \\
\hline $1.89 \mathrm{E}+10$ & 0.959749814 & 0.86630148 & 0.8029341 & 0.74658795 & 0.723594764 \\
\hline $1.9 \mathrm{E}+10$ & 0.96162359 & 0.84663515 & 0.7914151 & 0.74820368 & 0.723134248 \\
\hline $1.91 \mathrm{E}+10$ & 0.964214209 & 0.82981291 & 0.7454263 & 0.68397694 & 0.667391938 \\
\hline $1.92 \mathrm{E}+10$ & 0.967166916 & 0.86147045 & 0.7788325 & 0.71405424 & 0.693948234 \\
\hline $1.93 \mathrm{E}+10$ & 0.970775354 & 0.87024855 & 0.8071883 & 0.76071255 & 0.734220593 \\
\hline $1.94 \mathrm{E}+10$ & 0.976486543 & 0.85131127 & 0.7819388 & 0.73663741 & 0.71114779 \\
\hline $1.95 \mathrm{E}+10$ & 0.982393967 & 0.85090853 & 0.7729403 & 0.71715682 & 0.695297501 \\
\hline $1.96 \mathrm{E}+10$ & 0.985113049 & 0.86586983 & 0.7947846 & 0.73829477 & 0.716480766 \\
\hline $1.97 \mathrm{E}+10$ & 0.987421463 & 0.86597929 & 0.7937538 & 0.73647454 & 0.714876268 \\
\hline $1.98 \mathrm{E}+10$ & 0.991318838 & 0.85936408 & 0.7807337 & 0.72097023 & 0.70159359 \\
\hline $1.99 \mathrm{E}+10$ & 0.996080315 & 0.87556701 & 0.7972391 & 0.73729423 & 0.717742128 \\
\hline $2 \mathrm{E}+10$ & 0.999347015 & 0.88701685 & 0.8103331 & 0.75426151 & 0.732082357 \\
\hline
\end{tabular}


Table 18. Dielectric Constant for W-26 ORNL Simulant

\begin{tabular}{|c|c|c|c|c|c|}
\hline cy & $\mathrm{e}^{\prime} @ 20 \mathrm{C}$ & @ 40C & @6 & C & 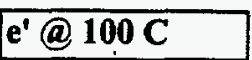 \\
\hline $1.53 \mathrm{E}+08$ & 345.701995 & 490.583217 & 644.365086 & 669.399334 & 805.6358985 \\
\hline $2.55 \mathrm{E}+08$ & 174.737904 & 235.471787 & 294.167976 & 293.734101 & 346.4452985 \\
\hline $3.58 \mathrm{E}+08$ & 119.126125 & 152.0259 & 180.772742 & 176.997863 & 199.5341105 \\
\hline $4.6 E+08$ & 97.834184 & 120.510603 & 139.994494 & 136.45117 & 149.325457 \\
\hline $5.62 \mathrm{E}+08$ & 83.5453045 & 99.642188 & 110.850949 & 105.435806 & 111.716193 \\
\hline $6.65 \mathrm{E}+08$ & 76.064806 & 88.682076 & 97.480558 & 92.6175845 & 96.6299045 \\
\hline $7.67 \mathrm{E}+08$ & 69.962281 & 79.4305305 & 84.7780175 & 78.7922065 & 78.9037465 \\
\hline $8.69 \mathrm{E}+08$ & 65.037774 & 71.869325 & 74.540559 & 68.10167 & 64.6150395 \\
\hline $9.71 \mathrm{E}+08$ & 62.313924 & 67.575165 & 68.8101615 & 62.04927 & 56.7427935 \\
\hline $1.07 \mathrm{E}+09$ & 59.7626555 & 63.6935525 & 63.6373215 & 56.3332105 & 50.0574945 \\
\hline $1.18 \mathrm{E}+09$ & 57.9754405 & 61.0579705 & 59.666669 & 51.790931 & 44.855854 \\
\hline $1.28 \mathrm{E}+09$ & 55.8613875 & 58.129707 & 56.1247795 & 48.2261105 & 41.405697 \\
\hline $1.38 \mathrm{E}+09$ & 55.130726 & 57.135986 & 55.1817855 & 47.6017115 & 40.617805 \\
\hline $1.48 \mathrm{E}+09$ & 53.7017705 & 55.055768 & 52.492507 & 44.349466 & 36.3194265 \\
\hline $1.59 \mathrm{E}+09$ & 53.1733245 & 54.047778 & 50.9347465 & 42.85892 & 33.62274 \\
\hline $1.69 \mathrm{E}+09$ & 52.374521 & 53.005198 & 49.7154075 & 41.6187865 & 32.105481 \\
\hline $1.79 \mathrm{E}+09$ & 51.6647705 & 52.043697 & 48.4212465 & 40.446707 & 31.300091 \\
\hline $1.89 E+09$ & 50.702617 & 50.582886 & 46.1795765 & 37.677039 & 28.340204 \\
\hline $1.99 \mathrm{E}+09$ & 50.055313 & 49.829117 & 45.6605465 & 37.1786005 & 28.415312 \\
\hline $2.1 \mathrm{E}+09$ & 49.963967 & 49.57374 & 45.3769095 & 36.9653075 & 27.6228685 \\
\hline $2.2 \mathrm{E}+09$ & 49.5865535 & 49.2229 & 45.2277515 & 37.1096425 & 27.2980935 \\
\hline $2.3 \mathrm{E}+09$ & 49.1856785 & 48.615655 & 44.182629 & 35.596634 & 24.88107 \\
\hline $2.4 \mathrm{E}+09$ & 48.827076 & 47.9925065 & 43.1148385 & 34.3280545 & 23.652766 \\
\hline $2.51 \mathrm{E}+09$ & 48.32423 & 47.4949595 & 42.727664 & 34.047026 & 24.4487615 \\
\hline $2.61 \mathrm{E}+09$ & 47.6925945 & 46.911021 & 41.91238 & 33.07986 & 23.826589 \\
\hline $2.71 \mathrm{E}+09$ & 47.238023 & 46.363947 & 41.3966015 & 32.668206 & 23.3809635 \\
\hline $2.81 \mathrm{E}+09$ & 46.873255 & 45.910074 & 40.8627785 & 32.1259175 & 22.1834605 \\
\hline $2.92 \mathrm{E}+09$ & 46.4751815 & 45.283062 & 39.942918 & 31.0137185 & 20.136323 \\
\hline $3.02 \mathrm{E}+09$ & 46.19987 & 45.024943 & 39.7775445 & 30.853594 & 20.1254335 \\
\hline $3.12 \mathrm{E}+09$ & 45.9603145 & 44.935757 & 39.774187 & 30.8409915 & 20.840077 \\
\hline $3.22 \mathrm{E}+09$ & 45.6163675 & 44.6725175 & 39.4906005 & 30.6557235 & 21.409104 \\
\hline $3.32 \mathrm{E}+09$ & 45.2610565 & 44.175814 & 39.031431 & 30.1776925 & 21.157171 \\
\hline $3.43 \mathrm{E}+09$ & 45.031571 & 43.9840785 & 39.0078535 & 30.314249 & 20.921724 \\
\hline $3.53 \mathrm{E}+09$ & 44.845768 & 43.801434 & 39.017758 & 30.4621615 & 20.5379805 \\
\hline $3.63 \mathrm{E}+09$ & 44.844783 & 43.8276245 & 39.141673 & 30.5916935 & 20.3569335 \\
\hline $3.73 E+09$ & 44.6121205 & 43.561751 & 38.7742035 & 30.1806845 & 20.1464955 \\
\hline $3.84 E+09$ & 44.331948 & 43.470548 & 38.7691785 & 30.2854975 & 21.2290065 \\
\hline $3.94 \mathrm{E}+09$ & 44.1099445 & 43.395354 & 38.971146 & 30.6450285 & 22.3240325 \\
\hline $4.04 E+09$ & 43.8595245 & 43.225818 & 38.8530575 & 30.6134 & 22.1584565 \\
\hline $4.14 \mathrm{E}+09$ & 43.6331265 & 42.8729075 & 38.456018 & 30.224908 & 21.0221325 \\
\hline $4.25 \mathrm{E}+09$ & 43.4601695 & 42.624899 & 38.2645955 & 30.033379 & 20.38068 \\
\hline $4.35 \mathrm{E}+09$ & 43.272276 & 42.480859 & 38.233692 & 30.101468 & 20.6264425 \\
\hline $4.45 \mathrm{E}+09$ & 42.8657075 & 42.1519215 & 37.878568 & 29.7614035 & 21.0220875 \\
\hline
\end{tabular}




\begin{tabular}{|c|c|c|c|c|c|}
\hline Frequency & $e^{\prime} @ 20 C$ & $e^{\prime} @ 40 \mathrm{C}$ & $\mathrm{e}^{\prime} @$ & $\mathrm{e}^{\prime} @$ & $e^{\prime} @ 100 \mathrm{C}$ \\
\hline $4.55 \mathrm{E}+09$ & 42.5551795 & 41.9079225 & 37.6980595 & 29.6867185 & .80344 \\
\hline $4.65 E+09$ & 42.396915 & 41.6558355 & 37.5709275 & 29.7575545 & 22.2521225 \\
\hline $4.76 \mathrm{E}+09$ & 42.0595955 & 41.1288505 & 36.7485005 & 28.6867235 & 20.3117915 \\
\hline $4.86 \mathrm{E}+09$ & 41.818203 & 40.675508 & 36.187616 & 28.0874805 & 18.792148 \\
\hline $4.96 \mathrm{E}+09$ & 41.545129 & 40.406919 & 35.9122205 & 27.8142295 & 18.3203435 \\
\hline $5.06 \mathrm{E}+09$ & 41.3436865 & 40.4947235 & 36.165368 & 28.1338455 & 19.301313 \\
\hline $5.17 \mathrm{E}+09$ & 41.0636675 & 40.3297 & 36.082914 & 28.0961175 & 20.020668 \\
\hline $5.27 \mathrm{E}+09$ & 40.6838945 & 40.082045 & 35.926054 & 28.149498 & 20.701627 \\
\hline $5.37 \mathrm{E}+09$ & 40.5731535 & 39.8946215 & 35.8712315 & 28.2044475 & 20.5666365 \\
\hline $5.47 \mathrm{E}+09$ & 40.3277705 & 39.5635775 & 35.532468 & 27.902346 & 19.4089835 \\
\hline $5.57 \mathrm{E}+09$ & 40.077512 & 39.2082805 & 35.0152355 & 27.3036125 & 18.0034095 \\
\hline $5.68 \mathrm{E}+09$ & 39.8360385 & 39.048474 & 34.9391065 & 27.2650055 & 18.244371 \\
\hline $5.78 \mathrm{E}+09$ & 39.6340665 & 39.1680335 & 35.1764475 & 27.6377425 & 19.604818 \\
\hline $5.88 \mathrm{E}+09$ & 39.4839135 & 39.3012 & 35.6790365 & 28.2925845 & 21.025458 \\
\hline $5.98 \mathrm{E}+09$ & 39.248206 & 39.1281005 & 35.6030375 & 28.2829885 & 21.032342 \\
\hline $6.09 \mathrm{E}+09$ & 39.046955 & 38.80039 & 35.264 & 28.1189375 & 20.3385545 \\
\hline $6.19 \mathrm{E}+09$ & 38.8906625 & 38.5093745 & 34.988673 & 27.8583905 & 19.328149 \\
\hline $6.29 \mathrm{E}+09$ & 38.5978255 & 38.478795 & 34.9925815 & 27.842022 & 19.1432685 \\
\hline $6.39 \mathrm{E}+09$ & 38.3461015 & 38.4852175 & 35.1484535 & 28.0514045 & 20.309645 \\
\hline $6.5 \mathrm{E}+09$ & 38.202163 & 38.8149275 & 35.9126515 & 29.103687 & 22.775801 \\
\hline $6.6 \mathrm{E}+09$ & 38.0932015 & 38.91569 & 36.2076225 & 29.4816925 & 23.684472 \\
\hline $6.7 \mathrm{E}+09$ & 37.8008135 & 38.3629785 & 35.639801 & 28.936747 & 22.496307 \\
\hline $6.8 \mathrm{E}+09$ & 37.630507 & 37.807511 & 35.0140085 & 28.312476 & 21.0413115 \\
\hline $6.9 \mathrm{E}+09$ & 37.436897 & 37.4599735 & 34.5767455 & 27.873547 & 20.13058 \\
\hline $7.01 \mathrm{E}+09$ & 37.2581015 & 37.323766 & 34.4909375 & 27.7447075 & 20.3577965 \\
\hline $7.11 \mathrm{E}+09$ & 36.9776905 & 37.2056045 & 34.485063 & 27.772817 & 21.5748405 \\
\hline $7.21 \mathrm{E}+09$ & 36.8883365 & 37.3329615 & 34.8750415 & 28.414561 & 23.417558 \\
\hline $7.31 \mathrm{E}+09$ & 36.71637 & 37.191194 & 34.830282 & 28.426324 & 23.2989915 \\
\hline $7.42 \mathrm{E}+09$ & 36.5831875 & 36.8645045 & 34.5703065 & 28.210457 & 22.104606 \\
\hline $7.52 \mathrm{E}+09$ & 36.3233025 & 36.345513 & 33.817279 & 27.3752245 & 20.3568155 \\
\hline $7.62 \mathrm{E}+09$ & 36.151693 & 36.1403075 & 33.583823 & 27.1788615 & 20.1297875 \\
\hline $7.72 \mathrm{E}+09$ & 36.040129 & 36.4111525 & 34.033425 & 27.665005 & 21.4249625 \\
\hline $7.83 E+09$ & 35.901976 & 36.6966715 & 34.450588 & 28.1654825 & 22.94381 \\
\hline $7.93 \mathrm{E}+09$ & 35.6642685 & 36.602428 & 34.4777305 & 28.2823035 & 23.653219 \\
\hline $8.03 E+09$ & 35.7622765 & 36.737997 & 34.8521725 & 28.8834675 & 23.7884655 \\
\hline $8.13 \mathrm{E}+09$ & 35.4850465 & 36.063342 & 33.952114 & 27.9051645 & 21.443122 \\
\hline $8.23 E+09$ & 35.334836 & 35.647067 & 33.5022615 & 27.517087 & 20.5595935 \\
\hline $8.34 \mathrm{E}+09$ & 35.143392 & 35.4494545 & 33.2334765 & 27.216731 & 20.7996095 \\
\hline $8.44 \mathrm{E}+09$ & 35.094779 & 35.7236975 & 33.6497855 & 27.657726 & 22.449247 \\
\hline $8.54 \mathrm{E}+09$ & 35.0427495 & 36.069719 & 34.272028 & 28.497695 & 24.1650215 \\
\hline $8.64 \mathrm{E}+09$ & 34.976751 & 36.255872 & 34.634597 & 28.981688 & 24.4463355 \\
\hline $8.75 \mathrm{E}+09$ & 34.861029 & 36.0461105 & 34.382334 & 28.7991325 & 23.2605685 \\
\hline $8.85 \mathrm{E}+09$ & 34.827521 & 35.7519315 & 34.081242 & 28.497346 & 21.9751235 \\
\hline $8.95 \mathrm{E}+09$ & 34.620509 & 35.4495305 & 33.687047 & 28.0351975 & 21.505554 \\
\hline $9.05 \mathrm{E}+09$ & 34.4586255 & 35.485418 & 33.868683 & 28.316676 & 23.2509185 \\
\hline
\end{tabular}




\begin{tabular}{|c|c|c|c|c|c|}
\hline requency $\mathrm{e}^{\prime}$ & $\mathrm{e}^{\prime} @ 20 \mathrm{C}$ & $\mathrm{e}^{\prime} @ 40 \mathrm{C}$ & $\mathrm{e}^{\prime} @ 60 \mathrm{c}$ & $\mathrm{e}^{\prime} @ 80 \mathrm{C}$ & $\bar{C}$ \\
\hline$E+09$ & 34.261845 & 35.9220115 & 34.539326 & 44695 & 03 \\
\hline $9.26 \mathrm{E}+09$ & 34.3025335 & 36.4172945 & 35.2675695 & 30.006073 & 26.531475 \\
\hline $9.36 \mathrm{E}+09$ & 34.257862 & 36.347264 & 35.3708405 & 30.213582 & 25.7566145 \\
\hline $9.46 \mathrm{E}+09$ & 34.3281205 & 36.1926775 & 35.1672325 & 30.004318 & 24.239152 \\
\hline $9.56 \mathrm{E}+09$ & 34.256604 & 35.4343295 & 34.2964965 & 29.1202405 & 22.9382615 \\
\hline $9.67 \mathrm{E}+09$ & 34.176068 & 35.317291 & 34.3004595 & 29.2246275 & 24.0480385 \\
\hline $9.77 \mathrm{E}+09$ & 33.778846 & 35.255005 & 34.2606695 & 29.090481 & 25.787109 \\
\hline $9.87 \mathrm{E}+09$ & 33.9293605 & 35.962686 & 35.3717245 & 30.4384875 & 28.5030335 \\
\hline $9.97 \mathrm{E}+09$ & 33.716695 & 36.1665365 & 35.7055445 & 30.936937 & 28.131191 \\
\hline $1.01 \mathrm{E}+10$ & 33.878123 & 36.223545 & 35.7826035 & 31.0967355 & 26.5079495 \\
\hline $1.02 \mathrm{E}+10$ & 33.949248 & 35.6410475 & 35.190879 & 30.5571515 & 24.919348 \\
\hline $1.03 \mathrm{E}+10$ & 33.878862 & 35.113996 & 34.6013265 & 29.9129815 & 24.5175125 \\
\hline $1.04 \mathrm{E}+10$ & 33.679454 & 34.9792645 & 34.417395 & 29.675649 & 25.880676 \\
\hline $1.05 \mathrm{E}+10$ & 33.592902 & 35.514128 & 35.1630595 & 30.504442 & 28.693159 \\
\hline $1.06 \mathrm{E}+10$ & 33.450101 & 35.989459 & 35.6703095 & 31.046193 & 29.407974 \\
\hline $1.07 \mathrm{E}+10$ & 33.6005445 & 36.0451745 & 35.7189335 & 31.055113 & 27.591997 \\
\hline $1.08 \mathrm{E}+10$ & 33.6623265 & 35.4291185 & 35.050395 & 30.401303 & 24.861439 \\
\hline $1.09 \mathrm{E}+10$ & 33.6403595 & 34.7555115 & 34.304012 & 29.7102715 & 23.7414815 \\
\hline $1.1 \mathrm{E}+10$ & 33.5530015 & 34.507765 & 34.083268 & 29.5873035 & 25.2512585 \\
\hline $1.11 \mathrm{E}+10$ & 33.4365395 & 35.165847 & 34.915097 & 30.431326 & 28.5455505 \\
\hline $1.12 \mathrm{E}+10$ & 33.202923 & 35.808519 & 35.660543 & 31.1490345 & 30.40134 \\
\hline $1.13 \mathrm{E}+10$ & 33.1534675 & 36.2802315 & 36.196744 & 31.6739125 & 29.7293255 \\
\hline $1.14 \mathrm{E}+10$ & 33.215673 & 35.9527475 & 35.860236 & 31.4161035 & 26.857306 \\
\hline $1.15 \mathrm{E}+10$ & 33.361628 & 35.5052265 & 35.491489 & 31.1962645 & 24.9397365 \\
\hline $1.16 \mathrm{E}+10$ & 33.296259 & 35.1616255 & 35.2072515 & 30.9817025 & 26.115625 \\
\hline $1.17 \mathrm{E}+10$ & 33.347749 & 35.742393 & 35.9165085 & 31.7746005 & 31.0850435 \\
\hline $1.18 \mathrm{E}+10$ & 33.3081515 & 36.651528 & 36.9438585 & 32.813984 & 35.221763 \\
\hline $1.19 \mathrm{E}+10$ & 33.0715505 & 36.7394895 & 37.1467155 & 32.887545 & 34.6525085 \\
\hline $1.2 \mathrm{E}+10$ & 32.8943885 & 36.2830685 & 36.639582 & 32.4098955 & 30.731031 \\
\hline $1.21 \mathrm{E}+10$ & 32.9330155 & 35.4093525 & 35.67763 & 31.497404 & 25.9281435 \\
\hline $1.22 \mathrm{E}+10$ & 33.093243 & 34.6502965 & 34.9411485 & 30.95024 & 23.546998 \\
\hline $1.23 \mathrm{E}+10$ & 33.6068135 & 35.028577 & 35.5549065 & 32.019446 & 27.648824 \\
\hline $1.24 E+10$ & 33.355484 & 35.931607 & 36.524467 & 32.947101 & 34.7605315 \\
\hline $1.25 \mathrm{E}+10$ & 33.158674 & 36.6104105 & 37.1469515 & 33.3681895 & 38.811808 \\
\hline $1.26 \mathrm{E}+10$ & 32.9751085 & 36.375771 & 36.928434 & 33.0394925 & 36.1238355 \\
\hline $1.27 \mathrm{E}+10$ & 32.55409 & 35.6462005 & 36.0935475 & 31.9426195 & 30.0346925 \\
\hline $1.28 \mathrm{E}+10$ & 32.5752675 & 34.994349 & 35.5958695 & 31.618592 & 24.644599 \\
\hline $1.29 \mathrm{E}+10$ & 32.863395 & 35.2554035 & 36.1866385 & 32.5667795 & 22.114886 \\
\hline $1.3 \mathrm{E}+10$ & 32.6308405 & 35.333262 & 36.310059 & 32.873042 & 23.7330635 \\
\hline $1.31 \mathrm{E}+10$ & 32.756229 & 36.1080245 & 37.1927055 & 33.9051455 & 32.158801 \\
\hline $1.32 \mathrm{E}+10$ & 32.462242 & 35.672331 & 36.475004 & 33.123593 & 36.248023 \\
\hline $1.33 \mathrm{E}+10$ & 32.4815515 & 34.916974 & 35.4145165 & 31.840392 & 33.474666 \\
\hline $1.35 \mathrm{E}+10$ & 32.2233425 & 33.982774 & 34.4279785 & 30.658815 & 27.9749295 \\
\hline $1.36 \mathrm{E}+10$ & 32.049771 & 33.948004 & 34.5532265 & 30.628022 & 22.8391595 \\
\hline $1.37 \mathrm{E}+10$ & 32.0298715 & 34.757027 & 35.534474 & 31.624472 & 9.8119 \\
\hline
\end{tabular}




\begin{tabular}{|c|c|c|c|c|c|}
\hline Frequency & $e^{\prime} @ 20 \mathrm{C}$ & $e^{\prime} @ 40 \mathrm{C}$ & $e^{\prime} @ 60 \mathrm{C}$ & $e^{\prime} @ 80 \mathrm{C}$ & $e^{\prime} @ 100 \mathrm{C}$ \\
\hline $1.38 \mathrm{E}+10$ & 32.067271 & 35.692791 & 36.8206825 & 33.140457 & 22.7064975 \\
\hline $1.39 \mathrm{E}+10$ & 31.9241365 & 35.635766 & 36.645089 & 33.1474375 & 29.8100335 \\
\hline $1.4 \mathrm{E}+10$ & 32.0972105 & 35.0078365 & 35.808645 & 32.372164 & 34.08630085 \\
\hline $1.41 E+10$ & 31.829064 & 33.839986 & 34.228287 & 30.5806465 & 31.087222 \\
\hline $1.42 \mathrm{E}+10$ & 31.7861575 & 33.402396 & 33.557136 & 29.6810525 & 26.401883 \\
\hline $1.43 E+10$ & 31.6755 & 34.5112325 & 34.980892 & 30.966653 & 23.100366 \\
\hline $1.44 E+10$ & 31.7474595 & 36.7187605 & 37.786938 & 33.712222 & 22.3254725 \\
\hline $1.45 \mathrm{E}+10$ & 31.3715215 & 37.417037 & 38.8678395 & 35.167251 & 26.948007 \\
\hline $1.46 \mathrm{E}+10$ & 31.4207705 & 36.7913415 & 38.4428775 & 35.356721 & 35.721633 \\
\hline $1.47 E+10$ & 31.3154335 & 34.779863 & 36.0959725 & 33.175379 & 36.289624 \\
\hline $1.48 \mathrm{E}+10$ & 31.7200135 & 33.0246865 & 33.6656045 & 30.493621 & 31.046679 \\
\hline $1.49 E+10$ & 31.986321 & 32.6588775 & 33.1608775 & 29.8327135 & 26.4062135 \\
\hline $1.5 \mathrm{E}+10$ & 31.368116 & 33.881642 & 34.803499 & 31.024449 & 23.1643035 \\
\hline $1.51 \mathrm{E}+10$ & 30.7834125 & 37.306303 & 38.862861 & 34.7928365 & 23.702627 \\
\hline $1.52 \mathrm{E}+10$ & 29.8448425 & 39.0158365 & 41.4629505 & 38.177663 & 31.529763 \\
\hline $1.53 \mathrm{E}+10$ & 30.5619615 & 39.44856 & 42.1535515 & 39.644898 & 42.427615 \\
\hline $1.54 \mathrm{E}+10$ & 30.332786 & 35.72576 & 37.4580185 & 34.9151915 & 38.2331445 \\
\hline $1.55 \mathrm{E}+10$ & 31.635466 & 33.4687225 & 34.4745845 & 31.760251 & 32.2766765 \\
\hline $1.56 \mathrm{E}+10$ & 31.9435365 & 30.3219675 & 31.0823995 & 28.2870285 & 25.389638 \\
\hline $1.57 \mathrm{E}+10$ & 32.5718495 & 30.542707 & 31.3833195 & 28.204 & 21.826739 \\
\hline $1.58 \mathrm{E}+10$ & 31.184581 & 33.5572245 & 34.7261795 & 30.9567505 & 21.7088275 \\
\hline $1.59 \mathrm{E}+10$ & 30.211003 & 38.510058 & 40.8120855 & 36.9834595 & 29.3326065 \\
\hline $1.6 \mathrm{E}+10$ & 28.3283195 & 37.6489565 & 39.9060895 & 37.1165465 & 37.41426 \\
\hline $1.61 \mathrm{E}+10$ & 30.15114 & 37.651821 & 39.737633 & 37.448373 & 40.0986205 \\
\hline $1.62 E+10$ & 31.8341255 & 34.7115825 & 36.690206 & 34.598447 & 35.1782315 \\
\hline $1.63 \mathrm{E}+10$ & 32.310045 & 31.5410425 & 33.00523 & 30.1818215 & 27.319928 \\
\hline $1.64 \mathrm{E}+10$ & 31.4703105 & 29.3878475 & 29.9696955 & 26.466105 & 20.9172455 \\
\hline $1.65 E+10$ & 30.1662155 & 31.405934 & 32.304656 & 28.4033675 & 21.302508 \\
\hline $1.66 \mathrm{E}+10$ & 29.05386 & 36.579302 & 38.5157115 & 34.477651 & 28.32124 \\
\hline $1.67 \mathrm{E}+10$ & 28.903188 & 39.2753945 & 41.476792 & 37.944541 & 36.6874925 \\
\hline $1.68 \mathrm{E}+10$ & 29.556481 & 38.233636 & 40.6448045 & 38.1015355 & 39.381906 \\
\hline $1.69 \mathrm{E}+10$ & 29.0631265 & 33.908907 & 36.173277 & 33.552495 & 32.8356395 \\
\hline $1.7 \mathrm{E}+10$ & 29.4185955 & 29.697436 & 30.686151 & 27.586462 & 24.232986 \\
\hline $1.71 \mathrm{E}+10$ & 29.8323975 & 28.9977305 & 29.853138 & 26.7605495 & 22.387553 \\
\hline $1.72 \mathrm{E}+10$ & 29.7168155 & 32.4226665 & 34.1420915 & 30.7803865 & 26.1486615 \\
\hline $1.73 E+10$ & 28.721835 & 35.606286 & 37.5571615 & 33.614816 & 29.340359 \\
\hline $1.74 \mathrm{E}+10$ & 28.649329 & 38.116672 & 40.2435215 & 36.711763 & 34.904348 \\
\hline $1.75 E+10$ & 28.475414 & 36.94195 & 39.4604775 & 36.6889515 & 35.7725215 \\
\hline $1.76 \mathrm{E}+10$ & 28.661208 & 34.0012355 & 36.240366 & 33.491088 & 30.655022 \\
\hline $1.77 \mathrm{E}+10$ & 28.6856555 & 30.767278 & 31.967506 & 28.812128 & 24.6515405 \\
\hline $1.79 \mathrm{E}+10$ & 28.9529415 & 31.0647335 & 32.3273635 & 29.117631 & 24.7890915 \\
\hline $1.8 \mathrm{E}+10$ & 28.332913 & 32.713531 & 34.5495795 & 31.130455 & 26.552096 \\
\hline $1.81 \mathrm{E}+10$ & 28.1598335 & 35.4193105 & 37.5755925 & 34.0775945 & 30.848636 \\
\hline $1.82 \mathrm{E}+10$ & 28.2547955 & 36.0685715 & 38.084371 & 34.9532555 & 32.9843355 \\
\hline $1.83 E+10$ & 28.1709365 & 35.0517455 & 37.3850015 & 34.7817945 & 32.583269 \\
\hline
\end{tabular}




\begin{tabular}{|r|r|r|r|r|r|}
\hline Frequency & $\mathbf{e}^{\prime} @ 20 \mathrm{C}$ & $\mathbf{e}^{\prime} @ 40 \mathrm{C}$ & $\mathrm{e}^{\prime} @ 60 \mathrm{C}$ & $\mathrm{e}^{\prime} @ 80 \mathrm{C}$ & $\mathrm{e}^{\prime} @ 100 \mathrm{C}$ \\
\hline \hline $1.84 \mathrm{E}+10$ & 27.773786 & 31.8204035 & 33.937958 & 31.4563075 & 28.244783 \\
\hline $1.85 \mathrm{E}+10$ & 28.1337625 & 31.099421 & 32.700455 & 29.9337855 & 26.422738 \\
\hline $1.86 \mathrm{E}+10$ & 28.0844415 & 31.613195 & 33.3774925 & 30.6791755 & 27.751853 \\
\hline $1.87 \mathrm{E}+10$ & 27.9514015 & 34.146481 & 36.53533 & 33.8940515 & 31.2572525 \\
\hline $1.88 \mathrm{E}+10$ & 27.5589305 & 35.035059 & 37.4534645 & 34.600178 & 32.489989 \\
\hline $1.89 \mathrm{E}+10$ & 27.9143185 & 35.061436 & 37.1463335 & 34.2159705 & 32.158544 \\
\hline $1.9 \mathrm{E}+10$ & 27.7823855 & 33.0384105 & 35.067258 & 32.432085 & 29.2558735 \\
\hline $1.91 \mathrm{E}+10$ & 27.562003 & 30.0011365 & 31.7249595 & 29.129817 & 25.373255 \\
\hline $1.92 \mathrm{E}+10$ & 27.5358995 & 28.6356825 & 30.21965 & 27.6210865 & 24.49769 \\
\hline $1.93 \mathrm{E}+10$ & 27.6261735 & 29.956717 & 31.7260945 & 29.085137 & 26.2273245 \\
\hline $1.94 \mathrm{E}+10$ & 27.221703 & 31.1733405 & 33.229066 & 30.5918235 & 28.0680125 \\
\hline $1.95 \mathrm{E}+10$ & 27.1608665 & 32.0061495 & 34.1849215 & 31.6728215 & 29.547383 \\
\hline $1.96 \mathrm{E}+10$ & 27.3172585 & 32.0890725 & 34.0805245 & 31.5279965 & 28.864023 \\
\hline $1.97 \mathrm{E}+10$ & 27.3953245 & 31.6164655 & 33.54125 & 30.998733 & 27.686517 \\
\hline $1.98 \mathrm{E}+10$ & 27.16034 & 30.8382955 & 32.600361 & 29.8031095 & 26.129447 \\
\hline $1.99 \mathrm{E}+10$ & 27.0407635 & 31.1335475 & 32.9544745 & 29.978679 & 26.536835 \\
\hline $2 \mathrm{E}+10$ & 27.050794 & 31.8738945 & 33.8388625 & 30.8887475 & 27.7232635 \\
\hline
\end{tabular}


Table 19. Dielectric Loss for the W-26 ORNL Simulant

\begin{tabular}{|c|c|c|c|c|c|}
\hline$\overline{\text { icy }}$ & $\mathrm{e}^{\prime \prime} @ 20 \mathrm{C}$ & $\mathrm{e}^{\prime \prime} @ 40 \mathrm{C}$ & $e^{\prime \prime @ 60 C ~}$ & $\mathrm{e}^{\prime \prime} @ 80 \mathrm{C}$ & C \\
\hline $1.53 \mathrm{E}+08$ & 1995.47473 & 2800.54816 & 3809.0626 & 4372.4805 & $\overline{10}$ \\
\hline $2.55 \mathrm{E}+08$ & 1232.12227 & 1733.58741 & 2361.4419 & 2700.8312 & 3044.44847 \\
\hline $3.58 \mathrm{E}+08$ & 890.360446 & 1254.13765 & 1708.1285 & 1950.1602 & 203.94815 \\
\hline $4.6 \mathrm{E}+08$ & 696.115989 & 981.260371 & 1336.5141 & 1525.3236 & 1726.3401 \\
\hline $5.62 \mathrm{E}+08$ & 581.504888 & 820.38307 & 1115.6543 & 1272.9505 & 1442.49645 \\
\hline $6.65 \mathrm{E}+08$ & 496.121363 & 699.376339 & 953.35315 & 1087.7391 & 1232.85179 \\
\hline $7.67 \mathrm{E}+08$ & 429.684258 & 605.277441 & 824.71895 & 940.40917 & 1065.25578 \\
\hline $8.69 E+08$ & 378.804483 & 533.235124 & 726.05905 & 827.95533 & 937.68438 \\
\hline $9.71 E+08$ & 340.375969 & 479.104957 & 652.78402 & 744.50195 & 843.607618 \\
\hline $1.07 E+09$ & 310.321317 & 436.613705 & 594.89581 & 678.47522 & 769.208219 \\
\hline $1.18 \mathrm{E}+09$ & 285.591197 & 401.607316 & 547.09229 & 623.98537 & 707.483404 \\
\hline $1.28 \mathrm{E}+09$ & 263.294344 & 369.796373 & 503.51354 & 574.18499 & 650.712954 \\
\hline $1.38 \mathrm{E}+09$ & 243.583772 & 341.511646 & 464.82622 & 530.16404 & 600.427811 \\
\hline $1.48 E+09$ & 227.078806 & 317.962799 & 432.39742 & 493.52438 & 558.570378 \\
\hline $1.59 E+09$ & 213.822089 & 298.932264 & 406.08274 & 463.07206 & 524.061116 \\
\hline $1.69 \mathrm{E}+09$ & 202.067169 & 282.250601 & 383.12867 & 436.87558 & 494.753846 \\
\hline $1.79 \mathrm{E}+09$ & 190.806937 & 266.318675 & 361.3548 & 411.87808 & 466.874168 \\
\hline $1.89 \mathrm{E}+09$ & 180.661554 & 251.797042 & 341.57182 & 389.26415 & 441.245231 \\
\hline $1.99 \mathrm{E}+09$ & 172.51965 & 239.910686 & 325.17777 & 370.53345 & 419.481586 \\
\hline $2.1 E+09$ & 165.10342 & 229.000367 & 310.12386 & 353.11532 & 399.333758 \\
\hline $2.2 \mathrm{E}+09$ & 157.727995 & 218.174033 & 295.22312 & 335.78729 & 379.765199 \\
\hline $2.3 \mathrm{E}+09$ & 150.798256 & 208.533032 & 282.14216 & 320.92251 & 363.319283 \\
\hline $2.4 \mathrm{E}+09$ & 144.95956 & 200.269992 & 270.78783 & 307.71688 & 348.680087 \\
\hline $2.51 \mathrm{E}+09$ & 140.270072 & 193.249573 & 260.74666 & 295.9541 & 335.168703 \\
\hline $2.61 E+09$ & 135.684496 & 186.590119 & 252.08259 & 286.17117 & 323.660604 \\
\hline $2.71 \mathrm{E}+09$ & 130.907952 & 179.466526 & 242.21804 & 274.78289 & 309.982174 \\
\hline $2.81 \mathrm{E}+09$ & 126.235178 & 172.626337 & 232.85997 & 264.05799 & 297.436032 \\
\hline $2.92 \mathrm{E}+09$ & 122.081698 & 166.623854 & 224.46294 & 254.43442 & 286.402003 \\
\hline $3.02 \mathrm{E}+09$ & 118.624562 & 161.656073 & 217.46069 & 246.28359 & 277.458483 \\
\hline $3.12 \mathrm{E}+09$ & 115.511774 & 157.204895 & 211.22361 & 239.11827 & 269.381288 \\
\hline $3.22 \mathrm{E}+09$ & 112.2696 & 152.393998 & 204.64721 & 231.51297 & 260.560848 \\
\hline $3.32 \mathrm{E}+09$ & 108.907019 & 147.19587 & 197.18821 & 222.79169 & 250.111501 \\
\hline $3.43 \mathrm{E}+09$ & 106.023374 & 142.699049 & 191.01717 & 215.75433 & 241.683405 \\
\hline $3.53 \mathrm{E}+09$ & 103.523541 & 139.023146 & 185.91825 & 209.80761 & 235.017044 \\
\hline $3.63 \mathrm{E}+09$ & 101.12838 & 135.65707 & 181.26126 & 204.73196 & 229.702849 \\
\hline $3.73 E+09$ & 98.609226 & 131.974918 & 175.9674 & 198.56096 & 222.925479 \\
\hline $3.84 \mathrm{E}+09$ & 96.361613 & 128.621341 & 171.22186 & 193.07313 & 216.644761 \\
\hline $3.94 \mathrm{E}+09$ & 94.5207335 & 125.774393 & 167.19167 & 188.5582 & 211.091525 \\
\hline $4.04 \mathrm{E}+09$ & 92.743612 & 122.990823 & 163.29012 & 184.02177 & 205.385483 \\
\hline $4.14 \mathrm{E}+09$ & $90: 8636815$ & 120.041079 & 159.07097 & 179.14415 & 199.561419 \\
\hline $4.25 \mathrm{E}+09$ & 88.9427765 & 117.218305 & 155.0918 & 174.59912 & 194.688449 \\
\hline $4.35 \mathrm{E}+09$ & 87.365378 & 114.891243 & 151.81746 & 170.96177 & 191.158484 \\
\hline $4.45 E+09$ & 85.9652525 & 112.805883 & 148.70653 & 167.2684 & 187.208081 \\
\hline
\end{tabular}




\begin{tabular}{|c|c|c|c|c|c|}
\hline \begin{tabular}{|l|l|} 
ncy & e \\
\end{tabular} & $\mathrm{e}^{\prime \prime @ 20 C}$ & $\mathrm{e}^{\prime \prime} @ 40 \mathrm{C}$ & $e^{\prime \prime @ 60 ~ C ~}$ & $\mathrm{e}^{\prime \prime} @ 80 \mathrm{C}$ & $\overline{00 \mathrm{C}}$ \\
\hline $4.55 \mathrm{E}+09$ & 84.5451515 & 110.483706 & 145.29192 & 163.25896 & 182.23869 \\
\hline $4.65 \mathrm{E}+09$ & 82.904851 & 107.874947 & 141.52005 & 158.88142 & 176.623849 \\
\hline $4.76 \mathrm{E}+09$ & 81.4729875 & 105.768715 & 138.59134 & 155.46043 & 172.343382 \\
\hline $4.86 \mathrm{E}+09$ & 80.1848065 & 103.837438 & 135.83717 & 152.22608 & 168.587461 \\
\hline $4.96 \mathrm{E}+09$ & 78.978226 & 102.092157 & 133.38909 & 149.34788 & 165.612961 \\
\hline $5.06 \mathrm{E}+09$ & 77.713024 & 100.315845 & 130.86976 & 146.39585 & 162.839819 \\
\hline $5.17 \mathrm{E}+09$ & 76.5660905 & 98.5387545 & 128.36612 & 143.55744 & 159.494901 \\
\hline $5.27 \mathrm{E}+09$ & 75.2444765 & 96.540483 & 125.34726 & 139.99151 & 154.738449 \\
\hline $5.37 \mathrm{E}+09$ & 74.178321 & 94.8462895 & 122.95523 & 137.20426 & 151.062335 \\
\hline $5.47 \mathrm{E}+09$ & 73.149705 & 93.353712 & 120.80852 & 134.66899 & 148.248122 \\
\hline $5.57 \mathrm{E}+09$ & 72.1077695 & 92.0042365 & 118.79612 & 132.21644 & 145.72272 \\
\hline $5.68 \mathrm{E}+09$ & 71.0589155 & 90.5406835 & 116.7443 & 129.75888 & 143.481246 \\
\hline $5.78 \mathrm{E}+09$ & 70.027991 & 89.045132 & 114.58454 & $127.2 \mathrm{~s}$ & 140.887973 \\
\hline $5.88 \mathrm{E}+09$ & 69.1464235 & 87.634103 & 112.65655 & 125.05776 & 138.393369 \\
\hline $5.98 \mathrm{E}+09$ & 68.2624035 & 86.055032 & 110.39789 & 122.35916 & 134.738908 \\
\hline $6.09 E+09$ & 67.3394325 & 84.553767 & 108.18755 & 119.80596 & 131.459111 \\
\hline $6.19 \mathrm{E}+09$ & 66.5578345 & 83.1801195 & 106.31706 & 117.65048 & 129.451479 \\
\hline $6.29 \mathrm{E}+09$ & 65.8167765 & 81.9871435 & 104.77701 & 115.91395 & 128.160924 \\
\hline $6.39 \mathrm{E}+09$ & 65.008606 & 80.796228 & 103.06859 & 113.92546 & 126.200124 \\
\hline $6.5 \mathrm{E}+09$ & 64.1706555 & 79.358371 & 101.10665 & 111.78799 & 123.765702 \\
\hline $6.6 \mathrm{E}+09$ & 63.3992225 & 78.23572 & 99.479761 & 110.01244 & 121.265697 \\
\hline $6.7 \mathrm{E}+09$ & 62.678402 & 77.0287635 & 97.706359 & 107.9579 & 118.250766 \\
\hline $6.8 \mathrm{E}+09$ & 61.9912925 & 75.8358595 & 95.964252 & 105.94129 & 115.747974 \\
\hline $6.9 E+09$ & 61.3075705 & 74.797076 & 94.458289 & 104.24377 & 114.274722 \\
\hline $7.01 \mathrm{E}+09$ & 60.633722 & 73.787405 & 93.07626 & 102.7547 & 113.205126 \\
\hline $7.11 \mathrm{E}+09$ & 59.911906 & 72.72847 & 91.554059 & 101.01832 & 111.149334 \\
\hline $7.21 \mathrm{E}+09$ & 59.1431215 & 71.571611 & 89.917113 & 99.228268 & 108.552959 \\
\hline $7.31 \mathrm{E}+09$ & 58.5903025 & 70.550087 & 88.543523 & 97.752274 & 106.280084 \\
\hline $7.42 \mathrm{E}+09$ & 58.01306 & 69.5260835 & 87.108997 & 96.065848 & 104.137153 \\
\hline $7.52 \mathrm{E}+09$ & 57.355174 & 68.7329425 & 85.835581 & 94.559949 & 102.498768 \\
\hline $7.62 \mathrm{E}+09$ & 56.7795335 & 67.9994145 & 84.788422 & 93.373072 & 101.834317 \\
\hline $7.72 \mathrm{E}+09$ & 56.1721335 & 67.2654295 & 83.939126 & 92.480343 & 101.446796 \\
\hline $7.83 \mathrm{E}+09$ & 55.6172205 & 66.561786 & 82.966013 & 91.372049 & 99.8923665 \\
\hline $7.93 \mathrm{E}+09$ & 55.015133 & 65.5931745 & 81.623978 & 89.750441 & 97.2552335 \\
\hline $8.03 \mathrm{E}+09$ & 54.4516745 & 64.5804745 & 80.196728 & 88.206758 & 95.143524 \\
\hline $8.13 \mathrm{E}+09$ & 54.039389 & 64.0233945 & 79.316279 & 87.09509 & 93.974286 \\
\hline $8.23 E+09$ & 53.441655 & 63.2734475 & 78.158704 & 85.630048 & 92.868581 \\
\hline $8.34 \mathrm{E}+09$ & 52.914313 & 62.6983935 & 77.43413 & 84.76946 & 92.583522 \\
\hline $8.44 \mathrm{E}+09$ & 52.384086 & 61.9194255 & 76.457368 & 83.734647 & 91.735559 \\
\hline $8.54 \mathrm{E}+09$ & 51.8697335 & 60.9220095 & 75.161492 & 82.3182 & 89.5883545 \\
\hline $8.64 E+09$ & 51.4014675 & 60.0404685 & 74.065714 & 81.099136 & 87.3804305 \\
\hline $8.75 \mathrm{E}+09$ & 50.9216915 & 59.385317 & 73.078585 & 79.859737 & 85.802431 \\
\hline $8.85 E+09$ & 50.5074695 & 58.9118765 & 72.321343 & 78.925545 & 85.409155 \\
\hline $8.95 E+09$ & 50.1185255 & 58.54322 & 71.745057 & 78.190047 & 85.3384775 \\
\hline $9.05 \mathrm{E}+09$ & 49.599212 & 57.749705 & 70.71292 & 77.03384 & 84.506581 \\
\hline
\end{tabular}




\begin{tabular}{|c|c|c|c|c|c|}
\hline requency & $e^{\prime \prime} @ 20 \mathrm{C}$ & @ $40 \mathrm{C}$ & @60C & $\overline{\mathbf{C}}$ & $\overline{\mathbf{C}}$ \\
\hline+09 & 152933 & 56.92958 & 4106 & 121 & 53 \\
\hline $9.26 \mathrm{E}+09$ & 48.7732975 & 56.0938355 & 68.632693 & 74.809753 & 80.988555 \\
\hline $9.36 \mathrm{E}+09$ & 48.384717 & 55.454628 & 67.697322 & 73.781567 & 79.135083 \\
\hline $9.46 \mathrm{E}+09$ & 48.0547925 & 55.202581 & 67.307935 & 73.297212 & 78.8667975 \\
\hline $9.56 \mathrm{E}+09$ & 47.638022 & 54.512664 & 66.266879 & 72.018711 & 78.4161885 \\
\hline $9.67 \mathrm{E}+09$ & 47.259627 & 54.0546245 & 65.574602 & 71.26143 & 78.473565 \\
\hline $9.77 \mathrm{E}+09$ & 46.8753395 & 52.991404 & 64.339209 & 69.940578 & 76.725931 \\
\hline $9.87 \mathrm{E}+09$ & 46.460552 & 52.005351 & 63.071471 & 68.695689 & 74.5180175 \\
\hline $9.97 \mathrm{E}+09$ & 46.0424455 & 50.9621605 & 61.813118 & 67.391056 & 72.1617465 \\
\hline $1.01 \mathrm{E}+10$ & 45.8788705 & 50.998943 & 61.749645 & 67.241966 & 71.7360225 \\
\hline $1.02 \mathrm{E}+10$ & 45.5232505 & 50.9213115 & 61.381987 & 66.677538 & 71.8656785 \\
\hline $1.03 \mathrm{E}+10$ & 45.174241 & 50.5068585 & 60.82171 & 66.004388 & 72.4768495 \\
\hline $1.04 \mathrm{E}+10$ & 44.778562 & 49.8692595 & 59987899 & 65.061468 & 71.93815 \\
\hline $1.05 \mathrm{E}+10$ & 4.5918825 & 49.301328 & 59.290118 & 64.388217 & 20.2152495 \\
\hline $1.06 \mathrm{E}+10$ & 44.06705 & $=48.63214$ & 58518881 & 6304843 & 67971714 \\
\hline $1.07 \mathrm{E}+10$ & 43.973545 & 48.9188995 & 58.628253 & 63.602952 & 67.2879295 \\
\hline $1.08 \mathrm{E}+10$ & 43.8389455 & 49.0683965 & 58.526817 & 63.367874 & 67.4038915 \\
\hline $1.09 \mathrm{E}+10$ & 43.53033 & 48.836883 & 58.125103 & 62.807048 & 5156 \\
\hline $1.1 \mathrm{E}+10$ & 3.1071875 & 48.0494225 & 57.201915 & 61.778834 & 68.78188 \\
\hline $1.11 \mathrm{E}+10$ & 42.873945 & 47.394627 & 56.542215 & 61.203932 & 68.3496395 \\
\hline $1.12 \mathrm{E}+10$ & 42.393499 & 46.5756675 & 55.556732 & 60.179715 & 65.3218135 \\
\hline $1.13 \mathrm{E}+10$ & 42.4779845 & 46.767513 & 55.720724 & 60.400564 & 63.8115395 \\
\hline $1.14 \mathrm{E}+10$ & 42.217363 & 46.6454425 & 55.357493 & 59.866972 & 63.2672335 \\
\hline $1.15 \mathrm{E}+10$ & 42.0850115 & 46.41573 & 54.892826 & 59.232019 & 64.352204 \\
\hline $1.16 \mathrm{E}+10$ & 41.9713905 & 45.910506 & 54.337972 & 58.465549 & 66.264934 \\
\hline $1.17 \mathrm{E}+10$ & 41.4144765 & 44.6436515 & 52.905573 & 56.957811 & 66.3424675 \\
\hline $1.18 \mathrm{E}+10$ & 40.985208 & 43.623424 & 51.787598 & 55.836121 & 63.7481755 \\
\hline $1.19 \mathrm{E}+10$ & 41.107887 & $44: 008415$ & 52.082014 & 56.175558 & 60.478179 \\
\hline $1.2 \mathrm{E}+10$ & 41.001224 & 44.1596595 & 52.099112 & 56.066254 & 58.0392695 \\
\hline $1.21 \mathrm{E}+10$ & 40.922198 & 44.47132 & 52.18389 & 55.961523 & 58.822153 \\
\hline $1.22 \mathrm{E}+10$ & 40.698894 & 43.9301065 & 51.350502 & 54.985413 & 61.482985 \\
\hline $1.23 \mathrm{E}+10$ & 40.6155235 & 42.6730015 & 49.788254 & 53.309525 & 64.6220305 \\
\hline $1.24 \mathrm{E}+10$ & 40.4476345 & 41.744306 & 49.022796 & 52.49084 & 66.233752 \\
\hline $1.25 \mathrm{E}+10$ & 40.1564735 & 41.490277 & 48.725561 & 51.994001 & 62.1714945 \\
\hline $1.26 \mathrm{E}+10$ & 39.890146 & 41.316048 & 48.445338 & 51.688265 & 55.809568 \\
\hline $1.27 \mathrm{E}+10$ & 39.721473 & 42.0653875 & 49.217318 & 52.398741 & 53.052652 \\
\hline $1.28 \mathrm{E}+10$ & 39.629122 & 42.162654 & 49.196045 & 52.332354 & 54.510512 \\
\hline $1.29 \mathrm{E}+10$ & 40.0605835 & 42.202648 & 49.185243 & 52.58059 & 60.428067 \\
\hline $1.3 \mathrm{E}+10$ & 39.8387685 & 41.236056 & 48.039721 & 51.434877 & 65.572664 \\
\hline $1.31 \mathrm{E}+10$ & 39.973201 & 40.5593835 & 47.237931 & 50.560919 & 67.6450475 \\
\hline $1.32 \mathrm{E}+10$ & 39.5238715 & 40.0175825 & 46.539991 & 49.465453 & 61.0469335 \\
\hline $1.33 \mathrm{E}+10$ & 39.1081975 & 40.3504115 & 46.746077 & 49.283749 & 53.4126915 \\
\hline $1.35 \mathrm{E}+10$ & 38.838014 & 40.635992 & 47.078738 & 49.458658 & 49.234571 \\
\hline $1.36 \mathrm{E}+10$ & 38.7106145 & 41.070331 & 47.646765 & 50.123192 & 50.715241 \\
\hline $1.37 \mathrm{E}+10$ & 38.6586675 & 40.998445 & 47.75737 & 50.524495 & 55.732923 \\
\hline
\end{tabular}




\begin{tabular}{|c|c|c|c|c|c|}
\hline cy $1 \mathrm{e}$ & $\mathrm{e}^{11} @ 20 \mathrm{C}$ & @ & E & 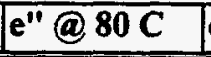 & $\bar{E}$ \\
\hline+10 & 38.9011095 & 39.7681525 & 46.423811 & 49.488166 & $\overline{4834}$ \\
\hline $39 \mathrm{E}+10$ & 38.8670705 & 38.956454 & 45.348536 & 48.104417 & 60.799852 \\
\hline $1.4 \mathrm{E}+10$ & 38.6308455 & 38.543461 & 44.711308 & 47.07776 & 55.156934 \\
\hline $1.41 \mathrm{E}+10$ & 38.0910145 & 38.996114 & 45.123406 & 47.070434 & 49.2815085 \\
\hline $1.42 \mathrm{E}+10$ & 37.537476 & 40.1774175 & 46.335309 & 48.0603 & 6.8978515 \\
\hline $1.43 \mathrm{E}+10$ & 37.3365805 & 41.5079745 & 8.033288 & 50.04613 & 50.2042805 \\
\hline $1.44 \mathrm{E}+10$ & 37.856192 & 41.929719 & 48.800478 & 51.341964 & 57.1048645 \\
\hline $1.45 \mathrm{E}+10$ & 38.12426 & 39.6018345 & 45.899774 & 48.538066 & 60.2244325 \\
\hline $1.46 E+10$ & 38.681799 & 36.692822 & 42.164516 & 44.643621 & 56.0834595 \\
\hline $1.47 \mathrm{E}+10$ & 37.813582 & 34.7002615 & 39.883225 & 41.982213 & 88.0986155 \\
\hline $1.48 E+10$ & 37.3536695 & 35.71931 & 40.770063 & 42.321443 & 43.9276575 \\
\hline $1.49 \mathrm{E}+10$ & 36.442838 & 37.0786385 & 42.107409 & 43.426792 & 24962 \\
\hline $1.5 \mathrm{E}+10$ & 36.58015 & 39.9388555 & 45.716172 & 47.388642 & 48.8358005 \\
\hline $1.51 \mathrm{E}+10$ & 36.537178 & 40.130916 & 46.496221 & $49 \times 23549$ & 56.3546595 \\
\hline $1.52 \mathrm{E}+10$ & 37.4157645 & 36.5753095 & 42.313927 & 45.48448 & 14925 \\
\hline $1.53 \mathrm{E}+10$ & 37.5259395 & 31.3182475 & 36.061595 & 39.072532 & 50.731628 \\
\hline $1.54 \mathrm{E}+10$ & 37.906253 & $\overline{30.73}$ & 53 & 37.451769 & $3 \overline{65}$ \\
\hline $1.55 \mathrm{E}+10$ & 37.129832 & 32.0176125 & 36.124451 & 37.687579 & 38.376268 \\
\hline $1.56 \mathrm{E}+10$ & 35.9428365 & 34.9772465 & 38.981684 & 40.143146 & 39.334143 \\
\hline $1.57 \mathrm{E}+10$ & 35.5424175 & 38.505695 & 43.38548 & 44.796312 & $\overline{5762}$ \\
\hline $1.58 \mathrm{E}+10$ & 35.8760965 & 40.1254305 & 45.770057 & 47.783302 & 52.492549 \\
\hline $1.59 \mathrm{E}+10$ & 37.1730745 & 38.7134635 & 44.453507 & 47.381909 & 039 \\
\hline $1.6 \mathrm{E}+10$ & 36.185009 & 32.1292315 & 36.530882 & 39.132149 & 48.7440285 \\
\hline $1.61 \mathrm{E}+10$ & 36.61 & 45 & 03 & 431 & 111 \\
\hline $1.62 \mathrm{E}+10$ & 36.9025255 & 31.01232 & 34.388696 & 36.246926 & 37.8499855 \\
\hline $1.63 \mathrm{E}+10$ & 38.2226615 & 35.7791685 & 40.228644 & 41.899652 & 42.843245 \\
\hline $1.64 \mathrm{E}+10$ & 36.942878 & 38.4317905 & 43.196588 & 44.359587 & 45.751278 \\
\hline $1.65 \mathrm{E}+10$ & 36.483138 & 40.697126 & 45.806903 & 47.231446 & 51.611609 \\
\hline $1.66 \mathrm{E}+10$ & 35.272369 & 38.743843 & 44.349116 & 46.839416 & 55.4231725 \\
\hline $1.67 \mathrm{E}+10$ & 35.7261465 & 35.645326 & 40.413302 & 43.038184 & 50.9739595 \\
\hline $1.68 \mathrm{E}+10$ & 36.3412165 & 32.4986405 & 36.151995 & 38.296004 & 8965 \\
\hline $1.69 \mathrm{E}+10$ & 37.47512 & 33.72304 & 37.835568 & 39.918911 & 43.145502 \\
\hline $1.7 \mathrm{E}+10$ & 36.6583375 & 34.19963 & 38.444852 & 39.855693 & 41.910323 \\
\hline $1.71 E+10$ & 36.2272145 & 35.5257405 & 39.463082 & 40.502744 & 43.2201835 \\
\hline $1.72 \mathrm{E}+10$ & 35.6487485 & 36.829338 & 41.318633 & 42.975116 & 48.670153 \\
\hline $1.73 \mathrm{E}+10$ & 35.4481945 & 36.9547425 & 42.182641 & 44.474701 & 51.4562155 \\
\hline $1.74 \mathrm{E}+10$ & 34.625914 & 34.0436655 & 38.346306 & 40.662589 & 46.4676275 \\
\hline $1.75 \mathrm{E}+10$ & 35.430882 & 33.254085 & 37.003675 & 39.272544 & 44.4028825 \\
\hline $1.76 \mathrm{E}+10$ & 35.877382 & 34.0483235 & 38.19211 & 40.403156 & 45.13278 \\
\hline $1.77 \mathrm{E}+10$ & 35.426307 & 34.623965 & 38.697826 & 40.104732 & 43.5826635 \\
\hline $1.79 \mathrm{E}+10$ & 35.2390725 & 35.486174 & 39.392804 & 40.634213 & 45.2121045 \\
\hline $1.8 \mathrm{E}+10$ & 35.1774155 & 36.969032 & 41.363689 & 43.050349 & 49.8723095 \\
\hline $1.81 \mathrm{E}+10$ & 34.2756545 & 34.51586 & 39.004538 & 41.237886 & 47.9491195 \\
\hline $1.82 \mathrm{E}+10$ & 34.285686 & 32.6156615 & 36.426385 & 38.44855 & 43.1234095 \\
\hline $1.83 \mathrm{E}+10$ & 34.6562065 & 31.563577 & 34.89214 & 36.815162 & 41.4995905 \\
\hline
\end{tabular}




\begin{tabular}{|r|r|r|r|r|r|}
\hline Frequency & $\mathrm{e}^{\prime \prime} @ 20 \mathrm{C}$ & $\mathrm{e}$ " @ 40 C & $\mathrm{e}$ " @ 60 C & $\mathrm{e}$ " @ 80 C & $\mathrm{e}^{\prime \prime} @ 100 \mathrm{C}$ \\
\hline \hline $1.84 \mathrm{E}+10$ & 34.554583 & 30.633819 & 33.972425 & 35.915784 & 40.7878255 \\
\hline $1.85 \mathrm{E}+10$ & 34.496413 & 31.335426 & 34.804611 & 36.461367 & 41.060708 \\
\hline $1.86 \mathrm{E}+10$ & 34.418873 & 32.0467735 & 35.183921 & 36.590904 & 42.092247 \\
\hline $1.87 \mathrm{E}+10$ & 34.2947065 & 31.4104035 & 34.72762 & 36.740253 & 43.4087275 \\
\hline $1.88 \mathrm{E}+10$ & 34.0470765 & 31.4797795 & 34.913962 & 37.082391 & 42.4682545 \\
\hline $1.89 \mathrm{E}+10$ & 33.9948655 & 31.44513 & 34.526601 & 36.33892 & 40.4907025 \\
\hline $1.9 \mathrm{E}+10$ & 34.2320885 & 31.974246 & 35.043391 & 36.703731 & 40.877008 \\
\hline $1.91 \mathrm{E}+10$ & 34.26251 & 32.138114 & 35.284392 & 36.706631 & 40.8154795 \\
\hline $1.92 \mathrm{E}+10$ & 34.0938185 & 31.535049 & 34.460435 & 35.634846 & 39.9635345 \\
\hline $1.93 \mathrm{E}+10$ & 34.044546 & 31.832833 & 34.739246 & 35.903883 & 40.7288945 \\
\hline $1.94 \mathrm{E}+10$ & 34.0027325 & 31.560894 & 34.499468 & 35.94853 & 40.4455395 \\
\hline $1.95 \mathrm{E}+10$ & 33.7978915 & 30.246205 & 33.099169 & 34.754846 & 38.6083835 \\
\hline $1.96 \mathrm{E}+10$ & 33.6242475 & 30.5040175 & 33.314301 & 34.825342 & 38.395684 \\
\hline $1.97 \mathrm{E}+10$ & 33.7417765 & 30.6697515 & 33.591358 & 35.095746 & 38.822857 \\
\hline $1.98 \mathrm{E}+10$ & 33.780152 & 32.077014 & 35.227449 & 36.651308 & 40.793876 \\
\hline $1.99 \mathrm{E}+10$ & 33.639012 & 32.9907185 & 36.113314 & 37.462406 & 42.136153 \\
\hline $2 \mathrm{E}+10$ & 33.5254245 & 33.8248395 & 36.710416 & 37.917177 & 42.336676 \\
\hline
\end{tabular}


Table 20. Loss Tangent for W-26 ORNL Simulant

\begin{tabular}{|c|c|c|c|c|c|}
\hline requency & e' 20 C & $1 e^{\prime} 40 \mathrm{C}$ & $e^{\prime \prime} / e^{\prime} 60 \mathrm{C}$ & $1 e^{\prime} 80 \mathrm{C}$ & $/ e^{\prime} 100 \mathrm{C}$ \\
\hline+08 & 239561 & 6098 & 3423 & 34633 & 60871789 \\
\hline $2.55 \mathrm{E}+08$ & 7.051259299 & 7.3621873 & 8.0275287 & 9.19481678 & 8.787674356 \\
\hline $3.58 \mathrm{E}+08$ & 7.474098979 & 8.2494999 & 1490378 & 11.0179872 & 1.04547058 \\
\hline $4.6 \mathrm{E}+08$ & 7.115263403 & 8.1425231 & 9.546905 & 11.1785307 & 11.5609229 \\
\hline $5.62 \mathrm{E}+08$ & 6.960353918 & 8.2332904 & 10.064454 & 12.0732283 & 12.91215188 \\
\hline $6.65 \mathrm{E}+08$ & 6.522350987 & 7.8863325 & 9.7799311 & 11.7444119 & 12.75849128 \\
\hline $7.67 \mathrm{E}+08$ & 6.14165593 & 7.6202115 & 9.7279811 & 11.9353069 & 13.50069965 \\
\hline $8.69 \mathrm{E}+08$ & 5.824376508 & 7.4195093 & 9.7404561 & 12.1576362 & 14.51185958 \\
\hline $9.71 \mathrm{E}+08$ & 5.462277885 & 7.0899562 & 9.4867387 & 11.998561 & 14.86722042 \\
\hline $1.07 \mathrm{E}+09$ & 5.192562377 & 6.8549121 & 9.3482219 & 12.0439651 & 15.3664946 \\
\hline $1.18 \mathrm{E}+09$ & 4.926072049 & 6.5774757 & 9.1691442 & 12.048159 & 15.77237619 \\
\hline $1.28 \mathrm{E}+09$ & 4.713351302 & 6.361573 & 8.9713233 & 11.9061021 & $=15.7155416$ \\
\hline $1.38 \mathrm{E}+09$ & 4.418294292 & 5.9771725 & 8.4235444 & 11.1374995 & 14.78237956 \\
\hline $1.48 \mathrm{E}+09$ & 4.228516181 & 5.7752859 & 8.2373169 & 11.1280794 & 15.37938321 \\
\hline $1.59 \mathrm{E}+09$ & 4.02122852 & 5.530889 & 7.9726074 & 10.8045668 & 15.58650828 \\
\hline $1.69 \mathrm{E}+09$ & 3.858119657 & 5.3249608 & 7.7064372 & 10.4970763 & 15.41026112 \\
\hline $1.79 \mathrm{E}+09$ & 3.69317303 & 5.1172128 & 7.4627323 & 10.183229 & 14.91606422 \\
\hline $1.89 \mathrm{E}+09$ & 3.56316033 & 4.9779098 & 7.3965992 & 10.3316013 & 15.5695855 \\
\hline $1.99 \mathrm{E}+09$ & 3.446580186 & 4.8146686 & 7.1216355 & 9.96630979 & 14.7625191 \\
\hline $2.1 E+09$ & 3.304449775 & 4.6193885 & 6.834398 & 9.55261423 & 14.45663608 \\
\hline $2.2 \mathrm{E}+09$ & 3.180862227 & 4.4323685 & 6.5274774 & 9.04851851 & 13.91178466 \\
\hline $2.3 \mathrm{E}+09$ & 3.065897637 & 4.2894214 & 6.3858164 & 9.01552964 & 14.60223708 \\
\hline $2.4 \mathrm{E}+09$ & 2.96883556 & 1729429 & 6.2806179 & 8.9640058 & 14.74161992 \\
\hline $2.51 \mathrm{E}+09$ & 2.902686116 & 4.0688438 & 6.1025256 & 8.69250965 & 13.70902581 \\
\hline $2.61 \mathrm{E}+09$ & 2.844980388 & 3.9775327 & 6.0145138 & 8.65091853 & 13.58400919 \\
\hline $2.71 \mathrm{E}+09$ & 2.771241114 & 3.8708207 & 5.8511577 & 8.41132464 & 13.25788706 \\
\hline $2.81 \mathrm{E}+09$ & 2.693117376 & 3.7600971 & 5.698584 & 8.21946936 & 13.40800873 \\
\hline $2.92 \mathrm{E}+09$ & 2.626814862 & 3.6796066 & 5.6195929 & 8.20393138 & 14.22315301 \\
\hline $3.02 \mathrm{E}+09$ & 2.567638437 & 3.5903671 & 5.4669209 & 7.98233057 & 13.78645993 \\
\hline $3.12 \mathrm{E}+09$ & 2.513293811 & 3.4984365 & 5.3105702 & 7.75326127 & 12.92611767 \\
\hline $3.22 \mathrm{E}+09$ & 2.461169217 & 3.4113591 & 5.1821752 & 7.55203077 & 12.17056295 \\
\hline $3.32 \mathrm{E}+09$ & 2.406197014 & 3.3320466 & 5.0520363 & 7.38266148 & 11.8215947 \\
\hline $3.43 \mathrm{E}+09$ & 2.354423156 & 3.2443342 & 4.8968902 & 7.11725808 & 11.55179205 \\
\hline $3.53 \mathrm{E}+09$ & 2.308435001 & 3.1739405 & 4.764965 & 6.88748264 & 11.44304541 \\
\hline $3.63 \mathrm{E}+09$ & 2.25507568 & 3.0952412 & 4.6309021 & 6.69240367 & 11.28376474 \\
\hline $3.73 \mathrm{E}+09$ & 2.210368503 & 3.0296054 & 4.5382595 & 6.57907421 & 11.06522368 \\
\hline $3.84 \mathrm{E}+09$ & 2.17363814 & $2: 9588157$ & 4.4164429 & 6.37510189 & 10.20512952 \\
\hline $3.94 \mathrm{E}+09$ & 2.142844081 & 2.8983377 & 4.29014 & 6.15297853 & 9.455797245 \\
\hline $4.04 \mathrm{E}+09$ & 2.114560362 & 2.8453093 & 4.2027611 & 6.01115092 & 9.268943575 \\
\hline $4.14 \mathrm{E}+09$ & 2.082447186 & 2.7999286 & 4.136439 & 5.92703718 & 9.492919831 \\
\hline $4.25 \mathrm{E}+09$ & 2.046535426 & 2.7499961 & 4.0531411 & 5.8135022 & 9.552598294 \\
\hline $4.35 \mathrm{E}+09$ & 2.018968866 & 2.7045414 & 3.9707769 & 5.67951608 & 9.267641936 \\
\hline $4.45 \mathrm{E}+09$ & 2.005455118 & 2.6761741 & 3.9258752 & 5.62031273 & 8.905304052 \\
\hline
\end{tabular}




\begin{tabular}{|c|c|c|c|c|c|}
\hline requency & $e^{\prime \prime} / e^{\prime} 20 C$ & $e^{\prime \prime} / e^{1} 40 C$ & $1 / e^{\prime} 60 \mathrm{C}$ & $e^{11 / e^{\prime} 80 C}$ & "1/e' \\
\hline $4.55 \mathrm{E}+09$ & 1.986718244 & 2.6363441 & 3.8540955 & 5.49939406 & 225265 \\
\hline $4.65 \mathrm{E}+09$ & 1.955445367 & 2.5896719 & 3.7667436 & 5.33919609 & 7.937393343 \\
\hline $4.76 \mathrm{E}+09$ & 1.937084428 & 2.5716429 & 3.7713467 & 5.41924655 & 8.484893196 \\
\hline $4.86 \mathrm{E}+09$ & 1.917461793 & 2.5528246 & 3.7536923 & 5.41971299 & 8.971165005 \\
\hline $4.96 \mathrm{E}+09$ & 1.901022524 & 2.5266009 & 3.7143093 & 5.36947763 & 9.039839291 \\
\hline $5.06 \mathrm{E}+09$ & 1.879682984 & 2.4772572 & 3.6186486 & 5.20354926 & 8.436722362 \\
\hline $5.17 \mathrm{E}+09$ & 1.864570195 & 2.4433297 & 3.557532 & 5.1095118 & 7.966512431 \\
\hline $5.27 \mathrm{E}+09$ & 1.849490503 & 2.4085718 & 3.4890351 & 4.97314393 & 7.474699887 \\
\hline $5.37 \mathrm{E}+09$ & 1.828261168 & 2.3774205 & 3.4276836 & 4.86463202 & 7.345018934 \\
\hline $5.47 \mathrm{E}+09$ & 1.813879223 & 2.3595872 & 3.3999473 & 4.82643984 & 7.638118812 \\
\hline $5.57 \mathrm{E}+09$ & 1.799207733 & 2.3465512 & 3.392698 & 4.84245229 & 8.094173495 \\
\hline $5.68 \mathrm{E}+09$ & 1.783784688 & 2.3186741 & 3.3413648 & 4.75917289 & 7.864411768 \\
\hline $5.78 E+09$ & 1.76686364 & 2.2734134 & 32574223 & 4.60284591 & 7.186395329 \\
\hline $5.88 \mathrm{E}+09$ & 1.751255571 & 2.2298073 & 3.1574997 & 4.42016048 & 6.582180945 \\
\hline $5.98 \mathrm{E}+09$ & 1.739249012 & 2.1993153 & 31007997 & 4.32624592 & 6.406272183 \\
\hline $6.09 E+09$ & 1.72457577 & 2.1791989 & 3.0679318 & 4.26068574 & 6.463542456 \\
\hline $6.19 \mathrm{E}+09$ & 1.71140912 & 2.1599966 & 3.0386138 & 4.22316135 & 562141 \\
\hline $6.29 \mathrm{E}+09$ & 1.705193898 & 2.1307097 & 2.9942635 & 4.16327347 & 6.694829752 \\
\hline $6.39 \mathrm{E}+09$ & 1.695311999 & 2.0994094 & 2.932379 & 4.06131032 & 5.213802531 \\
\hline $6.5 \mathrm{E}+09$ & 1.679764978 & 2.0445322 & 2.8153491 & 3.84102507 & 5.434087763 \\
\hline $6.6 \mathrm{E}+09$ & 1.664318566 & 2.0103902 & 2.7474812 & 3.73155105 & 5.120050681 \\
\hline $6.7 \mathrm{E}+09$ & 1.658123098 & 2.0078932 & 2.7414956 & 3.73082353 & 5.256452337 \\
\hline $6.8 \mathrm{E}+09$ & 1.647367985 & 2.0058411 & 2.7407388 & 3.74185894 & 5.500986666 \\
\hline $6.9 \mathrm{E}+09$ & 1.637624253 & 1.9967199 & 2.7318444 & 3.73988174 & 5.676673076 \\
\hline $7.01 \mathrm{E}+09$ & 1.627396984 & 1.976955 & 2.6985715 & 3.7035784 & 5.560774984 \\
\hline $7.11 \mathrm{E}+09$ & 1.620217628 & 1.9547719 & 2.6548903 & 3.63730905 & 5.151803278 \\
\hline $7.21 \mathrm{E}+09$ & 1.603301399 & 1.9171158 & 2.5782654 & 3.49216261 & 4.635537104 \\
\hline $7.31 E+09$ & 1.595754224 & 1.8969568 & 2.542142 & 3.43879404 & 4.561574414 \\
\hline $7.42 \mathrm{E}+09$ & 1.585784727 & 1.8859899 & 2.5197635 & 3.40532759 & 4.7111065 \\
\hline $7.52 \mathrm{E}+09$ & 1.579018703 & 1.8910984 & 2.5382166 & 3.45421637 & 5.035108144 \\
\hline $7.62 \mathrm{E}+09$ & 1.570591272 & 1.8815395 & 2.5246805 & 3.43550342 & 5.058886836 \\
\hline $7.72 \mathrm{E}+09$ & 1.558599679 & 1.8473853 & 2.4663731 & 3.34286377 & 4.73498124 \\
\hline $7.83 E+09$ & 1.54914093 & 1.8138371 & 2.4082612 & 3.24411446 & 4.35378285 \\
\hline $7.93 E+09$ & 1.542584085 & 1.7920444 & $2: 3674406$ & 3.17337805 & 4.111712385 \\
\hline $8.03 E+09$ & 1.522600903 & 1.757866 & 2.3010539 & 3.05388396 & 3.99956542 \\
\hline $8.13 E+09$ & 1.522877785 & 1.775304 & 2.336122 & 3.12111005 & 4.382490852 \\
\hline $8.23 E+09$ & 1.512435349 & 1.7749973 & 2.3329382 & 3.11188637 & 4.517043637 \\
\hline $8.34 \mathrm{E}+09$ & 1.505668918 & 1.7686702 & 2.3300039 & 3.11460842 & 4.451214433 \\
\hline $8.44 E+09$ & 1.492646128 & 1.7332871 & 2.2721502 & 3.02753187 & 4.086353498 \\
\hline $8.54 \mathrm{E}+09$ & 1.480184467 & 1.689007 & 2.1930856 & 2.88859152 & 3.707356706 \\
\hline $8.64 \mathrm{E}+09$ & 1.469589542 & 1.6560205 & 2.1384893 & 2.79828889 & 3.574377456 \\
\hline $8.75 \mathrm{E}+09$ & 1.460705348 & 1.647482 & 2.125469 & 2.77299106 & 3.688750385 \\
\hline $8.85 \mathrm{E}+09$ & 1.450217186 & 1.6477956 & 2.1220278 & 2.76957526 & 3.886629124 \\
\hline $8.95 \mathrm{E}+09$ & 1.447654207 & 1.6514526 & 2.129752 & 2.78899576 & 3.968206422 \\
\hline $9.05 \mathrm{E}+09$ & 1.439384516 & 1.6274207 & 2.0878556 & 2.72044076 & 3.634548072 \\
\hline
\end{tabular}




\begin{tabular}{|c|c|c|c|c|c|}
\hline & $\mathrm{OC}$ & $\overline{40 C}$ & $e^{11 / e^{\prime} 60 C}$ & $80 \mathrm{C}$ & 200 \\
\hline 09 & 34625981 & 1.5848105 & 2.0201351 & 2.61700221 & 2802505 \\
\hline & 004 & 403076 & 60568 & 372 & 552546268 \\
\hline$\overline{E+09}$ & 12368262 & 5256892 & 139303 & 4199998 & 178 \\
\hline-09 & 692 & 52417 & 139389 & 4288877 & 253694581 \\
\hline $9.56 \mathrm{E}+09$ & 1.390623017 & 1.5384139 & 9321763 & 47314958 & 418575924 \\
\hline-09 & 382828095 & 305428 & 9117704 & 3840336 & .2632002 \\
\hline $9.77 \mathrm{E}+09$ & 1.387712875 & 1.5030888 & 1.877932 & 40424273 & 975359937 \\
\hline $9.87 \mathrm{E}+09$ & 1.369331792 & 1.446092 & 1.7831042 & 25686932 & 614389009 \\
\hline $9.97 \mathrm{E}+09$ & 1.36556817 & 4090971 & 1.731191 & 2.17833638 & 565186326 \\
\hline $1.01 \mathrm{E}+10$ & 1.354232951 & 78949| & 1.725689 & 16234807 & 706207906 \\
\hline $1.02 \mathrm{E}+10$ & 1.340920733 & 4287266 & 7442584 & 18205998 & 883930932 \\
\hline+10 & 333404912 & 383683 & 1.7577855 & 20654661 & 2.956125728 \\
\hline+10 & 51304 & 306 & 529 & 19241936 & 2.779608655 \\
\hline$E+10$ & 27419778 & 882173 & 8861479 & 2.11078167 & 2.447107671 \\
\hline+10 & 1739662 & & 405487 & .04903844 & 2.31133617 \\
\hline $1.07 \mathrm{E}+10$ & 1.30871525 & 1.3571553 & 1.6413775 & 2.048067 & .438675588 \\
\hline+10 & 85 & 37 & 1.669 & 802 & .711182225 \\
\hline $1.09 \mathrm{E}+10$ & 1.293991225 & 1.4051551 & 1.6944112 & 2.1139843 & .871141635 \\
\hline+10 & 84749071 & 1 & 78299 & 2.0880184 & .723899088 \\
\hline $1.11 \mathrm{E}+10$ & 82248272 & 77459 & 94202 & 2.01121474 & 394406074 \\
\hline $1.12 \mathrm{E}+10$ & 76800208 & 1.3006868 & .5579329 & 1.93199295 & 2.14864915 \\
\hline $1.13 \mathrm{E}+10$ & 81253145 & 1.2890632 & 1.539385 & 1.90694989 & 14641733 \\
\hline $1.14 \mathrm{E}+10$ & 71007304 & $\overline{97}$ & 113 & 1.90561415 & .35568055 \\
\hline $1.15 \mathrm{E}+10$ & 1.261479551 & & 76 & 9868947 & .58030809 \\
\hline$E+10$ & 1.26054373 & 6992 & 1.5433744 & 1.8870993 & 2.537367342 \\
\hline $8+10$ & 212 & 490392 & 1.4730155 & 1.7925579 & .13422469 \\
\hline$E+10$ & 85817 & 2212 & 1.4017918 & 1.70159529 & .80990870 \\
\hline $1.19 \mathrm{E}+10$ & 4299848 & 78505 & 1.4020624 & 1.70811041 & 1.745275641 \\
\hline $1.2 \mathrm{E}+10$ & 46450409 & 70872 & 19352 & 1.72991158 & .888620968 \\
\hline $1.21 \mathrm{E}+10$ & 42588854 & 559202 & 626501 & 1.77670269 & 2.26866042 \\
\hline $1.22 \mathrm{E}+10$ & 1.229824892 & 1.2678133 & 1.4696283 & 1.77657467 & 2.61107530 \\
\hline $1.23 \mathrm{E}+10$ & 1.208550269 & 1.2182339 & 03202 & 1.6649109 & .337243367 \\
\hline $1.24 \mathrm{E}+10$ & 1.212623223 & 17712 & 1.3421906 & 1.59318538 & .905429783 \\
\hline $5 E+10$ & 11039787 & 32918 & 1.3116974 & 1.55819064 & .60187060 \\
\hline $1.26 \mathrm{E}+10$ & 1.209704769 & 1.1358123 & 18709 & 1.56443882 & 1.54495133 \\
\hline $1.27 \mathrm{E}+10$ & 1.220168434 & 0805 & 636043 & 1.64040211 & 1.76637 \\
\hline $1.28 \mathrm{E}+10$ & 1.216540187 & 2048418 & 20717 & 1.65511336 & 2.21186443 \\
\hline $1.29 \mathrm{E}+10$ & 1.219003195 & 1.1970547 & 1.3592101 & 1.6145468 & 2.732461158 \\
\hline $1.3 \mathrm{E}+10$ & 1.220893115 & 70605 & 1.3230417 & 1.56465219 & 2.76292455 \\
\hline $1.31 \mathrm{E}+10$ & 1.220323652 & 1.1232789 & 1.2700859 & 1.49124617 & 2.10346920 \\
\hline $1.32 \mathrm{E}+10$ & 1.217533635 & 1.1218101 & 1.275942 & 1.49336013 & 1.68414518 \\
\hline $1.33 \mathrm{E}+10$ & 1.204012607 & 1556102 & 1.3199694 & 1.54783738 & 1.595615368 \\
\hline $1.35 \mathrm{E}+10$ & 1.205275772 & 1.1957821 & 1.3674558 & 1.61319534 & 1.75995335 \\
\hline $1.36 \mathrm{E}+10$ & 1.207828115 & 1.2098011 & 1.3789382 & 1.63651415 & 2.22053885 \\
\hline $1.37 \mathrm{E}+10$ & 1.206956684 & 1.1795728 & 1.3439729 & 1.59763915 & 2.81308989 \\
\hline
\end{tabular}




\begin{tabular}{|c|c|c|c|c|c|}
\hline Frequency & $e^{\prime \prime} / e^{\prime} 20 \mathrm{C}$ & $e^{11 / e^{\prime} 40 C}$ & $e^{\prime \prime} / e^{\prime} 60 \mathrm{C}$ & $e^{\prime \prime} / e^{\prime} 80 \mathrm{C}$ & $e^{\prime \prime /} / e^{\prime} 100 \mathrm{C}$ \\
\hline $1.38 \mathrm{E}+10$ & 1.21310945 & 1.1141788 & 1.260808 & 1.49328556 & 2.663708306 \\
\hline $1.39 \mathrm{E}+10$ & 1.21748228 & 1.0931841 & 1.2375065 & 1.4512258 & 2.039576775 \\
\hline $1.4 \mathrm{E}+10$ & 1.20355772 & 1.1009952 & 1.2486177 & 1.4542667 & 1.618155102 \\
\hline $1.41 \mathrm{E}+10$ & 1.196736872 & 1.1523679 & 1.3183074 & 1.53922299 & 1.585265756 \\
\hline $1.42 \mathrm{E}+10$ & 1.180937834 & 1.2028304 & 1.3807885 & 1.61922491 & 1.776307072 \\
\hline $1.43 \mathrm{E}+10$ & 1.178721109 & 1.2027381 & 1.3731293 & 1.61612976 & 2.173311042 \\
\hline $1.44 \mathrm{E}+10$ & 1.19241642 & 1.1419154 & 1.2914642 & 1.52294807 & 2.557834532 \\
\hline $1.45 \mathrm{E}+10$ & 1.21525059 & 1.0583904 & 1.1809191 & 1.38020642 & 2.23483809 \\
\hline $1.46 \mathrm{E}+10$ & 1.231090084 & 0.9973222 & 1.0968096 & 1.26266293 & 1.570013876 \\
\hline $1.47 \mathrm{E}+10$ & 1.20750626 & 0.9977113 & 1.1049217 & 1.26546294 & 1.325409585 \\
\hline $1.48 \mathrm{E}+10$ & 1.177605725 & 1.0815942 & 1.2110302 & 1.38787857 & 1.414890704 \\
\hline $1.49 \mathrm{E}+10$ & 1.139325714 & 1.1353311 & 1.2697918 & 1.4556769 & 1.629349926 \\
\hline $1.5 \mathrm{E}+10$ & 1.166157062 & 1.1787757 & 1.313551 & 1.5274612 & 2.108235221 \\
\hline $1.51 \mathrm{E}+10$ & 1.18691123 & 1.0757141 & 1.1964179 & 1.40901221 & $2: 377570195$ \\
\hline $1.52 \mathrm{E}+10$ & 1.253676058 & 0.9374478 & 1.0205238 & 1.19138983 & 73901 \\
\hline $1.53 \mathrm{E}+10$ & 1.227864236 & 0.7939009 & 0.8554818 & 0.98556268 & 1.19572189 \\
\hline $1.54 \mathrm{E}+10$ & 1.249679241 & 0.8602598 & 0.9389806 & 1.07264967 & 05886 \\
\hline $1.55 \mathrm{E}+10$ & 1.173677416 & 0.9566428 & 1.0478575 & 1.18662724 & 1.188978301 \\
\hline $1.56 \mathrm{E}+10$ & 1.125199037 & 1.1535283 & 1.2541401 & 1.41913618 & 1.549220316 \\
\hline $1.57 \mathrm{E}+10$ & 1.091200471 & 1.2607165 & 1.3824376 & 1.58829641 & 2.071118457 \\
\hline $1.58 \mathrm{E}+10$ & 1.150443435 & 1.1957315 & 1.3180274 & 1.54355032 & 2.418027828 \\
\hline $1.59 \mathrm{E}+10$ & 1.230448208 & 1.0052819 & 1.0892241 & 1.28116487 & 062326 \\
\hline $1.6 \mathrm{E}+10$ & 1.277344002 & 0.8533897 & 0.9154212 & 1.05430469 & 1.302819527 \\
\hline $1.61 \mathrm{E}+10$ & 1.214541623 & 0.7878531 & 0.8370379 & 0.94715545 & 0.990086779 \\
\hline $1.62 \mathrm{E}+10$ & 1.159212792 & 0.8934286 & 0.9372718 & 1.04764603 & 1.075949071 \\
\hline $1.63 \mathrm{E}+10$ & 1.182996232 & 1.1343686 & 1.2188566 & 1.38824131 & 1.568204902 \\
\hline $1.64 \mathrm{E}+10$ & 1.173896203 & 1.3077443 & 1.4413422 & 1.67609049 & 2.187251567 \\
\hline $1.65 \mathrm{E}+10$ & 1.209403878 & 1.2958419 & 1.417966 & 1.66288189 & 2.422794959 \\
\hline $1.66 \mathrm{E}+10$ & 1.214033832 & 1.0591739 & 1.1514552 & 1.35854428 & 1.956947242 \\
\hline $1.67 \mathrm{E}+10$ & 1.236062489 & 0.907574 & 0.9743594 & 1.13423914 & 1.389409742 \\
\hline $1.68 \mathrm{E}+10$ & 1.229551532 & 0.8500013 & 0.8894616 & 1.00510394 & 1.084810281 \\
\hline $1.69 \mathrm{E}+10$ & 1.289438698 & 0.9945186 & 1.0459536 & 1.18974492 & 1.313983911 \\
\hline $1.7 E+10$ & 1.246094073 & 1.1516021 & 1.2528405 & 1.44475549 & 1.729474156 \\
\hline $1.71 \mathrm{E}+10$ & 1.214358132 & 1.2251214 & 1.3219073 & 1.51352438 & 1.930545223 \\
\hline $1.72 \mathrm{E}+10$ & 1.199615366 & 1.1359133 & 1.2101963 & 1.39618505 & 1.86128659 \\
\hline $1.73 \mathrm{E}+10$ & 1.234189755 & 1.0378713 & 1.1231584 & 1.32306841 & 1.753769117 \\
\hline $1.74 \mathrm{E}+10$ & 1.208611692 & 0.8931437 & 0.9528566 & 1.10761743 & 1.331284787 \\
\hline $1.75 \mathrm{E}+10$ & 1.244262226 & 0.9001713 & 0.9377402 & 1.07041882 & 1.241256714 \\
\hline $1.76 \mathrm{E}+10$ & 1.251774943 & 1.0013849 & 1.0538555 & 1.20638528 & 1.472280137 \\
\hline $1.77 \mathrm{E}+10$ & 1.234983353 & 1.1253503 & 1.2105363 & 1.39193926 & 1.767948883 \\
\hline $1.79 \mathrm{E}+10$ & 1.217115453 & 1.1423299 & 1.2185591 & 1.3955192 & 1.823870976 \\
\hline $1.8 \mathrm{E}+10$ & 1.24157426 & 1.1300838 & 1.197227 & 1.38290136 & 1.878281455 \\
\hline $1.81 \mathrm{E}+10$ & 1.217182428 & 0.9744927 & 1.0380285 & 1.21011727 & 1.554335158 \\
\hline $1.82 \mathrm{E}+10$ & 1.213446617 & 0.9042682 & 0.9564654 & 1.0999991 & 1.307390579 \\
\hline $1.83 \mathrm{E}+10$ & 1.230211374 & 0.9004852 & 0.9333192 & 1.05846068 & 1.273647236 \\
\hline
\end{tabular}




\begin{tabular}{|r|r|r|r|r|r|}
\hline Frequency & $e^{\prime \prime} / \mathrm{e}^{\prime} 20 \mathrm{C}$ & $\mathrm{e}^{\prime \prime} / \mathrm{e}^{\prime} 40 \mathrm{C}$ & $\mathrm{e}^{\prime \prime} / \mathrm{e}^{\prime} 60 \mathrm{C}$ & $\mathrm{e}^{\prime \prime} / \mathrm{e}^{\prime} 80 \mathrm{C}$ & $\mathrm{e}^{\prime \prime} / \mathrm{e}^{\prime} 100 \mathrm{C}$ \\
\hline \hline $1.84 \mathrm{E}+10$ & 1.244143776 & 0.9627099 & 1.0010156 & 1.14176732 & 1.444083514 \\
\hline $1.85 \mathrm{E}+10$ & 1.226157113 & 1.0075887 & 1.0643464 & 1.21806734 & 1.553991415 \\
\hline $1.86 \mathrm{E}+10$ & 1.225549492 & 1.0137151 & 1.0541211 & 1.19269516 & 1.51673645 \\
\hline $1.87 \mathrm{E}+10$ & 1.226940499 & 0.9198723 & 0.9505216 & 1.08397348 & 1.388756977 \\
\hline $1.88 \mathrm{E}+10$ & 1.235428077 & 0.8985222 & 0.9321958 & 1.07173988 & 1.307118156 \\
\hline $1.89 \mathrm{E}+10$ & 1.217828961 & 0.896858 & 0.9294753 & 1.06204556 & 1.259096261 \\
\hline $1.9 \mathrm{E}+10$ & 1.232150799 & 0.9677901 & 0.9993194 & 1.13171048 & 1.39722398 \\
\hline $1.91 \mathrm{E}+10$ & 1.243106678 & 1.0712299 & 1.1121966 & 1.26010508 & 1.608602424 \\
\hline $1.92 \mathrm{E}+10$ & 1.238158881 & 1.1012501 & 1.140332 & 1.29013193 & 1.631318483 \\
\hline $1.93 \mathrm{E}+10$ & 1.232329407 & 1.0626276 & 1.0949739 & 1.23444089 & 1.552918389 \\
\hline $1.94 \mathrm{E}+10$ & 1.249103794 & 1.0124322 & 1.0382316 & 1.17510256 & 1.440983379 \\
\hline $1.95 \mathrm{E}+10$ & 1.24435984 & 0.9450123 & 0.9682388 & 1.09730818 & 1.306660001 \\
\hline $1.96 \mathrm{E}+10$ & 1.230879281 & 0.9506045 & 0.9775173 & 1.10458468 & 1.330226351 \\
\hline $1.97 \mathrm{E}+10$ & 1.231661866 & 0.9700563 & 1.0014939 & 1.13216711 & 1.402229721 \\
\hline $1.98 \mathrm{E}+10$ & 1.243730822 & 1.0401682 & 1.0805846 & 1.22978134 & 1.561222325 \\
\hline $1.99 \mathrm{E}+10$ & 1.244011176 & 1.0596518 & 1.0958546 & 1.24963497 & 1.587836417 \\
\hline $2 \mathrm{E}+10$ & 1.239350849 & 1.0612082 & 1.0848596 & 1.22754013 & 1.527117325 \\
\hline
\end{tabular}


CORRELATION GRAPHS FOR THE

DIELECTRIC CONSTANT AND DIELECTRIC LOSS

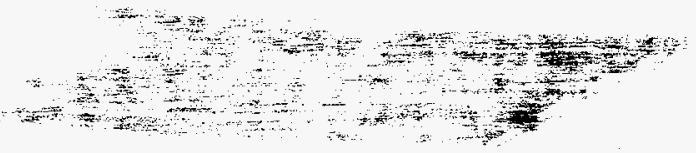

$=0$ ats 
H:IdaniexpdatalHan 4 me $x / s$

Rank 1 Eqn $436114762 z^{\wedge}(-1)=a+b x^{\wedge}(0.5) \ln x+c(\ln x)^{\wedge} 2+d x^{\wedge}(0.5)+e \ln x+f \ln x+g y^{\wedge}(1.5)+h y^{\wedge} 2+i y^{\wedge} 2 \ln y$ $m^{2}=0.98334083$ DF Adj $m=0.98302651$ FitStdErr $=5.3188438$ Fstat $=3526.8841$ $a=255.19972 b=6.5224812 e-06 \quad c=0.27752451 d=-0.00018758534 \quad \theta=14.42818$ $f=1526.7139 \mathrm{~g}=0.0013395486 \mathrm{~h}=0.00041664411 \mathrm{i}=6.2420861 \mathrm{e} \cdot 05$

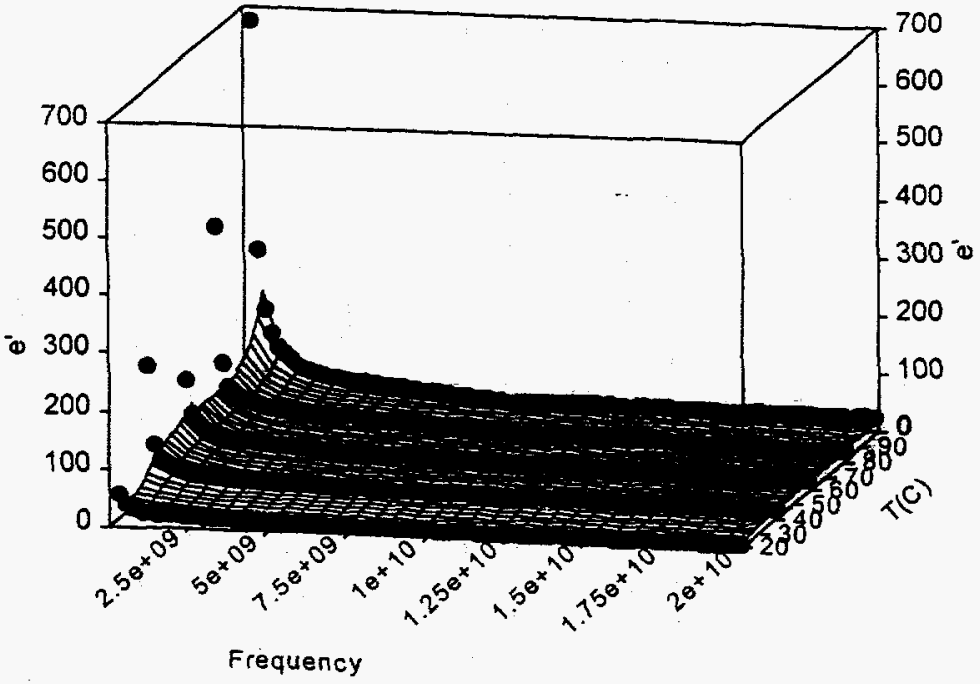

Figure C.1.a. Correlation data for the dielectric constant. Simulant: Hanford 4 Molar.

HildanlexpdataH Han4me.xs

Rank 1 Eqn $436114762 z^{\wedge}(-1)=a+b x^{\wedge}(0.5) \ln x+c(\ln x)^{\wedge} 2+d x^{\wedge}(0.5)+e \ln x+f / \ln x+g y^{\wedge}(1.5)+h y^{\wedge} 2+i y^{\prime} 2 \ln y$ $\mu^{\wedge} 2=0.98334083$ DF Adj $\mu^{\wedge} 2=0.98302651$ FitStdE $r=5.3188438$ Fstat $=3526.8641$ $z=255.19972 \quad b=6.5224812 e-06 \quad c=027752451 d=0.00018758534 e=1442818$

$=1526.7139 \mathrm{~g}=0.0013395486 \mathrm{~h}=0.00041664411 \mathrm{i}=-6.2420861 \mathrm{e}-05$

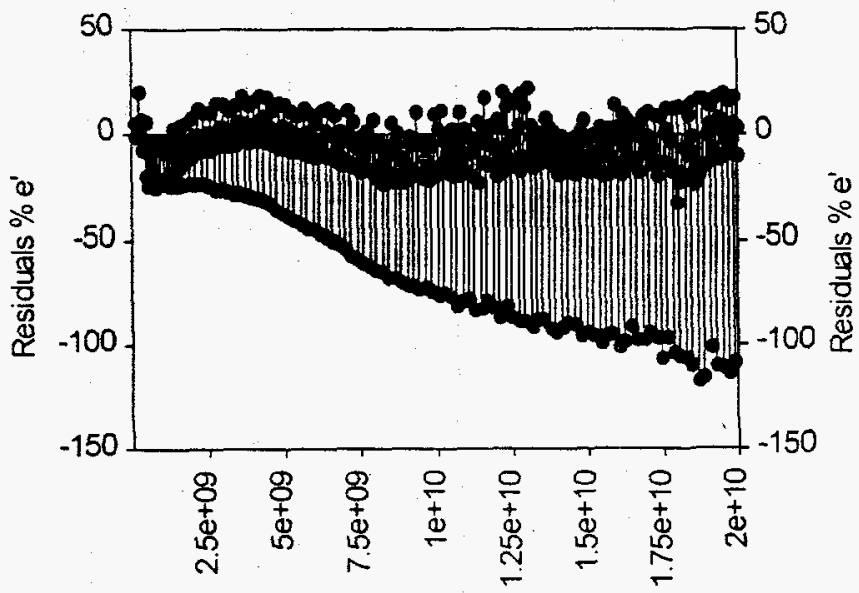

Frequency

Figure C.1.b. Residual percentage (\%) for dielectric constant. Simulant: Hanford 4 Molar. 
H: daniexpdatalHan 4 mee $x$ ls

Rank $53 \operatorname{Eqn} 303 \quad z=a+b / x+c y+d / x^{\wedge} 2+e y^{\wedge} 2+f y / x$

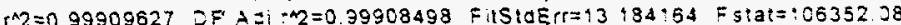
$a=16259991 \quad b=1.5297721 e+11 c=014703638$

$d=.1 .3098 \cdot 29 e+16 \theta=-0.00068643355:=8.845: 048 \theta+09$

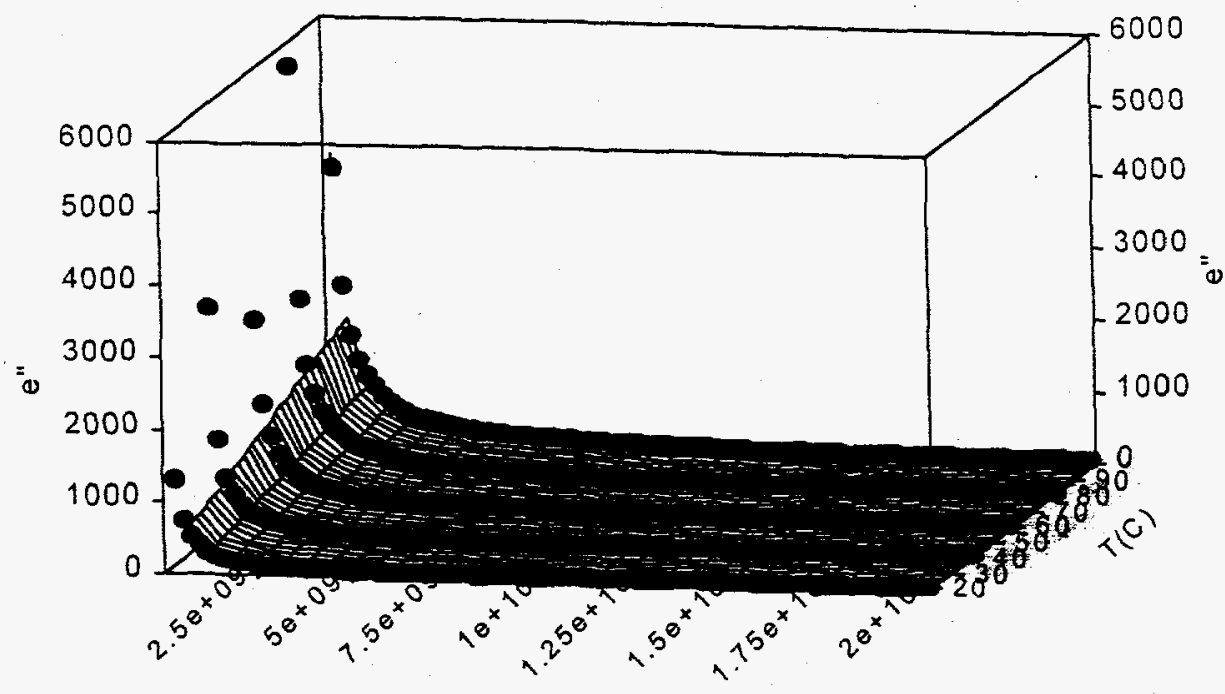

Frequency

Figure C.1.c. Correlation data for the dielectric loss. Simulant: Hanford 4 Molar.

H:ldanlexpdatalHan 4 mee.xls

Rank 53 Ean $303 z=a+b / x+c y+d / x^{\wedge} 2+e y^{\wedge} 2+f y / x$

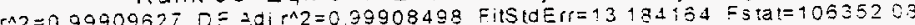
$a=: 3059991 \mathrm{~b}=1.5297721 \mathrm{e}+11 \mathrm{c}=014723638$

$d=-1.0053129 e+16 e=-0.00058643355 i=33451043 e+09$

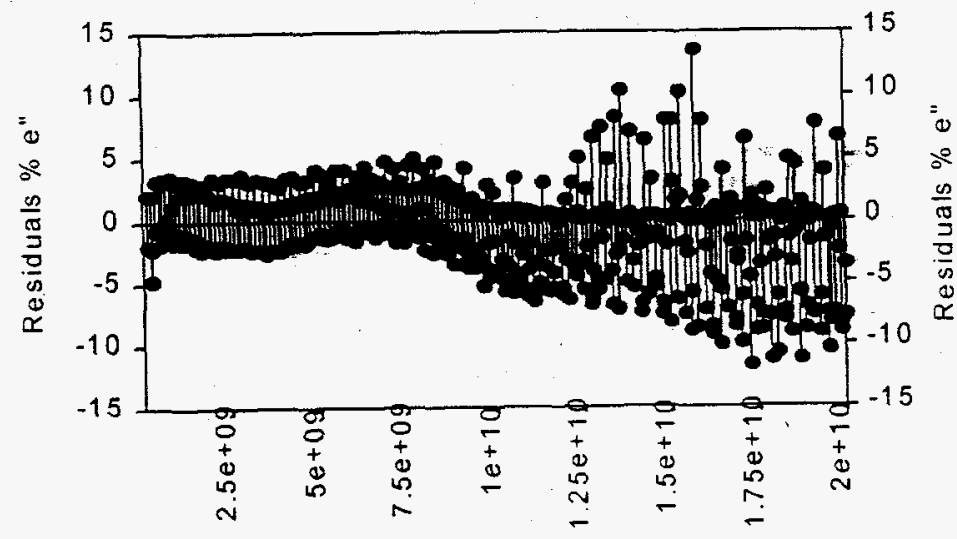

Frequency

Figure C.1.d. Residual percentage (\%) for the dielectric loss. Simulant: Hanford 4 Molar. 
$H:$ danlexpdatalHan6me $x / s$

Rank 1 Eqn $436114762 z^{\wedge}(-1)=a+b x^{\wedge}(0.5) \ln x+c(\ln x)^{\wedge} 2+d x^{\wedge}(0.5)+e \ln x+f \ln x+g y^{\wedge}(1.5)+h y^{\wedge} 2+i y^{\wedge} 2 \ln y$

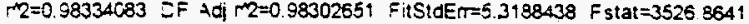
$a=255.19972 b=6.5224812 \theta-06 \quad c=0.27752451 d=0.00018758534 \mathrm{e}=14.42818$

$f=-1526.7139,=-0.0013395486 n=0.00041664411 i=-6.2420861 e-05$

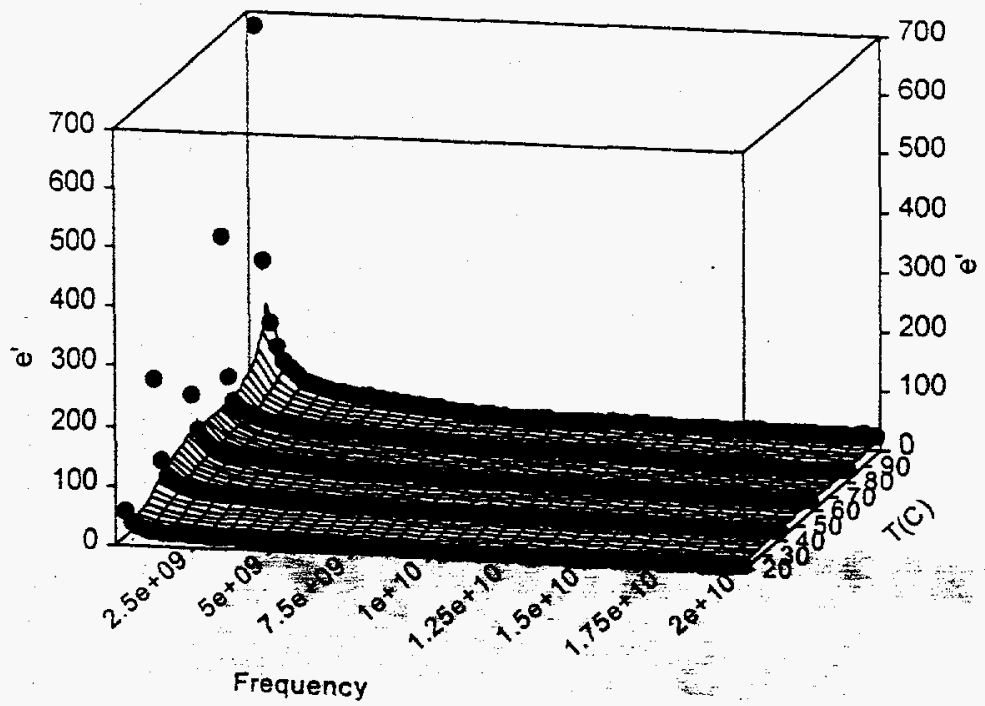

Figure C.2.a. Correlation data for the dielectric constant. Simulant: Hanford 6 Molar.

H: IdanlexpdataiHan6me.xls

Rank 1 Eqn $436114762 z^{\wedge}(-1)=a+b x^{\wedge}(0.5) \ln x+c(\ln x)^{\wedge} 2+d x^{\wedge}(0.5)+e \ln x+f / \ln x+g y^{\wedge}(1.5)+h y^{\wedge} 2+i y^{\wedge} 2 \ln y$ $r^{\wedge} 2=0.98334083$ DF Adj $r^{\wedge} 2=0.98302651$ FitStdErr $=5.3188438$ Fstat $=3526.8641$ $g=255.19972 b=65224812 \mathrm{e}-06 \mathrm{c}=0.27752451 d=-0.00018758534 \mathrm{e}=14.42818$

$f=-1526.7139 \mathrm{~g}=-0.0013395486 \mathrm{~h}=0.0004166441 \mathrm{i}=-6.2420861 \mathrm{e}-05$

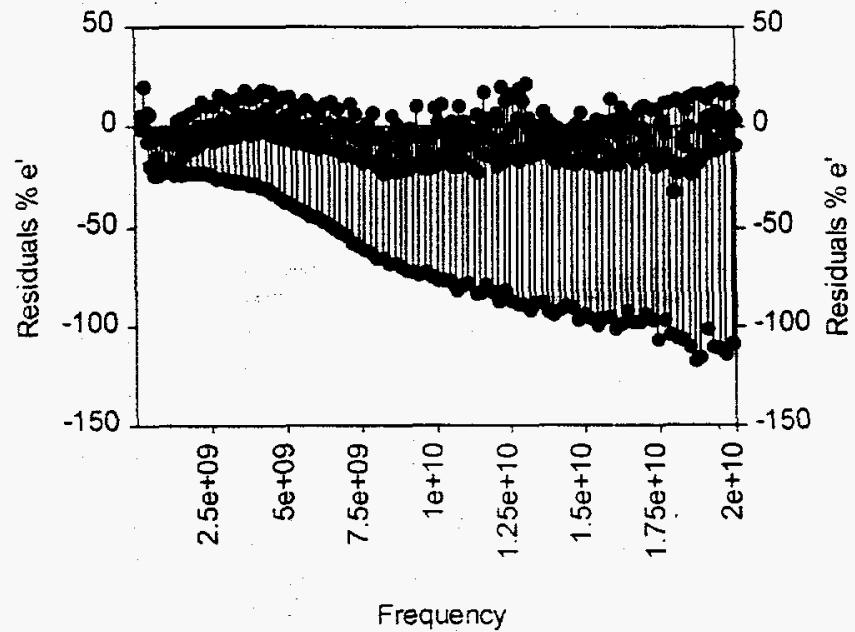

Figure C.2.b. Residual percentage (\%) for the dielectric constant. Simulant: Hanford 6 Molar. 


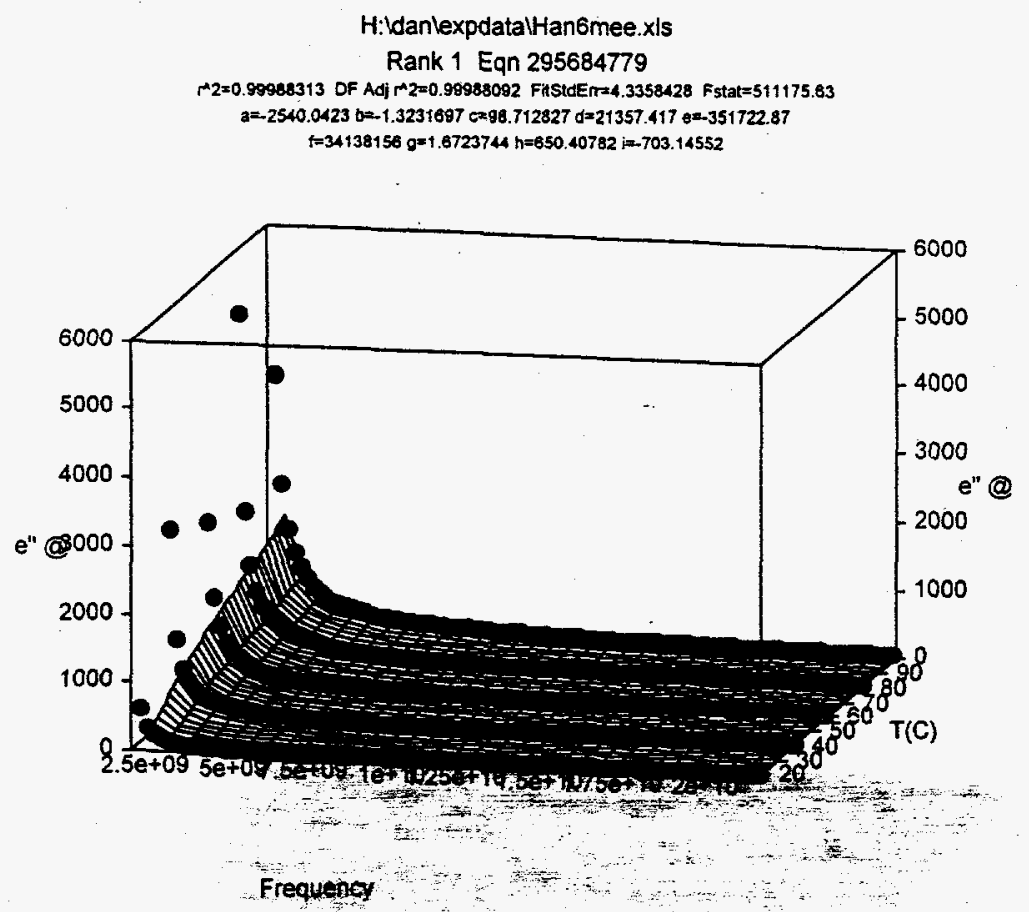

Figure C.2.c. Correlation data for the dielectric loss. Simulant: Hanford 6 Molar.

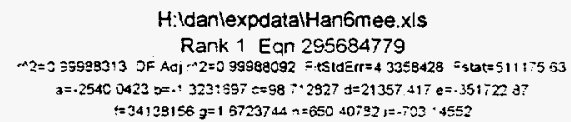

H:ldanlexpdatalHan6mee.xls

Rank 1 Eqn 295684779

: $323: 69 ; \quad<=58,2927 d=21357,4$

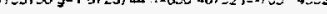

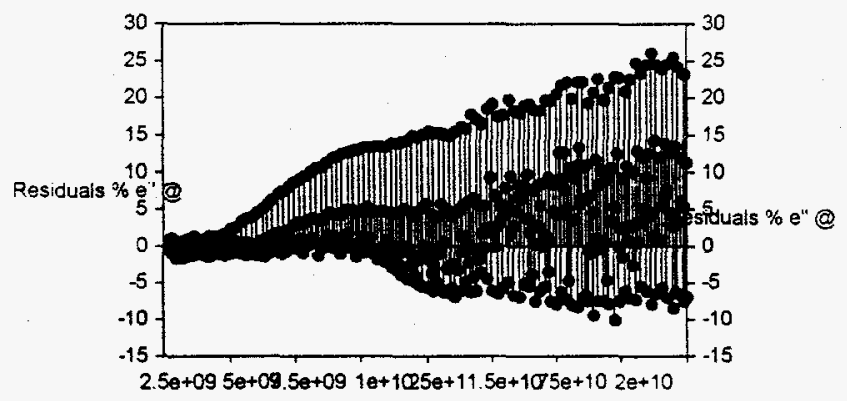

Frequency

Figure C.2.d. Residual percentage (\%) for the dielectric loss. Simulant: Hanford 6 Molar. 
H:IdanlexpdatalHan4me.xis

Rank 1 Eqn $434619096 z^{\wedge}(-1)=a+b e^{\wedge}\left(x^{\prime} w x\right)+c(\ln x)^{\wedge} 2+d x^{\wedge}(0.5)+e \ln x+f y \ln y^{\prime}+g y^{\wedge}(1.5)+h y^{\wedge} 2+i y^{\wedge} 2 \ln y$

$M_{2}=0.96247294$ DF Ad ${ }^{2} 2=0.96212295$ FitstdEr $=10.982596$ Fstat $=3096.9281$

$a=0.98518516 b=0.053542187 c=0.002910648 d=1.3311531 \theta-07 \quad \theta=0.10934245$

$f=0.0075879732 g=0.0078828198 h=0.0010015116 i=0.00012192781$

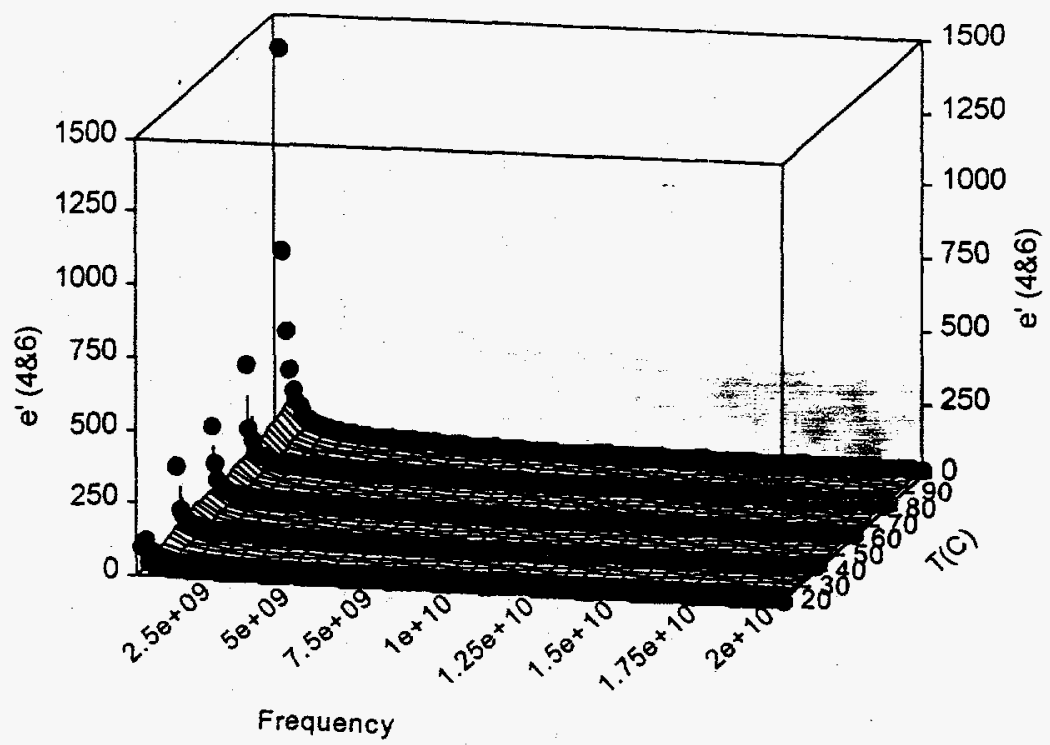

Figure C.3.a. Correlation data for the dielectric constant. Simulants: Hanford 4 and 6 Molar.

H: danlexpdatalHan4me.xls

Rank 1 Eqn $434619096 z^{\wedge}(-1)=a+b e^{\wedge}\left(x^{\prime} w x\right)+c(\ln x)^{\wedge} 2+d x^{\wedge}(0.5)+e \ln x+f y \ln y+g y^{\wedge}(1.5)+h y^{\prime} 2+i y^{\wedge} 2 \ln y$

$m=0.96247294$ DF Adj $\mu^{n}=096212295$ FitStaE $r=10982596$ F $5: a t=3095.928$

$a=0.98518516 b=0053542187 \quad s=0.002910648 d=1.3311531 e-07 \quad e=0 \quad 10934245$

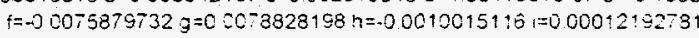

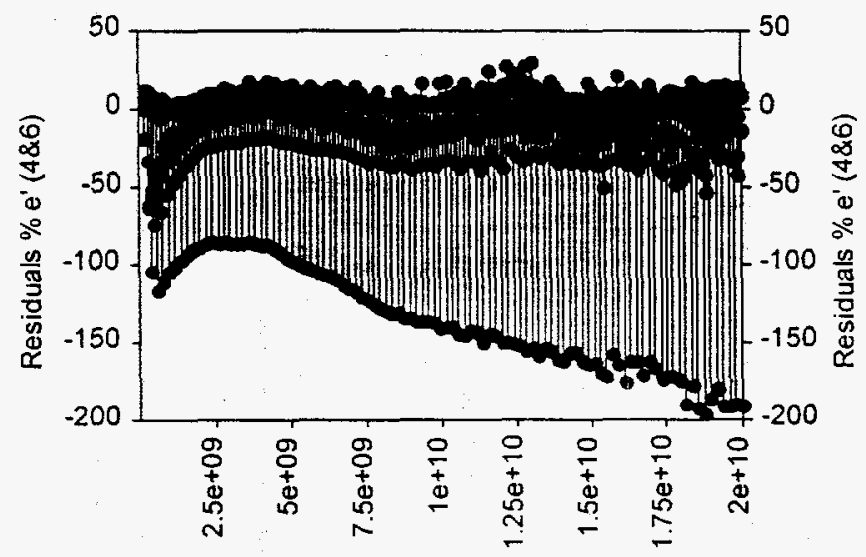

Frequency

Figure C.3.b. Residual percentage (\%) for the dielectric constant. Simulants: Hanford 4 and 6 Molar. 
$H$ : daniexpdatalHan 4 mee $x / s$

Rank 1 Ean 151232687 lnz $z=a+b \ln x+c / y^{\wedge}(0.5)$

$r 2=0.99198704$ of $A=j \mathrm{r} N=0.99196229$ FitStdErr $=38.837175$ F stat $=60155.781$ $a=27.585923 \quad b=-0.94664965$

$c=-11.262194$

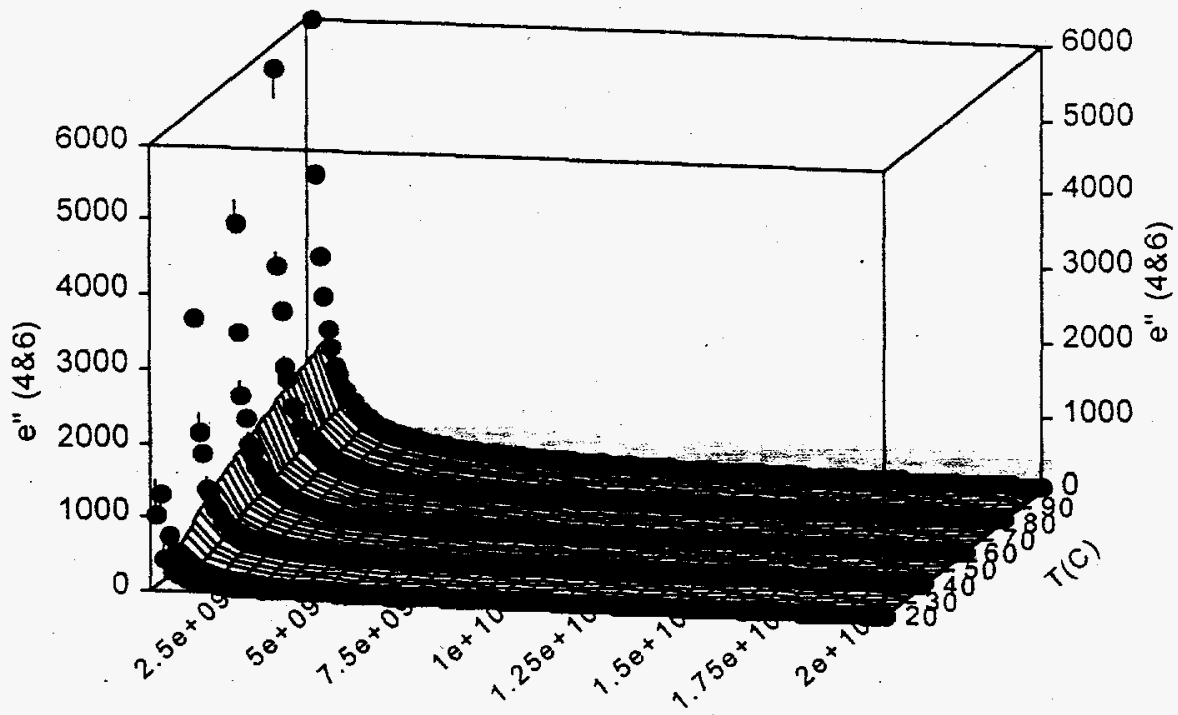

Frequency

Figure C.3.c. Correlation data for the dielectric loss. Simulants: Hanford 4 and 6 Molar.

$H:$ ldanlexpdatalHan 4 mee.xis

Rank 1 Eqn $151232687 \ln z=a+b \ln x+c / y^{\wedge}(0.5)$

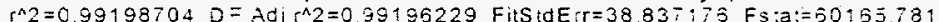
$a=27685923 b=-0.94664955$ $c=-11.252194$

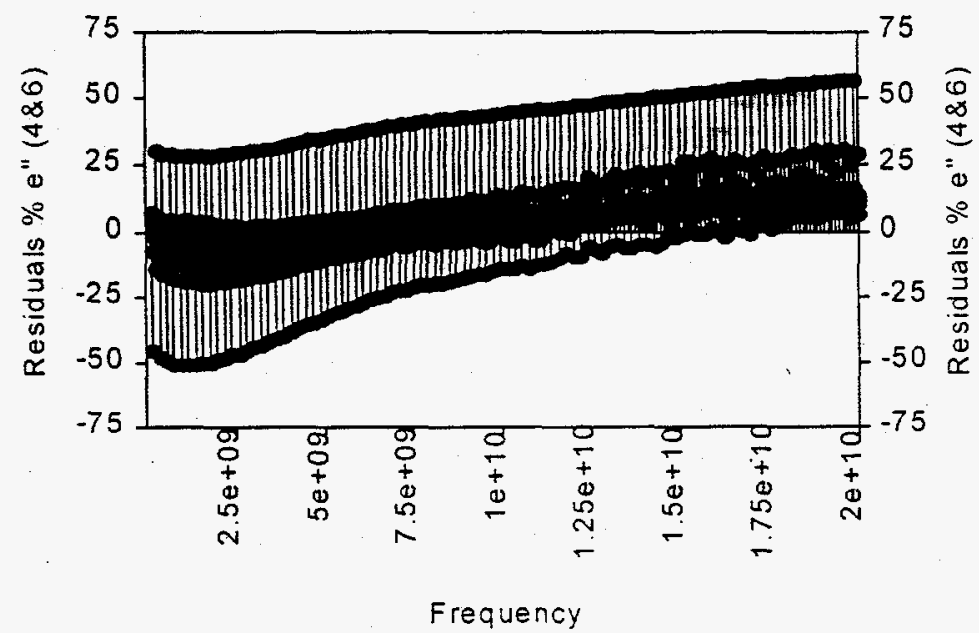

Figure C.3.d. Residual percentage (\%) for the dielectric loss. Simulants: Hanford 4 and 6 Molar. 


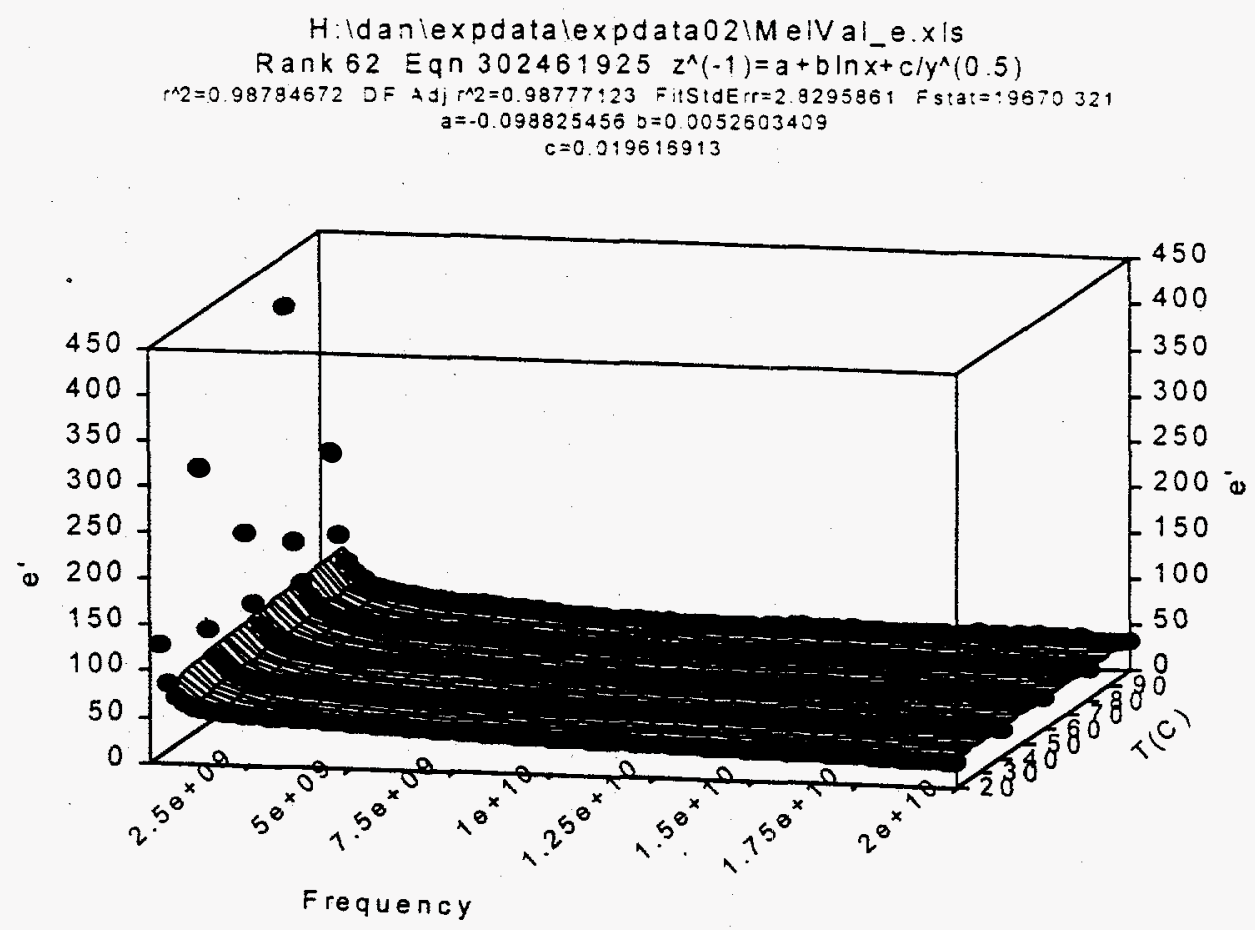

Figure C.4.a. Correlation data for the dielectric constant. Simulant: Melton Valley.

$H:$ idanlexpdatalexpdata 02 MeIVal_e.xls

Rank 62 Eqn $\left.302461925 z^{\wedge}(-1)=a+b \ln x+c / y^{\wedge}(0) 5\right)$

$r^{\wedge} 2=0.98784672$ OF Adi $r^{\wedge} 2=0.98777123$ FitStdErr=2.829586i Fstat=19570 32 $a=-00988254 \equiv 5 b=0.0052503403$ $c=00: 9515913$

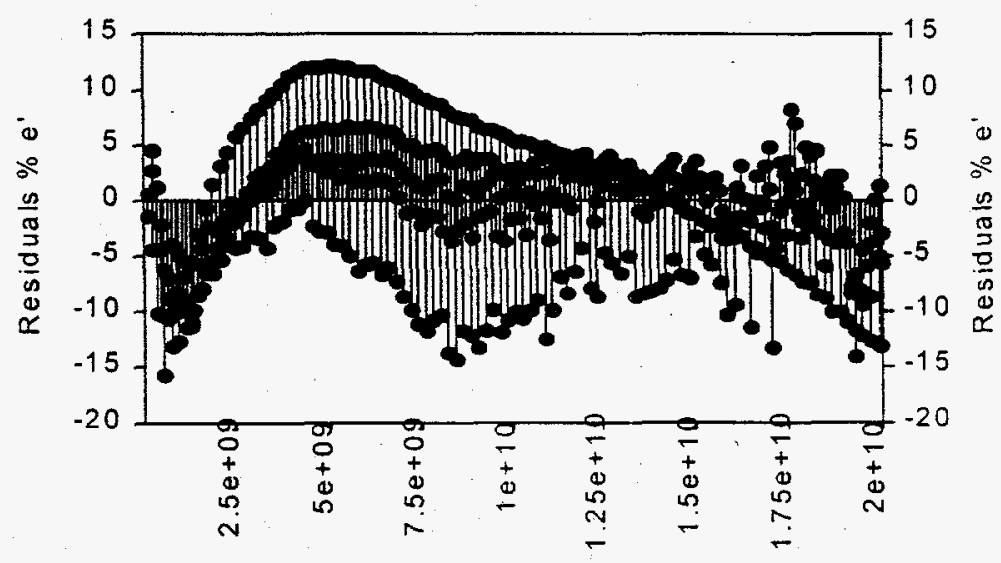

Frequency

Figure C.4.b. Residual percentage (\%) for the dielectric constant. Simulant: Melton Valley. 
$H:$ Idanlexpdatalexpdata021MelVal_ee. $x$ Is

Rank 3 Eqn $303 z=a+b / x+c y+d / x^{\wedge} 2+e y^{\wedge} 2+f y / x$

$r^{\wedge} 2=0.99974713$ DF Adj $r^{\wedge} 2=0.99974397$ FitStdE rr $=4.7107903$ F stat $=380342.72$

$a=25.50155 b=1.4974987 e+11 c=-0.36620891$

$\delta=-2.7+22719 \theta+18 \theta=0.0020865+38 f=5.5069923 e+09$

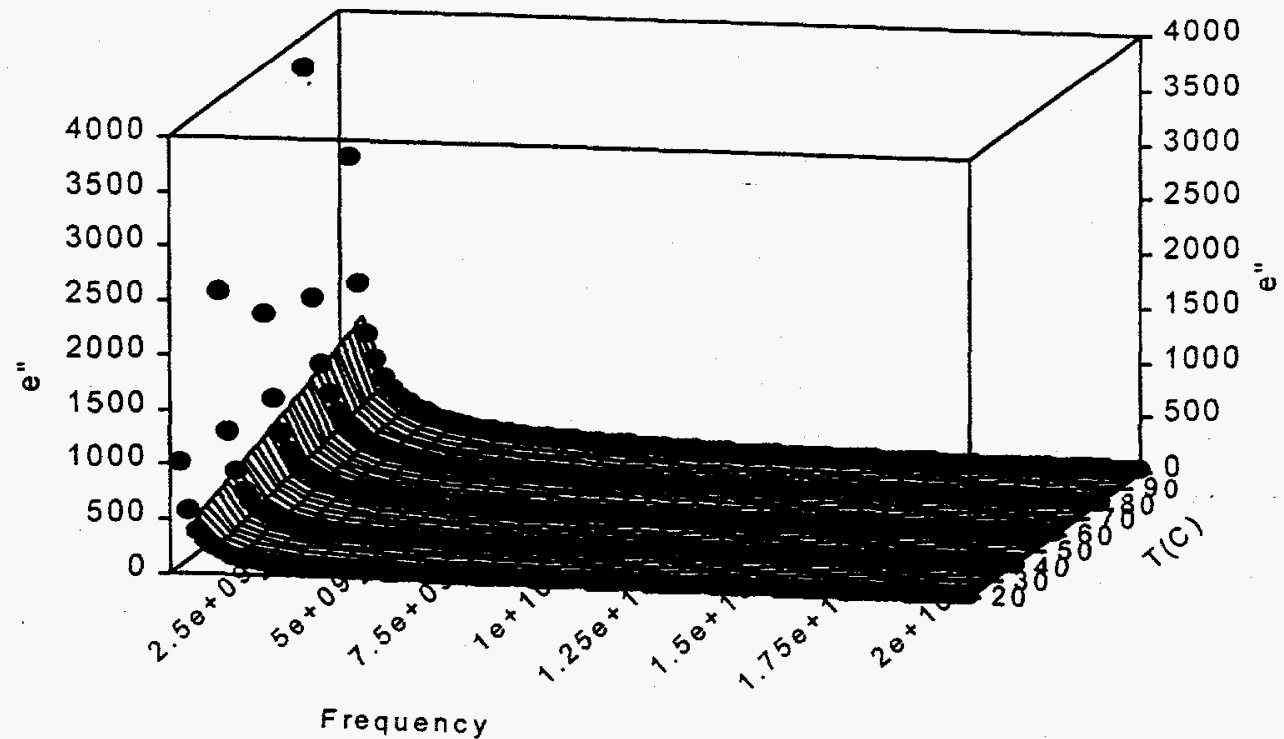

Figure C.4.c. Correlation data for the dielectric loss. Simulant: Melton Valley.
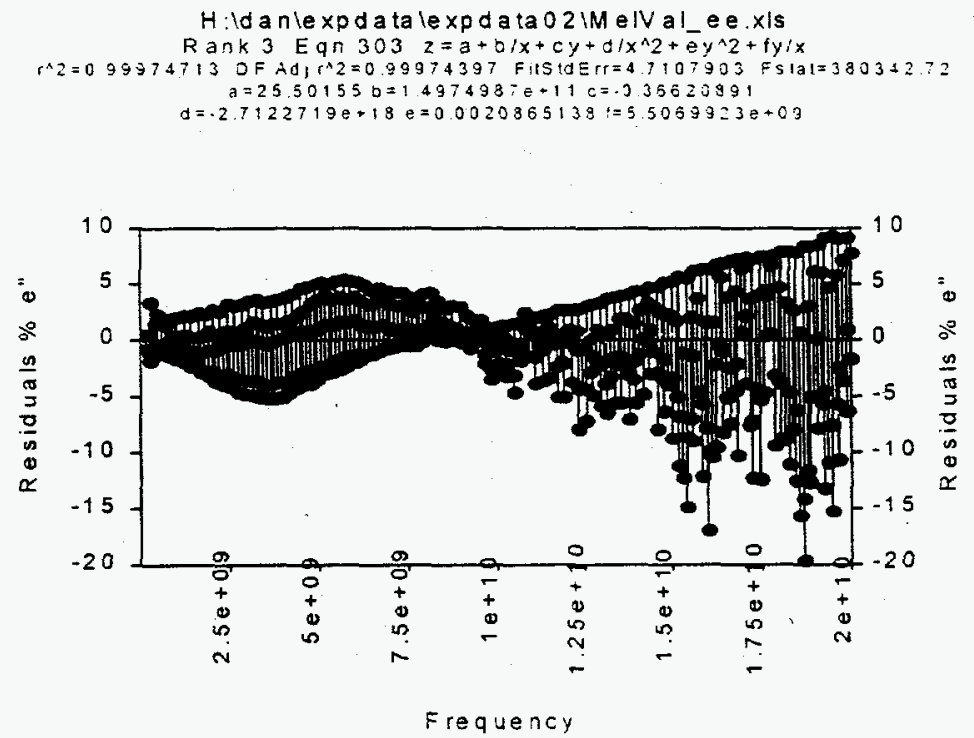

Figure C.4.d. Residual percentage (\%) for the dielectric loss. Simulant: Melton Valley. 
H:Idanlexpdatalexpdata021Saic_e.xls

Rank 1. Eqn $169 \quad z=a+b / x+c / x^{\wedge} 2+d y+e y^{\wedge} \overline{2}+f y^{\wedge} 3+g y^{\wedge} 4$

$\mu^{\wedge}=0.9989215$ DF Adj $\mathrm{r}^{\wedge}=0.99890578$ FitStdE $r \mathrm{r}=0.34293834$ Fstat $=73795.438$ $a=7.0776274 \quad b=5.80419378+10 \quad c=-2.0212419 \theta+19 d=1.281346$ $\theta=-0.038922062 f=0.00058758662 g=-3.277884 e-06$

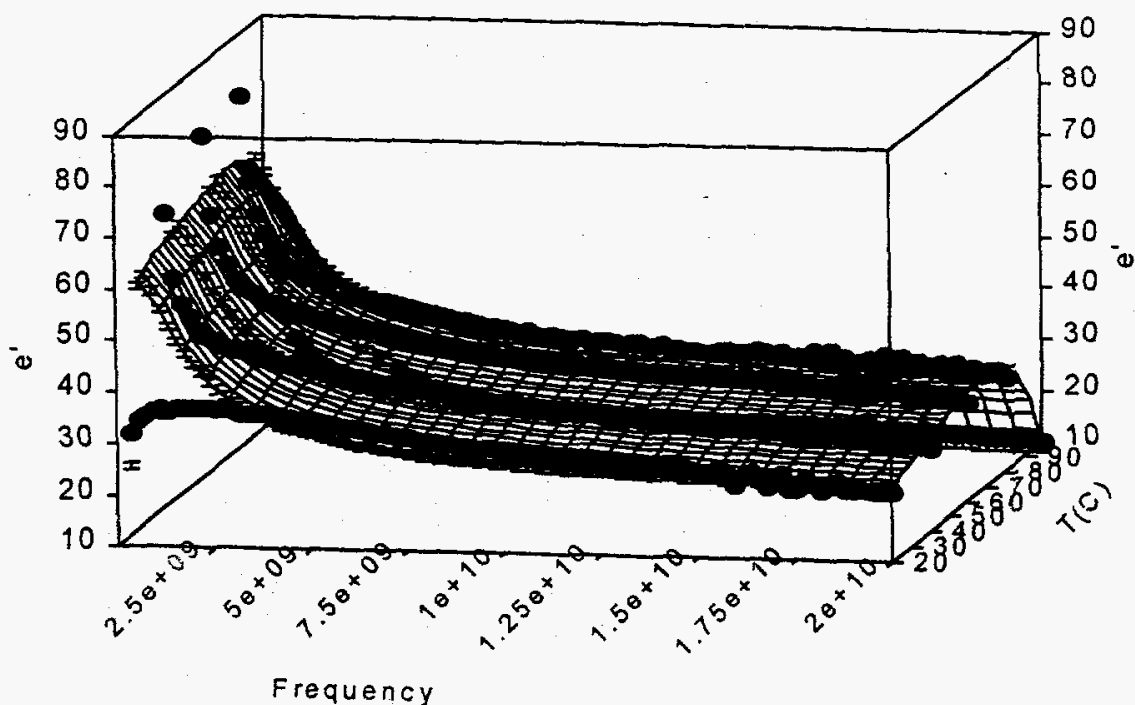

Figure C.5.a. Correlation data for the dielectric constant. Simulant: SAIC.

H ida nlexpdatalexpdata 02 is a ic e exls

Rank 1 E $0169 z=a+b / x+c / x^{\wedge} 2+d y+e y^{\wedge} 2+f y^{\wedge} 3+g y^{\wedge} 4$ $r=000802, j=F$

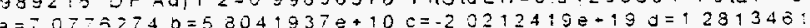
$e=.0038922062:=0.00053768062 \mathrm{~g}=.32773840 .05$

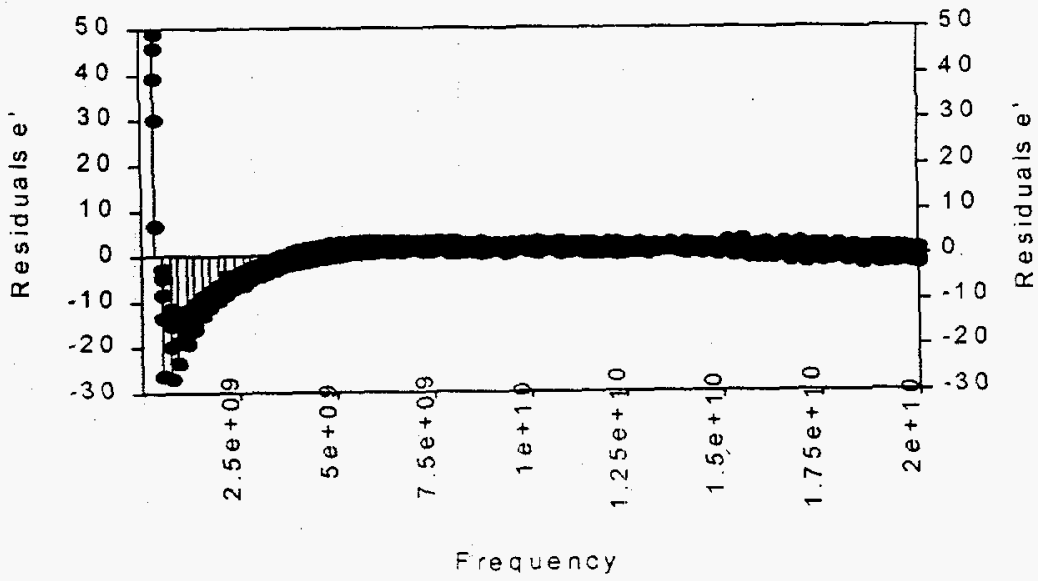

Figure C.5.b. Residual percentage (\%) for the dielectric constant. Simulant: SAIC. 
H: \danlexpdatalSAIC_ee.xls

Rank I Eqn $180868132 \ln z=a+b(\ln x)^{\wedge} 2+c x^{\wedge}(0.5)+d \ln x+e y+f y \ln y+g y^{\wedge}(0.5) \ln y+$ hy $/$ ny $r^{\wedge} 2=0.99929793$ DF Adj r^2=0.99928613 FitSidErr=2.3863999 Fstat $=96992.316$ $\mathrm{d}=5013.1051 \quad b=-0.06601026 \mathrm{c}=1.9450067 \mathrm{e}-05 \mathrm{~d}=1.4620443$ $=30619584 f=3.8499309 \mathrm{~g}=649.28179 \mathrm{~h}=-2936.169$

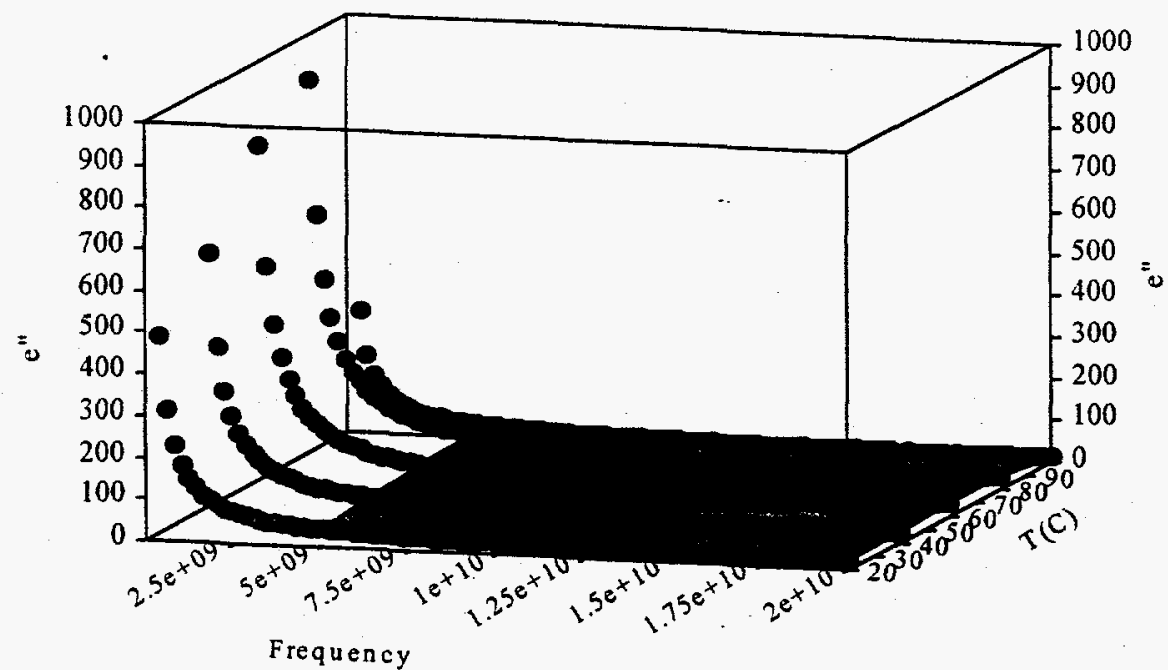

Figure C.5.c. Correlation data for the dielectric loss. Simulant: SAIC.

H:IdanlexpdatalSAIC_ee.xis

Rank 1 Eqn $180868132 \ln z=a+b(\ln x)^{\wedge} 2+c x^{\wedge}(0.5)+d \ln x+e y+f y \ln y+g y^{\wedge}(0.5) \mid \ln y+h y / \ln y$ $r^{\wedge} 2=0.99929793$ DF Adj $\kappa^{\wedge} 2=099928613$ Fis ${ }^{2}$ de $r=23863999$ Fstat=96992 315 $a=5013.1051 b=-0.06601026 c=1.9450067 \mathrm{e}-05 d=1.4620443$ $\theta=306.19584 f=-3.3499309 \mathrm{~g}=0.49 .28179 \mathrm{~h}=-2936.159$

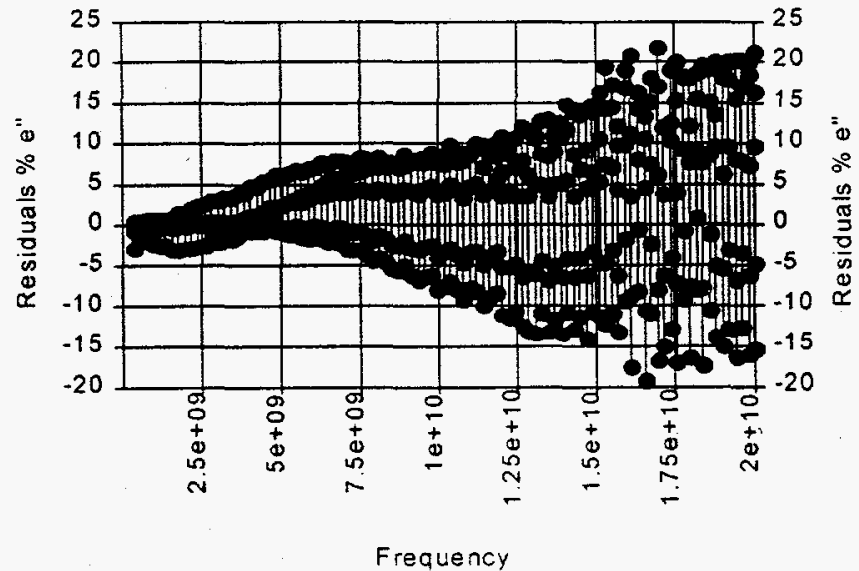

Figure C.5.d. Residual percentage (\%) for the dielectric loss. Simulant: SAIC. 
H:Idanlexpdatalexpdata02IRevORNL_e.xis

Rank 1 Eqn $312 z=a+b / x+c y+d / x^{\wedge} 2+e y^{\wedge} 2+f y / x+g / x^{\wedge} 3+h y^{\wedge} 3+i y^{\wedge} 2 / x+j y / x^{\wedge} 2$ ${ }^{\wedge} 2=0.97682831$ DF Adj $t^{\wedge} 2=0.97613523$ FitStdErr=2.5345411 Fstat $=2205.4146$ $a=29.62162 b=1.5229367 e+10 \quad c=-0.6076328 \quad d=-7.1666987 e+18 \quad \theta=0.01043381$

$i=7.2569132 \theta+08 g=9.2936058 \theta+28 \quad h=-6.0376109 \theta-05 \quad i=-7492753.4 j=9.6796559 \theta+18$

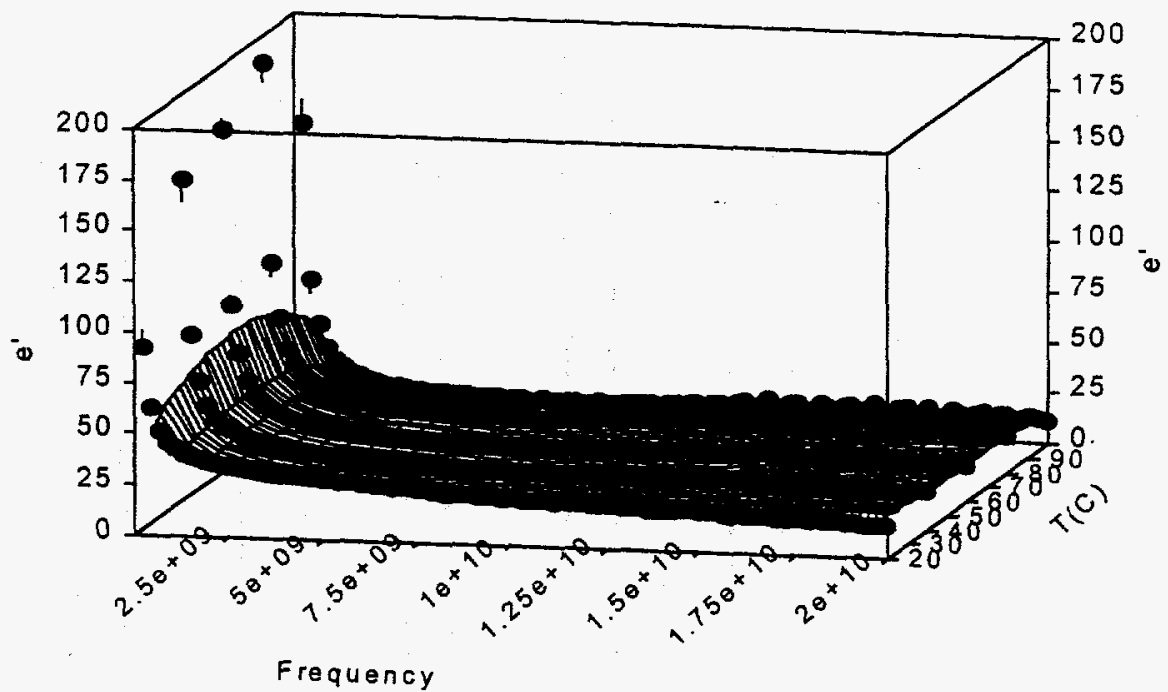

Figure C.6.a. Correlation data for the dielectric constant. Simulant: Revised ORNL.

H:Idanlexpdatalexpdata02'RevORNL_exls Rank 1 Eqn $312 z=a+b / x+c y+d / x^{\wedge} 2+e y^{\wedge} 2+f y / x+g / x^{\wedge} 3+h y^{\wedge} 3+i y^{\wedge} 2 / x+j y / x^{\wedge} 2$ $r^{\wedge} 2=097552831$ DF Adj $r^{\wedge} 2=097513523$ FitSldE $r=25345411$ Fslat $=22054145$ $a=2962162 b=1.5229367 e+10 c=-0.5075328 d=.7 .1566987 e+18 e=00104338$ : $f=7.2559132 e+08 g=9.2936058 e+25 h=-5.0375109 e-05 i=-7492753+i=96795559 e+16$

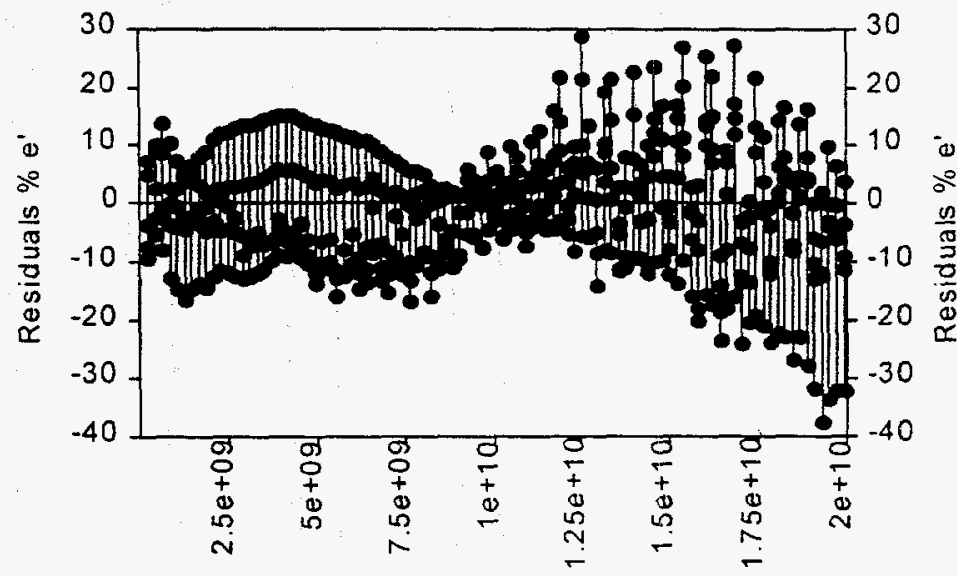

Frequency

Figure C.6.b. Residual percentage (\%) for the dielectric constant. Simulant: Revised ORNL. 


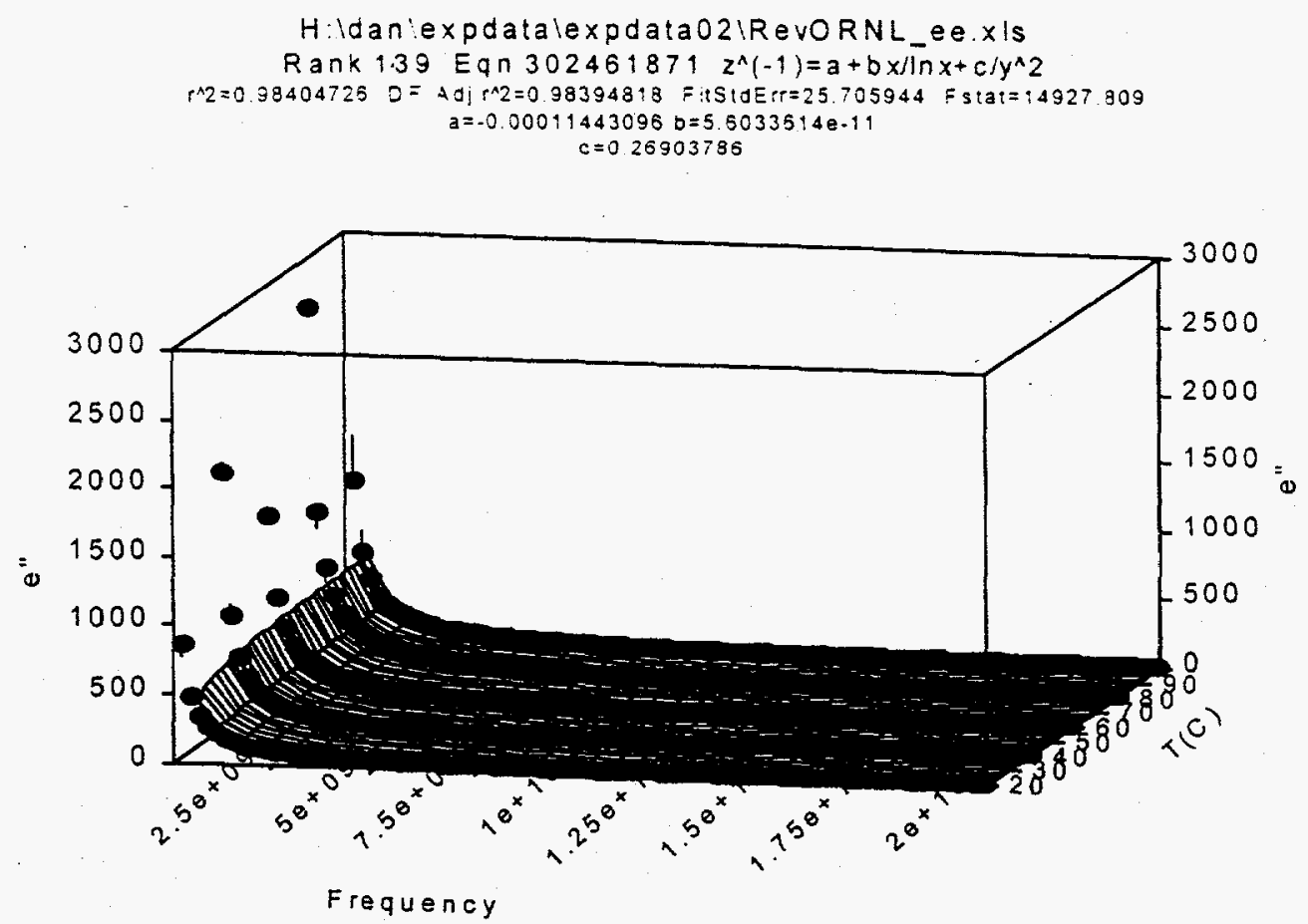

Figure C.6.c. Correlation data for the dielectric loss. Simulant: Revised ORNL.
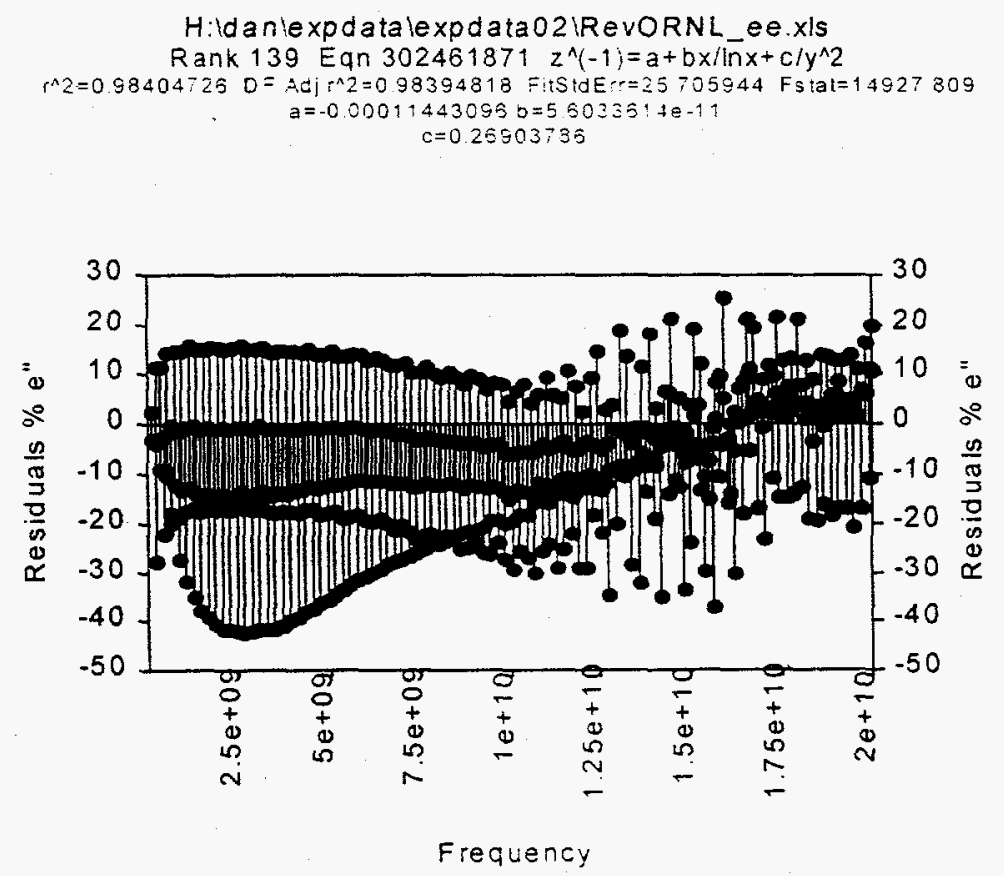

Figure C.6.d. Residual percentage (\%) for the dielectric loss. Simulant: Revised ORNL. 
H: Idanlexpdatalexpdata02lw26_e.x/s

Rank 5 Eqn $303 z=a+b / x+c y+d / x^{\wedge} 2+e y^{\wedge} 2+f y / x$

$r^{\wedge} 2=0.97906317$ DF Adj $r^{\wedge} 2=0.97880146$ FitstdErr $=6.2457247$ Fstat $=4498.5741$

$a=32.094252 b=-4.5291897 e+09 c=0.2160064$

$d=9.3061996 e+18 e=-0.0034957434 f=4.7096742 e+08$

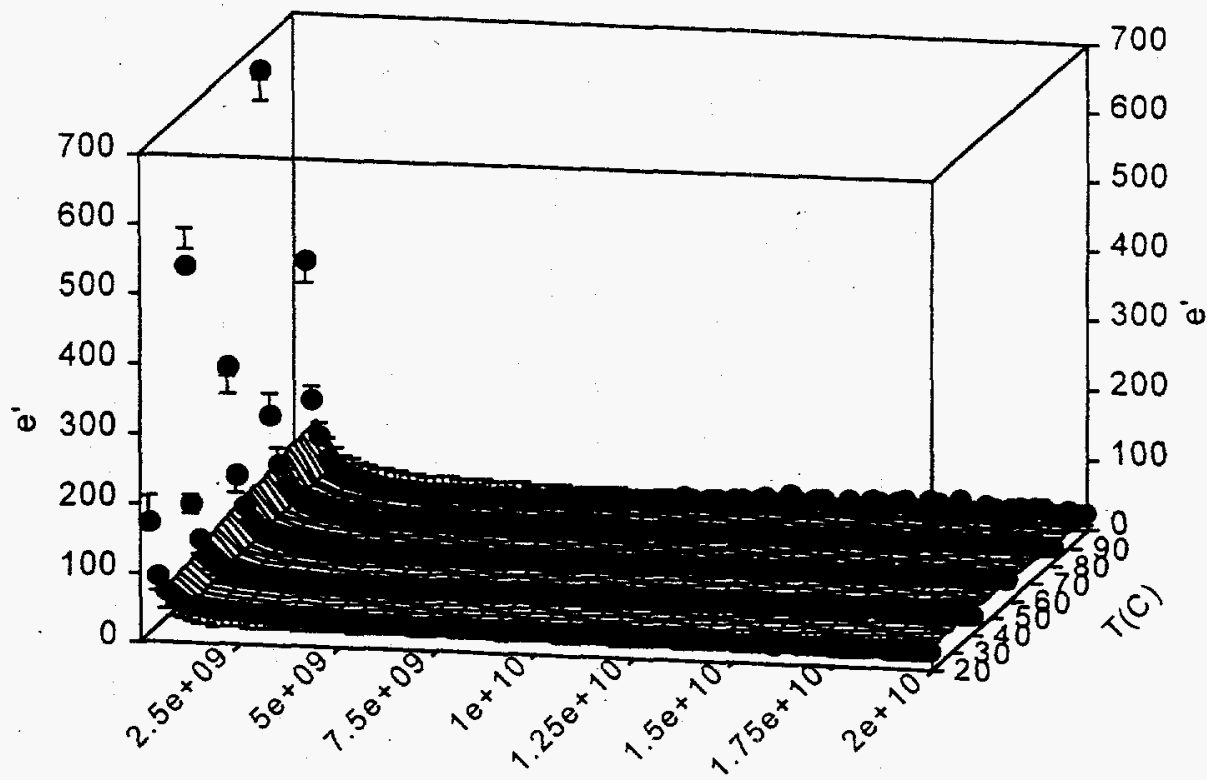

Frequency

Figure C.7.a. Correlation data for the dielectric constant: Simulant: W26 ORNL.
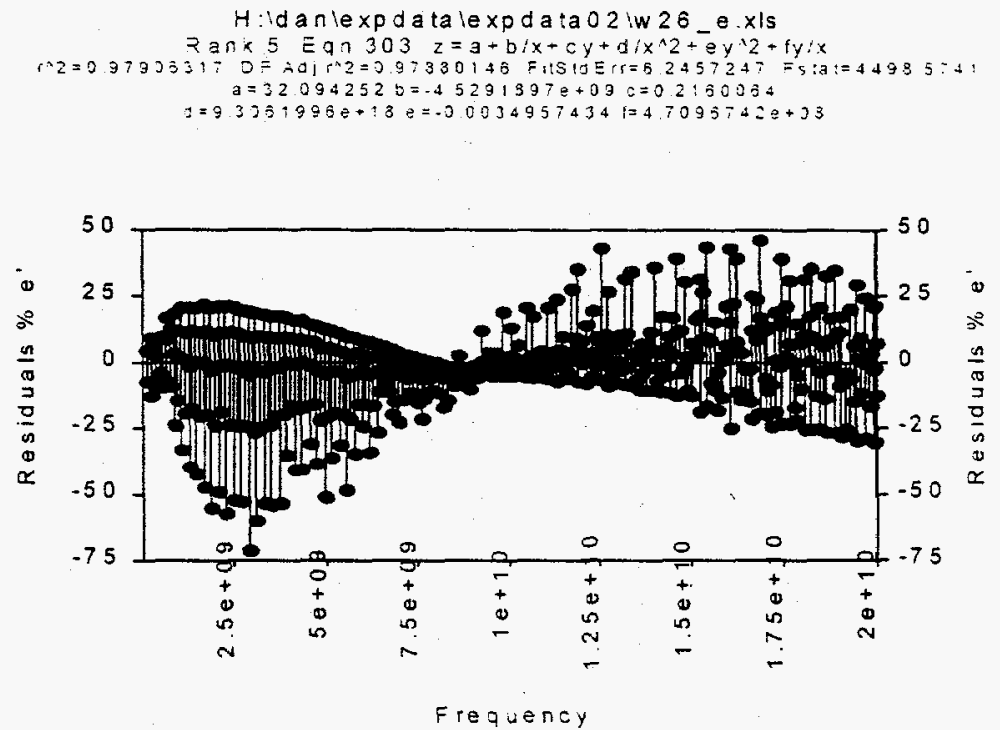

Figure C.7.b. Residual percentage (\%) for the dielectric constant. Simulant: W26 ORNL. 
$\mathrm{H}:$ Idanlexpdatalexpdata02lw26_ee.xls

Rank 1 Eqn $151232595 \ln z=a+b(\ln x)^{\wedge} 2+c \ln y$

$r^{N}=0.99879052$ DF Adj $r^{\wedge}=0.99878301$ FitStdEr $r=12.243843$ Fstat $=199844.7$ $a=14.206339 b=0.02354248$

$c=0.5741837$

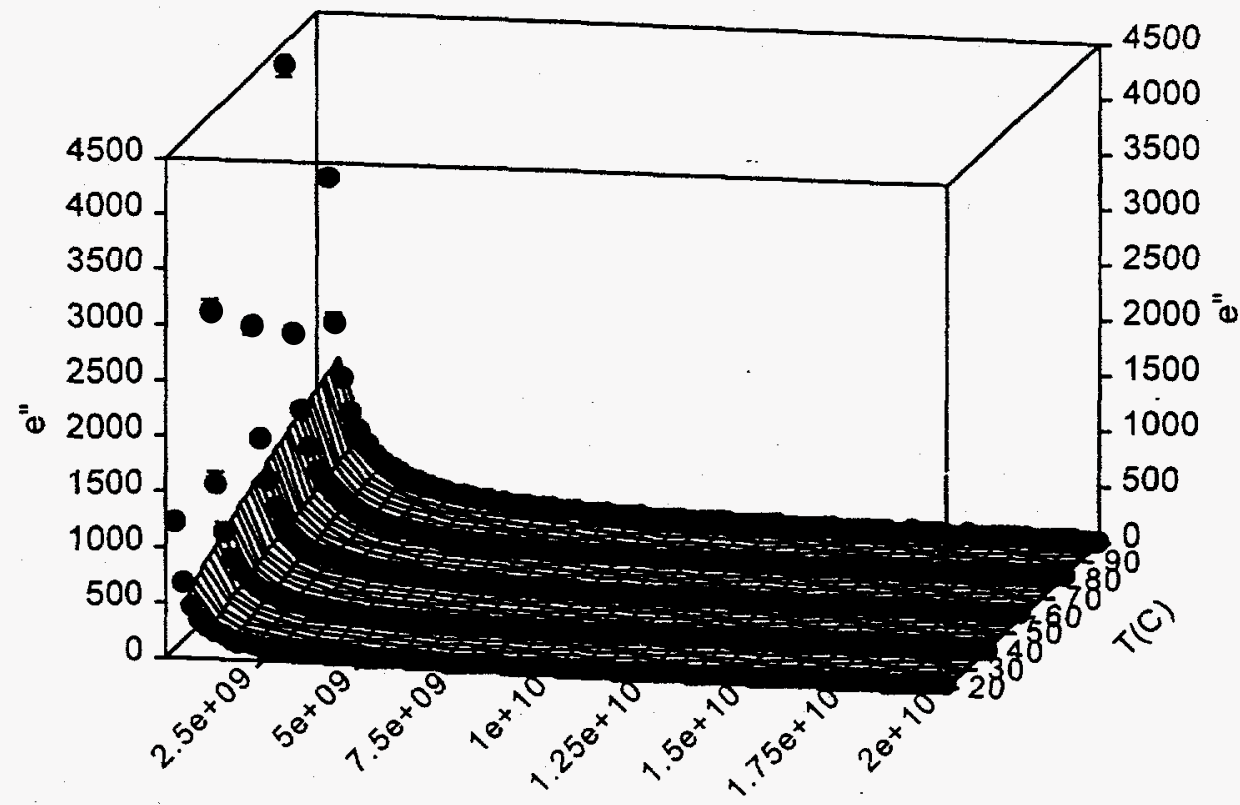

Frequency

Figure C.7.c. Correlation data for the dielectric loss. Simulant: W26 ORNL.

H:ldanlexpdatalexpdata 02 iw 26 _ee.xis

Rank 1 Eqn 151232595 lnz $=a+b(\ln x)^{\wedge} 2+c \ln y$ $r^{\wedge} 2=099879052$ DF Adj $r^{\wedge} 2=0.99878301$ FitSidErr=12 243843 Fs:at=1998447 $a=t+206339 b=-002354248$

$$
c=05741837
$$

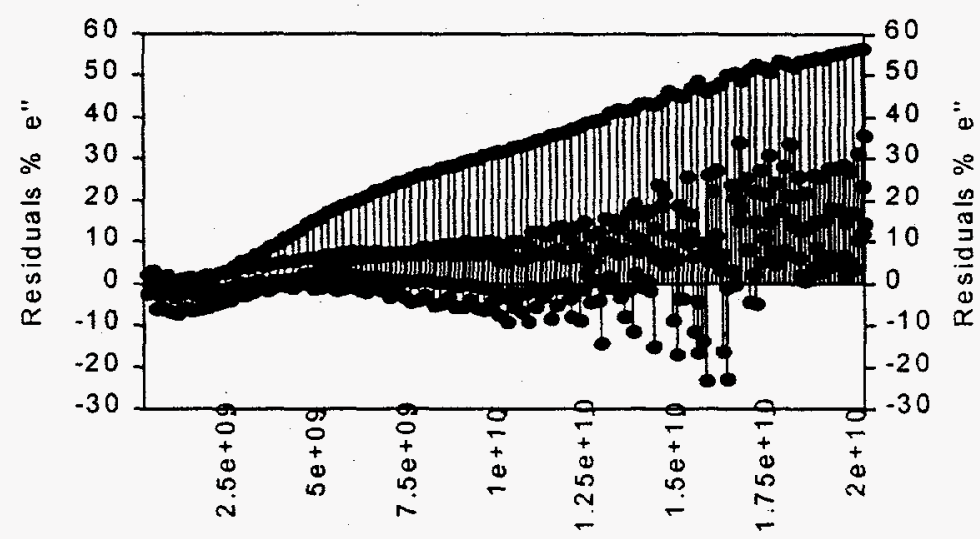

Frequency

Figure C.7.d. Residual percentage (\%) for the dielectric loss. Simulant: W26 ORNL. 
H:Idanlexpdatalexpdata021SavRiv_e.xls

Rank 1 Eqn $317 z=a+b \ln x+c / y+d(\ln x)^{\wedge} 2+e / y^{\wedge} 2+f(\ln x) / y+g(\ln x)^{\wedge} 3+h / y^{\wedge} 3+i(\ln x) / y^{\wedge} 2+j(\ln x)^{\wedge} 2 / y$ $\mathrm{r} M=0.99075073$ of $A d j \mathrm{r} M=0.99056784$ FitStdE $\pi=0.95812876$ F stat $=5719.128$ $a=13752.066 b=-1863.2924 \quad C=-39962.533 d=84.328773 \theta=-252217.15$ $f=4347.7517 g=-1.2729542 h=2115960.3 i=3082.3905 j=-104.92806$

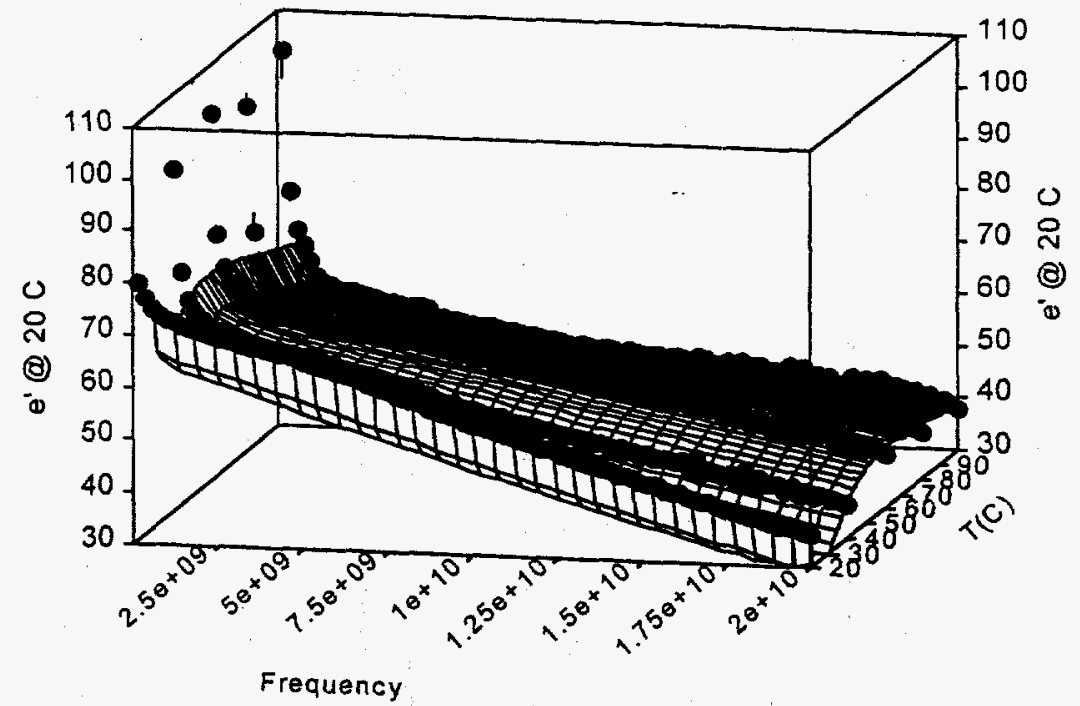

Figure C.8.a. Correlation data for the dielectric constant. Simulant: Savannah River.

H: danlexpdatalexpdata021SavRiv e.x/s

Rank 1 Eqn $317 z=a+b \ln x+c / y+d(\ln x)^{\prime 2} 2+e / y^{\prime 2} 2+f(\ln x) / y+g(\ln x)^{\prime \prime} 3+h / y^{\prime} 3+i(\ln x) / y^{\prime} 2+j(\ln x)^{\prime 2} 2 / y$ $r^{\wedge} 2=0.99076073$ DF Adj $r^{\wedge} 2=0.99056784$ FitStdE $r=0.95812876$ Fstat $=57: 9128$

$a=13752.066 b=1863.2924 c=39962.533 d=84.328773 e=252217.15$

$f=4347.7517 g=1.2729542 h=2115960.3 i=3082.3905 j=104.92806$

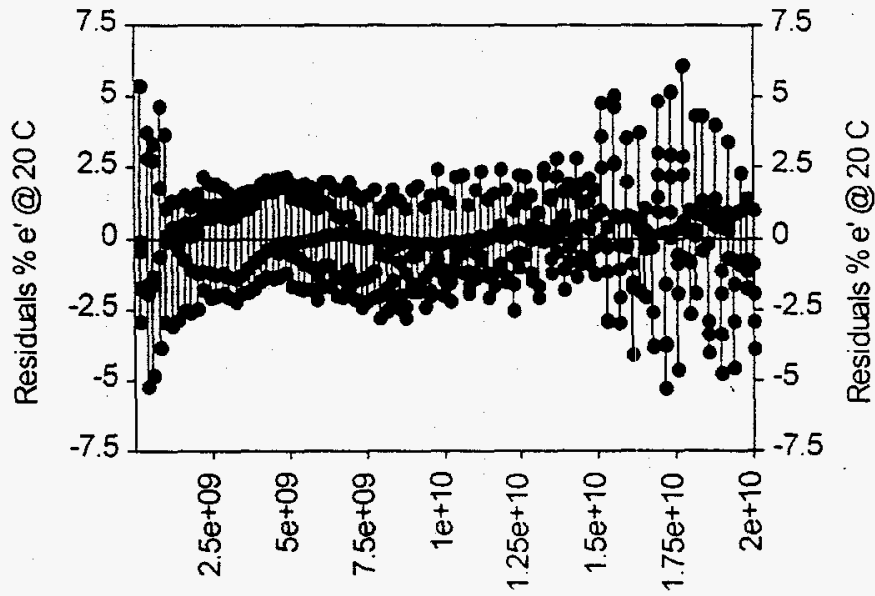

Frequency

Figure C.8.b. Residual percentage (\%) for the dielectric constant. Simulant: Sayannah River. 
H:idanlexpdatalexpdata021SavRiv_ee. $x$ Is

Rank 1 Eqn $318 \quad z=a+b / x+c / y+d / x^{\wedge} 2+e / y^{\wedge} 2+f /(x y)+g / x^{\wedge} 3+h / y^{\wedge} 3+i /\left(x y^{\wedge} 2\right)+i /\left(x^{\wedge} 2 y\right)$ $r^{M}=0.99881622$ of $A=j r^{N}=0.39879151$ FitStdErr $=3.0688293$ F stat $=45000068$ $a=0.50675443 \quad b=1.5528357 \theta+11 \quad c=2465.6128 d=1.6479615 \theta+19 \theta=.78892 .928$ $f=-5.354567 e+12 y=-1.9014567 e+27 h=838793.76 i=5.5711478 e+13 j=4.3904035 e+19$

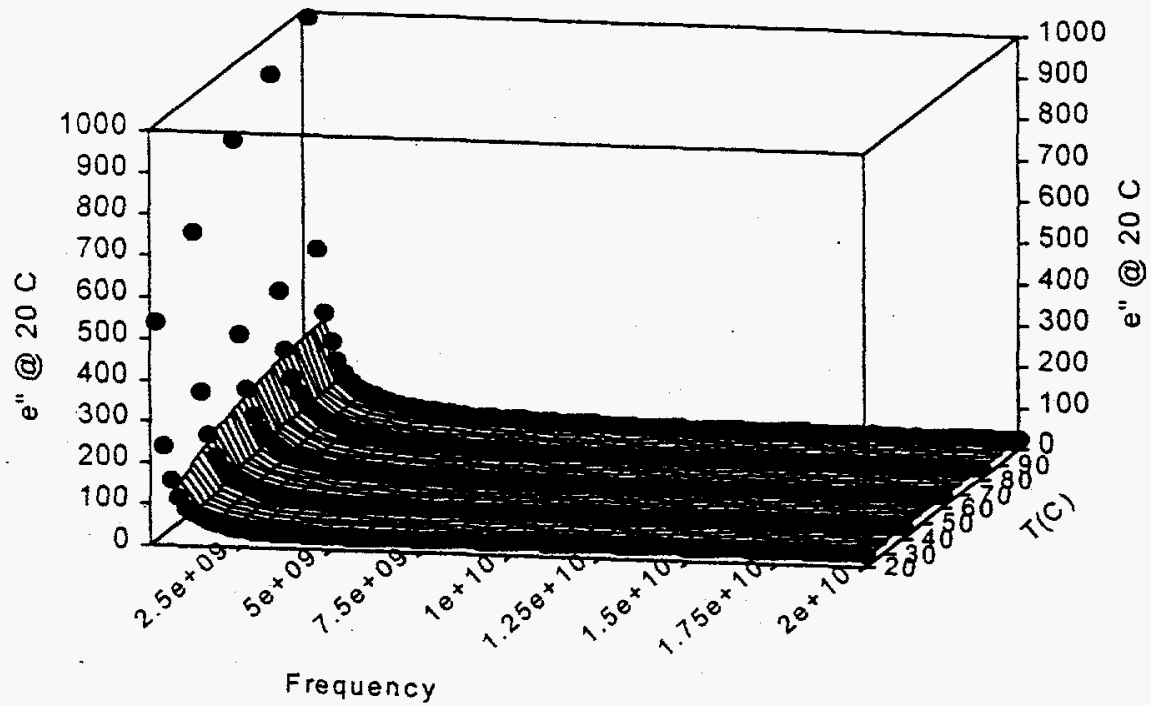

Figure C.8.c. Correlation data for the dielectric loss. Simulant: Savannah River.

H:Idanlexpdatalexpdata021SavRiv_ee.xls

Rank: Eqn $318 \quad z=a+s i x+c / y+d / x^{\wedge} 2+e / y^{\wedge} 2+f /(x y)+g / x^{\wedge} 3+h / y^{\wedge} 3+i /\left(x y^{\wedge} 2\right)+j /\left(x^{\wedge} 2 y\right)$

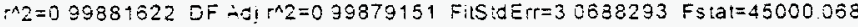

$\theta=050575443 b=1 \delta 523357 \theta+11 c=24553128 \gamma=10479515 e+19 e=-78302928$

$f=-5.354557 \mathrm{e}+12 \mathrm{~g}=-130: 4567 \mathrm{z}+27 \mathrm{n}=33879375:=5 \quad 5711473 \mathrm{z}+13 \mathrm{i}=4.3904035 \mathrm{e}+19$

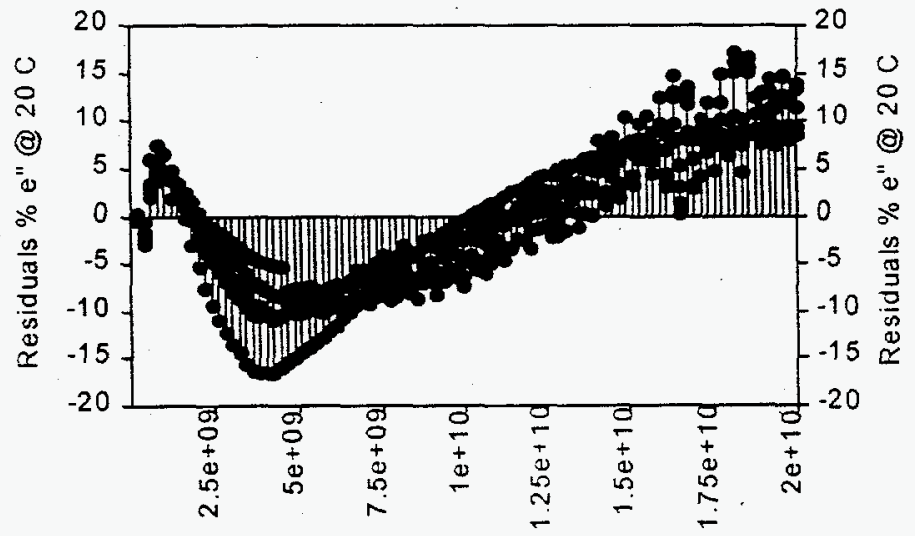

Frequency

Figure C.8.d. Residual percentage (\%) for the dielectric loss. Simulant: Savannah River. 